\title{
FLOODS OF APRIL 1979, MISSISSIPPI, ALABAMA, AND GEORGIA
}

Report prepared jointly by the U.S. Geological Survey

and the National Oceanic and Atmospheric Administration
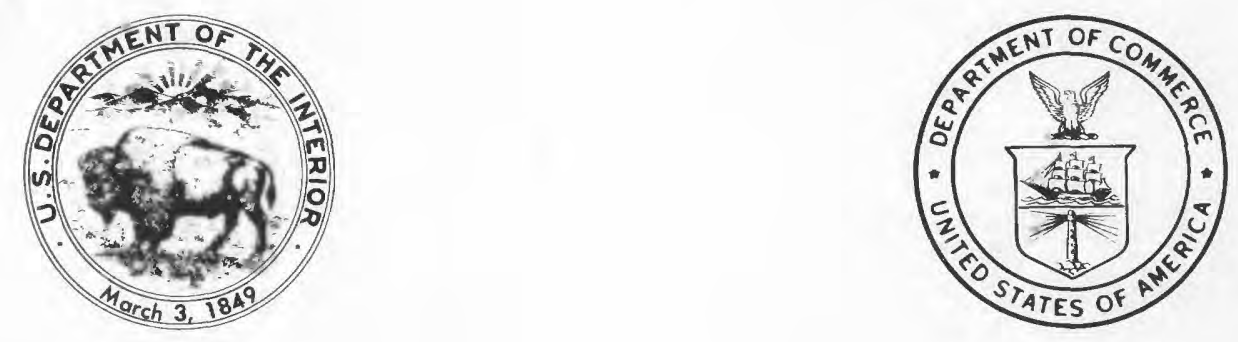
Floods of April 1979, MISSISSIPPI, ALABAMA, AND GEORGIA 


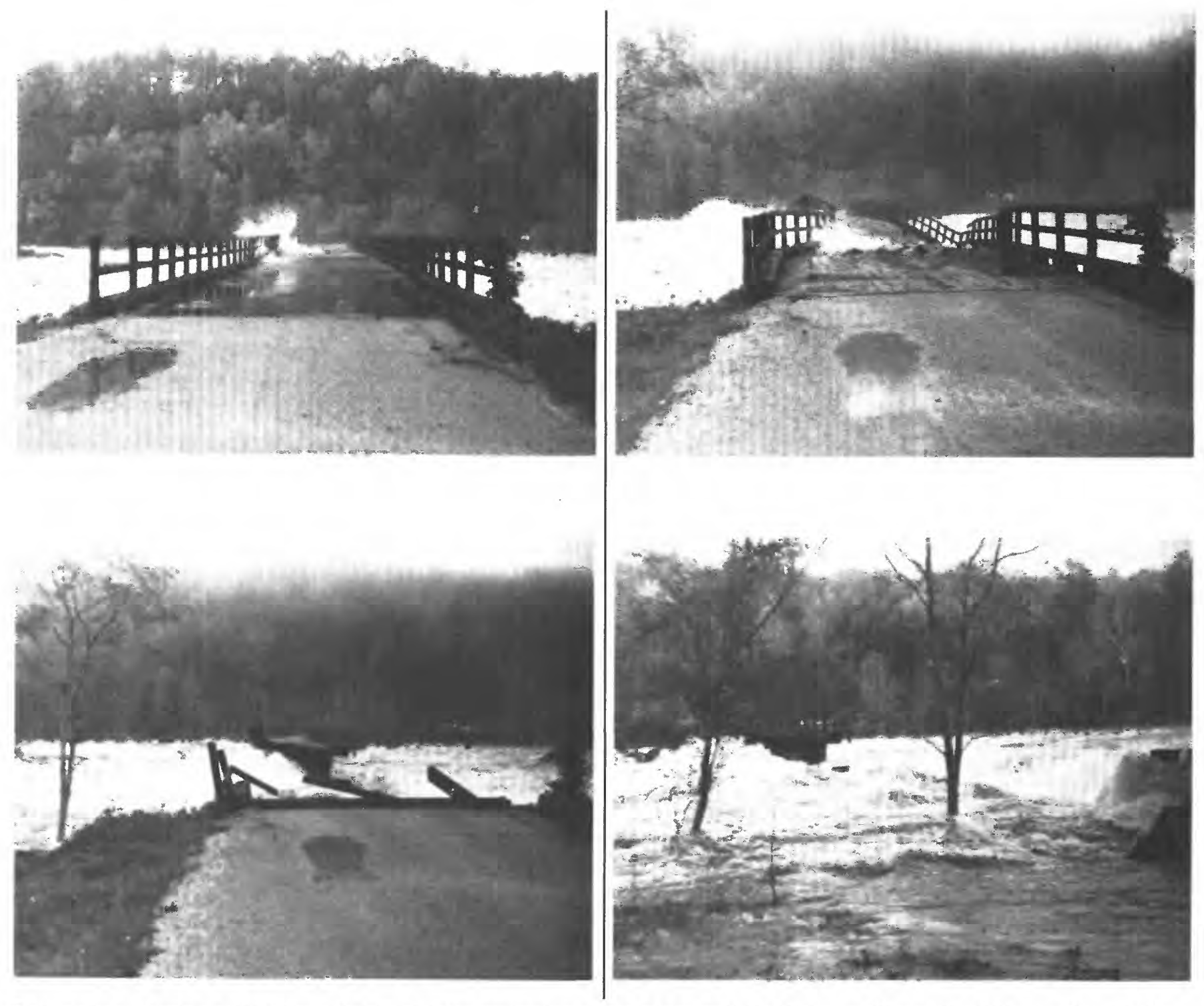

FronTISPIECE.-Sequence of photographs showing the destruction of the bridge on State Highway 50 over the Tallapoosa River below Martin Dam near Tallassee, Ala., about 1630 CST, April 14, 1979. Photographs courtesy of H. H. Weldon, Electic, Ala. 


\section{FLOODS OF APRIL 1979, MISSISSIPPI, ALABAMA, AND GEORGIA}

By GEORGE W. EDELEN, JR., K. V. WILSON, and JOE R. HARKINS, U.S. Geological Survey, and JOHN F. MILLER and EDWIN H. CHIN, National Weather Service, National Oceanic and Atmospheric Administration

U. S. GEOLOGICAL SURVEY PROFESSIONAL PAPER 1319

Report prepared jointly by the U.S. Geological Survey

and the National Oceanic and Atmospheric Administration
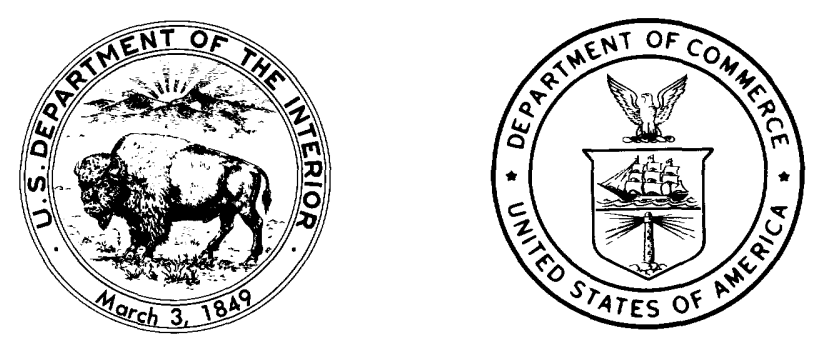

UNITED STATES GOVERNMENT PRINTING OFFICE， WASHINGTON：1986 


\author{
UNITED STATES \\ DEPARTMENT OF THE INTERIOR \\ DONALD PAUL HODEL, Secretary
}

GEOLOGICAL SURVEY

Dallas L. Peck, Director

\author{
UNITED STATES \\ DEPARTMENT OF COMMERCE \\ MALCOLM BALDRIDGE, Secretary
}

NATIONAL OCEANIC AND ATMOSPHERIC ADMINISTRATION

Anthony J. Calio, Administrator

\footnotetext{
Library of Congress Cataloging in Publication Data

Main entry under title:

Floods of April 1979, Mississippi, Alabama, and Georgia.

(Geological Survey professional paper ; 1319)

"Report prepared jointly by the U.S. Geological Survey and the National Oceanic and Atmospheric Administration."

Bibliography: p. $\mathbf{4 5}$

1. Floods-Mississippi. 2. Floods-Alabama. 3. Floods-Georgia. I. Edelen, George W. II. Geological Survey (U.S.) III. United States. National Oceanic and Atmospheric Administration. IV. Series. GB1399.4.M7F55 $1984 \quad 551.48^{\prime} 0976 \quad 84-600097$
}

For sale by the Books and Open-File Reports Section, U.S. Geological Survey, Federal Center, Box 25425, Denver, CO 80225 


\section{CONTENTS}

\begin{tabular}{|c|c|c|c|}
\hline & Page & & \\
\hline - & VIII & Reservoirs & \\
\hline Abstract - - & 1 & Major river basins of eastern Gulf of Mexico -- & \\
\hline Introduction - - & 1 & Coosa River basin - & \\
\hline Acknowledgments - & 3 & Tallapoosa River basin - & \\
\hline Meteorological setting - & 4 & Alabama River basin - & \\
\hline Antecedent conditions & 4 & Tombigbee River basin - & \\
\hline Early spring storms & 4 & Tombigbee River upstream from Gainesville, Ala. ---- & \\
\hline March 3-4 & 4 & Tombigbee River downstream from Gainesville, Ala.-- & \\
\hline March $10-11,14$, and 21 & 5 & Pascagoula River basin - & \\
\hline March 23-24 - & 5 & Pearl River basin - & \\
\hline April 1-4 - & 7 & Lower Mississippi River basin - & \\
\hline April 8-9 & 9 & n - & \\
\hline Major storm event: April 11-13 & 11 & - & \\
\hline res - & 11 & 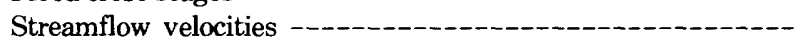 & \\
\hline features - & 11 & $k$ discharges -- - & \\
\hline eather features - & 14 & h bridge openings -- & \\
\hline y - - & 15 & -_- & \\
\hline stribution - - - & 17 & 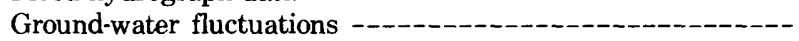 & \\
\hline loods - & 19 & lls - - - - - - - - - & \\
\hline floods -- & 23 & Salinity and temperature data, Mobile Bay and Gulf of Mexico - & \\
\hline damages - & 23 & Aerial photography & \\
\hline Flood frequency & 24 & Selected references - & \\
\hline
\end{tabular}

\section{ILLUSTRATIONS}

Frontispiece. Sequence of photographs showing the destruction of the bridge on State Highway 50 over the Tallapoosa River below Martin Dam near Tallassee, Ala., about 4:30 p.m., April 14, 1979.

1. Area affected by floods on the Alabama, Big Black, Chattahoochee, Chickasawhay, Coosa, Pearl, and Tombigbee Rivers and their tributaries in April 1979

2. Significant meteorological features associated with the storm of March 3-4, 1979

3. Isohyetal analysis of storm rainfall March 3-4, 1979

4. Significant meteorological features associated with the storm of March 23

5. Isohyetal anetysis of storm rainfall, March 23-24, 1979

5. Isohyetal analysis of storm rainfall, March 23-24, 1979 - Significant meteorological features associated with the storm of April 1-4, 1979

7. Isohyetal analysis of storm rainfall, April 1-4, 1979

8. 500-mb analyses:

A. 0600 CST, April 11, 1979

B. 1800 CST, April 11, 1979

C. 0600 CST, April 12, 1979

D. 1800 CST, April 12, 1979

E. 0600 CST, April 13, 1979

9. 850-mb analyses:

F. 1800 CST, April 13, 1979

A. 0600 CST, April 11, 1979

B. 1800 CST, April 11, 1979

C. 0600 CST, April 12, 1979

D. 1800 CST, April 12, 1979

E. 0600 CST, April 13, 1979

F. 1800 CST, April 13, 1979 
Figure 10. Surface analyses: Page

A. 0600 CST, April 11, 1979

B. 1800 CST, April 11, 1979 -

C. 0600 CST, April 12, 1979 - 18

D. 1800 CST, April 12, 1979 -

E. 0600 CST, April 13, 1979 -

F. 1800 CST, April 13, 1979

11. Analysis of hourly observations at 1500 CST, April 12, 1979

12. GOES visual image for 0730 CST, April 12, 1979, with major features of surface weather map superimposed --

13. Enhanced GOES infrared images (Mb curve):

A. 0000 CST, April 12, 1979

B. 0230 CST, April 12, 1979

14. Radar summary map:

A. 0535 CST, April 12, 1979

B. 1435 CST, April 12, 1979 -

15. Rawinsonde plot, 1800 CST, April 12, 1979:

A. Jackson, Miss. -

B. Centreville, Ala. -..-

16. Isohyetal analysis of storm rainfall, April 11-14, 1979 -

17. Rainfall mass curves - -

18. Map showing location of flood determination sites -

19. Comparison of April 1979 peak discharges with maximum known flood peaks in Mississippi and Alabama -................

20-23. Hydrographs of discharge at selected gaging stations:

20. Coosa River basin in Alabama, April 1-16, 1979

21. Tallapoosa River basin in Alabama, April 3-22, 1979

22. Cahaba River basin in Alabama, April 10-22, 1979

23. Noxubee River at Macon, Miss., and near Geiger, Ala., and Sucarnoochee River at Livingston, Ala., April 2-23, 1979 -

24. Photograph showing overflow of Tombigbee River at Demopolis, Ala., April 19, 1979 -

25-27. Hydrographs of discharge at selected gaging stations:

25. Valley Creek in the Black Warrior River basin in Alabama, April 2-21, 1979

26. North River and Black Warrior River in Alabama, April 2-21, 1979

27. Pearl River basin at and upstream from Jackson, Miss., March 2 to April 28, 1979

28-31. Photographs showing:

28. Housing development in flooded area along Hanging Moss Creek in northern part of Jackson, Miss., April 16, 1979 -

29. Inundated residential area in the vicinity of Westbrook Road in northern part of Jackson, Miss., April 16, 1979 --

30. Business district of Jackson, Miss., inundated by Pearl River overflow, April 16, 1979

31. Flooded fairgrounds enclosed by levee, Jackson, Miss., near crest of Pearl River flood, April 16, 1979 -.....- 38

32, 33. Hydrographs of discharge at selected gaging stations:

32. Pearl River near Monticello, Miss., and near Bogalusa, La., April 1-30, 1979

33. Big Black River at West, Miss., and near Bovina, Miss., April 1-30, 1979

34. Graph showing changes in point velocity, mean velocity, stage, and discharge of Alabama River near Montgomery,

Ala., April 13-20, 1979 -_.

35-43. Graphs showing velocity distribution and cross sections:

35. Alabama River at U.S. Highway 31 north, near Montgomery, Ala., April 15, 1979

36. Mulberry Creek at highway bridge at Jones, Ala., April 14, 1979

37. Hashuqua Creek near Macon, Miss., April 12, 1979

38. Noxubee River at U.S. Highway 45 bypass near Macon, Miss., April 14, 1979

39. Noxubee River at State Highway 17 near Geiger, Ala., April 15, 1979

40. Tombigbee River at Gainesville, Ala. (main channel), April 15, 1979

41. North River near Samantha, Ala., April 13, 1979

42. Pearl River at Interstate Highway 55 at Jackson, Miss., A pril 17, 1979

43. Zilpha Creek at State Highway 35 near Kosciusko, Miss., April 12, 1979

44, 45. Hydrographs of water levels in observation wells:

44. At Centreville, Ala. (Centreville Gin and Cotton Co.), March-April 1979

45. Near Pickensville, Ala., in the Tombigbee River basin, March-April 1979 -_-

46. Map showing location of specific-conductance sampling sites along the Intracoastal Waterway at the mouth of Mobile Bay, April 28-29, 1979 -_-

47. Map showing location of flight lines along streams where aerial photographs were obtained on or near the crest of the flood, April 1979 


\section{TABLES}

[All tables appear at end of report]

TABLE 1. Supplementary rainfall data, storm of April 11-13, 1979

2. Summary of flood stages and discharges -

3. Summary of flood damages on main streams and principal tributaries, March 1979 and April 1979 floods --_- 67

4. Summary of stages and contents of storage reservoirs -

5. Flood-crest stages -

6. Streamflow velocities, Alabama River near Montgomery, Ala., April 12-20, 1979

7. Gage height, discharge, and accumulated runoff, flood of April 1979

8. Ground-water levels in selected observation wells in Alabama and Mississippi, April 1979

9. Specific conductance and temperature of samples at selected sites along the Intracoastal Waterway at the mouth of Mobile Bay, April 28-29, 1979 -_

10. Aerial photographs obtained at or near the crest of the flood, April 1979

\section{CONVERSION OF INCH-POUND UNITS TO INTERNATIONAL SYSTEM OF UNITS (SI)}

Most units of measure used in this report are inch-pound units. The following factors may be used to convert inch-pound units to the International System of Units (SI).

\begin{tabular}{|c|c|c|c|c|c|}
\hline Inch-pound & to & $S I$ & $S I$ & to & Inch-pound \\
\hline \multicolumn{6}{|c|}{ Length } \\
\hline inch (in.) & $=$ & $25.4 \mathrm{~mm}$ & millimeter $(\mathrm{mm})$ & $=$ & 0.03937 in. \\
\hline foot $(\mathrm{ft})$ & $=$ & $0.3048 \mathrm{~m}$ & meter $(\mathrm{m})$ & $=$ & $3.2808 \mathrm{ft}$ \\
\hline mile (mi) & $=$ & $1.6093 \mathrm{~km}$ & kilometer $(\mathrm{km})$ & $=$ & $0.6214 \mathrm{mi}$ \\
\hline \multicolumn{6}{|c|}{ Area } \\
\hline square mile (mi $\left.{ }^{2}\right)$ & $=$ & $2.5900 \mathrm{~km}^{2}$ & square kilometer $\left(\mathrm{km}^{2}\right)$ & $=$ & $0.3861 \mathrm{mi}^{2}$ \\
\hline acre & $=$ & $4046.86 \mathrm{~m}^{2}$ & square meter $\left(\mathrm{m}^{2}\right)$ & $=$ & 0.000247 acre \\
\hline \multicolumn{6}{|c|}{ Volume } \\
\hline cubic foot $\left(\mathrm{ft}^{3}\right)$ & $=$ & $0.0283 \mathrm{~m}^{3}$ & cubic meter $\left(\mathrm{m}^{3}\right)$ & $=$ & $35.3147 \mathrm{ft}^{3}$ \\
\hline acre-foot (acre-ft) & $=$ & $1233 \mathrm{~m}^{3}$ & $\mathrm{~m}^{3}$ & $=$ & 0.00081 acre-ft \\
\hline \multicolumn{6}{|c|}{ Velocity } \\
\hline mile per hour (mph) & $=$ & $1.6093 \mathrm{~km} / \mathrm{h}$ & kilometer per hour $(\mathrm{km} / \mathrm{h})$ & $=$ & $0.6214 \mathrm{mph}$ \\
\hline foot per second $(\mathrm{ft} / \mathrm{s})$ & $=$ & $0.3048 \mathrm{~m} / \mathrm{s}$ & meter per second $(\mathrm{m} / \mathrm{s})$ & $=$ & $3.2808 \mathrm{ft} / \mathrm{s}$ \\
\hline \multicolumn{6}{|c|}{ Flow rate } \\
\hline cubic foot per second $\left(\mathrm{ft}^{3} / \mathrm{s}\right)$ & $=$ & $0.02832 \mathrm{~m}^{3} / \mathrm{s}$ & cubic meter per second $\left(\mathrm{m}^{3} / \mathrm{s}\right)$ & $=$ & $35.3147 \mathrm{ft}^{3} / \mathrm{s}$ \\
\hline$\left(\mathrm{ft}^{3} / \mathrm{s}\right) / \mathrm{mi}^{2}$ & $=$ & $0.01094\left(\mathrm{~m}^{3} / \mathrm{s}\right) / \mathrm{km}^{2}$ & $\left(\mathrm{~m}^{3} / \mathrm{s}\right) / \mathrm{km}^{2}$ & $=$ & $91.40768\left(\mathrm{ft}^{3} / \mathrm{s}\right) / \mathrm{mi}^{2}$ \\
\hline \multicolumn{6}{|c|}{ Pressure } \\
\hline [The National Weather Service us & . & ) as customary unit for & ospheric pressure.] & & \\
\hline inch of mercury at $32^{\circ} \mathrm{F}$ (in. $\mathrm{Hg}$ ) & $=$ & $33.8639 \mathrm{mb}$ & $\mathrm{mb}$ & $=$ & 0.02953 in. $\mathrm{Hg}$ \\
\hline \multicolumn{6}{|c|}{ Temperature } \\
\hline degrees Fahrenheit $\left({ }^{\circ} \mathrm{F}\right)$ & $=$ & $9 / 5\left({ }^{\circ} \mathrm{C}\right)+32$ & degrees Celsius $\left({ }^{\circ} \mathrm{C}\right)$ & $=$ & $5 / 9\left({ }^{\circ} \mathrm{F}-32\right)$ \\
\hline
\end{tabular}




\section{GLOSSARY}

Acre-foot (acre-ft). The volume of water required to cover 1 acre to a depth of $1 \mathrm{ft}$. It equals $43,560 \mathrm{ft}^{3}$ (cubic feet), $325,851 \mathrm{gal}$ (gallons), or $1,233 \mathrm{~m}^{3}$ (cubic meters).

Aquifer. A water-bearing formation.

Contents. The volume of water in a reservoir or lake. Content is computed on the basis of a level pool or reservoir backwater profile and does not include bank storage.

Cubic feet per second $\left(\mathrm{ft}^{3} / \mathrm{s}\right)$. A rate of discharge. One cubic foot per second is equal to the discharge of a stream of rectangular cross sec $1 \mathrm{ft}$ wide and $1 \mathrm{ft}$ deep, flowing at an average velocity of $1 \mathrm{ft} / \mathrm{s}$. It equals $28.32 \mathrm{~L} / \mathrm{s}$ (liters per second) or $0.02832 \mathrm{~m}^{3} / \mathrm{s}$ (cubic meters per second).

Cubic feet per second per square mile $\left[\left(\mathrm{ft}^{3} / \mathrm{s}\right) / \mathrm{mi}^{2}\right]$. The average number of cubic feet per second flowing from each square mile of area drained by a stream, assuming that the runoff is distributed uniformly in time and area. One $\left(\mathrm{ft}^{3} / \mathrm{s}\right) / \mathrm{mi}^{2}$ is equivalent to $0.0733\left(\mathrm{~m}^{3} / \mathrm{s}\right) / \mathrm{km}^{2}$ (cubic meters per second per square kilometer).

Cyclone. An atmospheric low-pressure system around which the wind blows in a counterclockwise direction in the Northern Hemisphere and clockwise in the Southern Hemisphere.

Dewpoint (or dewpoint temperature). The temperature to which a given parcel of air must be cooled at constant pressure and constant water-vapor content in order for saturation to occur.

Drainage area of a stream at a specific location. The area measured in a horizontal plane, bounded by topographic divides. Drainage area is given in square miles. One square mile is equivalent to $2.590 \mathrm{~km}^{2}$ (square kilometers).

Fall line. A narrow zone between resistant rocks and the softer formations of the coastal plain, characterized by steepened gradients and by waterfalls, locally.

Flood. Any high streamflow that overtops natural or artificial banks of a stream and overflows onto land not usually under water or ponding caused by precipitation at or near the point it fell.

Flood peak. The highest value of the stage or discharge attained by a flood.

Flood profile. A graph of the elevation of water surface of a river in a flood-plotted as ordinate; against distance-plotted as abscissa.

Flood stage. The approximate elevation of the stream when overbank flooding begins.

Front. The interface or transition zone between two airmasses of different density.

Gage height. The water-surface elevation referred to some arbitrary gage datum.

Gaging station. A particular site on a stream, canal, lake, or reservoir where systematic observations of gage height or discharge are made.

GOES. Geostationary Operational Environmental Satellite.

GMT. Greenwich mean time.

High. A center of high barometric pressure.

Hydrograph. A graph showing stage, flow, velocity, or ground-water level of water, with respect to time.

Instability. Areas of instability as referred to in this report are areas where the lifted index is less than four.

Isohyet. A line connecting points of equal precipitation.
$K$ index. A measure of the airmass moisture content and stability. $\mathrm{K}=\left(\mathrm{T}_{850}-\mathrm{T}_{500}\right)+\mathrm{T}_{\mathrm{d}, 850}-\left(\mathrm{T}_{700}-\mathrm{T}_{\mathrm{d}, 700}\right)$, where $\mathrm{T}$ and $\mathrm{T}_{\mathrm{d}}$ are temperature and dewpoint, respectively, in degrees Celsius $\left({ }^{\circ} \mathrm{C}\right.$ ); subscripts denote pressure levels.

Knot. A velocity of 1 nautical mile per hour.

Lifted index. Difference in degrees Celsius between the observed 500-millibar (mb) temperature and the computed temperature a parcel characterized by the mean temperature and dewpoint of the 50 -mb-thick surface layer would have if it were lifted from $25 \mathrm{mb}$ above the surface to $500 \mathrm{mb}$.

Low. Center of low barometric pressure.

Millibar (mb). A unit of pressure equal to 1,000 dynes per square centimeter.

National Geodetic Vertical Datum (NGVD). Formerly called Sea Level Datum of 1929. A geodetic datum derived from a general adjustment of the first order level nets of both the United States and Canada. In the adjustment, sea levels from selected tide stations in both countries were held as fixed. The year indicates the time of the last general adjustment. This datum should not be confused with mean sea level.

Nautical mile. A distance of $6,080.20$ feet $(1.853 \mathrm{~km})$.

Occluded front (occlusion). A composite of two fronts, formed as a cold front overtakes a warm front or a quasi-stationary front. This is a common process in the late stages of cyclone development.

Planck's law. One of the fundamental laws of physics that gives the intensity of radiation emission at a specific wavelength as a function of the temperature of a black-body.

Precipitable water. The amount of water contained in an atmospheric column if all the vapor between two levels (usually the surface and $500 \mathrm{mb}$ ) were condensed.

Radiosonde. A balloon-borne instrument package for measuring and transmitting meteorological data.

Rawinsonde. A meteorological data-collection system including a radiosonde and reflectors for measuring winds by radar.

Recurrence interval. As applied to flood events, the average number of years within which a given flood peak will be exceeded once.

Runoff. That part of the precipitation that appears in surface streams.

Sounding. A single complete radiosonde observation of the upper atmosphere.

Specific conductance. The measure of the ability of water to carry an electric current and, therefore, an indication, within rather wide limits, of the dissolved-solids concentration or salinity of a solution. Specific conductance is expressed in mhos/centimeter. In most waters, conductance is so low that micromho is used as the unit of expression.

Stage-discharge relation. The relation between gage height and the amount of water flowing in a stream channel.

Time of day is expressed in 24-hour time. For example, 12:30 a.m. is 0030 hours, and 1:00 p.m. is 1300 hours. Central standard time (CST) is used throughout this report unless stated otherwise.

Trough. An elongated area of relatively low atmospheric pressure. 


\title{
FLOODS OF APRIL 1979, MISSISSIPPI, ALABAMA, AND GEORGIA
}

\author{
By GeORge W. EDELEN, JR., K. V. WILSON, and \\ JOE R. HARKINS, of the U.S. GEOLOGICAL SURVEY \\ and JoHn F. Miller and EDWin H. Chin of the NAtional Weather Service, \\ NATIONAL OCEANIC and ATMOSPHERIC ADMINISTRATION
}

\section{ABSTRACT}

A major storm brought large amounts of rainfall over the southeastern United States April 11-13. 1979. Heaviest rain fell over north-central Mississippi and Alabama. Although the storm extended into the headwaters of the Chattahoochee River basin in northwestern Georgia, most flooding there was only moderate. A maximum of 21.5 inches was observed at a site 14 miles southeast of Louisville, Miss. Areal average rainfall exceeded 12 and 8 inches over the upper Pearl and upper Tombigbee River basins, respectively. Owing to a series of antecedent storms in March and April over the MississippiAlabama area, soils were saturated and many rivers were already bankfull. Additional rains April 21-23 in Mississippi and April 24-26 in Alabama averaged less than 2 inches over the flooded area. A maximum of 6.4 inches was reported at Ruth, Miss., about 65 miles south of Jackson, where little or no rain fell during the major storm of April 11-13.

Floods in Mississippi and Alabama caused by the series of storms were the maximum of record at 60 streamflow gaging stations in the Coosa, Alabama, Tombigbee, Chickasawhay, Pearl, and Big Black River basins.

On the Pearl River, peak discharges at main stem gaging stations generally approached or exceeded those of the great flood of 1874 , and recurrence intervals generally were greater than 100 years.

On some streams, maximum stages and discharges produced by the March 3-4 storm, although greater than those previously observed, were exceeded during the April 11-13 storm. Other storms in April extended the flood duration and added materially to the flood volume.

A comparison with the greatest known floods indicates that floods generally one-third greater than those in 1979 may occur in large basins and that floods two or three times greater may occur in small basins. Floods much greater than those observed in April 1979 or than the greatest known floods in the area are likely to occur if the probable maximum precipitation occurs.

Nine lives were reported lost. Estimated damages from the March and April flooding totaled nearly $\$ 400$ million. During April 1979, 75 percent of the total damage occurred in the Pearl River Basin, and 65 percent of the damage occurred in Jackson, Miss., and vicinity. Seventeen thousand people were driven from their homes in Jackson, Miss.
The report presents analyses of the meteorological settings of the storms, the distribution of rainfall, and supplementary rainfall data that have not been published elsewhere. It also gives summaries of flood stages and discharges at 221 streamflow gaging stations, stages and contents of 10 reservoirs, flood-crest stages and hydrograph data (gage height, discharge, and accumulated runoff at selected times) at 46 gaging stations, ground-water fluctuations in 11 observation wells, and water salinity and temperature at 22 sites along the Intracoastal Waterway in Mobile Bay. The availability of aerial photography obtained during the flood is summarized, and flood damages are discussed.

\section{INTRODUCTION}

During March and April 1979, a series of storms in Mississippi and Alabama resulted in recordbreaking floods on streams in the Coosa, Alabama, Tombigbee, Chickasawhay, Pearl, and Big Black River basins. The first storm, March 3-4, produced maximum stages and discharges greater than previously observed on several streams in the Coosa, Tombigbee, and Chickasawhay River basins. Some of these floods were exceeded during the major storm of the series, April 11-13, which produced most of the recordbreaking floods.

The storms of March 23-24 and April 1-4 produced bankfull stages on many streams.

A major rainstorm occurred April 11-13, 1979, over the Southeastern United States. Rain fell over large areas of Mississippi, Alabama, Georgia, South Carolina, Tennessee, and Arkansas. However, the heaviest rain fell in central and northern Mississippi and Alabama (henceforth referred to as "the two-State region" or "the region"), over the Tombigbee and Pearl River basins. 


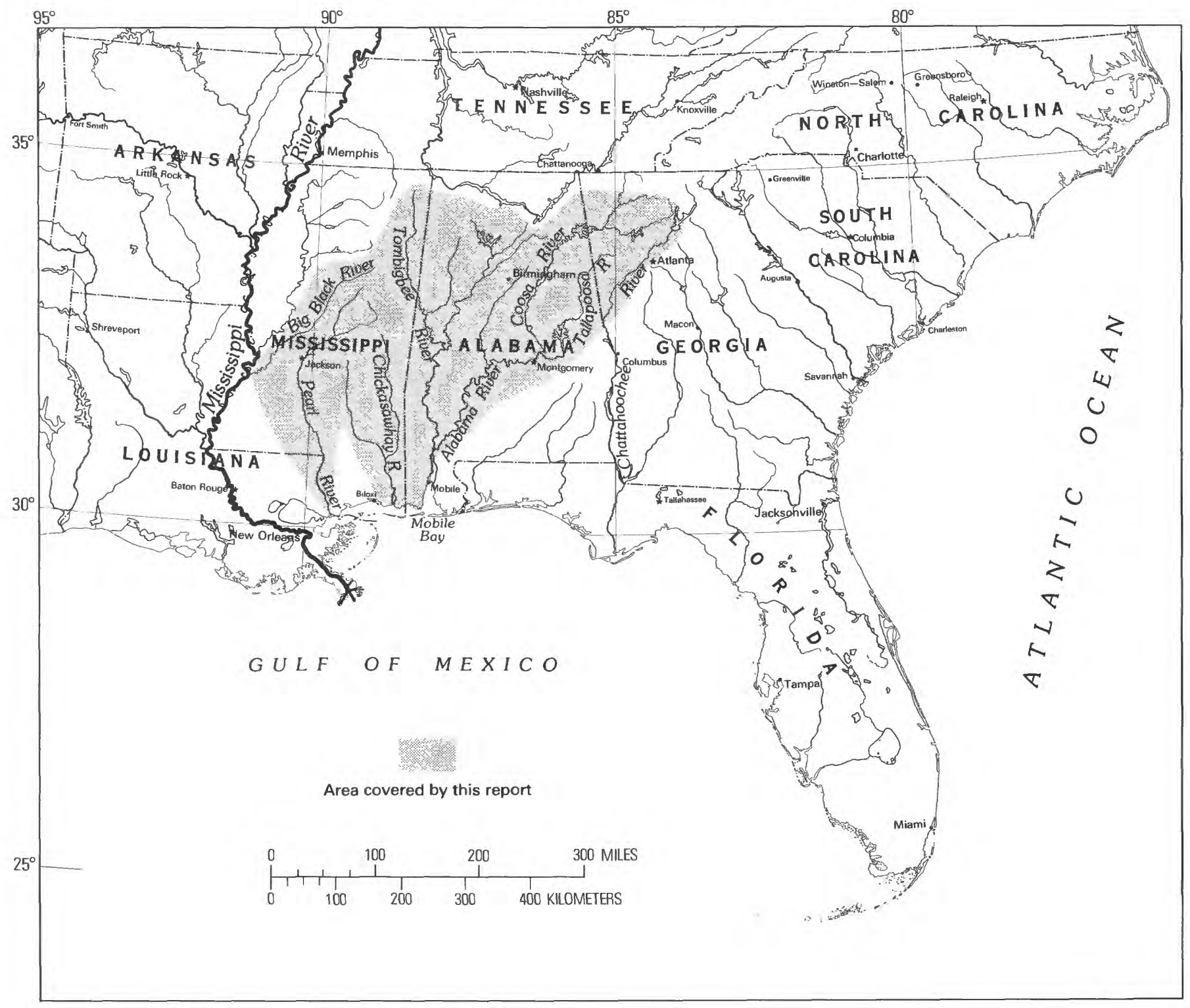

Figure 1.-Area affected by floods on the Alabama, Big Black, Chattahoochee, Chickasawhay, Coosa, Pearl, and Tombigbee Rivers and their tributaries in April 1979.

Stages were still high on many streams when the extremely heavy rains fell. The most severe flooding occurred in the middle reaches of the Tombigbee River and along the Pearl River at Jackson, Miss. Moderate flooding occurred along the Pascagoula River and the Yazoo River, a tributary to the lower Mississippi River. Although the storm extended into the headwaters of the Chattahoochee River basin in northwestern Georgia, most flooding there was only moderate.

Minor storms, April 21-26, caused small streams in the lower Pearl River basin to rise coincidentally with the arrival of floodwaters from the April 11-13 storm. The April 21-26 runoff added materially to flood volumes and extended the duration of flooding along the lower Pearl River. Figure 1 is a map showing major rivers and areas affected by the floods.

Peak flows in March and April at more than 60 streamflow gaging stations in Alabama and Mississippi were greater than those previously recorded. Maximum stages and discharges of record occurred along the main stems of the Coosa, Tombigbee, Pearl, and Big Black Rivers.

Nine lives were reported lost. Damages estimated by the U.S. Army Corps of Engineers (1980a, b) totaled nearly $\$ 400$ million.

All rivers in Mississippi and Alabama drain into the Gulf of Mexico. The major rivers are the Alabama and 
Tombigbee Rivers, which drain into Mobile Bay through the Mobile and Tensaw Rivers, and the Pascagoula, Pearl, and lower Mississippi Rivers.

The states of Mississippi and Alabama, bounded on the north by $35^{\circ} \mathrm{N}$. latitude and on the south by the Gulf of Mexico, have ample rainfall. Mean annual total precipitation over the two-State region exceeds 50 in., and the number of days with appreciable rain $(.01 \mathrm{in}$. or more) exceeds 100 each year. Rainfall is distributed throughout the year, with a maximum in March and a minimum in October.

The Tombigbee River rises in the fall-line hills in northeastern Mississippi and flows southeastward to western Alabama and then southward to join the Alabama River to form the Mobile and Tensaw Rivers, which flow into Mobile Bay. The Tombigbee is 442 miles long; about 350 miles are navigable because of a series of improvements in the form of dams and locks. Unlike the Pearl River in Mississippi. the Tombigbee River is an important artery for manufactured goods; it can be accessed by the Birmingham industrial area through the Black Warrior River tributary. The Tombigbee drainage basin is 22,100 square miles in area and is shaped like an inverted triangle. At the northernmost headwater region the drainage width is 170 miles; the width reduces to 80 miles at the midpoint of the river course near Demopolis, Ala, and narrows further as the river flows south, reaching a vertex near Mobile, Ala. Land use adjoining the Tombigbee River is predominantly agricultural. There is no city along the Tombigbee River main steam that compares in size with metropolitan Jackson, Miss., on the Pearl River.

At Demopolis, Ala., the Tombigbee River crested April 18, at 37.03 feet, more than 24 feet above flood stage and 1.3 feet above the previous record, established in 1961.

The Pearl River is the principal outlet for runoff from south-central Mississippi. Its watershed extends 240 miles from headwaters to the Gulf of Mexico and has a maximum width of about 50 miles. The total area drained is about 8,670 square miles. Except in its final 60-mile reach, where it forms the boundary between Louisiana and Mississippi, the Pearl River lies within the State of Mississippi. Its gradient is relatively flat, only about 1 in 5,000. It is mostly a shallow, meandering stream, nonnavigable except for recreational boating. Land use along the Pearl River is predominantly rural, agricultural, and woodlands except for the Jackson, Miss., metropolitan area. Jackson is located on the west side of the Pearl River about two-fifths of the way from its source. It lies on a rolling upland dissected by some 14 tributary creeks which drain eastward to the Pearl River. These creeks pose a double threat to the adjoining metropolitan area: flash floods from urban runoff within each tributary watershed and backwater from the Pearl River main stem. The natural flood plain at Jackson, about 2 miles in width, has been modified extensively by levees. About 10 miles upstream from Jackson is the Ross Barnett Dam, built in 1962 to provide water supply and recreation for Jackson. The reservoir behind this dam is maintained at a high level, to within a foot or two of capacity, to provide maximum recreational benefits; therefore, the flood control capacity of the system is minimal.

The April 1979 Pearl River flood set a new stage record of 43.28 feet at Jackson. The levee was overtopped. Nearly 2,000 dwellings and 298 commercial buildings were flooded. Seventeen thousand people were driven from their homes. A new $\$ 54$ million sewage treatment plant was damaged substantially and raw sewage flowed directly into the Pearl River. Vital public services-water supply, electric power, telephone, and fire protection-were curtailed or hampered.

The purpose of this report is to present an analysis of the meteorological settings associated with the storm; stages, discharges, and accumulated runoff of the flood; contents of reservoirs, flood-crest elevations, and magnitude and frequency of peak discharges for comparison with previous large floods; ground-water levels; and a summary of flood damages.

\section{ACKNOWLEDGMENTS}

The meteorological and rainfall analyses contained in this report are based on data obtained by the National Weather Service and represent the collective effort of many professional people.

The supplementary rainfall data were obtained by a field survey team that included Roy Roberts (National Weather Service Lower Mississippi River Forecast Center, Slidell, La.) Rovert Ellis and Robert Manning (National Weather Service, Southern Region), cooperative program managers from Mississippi, Alabama, and Arkansas, and personnel from the Mississippi Department of Natural Resources.

Discharge records and other streamflow data appearing in this report were obtained as part of cooperative programs between the U.S. Geological Survey and the States of Alabama, Georgia, Mississippi, and Louisiana; county and municipal agencies within those States; and agencies of the Federal Government. The cooperation of the Mobile and Vicksburg Districts of the U.S. Army Corps of Engineers in providing photography and information on reservoir operation, flood heights, salinity, temperature, and flood damages is gratefully acknowledged. Other Federal and State agencies, municipalities, universities, corporations, and individuals assisted, financially or otherwise, in the data- 
collection effort. Credit for this assistance is given in the appropriate places in the text.

\section{METEOROLOGICAL SETTING}

\section{ANTECEDENT CONDITIONS}

The 1978-1979 winter season was much wetter than normal over the two-State region. The December 1978 precipitation over central and northern Mississippi and northwestern Alabama was more than one and one-half times normal. Jackson, Miss., had a monthly total of 8.4 in., or 3.4 in. above normal.

Beginning in January 1979 a series of storms swept through the two-State region. The storm of January 1-3 was characterized by rapid movement of a Low from the lower Mississippi Valley to Quebec. The passage of the associated cold front caused precipitation to fall over the whole region. Jackson, Miss., had 2.74 in., and Birmingham, Ala., had 1.51 in. Subsequent storms that produced significant amounts of precipitation occurred during the periods January $6-8$ and $18-21$ and on January 24, 27, and 31. All of Mississippi, except the northernmost part, and western and southern Alabama had more than one and one-half times the normal precipitation in January, and the midsection of the Pearl River drainage basin, centered around Jackson, had more than three times the normal January amount. Jackson itself received $14.1 \mathrm{in}$. in January 1979, compared with a normal of 4.4 in.

Cyclogenesis occurred on February 5 and 6 off the Texas coast in the Gulf of Mexico. The surface Low moved eastward, and the southerly flow in advance of the center was lifted by the associated warm front, causing rain to fall over Mississippi and Alabama on February 6 and 7. Other periods of appreciable precipitation over the region were February 18-19 and 21-25. For the month, the central and southern parts of the two-State region had precipitation more than 50 percent above the February normal. Although departures were less for the northern half of Mississippi, precipitation was still abóve normal. Total February precipitation at Jackson, Miss., was 8.4 in., or 3.8 in. above normal. Thus, after three consecutive wet months in the winter season over the central part of the region, particularly over the middle Pearl River basin, the soil was thoroughly saturated. Streamflows were more than ample in the Pearl River, in several headwater tributaries of the Pascagula River, and in the Tombigbee River, and reservoirs in the region were nearly filled even before spring season.

\section{EARLY SPRING STORMS}

The series of storms continued into the spring. Prior to the large storm of April 11-13 which produced the major flooding, there were seven spring storms that brought significant amounts of precipitation to the region. These storms occurred on March 3-4, March 10-11, March 14, March 21, March 23-24, April 1-4, and April 8-9. Among these storms, the more important rainfall periods were associated with the storms of March 3-4, March 23-24, and April 1-4. The weather situations accompanying each of these storms are discussed briefly, with the three most significant storms discussed in some detail.

\section{MARCH 3-4}

On February 28, a surface Low moved across the northern Pacific coast of the United States. Significant meteorological features associated with the storm are shown in figure 2. The surface system was over Nevada by the morning of March 1 and continued toward the southeast crossing the intermountain region. Aloft, at $500 \mathrm{mb}$, a deep short wave trough moved onshore the night of February 28-March 1 and continued its eastward movement. By the evening of the $2 \mathrm{~d}$, the trough was over the eastern slope of the Rocky Mountains. The trough induced the re-formation of the surface Low in southeastern Colorado and the Oklahoma-Texas panhandle region. The Low stagnated briefly while its cyclonic circulation reorganized, then moved slowly eastward. By the morning of the $3 \mathrm{~d}$, the storm had become a well-organized system with a central pressure of $1,000 \mathrm{mb}$ located over eastern Oklahoma. The warm front extended eastward from this system, across central Arkansas, southern Kentucky, and eastern Tennessee to western North Carolina. The cold front extended southward along the Oklahoma-Arkansas and Texas-Louisiana borders. Across the Southeastern United States, warm moist air was advected from the Gulf of Mexico. At 0600 CST, March 3, the precipitable water in this air flow was 1.56 in. at Boothville, La., and 1.43 in. at Jackson, Miss. These values compare with the climatological values of the semimonthly maxima of 1.91 in. at Boothville and 1.68 in. at Jackson. The $\mathrm{K}$ index on the morning of the $3 \mathrm{~d}$ was 30 at Boothville and 40 at Jackson, indicating thunderstorm probabilities of 50 percent at the former and near 100 percent at the latter. As warm maritime air from the Gulf of Mexico moved into the region, thundershowers were prevalent. From eastern Oklahoma, the Low turned northeastward and by the morning of the 4 th was located over the eastcentral coast of Lake Michigan. The 500-mb trough continued a steady eastward movement and passed over the region by the evening of the 4 th. The eastward movement of these systems brought an end to the rain.

The rain in the March 3-4 storm began over southern Mississippi during the evening of the $2 \mathrm{~d}$ and then spread northeastward to large areas of the Pearl, Pascagoula, Tombigbee, and Alabama River basins. Much of 


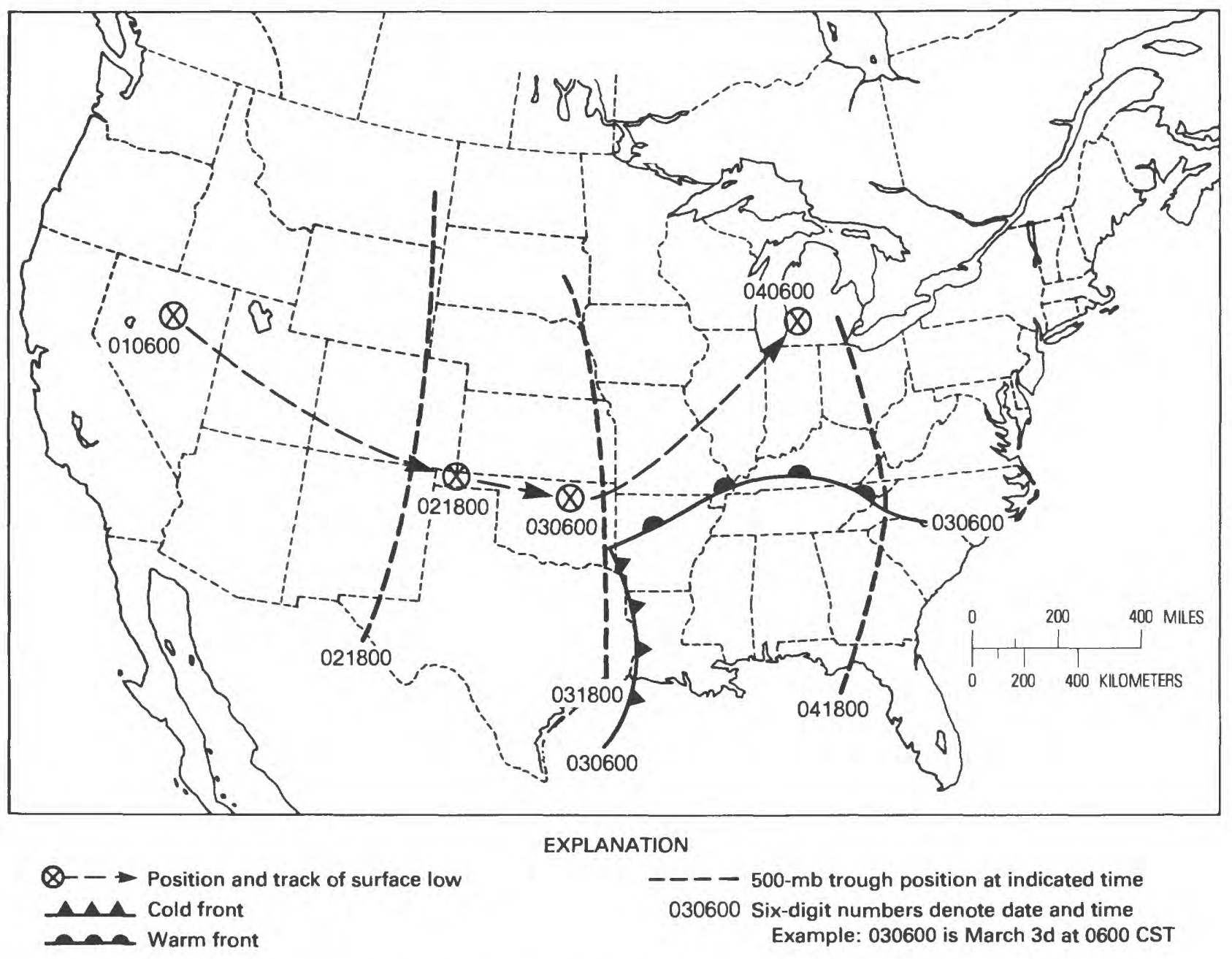

Figure 2.-Significant meteorological features associated with the storm of March 3-4, 1979.

the rain fell on the $3 \mathrm{~d}$. The greatest rainfall total for the storm was 13.08 in., at the Hickory $1 \mathrm{E}$, Miss., station. The largest single-day amount was $8.52 \mathrm{in}$. on the $3 \mathrm{~d}$ at Codin, Ala., on the Gulf coast. The axis of heaviest rainfall (fig. 3) extended between these two stations and continued northeastward across Alabama. Rain was widespread over the two-State area. Areal average rainfall was greatest, amounting to more than $5 \mathrm{in}$., over the Chickasawhay and Tombigbee River basins.

\section{MARCH 10-11, 14, AND 21}

Weak weather systems crossed the Southeastern United States on March 10-11 and March 14, and again on March 21. These storms caused rather widespread but light rain over Mississippi and Alabama. On March 10-11, rain over the coastal regions of Alabama averaged about $1 \mathrm{in}$., while over the rest of the two-State area rainfall averaged less than $0.5 \mathrm{in}$. The rainfall on March 14 and 21 was primarily centered over the northern portion of the two-State area and in each case aver- aged only about $0.25 \mathrm{in}$. Though the rainfall in these storms was light, it served to maintain a high level of soil moisture.

\section{MARCH 23-24}

The second significant early spring storm occurred on March 23-24. The chain of events began when a Low crossed the California coast on March 18 and moved slowly eastward across Arizona and New Mexico. By late afternoon on March 21, the Low was in central New Mexico (fig. 4). At this time, it started to move in a northeasterly direction. The Low crossed southeastern Colorado, Kansas, and Missouri, and by the morning of the $23 \mathrm{~d}$ it was centered in southeastern Iowa. The cold frontal system stretched southward from the surface Low. The primary front extended through Missouri, Arkansas, and Louisiana, crossing the Gulf of Mexico coast near Lake Charles. A secondary front and instability line/squall line existed in advance of the primary front. Most of the rainfall associated with the system 

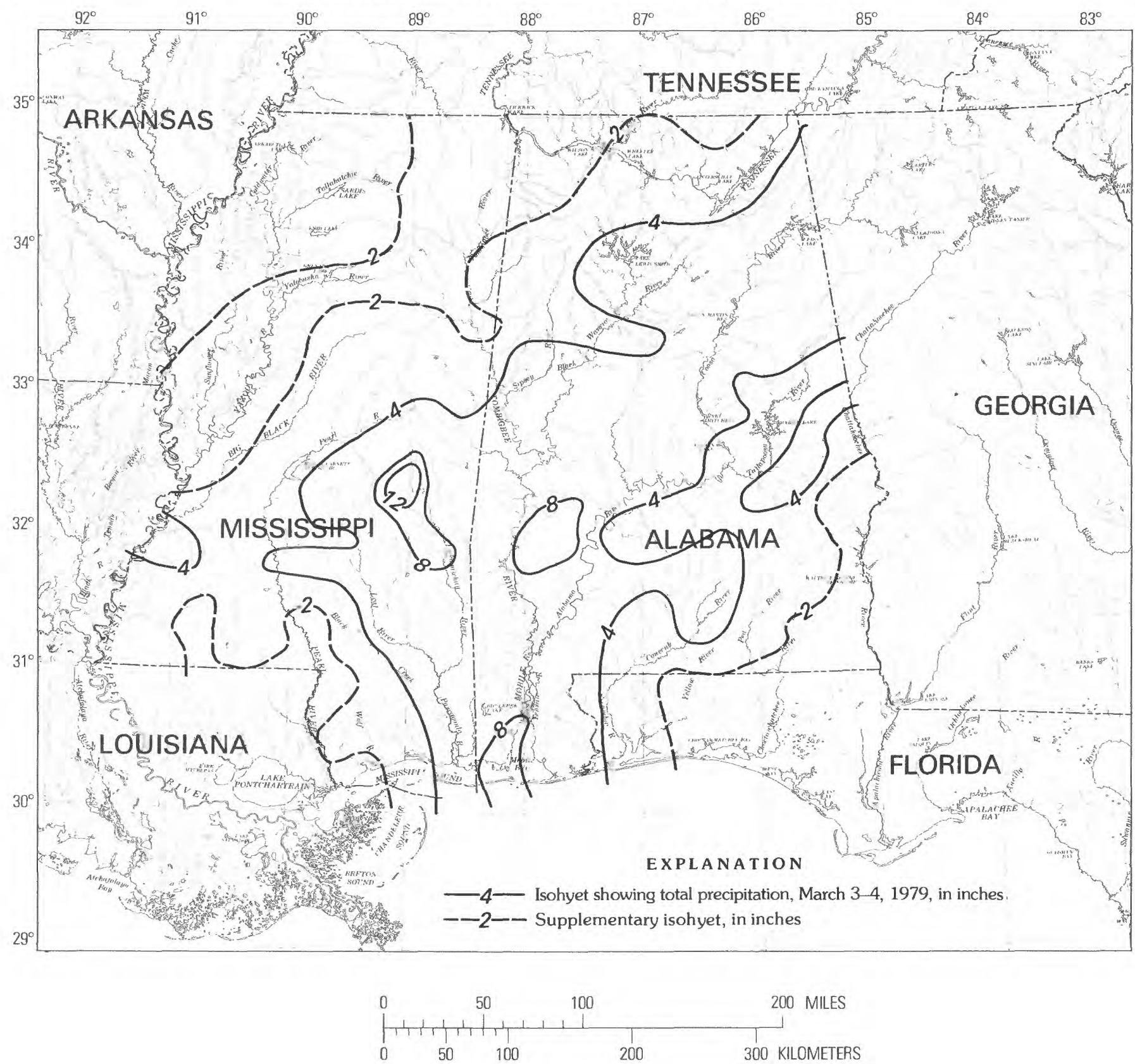

FIGURE 3.-Isohyetal analysis of storm rainfall. March 3-4, 1979.

was a result of the secondary front and instability line/ squall line. By the morning of the 24th, the Low had moved over central Lake Michigan and the frontal system had moved through the two-State region. The cold front extended from Lake Michigan through Michigan, Ohio, West Virginia, Virginia, North and South Carolina, crossing the coast near Charleston, S.C. Some lingering rainfall was occurring in northeastern Alabama, but the significant storm rainfall over the basin had ended.
The 500-mb circulation had large meridional components prior to the storm. By the morning of the $22 \mathrm{~d}$, the flow aloft had split into two separate waves, with one remaining offshore and the other centered over northern New Mexico. The easternmost 500 -mb closed Low and trough were associated with the surface system that caused the rain over the two-State region. By the morning of the $23 \mathrm{~d}$ the Low had reached approximately $95^{\circ}$ W. This position placed the two-State region under a trough-to-downwind ridge flow pattern. Both mass and 


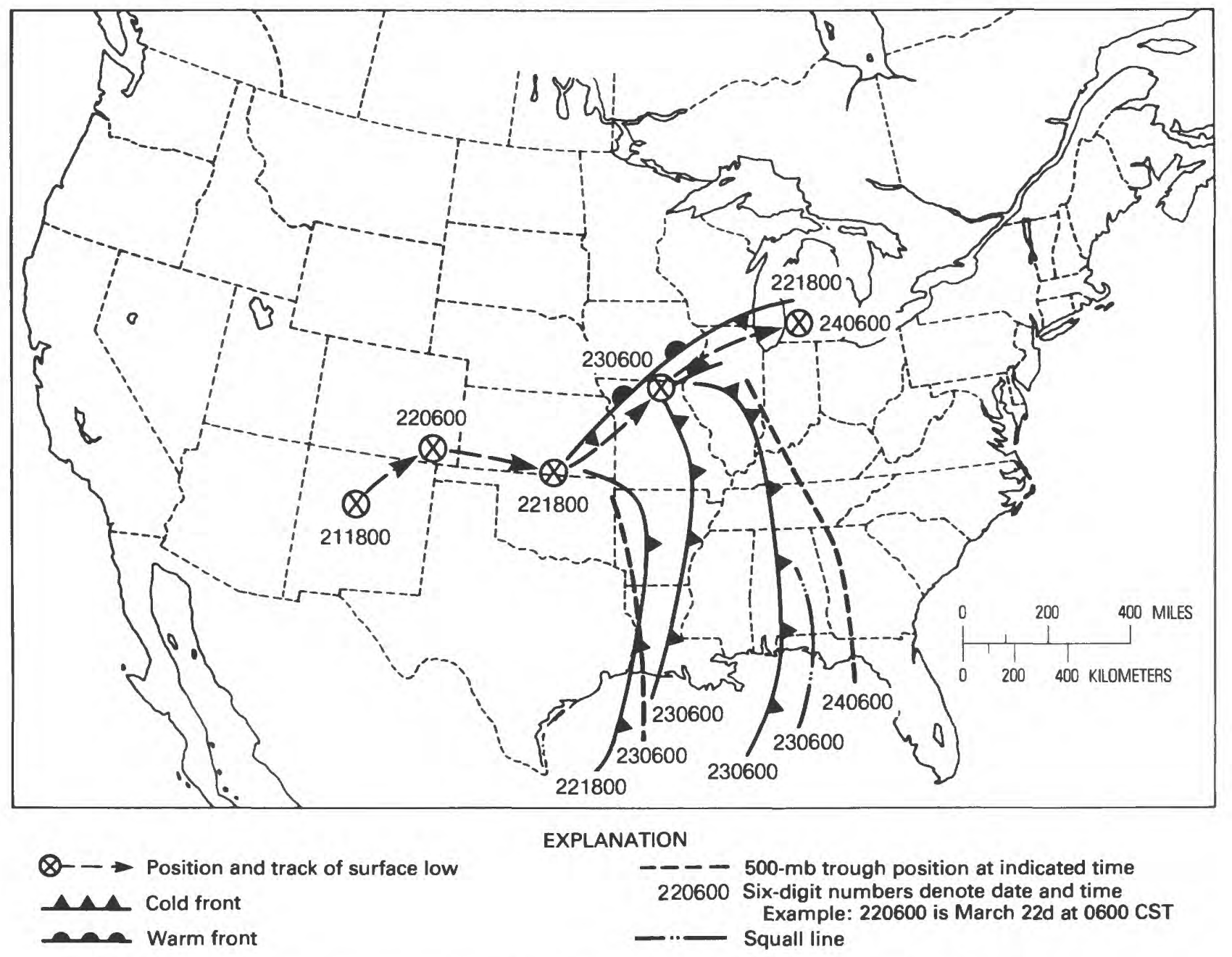

FIGURE 4.-Significant meteorological features associated with the storm of March 23-24, 1979.

isobaric convergence (divergence) existed in a deep layer below (above) about $500 \mathrm{mb}$. This condition is very favorable for intensification of weather systems, and in fact both the surface and $500-\mathrm{mb}$ Lows deepened as they moved to the northeast.

In the warm, moist airflow in advance of the surface and upper air systems, precipitable water was only moderate. At Jackson, Miss., the observed amount from surface to $500 \mathrm{mb}$ at $1200 \mathrm{GMT}$ was $0.95 \mathrm{in}$. on the $22 \mathrm{~d}$ and $0.43 \mathrm{in}$. on the $23 \mathrm{~d}$. These can be compared with the March mean at Jackson of .63 in. (standard deviation, .34). At Centreville; Ala., the observed amounts of precipitable water for the same layer were 0.91 and $1.12 \mathrm{in}$. on those dates; climatological values are not available for Centreville.

Rain began in the early morning of the $22 \mathrm{~d}$ over a large part of Mississippi and spread to Alabama. The period of rainfall over Mississippi was March 22-23, with the $22 \mathrm{~d}$ the rainier day, while the period of rainfall over Alabama was March 22-24, with the 23d the rain- iest day. A maximum storm precipitation of 4.42 in. fell at Dayton, Ala., $\left(32^{\circ} 22^{\prime} \mathrm{N}, 87^{\circ} 39^{\prime} \mathrm{W}\right)$ in the Tombigbee River drainage basin. The average depth of rain was less than 1.0 in. over the Pearl River basin and approximately 1.5 in. over the Tombigbee River basin. A generalized isohyetal map for this storm is shown in figure 5 .

\section{APRIL 1-4}

The third significant spring storm occurred during the period April 1-4. At 1800 on March 30, the 500-mb circulation over North America was characterized by a long wave trough over the Western United States, with the trough axis extending from western Montana to eastern Arizona. Meanwhile, off the west coast, a short wave impulse appeared and moved rapidly southeastward along the major trough. By the morning of April 1, this short wave trough was passing over the OklahomaTexas panhandle region (fig. 6), while the major long wave trough was still over the Rocky Mountains. The 

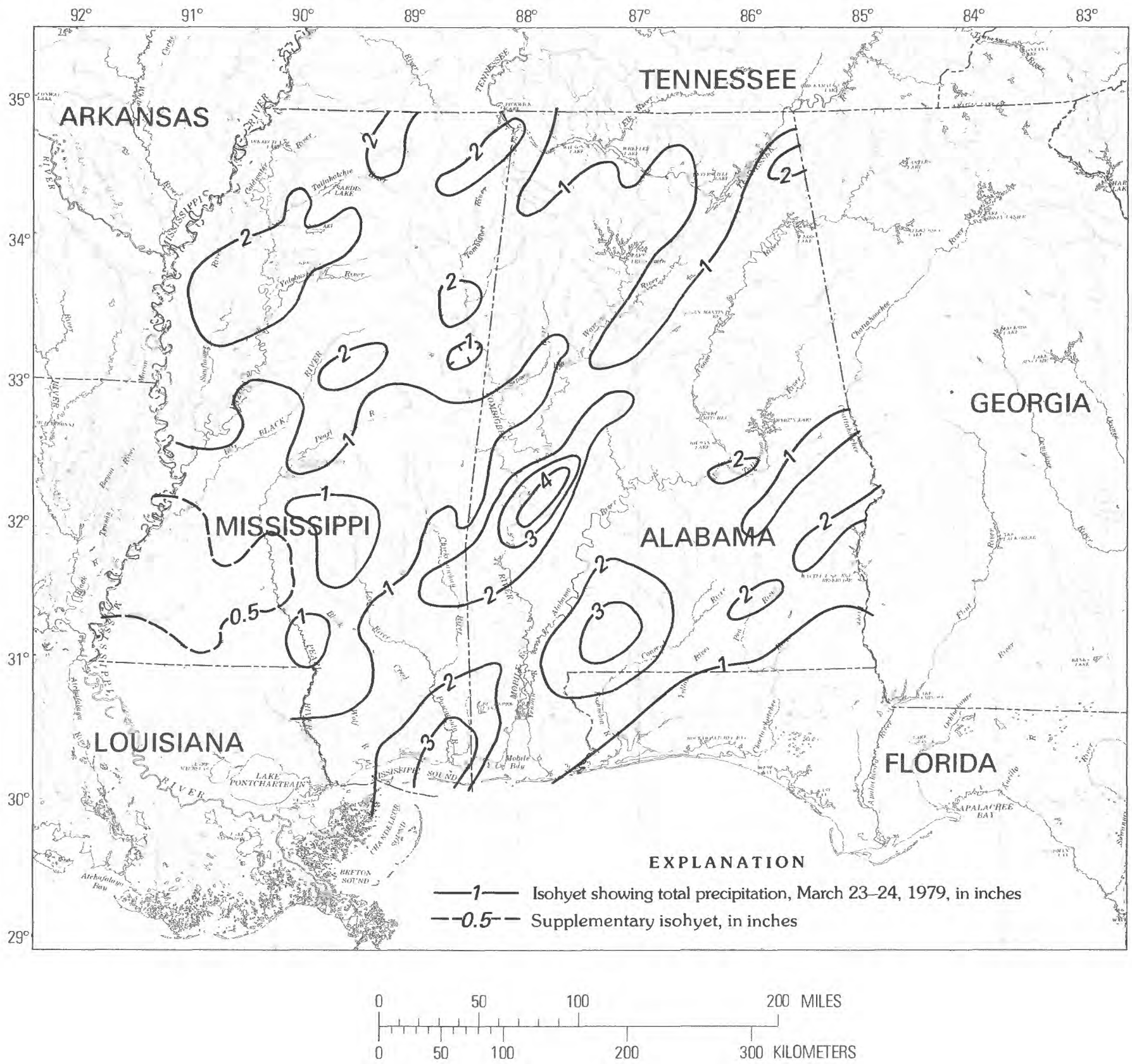

FIGURE 5.-Isohyetal analysis of storm rainfall, March 23-24, 1979.

passage of the upper air short wave trough over an existing diffuse surface frontal system over the Southeastern United States triggered frontal wave formation and cyclogenesis. A weak depression located over north central Texas on April 1 intensified, and by that evening it had become a well-formed system centered on the Arkansas-Missouri border (fig. 6). The Low continued to deepen, and its cyclonic circulation expanded over an even larger region as it moved rapidly northeastward to the Great Lakes region and then to the Hudson Bay coast of Quebec by the evening of the 3d. Simultane- ously, the 500-mb short wave disturbance propogated rapidly toward Quebec. A section of the preexisting weak surface frontal system was already in place over northernmost Mississippi on the evening of March 31, with precipitation generally to the north of the front. As the frontal wave developed and the closed Low formed, a squall line was generated ahead of the cold front that extended from the center of the Low southward into the Gulf of Mexico. By the morning of the 2d, the cold front was moving through northwestern Mississippi while the squall line was already in western Alabama. The 


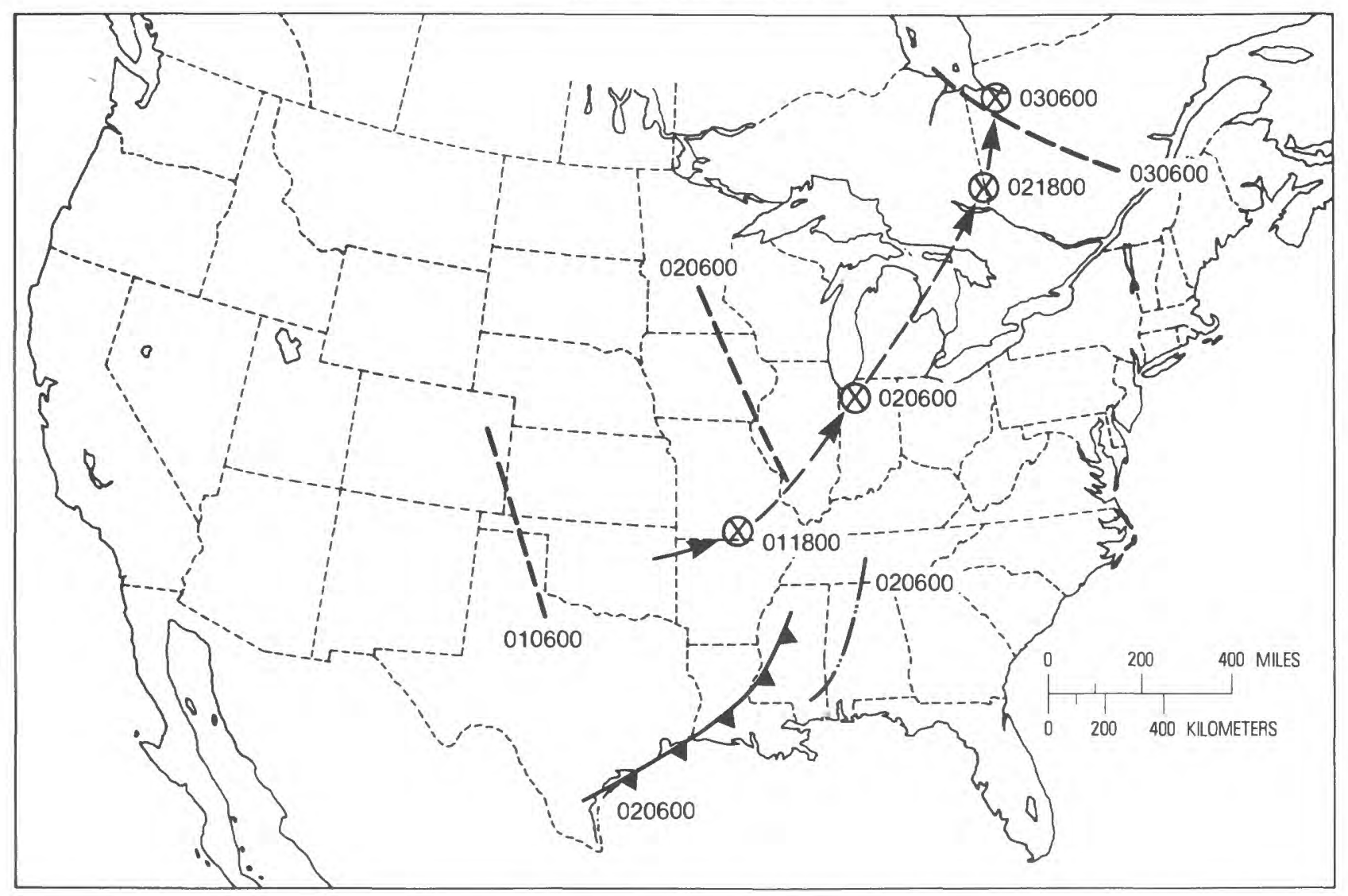

EXPLANATION

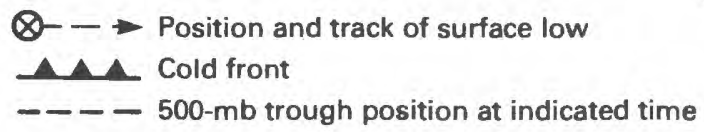

010600 Six-digit numbers denote date and time Example: 010600 is April 1st at 0600 CST

Figure 6.-Significant meteorological features associated with the storm of April 1-4, 1979.

precipitable water in the warm, moist airflow in advance of the cold front was 1.46 and 1.13 in. at Jackson, Miss., and Centreville, Ala., respectively, at $1200 \mathrm{GMT}$ on April 2, and 1.11 and 1.22 in., respectively, at 1200 GMT on April 3. These values at Jackson compare with $1.74 \mathrm{in}$. for the climatological April 1-15 semimonthly maximum. The $\mathrm{K}$ index was 38 at Jackson and 30 at Centreville on the $2 \mathrm{~d}$, indicating a thunderstorm probability of 80 to 90 percent at the former and 40 to 60 percent of the latter.

Rain started over northwestern Mississippi on the morning of March 31 and spread southeastward. Precipitation fell over the whole two-State region during the period March 31-April 4. However, most of the rain fell between April 1 and 3 over Mississippi and between April 2 and 4 over Alabama. Maximum amounts for the storm were centered over the Alabama River basin, where an average of 5 in. was received. A point maximum of 11.03 in. was observed at the Camden $3 \mathrm{NW}$, Ala., station, on April 1-4; 8.06 in. of that fell on the $3 \mathrm{~d}$.
The Tombigbee and Chickasawhay River basins received average rainfalls of 3.5 and 4 in., respectively. A generalized isohohyetal analysis for this storm is shown in figure 7.

\section{APRIL 8-9}

The next period of rain over the region occurred April 8-9, with amounts considerably less than those contributed by the three significant spring storms previously described. On April 6, a Low over the border region of British Columbia and Alberta in western Canada began to move southeastward, and by noon of the 7th it had reached the Dakotas. Once over the Iowa-Nebraska border region, the Low began to curve northeastward. It was over the Iowa-Illinois area on the morning of the 8 th and over the Indiana-Ohio area on the evening of the 8 th. Then it turned and moved toward New Brunswick. The associated 500-mb trough initially lagged behind 

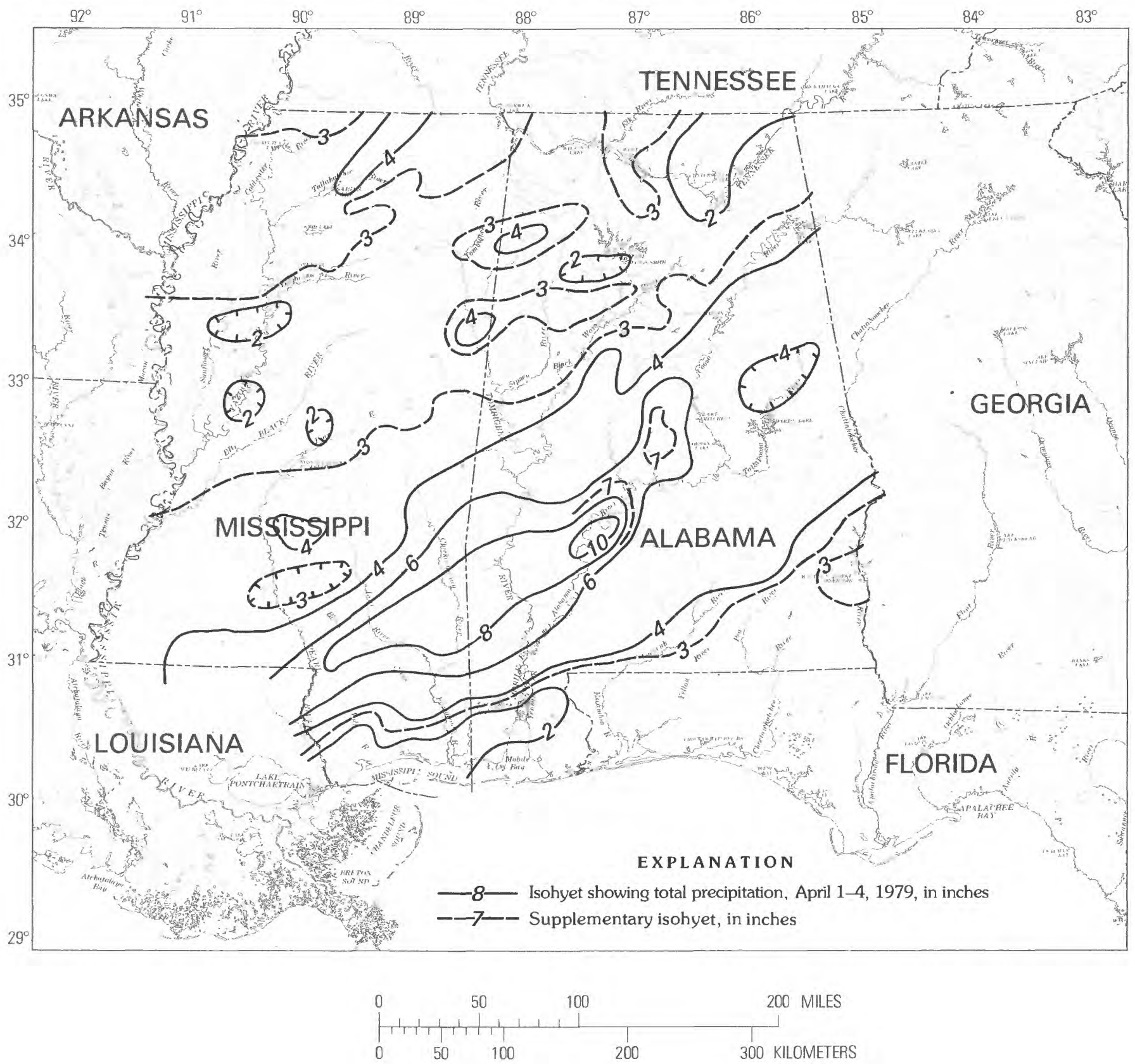

FIGURE 7.-Isohyetal analysis of storm rainfall, April 1-4, 1979.

the surface Low and was over western Montana and Idaho when the surface Low was already over the Dakotas. But by the morning of the 8th, the upper level trough had caught up with the surface system. Much of the wave undulation of the $500-\mathrm{mb}$ circulation pattern occurred to the north of the region of interest. The $500-\mathrm{mb}$ flow over Mississippi-Alabama was predominantly zonal and was only marginally affected by the passing of the short wave trough early on the morning of the 9th. This situation was in marked contrast to those associated with the three significant earlier storms, when the two-State region was under a pronounced $500-\mathrm{mb}$ trough-to-ridge pattern sometime during the storms.

The precipitable water in the southerly airflow over the region at 1200 GMT on April 8 was 1.22 in. and 0.75 in. at Jackson, Miss., and Centreville, Ala., respectively. As the Low turned toward the northeast on the morning of the 8th, the associated cold front moved eastward toward the two-State region. The distance of this surface system from the two-State region, the primarily zonal flow aloft over the region, and the lower moisture 
content of the air than in the previous storms resulted in generally lower precipitation amounts. Scattered moderate rain began to fall over the northern two-thirds of the region on the morning of the 8th and continued intermittently throughout the day. At a few stations, the rain extended into the 9th. A maximum of $2.99 \mathrm{in}$. was observed at the Collinsville 7SE, Miss., station in the Chickasawhay River basin.

\section{MAJOR STORM EVENT:} APRIL 11-13

The cumulative effect of these antecedent storms was to saturate the soil in the region. Many rivers were at high stages or exceeded bankfull along some sections before the outbreak of the major storm of April 11-13. For example, the Pearl River at Jackson, Miss., was already 10 feet above flood stage before heavy rain began on the 11th.

The storm that was the direct cause of the floods on the Pearl and Tombigbee Rivers occurred on April 11-13. Precipitation was heavy through most of the two-State area but was concentrated primarily in the Pearl and Tombigbee River basins. The prolonged, large precipitation event resulted from a large, slow-moving weather system that for a period of several days brought warm, moist air over the Southeastern States. The discussion of meteorological conditions will proceed from the $500-\mathrm{mb}$ to the $850-\mathrm{mb}$ level, and then cover the surface features.

\section{0-MB FEATURES}

Prior to the storm, on the evening of the 9th, a deep, long wave trough at the $500-\mathrm{mb}$ level became established over the Western United States. This trough advanced very slowly eastward, and by the morning of the 11th a cutoff Low was located on the Colorado-New Mexico border at approximately $104^{\circ} \mathrm{W}$ (fig. 8A). The associated downwind ridge extended southeastward from Michigan through Ohio and West Virginia to the Atlantic coast of North Carolina. This circulation pattern placed large portions of the Southeastern United States under a trough-to-ridge pattern and resulted in a southwesterly flow over the two-State area. Windspeeds were about 40 knots over the region, and dewpoint depressions at the 500 -mb level averaged about 5 degrees. The 500 -mb pattern changed very little during the next 12 hours. The center of the cutoff Low moved northeastward to the Kansas-Nebraska border at a speed of only about 10 miles per hour, and the trough now extended southward from this locaton into extreme western Texas (fig. 8B). Over the two-State area, southwesterly winds still prevailed, with some increase in windspeed. The 500-mb Low continued to move northward, with very little eastward component. By the morning of the 12th, it was centered in east-central
South Dakota (fig. 8C). During the next 12 hours movement slowed, and by the evening of the 12th the Low had moved to southern North Dakota (fig. $8 D$ ). From the morning of the 13th, the Low progressed slowly to eastern North Dakota, reaching the MinnesotaManitoba border by the evening of the 13th (figs. $8 E$, $8 F)$.

The very slow progression of the Low and the predominant northward (rather than northeastward) trajectory was matched by a corresponding slow progression of the associated trough. From a position extending from the Kansas-Nebraska border to extreme western Texas on the morning of the 12th, the trough progressed very slowly eastward, reaching the Mississippi-Alabama regon on the night of the 13th. Therefore, the two-State region was under a prolonged $500-\mathrm{mb}$ trough to downwind ridge pattern with a strong southwesterly flow-a most favorable prerequisite for flood-producing storms to develop.

\section{0-MB FEATURES}

At the $850-\mathrm{mb}$ level, the major feature associated with the deep 500-mb trough was a Low over Wyoming on the evening of the 9th. The Low progressed southeastward through Colorado and brushed the Texas Panhandle by the evening of the 10th before slowing down and beginning to turn northeastward. Prior to the storm period, the $850-\mathrm{mb}$ wind over the two-State region was westerly. As the Low approached, the wind direction began to change and became predominantly southerly by the evening of the 10th. By the morning of the 11th, the 850-mb Low was located over the Colorado-Kansas border region; it deepened as the circulation pattern over the Southeastern United States became more meridional (fig. 9A). Warm, moisture-laden air from the Gulf of Mexico was advected into the region by southerly winds reaching 40 to 45 knots as the $850-\mathrm{mb}$ contour gradient tightened. The $850-\mathrm{mb}$ Low continued to deepen, and by the evening of the 11th, when it was centered over the Kansas-Nebraska border (fig. 9B), its central height was 1,230 meters, a decrease of 110 meters in 2 days. A tongue of warm air extended northward from the Gulf of Mexico over the Pearl and Tombigbee River basins, with temperatures exceeding $16^{\circ} \mathrm{C}$ (shaded regon in fig. 9B). Dewpoint depressions in this tongue of warm air ranged between 2 and 6 degrees, indicative of the high moisture content.

On the evening of the 11th, the $850-\mathrm{mb}$ Low took a mostly northward track, with only limited eastward drift. By the morning of the 12th, the Low was centered over eastern South Dakota (fig. 9C), and 24 hours later it was over the North Dakota-Minnesota border (figs. $9 D, 9 E)$. By the evening of the 13th, it was over the Minnesota-Manitoba border (fig. $9 F$ ). The strong southerly flow over the Mississippi-Alabama region persisted 

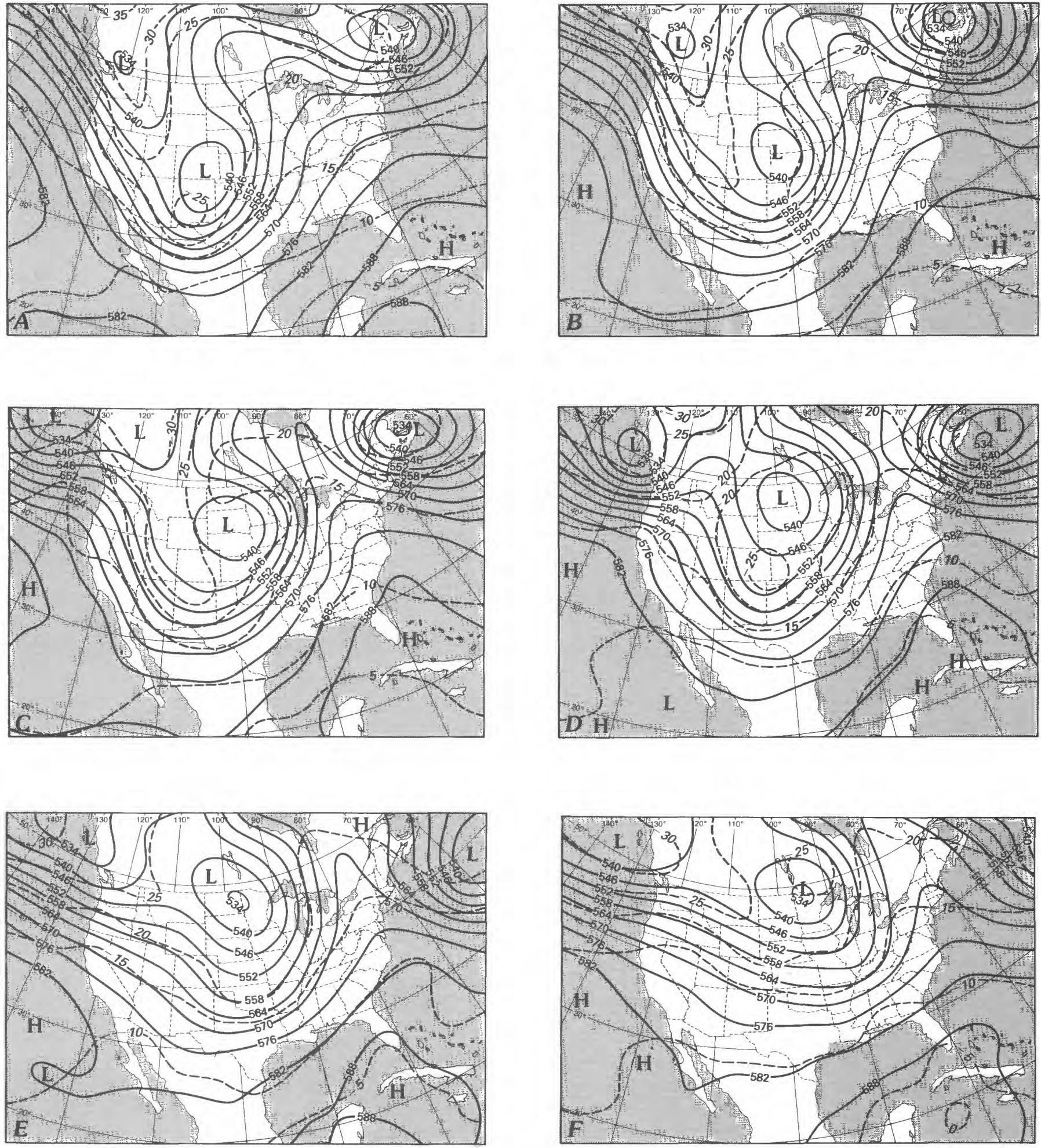

EXPLANATION

-570 - Isobar, in tens of meters

L Center of low pressure

$--5--$ Isotherm, in degrees Celsius $\left({ }^{\circ} \mathrm{C}\right)$

$\mathrm{H}$ Center of high pressure

Figure: 8.-500-mb analyses. A, 0600 CST, April 11, 1979. B, 1800 CST, April 11, 1979. C, 0600 CST, April 12, 1979. D, 1800 CST, April 12, 1979. E, 0600 CST, April 13, 1979. F, 1800 CST, April 13, 1979. 

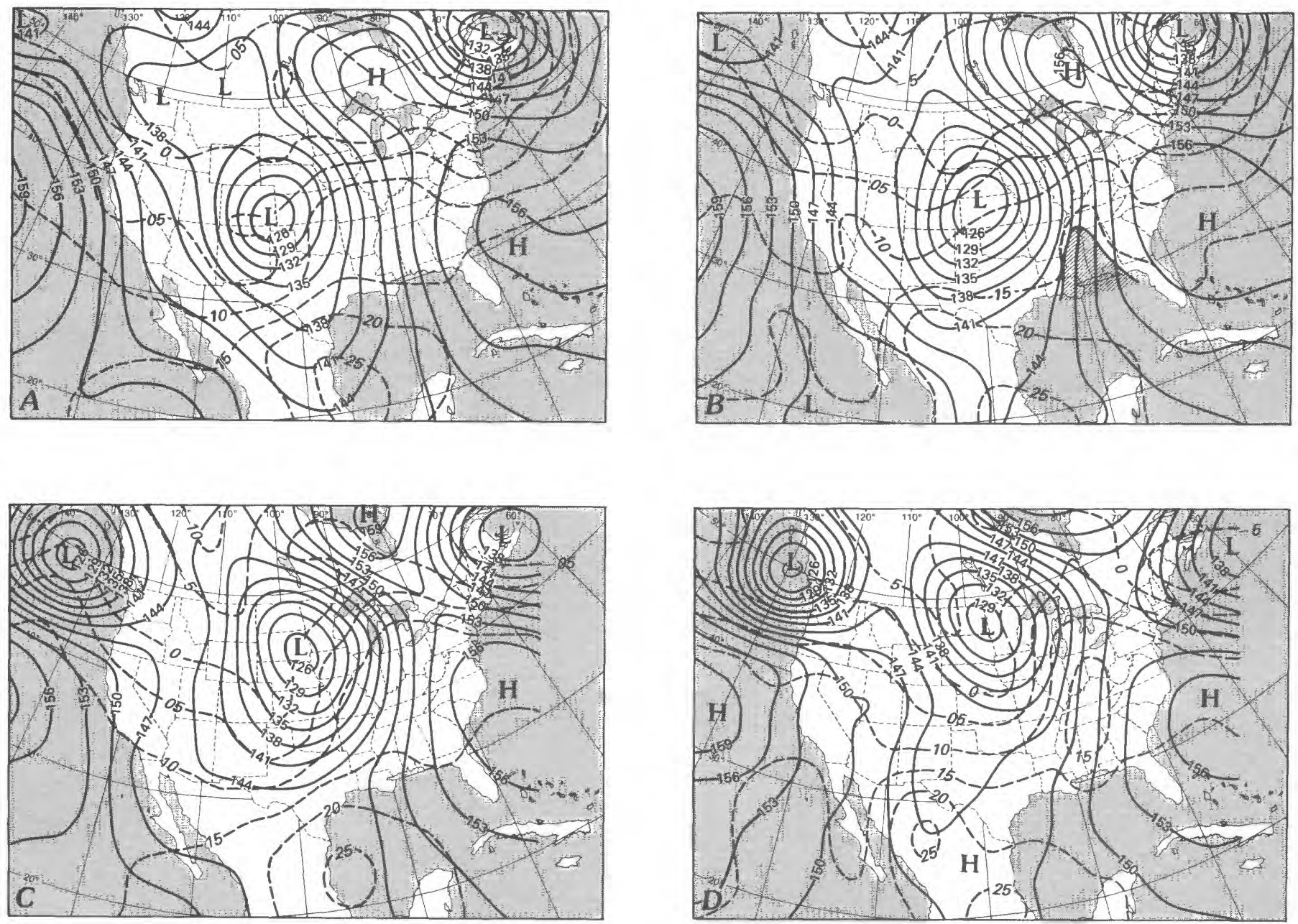

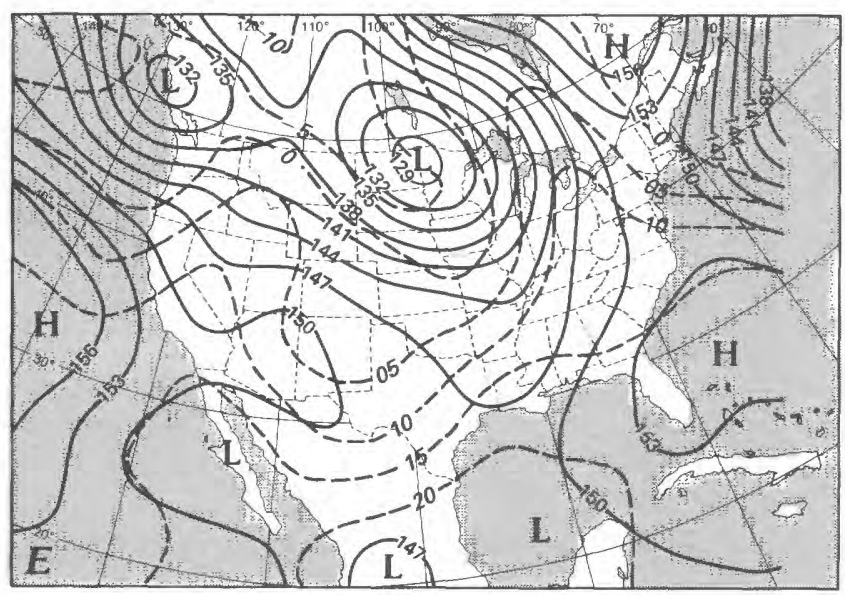

EXPLANATION

- 153 - Isobar, in tens of meters

$--10--$ Isotherm, in degrees Celsius $\left({ }^{\circ} \mathrm{C}\right)$

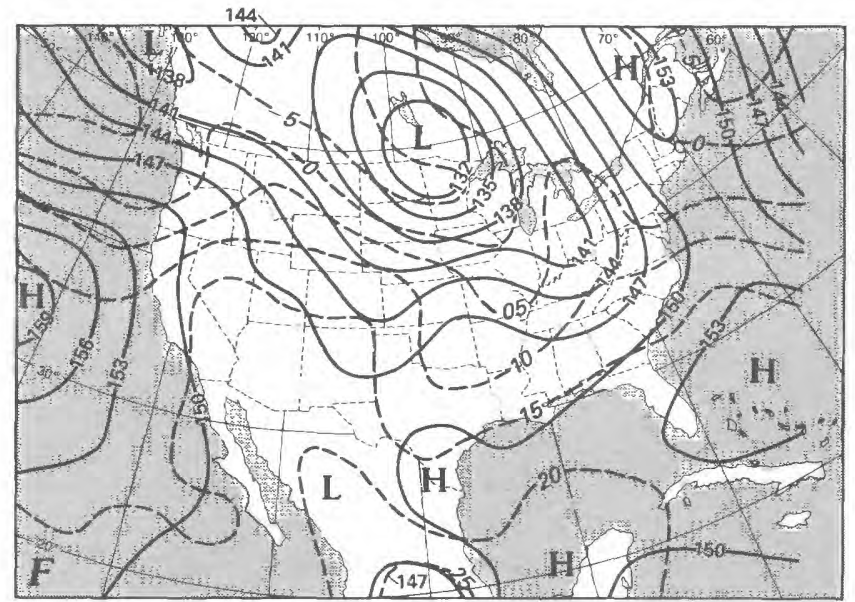

L Center of low pressure

H Center of high pressure

Temperature $16^{\circ} \mathrm{C}$ or higher

Figure 9.-850-mb analyses. A, 0600 CST, April 11, 1979. B, 1800 CST, April 11, 1979. C, 0600 CST, April 12, 1979. D, 1800 CST, April 12, 1979. E, 0600 CST, April 13, 1979. F, 1800 CST, April 13, 1979. 
from the morning of the 11th until the morning of the 13th, when the circulation pattern began to change significantly. By the evening of the 13th, the region of interest came under a ridge-to-downwind trough flow pattern and the $850-\mathrm{mb}$ wind became northwesterly, signifying that the storm had ended.

The average precipitable water over the two-State region increased from $0.6 \mathrm{in}$. on the morning of the $12 \mathrm{th}$ to more than $1.4 \mathrm{in}$. on the morning of the $13 \mathrm{th}$. Then, as northwesterly flow replaced southerly flow, the precipitable water decreased, to about $0.3 \mathrm{in}$. on the morning of the 14th. Specifically, the precipitable water in the airflow on the morning of the 13 th was 1.47 and $1.55 \mathrm{in}$. at Jackson and Centreville, respectively. The $1.47 \mathrm{in}$. at Jackson can be compared with the mean of record there of semimonthly maxima of 1.29 (standard deviation, .21) and a maximum semimonthly value of 1.74 . Comparative statistics for Centreville are not available. The stability condition, as depicted by the daily lifted index analysis, indicated less than +4 (unstable condition) over the two-State region, from the morning of the 11th and throughout the duration of the storm.

\section{SURFACE WEATHER FEATURES}

On the evening of the 9 th, the surface Low associated with this system was located over southern Montana, to the lee of the Continental Divide. By the evening of the 10th it had moved southward through Wyoming to eastern Colorado and deepened. The central pressure dropped from 992 to $985 \mathrm{mb}$. By the morning of the 11th, the Low was located in southeastern Colorado and had a central pressure of $982 \mathrm{mb}$ (fig. 10A). By this time, occlusion of the frontal system had already occurred. The air near the center of the Low became well mixed with the occluded front, extending from the Center of the Low through Colorado, Kansas, and Oklahoma into Texas to the confluence of the warm and cold fronts. The warm front extended eastward through southern Oklahoma, Arkansas, central Mississippi, and southwestern Alabama to northern Florida, while the cold front extended through east-central Texas southward into northern Mexico. The warm front, under the influence of the strong southerly flow from the surface to above $500 \mathrm{mb}$, moved rapidly northward during the day. This was the same warm front that passed through the Red River Valley near the Texas-Oklahoma border region on the afternoon of the 10th, triggering severe thunderstorms and tornadoes that touched down at Veron and Wichita Falls, Tex., causing considerable destruction. The surface Low began a curvature to the northeast and by the evening of the 11 th was centered in north-central Kansas (fig. 10B). The occluded front became indistinct near the center of the Low but was clearly defined from north of the Low into south-central Missouri. The cold front extended from this location southward through central Arkansas to eastern Texas. The warm front extended from the confluence of the fronts northeastward through southern Illinois, Indiana, and Ohio to West Virginia. The two-State area now was whally within the warm sector of this storm and would remain in it until the rain ended with the passage of the cold front some 2 days later. As the cold front was progressing slowly eastward, a squall line had developed in advance of the front in the warm, moist, convectively unstable air in the warm sector. At 1800 CST on the 11th, the squall line extended from the Arkansas-Mississippi border to central Louisiana and was moving very slowly eastward across Tennessee and northern Mississippi and Alabama. Most of the rain during this storm was associated with this and other squall lines that developed in the warm sector of the storm.

By the morning of the 12th, the Low had moved to southeastern South Dakota while the associated cold front, after reaching western Mississippi, had become a weak, quasi-stationary front (fig. 10C). Ahead of this front, a major squall line in the warm sector now extended form central-eastern Mississippi to northeastern Alabama and northeastward. The movement of the Low slowed, and it had reached the North Dakota-Minnesota border region by 1800 (fig. $10 \mathrm{D}$ ).

The northern portion of the instability line had moved eastward more rapidly than the southern end during the day on the 12th. By 1800 it was oriented east-northeast to west-southwest from south of McComb, Miss., to just south of Atlanta, Ga. (fig. 10D). An analysis of hourly surface observations at 1500 on April 12 for the region is shown in figure 11 . At this time, heavy rain was falling in central-western Alabama. A very interesting feature was the presence of a sharp surface trough oriented nearly east-west. The squall line was alined with this deep trough and took on the characteristics of a front, acting as a boundary between the warm maritime airmass to the south and the continental airmass to the north. Most of the thunderstorms and rains occurred to the north of the trough as incoming maritime air was lifted along this line of low level convergence.

The Low continued a very slow drift northward for the next 24 hours and was over the Minnesota-Canada border by the evening of the 13th (figs. 10E, 10F). The two-State region was still in the warm sector of the primary system as a weak Low developed in northern Mississippi by the morning of the 13th and then moved northward along the cold front. The squall line, meanwhile, persisted over the two-State region (fig. 10C). The southerly inflow weakened noticeably as the cold front started to sweep past the region.

Beginning on the morning of the 13th, the upper air circulation pattern over the Southeastern United States gradually shifted from southwesterly to a more zonal 
front. As the cooler and drier airmass from the interior of the continent replaced the maritime airmass, the 850-mb temperature at Jackson, Miss., decreased (from $16^{\circ} \mathrm{C}$ to $11^{\circ} \mathrm{C}$ ) and the dew point dropped (from $15^{\circ} \mathrm{C}$ to $1^{\circ} \mathrm{C}$ ) during the 12-hour period ending at 1800, April 13 . At the surface, the cold front had moved across Mississippi and northwestern Alabama by then, and a High developed covering an area extending from central Texas to central Mississippi (fig. 10F). Surface winds became predominantly northerly over the MississippiAlabama as the storm ended and clear weather returned.

\section{SATELLITE IMAGERY}

A visible imagery photograph from GOES East taken at $0730 \mathrm{CST}$, April 12, with major features of the surface map superimposed, is shown in figure 12 . The visible range photograph is the result of reflected sunlight sensed in the $0.55-0.70 \mu \mathrm{m}$ band. Clouds, particularly large cumulonimbus towers with a reflectivity of 92 percent, appear very white on such photographs. Water surfaces, which are very poor reflectors, with a reflectivity of 9 percent, appear very dark. Between these two extremes are various shades of gray representing different surface reflectivity characteristics. It is evident that little active weather was associated with the stationary front that extended from Iowa, Illinois, Missouri, and Arkansas southwestward to the Texas coast. Most of the strong convective activity occurred along the squall line ahead of the front in the warm sector. At the time this satellite picture was taken, the very intense major burst of rain around Louisville, Miss., in the Pearl River headwaters was near its end, while heavy rain had just started to fall around Pickensville, Ala., in the Tombigbee River headwaters.

As the thunderstorm downdrafts were chilled by the evaporation of raindrops and became colder than the environment, the denser and colder airmass gradually spread out from the squall line to form a boundary. Such a boundary could assume the characteristics of a cold front and provide the necessary lifting to a warm, moist, convectively unstable maritime airmass coming in from the Gulf of Mexico. This would lead to condensation and continuous replenishment of cloud material and thus would prolong the rainfall. The narrow clear strip just ahead of part of the squall line in figure 12 represents such a boundary created by squall line downdrafts and raindrop evaporation (in this case, the boundary was not obscured by higher clouds). Ahead of this major squall line but oriented similarly, in the central Alabama-Georgia region, is a less well-defined clear strip. This represents a secondary squall line whose associated thunderstorm clouds are much thinner than those along the main squall line.

Two infrared pictures taken by the GOES East satellite are shown in figure 13. Every object having a temperature above absolute zero radiates electromagnetic energy in a spectrum that is a function of its temperature. The transfer of infrared radiation in clouds is dominated by water droplet absorption. If the liquid path through a cloud is greater than $30 \mathrm{gm}^{-2}$, which is the case for the great majority of clouds except thin cirrus and clouds with very high base, the cloud is optically thick. The intensity of the emitted radiation will be close to that given by the Planck's law at the cloud top temperature with no contribution from the underlying surface of the earth and internal cloud structure. The infrared sensor aboard GOES measures the outgoing long wave radiation emitted from the surface of the Earth in a cloud-free area and from the tops of clouds in the atmospheric transparent window band 10.5-12.6 $\mu \mathrm{m}$. This radiametric information is eventually transmitted to a central facility in Marlow Heights, Md., where it is converted by computer processing into shades of gray. The computer is capable of producing and recognizing 255 distinctive shades between black and white. This is far beyond the ability of human eyes to distinguish and digest properly in an operational environment. Therefore, the infrared image is usually enhanced to facilitate interpretation. In the enhancement process, any shade of gray may be assigned to any temperature when more contrast is needed to highlight a certain temperature range of specific interest. Thus, the enhancement process increases the contrast between features of interest and their backgrounds. There are different enhancement curves designed for hurricane detection, for viewing convective activity, for determining the extent of ice and snow covers, for viewing coastal upwelling, and so on. The two photos in figure 13 were enhanced according to the Mb curve, whose specification is listed in "The GOES/SMS User's Guide" (NOAA and NASA, undated). The $\mathrm{Mb}$ enhancement gives good definition to the low and middle clouds, but its main purpose is to highlight convective thunderstorms. Cloud-top temperatures between $-42^{\circ}$ and $-52.2^{\circ} \mathrm{C}$ are shown in light gray, between -53.2 and $-58.2^{\circ} \mathrm{C}$ in dark gray, between -59.2 and $-62.2^{\circ} \mathrm{C}$ in black, between -63.2 and $-80.2^{\circ} \mathrm{C}$ in medium gray, and below $-80.2^{\circ} \mathrm{C}$ (characteristic of overshooting cumulonimbus turrets) in vivid white.

The relationship between cloud-top temperature of convective thunderstorms and the amount of precipitation is complex and depends on many concurrent meteorological factors. Scofield and Oliver (1977), using the Mb-enhanced GOES infrared images, developed an empirical technique for estimating rainfall from shortlived isolated thunderstorms that produce heavy rain because of large updrafts. For thunderstorms in a saturated environment that is stationary over an area for more than 1 hour, as was the case for the storm over Louisville, Miss., on April 12, the rainfall rate will be 

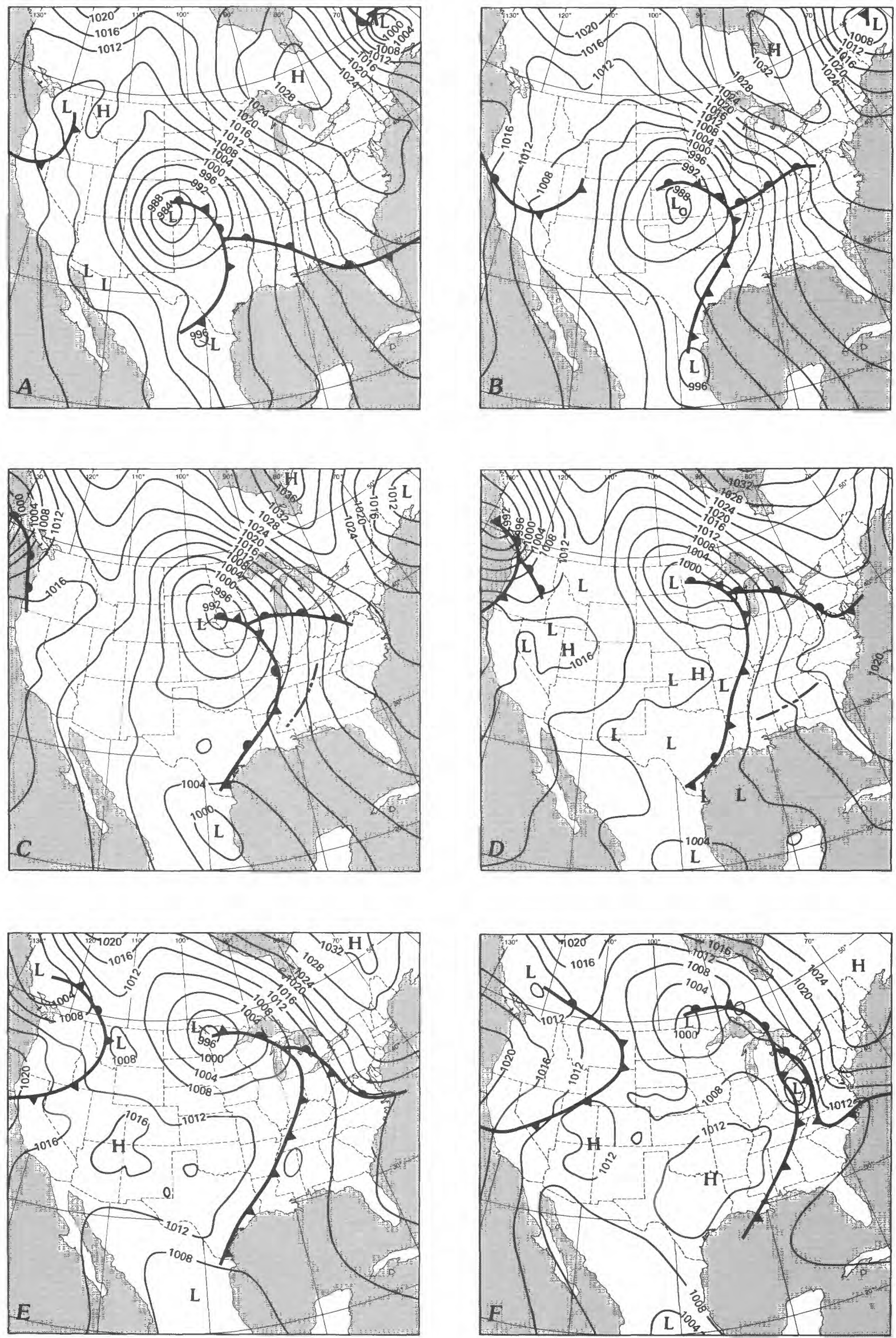
EXPLANATION

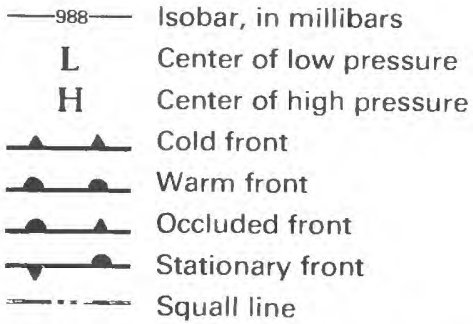

Figure 10.-Surface analyses. $A, 0600$ CST, April 11, 1979, $B, 1800$ CST, April 11, 1979. C, 0600 CST, April 12, 1979. D, 1800 CST, April 12, 1979.E, 0600 CST, April 13, 1979. F, 1800 CST, April 13, 1979.

greater than that expected from a short-lived storm, and adjustments must be made (Scofield and Oliver, 1980).

Qualitatively, an estimate of the amount of convective rainfall depends on cloud-top growth, the existence of any overshooting top cloud, the merge factor, the saturated environment factor, and the observed surface to $500-\mathrm{mb}$ precipitable water. Heavy precipitation is associated with expanding, overshooting cold top, with the merging of cold tops, with persistent white contour over a geographical area, and with high precipitable water content in the atmospheric environment. Each of these empirical factors has its meteorological basis. For example, a convective storm with rapidly expanding cold top indicates strong rising motion and vigorous growth and, consequently, heavy rainfall. Another storm of exactly the same size but with contracting cold top is in a stage of dissipation. Rainfall rates of the two storms could differ by a factor of 20 .

At 0000 CST on April 12, there were two cumulonimbus overshootings with cloud-top temperature less than $-80^{\circ} \mathrm{C}$ over the State of Mississippi (fig. 13A). A smaller top in the northern part of the State covered areas of Yalobusha and Calhoun Counties. Sarepta $1 \mathrm{NNE}\left(34^{\circ} 08^{\prime}\right.$ N., $89^{\circ} 17^{\prime}$ W.), in Calhoun County, had $2.1 \mathrm{in}$. of rain during the first hour of April 12. The much more extensive overshooting tops were in north-central Mississippi and covered Winston, Attala, and Holmes Counties and adjacent areas to the north. By 0230 these two tops had merged and expanded northeastward into northwestern Alabama (fig. 13B). At this time, Louisville, Miss. $\left(33^{\circ} 08^{\prime}\right.$ N., $89^{\circ} 04^{\prime}$ W.), in Winston County had already received 3 in. of rain, while at Pickensville 1E, Ala. $\left(33^{\circ} 14^{\prime}\right.$ N., $88^{\circ} 16^{\prime}$ W.), rain had just begun to fall. The great expansion of the white top area in the $2 \frac{1}{2}$-hour period indicates vigorous growth of convective storm activity as the Pearl, Noxubee, and Tombigbee River headwaters were inundated with heavy rainfall.

\section{PRECIPITATION DISTRIBUTION}

Rain began to fall over western Mississippi on the evening of the 11th. As warm, moist maritime air con-

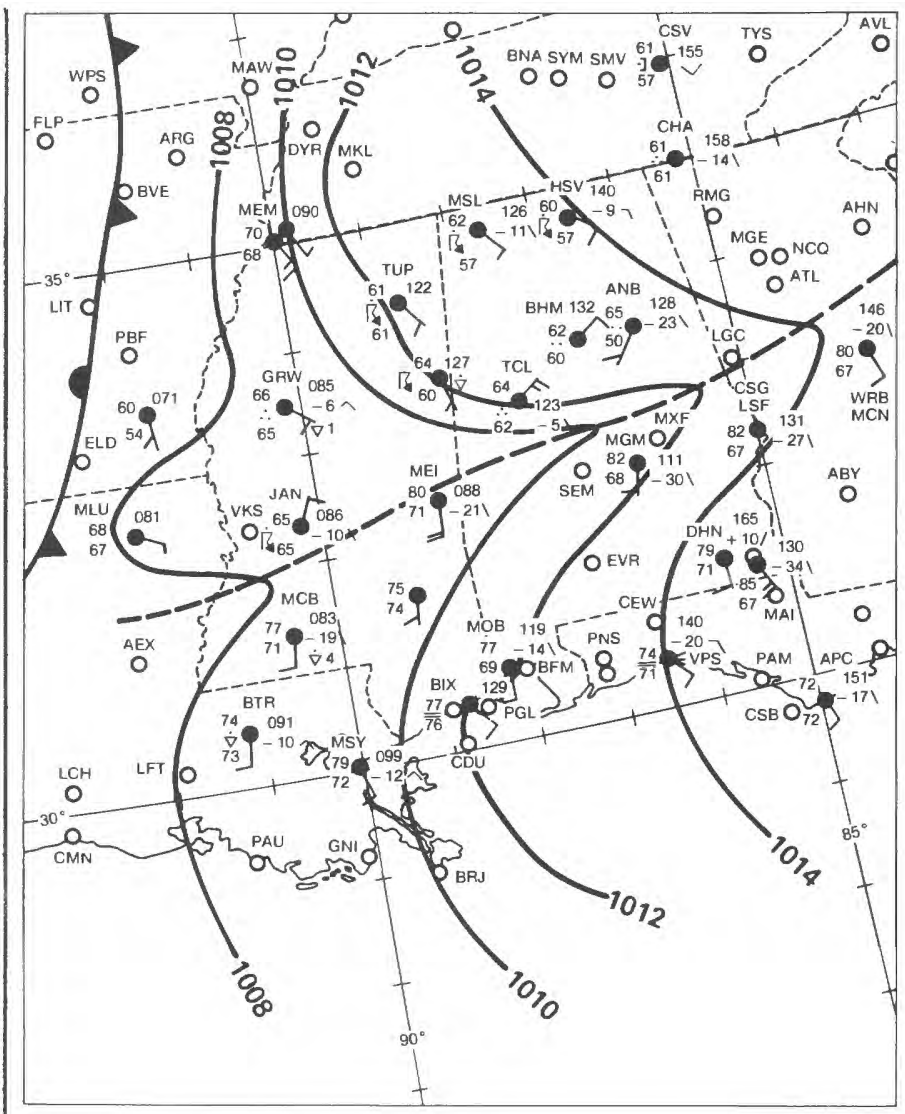

EXPLANATION

1008 - Isobar, in millibars. - _ - Surface trough Interval $2 \mathrm{mb}$ Stationary front

For other symbols, refer to specimen station model in daily weather maps published by National Oceanic and Atmospheric Administration

Figure 11.-Analysis of hourly observations at 1500 CST, April 12, 1979. The deep surface trough extending across the central region of Mississippi and Alabama is a major feature.

verged into the warm sector and was lifted, numerous thundershowers occurred in the unstable environment, and some cumulonimbus turrets reached to a height of $15 \mathrm{~km}$ along the squall line. By midnight, the rain had extended over central and northern Mississippi. Heavy rains were falling over the headwaters of the Pearl, Noxubee, and Tombigbee Rivers, and by the morning of the 12th, rain had spread to adjacent areas of Alabama. The time distribution pattern demonstrates that two different rainfall intensities existed over the Pearl River headwaters. Starting late on the night of the $11 \mathrm{th}$, and mainly during the early morning of the 12th, extremely intense rain fall; this was followed by prolonged heavy rain ending early on the morning of the 13th. For example, Louisville, Miss., in a 31-hour period ending at 0400 on April 13, had a total of 18.7 in. of rain, but $9.1 \mathrm{in}$. of this fell during a 5-hour period ending at 0600 on the 12th. Over the Tombigbee and Alabama Rivers headwaters farther to the east, rain began and ended later, 


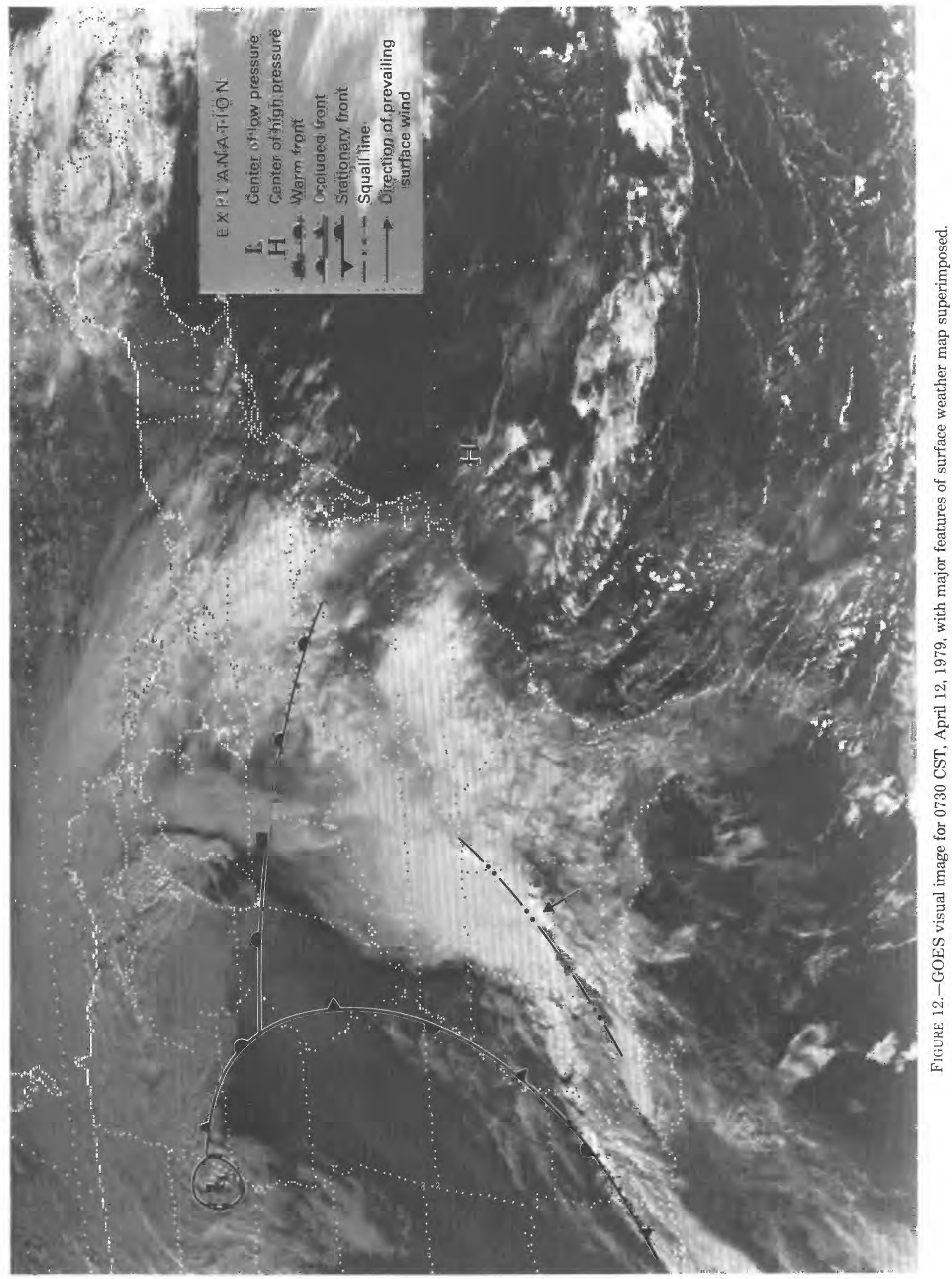


and differences in the rainfall intensity pattern became less distinguishable.

Radar summary maps of the United States east of $100^{\circ} \mathrm{W}$. for 0535 and $1435 \mathrm{CST}$, April 12, are shown in figures $14 A$ and $14 B$. At $0535 \mathrm{CST}$, a solid line of echoes in intensity 5 , corresponding to a rainfall rate of 4.57.1 in./hour, extended from southeast of Louisville, Miss. $\left(38^{\circ} 08^{\prime}\right.$ N., $89^{\circ} 04^{\prime}$ W.), through north-central Alabama. This was near the end of the period when torrential rain had been falling at Louisville 14SE, Miss. At 1435 , a closed area with echo intensity 3 , representing a rainfall rate of 1.1-2.2 in./hour, over east-central Mississippi and north-central Alabama corresponded to the area north of the trough line in figure 11 where rain was prevalent. A cumulonimbus tower having a cloudtop height greater than $15 \mathrm{~km}$ was located near the maximum precipitation center just to the southeast of Louisville, Miss.

As the heavy rain progressed eastward into northwestern Alabama, by 0600 CST, April 12, the squall line reached a position extending from northeastern Alabama to southwestern Mississippi. By 0800, precipitation had reached all of Alabama except the southeast and coastal areas. The headwaters of the Black Warrior, Coosa, and Tallapoosa Rivers in central Alabama had been receiving considerable rain. By late morning, moderate rain began to spread over northwestern Georgia. Rawinsonde observations for Jackson, Miss., and Centreville, Ala., for 1800 on April 12 are shown in figures $15 A$ and $15 B$. The $\mathrm{K}$ indices associated with the soundings were 38 and 39 for Jackson and Centreville, respectively, indicating a thunderstorm probability of 80-90 percent. The wind shears through the cloud layer for both soundings were weak, a condition favoring a longer mature stage once a thunderstorm formed. At 1800 on the 12th, storm precipitation had occurred and was near its end at Jackson but was still in progress around Centreville.

Most of the rain of the storm fell on the 12th and was a consequence of thundershowers associated with a major squall line. The rain continued over the entire northcentral portion of the two-State region throughout the 12th, with only occasional respite before tapering off. Rain essentially ended in Mississippi by noon of April 13 , but continued into the afternoon over some areas of Alabama. Heaviest rain occurred in east-central Mississippi near Louisville, Miss., in the headwater region of the Pearl and Noxubee Rivers. Isohyetal analysis of the total storm rainfall is shown in figure 16 . The maximum point rainfall of more than $21.5 \mathrm{in}$. in about 32 hours was located 14 miles east-southeast of Louisville, Miss. Areal average rainfall over the Pearl River headwaters above Carthage amounted to more than $12 \mathrm{in.}$ Louisville itself, which is 80 miles northeast of Jackson, Miss., received a storm total of $18.7 \mathrm{in}$. Jackson recorded a storm total of $8.60 \mathrm{in}$., of which $4.16 \mathrm{in}$. fell in the 1-hour period ending at 2300 on April 11. The monthly total rainfall of
14.38 in. was 9.73 in. above normal and made April 1979 the wettest April on record for Jackson. To the south of Jackson, the storm rainfall decreased rapidly, dropping to 3 in. about 35 miles southward and to only $0.7 \mathrm{in}$. at Brookhaven, $52 \mathrm{mi}$. south by west from Jackson. Very little rain fell over the southern one-third of the Pearl River basin. A secondary precipitation maximum of 17.3 in. was located 1 mile east of Pickensville, Ala., in the headwaters of the Tombigbee River, just across the Mississippi border in central Alabama. The areal average rainfall over the Tombigbee River basin above Livingston exceeded 8 in. It should be pointed out that both the primary and secondary point maxima of $21.6+$ in. and $17.3 \mathrm{in}$. far exceeded the 100-year 4-day rainfall of 13 in. over the region (Miller, 1964).

In figure 16, it is seen that the axis joining the primary precipitation center at Louisville $14 \mathrm{SE}$, Miss., and the secondary center at Pickensville $1 \mathrm{E} \mathrm{Ala}$, was nearly zonal and took a direction southwest-northeast. So was the orientation of the area receiving 6 in. or more of rain. This phenomenon could be explained by the fact that during the early morning of the 12 th, the very intense thunderstorms that brought heavy rain over Louisville, Miss., took a nearly eastward track. Furthermore, the instability line over central Mississippi and Alabama, along which the incoming maritime air was lifted and processed into rainfall, was alined along a surface trough that had a zonal orientation throughout the late morning and afternoon of the 12th.

Selected rainfall mass curves are shown in figure 17. Time distributions of rainfall for the two reported rainfall maxima at Louisville $14 \mathrm{SE}$, Miss., and Pickensville $1 \mathrm{E}, \mathrm{Ala}$, were estimated using data from the nearest recording gages. Supplementary rainfall data for the storm of April 11-13 are published in table 1 (at end of report) for convenient reference.

Later in the month rain fell again, over Mississippi during the period April 21-23 and over Alabama on April 24-26. The area receiving the most rain was the lower Pearl River drainage basin, where very little or no rain fell during the major storm of April 11-13. A maximum of $6.4 \mathrm{in}$. was reported at Ruth, Miss., about 65 miles south of Jackson. However, when averaged over the still-flooded areas of the two-State region, less than 2 in. was added. The main effect of this postflood rain was to retard floodwater recession and thus to prolong the period of flooding. The Pearl River at Monticello, Miss., was above flood stage for 27 days during the month of April, returning to its normal channel in early May.

\section{GENERAL DESCRIPTION OF FLOODS}

The area affected by the floods of March-April 1979 in Alabama, Mississippi, and adjacent parts of Georgia and Louisiana is shown in figure 1. Streams throughout the area were high in early March as a result of storms 


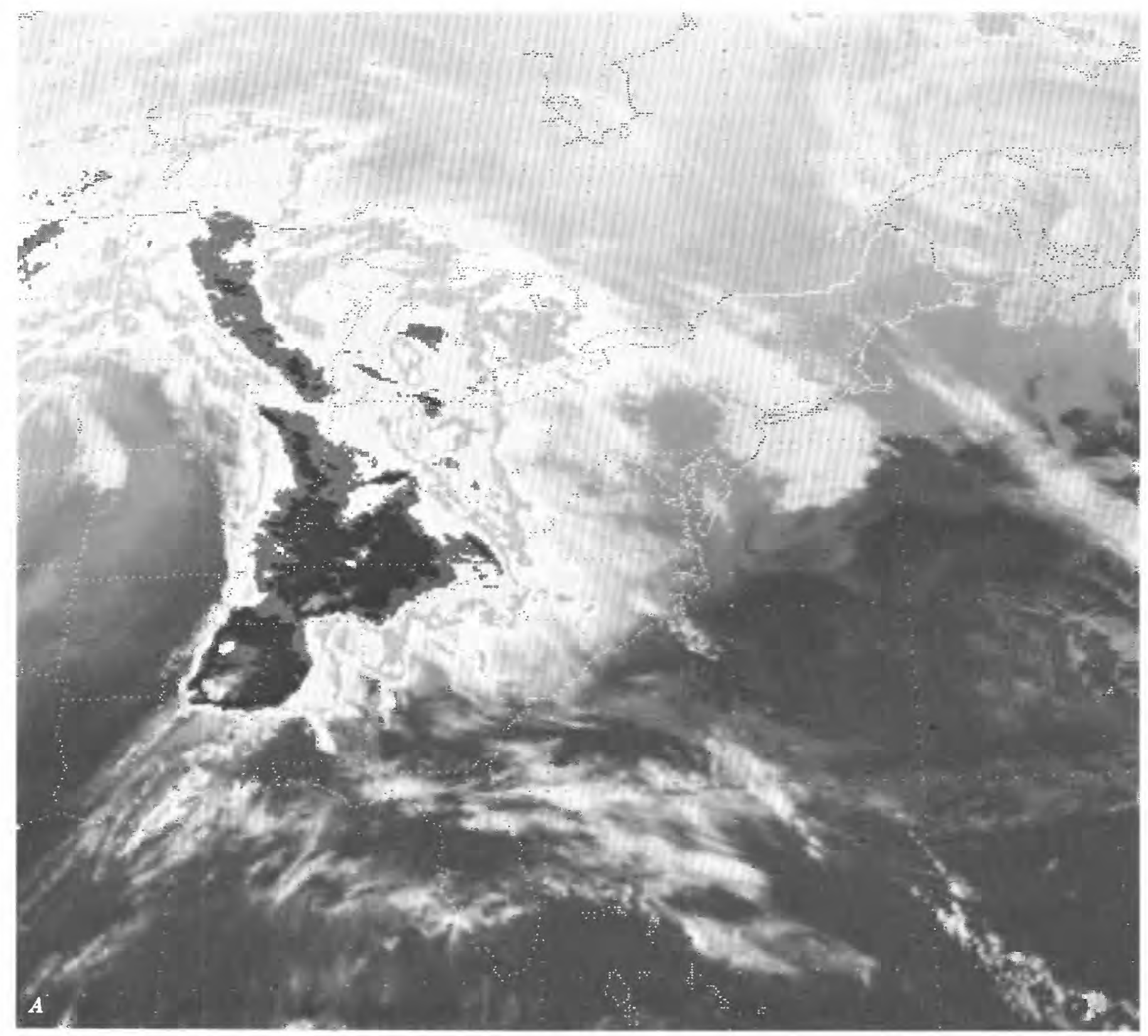

FIGURE 13A-GOES infrared images enhanced by Mb curve:0000 CST, April 12, 1979.

centered over the Chunky River basin in east-central Mississippi and the Satilpa Creek basin in west-central Alabama. Successive storm periods in March and April over central Alabama and Mississippi increased soil moisture conditions favorable for high runoff yields and culminated in widespread, recordbreaking floods in midApril.

Data at 221 streamflow gaging sites are presented in table 2 (at end of report). The first column in table 2 lists a number assigned to each site, for use only in this report. For convenience, these site numbers are used throughout this report in illustrations, tables, and discussions.

Flood data in table 2 are presented in the downstream order used in the annual water-resources data reports. Gaging station records are listed in a downstream direction along the mainstream, and stations on tributaries are listed between stations on the mainstream in the order in which those tributaries enter the mainstream. Stations on tributaries entering above all mainstream 


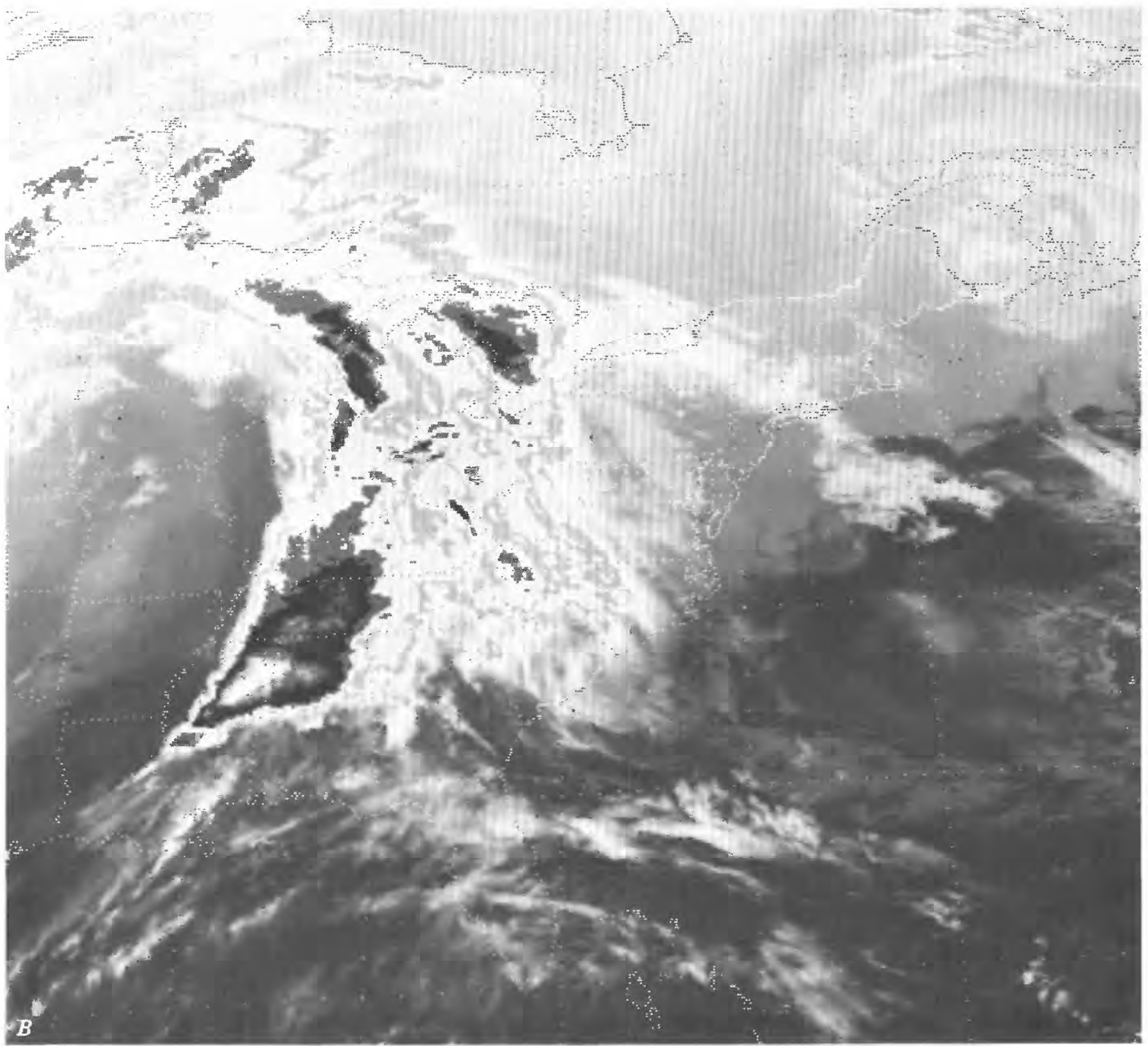

FIGURE $13 B$ - GOES infrared images enhanced by Mb curve: 0230 CST, April 12, 1979.

stations are listed before the first mainstream station. Stations on tributaries to tributaries are listed in a similar manner.

Each gaging station has been assigned a permanent station number (column 2) conforming to the downstream order. The 8-digit permanent station number (for example 02441500) includes a 2-digit part number ("02") plus a 6-digit "downstream order number" ("441500"). In this report, the records are listed in downstream order by part. The part number refers to an area whose boundaries coincide with certain natural drainage lines. Records in this report are in Part 2 (South Atlantic slope and Eastern Gulf of Mexico basins) and Part 7 (Lower Mississippi River basin).

Datum of gage above National Geodetic Vertical Datum (NGVD) is the elevation of the "zero" reading of the gage.

The location of each gaging station site is shown in figure 18. The site numbers on that map correspond to those in table 2. 

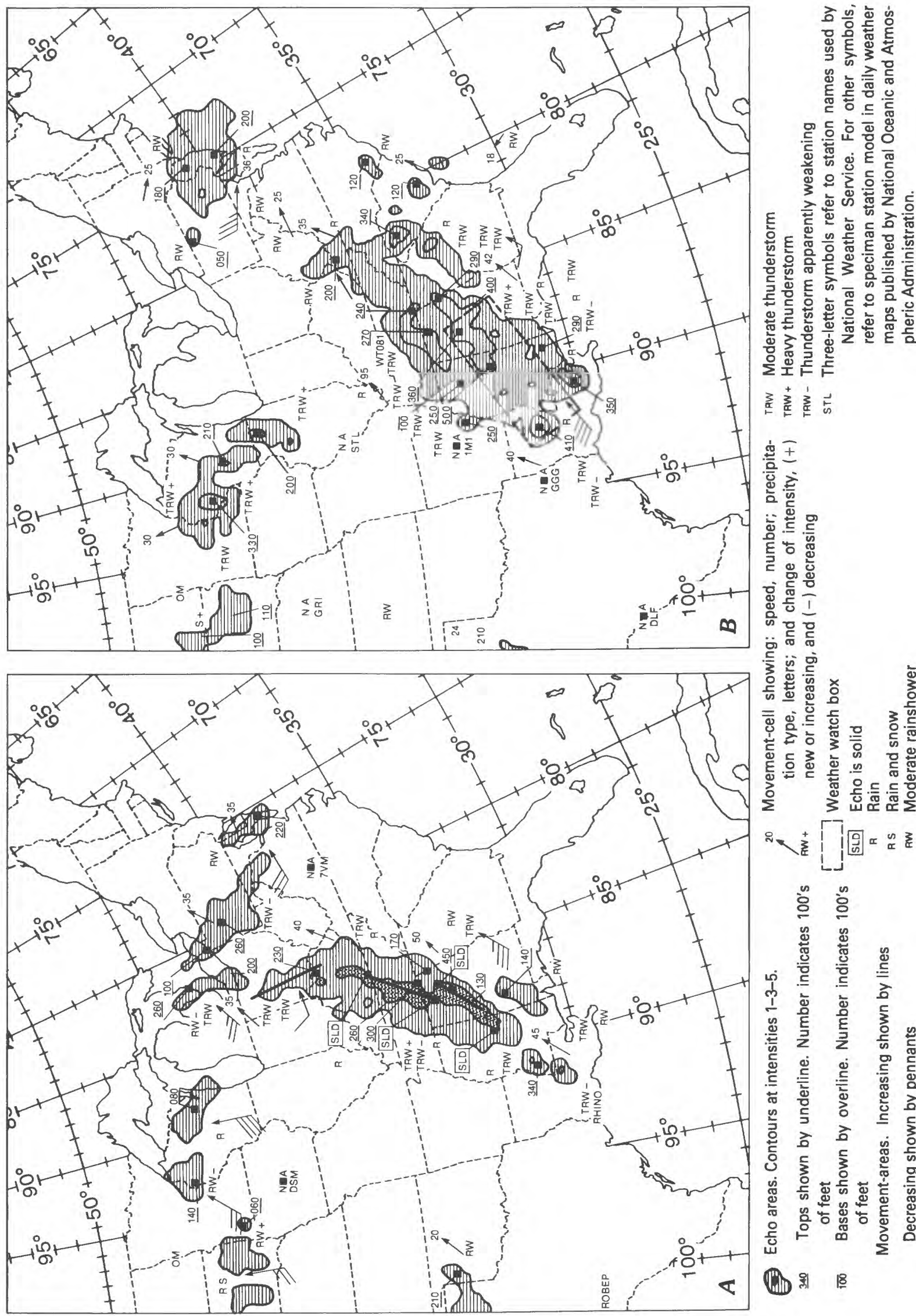

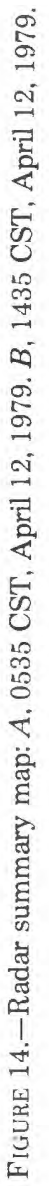



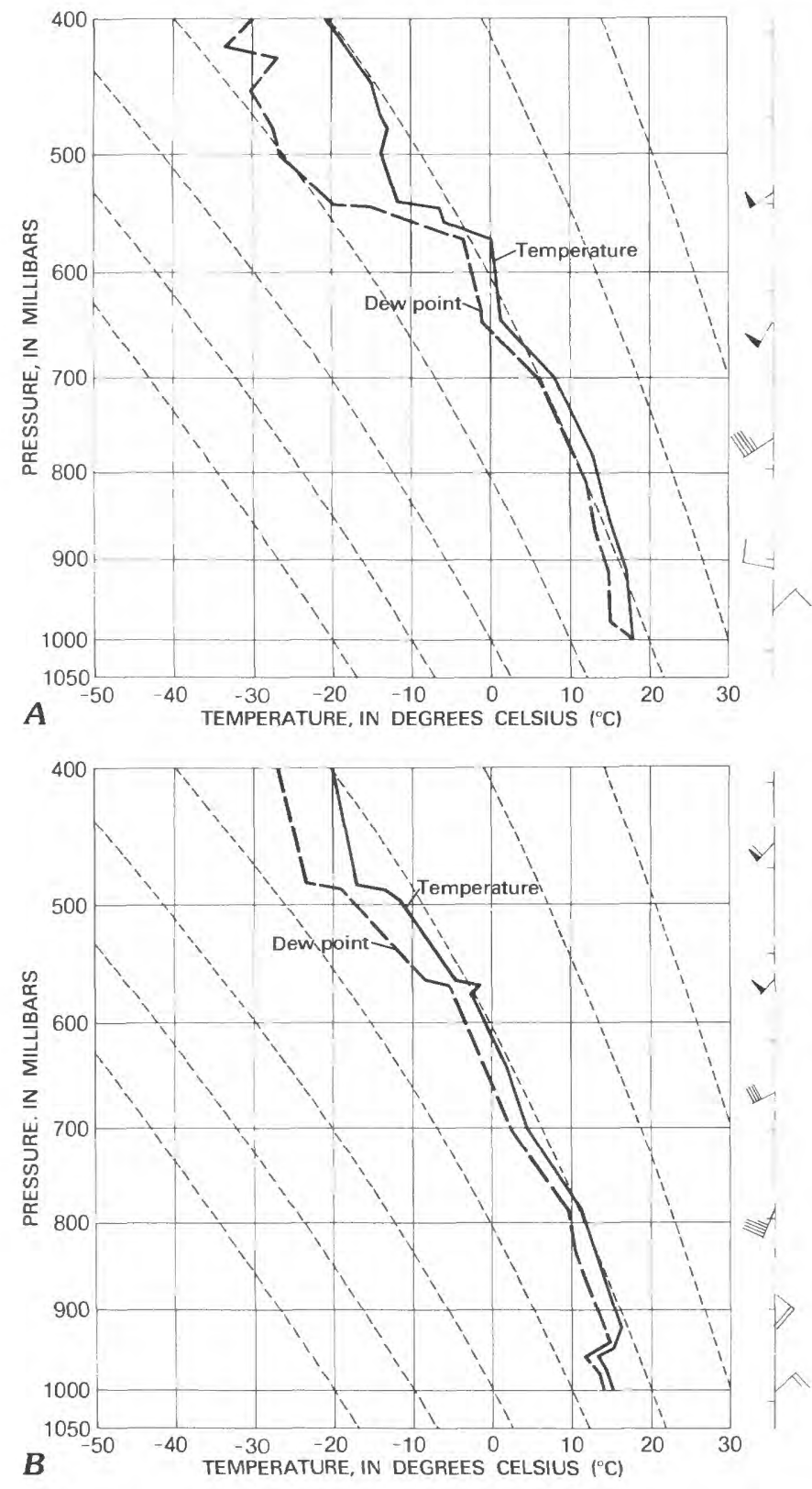

\begin{abstract}
$\checkmark$ Direction and speed of wind. Pennants and barbs on shaft indicate wind speed. Pennants $=50$ knots, long barb $=10$ knots, short barb $=5$ knots. North is at top. Example shown indicates 60-knot northeasterly wind
\end{abstract}

Figure 15,-Rawinsonde plot, 1800 CST, April 12, 1979. A, Jackson, Miss. $B$, Centreville, Ala.

\section{MAGNITUDE OF FLOODS}

Peak discharges at about one-fourth of the streamflow stations were the greatest recorded since the stations were established. In the Pearl River basin above Jackson, record floods occurred at most gaged sites. Figure 19, which relates flood discharge rates to corresponding drainage areas, provides a comparison of flood discharges in 1979 with those of the greatest known floods in the area. Curves A and B (fig. 19), developed by Crippen and Bue (1977), are defined by the greatest known floods through September 1974, in areas above and below the fall line, respectively, in the regions that include the area of this report. The curves provide a guide for estimating potential maximum floodflows. Curve C (fig. 19) is an enveloping curve through the greatest discharge rates during the 1979 floods. The curves indicate that floods generally about one-third greater than those in 1979 may occur in large basins and that floods two or three times greater may occur in small basins. However, the all-season probable maximum precipitation (PMP) over the central MississippiAlabama region is 27 in. in 72 hours over a 5,000-squaremile area (Schreiner and Riedl, 1978). Therefore, potential floods much greater than those observed in April 1979 or those indicated by an envelope curve of historic floods are likely to occur if precipitation is near or equal to the magnitude of the PMP.

\section{FLOOD DAMAGES}

Flood damages provide a measure of the relative magnitude of floods. Exact amounts of flood damage for this flood, which extended over a wide area, are not known. Estimates of flood damage were obtained from the U.S. Army Corps of Engineers, Mobile District (1980a), and Vicksburg District (1980b). Summaries of estimated damages on main streams and principal tributaries for the floods of March and April 1979 are shown in table 3 (at end of report). In the area of this report, estimated flood damages were $\$ 41,916,000$ for March 1979 and $\$ 344,239,000$ for April 1979.

During April 1979, 75 percent of the total flood damage occurred in the Pearl River basin, and 65 percent of the total damage occurred in Jackson, Miss., and vicinity (Hinds and Rankin Counties).

At least nine lives were lost.

The Federal Emergency Management Agency reported that within the areas declared eligible for Federal disaster relief assistance, 5,549 flood insurance policies were in force in Mississippi, with $\$ 130,076,100$ of coverage, and 1,450 flood insurance policies were in force in Alabama, with $\$ 45,853,000$ of coverage, prior to the April 1979 flood. 

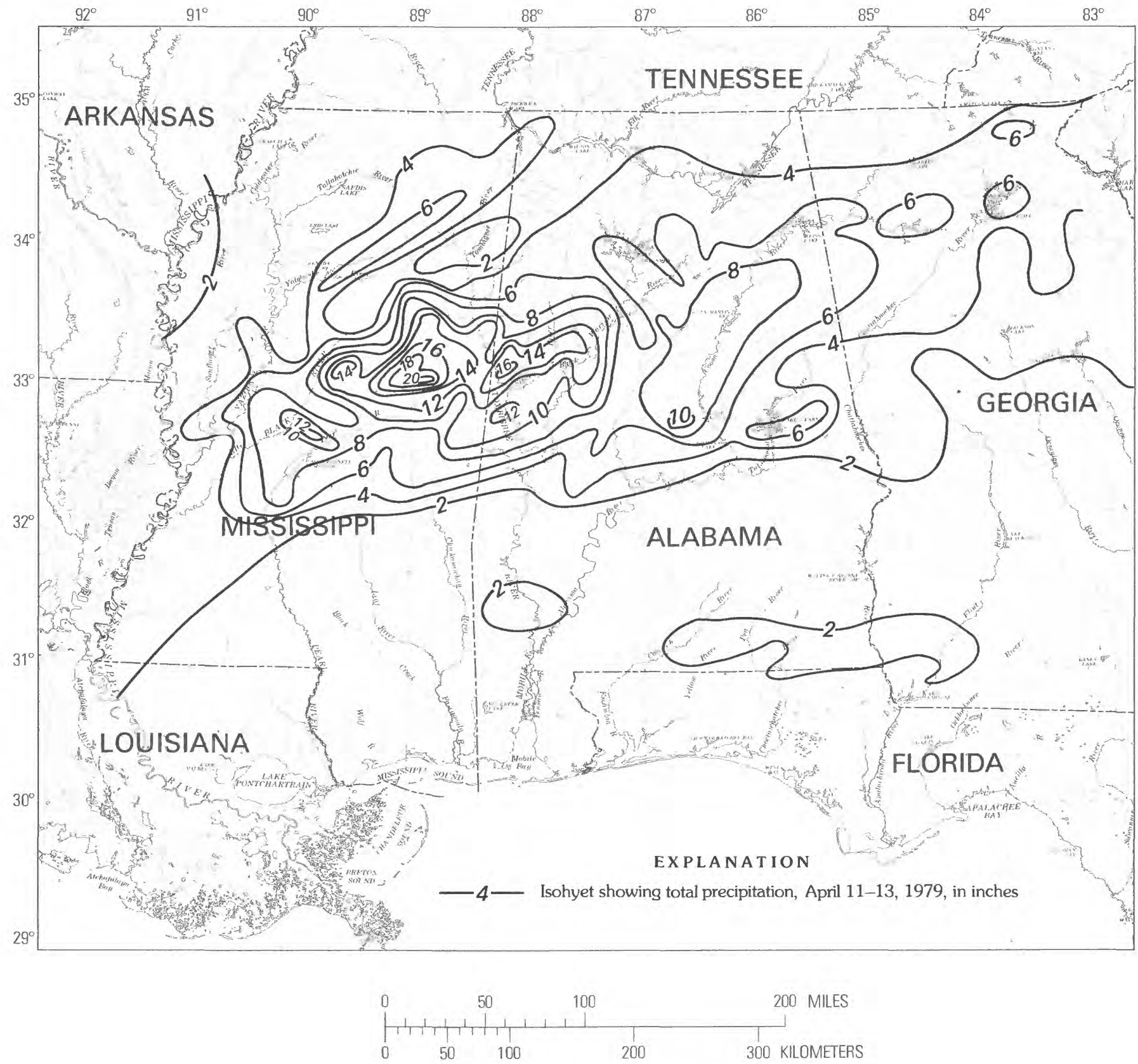

FIGURE 16.-Isohyetal analysis of storm rainfall, April 11-14, 1979.

\section{FLOOD FREQUENCY}

Knowledge of the magnitude and probable frequency of recurrence of floodflows is useful in designing and locating structures to be situated on the flood plain so as to minimize flood losses and in providing a technical basis on which to develop criteria for flood-plain management.
Frequency of flooding was derived from a statistical evaluation of historical records of floodflows from a network of streamflow gaging stations distributed throughout the flood area (fig. 18). The techniques generally used to determine flood-frequency relations are those described by the U.S. Water Resources Council (1977). Recurrence intervals at most sites in Mississippi were obtained from flood-frequency relations described 

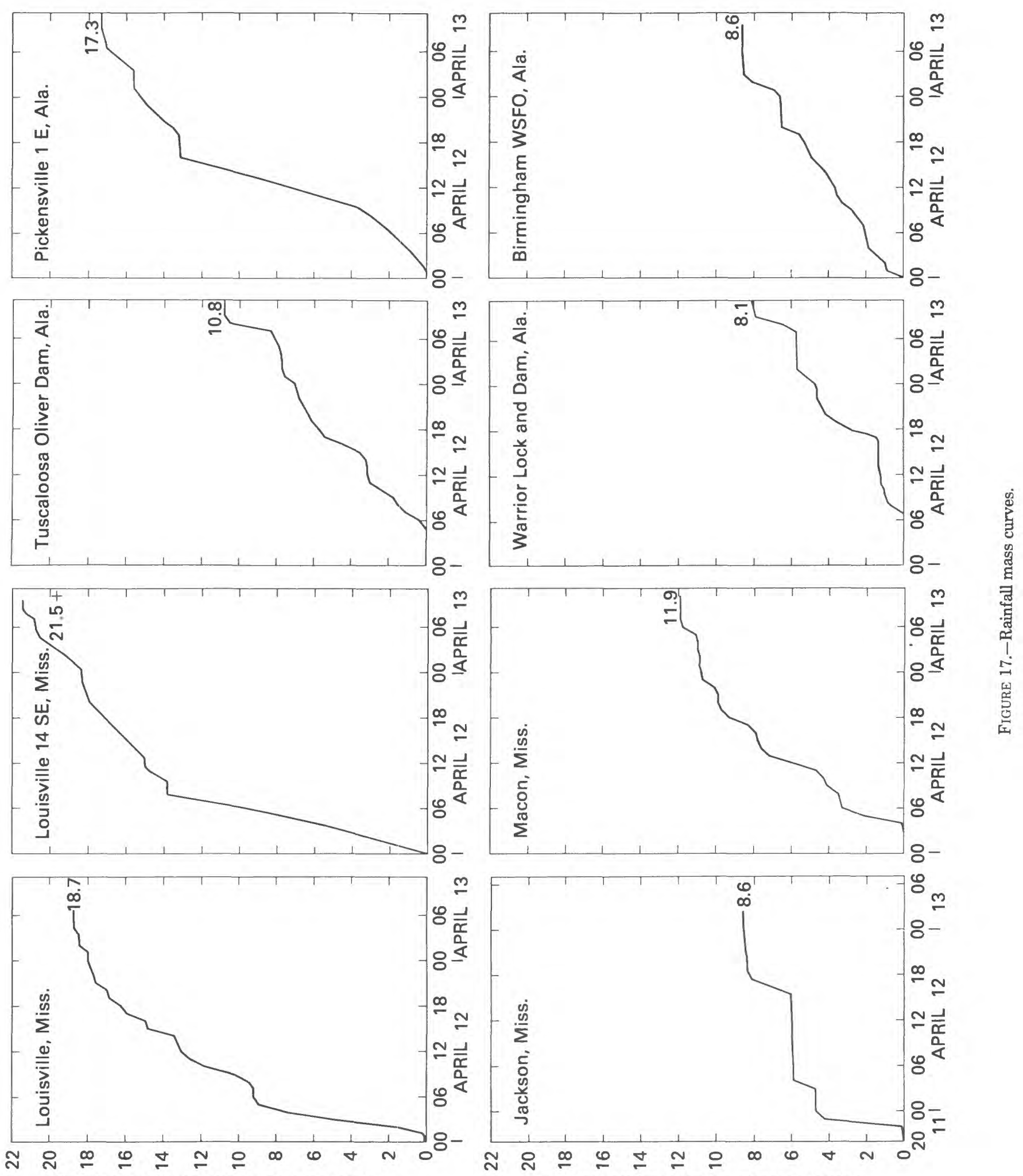

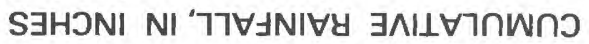

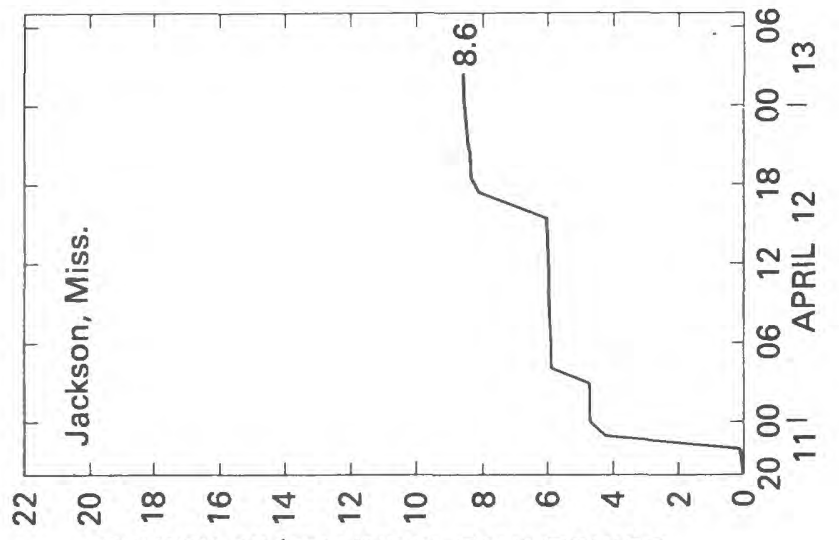

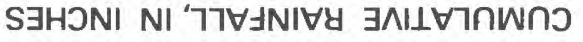




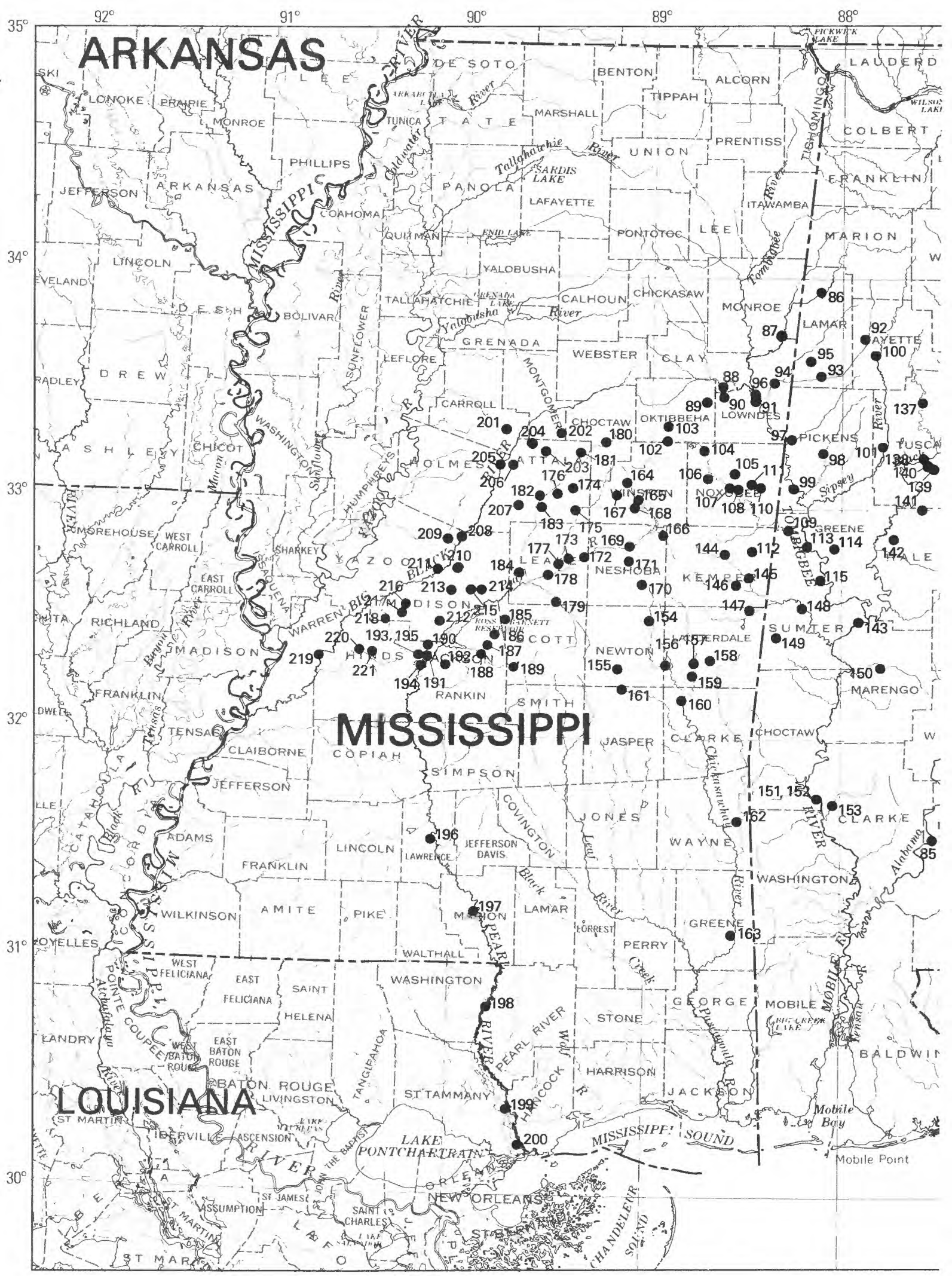

FIGURE 18.-Location of flood determination sites. 


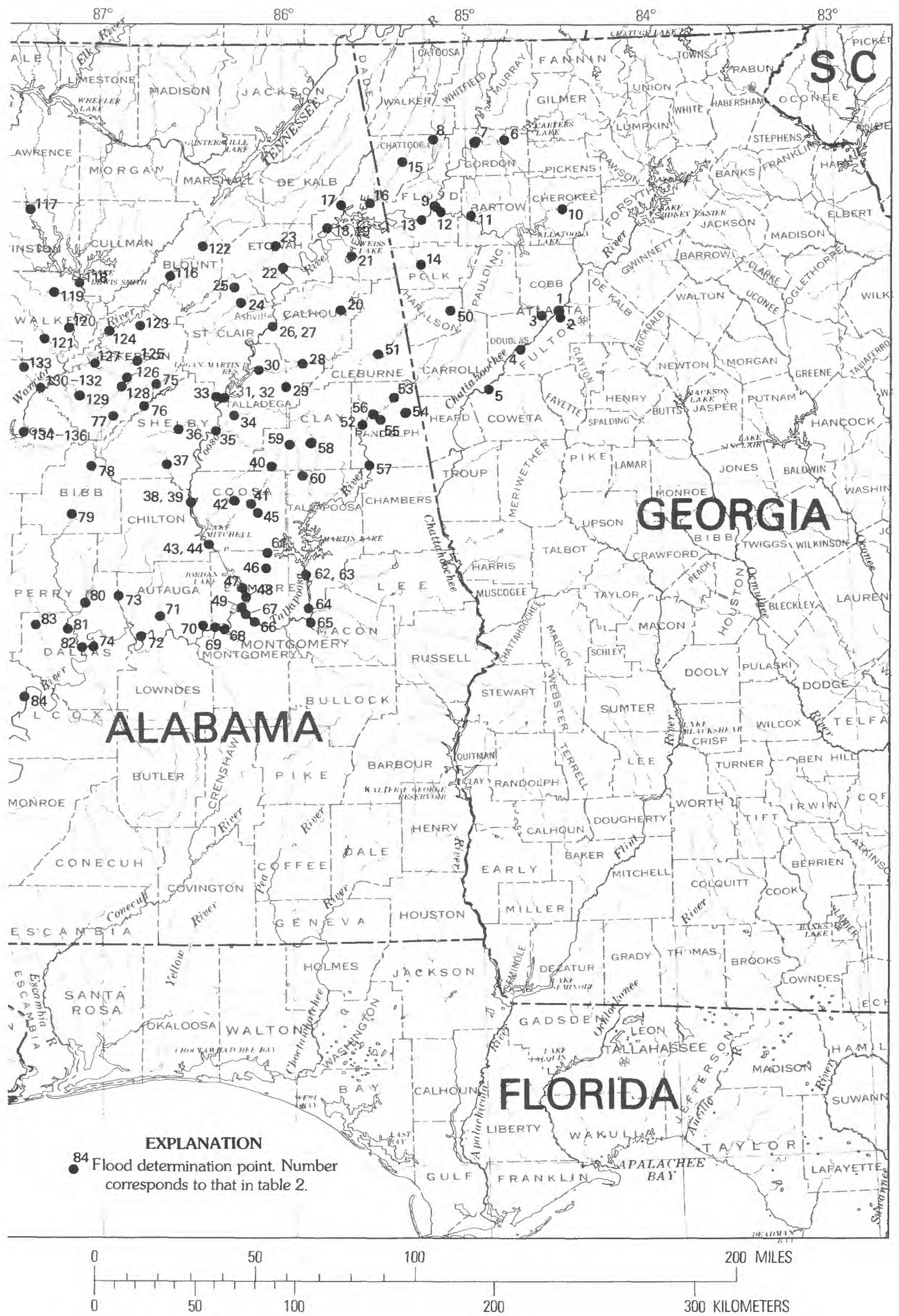




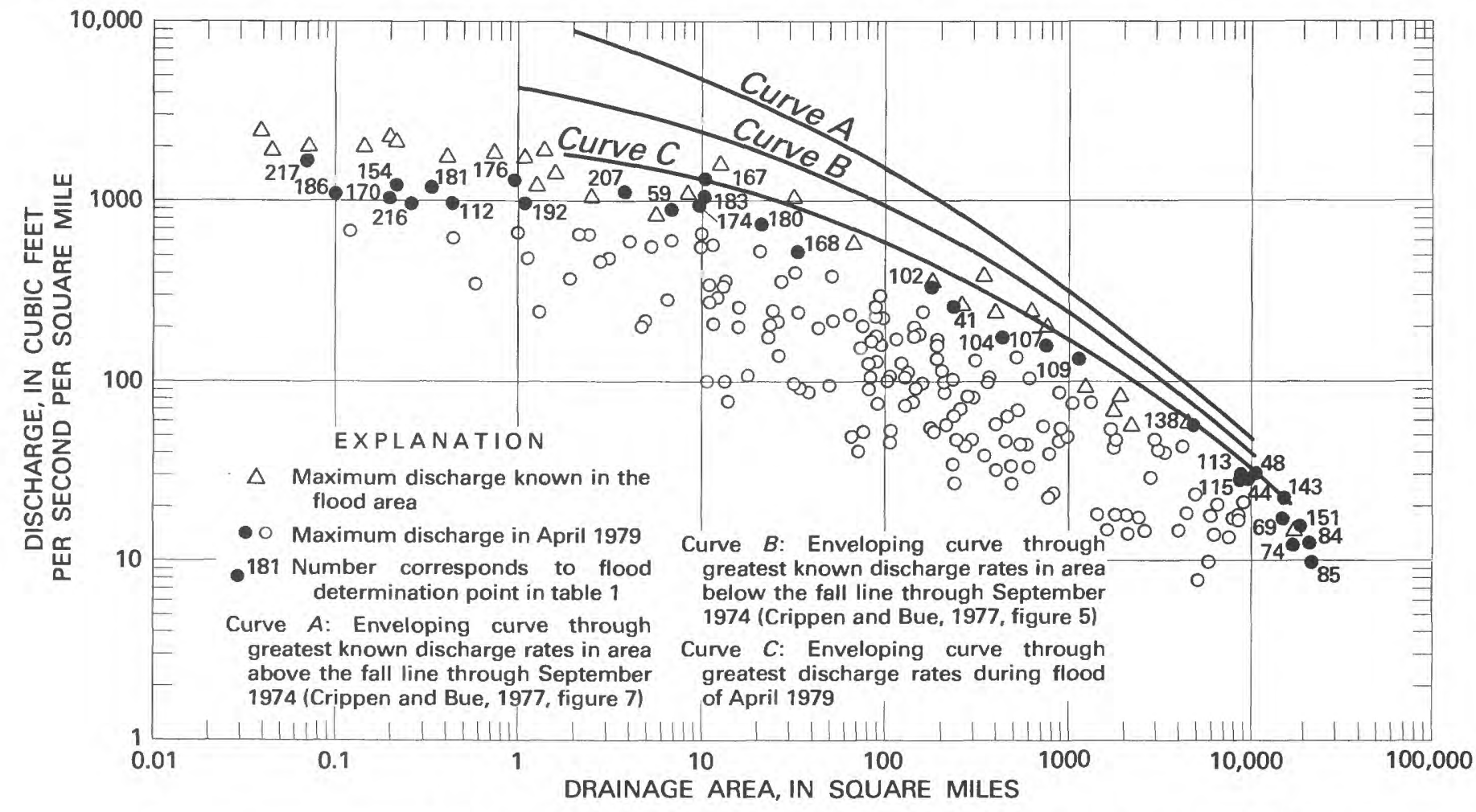

Figure 19.-Comparison of April 1979 peak discharges with maximum known flood peaks in Mississippi and Alabama.

by Colson and Hudson (1976), and in Alabama by Olin and Bingham (1977). At sites where the 1979 flood events significantly affected flood-frequency relations, 1979 peak discharges were combined with those of other floods to determine the floodflow potential.

Recurrence interval, as applied to flood events, is the average number of years within which a given flood peak will be exceeded once. Frequencies of floodflows may also be stated in terms of their probabilities of occurrence, which for large floods are virtually the reciprocals of the recurrence intervals. Thus, a flood with a 25-year recurrence interval would have a 4-percent chance of being exceeded in any given year, and a flood with a 100-year recurrence interval would have a 1-percent chance of being exceeded in any given year. Recurrence intervals are average figures-the average number of years that will lapse between occurrences of floods that exceed a given magnitude. The occurrence of a major flood in one year does not reduce the probability of that flood being exceeded in the next year, or later in the same year.

In the area of this report, the lengths of available streamflow records generally are adequate to reliably define flood-frequency relations for recurrence intervals of up to 100 years. The 100-year (1-percent chance) flood discharges at most sites are shown in table 2 for comparison with the discharges of the March-April 1979 floods. The 100-year flood discharge is not shown for sites with less than 10 years of record or for sites on streams materially affected by regulation or diversion.

Estimates of 100-year discharges for some streams in small drainage basins were based both on observed peak discharges and on synthetic discharge data generated with a calibrated rainfall-runoff model. Peak discharges based on modeling techniques are identified in table 2 by appropriate footnote.

\section{RESERVOIRS}

Many reservoirs are located on the main stems of the Coosa, Tombigbee, and Alabama Rivers. A summary of stages and contents of selected reservoirs in the Coosa, Tallapoosa, Black, Warrior, Chickasawhay, and Pearl River basins is presented in table 4 (at end of report).

Many relatively small Soil Conservation Service flood-control reservoirs are located in the Tombigbee, Pearl, and Black River basins. Emergency spillways of some of these reservoirs were overtopped. The Soil Conservation Service reports that substantial reductions of peak stages existed in the reaches just below the reservoirs. Storage in the reservoirs in the Pearl River basin, about 18,000 acre-feet, had little effect on Pearl River at Jackson, which discharged about 250,000 acre-feet per day for several days. 


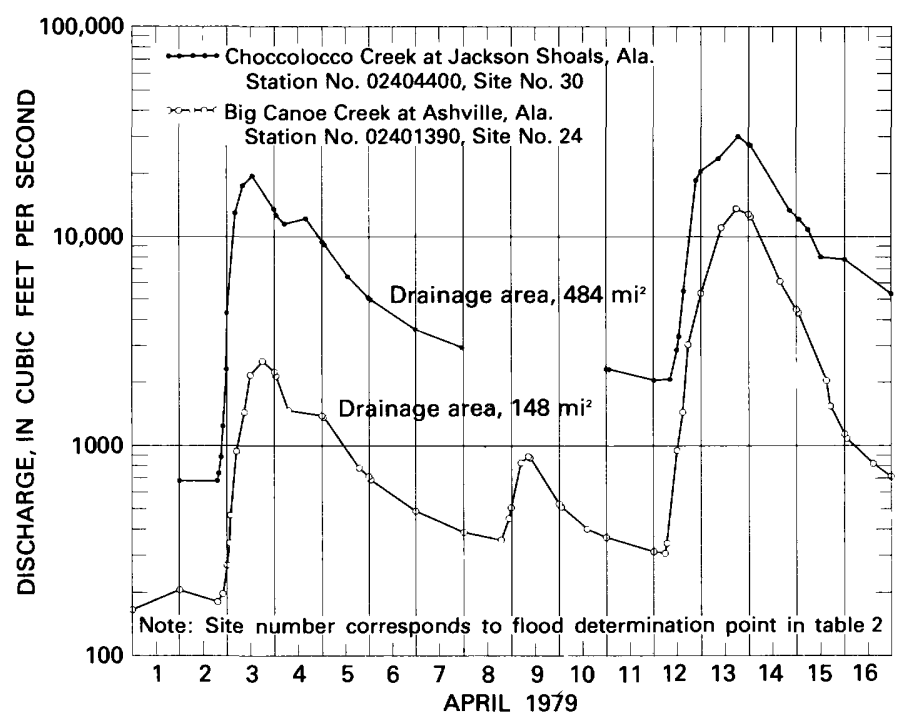

FIGURE 20.-Discharge at gaging stations in the Coosa River basin in Alabama, April 1-16, 1979.

Bluff and Loakfoma Lakes, adjacent lakes in the Noxubee National Wildlife Refuge, 38 miles upstream from Macon, Miss., were washed out near the crest of the flood. They are shallow lakes located on the Noxubee River flood plain. Estimated total storage is less than 5,000 acre-feet. Levees surrounding the two lakes developed six crevasses during the flood.

Storage in Okatibbee Reservoir on Okatibbee Creek near Meridian, Miss., during both the March and April floods caused substantial reductions in peak stages along Okatibbee Creek and upper Chickasawhay River, according to the U.S. Army Corps of Engineers. Okatibbee Reservoir crested March 5 at elevation 350.7 feet (table 4), fell to 341.9 feet April 1, and crested again April 15 at 355.2 feet (3.8 feet below the crest of the emergency spillway). The peak inflow of $15,100 \mathrm{ft}^{3} / \mathrm{s}$ April 13 was reduced to an outflow of $1,240 \mathrm{ft}^{3} / \mathrm{s}$, according to the U.S. Army Corps of Engineers.

The Ross Barnett Reservoir on the Pearl River just upstream from Jackson, Miss., is primarily a watersupply and recreation reservoir with a relatively small capacity for storing floodflows. On April 14, prior to the arrival of the flood crest, the reservoir was drawn down to pool elevation of 296.5 feet. Floodwaters were stored in the reservoir, and on April 16 the pool elevation crested at 299.8 feet. This represented an increase in storage of about 120,000 acre-feet during the passage of the flood wave.

\section{MAJOR RIVER BASINS OF EASTERN GULF OF MEXICO}

\section{COOSA RIVER BASIN}

Recurrence intervals of peak discharges in the Coosa River basin in Georgia, upstream from Weiss Reservoir, were in the 10- to 20-year flood range. Little River, just upstream from Weiss Dam in Alabama, had a peak discharge slightly less than that of the 100-year flood.

Severe flooding occurred along the Coosa River and its tributaries downstream from Weiss Dam near Centre, Ala., to its confluence with the Tallapoosa River near Montgomery, Ala. Along the Coosa River main steam, recurrence intervals of peak flows were approximately 5 years at Gadsden (site 22), 50 years at Childersburg (site 35), and more than 100 years at Jordan Dam (site 48). Tributaries to the Coosa River between Weiss Dam and Jordan Dam near Wetumpka, Ala., recorded peak discharges generally approaching 100-year floods. An exception was Hatchet Creek basin, where peak flows were nearly double those of a 100-year flood.

Hydrographs of discharge April 1-16, 1979, at streamflow gaging stations on Big Canoe Creek at Ashville, Ala. (site 24), and Choccolocco Creek at Jackson Shoals, Ala. (site 30), are shown in figure 20.

\section{TALLAPOOSA RIVER BASIN}

The flood of April 1979 in the Tallapoosa River basin was characterized by heavy rainfall and high runoff yields. The combination of high antecedent streamflow, saturated soils, and intense rainfall resulted in widespread flooding. At the gaging station, Tallapoosa River at Wadley, Ala. (site 57), a new maximum (period 1924-79) occurred on March 4, 1979, and that in turn was exceeded by the April 14, 1979, flood peak. Lesser peaks occurred April 3, 4, and 9.

In the Alabama counties of Clay, Randolph, and Tallapoosa, peak discharges were in the 100-year flood range. The high unit runoff in this part (middle third) of the basin is documented at two discontinued gaging stations. Harbuck Creek near Hackneyville, Ala. (site 59), and Hillabee Creek near Hackneyville, Ala. (site 60).

Along the main stem Tallapoosa River, the peak discharge at Heflin, Ala. (site 51), was that of a 25-year flood, and at Wadley, Ala. (site 57), was greater than the 100-year flood. The peak discharge passing Martin Dam (site 63), $142,000 \mathrm{ft}^{3} / \mathrm{s}$, attenuated to $128,000 \mathrm{ft}^{3} / \mathrm{s}$ at the gaging station Tallapoosa River below Tallassee, Ala. (site 64), which equaled the February 1961 flood and was exceeded only by the December 1919 flood (the highest since 1886).

Hydrographs of discharge of the Tallapoosa River, April 3-22, at gaging stations near Heflin, Ala., and at Wadley, Ala., are shown in figure 21.

\section{ALABAMA RIVER BASIN}

The combined flows of the Coosa and Tallapoosa Rivers resulted in a peak discharge in the 50-year frequency range on the Alabama River at Montgomery, 


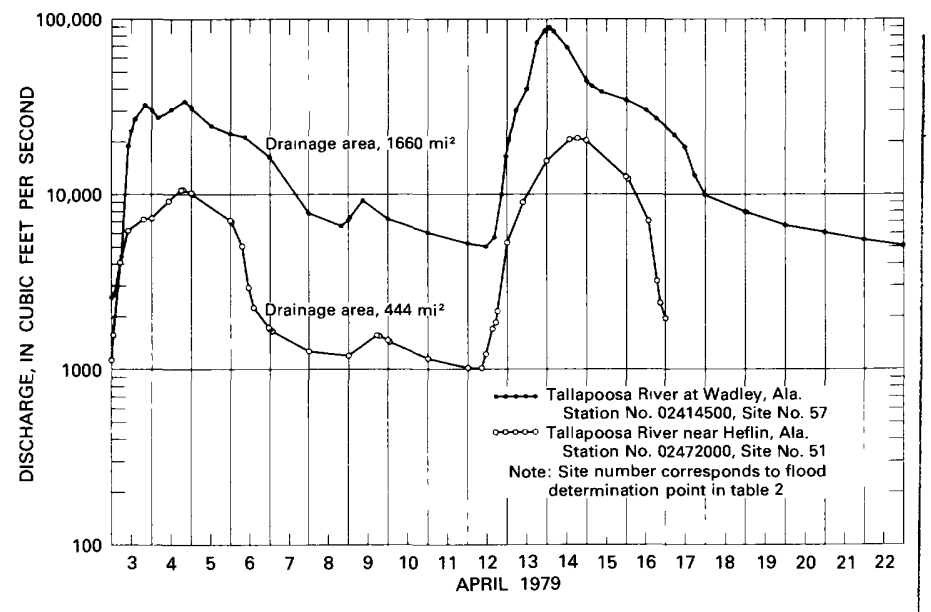

Figure 21.-Discharge at gaging stations in the Tallapoosa River basin in Alabama, April 3-22, 1979.

Ala. (site 68). The April 1979 peak discharge at the gaging station near Montgomery (site 69) was exceeded only by the April 1, 1886, March 3, 1888, and February 26, 1961, floods. The April 1979 flood was the fourth highest flood since 1833 .

Discharge of the Alabama River was measured near the peak on April 18, 1979, at Selma, Ala. (site 74). The measured discharge (less than the peak discharge) was within 10 percent of the 50-year flood at Selma. This discharge was exceeded only by the floods of March 1, 1961, and April 8, 1886.

Peak discharge at the most downstream gaging station at Claiborne, Ala. (site 85), was the fourth highest peak since 1886 and was in the 25-year flood range.

In the Cahaba River basin, in central Alabama, flood magnitudes varied widely. Recurrence intervals of peak discharges on Shades Creek, a tributary to the Cahaba River upstream from Centreville, draining part of the urban and industrial areas of Birmingham, ranged from about 10 years in the upstream part of the basin to 100 years downstream from Greenwood in the lower part.

Peak discharges on the Cahaba River at Centreville (site 79) and Marion Junction (site 81) were the fourth greatest floods during the period of record (1902-1979) and were in the 25-year flood range.

Hydrographs of discharge at gaging stations on the Cahaba River near Cahaba Heights, at Centreville, and near Marion Junction, Ala., were shown in figure 22.

\section{TOMBIGBEE RIVER BASIN}

\section{TOMBIGBEE RIVER UPSTREAM FROM GAINESVILLE, ALA.}

In the Tombigbee River basin, the greatest rains fell in the middle third. Severe flooding occurred along the Tombigbee River from Columbus, Miss., downstream to the mouth and on tributaries upstream from Choctaw County, Ala. Upstream from Columbus, Miss., flooding was not severe. Tributaries in northern Alabama flowing westward into Mississippi, the Buttahatchee River and Luxapallila Creek, experienced only minor flooding in the upstream reaches.

Recorded peak discharges on the Buttahatchee River were in the 2-year frequency range. In the adjacent basin to the south, Luxapallila Creek, a major left-bank tributary of the Tombigbee River at Columbus, Miss., had a peak flow of $40,400 \mathrm{ft}^{3} / \mathrm{s}$, with a recurrence interval greater than 50 years at the gaging station near Columbus (site 96). Yellow Creek, which flows into Luxapallila Creek just downstream from the Steens, Miss., gaging station contributed substantially to the floodflow.

Along the main stem Tombigbee River, peak discharges upstream from Cochrane, Ala., were in the 10-year range. In contrast, the peak discharge at Gainesville, Ala., augmented by inflow between Cochrane and Gainesville, exceeded that of a 100-year flood and was the greatest known since at least 1892.

Tributary inflow between Cochrane and Gainesville was documented at two gaging stations-Sipsey River near Elrod, Ala. (site 101), and Noxubee River near Geiger, Ala. (site 109).

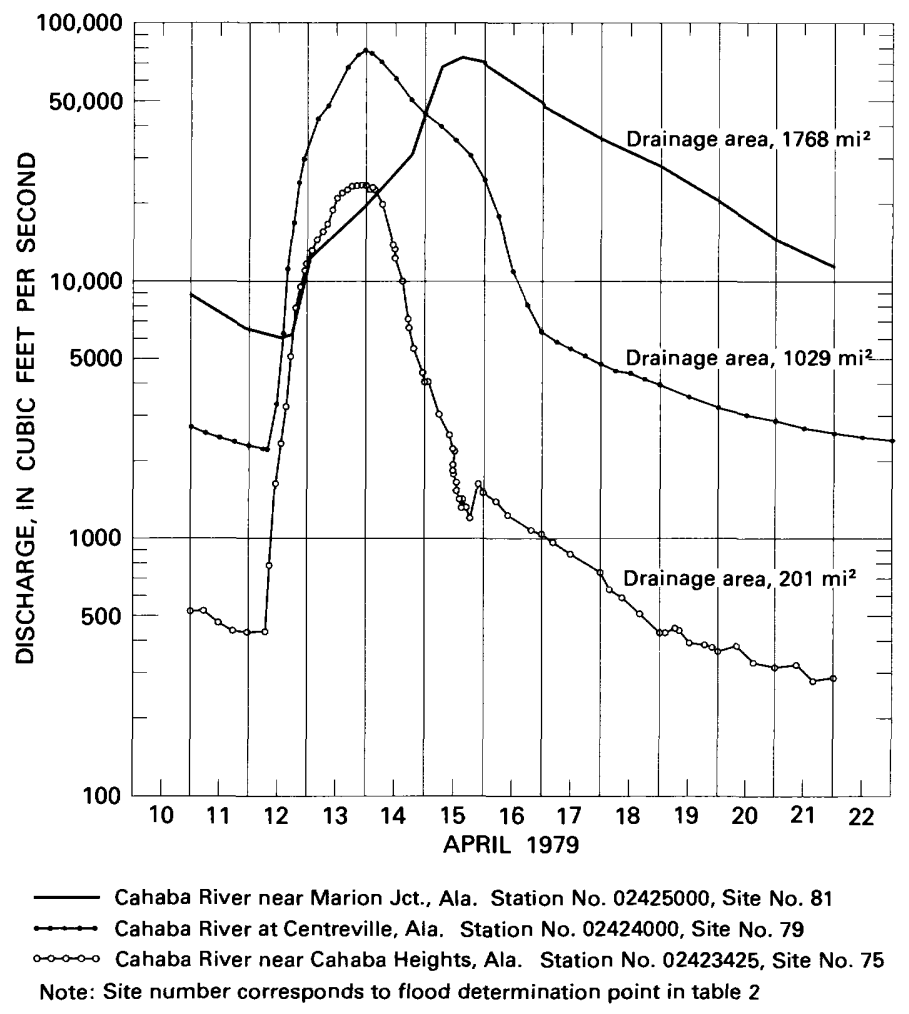

Figure 22.-Discharge at gaging stations in the Cahaba River basin in Alabama, April 10-22, 1979. 


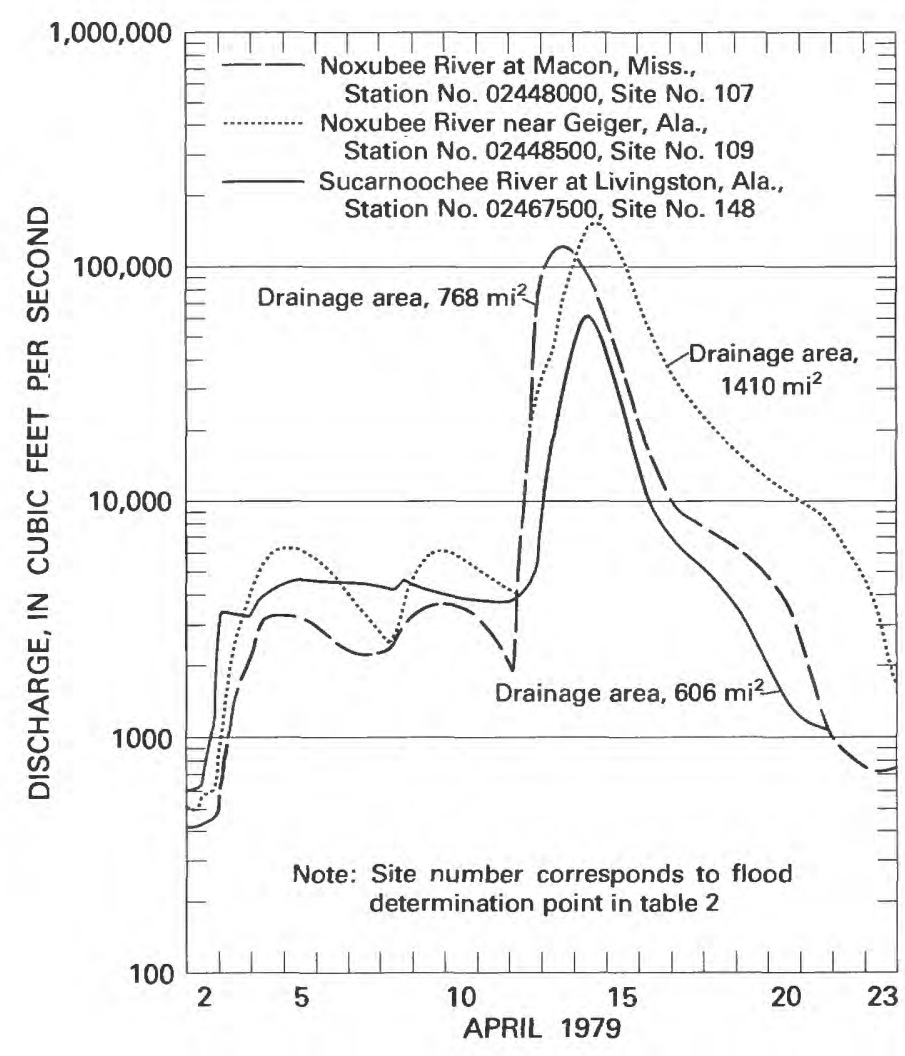

FIGURE 23.-Discharge at gaging stations on the Noxubee River at Macon, Miss., and near Geiger, Ala., and on the Sucarnoochee River at Livingston, Ala., April 2-23, 1979.

Sipsey River, located between the Tombigbee and Black Warrior Rivers, flows southwestward to the Tombigbee River. The peak discharge of the Sipsey River at the gaging station near Elrod (site 101) was the second highest known flood since 1900 and was exceeded only by the flood of February 23, 1961. The recurrence interval of the peak discharge April 13, 1979, was about 25 years.

Noxubee River, which flows southeastward from Mississippi to its confluence with the Tombigbee River in Alabama, experienced the heaviest rainfall of the storm, over most of its drainage area. At the gaging station at Macon, Miss. (site 107), the stream reached a stage of 38.97 feet (discharge $125,000 \mathrm{ft}^{3} / \mathrm{s}$ ), which is 6 feet higher than the flood of March 1951, the previous maximum of record. At Geiger, Ala. (site 109), near the mouth, the runoff resulted in a peak discharge more than four times the maximum known flood and more than double that of a 100-year flood. Tributaries to the Noxubee River had lesser floods, with peak discharges having recurrence intervals generally ranging from 20 to 100 years.

Hydrographs of discharge for the period April 2-23, 1979, at gaging stations on the Noxubee River at
Macon, Miss., and Geiger, Ala., and on the Sucarnoochee River at Livingston, are shown in figure 23.

\author{
TOMBIGBEE RIVER DOWNSTREAM \\ FROM GAINESVILLE, ALA.
}

In the reach of the Tombigbee River between Gainesville and Demopolis, Ala., the flood was the highest known at Epes, Ala. (site 115), since 1892. The Black Warrior River flows into the Tombigbee River just upstream from Demopolis. The combined flow of the Tombigbee and Black Warrior Rivers produced the maximum known flood since 1874 , and probably since 1812 , on the Tombigbee River at Demopolis lock and dam gaging station (site 143) (fig. 24). The April 1979 peak discharge at Demopolis exceeded that of a 100-year flood by nearly a third.

The lower half of the Black Warrior River basin received the heaviest rainfall, and severe flooding occurred along the Black Warrior River and its tributaries. In the Tuscaloosa-Oliver lock and dam area at Northport, Ala. (site 138), the flood on Black Warrior River was the greatest since 1900 and the peak discharge $\left(272,000 \mathrm{ft}^{3} / \mathrm{s}\right)$ exceeded that of the 100-year flood by 9 percent. Peak discharges on tributaries of the Black Warrior River approached those of a 100-year flood.

Hydrographs of discharge of Valley Creek, April 2-21, 1979, near Bessemer (site 128) and near Oak Grove, Ala. (site 129), are shown in figure 25.

Hydrographs of discharge of North River near Samantha, Ala. (site 137), and Black Warrior River at Northport, Ala. (site 138), for the period April 12-21, 1979, are shown in figure 26.

The flood on the Tombigbee River from Demopolis downstream to Coffeeville, Ala., was the greatest known since 1874 and exceeded the 100-year flood discharge at Demopolis (site 143) and at Coffeeville, Ala. (site 151). Sucarnoochee River, a large right-bank tributary flowing into the Tombigbee River near Demopolis, Ala, had peak discharges with recurrence intervals greater than 100 years at all gaged sites downstream from State Highway 16 near Dekalb, Miss.

The April 1979 peak flow of the Sucarnoochee River at Livingston, Ala. (site 148), was nearly double that of the maximum known flood (1939-79) in February 1961 and a third greater than the 100-year flood discharge. A hydrograph of the discharge of the Sucarnoochee River at Livingston, Ala., is shown in figure 23. Pawticfaw and Ponta Creeks, right-bank tributaries to Sucarnoochee Creek, had peak discharges with recurrence intervals in excess of 100 years at their crossings of U.S. Highway 45 (sites 146 and 147, respectively). 


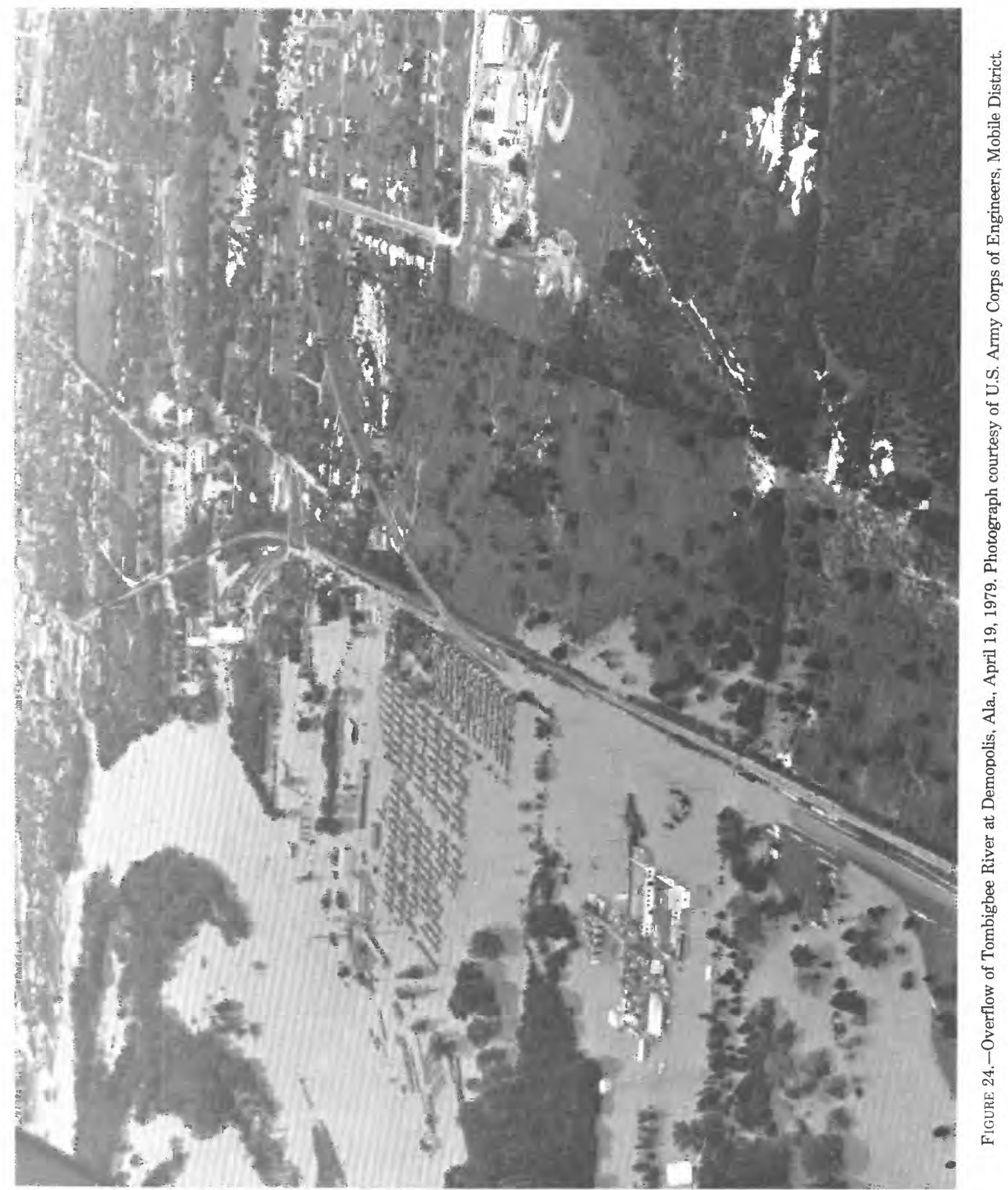




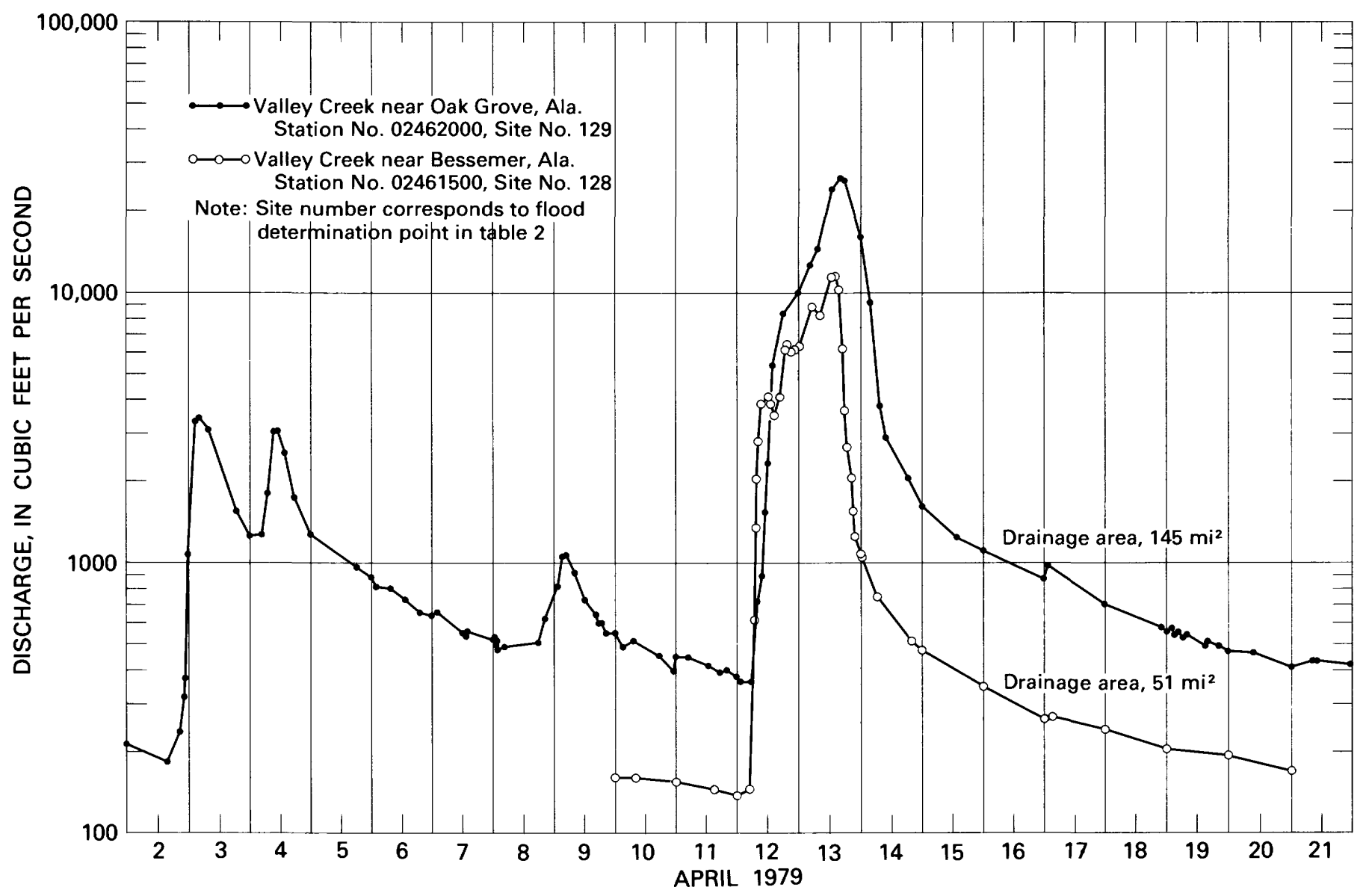

Figure 25.-Discharge at gaging stations on Valley Creek in the Black Warrior River basin in Alabama, April 2-21, 1979.

\section{PASGAGOULA RIVER BASIN}

In the Pascagoula River basin the March floods generally were greater than those in April. The heaviest rains, March 3-4, were centered over the upstream part of the Chickasawhay River. The peak discharge of Chunky Creek, head of Chickasawhay River, March 4, near Chunky, Miss. (site 156), was the greatest since records began in 1939, exceeding the 100-year flood discharge.

The stage of the flood on Sowashee Creek at Meridian, Miss. (site 158), on April 13 was the highest since records began in 1939 , but it was only 0.8 foot higher than that reached on March 4. The March 5 flood on Chickasawhay River at Enterprise, Miss. (site 160), was a foot lower than the previous maximum (since 1900) flood in 1961 and was equaled a few weeks later, April 14.

\section{PEARL RIVER BASIN}

Pearl River is formed by the confluence of Tallahaga, Nanih Waiya, and Bogue Chitto Creeks about 10 miles east of Philadelphia, Miss. Recurrence intervals of peak discharges on most streams in the northern and eastern parts of the basin were much greater than 100 years. Exceptions were Tuscolameta Creek and eastern tributaries downstream from Tuscolameta Creek, where flood peaks generally had recurrence intervals of less than 10 years.

Floods on Pearl River at all gaged sites from Burnside, Miss. (site 169), downstream to Jackson (site 194) were the greatest known since at least 1874 . Recurrence intervals of peak discharges near Monticello (site 196), and Columbia, Miss. (site 197), and near Bogalusa, La. (site 198), were equal to or greater than 100 years, although the April 1979 flood may not have exceeded the great flood of 1874 .

On Lobutcha Creek and Yockanookany River, rightbank tributaries to the Pearl River upstream and downstream, respectively, from Carthage, Miss., (site 178), peak flows were two to three times those of a 100-year flood.

The three gaging stations that measure most of the inflow into Ross Barnett Reservoir immediately upstream from Jackson-Pearl River at Carthage (site 178), 


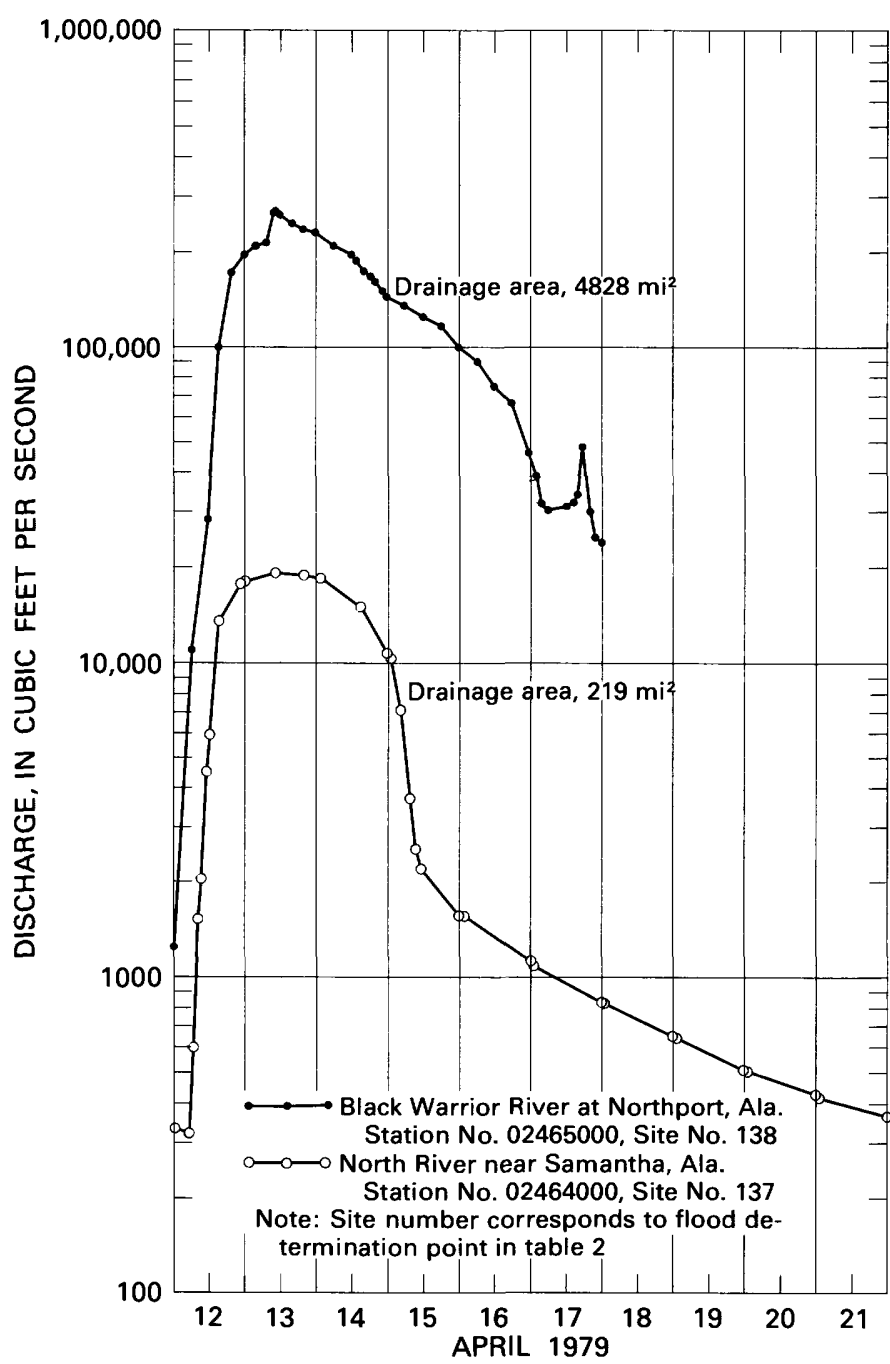

FiguRE 26.-Discharge at gaging stations on North River and Black Warrior River in Alabama, April 2-21, 1979.

Yockanookany River near Ofahoma (site 184), and Tuscolameta Creek at Walnut Grove (site 179), had peak discharges of $102,000 \mathrm{ft}^{3} / \mathrm{s}, 46,500 \mathrm{ft}^{3} / \mathrm{s}$, and $23,600 \mathrm{ft}^{3} / \mathrm{s}$, respectively. A near-peak discharge of $143,000 \mathrm{ft}^{3 /} / \mathrm{s}$ was measured in Ross Barnett Reservoir at State Highway 43 about 10 miles upstream from the Ross Barnett Reservoir dam. A peak discharge of $145,000 \mathrm{ft}^{3} / \mathrm{s}$ in the vicinity of Jackson, representing natural flow of the Pearl River without the effects of storage in Ross Barnett Reservoir, was estimated on the basis of reservoir inflow and discharge measurements made at State Highway 43 and at the gage at U.S. Highway 80 . The exceedance probability of a peak discharge of $145,000 \mathrm{ft}^{3} / \mathrm{s}$ estimated from a frequency relation based on the period of known floods, 1874-1980, is about 0.2 percent-equivalent to an average recurrence interval of about 500 years. The peak flow at the gaging station at U.S. Highway 80 (site 194) in Jackson, based on a series of discharge measurements, attenuated to $128,000 \mathrm{ft}^{3} / \mathrm{s}$ on April 17, 1979. Hydro- graphs of discharge at gaging stations on Pearl River, Tuscolameta Creek, and Yockanookany River are shown in figure 27.

The Pearl River at Jackson, Miss. (site 194), crested at 43.28 feet April 17, nearly 6 feet higher than the previous record stage of 37.5 feet in 1902. The 43.28-foot stage resulted in extensive flooding along the Pearl River and its tributaries in Jackson, Miss., and vicinity, both in the business district and in residential areas (figs. 28 to 31 ).

At Monticello, Miss. (site 196), the Pearl River crested on April 20 with a peak discharge of $122,000 \mathrm{ft}^{3} / \mathrm{s}$ at a stage of 34.08 feet, a foot higher than the flood of April 1902. At Columbia, Miss. (site 197), the Pearl River crested with a discharge of $120,000 \mathrm{ft}^{3} / \mathrm{s}$ at a stage of 27.8 feet, nearly as high as that of a flood in 1900 but 1 to 3 feet lower than the extreme flood of 1874 . Watersurface differentials of 4 feet developed between the upstream and downstream sides of the U.S. Highway 98 crossing of the east flood plain at Columbia, and the highway embankment was cut in two places by the floodflow.

The Pearl River at Bogalusa, La. (site 198), crested April 24 at a stage of 23.2 feet and a discharge of $129,000 \mathrm{ft}^{3} / \mathrm{s}$. The increase in discharge between Columbia and Bogalusa resulted from moderately heavy rain in the vicinity (3.41 in. at Bogalusa) April 21-23. The flood at Bugalusa exceeded the previous maximum (records since 1938) of April 17, 1974, by 1.1 feet.

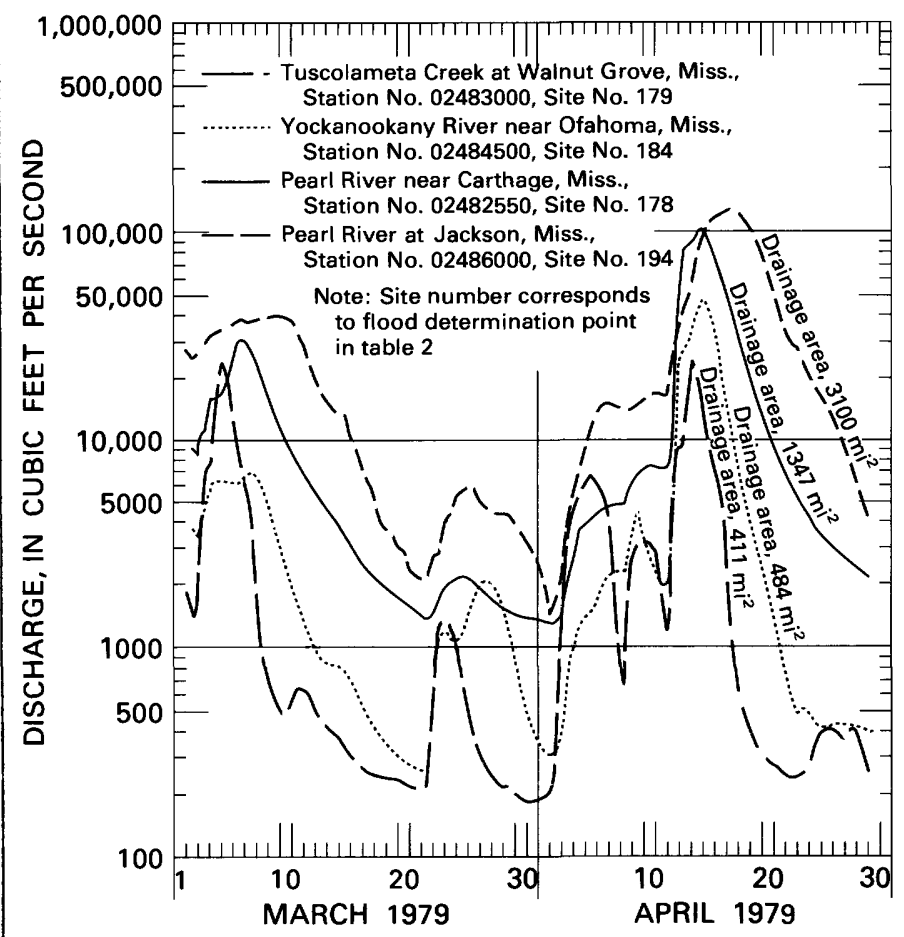

Figure 27.-Discharge at gaging stations in the Pearl River basin at and upstream from Jackson, Miss., March 2 to April 28, 1979. 


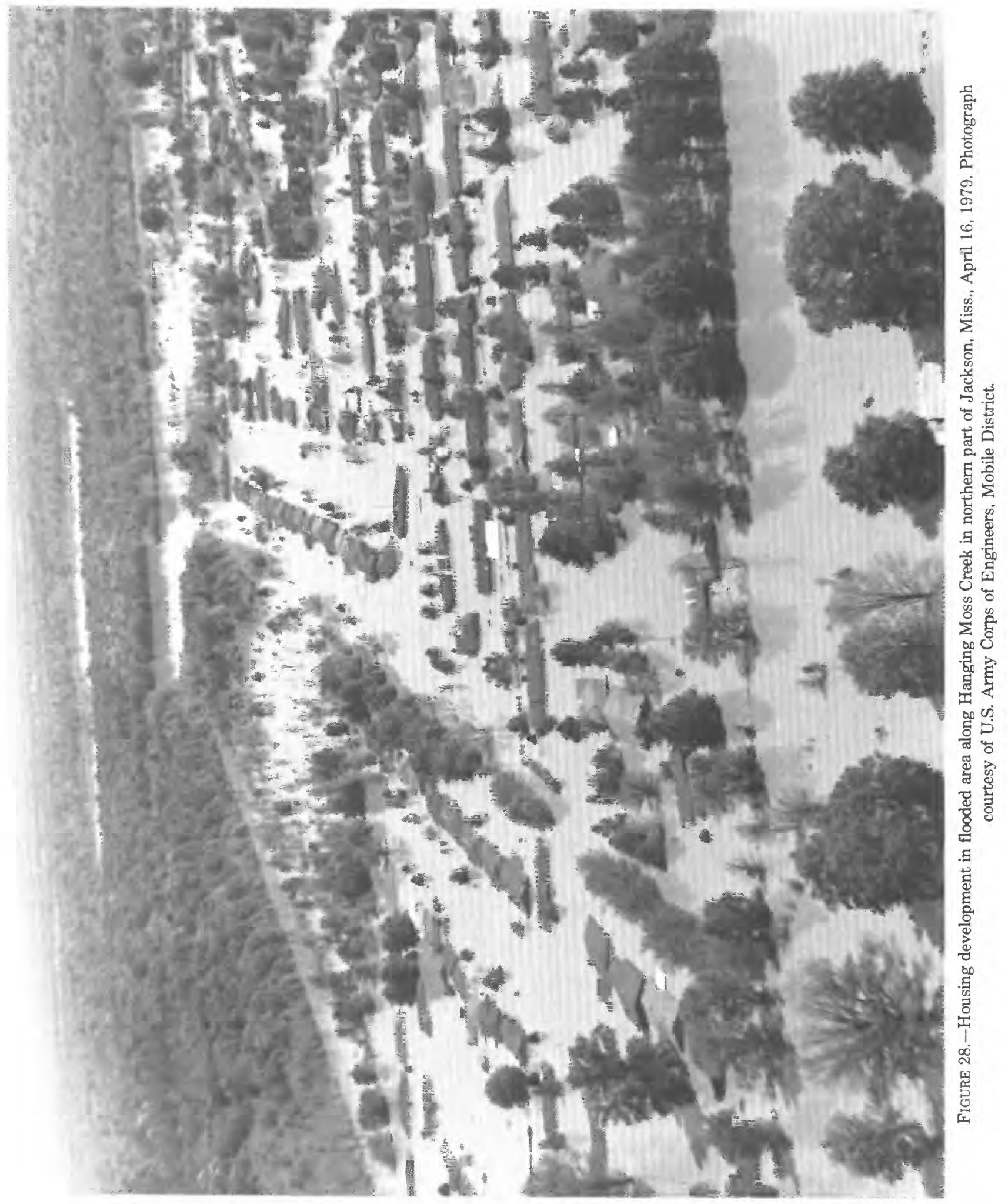




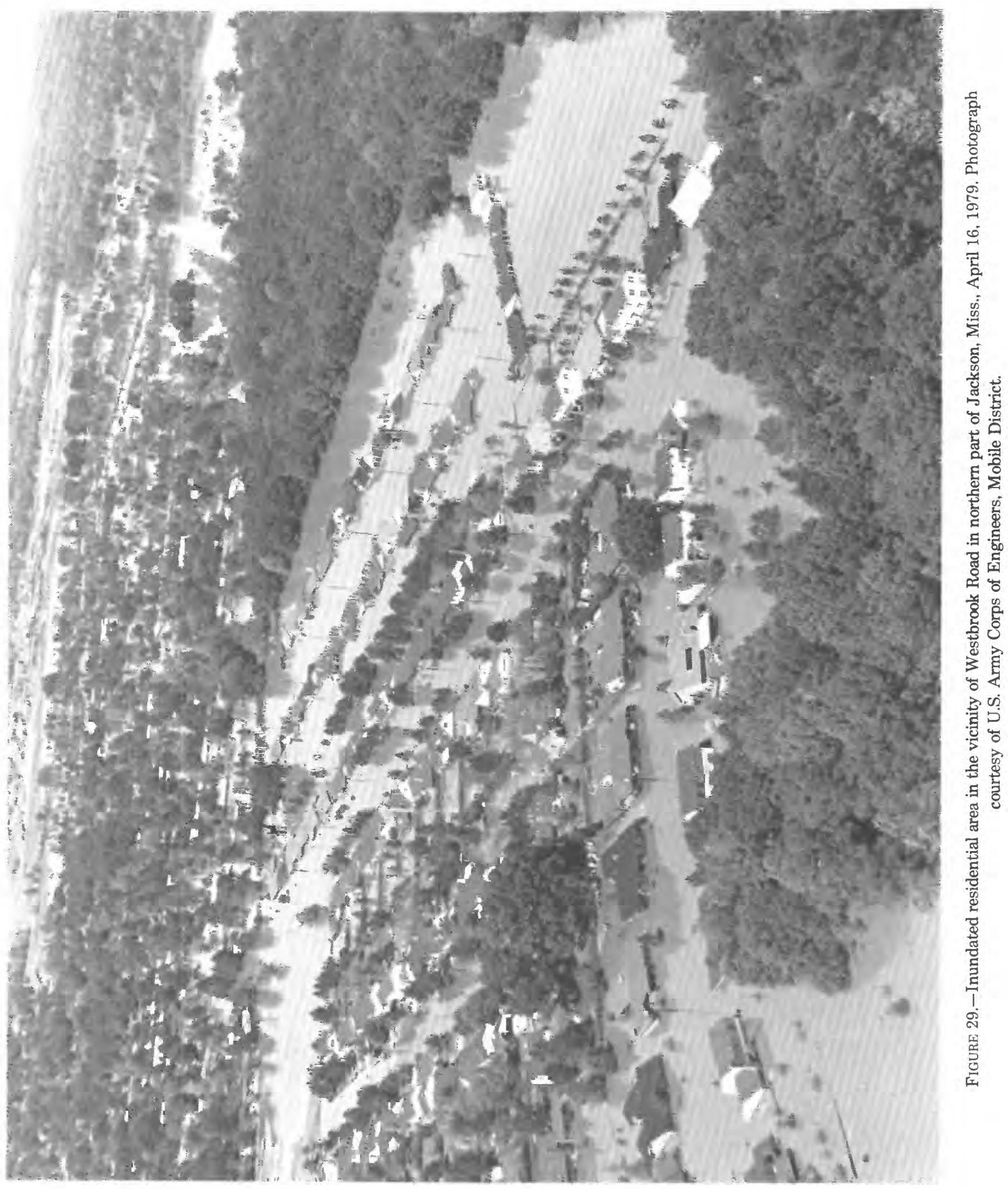




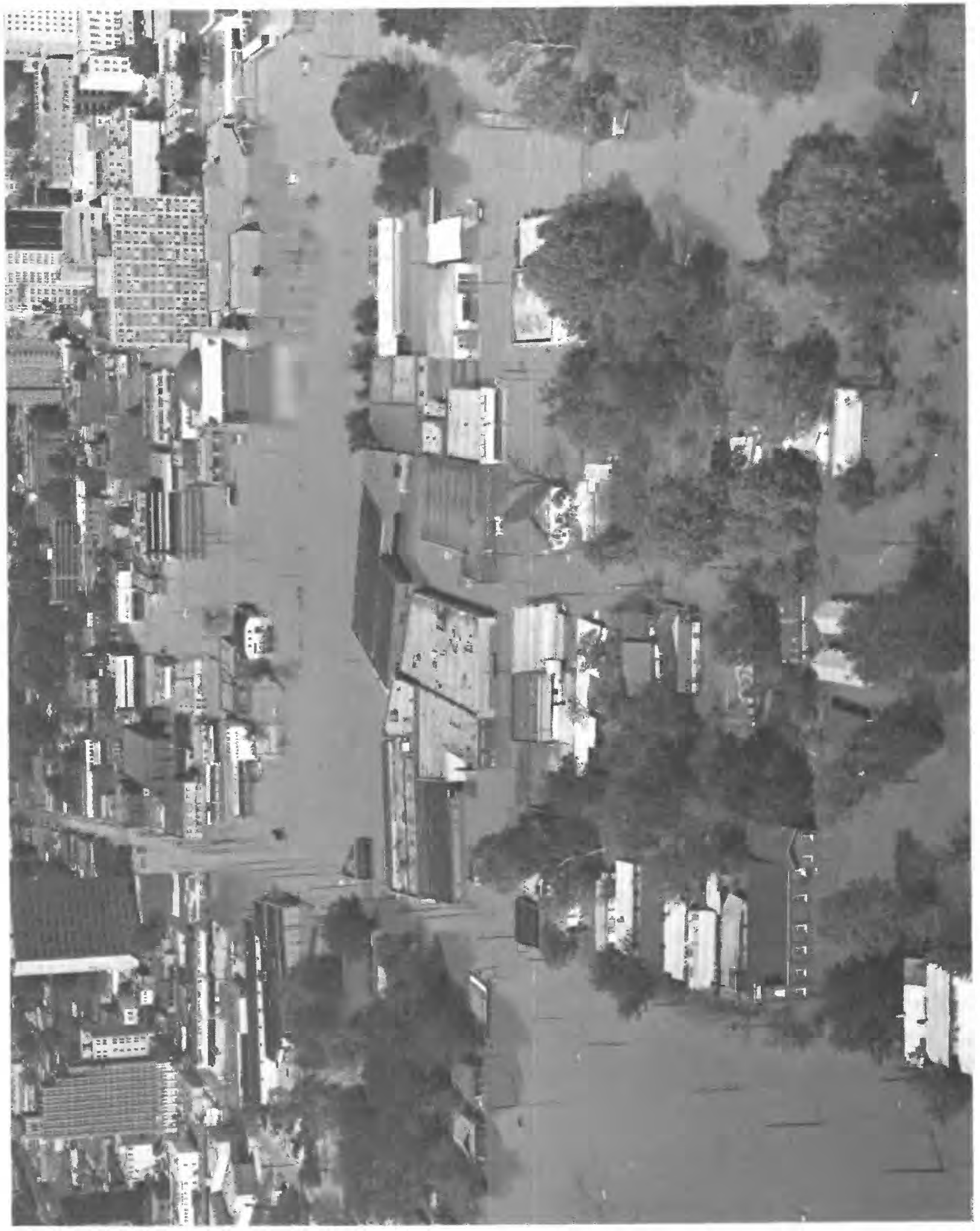



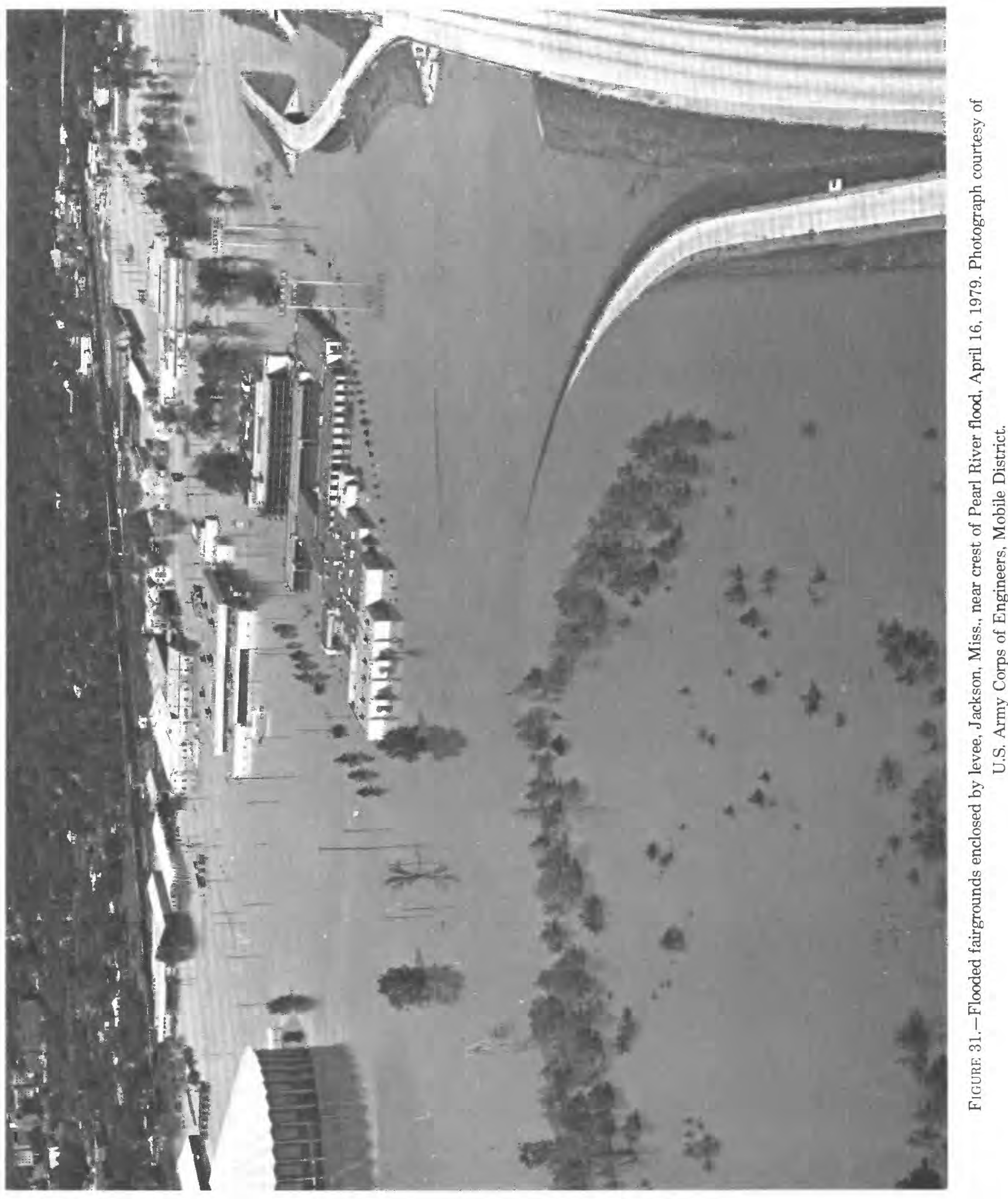


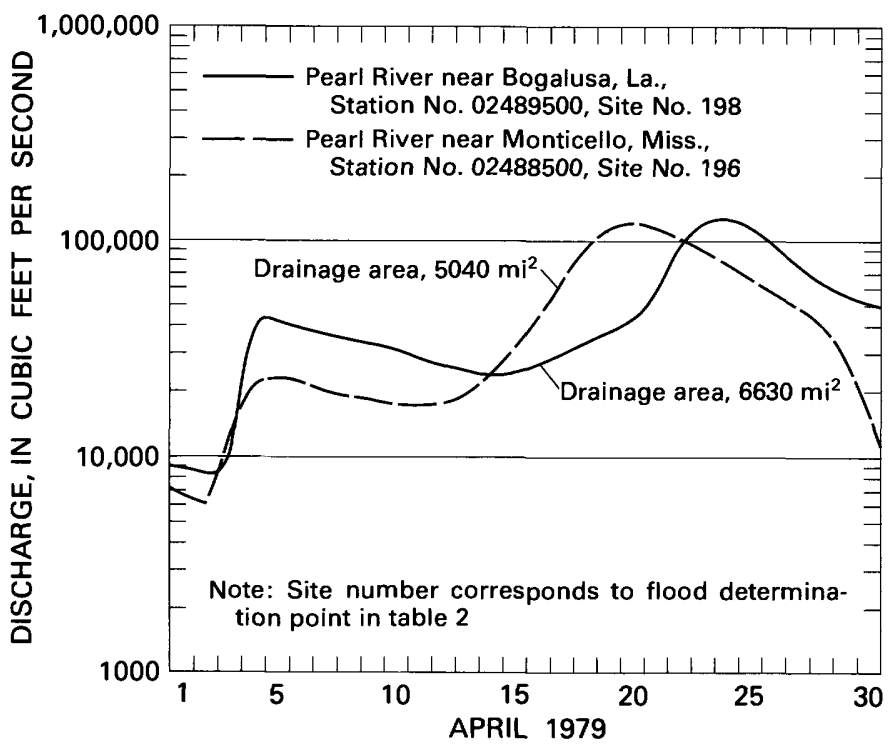

Figure 32.-Discharge at gaging stations at Pearl River near Monticello, Miss., and near Bogalusa, La., April 1-30, 1979.

Hydrographs of discharge of the Pearl River, April 1-30, 1979, at gaging stations near Monticello, Miss. (site 196), and Bogalusa, La. (site 198), are shown in figure 32.

A discharge of $155,000 \mathrm{ft}^{3} / \mathrm{s}$ was measured at the Interstate 59 crossing of Pearl River near Pearl River, La. (site 199), on April 26 near the crest. A discharge of $152,000 \mathrm{ft}^{3} / \mathrm{s}$ was measured at the Interstate 10 crossing of Pearl River near Slidell, La. (site 200), on April 26.

\section{LOWER MISSISSIPPI RIVER BASIN}

\section{BIG BLACK RIVER BASIN}

The major flood along the Big Black River, which flows into the Mississippi River about 25 miles downstream from Vicksburg, Miss., occurred April 12-16, 1979. Runoff from tributaries in the central part of the basin contributed substantially to the flood. Extreme floods occurred on medium-sized tributaries draining up to 160 square miles. Zilpha Creek near Kosciusko (site 204) had a peak discharge approaching that of a 100-year flood, and Doaks Creek near Canton, Miss. (site 210), had a peak flow more than twice that of a 100-year flood. Major floods occurred also on Long Creek near Kosciusko (site 207) and Bear Creek near Canton, Miss. (site 213).

Recurrence intervals of peak flows on the main stem of Big Black River ranged from about 25 years at the gaging staton at West, Miss. (site 205), to more than 100 years downstream near Bovina, Miss. (site 219).

Big Black River at West (site 205) crested at a stage of 24.27 feet (discharge $48,000 \mathrm{ft}^{3} / \mathrm{s}$ ). Big Black River at
Pickens (site 208) crested at a stage of 23.6 feet and at State Highway 16 near Canton (site 211) crested at an elevation of 193.22 feet (discharge $85,800 \mathrm{ft}^{3} / \mathrm{s}$ ). The increase in flood magnitude resulted from extreme tributary inflow from Big Cypress and Doaks Creeks between Pickens and Canton. At the U.S. Highway 80 gage near Bovina, Miss. (site 219), Big Black River crested at 40.56 feet (discharge $81,200 \mathrm{ft}^{3} / \mathrm{s}$ ), the greatest flood since at least 1912.

Hydrographs of discharge of the Big Black River, April 1-30, 1979, at gaging stations near West (site 205) and Bovina, Miss. (site 219), are shown in figure 33.

\section{FLOOD-CREST STAGES}

Flood-crest elevations at many ungaged points along streams were obtained by leveling to floodmarks identified during or immediately following the floods. Floodcrest stages provide a means to determine the extent of overflows and are useful in land-use management of flood-plain lands.

Both the U.S. Geological Survey and the U.S. Army Corps of Engineers (Mobile and Vicksburg Districts) participated in flagging the floodmarks. Most of the elevations were determined by the Corps of Engineers.

Records of flood-crest stages in the Mobile, Pascagoula, Pearl, and Big Black River basins are presented in table 5 (at end of report). Data on both main-stem streams and several tributaries are included.

Points of measurement are referred to distance in miles upstream from the mouth of the stream. River mileage was determined by the U.S. Army Corps of Engineers (1972) unless otherwise noted. Flood-crest

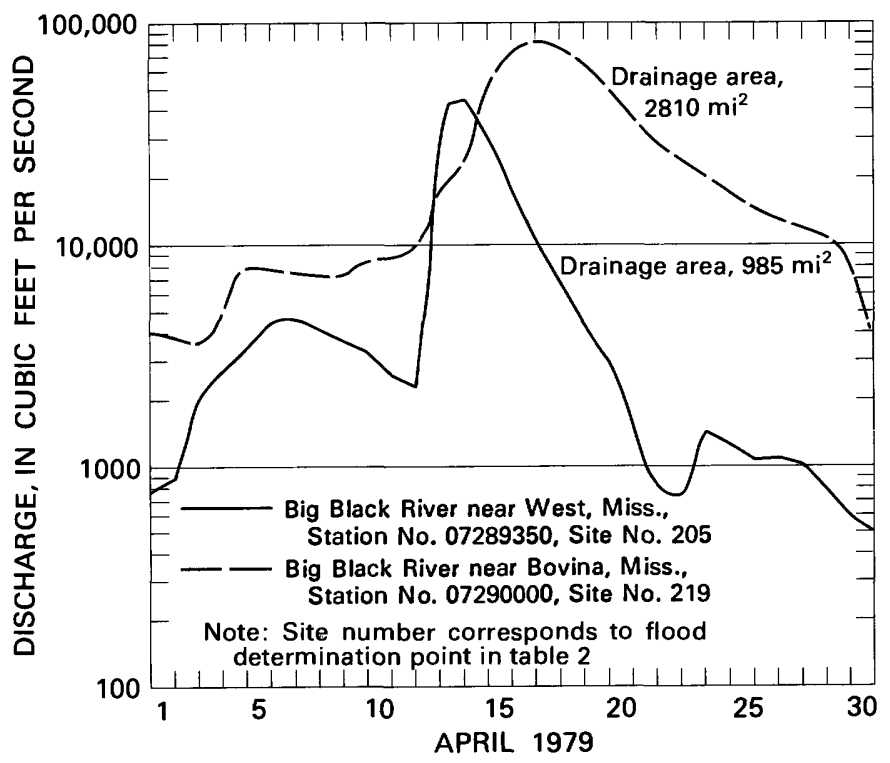

Figure 33.-Discharge at gaging stations on Big Black River at West, Miss., and near Bovina, Miss., April 1-30, 1979. 


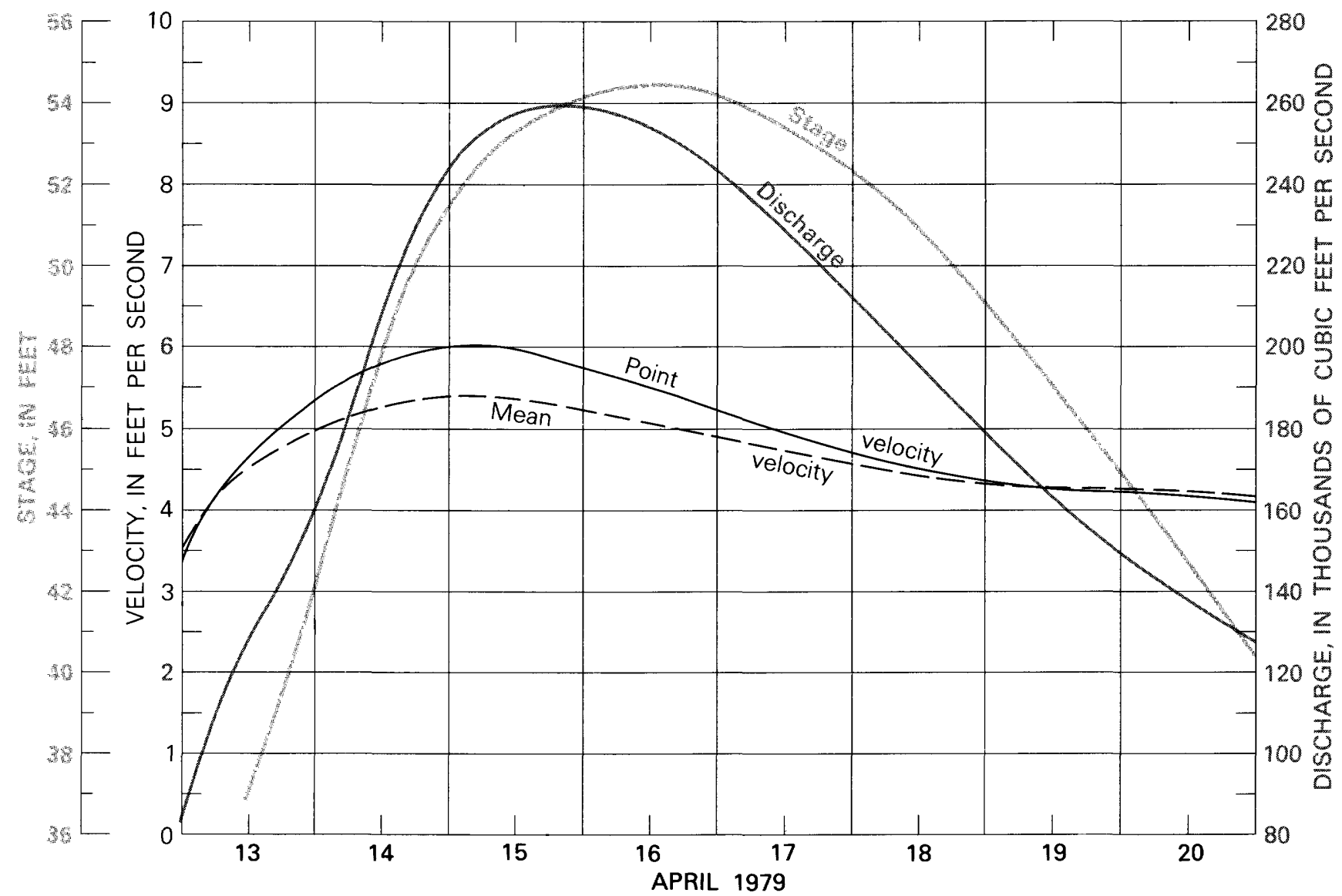

Figure 34.-Changes in point velocity, mean velocity, stage, and discharge of Alabama River near Montgomery, Ala., April 13-20, 1979. Meter location for point velocity is at stage $15.0 \mathrm{ft}, 25 \mathrm{ft}$ above streambed at upstream side of the center bridge pier on U.S. Highway 31.

elevations are water-surface elevations in feet above National Geodetic Vertical Datum of 1929 (NGVD of 1929).

Flood-crest elevations at U.S. Geological Survey gaged sites may be determined from Table 2, "Summary of Flood Stages and Discharges" by adding the gage height of the flood to the datum of the gage (NGVD of 1929), where the datum is known.

Additional records of flood-crest stages and other detailed information may be obtained from the U.S. Corps of Engineers and the U.S. Geological Survey.

\section{STREAMFLOW VELOCITIES}

\section{VELOCITY CHANGES DURING PEAK DISCHARGES}

Velocity changes with respect to time and stage during the passage of a flood wave are of great interest to hydrologists. The gaging station, Alabama River near Montgomery, Ala. (site 69), records a point velocity that has been calibrated to the mean velocity in the cross section at the gage (table 6) (at end of report). The relations of point velocity, mean velocity, stage, and discharge, to time every 6 hours for the period April 13-20, 1979, are shown in figure 34 .

\section{VELOCITY DISTRIBUTION THROUGH BRIDGE OPENINGS}

Velocity distribution through channel constrictions during peak flows is of great interest to designers of bridges, culverts, and other hydraulic structures. The distribution of velocities is an integral part of a velocitymeter discharge measurement of flow.

Stream velocities obtained at or near the crest of the floods exceeded 10 feet per second at some sites. Velocities at bridges were far from uniform, varying greatly both in vertical and horizontal directions. Maximum velocities were $11 / 2$ to 2 times the average velocities at the bridge openings. Variations were related to bridge and channel geometry and to the extent of channel (or flood plain) contraction at the bridge.

Velocity distribution diagrams are shown for nine selected bridges, five in Alabama and four in Mississippi 

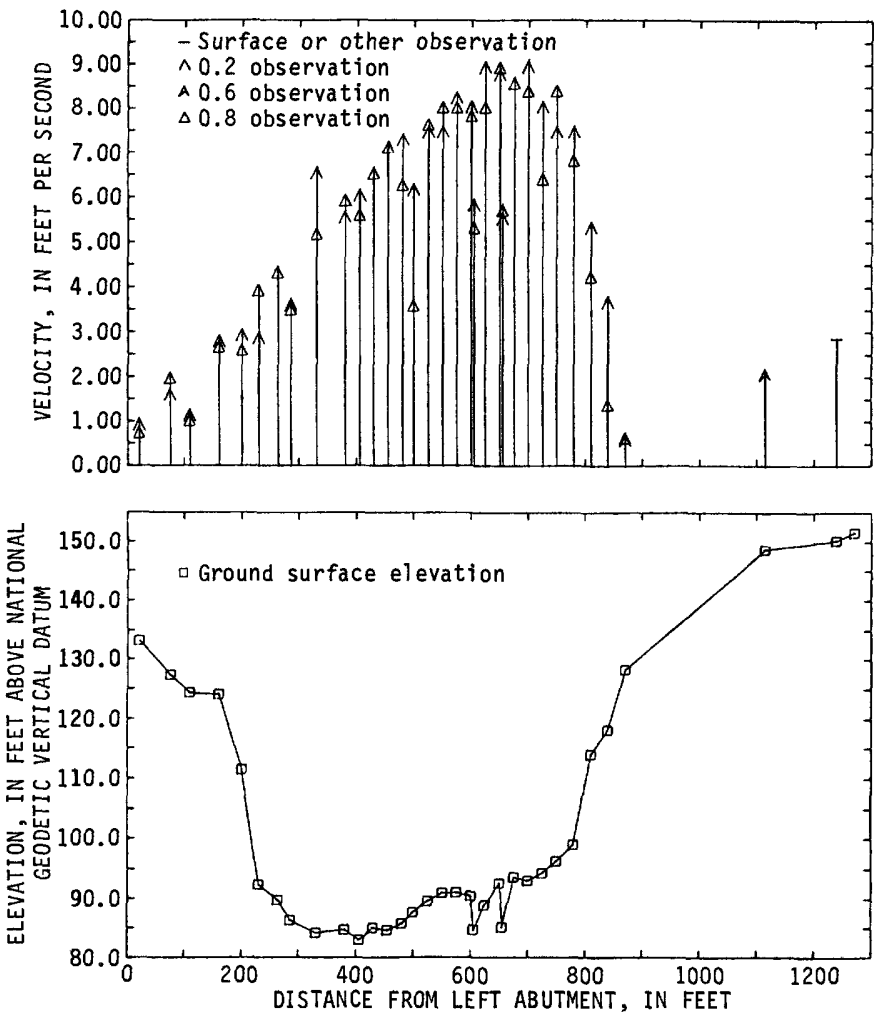

FIgURE 35.-Velocity distribution and cross section: Alabama River at U.S. Highway 31 north, near Montgomery, Ala., April 15, 1979. Water-surface elevation, $151.50 \mathrm{ft}$; total discharge, $261,000 \mathrm{ft}^{3} / \mathrm{s}$.

(figs. 35-43). The velocity diagrams for the highly contracted bridges (Hashuqua and Zilpha Creeks and Noxubee River in Mississippi) show higher velocities in the overflow areas (on the flood plain) than in the channels. Moderately contracted bridges (Noxubee, Tombigbee, and Alabama Rivers in Alabama) show fairly high velocities near one abutment but not as great as those in the channels. Velocities near the abutment are low on Mulberry Creek and North River in Alabama.

\section{FLOOD HYDROGRAPH DATA}

Gage height, discharge, and accumulated runoff at selected times during the flood at 47 gaging stations in Alabama, Mississippi, and Louisiana are shown in table 7 (at end of report). The period begins prior to the major rise and extends to the end of the gaged record or to an arbitrary cutoff point on the recession, when the discharge approaches that of the antecedent flow. The period for some gaging stations starts March 1, 1979, to define flow conditions prior to the floods of March 1979, whose peak flows exceeded those in April 1979 at some stations. The intervals selected for presenting momentary stage and discharge information provide sufficient detail to define the flood hydrograph. Runoff in inches shows the depth to which the drainage basin would be
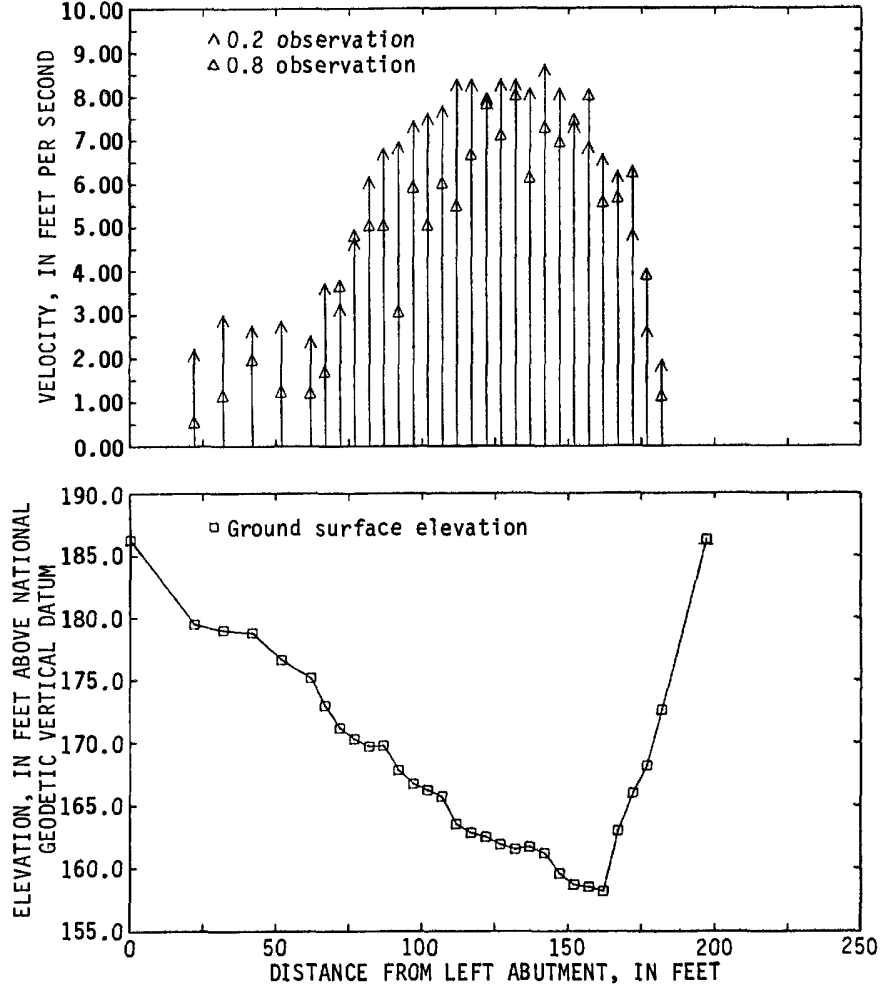

FIGURE 36.-Velocity distribution and cross section: Mulberry Creek at highway bridge at Jones, Ala., April 14, 1979. Water-surface elevation, $186.35 \mathrm{ft}$; total discharge, $15,600 \mathrm{ft}^{3} / \mathrm{s}$.
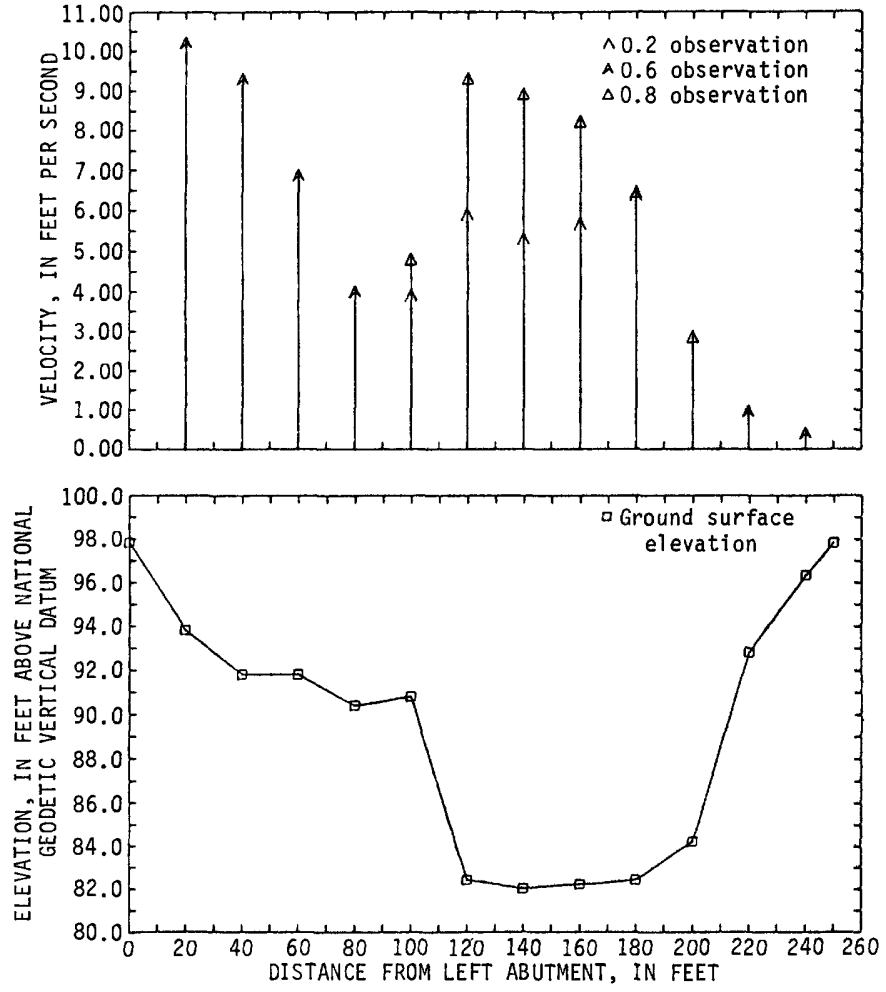

FIGURE 37.-Velocity distribution and cross section: Hashuqua Creek near Macon, Miss., April 12, 1979. Gage height, $97.85 \mathrm{ft}$; total discharge, $13,900 \mathrm{ft}^{3} / \mathrm{s}$. 

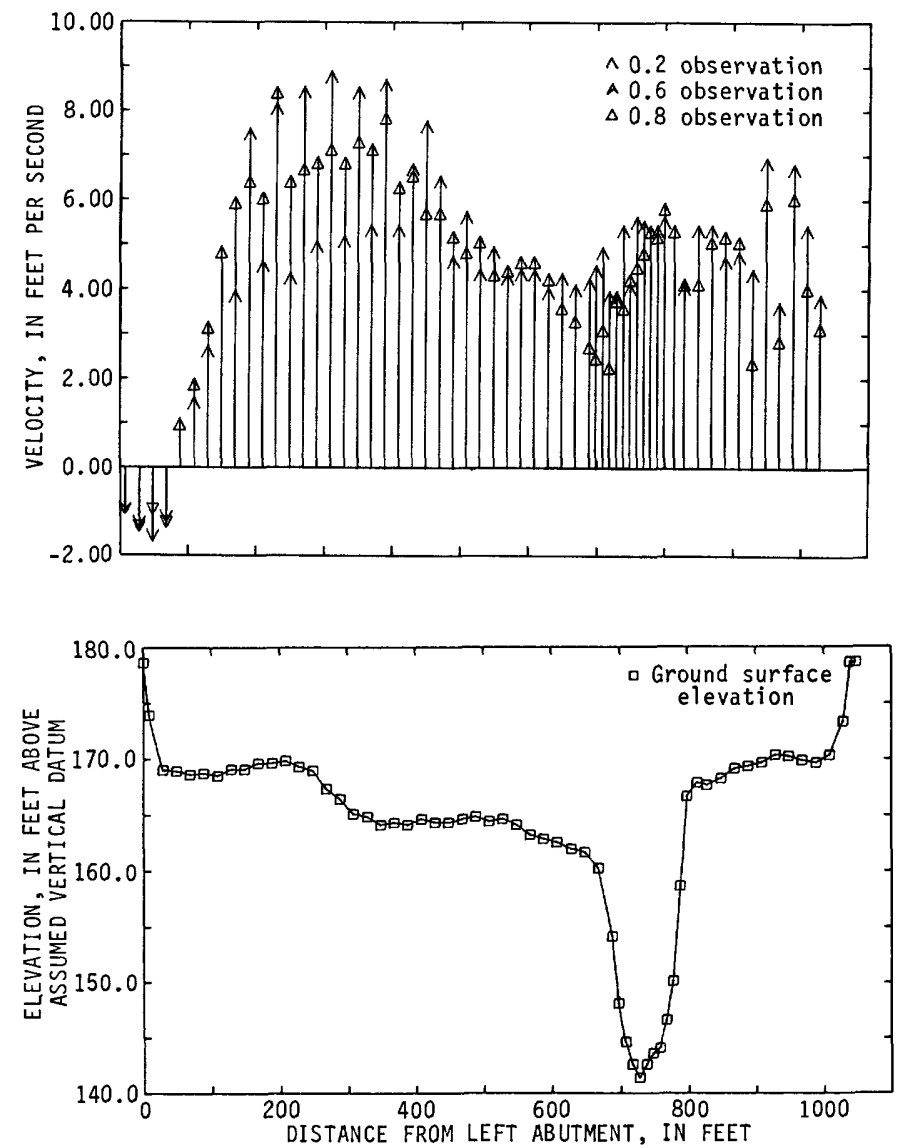

FIGURE 38.-Velocity distribution and cross section: Noxubee River at U.S. Highway 45 bypass near Macon, Miss., April 14, 1979. Water-surface elevation, $36.25 \mathrm{ft}$; main channel discharge, 69,400 $\mathrm{ft}^{3} / \mathrm{s}$; total discharge, $81,700 \mathrm{ft}^{3 / s}$.

covered if all the runoff during a given period were uniformly distributed. The runoff for the March or April storm can be roughly approximated by subtracting the accumulated runoff at the beginning of the flood from the runoff at the end of the flood period.

\section{GROUND-WATER FLUCTUATIONS}

Descriptions of selected ground-water observation wells influenced by the storm of April 1979 are shown in table 8 (at end of report). These wells tap unconsolidated aquifers of Late Cretaceous and Quaternary age in the Black Warrior and Tombigbee River basins and consolidated aquifers of Late Cambrian, Early Ordovician, and Early Mississippian ages and metamorphic rocks in the Coosa and Tallapoosa basins.

The water-level fluctuations, shown on the hydrograph of well $1 \mathrm{~W}$ (fig. 44) and other wells, during the storm of April 1979 were influenced by local precipitation and direct infiltration and loading effects from nearby streams. An absence of rises in the water levels in wells $3 \mathrm{~W}$ and $5 \mathrm{~W}$ until several days after the storm may indicate a lag in recharge to the aquifer from the outcrop area to the vicinity of the wells. The variations in water level in these observation wells are shown in table 8.
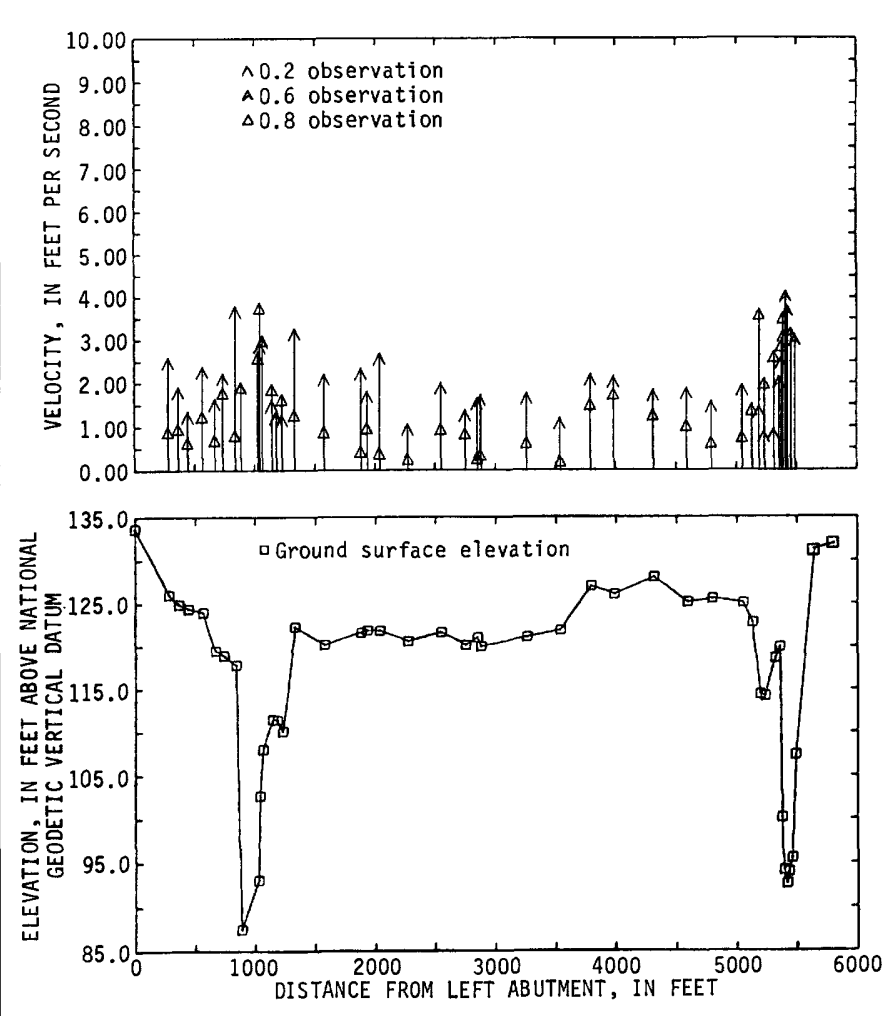

Figure 39.-Velocity distribution and cross section: Noxubee River at State Highway 17 near Geiger. Ala., April 15, 1979. Watersurface elevation, $133.62 \mathrm{ft}$; total discharge, $125,000 \mathrm{ft}^{3 /} / \mathrm{s}$.
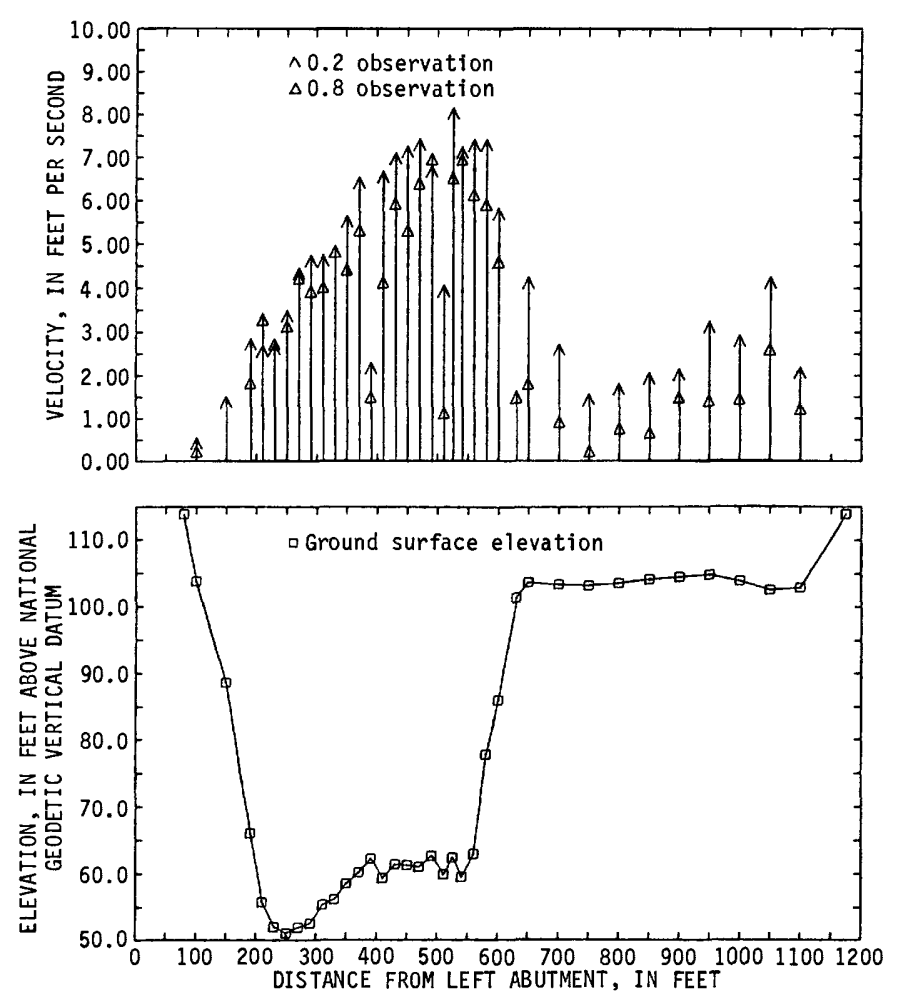

Figure 40.-Velocity distribution and cross section: Tombigbee River at Gainesville, Ala. (main channel), April 15, 1979. Watersurface elevation, $113.88 \mathrm{ft}$; total discharge, $124,000 \mathrm{ft}^{3 /} / \mathrm{s}$. 

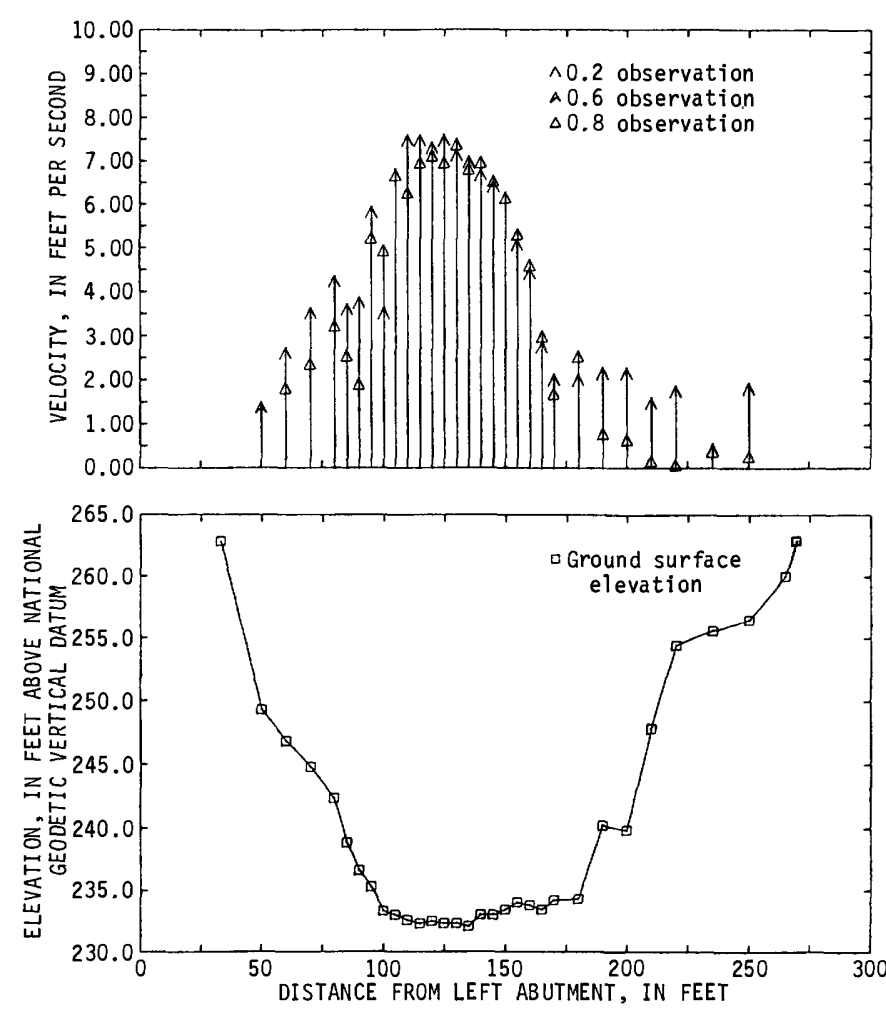

FIGURE 41.-Velocity distribution and cross section: North River near Samantha, Ala., April 13, 1979. Water-surface elevation, $262.84 \mathrm{ft}$; total discharge, $18,500 \mathrm{ft}^{3} / \mathrm{s}$.

Water-level fluctuations in observation wells near the Tombigbee River were influenced primarily by the stage of the river during the flood, and this influence decreased with distance from the river. The rise in water levels in response to high river stages and to local precipitation during the flood and resultant recharge to the adjacent alluvial deposits of Quaternary age is shown on the hydrograph for observation well $15 \mathrm{~W}$ (fig. 45). The decline of water levels March 5 to April 1, and April 13-30, resulted from water draining from the alluvial deposits subsequent to lowering of the stage of the river.

The water levels in observation wells tapping the Eutaw Formation of Late Cretaceous age near the Tombigbee River were influenced during the flood in part by direct infiltration from the river to the aquifer, in part by recharge on outcrop areas, and in part by loading effects from high river stages. Wells $8 \mathrm{~W}, 9 \mathrm{~W}, 11 \mathrm{~W}$, and $12 \mathrm{~W}$, which tap the Eutaw Formation, are separated from the river by the relatively impermeable Mooreville Chalk of Late Cretaceous age, suggesting that the fluctuations shown on the hydrographs are caused principally by loading effects from an increase in the volume of water in the river and in the adjoining alluvial deposits.
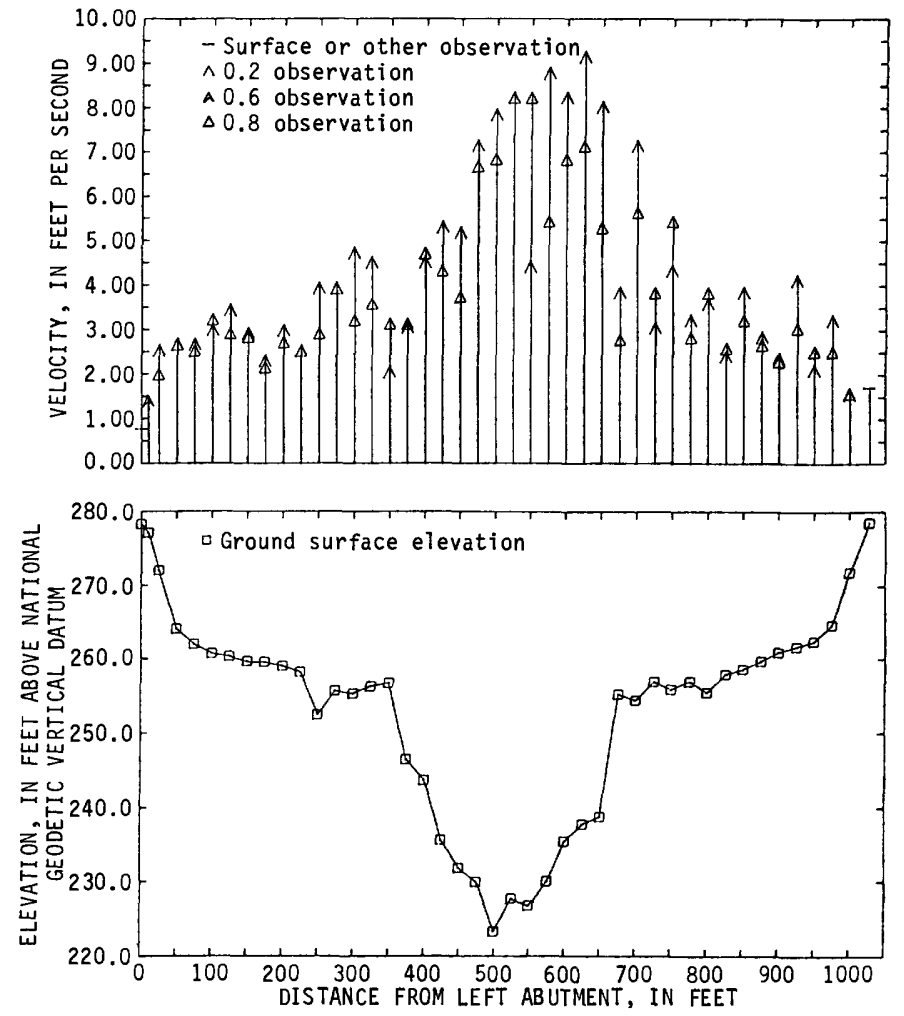

Figure 42.-Velocity distribution and cross section: Pearl River at Interstate Highway 55 at Jackson, Miss., April 17, 1979. Gage height, $43.21 \mathrm{ft}$; total discharge, $128,000 \mathrm{ft}^{3} / \mathrm{s}$.
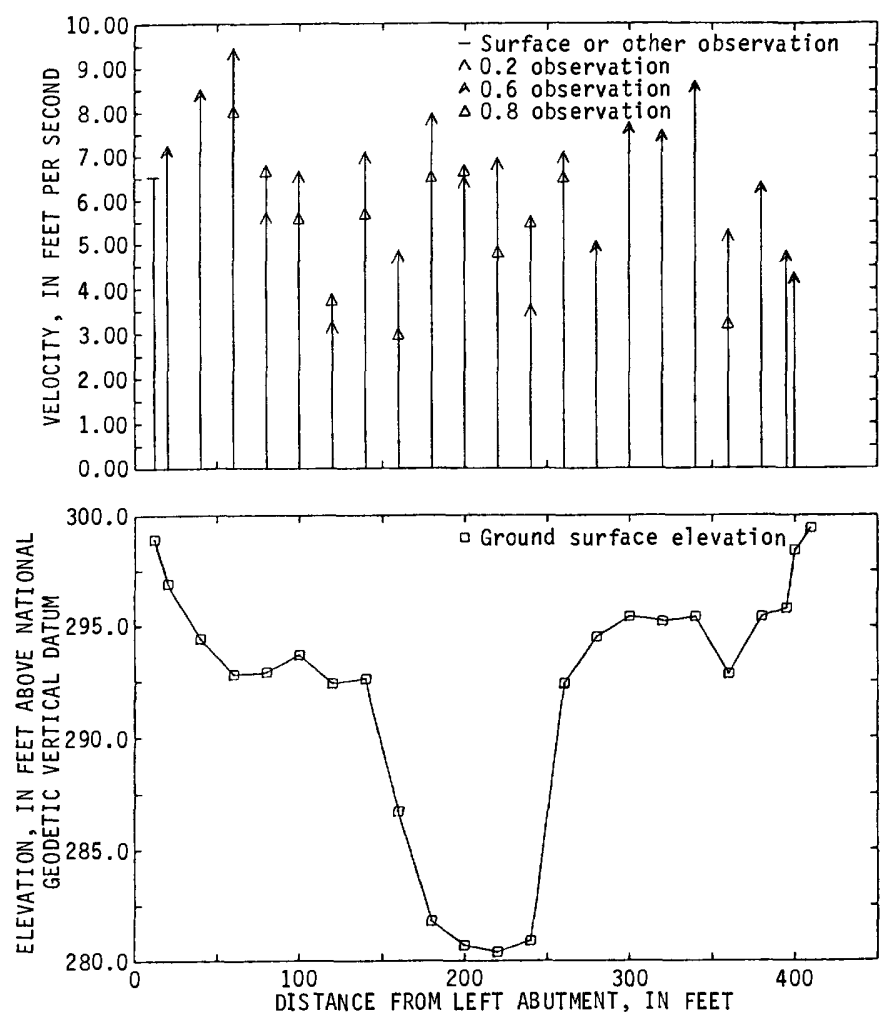

Figure 43.-Velocity distribution and cross section: Zilpha Creek at State Highway 35 near Kosciusko, Miss., April 12, 1979. Watersurface elevation, $291.41 \mathrm{ft}$; total discharge, $21,400 \mathrm{ft}^{3} / \mathrm{s}$. 


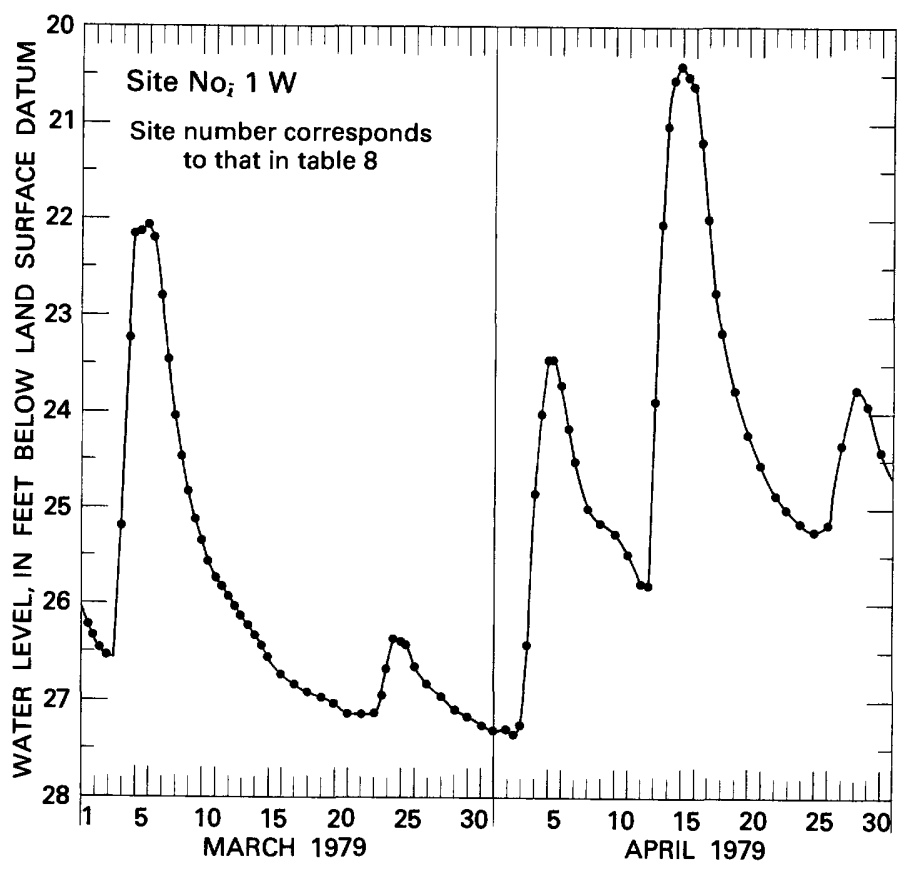

Figure 44.-Water level in observation well 3256220870755501 , at Centreville, Ala. (site 1W) (Centreville Gin and Cotton Co.), MarchApril 1979.
Specific conductance readings from 10 feet to the surface at site 16 were about 300 micromhos per centimeter, indicating the "wedge" effect of freshwater floodflow over the more saline Gulf water.

The temperature was relatively constant from water surface to the floor of the waterway. Generally, temperatures at the surface were higher, $0.5^{\circ}$ to $1.0^{\circ}$ (Celsius), than the temperatures near the floor of the waterway.

\section{AERIAL PHOTOGRAPHY}

Aerial photographs were taken April 14-19, 1979, at or near the crest of the flood on several streams in the Mobile and Pearl River basins. The photographs are useful in identifying inundated areas and analyzing hydraulic conditions.

Flight lines along streams where aerial photographs were obtained are listed in table 10 (at end of report). The table furnishes information on date the photographs were taken, flight heights, and type of film used. The date, time, and altitude are also shown on each photograph. The approximate locations of the flight lines are shown in figure 47.

The photographs listed in table 10 were obtained by the U.S. Geological Survey or the Corps of Engineers and are on file in the U.S. Geological Survey District Offices in Tuscalcosa, Ala., or Jackson, Miss.

Aerial photographs were obtained near the crest of the flood at 15 highway and railroad crossings of Pearl River (5 on April 14 and 10 on April 19). These photographs, together with stage and discharge data, are useful in analyzing the hydraulics at these crossings. tude. The system provides the geographic location of the well and a unique number for each well. The number consists of 15 digits. The first 6 digits denote the degrees, minutes, and seconds of latitude, the next 7 digits denote degrees, minutes, and seconds of longitude, and the last 2 digits (assigned sequentially) identify the wells within a 1-second grid.

\section{SALINITY AND TEMPERATURE DATA, MOBILE BAY AND GULF OF MEXICO}

The U.S. Geological Survey, in cooperation with the Corps of Engineers, Mobile District, collected salinity and temperature data along the Intracoastal Waterway in Mobile Bay during the flood period April 28-29, 1979. The data are summarized in table 9 (at end of report), and the locations of sites are shown in figure 46. Specific conductance readings indicate that salinity, ranging from 20 to 3,000 micromhos per centimeter, is relatively low in the waterway sampled except for the Mobile Ship Channel. The specific conductance in the ship channel (site 16, fig. 46) at a depth of 35 feet was 45,000 micromhos per centimeter (compared with about 55,000 micromhos per centimeter in the Gulf of Mexico).

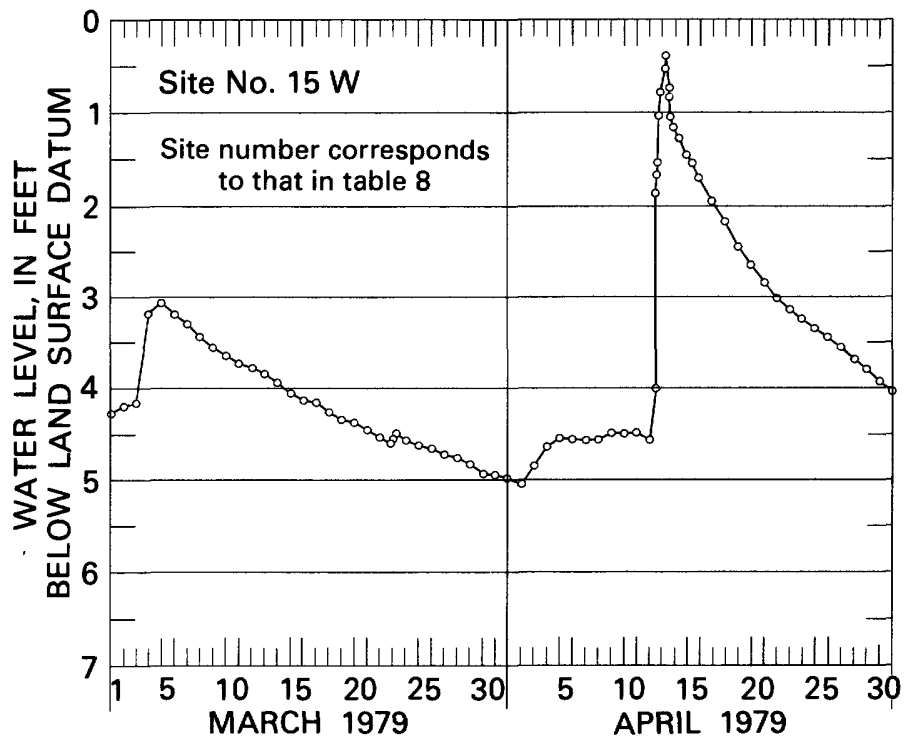

Figure 45.-Water level in observation well 331426088192202 , at site 3.4 miles west of Pickensville, Ala. (site $15 \mathrm{~W}$ ), at river mile 292.0 above the mouth of the Tombigbee River, March-April 1979 


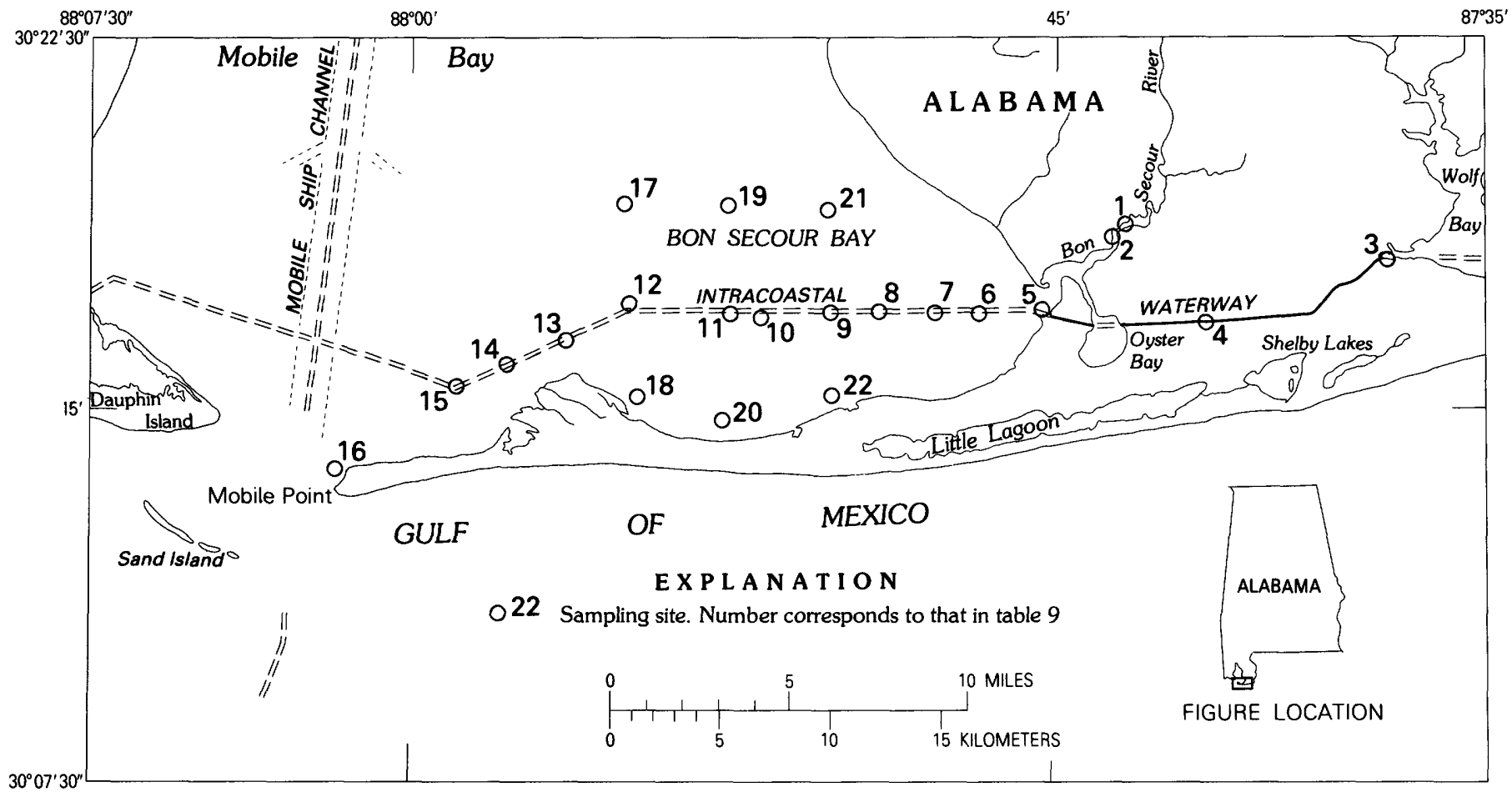

FIGURE 46.-Location of specific-conductance sampling sites at selected sites along the Intracoastal Waterway at the mouth of Mobile Bay, April 28-29, 1979.

Aerial photographs were taken April 16 from a height of 1,200 feet along Alabama Highway 17 crossing Noxubee River near Geiger, Ala. April 18 photographs were taken from a height of 1,900 feet along U.S. Highway 80 at Selma, Ala.

Low-altitude photographs of the Jackson, Miss., vicinity on April 16 near the time of the crest are shown in figures 28 through 31 .

Video-tape photographs taken from a helicopter (at low altitude), of Jackson, Monticello, and Columbia, Miss., and Bogalusa, La., were obtained by the U.S. Army Corps of Engineers, Mobile District, April 15-23. The tapes include inspections of the levees at Jackson beginning on April 15 and end with the crest of flood at Columbia on April 23. Video-tape photography is useful for analyzing flow through and over highways, railroads, levees, and landfills.

Video tapes of Interstate Highway 65 bridges spanning the Alabama River at Montgomery, Ala., April 16, and U.S. Highway 80 at Selma, April 18, and the inundated area along the Tombigbee River at Demopolis, April 18, were made at low altitudes.

\section{SELECTED REFERENCES}

Colson, B. E., and Hudson, J. W., 1976, Flood frequency of Mississippi streams: Mississippi State Highway Department, $34 \mathrm{p}$.

Crippen, J. R., and Bue, Conrad D., 1977, Maximum floodflows in the conterminous United States: U.S. Geological Survey WaterSupply Paper 1887, 52 p., figs. 3,5 .

Miller, J. F., 1964, Two to ten-day precipitation for return periods of 2 to 100 years in the contiguous United States: U.S. Weather Bureau Technical Paper 49, $29 \mathrm{p}$.

National Oceanic and Atmospheric Administration, 1979, Climatological data, Alabama, v. 85, no. 4: Ashville, N.C., National Climatic Center, 16 p.

National Oceanic and Atmospheric Administration (NOAA) and National Aeronautics Space Administration (NASA), The GOES/ SMS User's Guide (updated continuously by amendments distributed by NOAA).

Olin, D. A., and Bingham, R. H., 1977, Flood frequency of small streams in Alabama: Ala. Highway Dept. Research Report HPR No. 30,44 p.

Schreiner, L. C., and Riedl, J. T., 1978, Probable maximum precipitation estimates, United States, east of 105th meridian: U.S. Department of Commerce, National Oceanic and Atmospheric Administration, and U.S. Army Corps of Engineers Hydrometeorological Report No. 51, 87 p. 


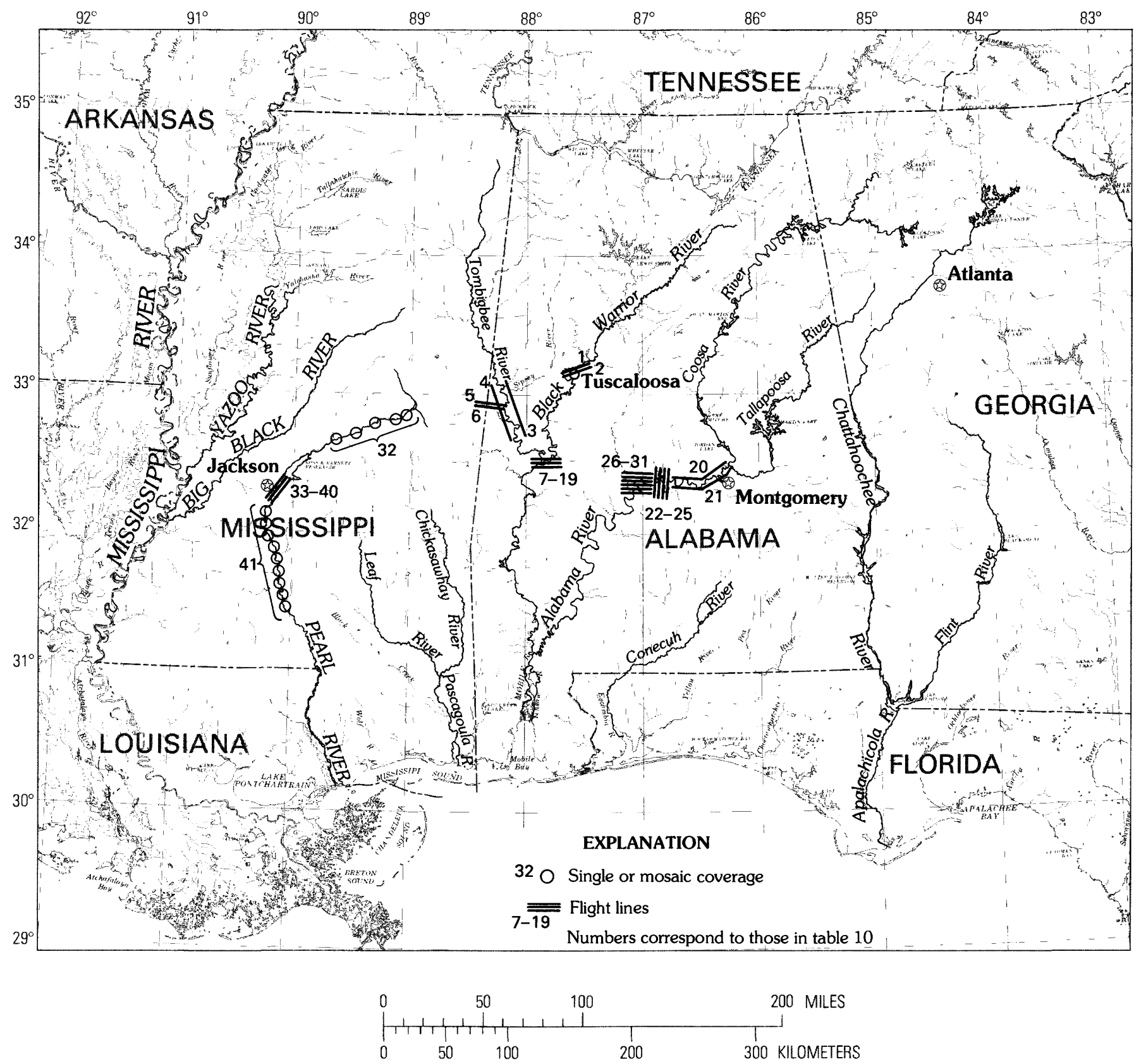

FIGURE 47.-Flight lines along streams where aerial photographs were obtained on or near the crest of the flood, April 1979.

Scofield, R. A., and Oliver, V. J., 1977, A scheme for estimating convective rainfall from satellite imagery: NOAA/NESS Technical Memorandum 86, $47 \mathrm{p}$.

1980, Some improvements to the Scofield/Oliver technique: Preprint volume, Second Conference on Flash Floods, Atlanta, Ga., March 8-10, 1980: American Meteorological Society.

U.S. Army Corps of Engineers, Mobile District, 1972, Stream mileage tables with drainage areas: Mobile, Ala., 164 p. 1980a, Post disaster report on spring floods of 1979 in the Mobile District: Mobile, Ala., 83 p., 80 pl.

$1980 \mathrm{~b}$, Vicksburg District, Post flood report flood of 1979: Vicksburg, Miss., U.S. Army Waterways Experiment Station, 93 p., 34 pl.

U.S. Water Resources Council, 1977, Guidelines for determining flood flow frequency: Washington, D.C., Water Resources Council, Hydrology Committee, Bulletin 17A, 163 p. 
TABLES 1-10 



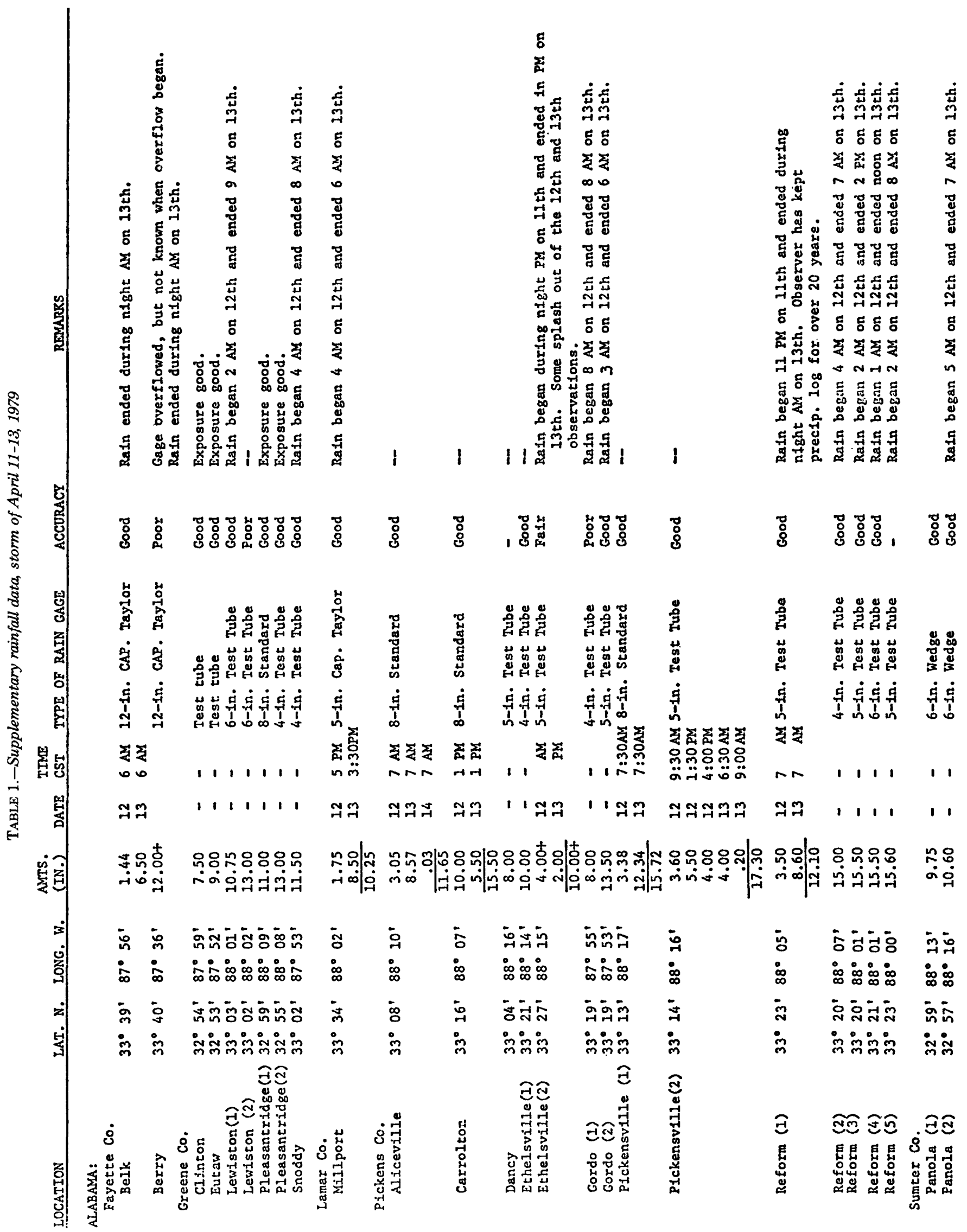




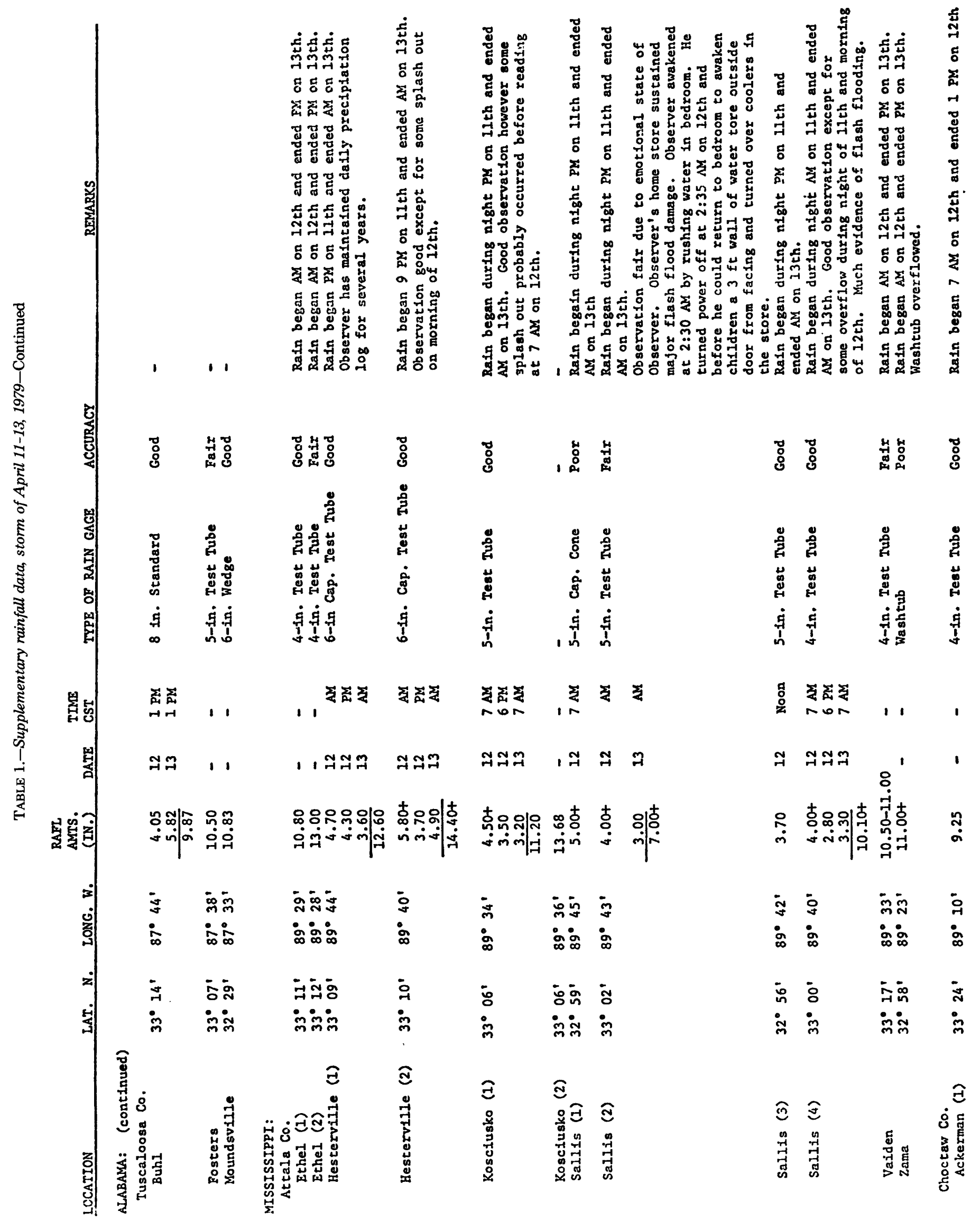


TABLES

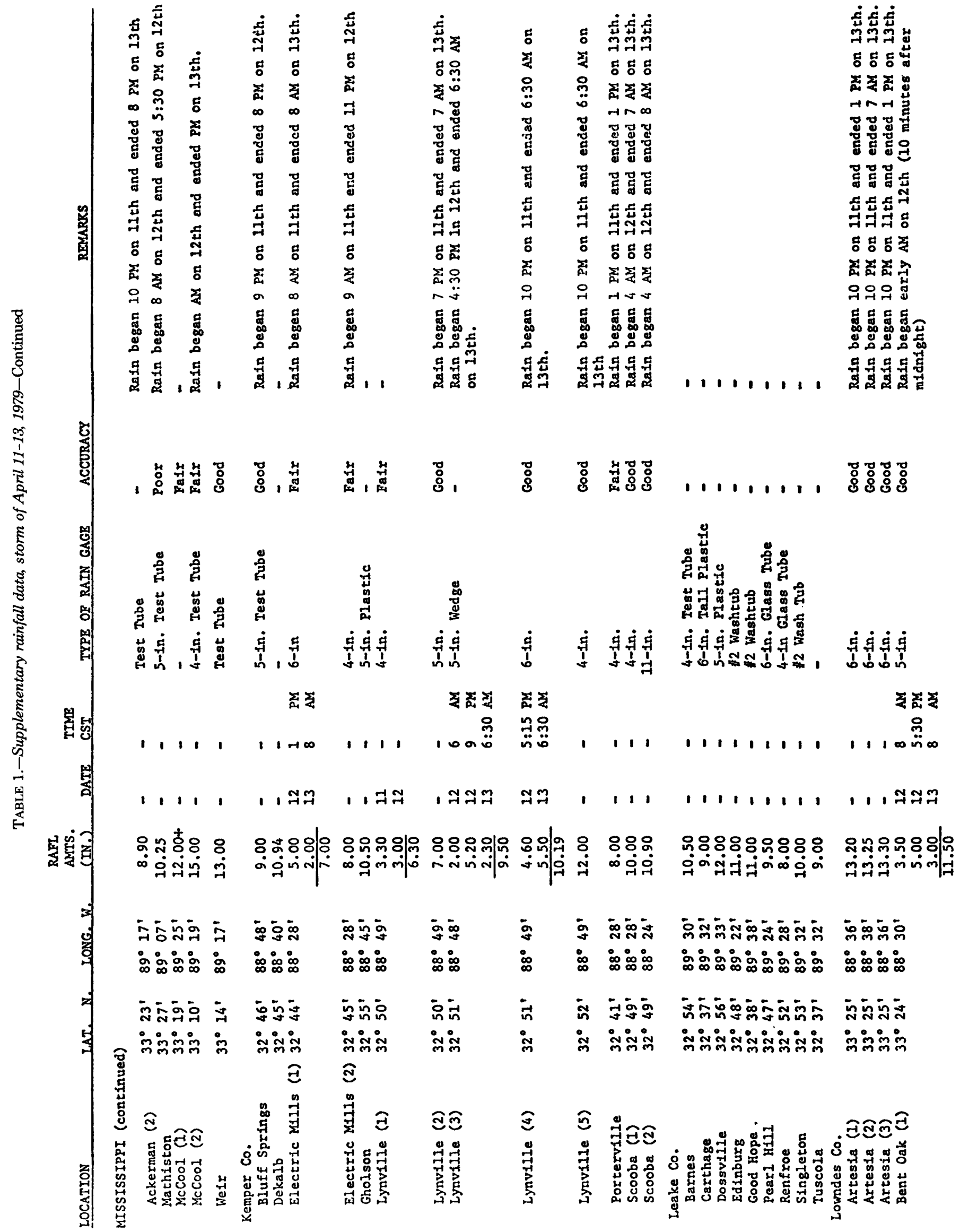




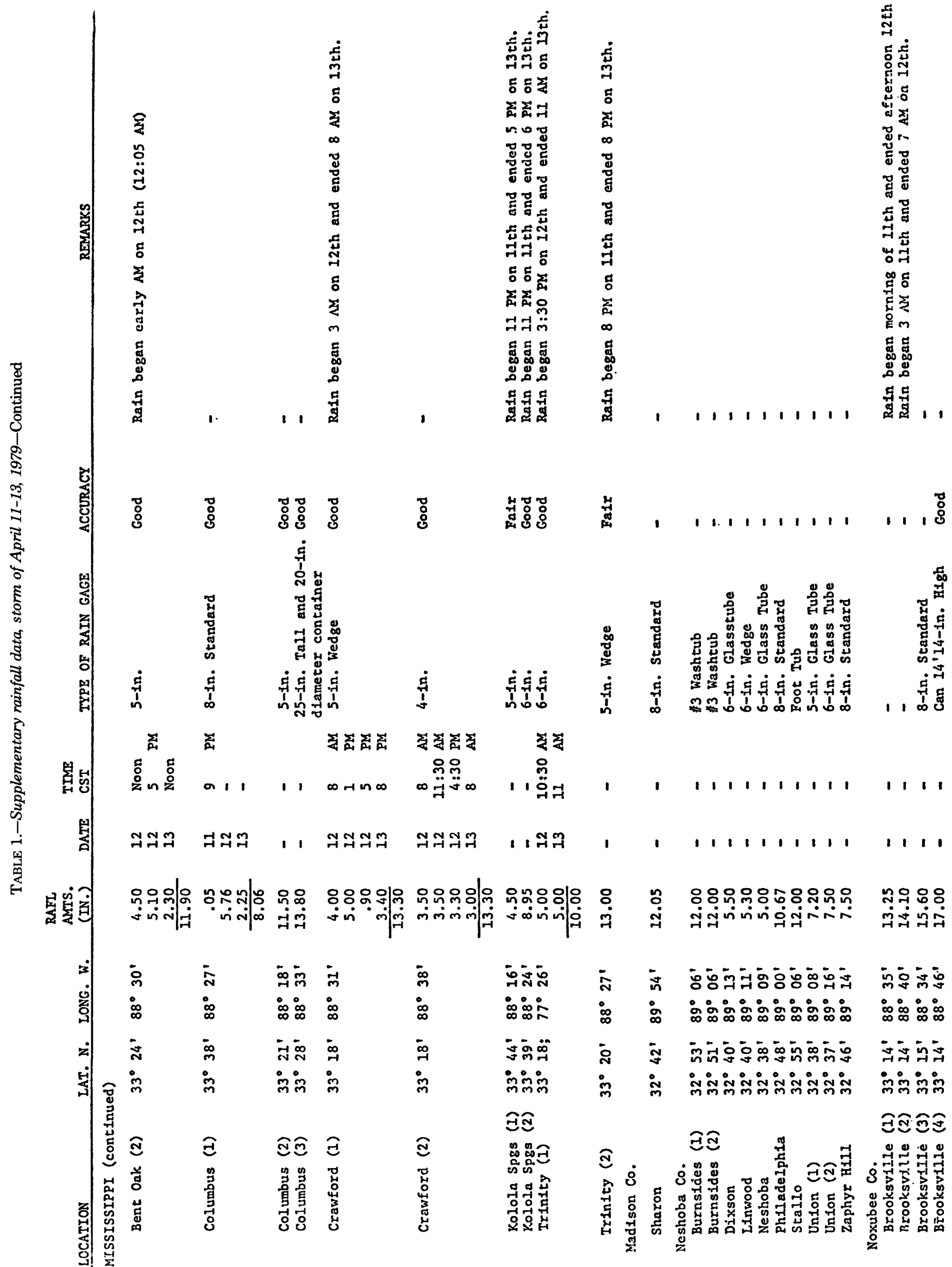




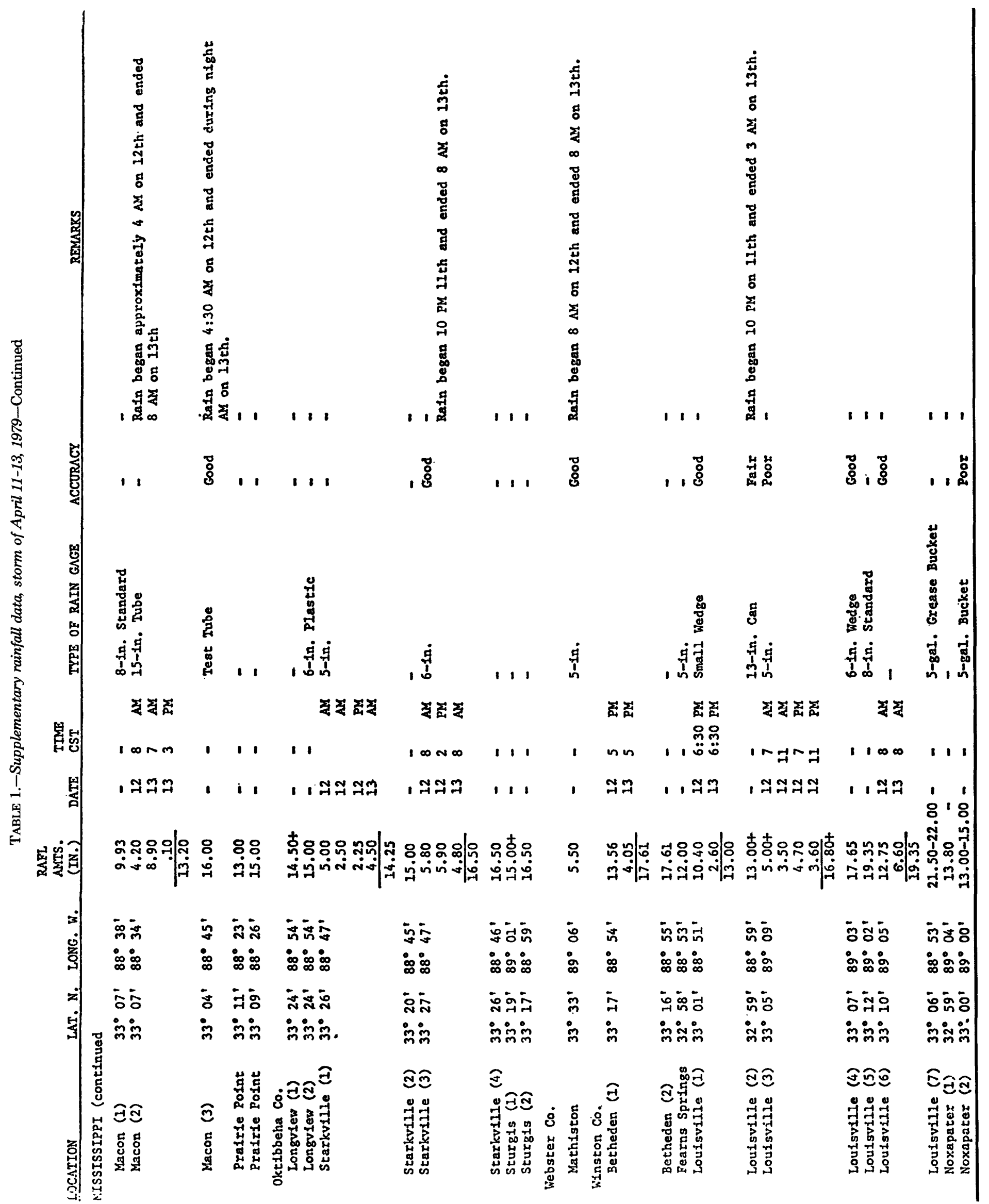




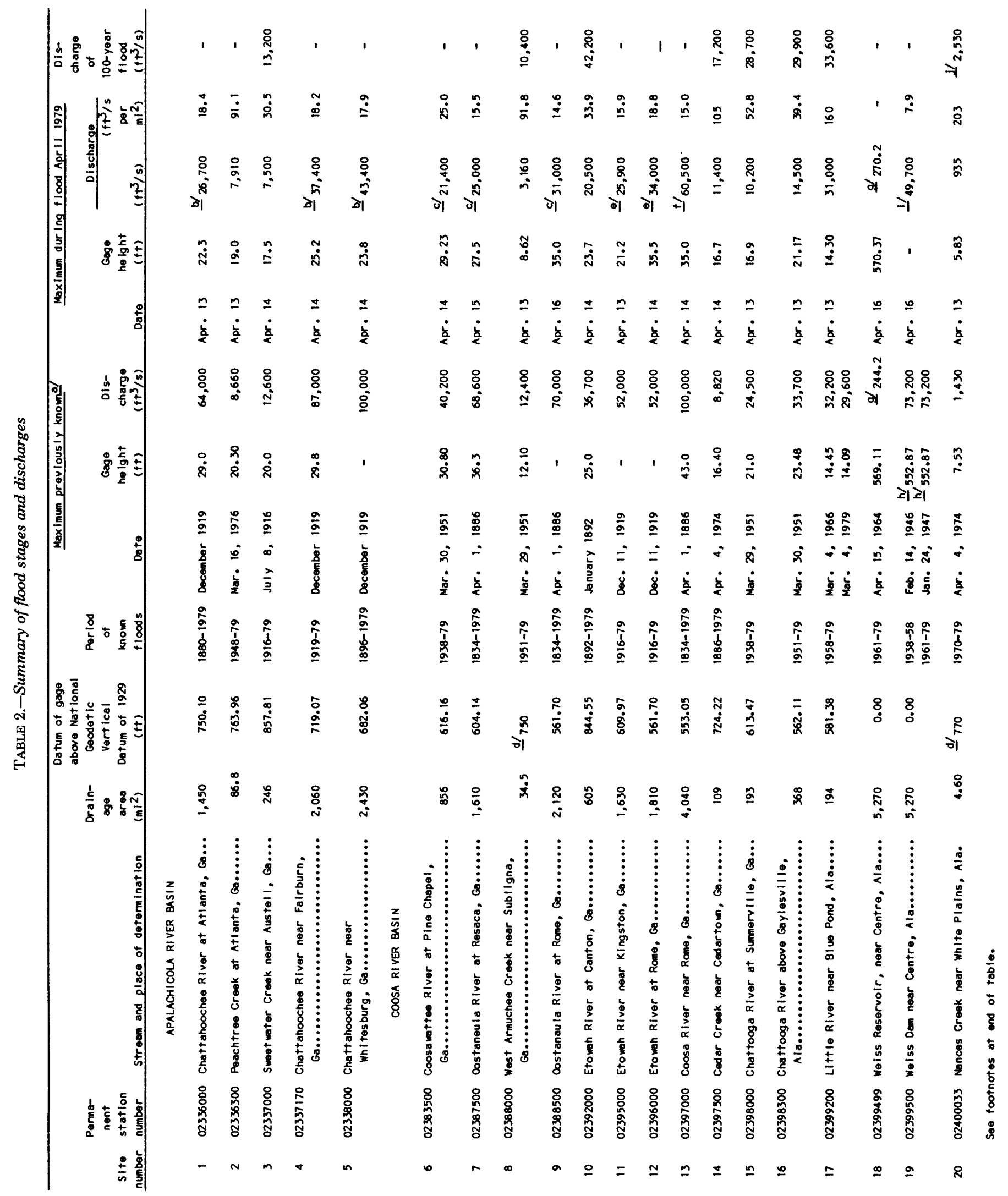




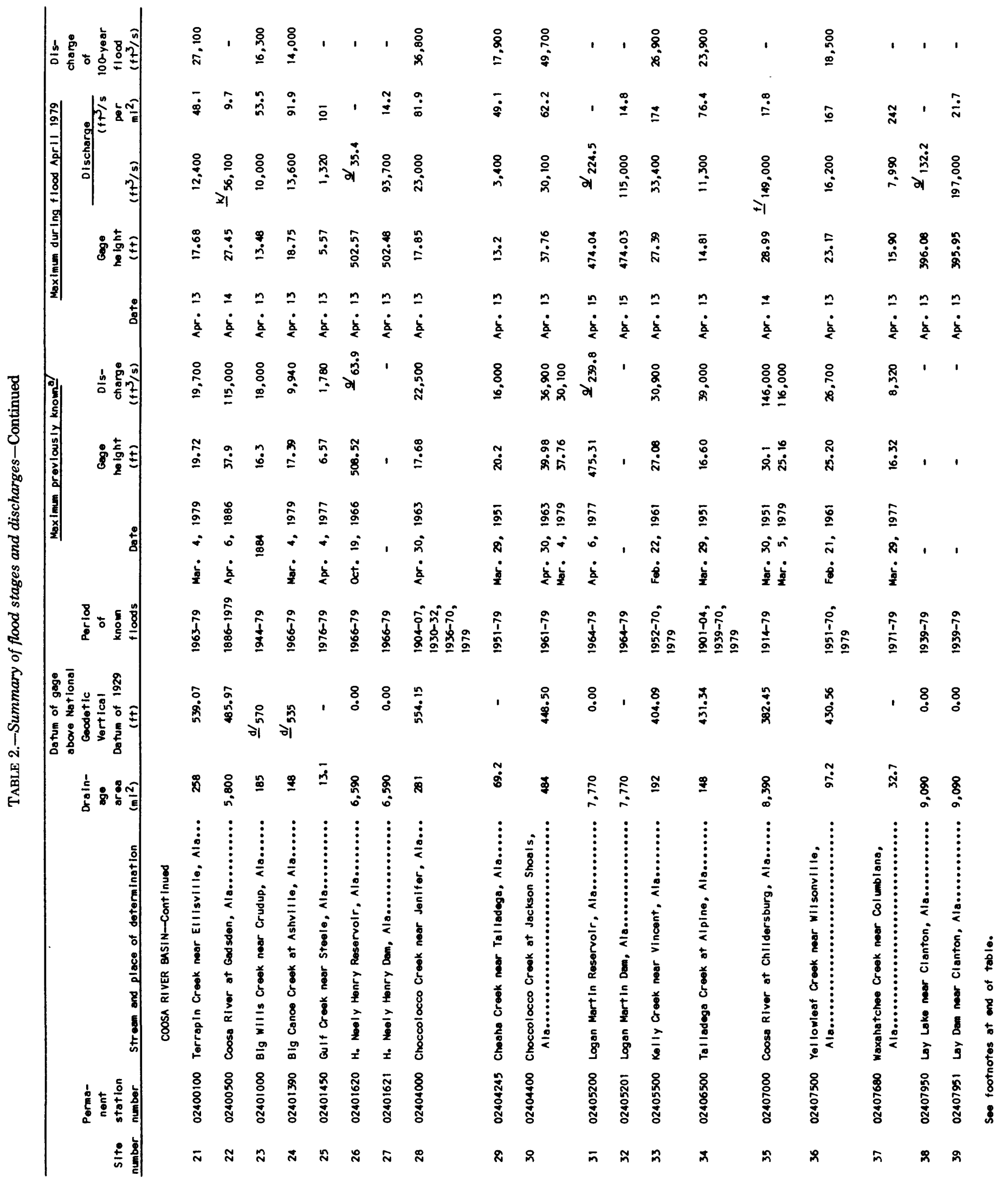


FLOODS OF APRIL 1979, MISSISSIPPI, ALABAMA, AND GEORGIA

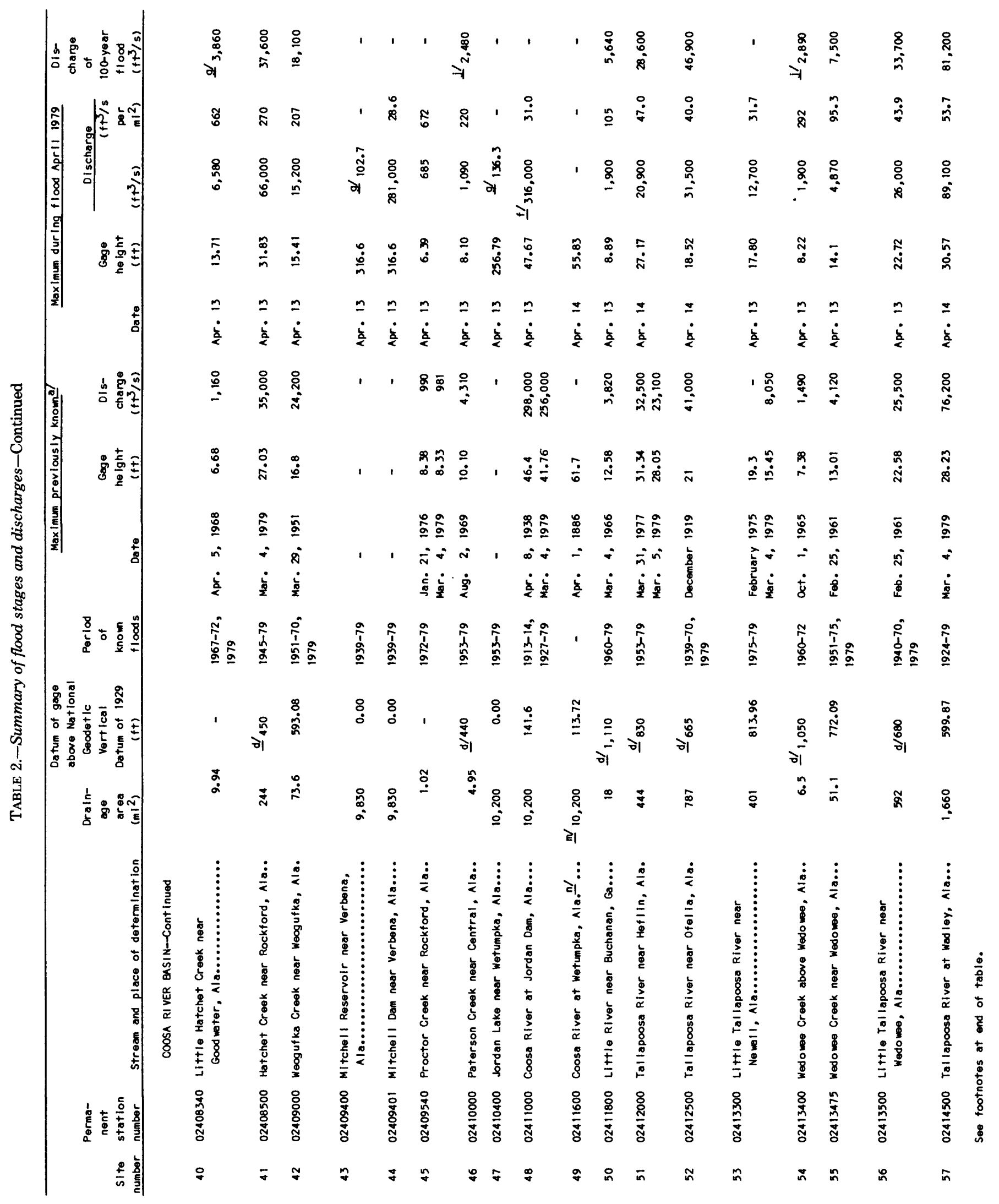




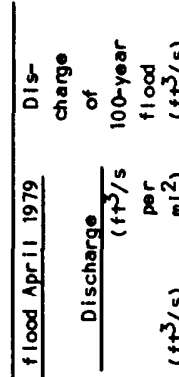

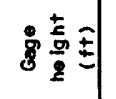

$\left|\frac{5}{x}\right|$

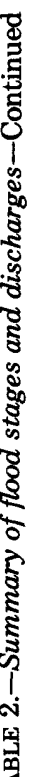

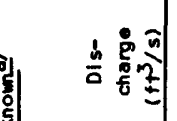

$\frac{8}{8} \div \frac{2}{2} \div \frac{1}{2}$

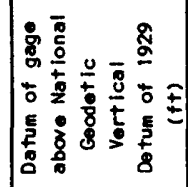

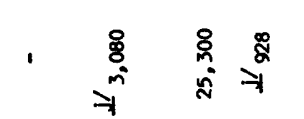

, $\quad \stackrel{8}{\square}$

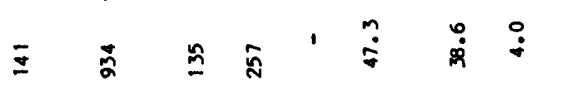

竞

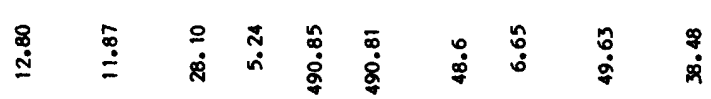

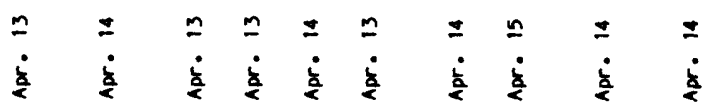

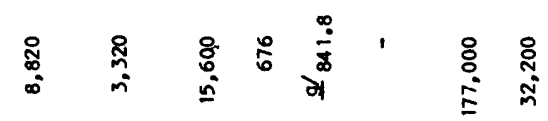

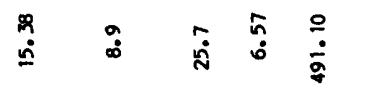

$\stackrel{\square}{a}$

+ त क

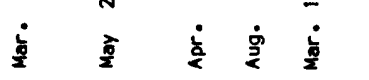

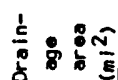

$\stackrel{\infty}{\dot{0}}$

突

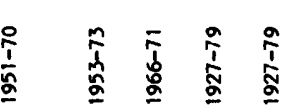

,

$\dot{8}$

$\stackrel{2}{2}$

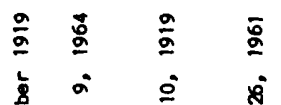

$\dot{8} \dot{8} \dot{8}$

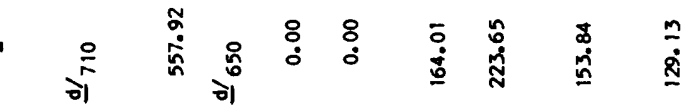

$3 \stackrel{2}{-}$

:

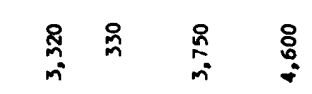

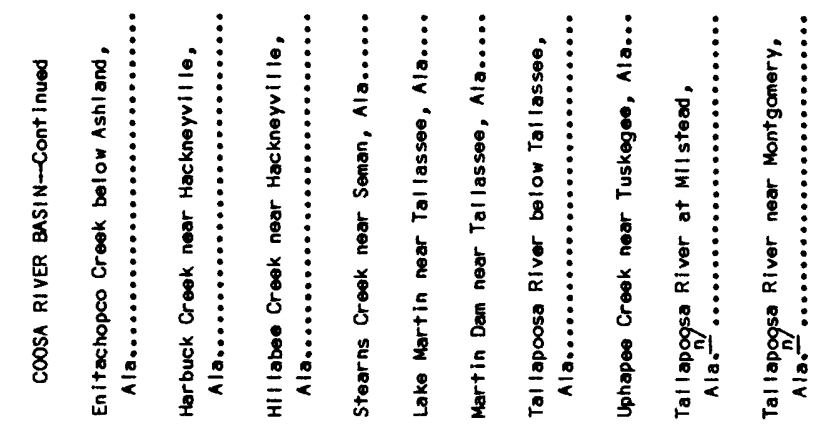

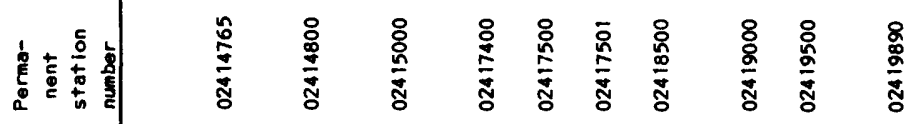

辛量

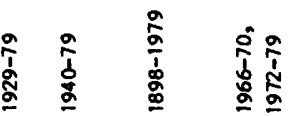

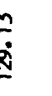

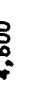

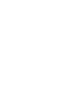

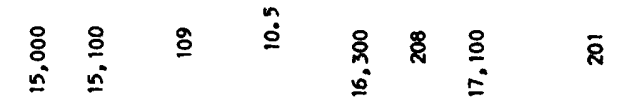

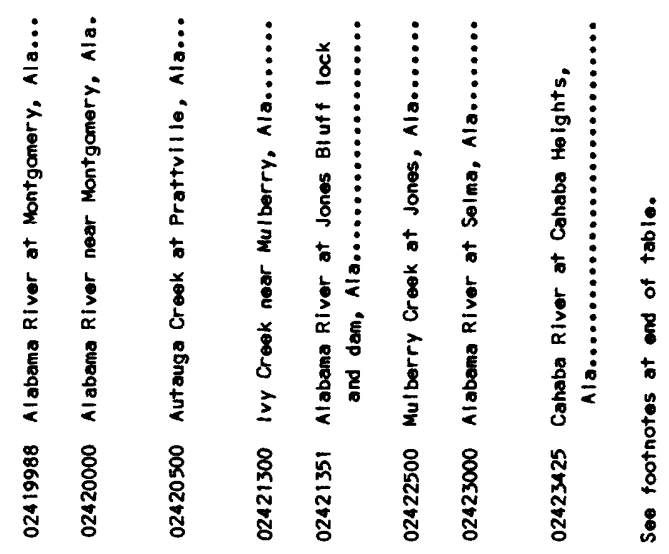




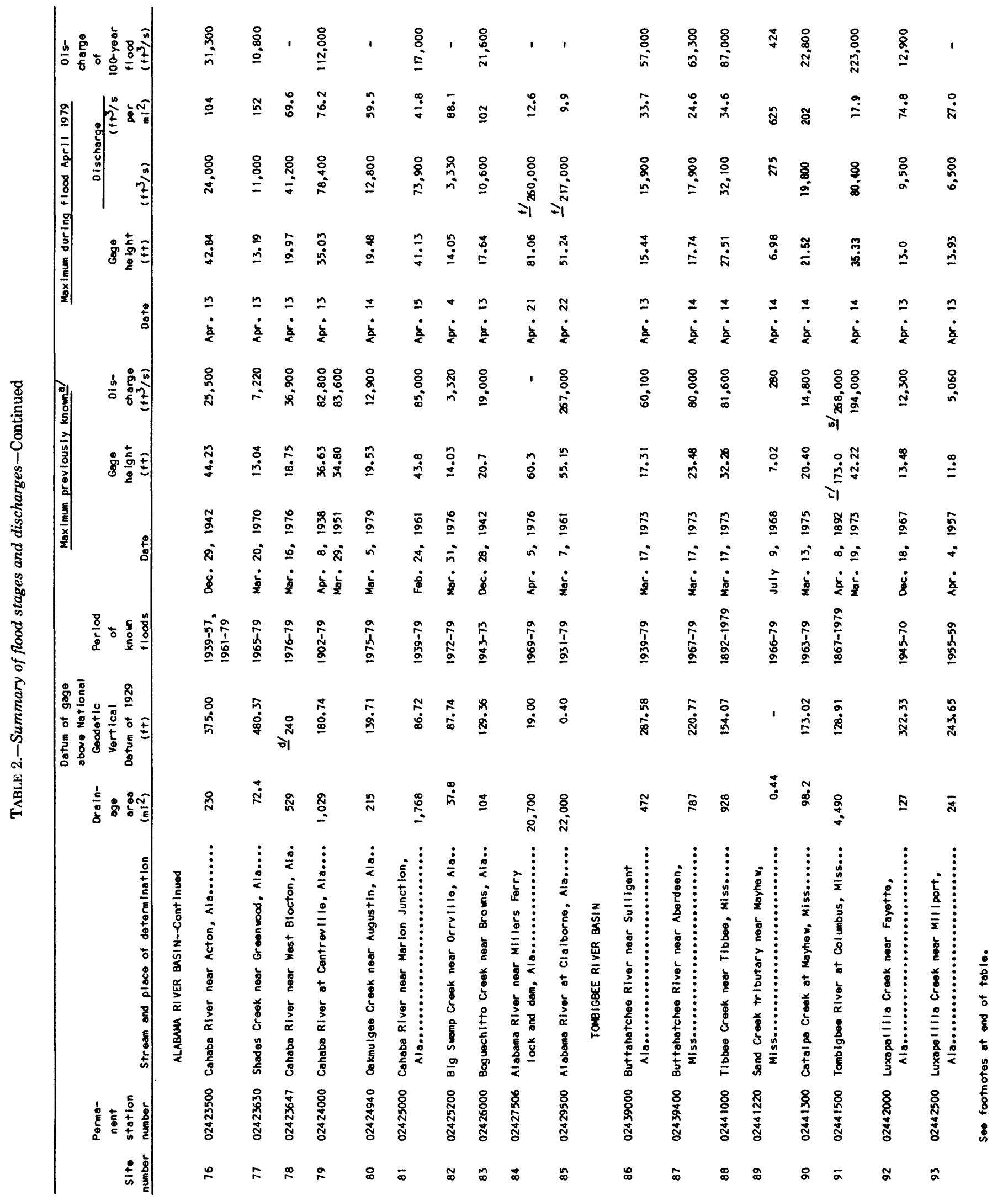




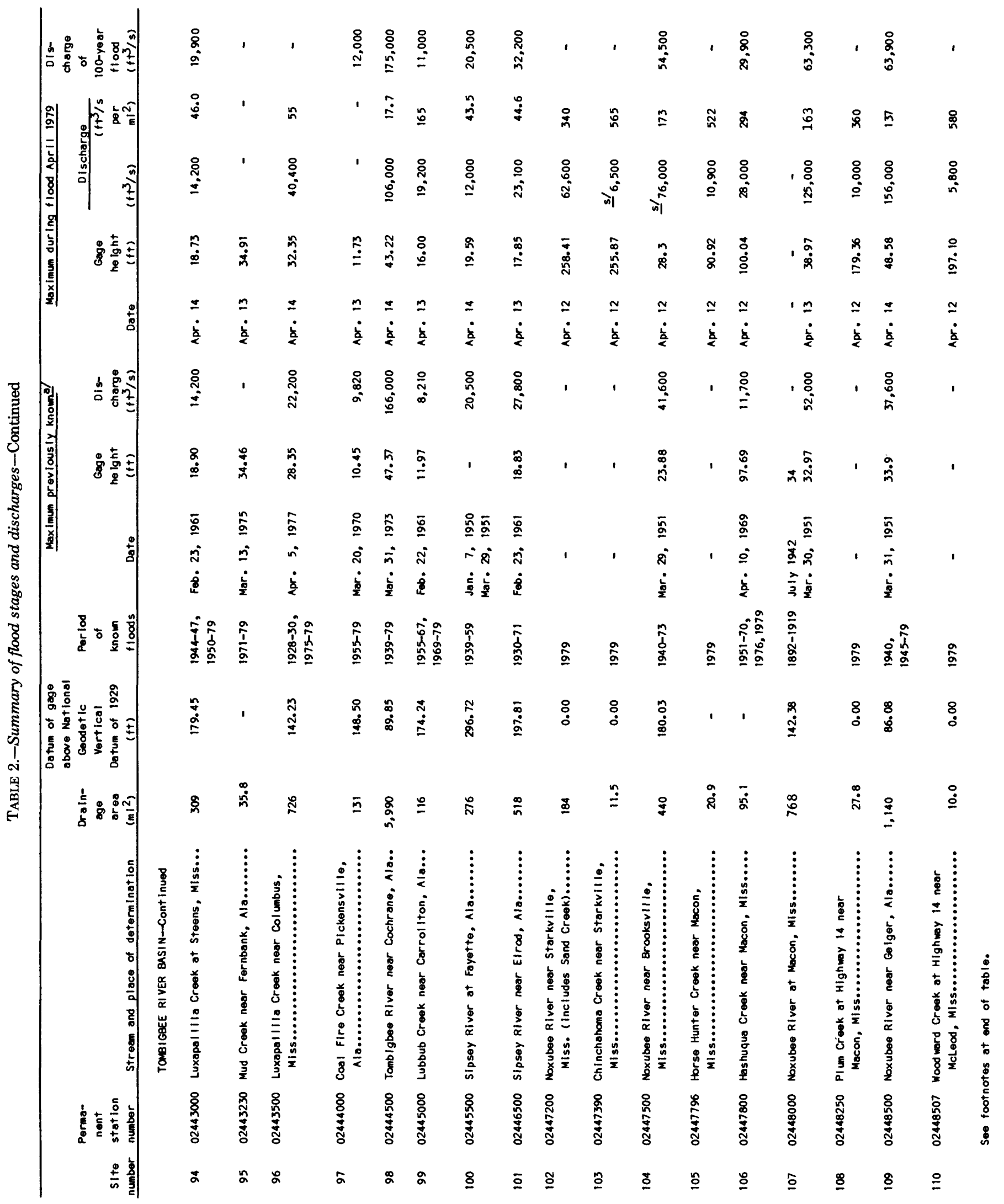




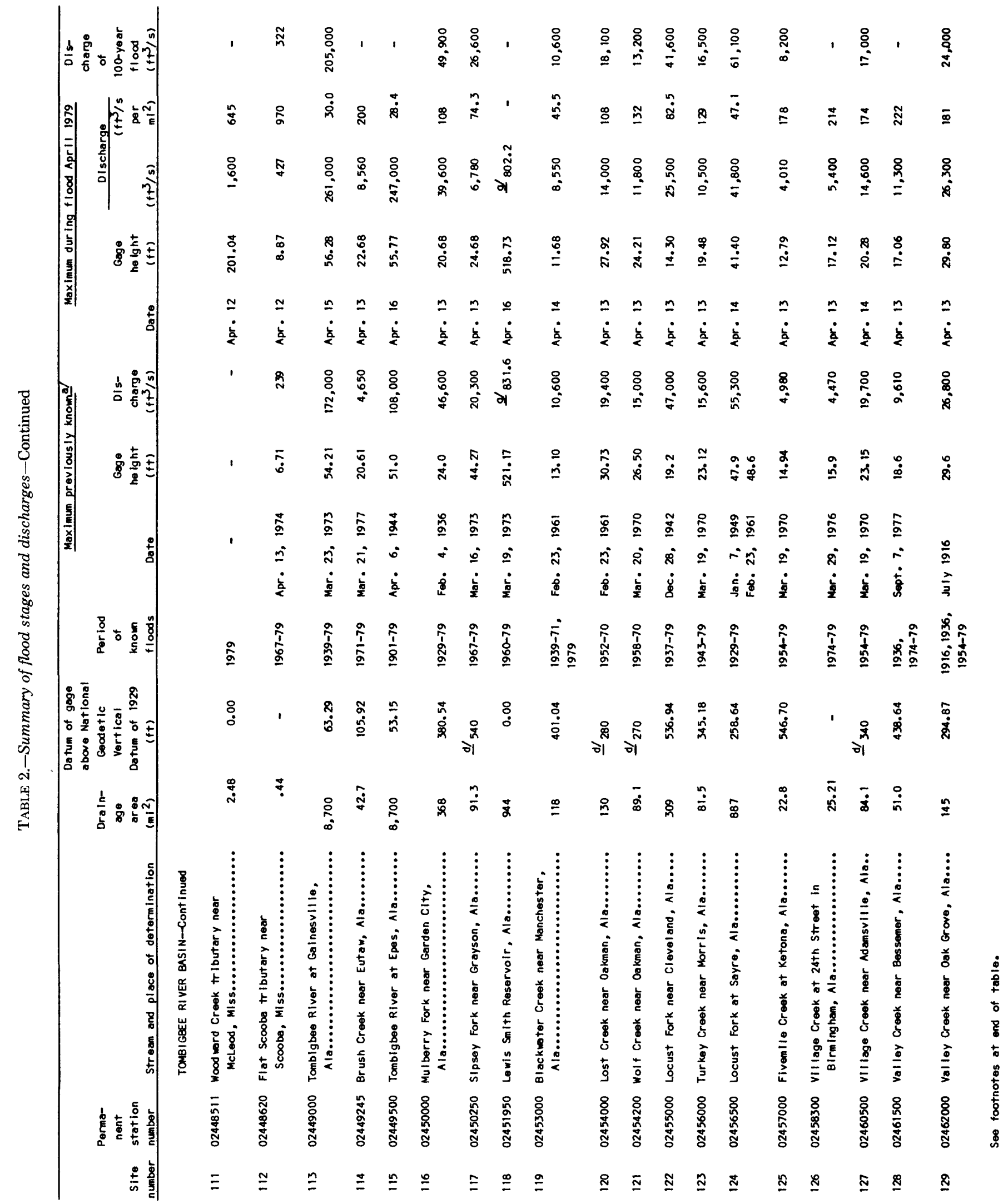




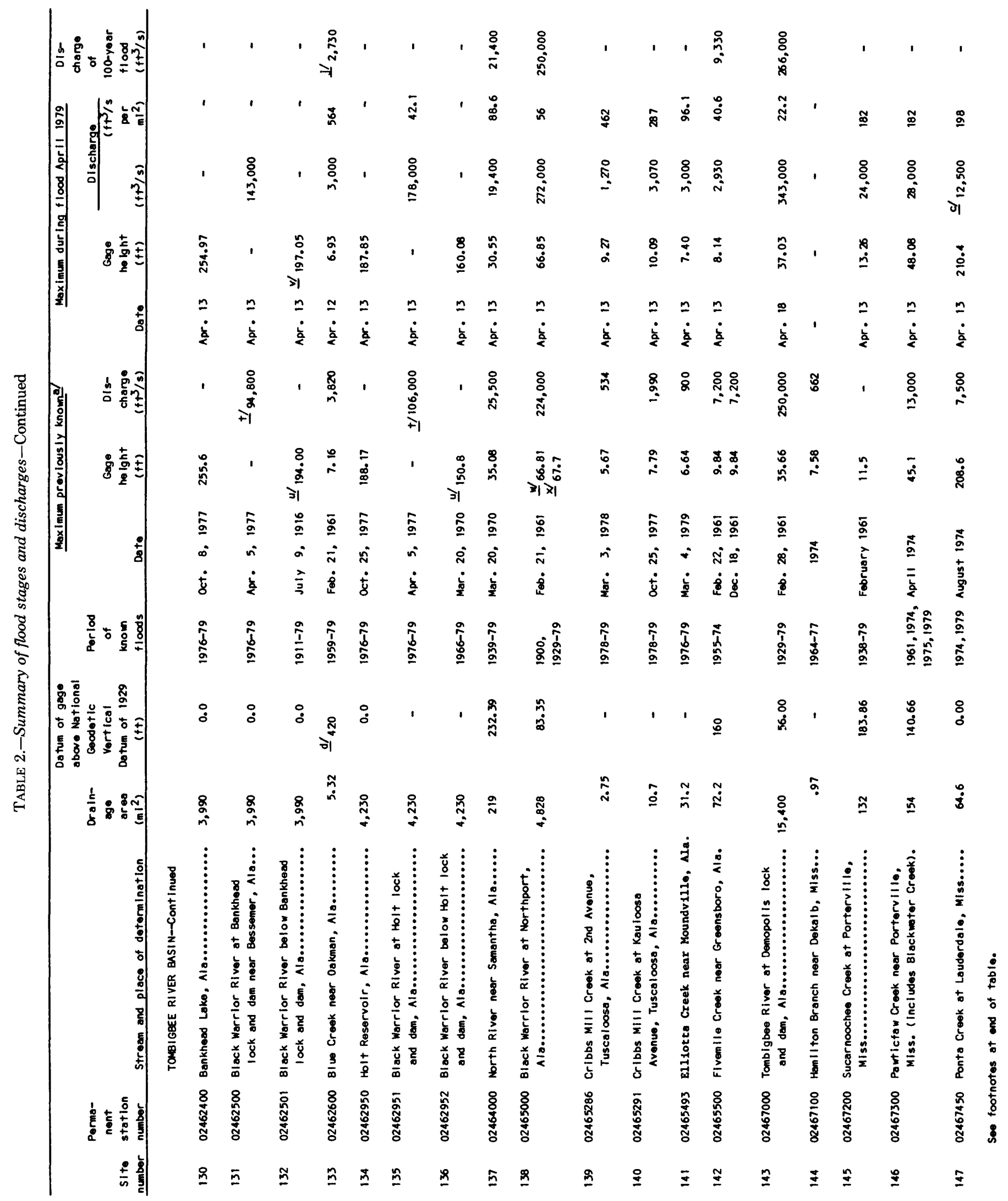




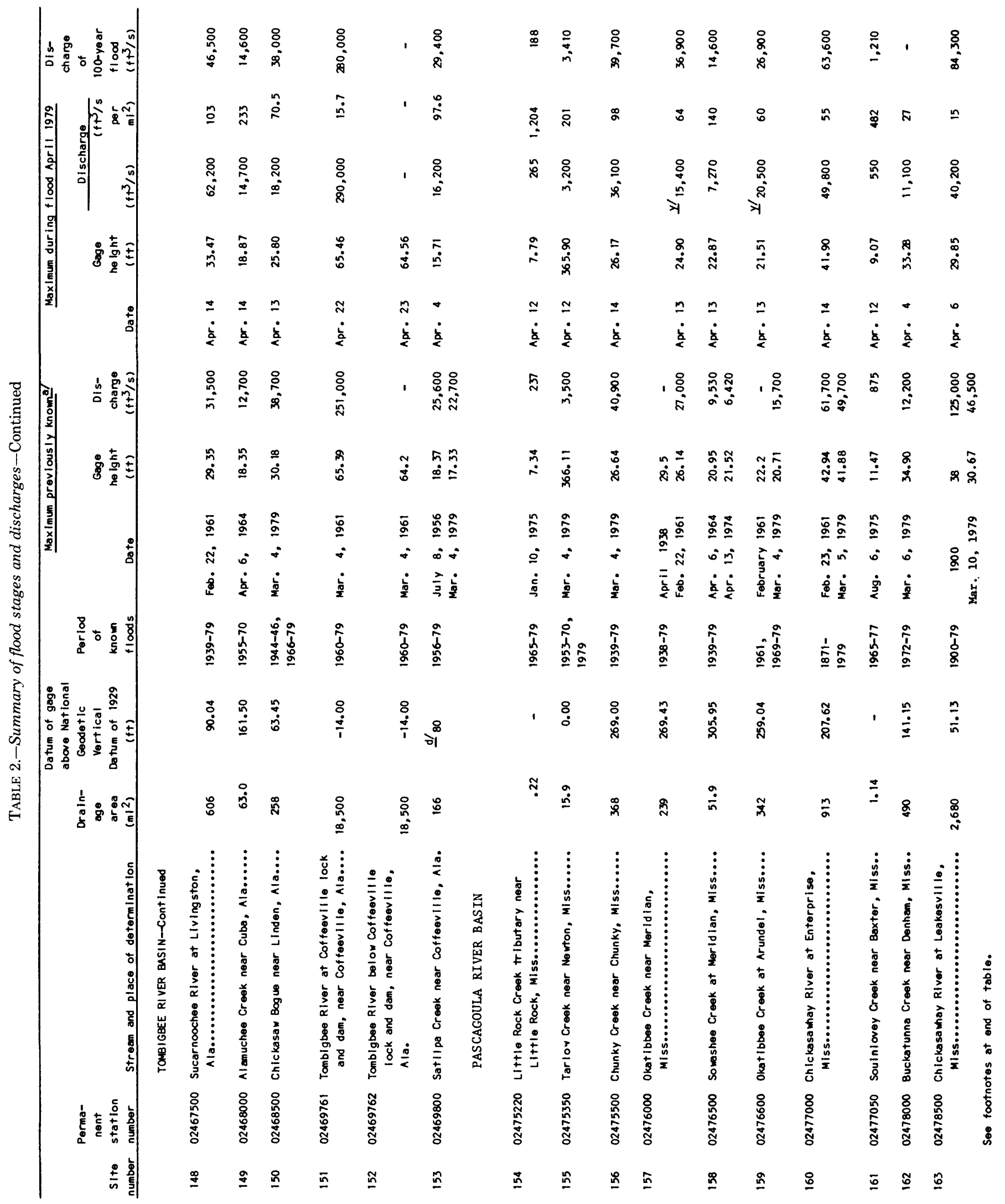




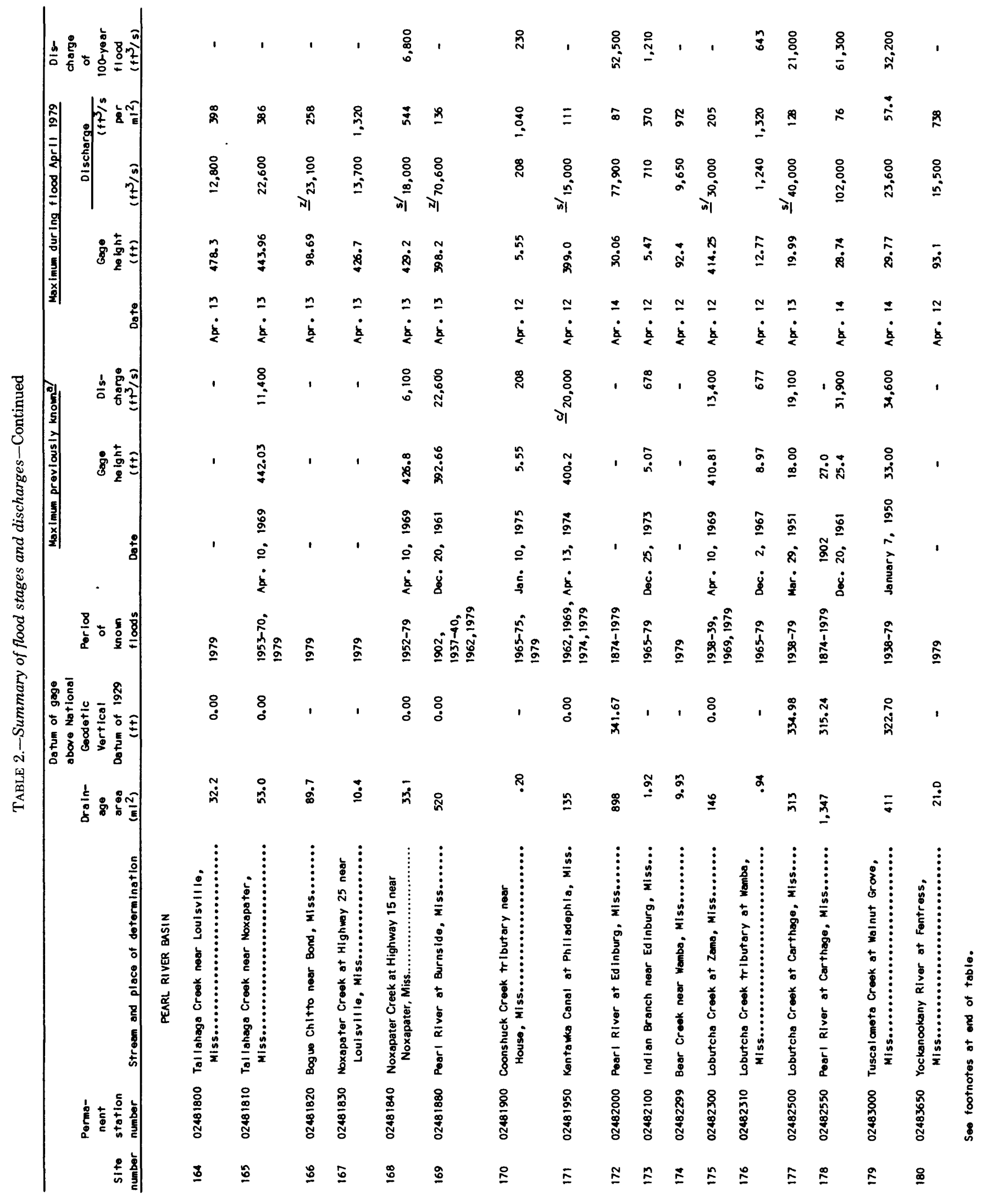




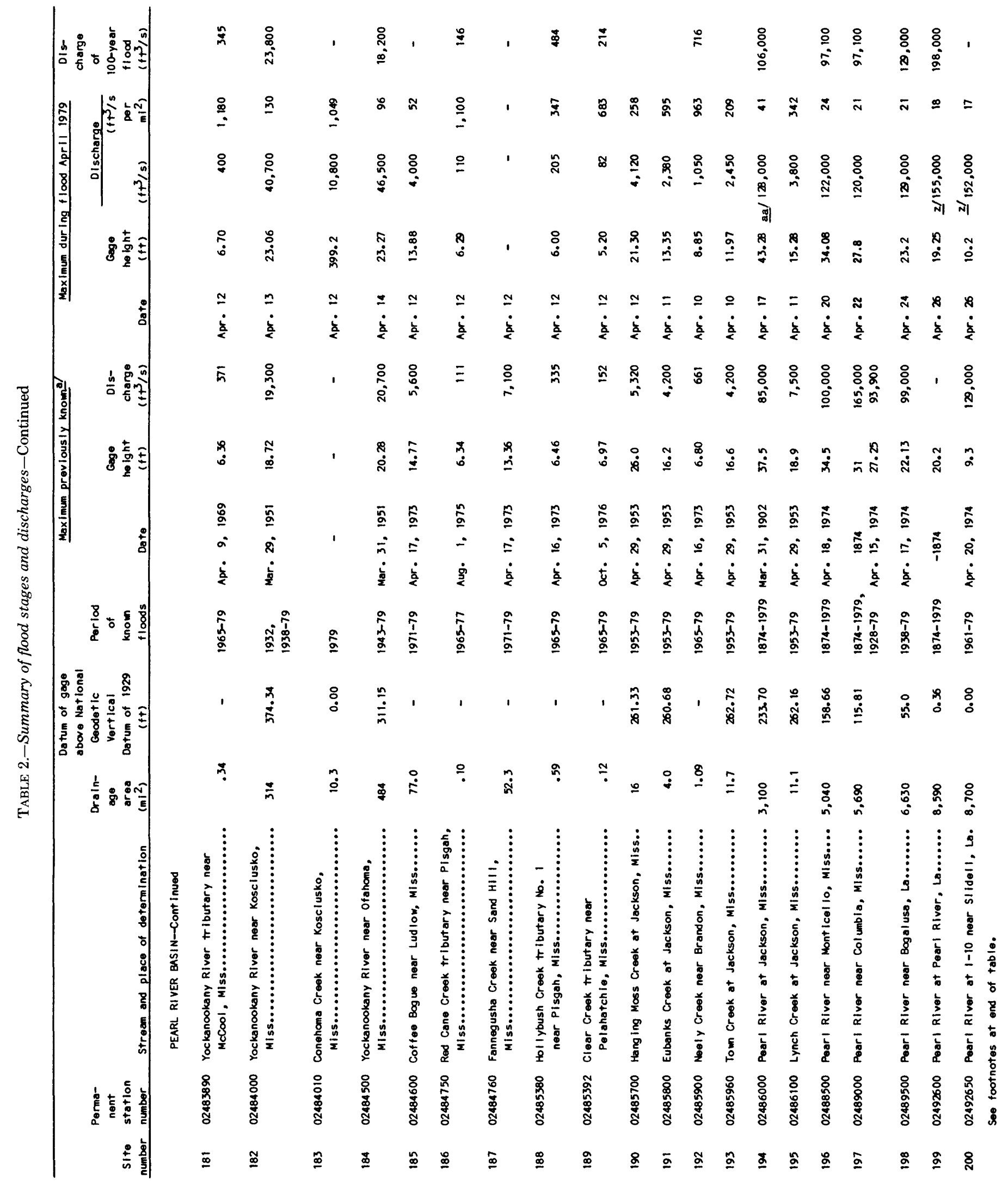




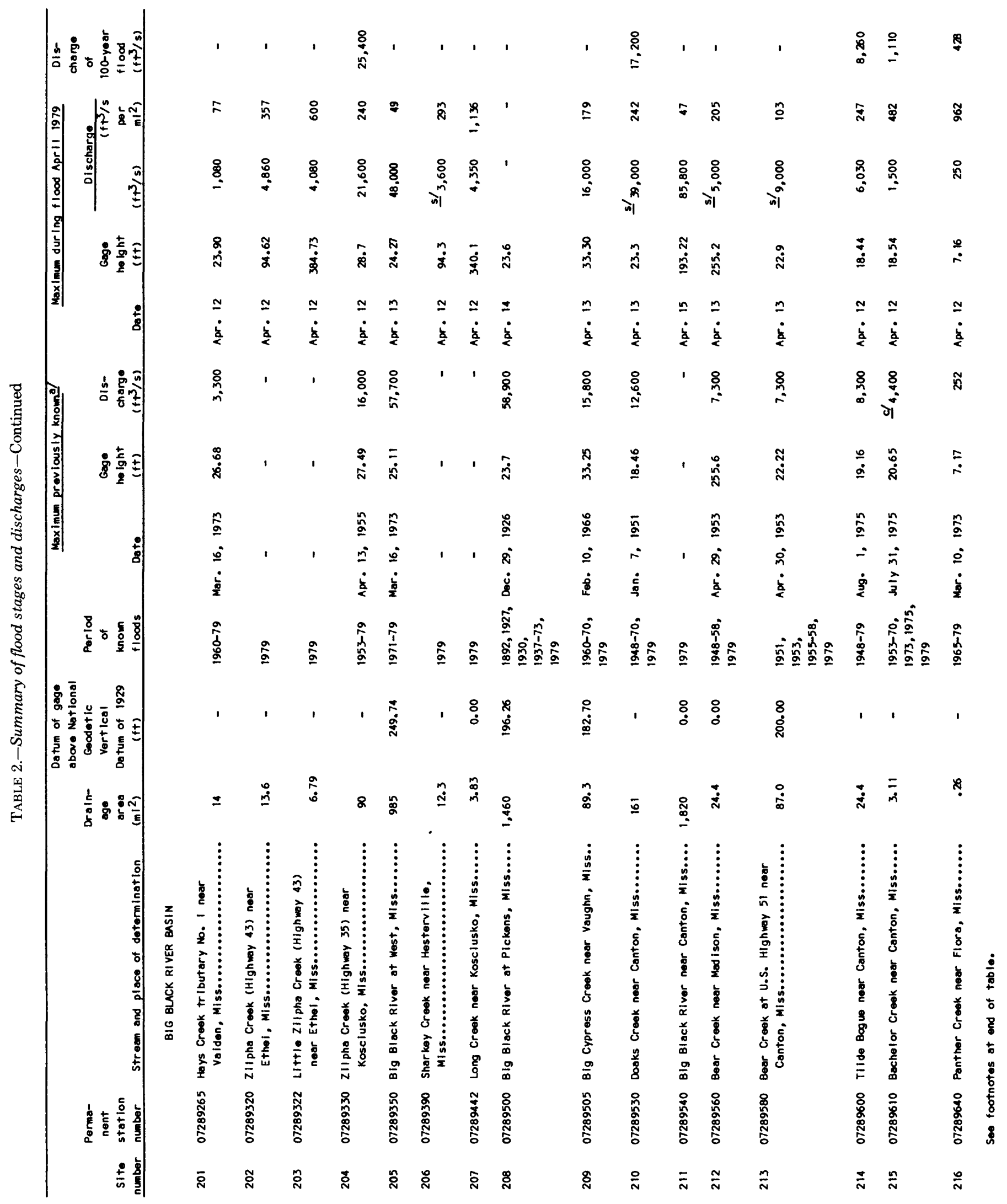




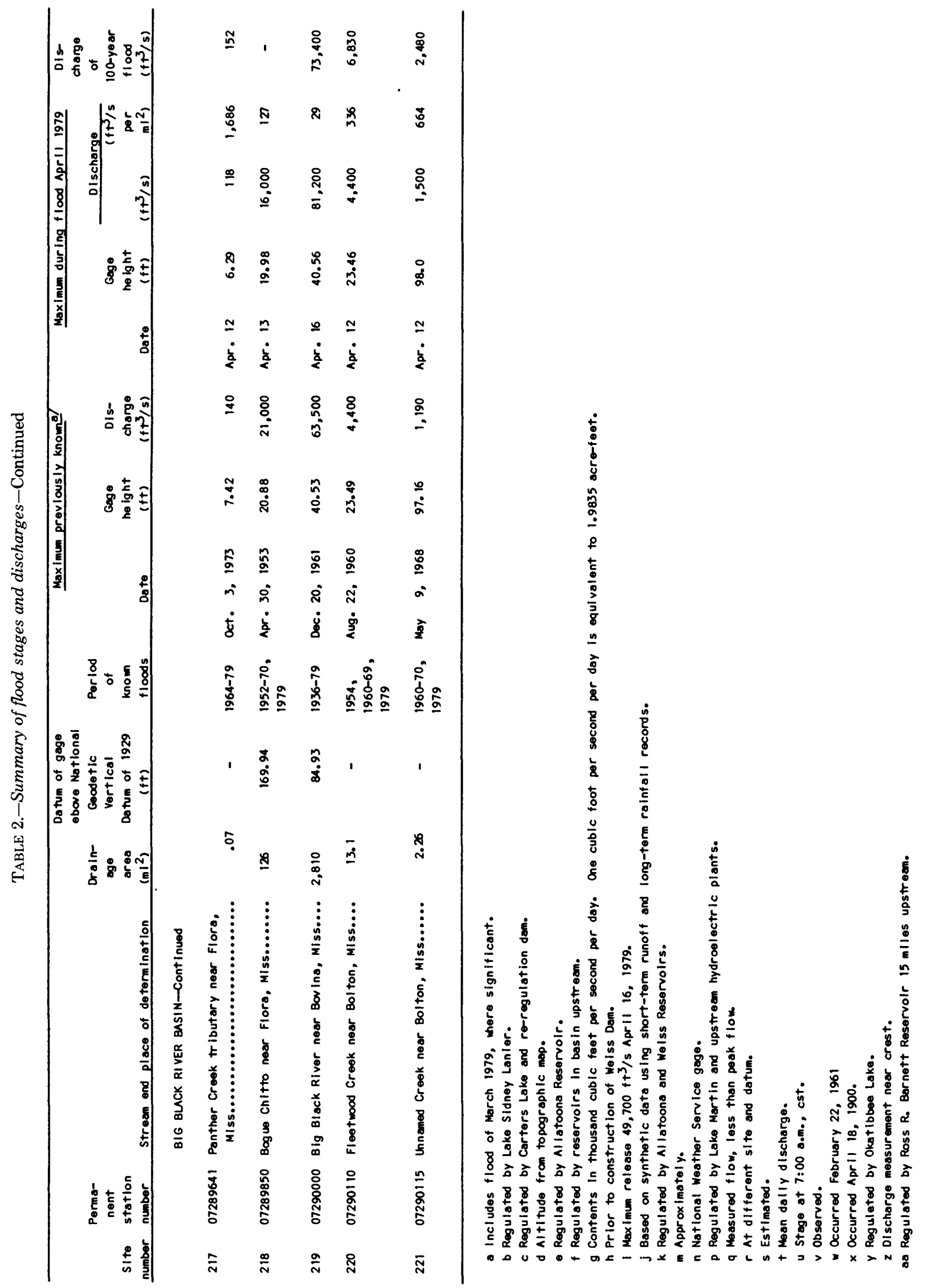


TABLE 3.-Summary of flood damages on main streams and principal tributaries March 1979 flood

Basin and Stream

Alabama-Coosa River Basin

Alabama River
Coosa River
Tallapoosa River

Escambia-Conecuh River Basin

Escambia River
Conecuh River

Total

Tombigbee River Basin Tombigbee River

Choctawhatchee River Basin Choctawhatchee River

Total

Coastal (Baldwin and Santa Rosa Counties)

Total

Pascagoula River Basin

Pascagoula River

Total

Pearl River Basin

Pearl River
Total

Flood damages in dollars

\section{Roads and}

Agriculture Railroads Urban \& Other Total

$\begin{array}{rrrr}110,000 & 91,700 & 4,300 & 206,000 \\ 1,514,300 & 1,313,000 & 11,989,700 & 14,817,000 \\ 200,000 & \frac{1,285,000}{2,689,700} & \frac{25,000}{12,019,000} & \frac{1,510,000}{16,533,000}\end{array}$

53,000

674,500

$13,189,000$

$13,916,500$

55,500

$\frac{--}{13,189,000}$

$\frac{55,500}{13,972,000}$

$\frac{--}{53,000}$

$\frac{55,500}{730,000}$

50,000

335,000

203,000

$\frac{203,000}{203,000}$

588,000

58,000
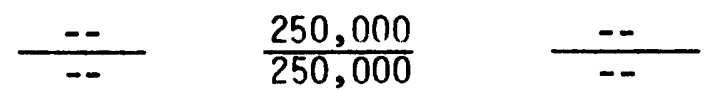

250,000

250,000

$\frac{2,023,000}{2,023,000}$

$\frac{1,020,000}{1,020,000}$

$\frac{3,750,000}{3,750,000}$

$\frac{6,793,000}{6,793,000}$

$\frac{6,793,000}{6,793,000}$

11,000

177,000

182,000

370,000

$\overline{182,000}$

$\frac{370,000}{370,000}$

Total

921,000

$\frac{1,038,000}{1,038,000}$

$1,451,000$

$\frac{3,410,000}{3,410,000}$

TOTAL

$6,239,700$

$30,794,000$

$41,916,000$ 


\begin{tabular}{|c|c|c|c|c|c|}
\hline \multirow[b]{2}{*}{ Basin and Stream } & & \multicolumn{4}{|c|}{ Flood damages in dollars } \\
\hline & & Agriculture & $\begin{array}{l}\text { Roads and } \\
\text { Railroads }\end{array}$ & Urban \& Other & Total \\
\hline \multicolumn{6}{|l|}{ Alabama-Coosa River Basin } \\
\hline $\begin{array}{l}\text { Alabama River } \\
\text { Coosa River } \\
\text { Tallapoosa River }\end{array}$ & Total & $\begin{array}{r}2,373,900 \\
1,365,000 \\
425,000 \\
4,163,900\end{array}$ & $\begin{array}{l}1,148,300 \\
2,014,300 \\
1,484,000 \\
4,646,600\end{array}$ & $\begin{array}{r}13,503,500 \\
5,261,900 \\
1,446,800 \\
20,212,200\end{array}$ & $\begin{array}{r}17,025,700 \\
8,641,200 \\
3,355,800 \\
29,022,700\end{array}$ \\
\hline $\begin{array}{l}\text { Apalachicola River Basin } \\
\text { Apalachicola River } \\
\text { Chattahoochee River } \\
\text { Flint River }\end{array}$ & Total & $\begin{array}{r}0 \\
0 \\
5 n, 000 \\
50,000\end{array}$ & $\begin{array}{r}0 \\
276,700 \\
410,000 \\
686,700\end{array}$ & $\begin{array}{r}0 \\
1,075,700 \\
205,000 \\
1,280,700\end{array}$ & $\begin{array}{r}0 \\
1,352,400 \\
\quad 665,000 \\
2,017,400\end{array}$ \\
\hline \multicolumn{6}{|l|}{ Tombigbee River Basin } \\
\hline $\begin{array}{l}\text { Tombigbee River } \\
\text { Black Warrior River }\end{array}$ & Total & $\begin{array}{r}9,346,100 \\
750,000 \\
10,096,100\end{array}$ & $\begin{array}{r}11,854,200 \\
879,900 \\
12,734,100\end{array}$ & $\begin{array}{r}17,031,300 \\
4,543,700 \\
21,575,000\end{array}$ & $\begin{array}{r}38,231,600 \\
6,173,600 \\
44,405,200\end{array}$ \\
\hline$\frac{\text { Mobile River Basin }}{\text { Mobile River }}$ & Total & $\frac{n}{0}$ & $\frac{580,000}{580,000}$ & $\frac{1,609,800}{1,609,800}$ & $\frac{2,189,800}{2,189,800}$ \\
\hline $\begin{array}{l}\text { Pascagoula River Basin } \\
\text { Pascagoula River } \\
\text { Leaf River } \\
\text { Chickasawhay River }\end{array}$ & Total & $\begin{array}{r}82,000 \\
637,0 n 0 \\
719,000\end{array}$ & $\begin{array}{r}304,000 \\
143,800 \\
1,387,800 \\
1,835,600\end{array}$ & $\begin{array}{r}216,000 \\
468,500 \\
1,167,800 \\
1,852,300\end{array}$ & $\begin{array}{r}520,000 \\
694,300 \\
3,192,600 \\
4,406,900\end{array}$ \\
\hline$\frac{\text { Pearl River Basin }}{\text { Pearl River }}$ & Total & $\frac{5,447,000}{5,447,000}$ & $\frac{12,236,000}{12,236,000}$ & $\frac{239,914,000}{239,914,000}$ & $\frac{257,597,000}{257,597,000}$ \\
\hline \multirow[t]{2}{*}{$\begin{array}{l}\frac{\text { Big Black River Basin }}{\text { Big Black River and }} \\
\text { southwest Tributaries }\end{array}$} & & $3,36 \cap, \cap \cap 0$ & -- & $1,240,0001 /$ & $4,600,000$ \\
\hline & TOTAL & $23,836,000$ & $32,719,000$ & $287,684,000$ & $344,239,000$ \\
\hline
\end{tabular}

1/ Includes Roads and Railroads 
TABLE 4.-Summary of stages and contents of storage reservoirs [Measurements taken at $2400 \mathrm{CST}$ on indicated dates]

Weiss Lake near Leesburg, Ala. 02399499

\begin{tabular}{|c|c|c|c|c|c|c|}
\hline & \multicolumn{3}{|c|}{ March 1979} & \multicolumn{3}{|c|}{ April 1979} \\
\hline Day & $\begin{array}{l}\text { Elevation } \\
\text { 1/ NGVD } \\
\text { of } 1929 \\
\text { (feet) }\end{array}$ & $\begin{array}{c}\text { Contents } \\
2 /\left(\mathrm{ft}^{3} / \mathrm{s} / \mathrm{day}\right)\end{array}$ & $\begin{array}{l}\text { Change in } \\
\text { storage } \\
\text { 2/ }(\mathrm{ft} 3 / \mathrm{s} / \text { day })\end{array}$ & $\begin{array}{l}\text { Elevation } \\
\text { 1/ NGVD } \\
\text { of } 1929 \\
\text { (feet) }\end{array}$ & $\begin{array}{l}\text { Contents } \\
2 /(\mathrm{ft} 3 / \mathrm{s} / \mathrm{day})\end{array}$ & $\begin{array}{l}\text { Change in } \\
\text { storage } \\
\text { 2/ }(\mathrm{ft} 3 / \mathrm{s} / \text { day })\end{array}$ \\
\hline $\begin{array}{l}1 \\
2 \\
3 \\
4 \\
5\end{array}$ & $\begin{array}{l}563.26 \\
563.22 \\
563.87 \\
567.38 \\
569.50\end{array}$ & $\begin{array}{l}143,510 \\
142,930 \\
152,490 \\
210,960 \\
252,050\end{array}$ & $\begin{array}{r}-580 \\
+9,560 \\
+58,470 \\
+41,090\end{array}$ & $\begin{array}{l}56 ? .60 \\
562.30 \\
562.24 \\
563.36 \\
563.40\end{array}$ & $\begin{array}{l}134,160 \\
130,050 \\
129,230 \\
144,960 \\
145,540\end{array}$ & $\begin{array}{r}+550 \\
-4,110 \\
-820 \\
+15,730 \\
+580\end{array}$ \\
\hline $\begin{array}{r}6 \\
7 \\
8 \\
9 \\
10\end{array}$ & $\begin{array}{l}570.41 \\
570.42 \\
570.89 \\
569.57 \\
568.94\end{array}$ & $\begin{array}{l}271,080 \\
271,300 \\
281,460 \\
253,490 \\
240,760\end{array}$ & $\begin{array}{r}+19,030 \\
+220 \\
+10,160 \\
-27,960 \\
-12,730\end{array}$ & $\begin{array}{l}563.28 \\
563.22 \\
562.78 \\
562.56 \\
562.36\end{array}$ & $\begin{array}{l}143,800 \\
142,930 \\
136,670 \\
133,610 \\
130,860\end{array}$ & $\begin{array}{r}-1,740 \\
-870 \\
-6,260 \\
-3,060 \\
-2,750\end{array}$ \\
\hline $\begin{array}{l}11 \\
12 \\
13 \\
14 \\
15\end{array}$ & $\begin{array}{l}568.10 \\
566.95 \\
565.54 \\
564.31 \\
563.54\end{array}$ & $\begin{array}{l}224,410 \\
203,160 \\
178,850 \\
159,190 \\
147,540\end{array}$ & $\begin{array}{l}-16,350 \\
-21,250 \\
-24,310 \\
-19,660 \\
-11,600\end{array}$ & $\begin{array}{l}562.40 \\
562.99 \\
566.78 \\
569.10 \\
570.07\end{array}$ & $\begin{array}{l}131,410 \\
139,640 \\
200,130 \\
243,950 \\
263,870\end{array}$ & $\begin{array}{r}+550 \\
+8,230 \\
+60,490 \\
+43,820 \\
+19,920\end{array}$ \\
\hline $\begin{array}{l}16 \\
17 \\
18 \\
19 \\
20\end{array}$ & $\begin{array}{l}563.05 \\
562.56 \\
561.95 \\
561.57 \\
562.06\end{array}$ & $\begin{array}{l}140,490 \\
133,610 \\
125,340 \\
120,360 \\
126,810\end{array}$ & $\begin{array}{l}-7,100 \\
-6,880 \\
-8,270 \\
-4,980 \\
+6,450\end{array}$ & $\begin{array}{l}570.31 \\
570.16 \\
569.89 \\
569.43 \\
568.80\end{array}$ & $\begin{array}{l}268,950 \\
265,770 \\
260,100 \\
250,620 \\
237,990\end{array}$ & $\begin{array}{r}+5,080 \\
-3,180 \\
-5,670 \\
-9,480 \\
-12,630\end{array}$ \\
\hline $\begin{array}{l}21 \\
22 \\
23 \\
24 \\
25\end{array}$ & $\begin{array}{l}561.74 \\
561.32 \\
561.69 \\
562.38 \\
562.50\end{array}$ & $\begin{array}{l}122,580 \\
117,160 \\
121,920 \\
131,140 \\
132,780\end{array}$ & $\begin{array}{l}-4,230 \\
-5,420 \\
+4,760 \\
+9,220 \\
+1,640\end{array}$ & $\begin{array}{l}567.92 \\
566.76 \\
565.50 \\
564.46 \\
563.66\end{array}$ & $\begin{array}{l}221,000 \\
199,780 \\
178,190 \\
161,510 \\
149,360\end{array}$ & $\begin{array}{l}-16,990 \\
-21,220 \\
-21,590 \\
-16,680 \\
-12,150\end{array}$ \\
\hline $\begin{array}{l}26 \\
27 \\
28 \\
29 \\
30 \\
31\end{array}$ & $\begin{array}{l}562.46 \\
562.36 \\
562.44 \\
562.28 \\
562.40 \\
562.56\end{array}$ & $\begin{array}{l}132,230 \\
130,860 \\
131,960 \\
129,770 \\
131,410 \\
133,610\end{array}$ & $\begin{array}{r}-550 \\
-1,370 \\
+1,100 \\
-2,190 \\
+1,640 \\
+2,200\end{array}$ & $\begin{array}{l}563.26 \\
563.30 \\
563.39 \\
563.22 \\
563.46\end{array}$ & $\begin{array}{l}143,510 \\
144,080 \\
145,390 \\
142,930 \\
146,420\end{array}$ & $\begin{array}{r}-5,850 \\
+570 \\
+1,310 \\
-2,460 \\
+3,490\end{array}$ \\
\hline
\end{tabular}

1/ National Geodetic Vertical Datum of 1929.

. 2 One cubic foot per second per day is equivalent to 1.9835 acre-feet.

Note: Records furnished by Alabama Power Co. 
TABLE 4.-Summary of stages and contents of storage reservoirs-Continued

H. Neely Henry Reservoir near Ohatchee, Ala. 02401620

\begin{tabular}{|c|c|c|c|c|c|c|}
\hline \multirow[b]{2}{*}{ Day } & \multicolumn{3}{|c|}{ March 1979} & \multicolumn{3}{|c|}{ April 1979} \\
\hline & $\begin{array}{l}\text { Elevation } \\
\text { 1/ NGVD } \\
\text { of } 1929 \\
\text { (feet) }\end{array}$ & $\begin{array}{c}\text { Contents } \\
2 /(\mathrm{ft} 3 / \mathrm{s} / \text { day })\end{array}$ & $\begin{array}{l}\text { Change in } \\
\text { storage } \\
2 /\left(\mathrm{ft}^{3} / \mathrm{s} / \text { day }\right)\end{array}$ & $\begin{array}{l}\text { Elevatior } \\
\text { 1/ NGVD } \\
\text { of } 1929 \\
\text { (feet) }\end{array}$ & $\begin{array}{l}\text { Contents } \\
2 /\left(\mathrm{ft}^{3} / \mathrm{s} / \text { day }\right)\end{array}$ & $\begin{array}{l}\text { Change in } \\
\text { storage } \\
2 /\left(\mathrm{ft}^{3} / \mathrm{s} / \text { day }\right)\end{array}$ \\
\hline 1 & 505.00 & 45,610 & & 504.83 & 44,840 & $+1,780$ \\
\hline 2 & 504.96 & 45,430 & -180 & 503.92 & 40,660 & $-4,180$ \\
\hline 3 & 503.74 & 40,100 & $-5,330$ & 504.41 & 42,970 & $+2,310$ \\
\hline 4 & 502.50 & 35,160 & $-4,940$ & 502.49 & 35,120 & $-7,850$ \\
\hline 5 & 502.54 & 35,310 & +150 & 502.64 & 35,700 & +580 \\
\hline 6 & 502.41 & 34,820 & -490 & 504.36 & 42,750 & $+7,050$ \\
\hline 7 & 502.43 & 34,900 & +80 & 505.01 & 45,660 & $+2,910$ \\
\hline 8 & 502.58 & 35,470 & +570 & 505.00 & 45,610 & -50 \\
\hline 9 & 502.51 & 35,200 & -270 & 504.97 & 45,480 & -130 \\
\hline 10 & 502.42 & 34,560 & -640 & 504.83 & 44,840 & -640 \\
\hline 11 & 502.49 & 35,120 & +560 & 504.47 & 43,230 & $-1,610$ \\
\hline 12 & 502.44 & 34,930 & -190 & 502.50 & 35,160 & $-8,070$ \\
\hline 13 & 502.38 & 34,710 & -220 & 502.57 & 35,430 & +270 \\
\hline 14 & 502.54 & 35,310 & +600 & 502.53 & 35,270 & -160 \\
\hline 15 & 505.00 & 45,610 & $+10,300$ & 502.40 & 34,780 & -490 \\
\hline 16 & 505.00 & 45,610 & 0 & 502.50 & 35,160 & +380 \\
\hline 17 & 504.92 & 45,290 & -320 & 502.58 & 35,470 & +310 \\
\hline 18 & 504.57 & 43,670 & $-1,620$ & 502.54 & 35,310 & -160 \\
\hline 19 & 504.51 & 43,410 & -260 & 502.64 & 35,700 & +390 \\
\hline 20 & 501.31 & 30,840 & $-12,570$ & 502.56 & 35,590 & -310 \\
\hline 21 & 503.63 & 39,640 & $+8,800$ & 502.58 & 35,470 & +70 \\
\hline 22 & 503.18 & 37,810 & $-1,830$ & 502.44 & 34,930 & -540 \\
\hline 23 & 504.43 & 43,060 & $+5,250$ & 503.36 & 38,540 & $+3,610$ \\
\hline 24 & 504.25 & 42,270 & -790 & 504.99 & 45,570 & $+7,030$ \\
\hline 25 & 504.63 & 43,940 & $+1,670$ & 506.50 & 52,860 & $+7,290$ \\
\hline 26 & 504.42 & 43,010 & -930 & 506.56 & 53,170 & +310 \\
\hline 27 & 503.81 & 40,390 & $-2,620$ & 507.36 & 57,380 & $+4,210$ \\
\hline 28 & 503.78 & 40,270 & -120 & 507.59 & 58,640 & $+1,260$ \\
\hline 29 & 503.42 & 38,750 & $-1,520$ & 507.56 & 58,470 & -170 \\
\hline 30 & 503.63 & 39,640 & +890 & 507.47 & 57,980 & -490 \\
\hline 31 & 504.48 & 43,060 & $+3,420$ & & & \\
\hline
\end{tabular}

1/ National Geodetic Vertical Datum of 1929.

2) One cubic foot per second per day is equivalent to 1.9835 acre-feet.

Note: Records furnished by Alabama Power Co. 
TABLE 4.-Summary of stages and contents of storage reservoirs-Continued Logan Martin Reservoir near Childersburg, Ala. 02405200

\begin{tabular}{|c|c|c|c|c|c|c|}
\hline \multirow[b]{2}{*}{ Day } & \multicolumn{3}{|c|}{ March 1979} & \multicolumn{3}{|c|}{ April 1979} \\
\hline & $\begin{array}{l}\text { Elevation } \\
\text { 1/NGVD } \\
\text { of } 1929 \\
\text { (feet) }\end{array}$ & $\begin{array}{c}\text { Contents } \\
2 /\left(\mathrm{ft}^{3} / \mathrm{s} / \text { day }\right) \\
\end{array}$ & $\begin{array}{c}\text { Change in } \\
\text { storage } \\
2 /\left(\mathrm{ft}^{3} / \mathrm{s} / \text { day }\right)\end{array}$ & $\begin{array}{l}\text { Elevation } \\
\text { 1/NGDV } \\
\text { of } 1929 \\
\text { (feet) }\end{array}$ & $\begin{array}{c}\text { Contents } \\
2 /\left(\mathrm{ft}^{3} / \mathrm{s} / \text { day }\right) \\
\end{array}$ & $\begin{array}{c}\text { Change in } \\
\text { storage } \\
2 /\left(\mathrm{ft}^{3} / \mathrm{s} / \mathrm{day}\right)\end{array}$ \\
\hline $\begin{array}{l}1 \\
2 \\
3 \\
4 \\
5\end{array}$ & $\begin{array}{l}459.94 \\
459.91 \\
460.55 \\
468.79 \\
471.83\end{array}$ & $\begin{array}{l}103,370 \\
103,190 \\
107,070 \\
169,790 \\
199,910\end{array}$ & $\begin{array}{r}-180 \\
+3,880 \\
+62,720 \\
+30,120\end{array}$ & $\begin{array}{l}459.95 \\
459.81 \\
461.53 \\
463.74 \\
464.84\end{array}$ & $\begin{array}{l}103,430 \\
102,600 \\
113,260 \\
128,390 \\
136,570\end{array}$ & $\begin{array}{r}+1,600 \\
-830 \\
+10,660 \\
+15,130 \\
+8,180\end{array}$ \\
\hline $\begin{array}{r}6 \\
7 \\
8 \\
9 \\
10\end{array}$ & $\begin{array}{l}472.16 \\
470.86 \\
468.87 \\
467.09 \\
465.94\end{array}$ & $\begin{array}{l}203,430 \\
180,840 \\
170,530 \\
154,720 \\
145,200\end{array}$ & $\begin{array}{r}+3,520 \\
-13,590 \\
-19,310 \\
-15,810 \\
-9,520\end{array}$ & $\begin{array}{l}464.68 \\
463.23 \\
461.74 \\
461.24 \\
461.37\end{array}$ & $\begin{array}{l}135,350 \\
124,750 \\
114,630 \\
111,400 \\
112,230\end{array}$ & $\begin{array}{r}-1,220 \\
-10,600 \\
-10,120 \\
-3,230 \\
+830\end{array}$ \\
\hline $\begin{array}{l}11 \\
12 \\
13 \\
14 \\
15\end{array}$ & $\begin{array}{l}464.70 \\
463.57 \\
462.60 \\
461.75 \\
460.64\end{array}$ & $\begin{array}{l}135,500 \\
127,170 \\
120,300 \\
114,690 \\
107,630\end{array}$ & $\begin{array}{l}-9,700 \\
-8,330 \\
-6,870 \\
-5,610 \\
-7,060\end{array}$ & $\begin{array}{l}461.41 \\
464.61 \\
471.95 \\
474.04 \\
472.83\end{array}$ & $\begin{array}{l}112,490 \\
134,820 \\
201,180 \\
224,540 \\
210,760\end{array}$ & $\begin{array}{r}+260 \\
+22,330 \\
+66,360 \\
+23,360 \\
-13,780\end{array}$ \\
\hline $\begin{array}{l}16 \\
17 \\
18 \\
19 \\
20\end{array}$ & $\begin{array}{l}460.40 \\
459.77 \\
459.77 \\
459.75 \\
459.68\end{array}$ & $\begin{array}{l}106,150 \\
102,360 \\
102,360 \\
102,240 \\
101,830\end{array}$ & $\begin{array}{r}-1,480 \\
-3,790 \\
0 \\
-120 \\
-410\end{array}$ & $\begin{array}{l}470.77 \\
469.09 \\
467.62 \\
466.36 \\
465.43\end{array}$ & $\begin{array}{l}188,930 \\
172,580 \\
159,290 \\
148,610 \\
141,120\end{array}$ & $\begin{array}{r}-21,830 \\
-16,350 \\
-13,290 \\
-10,680 \\
-7,490\end{array}$ \\
\hline $\begin{array}{l}21 \\
22 \\
23 \\
24 \\
25\end{array}$ & $\begin{array}{r}459.47 \\
459.08 \\
3 / 459.99 \\
460.06 \\
459.82\end{array}$ & $\begin{array}{r}100,590 \\
98,340 \\
103,670 \\
104,090 \\
102,650\end{array}$ & $\begin{array}{r}-1,240 \\
-2,250 \\
+5,330 \\
+420 \\
-1,440\end{array}$ & $\begin{array}{l}464.32 \\
463.52 \\
463.16 \\
463.26 \\
463.46\end{array}$ & $\begin{array}{l}132,650 \\
126,810 \\
124,260 \\
124,960 \\
126,380\end{array}$ & $\begin{array}{r}-8,470 \\
-5,840 \\
-2,550 \\
+700 \\
+1,420\end{array}$ \\
\hline $\begin{array}{l}26 \\
27 \\
28 \\
29 \\
30 \\
31\end{array}$ & $\begin{array}{l}459.87 \\
459.81 \\
459.52 \\
459.35 \\
459.33 \\
459.68\end{array}$ & $\begin{array}{r}102,950 \\
102,600 \\
100,890 \\
99,900 \\
99,780 \\
101,830\end{array}$ & $\begin{array}{r}+300 \\
-350 \\
-1,710 \\
-990 \\
-120 \\
+2,050\end{array}$ & $\begin{array}{c}466.13 \\
466.33 \\
465.54 \\
463.94 \\
463.84 \\
\ldots \ldots\end{array}$ & $\begin{array}{c}146,730 \\
148,370 \\
142,000 \\
129,850 \\
129,120 \\
\ldots \ldots\end{array}$ & $\begin{array}{r}+20,350 \\
+1,640 \\
-6,370 \\
-12,150 \\
-730 \\
\ldots \ldots\end{array}$ \\
\hline
\end{tabular}

$1 /$ National Geodetic Vertical Datum of 1929.

2/ One cubic foot per second per day is equivalent to 1.9835 acre-feet.

3/ Forebay elevation for 0100 hours on Mar. 24.

Note: Maximum outflow discharge, 115,200 cubic feet per second, Apr. 15. Records furnished by Alabama Power Co. 
TABLE 4.-Summary of stages and contents of storage reservoirs-Continued Lay Lake near Clanton, Ala. 02407950

\begin{tabular}{|c|c|c|c|c|c|c|}
\hline \multirow[b]{2}{*}{ Day } & \multicolumn{3}{|c|}{ March 1979} & \multicolumn{3}{|c|}{ April 1979} \\
\hline & $\begin{array}{c}\text { Elevation } \\
\text { 1/NGVD } \\
\text { of } 1929 \\
\text { (feet) }\end{array}$ & $\begin{array}{c}\text { Contents } \\
2 /\left(\mathrm{ft}^{3} / \mathrm{s} / \mathrm{day}\right)\end{array}$ & $\begin{array}{c}\text { Change in } \\
\text { storage } \\
2 /\left(\mathrm{ft}^{3} / \mathrm{s} / \text { day }\right)\end{array}$ & $\begin{array}{l}\text { Elevation } \\
\text { 1/NGVD } \\
\text { of } 1929 \\
\text { (feet) }\end{array}$ & $\begin{array}{c}\text { Contents } \\
2 /\left(\mathrm{ft}^{3} / \mathrm{s} / \text { day }\right)\end{array}$ & $\begin{array}{c}\text { Change in } \\
\text { storage } \\
2 /\left(\mathrm{ft}^{3} / \mathrm{s} / \mathrm{day}\right)\end{array}$ \\
\hline 1 & 396.24 & 133,910 & $\ldots \ldots$ & 395.80 & 131,300 & -700 \\
\hline 2 & 396.27 & 134,090 & +180 & 396.07 & 132,890 & $+1,590$ \\
\hline 3 & 396.15 & 133,370 & -720 & 396.02 & 132,600 & -290 \\
\hline 4 & 396.02 & 132,600 & -770 & 395.73 & 130,880 & $-1,720$ \\
\hline 5 & 395.33 & 128,560 & $-4,040$ & 395.84 & 131,530 & +650 \\
\hline 6 & 395.76 & 131,060 & $+2,500$ & 395.90 & 131,890 & +360 \\
\hline 7 & 395.85 & 131,590 & +530 & 396.18 & 133,550 & $+1,660$ \\
\hline 8 & 395.85 & 131,590 & 0 & 395.90 & 131,890 & $-1,660$ \\
\hline 9 & 396.13 & 133,250 & $+1,660$ & 396.29 & 134,210 & $+2,320$ \\
\hline 10 & 395.90 & 131,890 & $-1,360$ & 396.16 & 133,430 & -780 \\
\hline 11 & 395.82 & 131,410 & -480 & 396.07 & 132,890 & -540 \\
\hline 12 & 396.07 & 132,890 & $+1,480$ & 395.78 & 131,180 & $-1,710$ \\
\hline 13 & 396.06 & 132,840 & -50 & 395.95 & 132,180 & $+1,000$ \\
\hline 14 & 396.03 & 132,660 & -180 & 395.75 & 131,000 & $-1,180$ \\
\hline 15 & 395.81 & 131,350 & $-1,310$ & 395.94 & 132,120 & $+1,120$ \\
\hline 16 & 395.96 & 132,240 & +890 & 396.07 & 132,890 & +770 \\
\hline 17 & 396.03 & 132,660 & +420 & 395.85 & 131,590 & $-1,300$ \\
\hline 18 & 395.69 & 130,650 & $-2,010$ & 395.79 & 131,240 & -350 \\
\hline 19 & 395.89 & 131,830 & $+1,180$ & 396.10 & 133,070 & $+1,830$ \\
\hline 20 & 396.06 & 132,840 & $+1,010$ & 396.06 & 132,840 & -230 \\
\hline 21 & 396.21 & 133,730 & +890 & 396.13 & 133,250 & +410 \\
\hline 22 & 396.01 & 132,540 & $-1,190$ & 396.12 & 133,190 & -60 \\
\hline 23 & 396.39 & 134,820 & $+2,280$ & 396.15 & 133,370 & +180 \\
\hline 24 & 395.93 & 132,060 & $-2,760$ & 396.04 & 132,720 & -650 \\
\hline 25 & 396.15 & 133,370 & $+1,310$ & 396.00 & 132,480 & -240 \\
\hline 26 & 396.39 & 134,820 & $+1,450$ & 395.79 & 131,240 & $-1,240$ \\
\hline 27 & 396.23 & 133,850 & -970 & 395.92 & 132,000 & +760 \\
\hline 28 & 396.24 & 133,910 & +60 & 395.74 & 130,940 & $-1,060$ \\
\hline 29 & 396.27 & 134,090 & +180 & 396.21 & 133,730 & $+2,790$ \\
\hline 30 & 396.40 & 134,880 & +790 & 396.34 & 134,520 & +790 \\
\hline 31 & 395.92 & 132,000 & $-2,880$ & $\ldots \ldots$ & $\ldots \ldots$ & $\ldots \ldots$ \\
\hline
\end{tabular}

$1 /$ National Geodetic Vertical Datum of 1929.

2/ One cubic foot per second per day is equivalent to 1.9835 acre-feet.

Note: Maximum outflow discharge, 197,000 cubic feet per second, Apr. 13. Records furnished by Alabama Power Co. 
TABLES

TABLE 4.-Summary of stages and contents of storage reservoirs-Continued

Mitchell Dam near Verbena, Ala. 02409400 ।

\begin{tabular}{|c|c|c|c|c|c|c|}
\hline & \multicolumn{3}{|c|}{ March 1979} & \multicolumn{3}{|c|}{ April 1979} \\
\hline Day & $\begin{array}{l}\text { Elevation } \\
\text { 1/ NGVD } \\
\text { of } 1929 \\
\text { (feet) }\end{array}$ & $\begin{array}{c}\text { Contents } \\
2 /\left(\mathrm{ft}^{3} / \mathrm{s} / \text { day }\right) \\
\end{array}$ & $\begin{array}{c}\begin{array}{c}\text { Change in } \\
\text { storage }\end{array} \\
2 /\left(\mathrm{ft}^{3} / \mathrm{s} / \text { day }\right) \\
\end{array}$ & $\begin{array}{l}\text { Elevation } \\
\text { 1/ NGVD } \\
\text { of } 1929 \\
\text { (feet) }\end{array}$ & $\begin{array}{l}\text { Contents } \\
2 /\left(\mathrm{ft}^{3} / \mathrm{s} / \text { day }\right)\end{array}$ & $\begin{array}{c}\text { Change in } \\
\text { storage } \\
2 /\left(\mathrm{ft}^{3} / \mathrm{s} / \mathrm{day}\right)\end{array}$ \\
\hline $\begin{array}{l}1 \\
2 \\
3 \\
4 \\
5\end{array}$ & $\begin{array}{l}311.8 \\
311.9 \\
312.2 \\
311.5 \\
312.1\end{array}$ & $\begin{array}{l}87,930 \\
88,230 \\
89,120 \\
87,040 \\
88,820\end{array}$ & $\begin{array}{r}-600 \\
+300 \\
+890 \\
-2,080 \\
+1,780\end{array}$ & $\begin{array}{l}310.8 \\
312.3 \\
311.9 \\
311.8 \\
312.8\end{array}$ & $\begin{array}{l}84,990 \\
89,420 \\
88,230 \\
87,930 \\
90,920\end{array}$ & $\begin{array}{r}-3,540 \\
+4,430 \\
-190 \\
-300 \\
+2,990\end{array}$ \\
\hline $\begin{array}{r}6 \\
7 \\
8 \\
9 \\
10\end{array}$ & $\begin{array}{l}312.2 \\
312.2 \\
311.9 \\
311.8 \\
312.0\end{array}$ & $\begin{array}{l}89,120 \\
89,120 \\
88,230 \\
87,930 \\
88,530\end{array}$ & $\begin{array}{r}+300 \\
0 \\
-890 \\
-300 \\
+600\end{array}$ & $\begin{array}{l}312.1 \\
312.0 \\
312.2 \\
312.0 \\
312.0\end{array}$ & $\begin{array}{l}88,820 \\
88,530 \\
89,120 \\
88,530 \\
88,530\end{array}$ & $\begin{array}{r}-2,100 \\
-290 \\
+590 \\
+590 \\
0\end{array}$ \\
\hline $\begin{array}{l}11 \\
12 \\
13 \\
14 \\
15\end{array}$ & $\begin{array}{l}312.2 \\
312.2 \\
311.8 \\
312.2 \\
312.2\end{array}$ & $\begin{array}{l}89,120 \\
89,120 \\
87,930 \\
89,120 \\
89,120\end{array}$ & $\begin{array}{r}+590 \\
0 \\
-1,190 \\
+1,190 \\
0\end{array}$ & $\begin{array}{l}312.0 \\
312.2 \\
316.2 \\
312.2 \\
312.1\end{array}$ & $\begin{array}{r}88,530 \\
89,120 \\
101,460 \\
89,120 \\
88,820\end{array}$ & $\begin{array}{r}0 \\
+590 \\
+12,340 \\
-12,340 \\
-300\end{array}$ \\
\hline $\begin{array}{l}16 \\
17 \\
18 \\
19 \\
20\end{array}$ & $\begin{array}{l}311.9 \\
311.9 \\
312.2 \\
312.2 \\
311.9\end{array}$ & $\begin{array}{l}88,230 \\
88,230 \\
89,120 \\
89,120 \\
88,230\end{array}$ & $\begin{array}{r}-890 \\
0 \\
+890 \\
0 \\
-890\end{array}$ & $\begin{array}{l}311.9 \\
311.8 \\
312.2 \\
311.8 \\
312.1\end{array}$ & $\begin{array}{l}88,230 \\
87,930 \\
89,120 \\
87,930 \\
88,820\end{array}$ & $\begin{array}{r}-590 \\
-300 \\
+1,190 \\
-1,190 \\
+890\end{array}$ \\
\hline $\begin{array}{l}21 \\
22 \\
23 \\
24 \\
25\end{array}$ & $\begin{array}{l}312.0 \\
311.9 \\
312.0 \\
311.9 \\
312.0\end{array}$ & $\begin{array}{l}88,530 \\
88,230 \\
88,530 \\
88,230 \\
88,530\end{array}$ & $\begin{array}{l}+300 \\
-300 \\
+300 \\
-300 \\
+300\end{array}$ & $\begin{array}{l}311.9 \\
312.0 \\
312.1 \\
312.2 \\
312.2\end{array}$ & $\begin{array}{l}88,230 \\
88,530 \\
88,820 \\
89,120 \\
89,120\end{array}$ & $\begin{array}{r}-590 \\
+300 \\
+290 \\
+300 \\
0\end{array}$ \\
\hline $\begin{array}{l}26 \\
27 \\
28 \\
29 \\
30 \\
31\end{array}$ & $\begin{array}{l}311.9 \\
312.2 \\
311.8 \\
312.3 \\
312.1 \\
312.0\end{array}$ & $\begin{array}{l}88,230 \\
89,120 \\
87,930 \\
89,420 \\
88,820 \\
88,530\end{array}$ & $\begin{array}{r}-300 \\
+840 \\
-1,190 \\
+1,490 \\
-600 \\
-290\end{array}$ & $\begin{array}{l}311.8 \\
312.1 \\
312.0 \\
312.1 \\
311.9\end{array}$ & $\begin{array}{l}87,930 \\
88,820 \\
88,530 \\
88,820 \\
88,230\end{array}$ & $\begin{array}{r}-1,190 \\
+890 \\
-290 \\
+290 \\
-590\end{array}$ \\
\hline
\end{tabular}

1) National Geodetic Vertical Datum of 1929.

$2 /$ One cubic foot per second per day is equivalent to 1.9835 acre-feet.

Note: Records furnished by Alabama Power Co. 
TABLE 4.-Summary of stages and contents of storage reservoirs-Continued Jordan Lake near Wetumpka, Ala. 02414000

\begin{tabular}{|c|c|c|c|c|c|c|}
\hline \multirow[b]{2}{*}{ Day } & \multicolumn{3}{|c|}{ March 1979} & \multicolumn{3}{|c|}{ April 1979} \\
\hline & $\begin{array}{l}\text { Elevation } \\
\text { 1/NGVD } \\
\text { of } 1929 \\
\text { (feet) }\end{array}$ & $\begin{array}{l}2 / \text { Contents } \\
\underline{3} /\left(\mathrm{ft}^{3} / \mathrm{s} / \mathrm{day}\right)\end{array}$ & $\begin{array}{c}\text { Change in } \\
\text { storage } \\
\underline{3} /\left(\mathrm{ft}^{3} / \mathrm{s} / \text { day }\right)\end{array}$ & $\begin{array}{l}\text { Elevation } \\
\text { 1/NGVD } \\
\text { of } 1929 \\
\text { (feet) }\end{array}$ & $\begin{array}{l}2 / \text { Contents } \\
\underline{3} /(\mathrm{ft} 3 / \mathrm{s} / \mathrm{day})\end{array}$ & $\begin{array}{c}\text { Change in } \\
\text { storage } \\
\underline{3} /\left(\mathrm{ft}^{3} / \mathrm{s} / \mathrm{day}\right)\end{array}$ \\
\hline 1 & 251.90 & 118,750 & $\ldots \ldots$ & 250.22 & 113,280 & $-3,030$ \\
\hline 2 & 251.31 & 116,800 & $-1,950$ & 250.90 & 115,460 & $+2,180$ \\
\hline 3 & 252.77 & 121,680 & $+4,880$ & 251.53 & 117,520 & $+2,060$ \\
\hline 4 & 251.78 & 118,350 & $-3,330$ & 251.71 & 118,120 & +600 \\
\hline 5 & 251.90 & 118,750 & +400 & 251.86 & 118,610 & +490 \\
\hline 6 & 251.95 & 118,910 & +160 & 251.81 & 118,450 & -160 \\
\hline 7 & 251.77 & 118,310 & -600 & 251.74 & 118,210 & -240 \\
\hline 8 & 251.80 & 118,410 & +100 & 251.76 & 118,280 & +70 \\
\hline 9 & 251.70 & 118,080 & -330 & 251.71 & 118,120 & -160 \\
\hline 10 & 251.60 & 117,750 & -330 & 251.84 & 118,550 & +430 \\
\hline 11 & 251.90 & 118,750 & $+1,000$ & 251.40 & 117,090 & $-1,460$ \\
\hline 12 & 251.82 & 118,480 & -270 & 251.77 & 118,310 & $+1,220$ \\
\hline 13 & 251.85 & 118,580 & +100 & 256.71 & 135,960 & $+17,650$ \\
\hline 14 & 251.91 & 118,780 & +200 & 251.90 & 118,750 & $-17,210$ \\
\hline 15 & 251.79 & 118,380 & -400 & 251.93 & 118,850 & +100 \\
\hline 16 & 251.90 & 118,750 & +370 & 251.92 & 118,810 & -40 \\
\hline 17 & 251.82 & 118,480 & -270 & 251.93 & 118,850 & +40 \\
\hline 18 & 251.86 & 118,610 & +130 & 251.95 & 118,910 & +60 \\
\hline 19 & 251.74 & 118,210 & -400 & $251 \cdot .72$ & 118,150 & -760 \\
\hline 20 & 251.84 & 118,550 & +340 & 251.81 & 118,450 & +300 \\
\hline 21 & 251.90 & 118,750 & +200 & 251.71 & 118,120 & -330 \\
\hline 22 & 251.70 & 118,080 & -670 & 251.89 & 118,710 & +590 \\
\hline 23 & 251.78 & 118,350 & +270 & 251.81 & 118,450 & -260 \\
\hline 24 & 251.72 & 118,150 & -200 & 251.75 & 118,250 & -200 \\
\hline 25 & 251.57 & 117,650 & -500 & 251.86 & 118,610 & +360 \\
\hline 26 & 251.87 & 118,650 & $+1,000$ & 251.71 & 118,120 & -490 \\
\hline 27 & 251.94 & 118,880 & +230 & 251.73 & 118,180 & +60 \\
\hline 28 & 251.75 & 118,250 & -630 & 251.74 & 118,210 & +30 \\
\hline 29 & 251.91 & 118,780 & +530 & 251.82 & 118,480 & +270 \\
\hline 30 & 251.82 & 118,480 & -300 & 251.86 & 118,610 & +130 \\
\hline 31 & 251.16 & 116,310 & $-2,170$ & $\ldots \ldots$ & $\ldots \ldots$ & $\ldots \ldots$ \\
\hline
\end{tabular}

1/ National Geodetic Vertical Datum of 1929.

2/ Includes Walter Bouldin Reservoir

3/ One cubic foot per second per day is equivalent to 1.9835 acre-feet.

Note: Maximum outflow discharge, 316,000 cubic feet per second, Apr. 13. Records furnished by Alabama Power Co. 
TABLES

TABLE 4.-Summary of stages and contents of storage reservoirs-Continued Lake Martin near Tallassee, Ala. 02417500

\begin{tabular}{|c|c|c|c|c|c|c|}
\hline \multirow[b]{2}{*}{ Day } & \multicolumn{3}{|c|}{ March 1979} & \multicolumn{3}{|c|}{ April 1979} \\
\hline & $\begin{array}{l}\text { Elevation } \\
\text { l/NGVD } \\
\text { of } 1929 \\
\text { (feet) }\end{array}$ & $\begin{array}{c}\text { Contents } \\
2 /\left(\mathrm{ft}^{3} / \mathrm{s} / \mathrm{day}\right)\end{array}$ & $\begin{array}{c}\text { Change in } \\
\text { storage } \\
2 /\left(\mathrm{ft}^{3} / \mathrm{s} / \mathrm{day}\right)\end{array}$ & $\begin{array}{l}\text { Elevation } \\
\text { 1/NGVD } \\
\text { of } 1929 \\
\text { (feet) }\end{array}$ & $\begin{array}{c}\text { Contents } \\
2 /(\mathrm{ft} 3 / \mathrm{s} / \mathrm{day})\end{array}$ & $\begin{array}{c}\text { Change in } \\
\text { storage } \\
2 /\left(\mathrm{ft}^{3} / \mathrm{s} / \text { day }\right)\end{array}$ \\
\hline 1 & 482.24 & 675,620 & $\ldots \ldots$ & 486.49 & 752,440 & $+3,360$ \\
\hline 2 & 482.21 & 675,100 & -520 & 486.30 & 748,890 & $-3,550$ \\
\hline 3 & 482.40 & 678,420 & $+3,320$ & 487.17 & 765,230 & $+16,340$ \\
\hline 4 & 484.70 & 719,440 & $+41,020$ & 489.16 & 803,450 & $+38,220$ \\
\hline 5 & 487.78 & 776,820 & $+57,380$ & 489.92 & 813,360 & $+9,910$ \\
\hline 6 & 489.15 & 803,250 & $+26,430$ & 490.05 & 820,920 & $+7,560$ \\
\hline 7 & 489.83 & 816,580 & $+13,330$ & 489.94 & 818,750 & $-2,170$ \\
\hline 8 & 489.86 & 817,170 & +590 & 489.75 & 815,010 & $-3,740$ \\
\hline 9 & 489.61 & 812,250 & $-4,920$ & 489.63 & 812,650 & $-2,360$ \\
\hline 10 & 489.39 & 807,940 & $-4,310$ & 489.39 & 807,940 & $-4,710$ \\
\hline 11 & 489.12 & 802,670 & $-5,270$ & 489.24 & 805,010 & $-2,930$ \\
\hline 12 & 488.88 & 798,000 & $-4,670$ & 489.32 & 806,570 & $+1,560$ \\
\hline 13 & 488.64 & 793,350 & $-4,650$ & 490.80 & 835,840 & $+29,270$ \\
\hline 14 & 488.45 & 789,680 & $-3,670$ & 490.52 & 830,250 & $-5,590$ \\
\hline 15 & 488.23 & 785,440 & $-4,240$ & 490.10 & 821,910 & $-8,340$ \\
\hline 16 & 487.92 & 779,490 & $-5,950$ & 490.05 & 820,920 & -990 \\
\hline 17 & 487.68 & 774,910 & $-4,580$ & 490.02 & 820,330 & -590 \\
\hline 18 & 487.40 & 769,590 & $-5,320$ & 489.90 & 817,960 & $-2,370$ \\
\hline 19 & 487.13 & 764,470 & $-5,120$ & 489.78 & 815,600 & $-2,360$ \\
\hline 20 & 486.93 & 760,700 & $-3,770$ & 489.88 & 817,570 & $+1,970$ \\
\hline 21 & 486.90 & 760,140 & -560 & 489.37 & 807,550 & $-10,020$ \\
\hline 22 & 486.44 & 751,510 & $-8,630$ & 489.16 & 803,450 & $-4,100$ \\
\hline 23 & 486.45 & 751,700 & +190 & 489.19 & 804,030 & +580 \\
\hline 24 & 486.50 & 752,630 & +930 & 489.15 & 803,250 & -780 \\
\hline 25 & 486.65 & 755,440 & $+2,810$ & 489.75 & 815,010 & $+11,760$ \\
\hline 26 & 486.55 & 753,570 & $-1,870$ & 490.00 & 819,940 & $+4,930$ \\
\hline 27 & 486.49 & 752,440 & $-1,130$ & 490.03 & 820,530 & +590 \\
\hline 28 & 486.58 & 754,130 & $+1,690$ & 489.98 & 819,540 & -990 \\
\hline 29 & 486.29 & 748,710 & $-5,420$ & 489.87 & 817,370 & $-2,170$ \\
\hline 30 & 486.12 & 745,540 & $-3,170$ & 489.78 & 815,600 & $-1,770$ \\
\hline 31 & 486.31 & 749,080 & $+3,540$ & $\ldots \ldots$ & $\ldots \ldots$ & $\ldots \ldots$ \\
\hline
\end{tabular}

1/ National Geodetic Vertical Datum of 1929.

2/ One cubic foot per second per day is equivalent to 1.9835 acre-feet.

Note: Maximum outflow discharge, 128,000 cubic feet per second, Apr. 14. Records furnished by Alabama Power Co. 
TABLE 4.-Summary of stages and contents of storage reservoirs-Continued Lewis Smith Reservoir near Jasper, Ala. 02451950

\begin{tabular}{|c|c|c|c|c|c|c|}
\hline \multirow[b]{2}{*}{ Day } & \multicolumn{3}{|c|}{ March 1979} & \multicolumn{3}{|c|}{ April 1979} \\
\hline & $\begin{array}{l}\text { Elevation } \\
1 / \text { NGVD } \\
\text { of } 1929 \\
\text { (feet) }\end{array}$ & $\begin{array}{c}\text { Contents } \\
2 /\left(\mathrm{ft}^{3} / \mathrm{s} / \text { day }\right) \\
\end{array}$ & $\begin{array}{c}\text { Change in } \\
\text { storage } \\
2 /\left(\mathrm{ft}^{3} / \mathrm{s} / \text { day }\right) \\
\end{array}$ & $\begin{array}{c}\text { Elevation } \\
1 / \text { NGVD } \\
\text { of } 1929 \\
(\mathrm{feet})\end{array}$ & $\begin{array}{c}\text { Contents } \\
2 /\left(\mathrm{ft}^{3} / \mathrm{s} / \text { day }\right) \\
\end{array}$ & $\begin{array}{c}\text { Change in } \\
\text { storage } \\
2 /\left(\mathrm{ft}^{3} / \mathrm{s} / \mathrm{day}\right) \\
\end{array}$ \\
\hline 1 & 507.81 & 698,760 & & 509.36 & 693,970 & $+3,380$ \\
\hline 2 & 509.96 & 700,360 & $+1,600$ & 509.87 & 699,400 & $+5,430$ \\
\hline 3 & 512.64 & 729,640 & $+29,280$ & 510.45 & 705,620 & $+6,220$ \\
\hline 4 & 515.33 & 760,320 & $+30,680$ & 510.55 & 706,700 & $+1,080$ \\
\hline 5 & 515.55 & 762,880 & $+2,560$ & 510.38 & 704,870 & $-1,830$ \\
\hline 6 & 516.12 & 769,580 & $+6,700$ & 510.14 & 702,290 & $-2,580$ \\
\hline 7 & 515.84 & 778,120 & $+8,540$ & 510.12 & 702,070 & -220 \\
\hline 8 & 515.30 & 759,970 & $-18,150$ & 510.06 & 701,430 & -640 \\
\hline 9 & 514.68 & 752,780 & $-7,190$ & 509.94 & 700,150 & $-1,280$ \\
\hline 10 & 514.16 & 746,810 & $-5,970$ & 509.89 & 699,610 & -540 \\
\hline 11 & 513.61 & 740,550 & $-6,260$ & 509.90 & 699,720 & +110 \\
\hline 12 & 513.01 & 733,780 & $-6,770$ & 512.61 & 729,310 & $+29,590$ \\
\hline 13 & 512.70 & 730,310 & $-3,470$ & 517.18 & 782,190 & $+52,880$ \\
\hline 14 & 512.35 & 726,410 & $-3,900$ & 518.20 & 794,520 & $+12,330$ \\
\hline 15 & 511.99 & 722,420 & $-3,990$ & 518.67 & 800,270 & $+5,750$ \\
\hline 16 & 511.60 & 718,130 & $-4,290$ & 518.43 & 797,330 & $-2,940$ \\
\hline 17 & 511.20 & 713,750 & $-4,380$ & 517.89 & 790,750 & $-6,580$ \\
\hline 18 & 510.74 & 708,750 & $-5,000$ & 517.32 & 783,870 & $-6,880$ \\
\hline 19 & 510.35 & 704,540 & $-4,210$ & 516.63 & 775,620 & $-8,250$ \\
\hline 20 & 510.60 & 707,240 & $+2,700$ & 515.95 & 767,570 & $-8,050$ \\
\hline 21 & 509.60 & 696,520 & $-10,720$ & 515.25 & 759,380 & $-8,190$ \\
\hline 22 & 509.16 & 691,860 & $-4,660$ & 514.54 & 751,170 & $-8,210$ \\
\hline 23 & 509.50 & 695,460 & $+3,600$ & 513.81 & 742,620 & $-8,550$ \\
\hline 24 & 509.82 & 698,860 & $+3,400$ & 513.10 & 734,790 & $-7,830$ \\
\hline 25 & 510.04 & 701,220 & $+2,360$ & 512.55 & 728,640 & $-6,150$ \\
\hline 26 & 509.77 & 698,330 & $-2,890$ & 512.33 & 726,190 & $-2,450$ \\
\hline 27 & 509.48 & 695,250 & $-3,080$ & 511.95 & 721,980 & $-4,210$ \\
\hline 28 & 509.33 & 693,650 & $-1,600$ & 511.57 & 717,800 & $-4,180$ \\
\hline 29 & 509.19 & 692,180 & $-1,470$ & 511.16 & 713,320 & $-4,480$ \\
\hline 30 & 509.04 & 690,590 & $-1,590$ & 510.75 & 708,860 & $-4,460$ \\
\hline 31 & 509.04 & 690,590 & 0 & & & \\
\hline
\end{tabular}

1/ National Geodetic Vertical Datum of 1929.

2/ One cubic foot per second per day is equivalent to 1.9835 acre-feet.

Note: Maximum outflow discharge 9,100 ft $3 /$ day Apri1, 21,23. Outflow April 12, 2,700 ft $3 / \mathrm{s}$; April 13 and 14 no outflow; Apri1 15, 1,400 ft $3 / \mathrm{s}$.

Records furnished by Alabama Power Company. 
TABLES

TABLE 4.-Summary of stages and contents of storage reservoirs-Continued Okatibbee Reservoir near Meridian, Miss. 02475976

\begin{tabular}{|c|c|c|c|c|c|c|}
\hline & \multicolumn{3}{|c|}{ March 1979} & \multicolumn{3}{|c|}{ April 1979} \\
\hline Day & $\begin{array}{l}\text { Elevation } \\
1 / \text { NGVD } \\
\text { of } 1929 \\
\text { (feet) } \\
\end{array}$ & $\begin{array}{c}\text { Contents } \\
2 /\left(\mathrm{ft}^{3} / \mathrm{s} / \text { day }\right) \\
\end{array}$ & $\begin{array}{c}\begin{array}{c}\text { Change in } \\
\text { storage } \\
2 /\left(\mathrm{ft}^{3} / \mathrm{s} / \mathrm{day}\right)\end{array} \\
\end{array}$ & $\begin{array}{l}\text { Elevation } \\
1 / \text { NGVD } \\
\text { of } 1929 \\
\text { (feet) } \\
\end{array}$ & $\begin{array}{l}\text { Contents } \\
2 /\left(\mathrm{ft}^{3} / \mathrm{s} / \text { day }\right) \\
\end{array}$ & $\begin{array}{c}\text { Change in } \\
\text { storage } \\
2 /\left(\mathrm{ft}^{3} / \mathrm{s} / \text { day }\right)\end{array}$ \\
\hline $\begin{array}{l}1 \\
2 \\
3 \\
4 \\
5\end{array}$ & $\begin{array}{l}344.07 \\
344.15 \\
347.33 \\
350.05 \\
350.66\end{array}$ & $\begin{array}{l}23,385 \\
23,543 \\
30,976 \\
38,567 \\
40,435\end{array}$ & $\begin{array}{r}\dddot{158} \\
7,433 \\
7,591 \\
1,867\end{array}$ & $\begin{array}{l}341.92 \\
342.15 \\
342.80 \\
343.71 \\
344.27\end{array}$ & $\begin{array}{l}19,265 \\
19,674 \\
20,879 \\
22,649 \\
23,802\end{array}$ & $\begin{array}{r}-142 \\
408 \\
1,204 \\
1,770 \\
1,152\end{array}$ \\
\hline $\begin{array}{r}6 \\
7 \\
8 \\
9 \\
10\end{array}$ & $\begin{array}{l}350.51 \\
350.27 \\
349.99 \\
349.66 \\
349.36\end{array}$ & $\begin{array}{l}39,982 \\
39,244 \\
38,363 \\
37,399 \\
36,524\end{array}$ & $\begin{array}{l}-453 \\
-737 \\
-880 \\
-963 \\
-875\end{array}$ & $\begin{array}{l}344.35 \\
344.31 \\
344.68 \\
345.33 \\
345.62\end{array}$ & $\begin{array}{l}23,985 \\
23,892 \\
24,697 \\
26,134 \\
26,802\end{array}$ & $\begin{array}{r}182 \\
-92 \\
804 \\
1,436 \\
667\end{array}$ \\
\hline $\begin{array}{l}11 \\
12 \\
13 \\
14 \\
15\end{array}$ & $\begin{array}{l}349.04 \\
348.71 \\
348.38 \\
348.02 \\
347.64\end{array}$ & $\begin{array}{l}35,608 \\
34,673 \\
33,775 \\
32,783 \\
31,791\end{array}$ & $\begin{array}{l}-915 \\
-934 \\
-898 \\
-991 \\
-992\end{array}$ & $\begin{array}{l}345.75 \\
349.41 \\
353.81 \\
355.24 \\
355.20\end{array}$ & $\begin{array}{l}27,092 \\
36,686 \\
50,970 \\
56,293 \\
56,134\end{array}$ & $\begin{array}{r}290 \\
9,593 \\
14,283 \\
5,323 \\
-159\end{array}$ \\
\hline $\begin{array}{l}16 \\
17 \\
18 \\
19 \\
20\end{array}$ & $\begin{array}{l}347.26 \\
346.85 \\
346.46 \\
346.06 \\
345.66\end{array}$ & $\begin{array}{l}30,802 \\
29,764 \\
28,799 \\
27,817 \\
26,897\end{array}$ & $\begin{array}{r}-988 \\
-1,037 \\
-965 \\
-982 \\
-919\end{array}$ & $\begin{array}{l}354.98 \\
354.73 \\
354.46 \\
354.18 \\
353.88\end{array}$ & $\begin{array}{l}55,297 \\
54,359 \\
53,371 \\
52,321 \\
51,239\end{array}$ & $\begin{array}{r}-836 \\
-938 \\
-987 \\
-1,049 \\
-1,081\end{array}$ \\
\hline $\begin{array}{l}21 \\
22 \\
23 \\
24 \\
25\end{array}$ & $\begin{array}{l}345.36 \\
345.23 \\
345.05 \\
344.73 \\
344.31\end{array}$ & $\begin{array}{l}26,203 \\
25,899 \\
25,493 \\
24,790 \\
23,896\end{array}$ & $\begin{array}{l}-694 \\
-304 \\
-406 \\
-702 \\
-893\end{array}$ & $\begin{array}{l}353.58 \\
353.30 \\
353.02 \\
352.76 \\
352.49\end{array}$ & $\begin{array}{l}50,174 \\
49,157 \\
48,187 \\
47,290 \\
46,363\end{array}$ & $\begin{array}{l}-1,065 \\
-1,017 \\
-\quad 970 \\
-\quad 897 \\
-\quad 926\end{array}$ \\
\hline $\begin{array}{l}26 \\
27 \\
28 \\
29 \\
30 \\
31\end{array}$ & $\begin{array}{l}343.86 \\
343.39 \\
342.90 \\
342.40 \\
342.12 \\
342.00\end{array}$ & $\begin{array}{l}22,945 \\
22,016 \\
21,055 \\
20,135 \\
19,622 \\
19,407\end{array}$ & $\begin{array}{l}-950 \\
-929 \\
-961 \\
-920 \\
-513 \\
-214\end{array}$ & $\begin{array}{l}352.23 \\
351.94 \\
351 . .62 \\
351.29 \\
350.94\end{array}$ & $\begin{array}{l}45,482 \\
44,530 \\
43,494 \\
42,405 \\
41,292\end{array}$ & $\begin{array}{l}-\quad 880 \\
-\quad 952 \\
-1,035 \\
-1,089 \\
-1,1112\end{array}$ \\
\hline
\end{tabular}

Note: Furnished by U.S. Army Corps of Engineers 
TABLE 4.-Summary of stages and contents of storage reservoirs-Continued Ross Barnett Reservoir near Jackson, Miss. 02485600

\begin{tabular}{|c|c|c|c|c|}
\hline \multirow[b]{2}{*}{ Day } & \multicolumn{2}{|c|}{ March 1979} & \multicolumn{2}{|c|}{ April 1979} \\
\hline & $\begin{array}{c}\text { Elevation above } \\
\text { National Geodetic } \\
\text { Vertical Datum at } \\
\text { Iy Meeks Bridge } \\
\text { (feet) }\end{array}$ & $\begin{array}{l}\text { 2/ Elevation above } \\
\text { National Geodetic } \\
\text { Vertical Datum at } \\
\quad \text { dam (feet) }\end{array}$ & $\begin{array}{c}\text { Elevation above } \\
\text { National Geodetic } \\
\text { Veytical Datum at } \\
\text { - Meeks Bridge } \\
\text { (feet) }\end{array}$ & $\begin{array}{l}\text { 2/ Elevation above } \\
\text { National Geodetic } \\
\text { Vertical Datum at } \\
\text { dam (feet }\end{array}$ \\
\hline $\begin{array}{l}1 \\
2 \\
3 \\
4 \\
5\end{array}$ & $\begin{array}{l}297.1 \\
297.2 \\
297.3 \\
297.4 \\
297.6\end{array}$ & $\begin{array}{l}297.2 \\
297.2 \\
297.3 \\
297.3 \\
297.6\end{array}$ & $\begin{array}{l}297.4 \\
297.4 \\
297.6 \\
297.9 \\
297.9\end{array}$ & $\begin{array}{l}297.4 \\
297.5 \\
297.6 \\
297.9 \\
297.9\end{array}$ \\
\hline $\begin{array}{r}6 \\
7 \\
8 \\
9 \\
10\end{array}$ & $\begin{array}{l}297.3 \\
297.4 \\
297.7 \\
297.9 \\
297.5\end{array}$ & $\begin{array}{l}297.3 \\
297.2 \\
297.6 \\
297.9 \\
297.5\end{array}$ & $\begin{array}{l}297.8 \\
297.7 \\
297.8 \\
297.8 \\
297.6\end{array}$ & $\begin{array}{l}297.8 \\
297.7 \\
297.8 \\
297.8 \\
297.7\end{array}$ \\
\hline $\begin{array}{l}11 \\
12 \\
13 \\
14 \\
15\end{array}$ & $\begin{array}{l}297.2 \\
297.3 \\
297.5 \\
297.4 \\
297.3\end{array}$ & $\begin{array}{l}297.2 \\
297.3 \\
297.5 \\
297.5 \\
297.3\end{array}$ & $\begin{array}{l}297.6 \\
298.1 \\
298.2 \\
297.1 \\
297.9\end{array}$ & $\begin{array}{l}297.5 \\
298.1 \\
298.2 \\
296.6 \\
296.9\end{array}$ \\
\hline $\begin{array}{l}16 \\
17 \\
18 \\
19 \\
20\end{array}$ & $\begin{array}{l}297.3 \\
297.3 \\
297.3 \\
297.3 \\
297.3\end{array}$ & $\begin{array}{l}297.4 \\
297.3 \\
297.4 \\
297.3 \\
297.4\end{array}$ & $\begin{array}{l}299.1 \\
300.0 \\
299.2 \\
297.9 \\
297.2\end{array}$ & $\begin{array}{r}3 / 298.8 \\
299.7 \\
299.1 \\
297.8 \\
297.2\end{array}$ \\
\hline $\begin{array}{l}21 \\
22 \\
23 \\
24 \\
25\end{array}$ & $\begin{array}{l}297.3 \\
297.4 \\
297.5 \\
297.4 \\
297.4\end{array}$ & $\begin{array}{l}297.4 \\
297.4 \\
297.4 \\
297.4 \\
297.4\end{array}$ & $\begin{array}{c}296.6 \\
296.2 \\
296.1 \\
\ldots \\
\ldots\end{array}$ & $\begin{array}{l}296.6 \\
296.3 \\
296.1 \\
296.0 \\
296.0\end{array}$ \\
\hline $\begin{array}{l}26 \\
27 \\
28 \\
29 \\
30 \\
31\end{array}$ & $\begin{array}{l}297.3 \\
297.4 \\
297.4 \\
297.4 \\
297.4 \\
297.4\end{array}$ & $\begin{array}{l}297.4 \\
297.4 \\
297.4 \\
297.4 \\
297.4 \\
297.4\end{array}$ & $\begin{array}{l}\cdots \\
\cdots \\
\cdots \\
\cdots\end{array}$ & $\begin{array}{l}296.0 \\
296.1 \\
296.2 \\
296.2 \\
296.3\end{array}$ \\
\hline
\end{tabular}


TABLE 4.-Summary of stages and contents of storage reservoirs-Continued

Ross Barnett Reservoir near Jackson, Miss. 02485600-Continued

Discharge measurements of (02485000) Pearl River at Meeks Bridge

near Canton, Mississippi, (State Highway 43).

\begin{tabular}{cccc} 
Date & Mean time & $\begin{array}{c}\text { Mean elevation } \\
\text { National Geodetic } \\
\text { Vertical Datum } \\
\text { (feet) }\end{array}$ & $\begin{array}{c}\text { Discharge } \\
\left(\mathrm{ft}^{3} / \mathrm{s}\right)\end{array}$ \\
April 15 & 1740 & 298.3 & 128,000 \\
April 16 & 1330 & 299.4 & 143,000 \\
April 17 & 1730 & 299.3 & 102,000 \\
April 18 & 1550 & 298.5 & 73,200 \\
April 19 & 1420 & 297.2 & 43,700 \\
\hline
\end{tabular}




\begin{tabular}{ccc}
\hline & Elevation above \\
& Distance & National geodetic \\
upstream & vertical datum \\
Stream and location & from mouth & of 1929 \\
(miles) & (feet) \\
\hline
\end{tabular}

COOSA RIVER BASIN

Coosa River:

Jordan Dam near Wetumpka, Ala. (upstream)

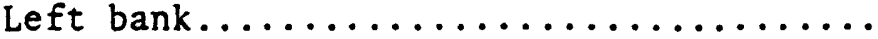

U.S. Geological Survey gaging station

(02411000) on right bank $0.5 \mathrm{mi}$ downstream

from Jordan Dam, near Wetumpka, Ala.....

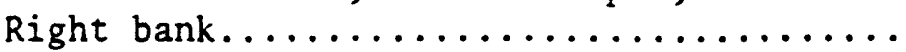

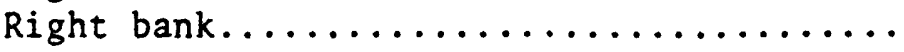

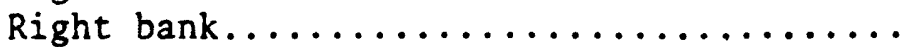

Right bank..................

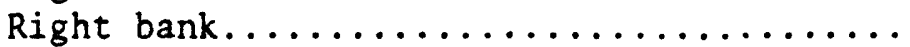

U.S. Geological Survey gaging station

(02411600) on downstream side of bridge

on State Highway 14, in Wetumpka, Ala....

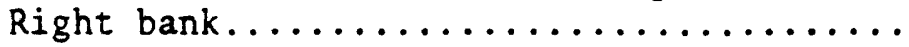

Mouth, at Alabama River, mile 314.4

(confluence of Coosa and Tallapoosa

Rivers) near Montgomery, Ala..........

\section{TALLAPOOSA RIVER BASIN}

Tallapoosa River:

Alabama Power Co. gage (02418500) on

left bank, $1.5 \mathrm{mi}$ downstream from

Benjamin Fitzpatrick Highway bridge at

Thurlow Dam, at Tallassee, Ala.........

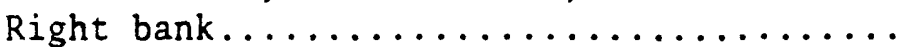

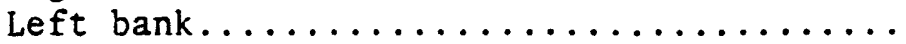

U.S. National Weather Service gage

(02419500) on Atlanta and West Point

Railroad bridge at Milstead, Ala........

Upstream side of Alabama Highway 229, $0.4 \mathrm{mi}$ northeast of main channel, right

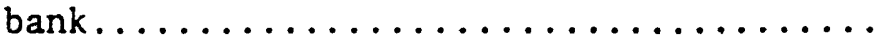

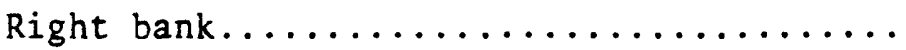

Downstream side of Alabama Highway 229,

$0.4 \mathrm{mi}$ northeast of main channel, right

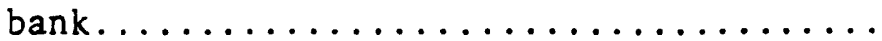

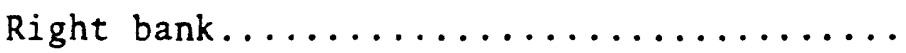

Left bank.
18.9

18.7

18.6

17.7

15.8

14.6

13.5

12.2

11.4

6.4

0.0
256.8

191.9

189.3

186.0

182.9

178.9

174.8

170.9

169.6

163.1
48.0

46.8

44.1

39.8

39.8

39.8

39.7

39.7

39.6
212.6

211.8

209.5

203.5

203.3

202.9

203.0

202.7

202.4

See footnotes at end of table. 
TABLE 5.-Flood-crest stages-Continued

\begin{tabular}{ccc}
\hline & Elevation above \\
& Distance & National geodetic \\
Stream and location & upstream & vertical datum \\
from mouth & of 1929 \\
\hline
\end{tabular}

TALLAPOOSA RIVER BASIN--Continued

Tallapoosa River--Continued

\begin{tabular}{|c|c|c|}
\hline Left bank. & 39.4 & 200.4 \\
\hline 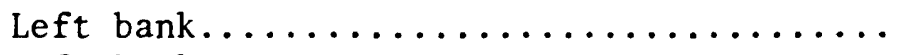 & 37.1 & 197.1 \\
\hline 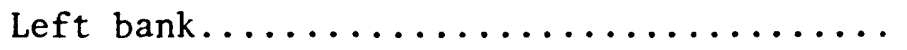 & 33.0 & 189.0 \\
\hline 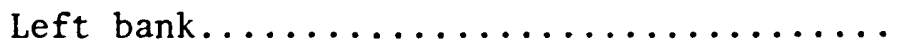 & 28.0 & 182.0 \\
\hline 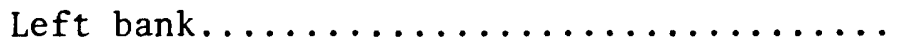 & 24.8 & 174.9 \\
\hline 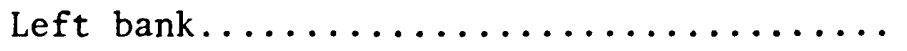 & 21.0 & 171.3 \\
\hline 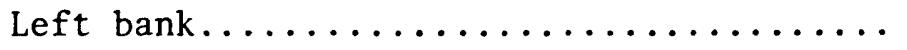 & 19.5 & 170.1 \\
\hline 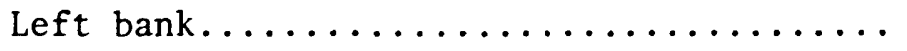 & 14.8 & 168.0 \\
\hline 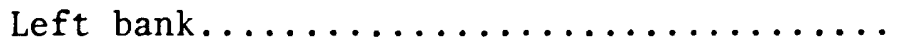 & 9.8 & 163.9 \\
\hline 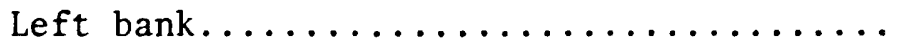 & 9.6 & 163.4 \\
\hline 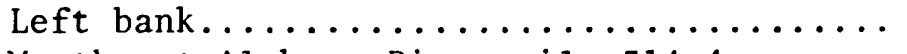 & 4.8 & 163.0 \\
\hline $\begin{array}{l}\text { Mouth, at Alabama River mile } 314.4 \\
\text { (confluence of Coosa and Tallapoosa }\end{array}$ & & \\
\hline Rivers) near Montgomery, Ala....... & 0.0 & - \\
\hline
\end{tabular}

ALABAMA RIVER BASIN

Alabama River:

Confluence of Coosa and Tallapoosa

Rivers near Montgomery, Ala...........

314.4

- - - -

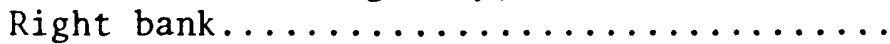

311.1

162.1

$304.8 \quad 159.4$

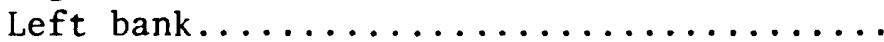

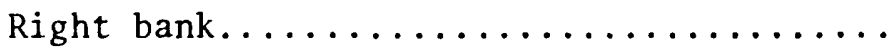

301.8

157.6

U.S. National Weather Service gage

(02419988) on left bank in abandoned

pumping station of the Riverview

Manufacturing Co. at 715 Shady St.,

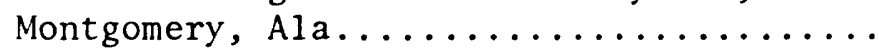

269.9

a/ 156.2

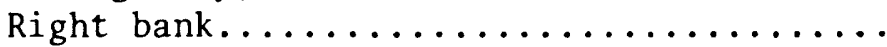

291.2

155.0

U.S. Geological Survey gaging station

(02420000) at bridge on U.S. Highway 31,

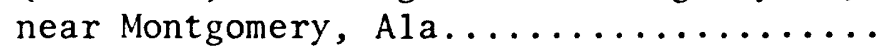

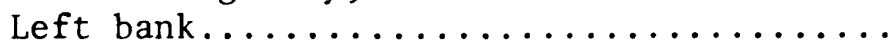

287.6

a/ 152.4

277.6

147.6

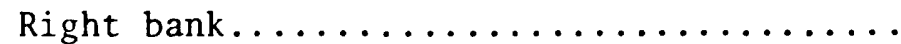

261.2

139.5

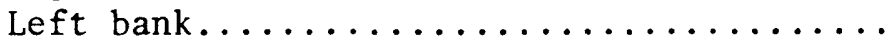

255.0

137.5

U.S. Army Corps of Engineers gage

(02421351) at downstream end of Jones

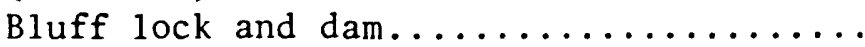

245.4

b/ 133.2

See footnotes at end of table. 
TABLE 5.-Flood-crest stages-Continued

\begin{tabular}{|c|c|c|}
\hline Stream and location & $\begin{array}{l}\text { Distance } \\
\text { upstream } \\
\text { from mouth } \\
\text { (miles) }\end{array}$ & $\begin{array}{l}\text { Elevation a } \\
\text { National geo } \\
\text { vertical da } \\
\text { of } 1929 \\
\text { (feet) }\end{array}$ \\
\hline \multicolumn{3}{|l|}{ ALABAMA RIVER BASIN--Continued } \\
\hline \multicolumn{3}{|l|}{ Alabama River--Continued } \\
\hline 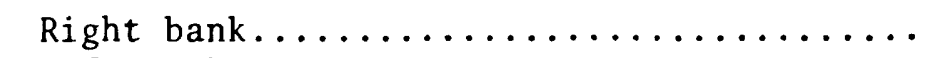 & 244.9 & 133.1 \\
\hline 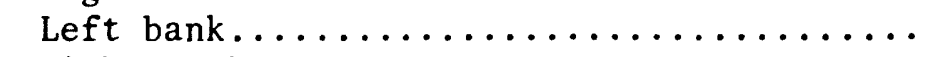 & 243.0 & 132.3 \\
\hline 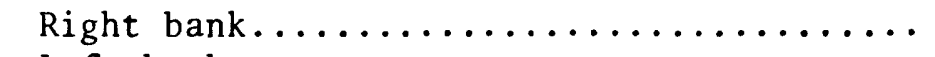 & 239.5 & 130.3 \\
\hline 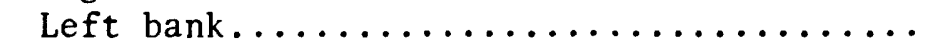 & 237.8 & 127.7 \\
\hline 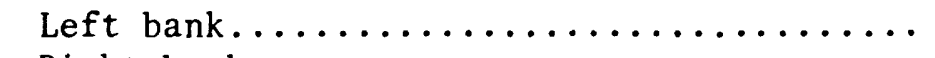 & 234.5 & 128.1 \\
\hline 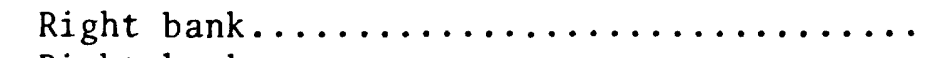 & 227.4 & 124.4 \\
\hline 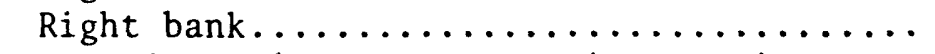 & 219.4 & 119.3 \\
\hline $\begin{array}{l}\text { U.S. Geological Survey gaging station } \\
(02423000) \text { at bridge on U.S. Highway } 80 \text {, }\end{array}$ & & \\
\hline 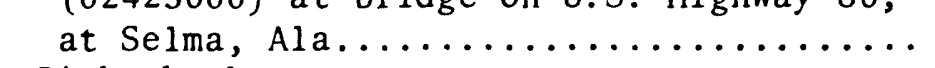 & 214.8 & c $/ 116.8$ \\
\hline 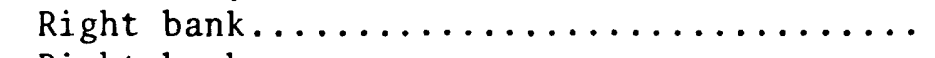 & 213.4 & 116.2 \\
\hline 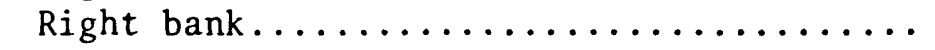 & 205.4 & 112.9 \\
\hline 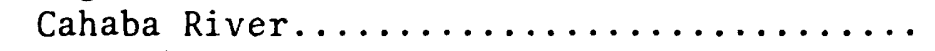 & 198.1 & ---- \\
\hline 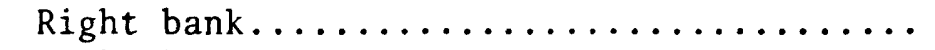 & 197.4 & 108.5 \\
\hline 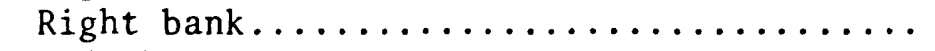 & 187.8 & 104.0 \\
\hline 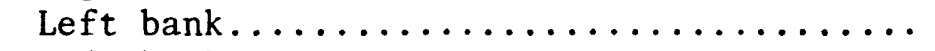 & 180.2 & 98.9 \\
\hline 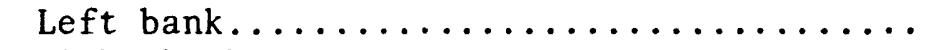 & 169.4 & 93.9 \\
\hline 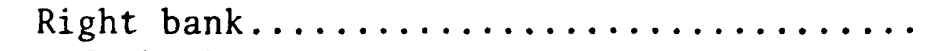 & 156.0 & 87.8 \\
\hline 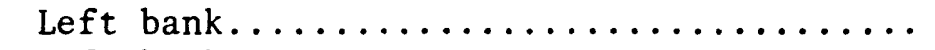 & 133.0 & 78.2 \\
\hline 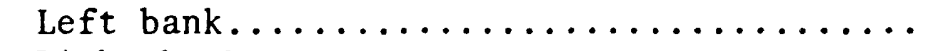 & 124.4 & $\frac{d}{d /} 73.2$ \\
\hline 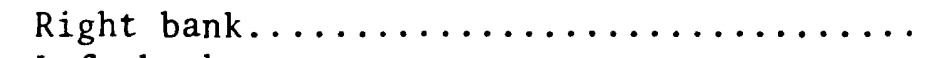 & 119.5 & d/ 73.9 \\
\hline 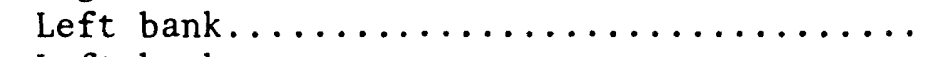 & 114.8 & 71.8 \\
\hline 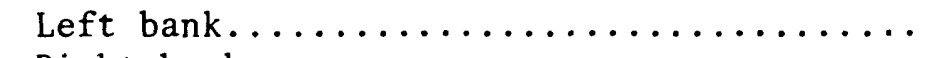 & 110.5 & 68.2 \\
\hline 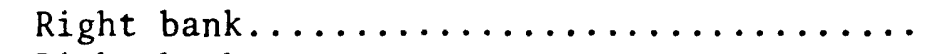 & 105.2 & 66.0 \\
\hline 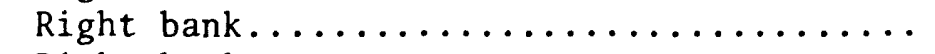 & 80.0 & 53.5 \\
\hline 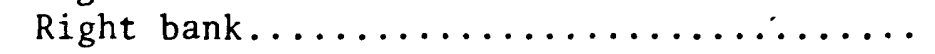 & 79.2 & 52.9 \\
\hline $\begin{array}{l}\text { U.S. Geological Survey gaging station } \\
(02429500) \text { downstream side of bridge }\end{array}$ & & \\
\hline on U.S. Highway 84 , at Claiborne, Ala... & 76.1 & ㄹ 51.6 \\
\hline 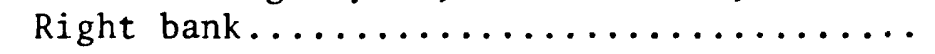 & 74.9 & 50.9 \\
\hline Right bank.................... & 70.7 & 49.5 \\
\hline Right bank.................... & 68.9 & 47.8 \\
\hline 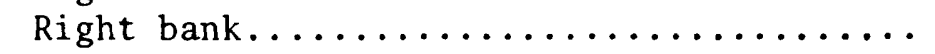 & 68.8 & 47.8 \\
\hline 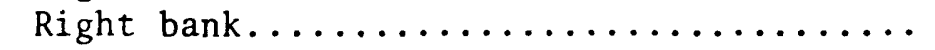 & 63.2 & 44.0 \\
\hline 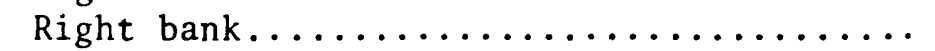 & 62.6 & 43.6 \\
\hline 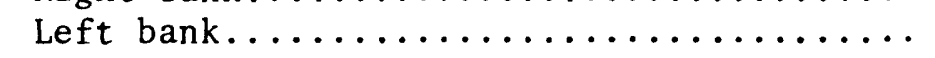 & 61.3 & 42.6 \\
\hline 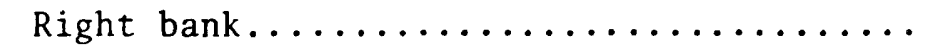 & 61.1 & 42.4 \\
\hline 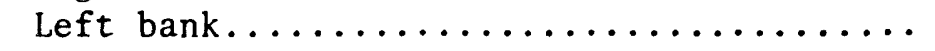 & 58.7 & 41.8 \\
\hline Left bank........... & 55.9 & 39.4 \\
\hline
\end{tabular}

See footnotes at end of table. 


\begin{tabular}{ccc}
\hline & Elevation above \\
& $\begin{array}{c}\text { Distance } \\
\text { upstream } \\
\text { Stream and location }\end{array}$ & $\begin{array}{c}\text { National geodetic } \\
\text { vertical datum }\end{array}$ \\
(miles) & of 1929 \\
(feet)
\end{tabular}

ALABAMA RIVER BASIN--Continued

Alabama River--Continued

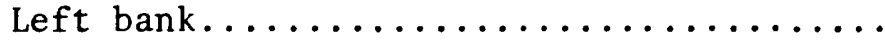

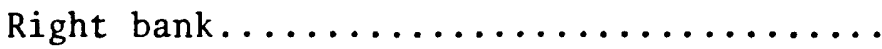

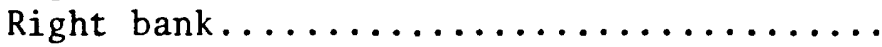

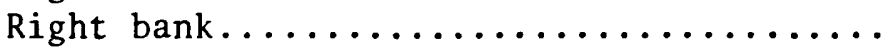

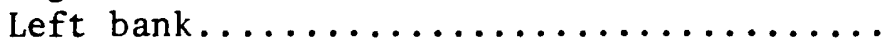

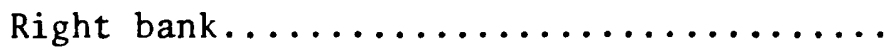

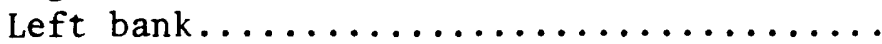

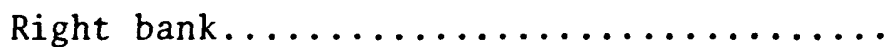

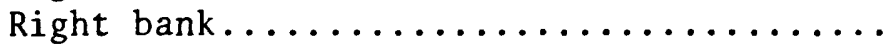

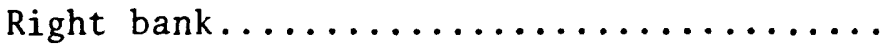

Mouth, at Mobile River mile 45.0

(confluence of Alabama and Tombigbee

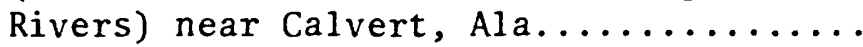

CAHABA RIVER BASIN

\section{Shades Creek:}

Left bank $200 \mathrm{ft}$ upstream from 5-barrel culvert and $100 \mathrm{ft}$ north of U.S. Highway

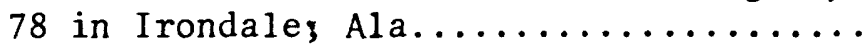
Right bank at downstream end of 5-barrel culvert and $100 \mathrm{ft}$ north of U.S. Highway 78 in Irondale, Ala................ Right bank $100 \mathrm{ft}$ upstream from U.S.

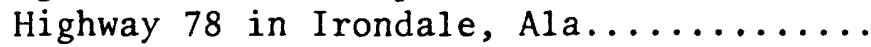
Left bank $60 \mathrm{ft}$ downstream from U.S.

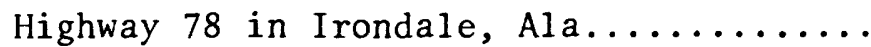
Right bank at downstream side of bridge on Elder Street in Birmingham, Ala...... Right bank at upstream side of culvert on Old Leeds Road in Mountain Brook, Ala.... Left bank $80 \mathrm{ft}$ downstream from 01d Leeds

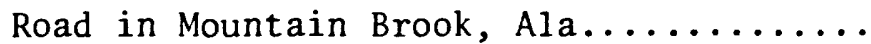
Left bank $5 \mathrm{ft}$ upstream from abutment of bridge on Beachwood Road in Mountain

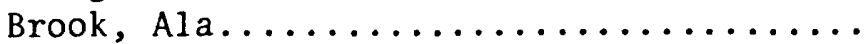
Left bank $100 \mathrm{ft}$ downstream from Beachwood Road in Mountain Brook, Ala.... Left bank on downstream side of Beachwood Road in Mountain Brook, Ala....
52.5

51.6

50.1

48.0

43.4

42.1

41.8

39.8

38.1

31.0

0.0
37.0

35.8

34.6

33.3

31.2

31.1

30.6

30.5

28.0

24.9

53.2

707.0

53.2

706.5

53.1

706.4

53.0

705.9

51.6

688.1

49.0

675.1

49.0

675.2

48.5

670.0

48.5

669.4

47.9

664.6

See footnotes at end of table. 


\begin{tabular}{ccc}
\hline & Elevation above \\
& Distance & National geodetic \\
& upstream & vertical datum \\
Stream and location & from mouth & of 1929 \\
(miles) & (feet) \\
\hline
\end{tabular}

CAHABA RIVER BASIN--Continued

Shades Creek--Continued

Right bank $20 \mathrm{ft}$ upstream from Lakeshore Drive in Mountain Brook, Ala........... Right bank at downstream end of bridge on Lakeshore Drive in Mountain Brook,

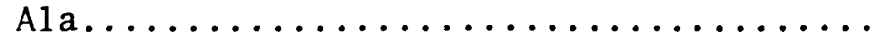

Right bank $100 \mathrm{ft}$ downstream from Lakeshore Drive in Mountain Brook, Ala... Left downstream wingwall at bridge on old U.S. Highway 31 near Homewood, Ala... Right bank at upstream side of bridge on Green Spring Highway in Homewood, Ala.... Right bank at downstream side of bridge on Green Spring Highway in Homewood, Ala. Right bank at Shades Creek filter plant near Homewood, Ala................. Right bank at downstream side of bridge on Oxmoor Road near Homewood, Ala.......

Right bank $35 \mathrm{ft}$ upstream from gravel road 0.1 mile east of Shannon Road (E $\frac{1}{2} \mathrm{SE}^{\frac{1}{4}}$ sec. 33, T. 18 S., R. 3 W.) near Homewood, Ala.................... Right bank $35 \mathrm{ft}$ downstream from gravel road 0.1 mile east of Shannon Road (E $\left.\frac{1}{2} \mathrm{SE}^{\frac{1}{4}} \mathrm{sec} .33, \mathrm{~T} .18 \mathrm{~S} ., \mathrm{R} .3 \mathrm{~W}.\right)$

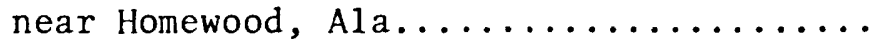
Right bank $25 \mathrm{ft}$ upstream from Alabama State Highway $150,0.6$ mile southeast of

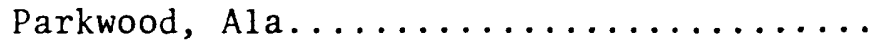
Right bank at downstream side of Alabama State Highway $150,0.6 \mathrm{mile}$ southeast of

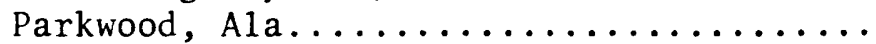
Right bank at upstream side of county road 0.8 mile southwest of Parkwood, Ala..... Right bank at downstream side of county road 0.8 mile southwest of Parkwood, Ala. Left bank upstream side of Morgan Road near Hopewel1, Ala................. Left bank downstream from Morgan Road near Hopewell, Ala.................

47.3 654.5

47.3 651.3

47.2 650.9

45.1 637.9

42.8 626.4

42.8 626.4

41.1 616.4

40.2 611.9

39.2 601.2

39.2 601.2

31.5 553.1

31.5 553.0

30.4 538.6

30.3 538.6

25.2 510.6

25.2 510.4

See footnotes at end of table. 


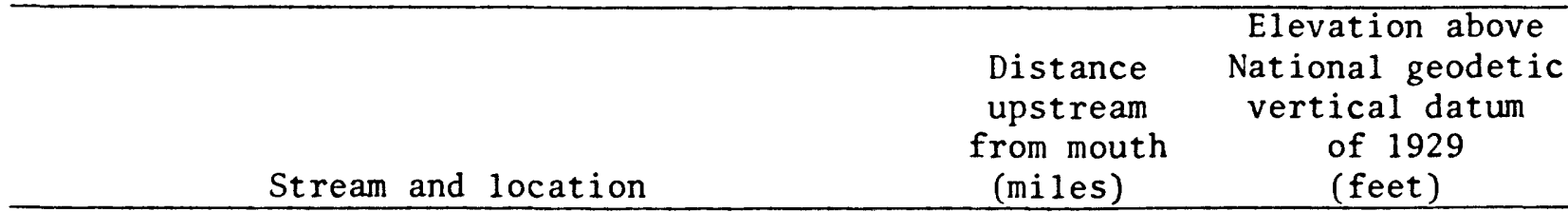

CAHABA RIVER BASIN--Continued

Shades Creek--Continued

U.S. Geological Survey gage (02423630)

on left bank on downstream side of

bridge on Dickey Spring Road near

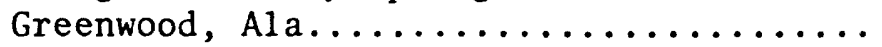

Mouth (at Cahaba River mile 104.2).......

20.8

493.6

TOMBIGBEE RIVER BASIN

Tombigbee River:

U.S. Geological Survey gaging station

(02441500) on left bank, 1200 feet

downstream from bridge on U.S. Highway

$45 \mathrm{E}$ and 82 , at Columbus, Miss..........

319.7

164.4

Left bank at sediment range $10 \mathrm{~B} f / . .$.

319.3

164.0

Right bank near sediment range 9 B f $/ . .$. .

318.5

i 63.8

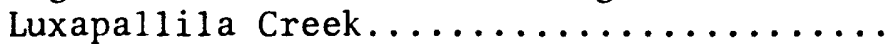

317.4

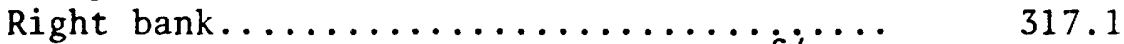

-..--

Right bank near sediment range $20 \mathrm{~A} f \ldots$.

316.0

163.2

Left bank near sediment range $19 \mathrm{~A} \mathrm{f} / . .$. .

315.0

162.6

Right bank at sediment range SA $12 \mathrm{f} / \ldots .$.

312.7

161.0

Left bank at sediment range $17 \mathrm{~A} / . . .$. .

312.2

158.4

Right bank at sediment range $16 \mathrm{~A} f / . .$. .

311.2

157.3

Right bank near sediment range 15 A f $/ . .$.

309.6

156.0

Right bank near sediment range $13 \mathrm{~A} \overline{\mathrm{f}} / \ldots$.

307.8

Left bank at sediment range $11 \mathrm{HB} / \ldots . .$.

307.3

306.0

Left bank at sediment range $8 \mathrm{HB}$ ff.......

302.6

Right bank at sediment range $3 \mathrm{HB} f / \ldots .$.

300.2

153.8

Right bank at sediment range $8 \mathrm{~A} f \ldots . .$.

297.8

152.7

Right bank near Southern Natural Gas

pipeline near Forreston, Miss..........

Right bank at sediment range $4 \mathrm{~A} f j . . .$.

Right bank near sediment range 3 A 1 i....

295.0

d/ 152.7

Left bank on staff gage at Pickensville

292.0

d/ 152.1

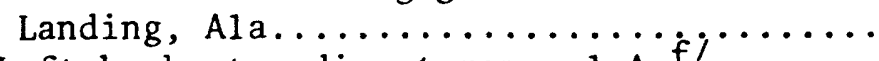

Left bank at sediment range i $\mathrm{A} f \mathrm{f} j \ldots . .$.

290.1

150.7

Left bank at sediment range 15 AG f/.....

288.0

286.2

285.0

283.0

148.8

145.0

143.7

Right bank near sediment range $B C D \frac{f}{f} \ldots . .$.

Left bank near sediment range 15 A $/ \ldots .$.

Left bank at sediment range $14 \mathrm{~A} / . . .$. .

281.8

143.0

141.1

139.7

139.5

140.0

139.6

See footnotes at end of table. 


\begin{tabular}{ccc}
\hline & Elevation above \\
& $\begin{array}{c}\text { Distance } \\
\text { upstream } \\
\text { from mouth } \\
\text { Stream and location }\end{array}$ & $\begin{array}{c}\text { National geodetic } \\
\text { vertical datum }\end{array}$ \\
of 1929 \\
(miles) & (feet) \\
\hline
\end{tabular}

TOMBIGBEE RIVER BASIN--Continued

Tombigbee River--Continued

U.S. Geological Survey gaging station (02444500) near left bank, downstream side of bridge on State Highway 17,

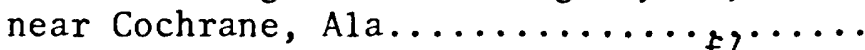

Right bank at sediment range $10 \mathrm{~A}$ f $\ldots . .$. Left bank near sediment range $9 \mathrm{~A}$ f $/ . . .$. Right bank near sediment range $8 \mathrm{~A}$ f $\ldots . .$. Right bank at sediment range $7 \mathrm{~A} / \ldots . .$. Left bank at sediment range $6 \mathrm{~A} f \mathrm{f} . . . .$. Right bank at sediment range $5 \mathrm{~A} f \ldots . . .$. Left bank near sediment range $4 \mathrm{~A}$ f $\ldots . . .$. Right bank near sediment range $3 \mathrm{~A}$ f $\% . .$. Right bank near sediment range 2 A $\underline{f} \ldots . .$. U.S. Geological Survey gaging station (02449000) near right bank on downstream side of bridge on State

Highway 39 at Gainesville, Ala......... Right bank at sediment range $1 \mathrm{C} \underline{\mathrm{f}} j \ldots . . .$.

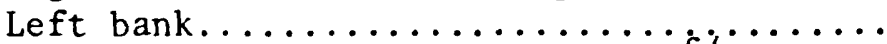
Left bank at sediment range $3 \mathrm{C}$ f $j \ldots . .$. Left bank....................... Left bank near sediment range $5 \mathrm{c}$ f $j \ldots . .$. Right bank near sediment range $6 \mathrm{C}$ f $j . . .$. U.S. Geological Survey gaging station (02449500 discontinued) at bridge on U.S. Highway 11 at Epes, Ala.......... Left bank near sediment range $7 \mathrm{C} \neq . . .$. Left bank near sediment range $8 \mathrm{C} / \ldots . .$. Left bank near sediment range $9 \mathrm{C} \underline{\mathrm{f}} / \ldots . .$. Left bank, Bluffport, Ala............. Right bank near sediment range $10 \mathrm{C} / . .$. Right bank near sediment range $11 \mathrm{C} / \mathrm{f} / . .$. Right bank near sediment range 12 C $f / \ldots$ Right bank near sediment range 12 CA f\%... Right bank near sediment range RB $5, \ldots$... Left bank at sediment range RB $10 \mathrm{f} / \ldots . .$. Right bank near sediment range RB 12 f $/ . .$. Right bank near sediment range 12 CE $\underline{\mathrm{f}} / \ldots$ Left bank near sediment range $13 \mathrm{C}$ f $/ . .$. . Left bank 100 feet upstream from Black

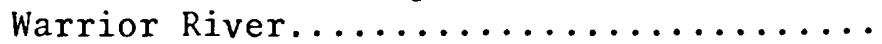

271.4

271.1

264.8

261.4

259.0

255.6

252.1

248.1

244.5

241.0

234.4

232.8

229.4

229.0

226.5

221.3

218.1

215.2

212.9

207.9

204.2

202.0

200.5

198.3

193.0

191.2

189.8

185.0

183.0

180.8

179.9

175.0
133.1

131.7

130.2

130.5

129.3

127.5

124.8

123.2

122.6

120.2

119.6

117.5

116.2

116.2

115.4

111.7

111.3

108.9

107.4

106.8

104.3

102.5

101.5

100.7

96.4

95.5

d/ 95.7

d/ 95.4

d/ 95.5

95.2

95.2

93.9

See footnotes at end of table. 
TABLE 5.-Flood-crest stages-Continued

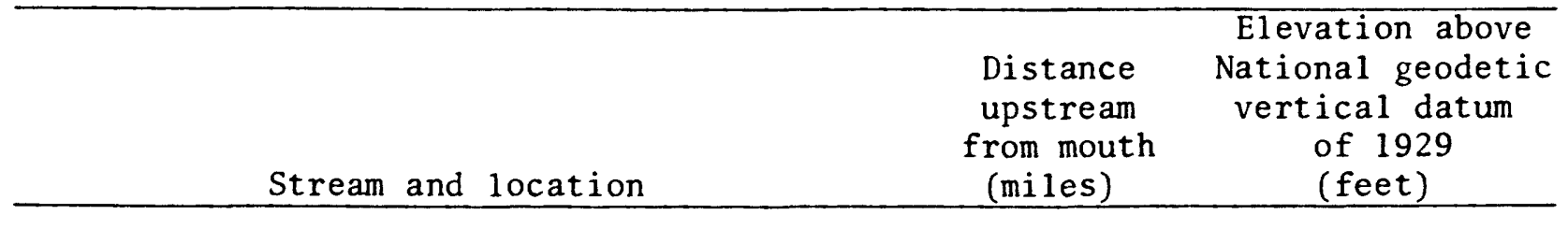

TOMBIGBEE RIVER BASIN--Continued

Tombigbee River--Continued

Left bank, old lock $4 \ldots \ldots \ldots \ldots \ldots \ldots \ldots$

174.5

93.8

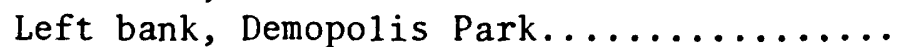

174.1

93.8

Left bank, Demopolis Park...............

174.0

93.6

Left bank near Alabama State grain

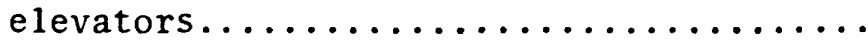

173.0

93.4

U.S. Geological Survey gaging station (02467000) on left bank, $100 \mathrm{ft}$

upstream from Demopolis lock and dam

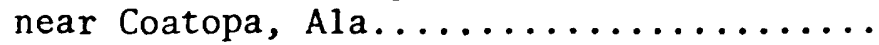

Left bank about 1000 feet downstream

from Demopolis lock and dam............

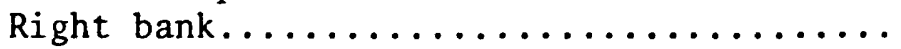

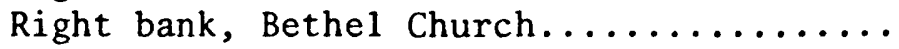

Left bank, Gulf States Company...........

Left bank about 0.15 mile upstream from

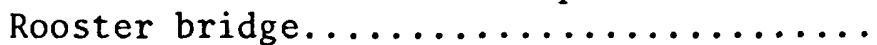

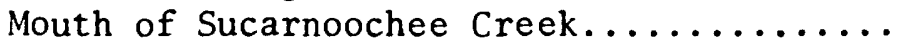

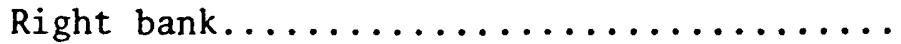

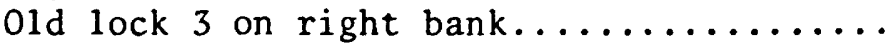

U.S. Geological Survey gaging station

(02469761) near right bank at

Coffeeville lock and dam near

Coffeeville, Ala...................

U.S. Geological Survey gaging station

(02469762) near right bank below

Coffeeville lock and dam near

Coffeeville, Ala..................

Mouth, at Mobile River mile 45.0

(confluence of Tombigbee and Alabama

Rivers)......................

$\begin{array}{lr}171.2 & 93.0 \\ 170.9 & 92.4 \\ 170.1 & 90.8 \\ 165.8 & 89.5 \\ 163.6 & 87.3 \\ & \\ 159.8 & 84.7 \\ 158.6 & --- \\ 155.7 & 82.9 \\ 148.9 & 77.9\end{array}$

74.7

51.5

Tombigbee River tributary streams:

Luxapallila Creek near Columbus, Miss.:

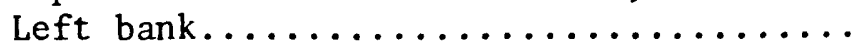

7.0

177.9

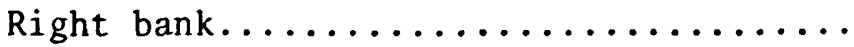

6.2

175.2

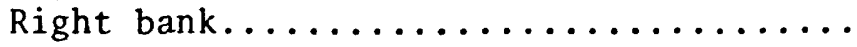

6.0

175.2

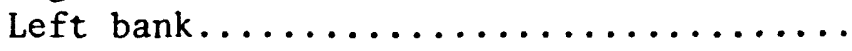

2.6

165.3

Mouth (at Tombigbee River at mile 317.4)

0.0

-..--

See footnotes at end of table. 


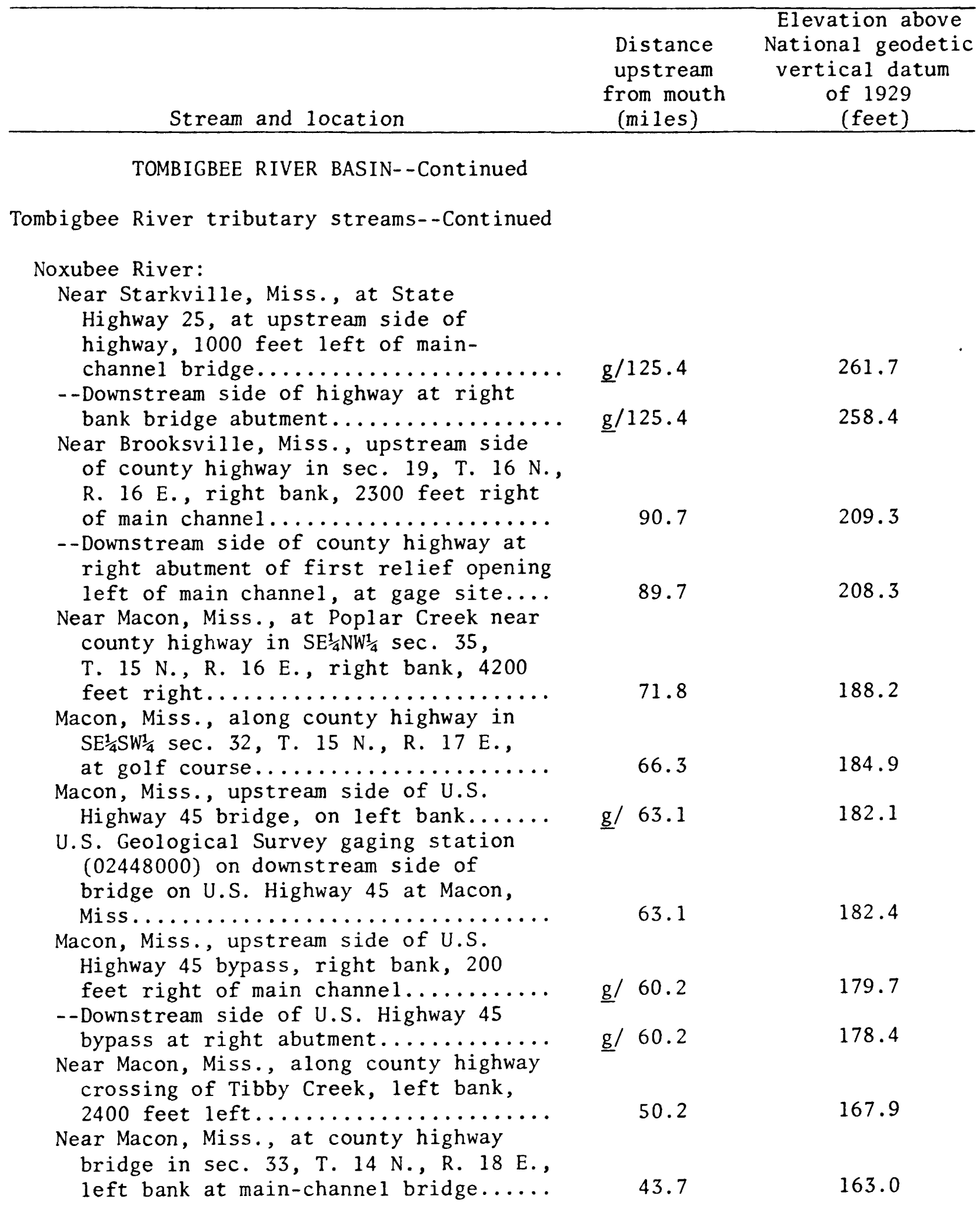

See footnotes at end of table. 
TABLE 5.-Flood-crest stages-Continued

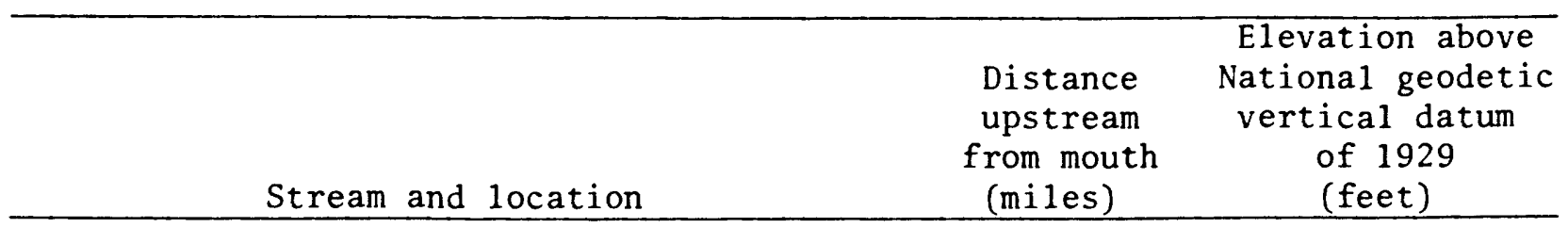

TOMBIGBEE RIVER BASIN--Continued

Tombigbee River tributary streams--Continued

Noxubee River--Continued

Near Paulette, Miss., at county highway in $\mathrm{NE}^{\frac{1}{4}} \mathrm{SE}^{\frac{1}{4}} \mathrm{sec} .17$, T. $13 \mathrm{~N}$.,

R. 18 E., right bank..............

- - In NW $\frac{1}{4} \mathrm{SW}_{\frac{1}{4}} \mathrm{sec} .16, \mathrm{~T} .13 \mathrm{~N} .$, R. $18 \mathrm{E}$. ,

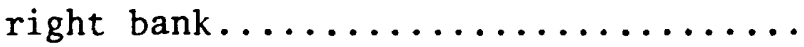

Near Geiger, Ala., upstream side of State Highway 17 (north bridge) left bank, 300 feet left of north channel..

--Downstream side of State Highway 17 at left end of bridge.............. Near Geiger, Ala., upstream side of State Highway 17 (south bridge) right bank, 500 feet right of main channe1......................

--Downstream side of State Highway 17, 600 feet right of main channe1.......

U.S. Geological Survey gaging station (02448500) near left bank on downstream side of bridge on State High-

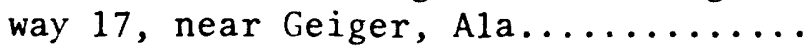

Near Geiger, Ala., downstream side of St. Louis-San Francisco Railroad, right bank, 3000 feet right of main

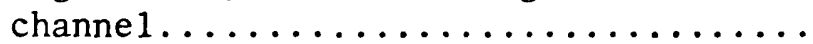

Near Gainesville, Ala., upstream side of county highway, 3800 feet left of

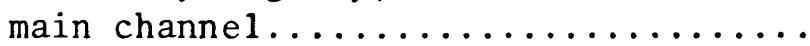

--Downstream side of county highway 3500 feet right of main channe $1 . . . \ldots$.

g/ 16.9

134.7

g/ $16.9 \quad 134.2$

g/ $16.9 \quad 135.0$

g/ $16.9 \quad 134.2$

$39.6 \quad 157.6$

g/ 16.9

16.9

134.7

g/ 14.7

133.6

$\begin{array}{lll}\text { g/ } & .3 & 122.7\end{array}$

g/ $.3 \quad 122.3$

Sucarnoochee Creek:

Near DeKalb, Miss., upstream side of State Highway 16, left bank, 2000

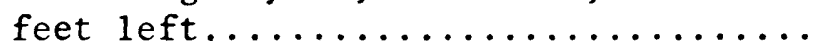

--Downstream side of State Highway 16... Near Porterville, Miss., downstream side of U.S. Highway 45 , left end of

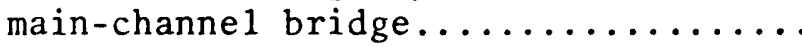

U.S. Geological Survey gaging station (02467500) on right bank 10 feet

downstream from bridge on U.S. Highway 11 at Livingston, Ala.......... Mouth, at Tombigbee River, mile 158.6... 


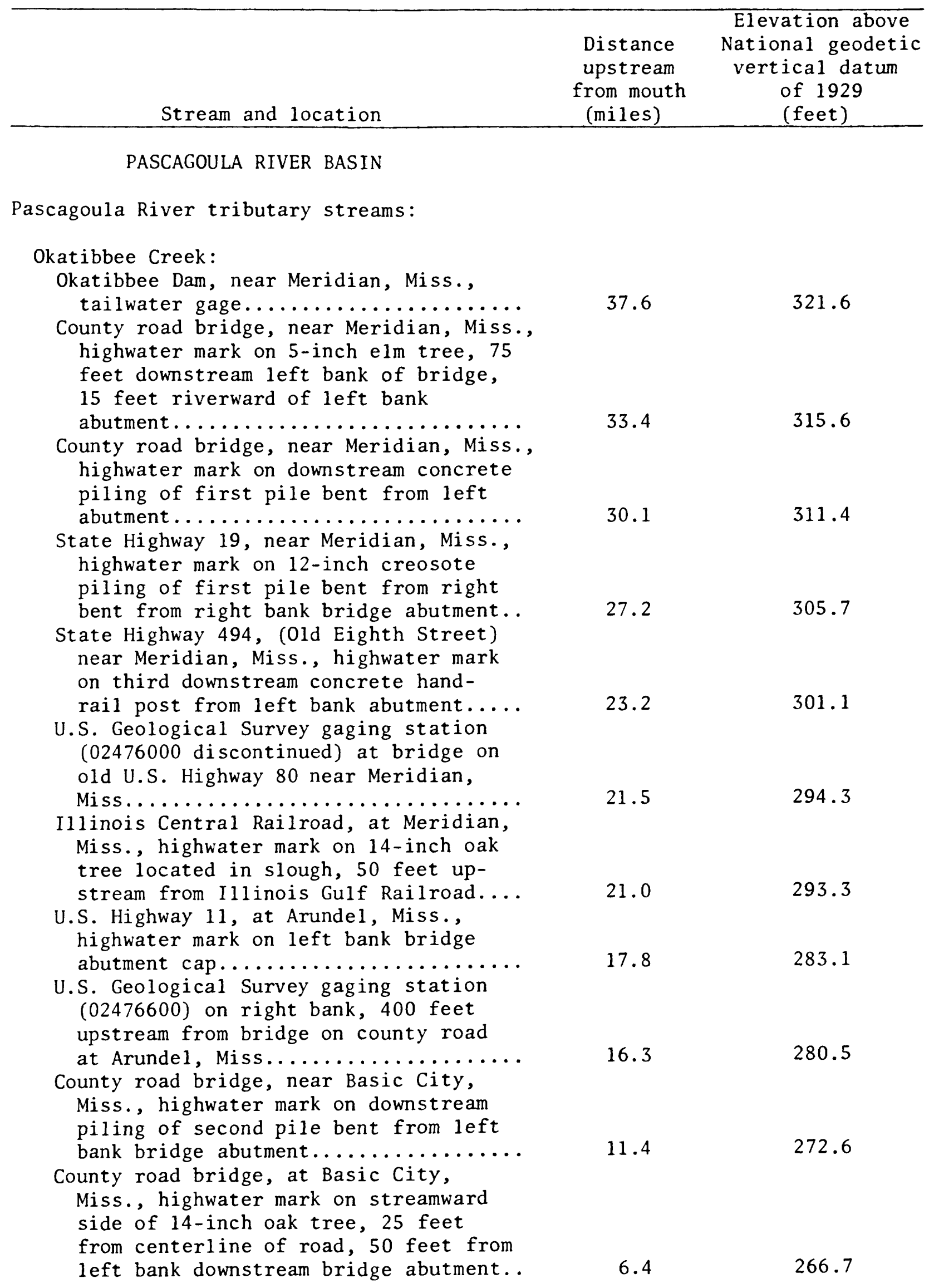

See footnotes at end of table. 
TABLE 5.-Flood-crest stages-Continued

(Data furnished by Corps of Engineers, Mobile District)

\begin{tabular}{|c|c|c|}
\hline Stream and location & $\begin{array}{l}\text { Distance } \\
\text { upstream } \\
\text { from mouth } \\
\text { (miles) }\end{array}$ & $\begin{array}{c}\text { Elevation above } \\
\text { National geodetic } \\
\text { vertical datum } \\
\text { of } 1929 \\
\text { (feet) }\end{array}$ \\
\hline
\end{tabular}

PASCAGOULA RIVER BASIN--Continued

Pascagoula River tributary streams--Continued

Okatibbee Creek--Continued

County road bridge, near Enterprise, Miss., highwater mark on upstream pile located on first pile bent from right bank abutment...........

257.4

Mouth, at Enterprise, Miss., at

Chickasawhay River mile 158.7........

0.0

Sowashee Creek at Meridian, Miss.

Hawkins Crossing road bridge, highwater mark on upstream and downstream curb

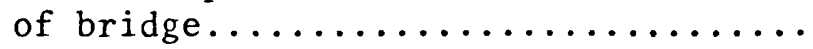

Southern Railroad bridge, highwater mark on second bent from left bank abutment on downstream pile................

U.S. Highway 11 and 80 bridge, highwater mark on upstream end of right bank concrete abutment................

U.S. Geological Survey gaging station (02476500) upstream side of bridge on U.S. Highway 45 at Meridian, Miss..... Meridian and Bigbee Railroad, highwater mark on downstream piling of first bent from left abutment............

18 th Avenue bridge, highwater mark on

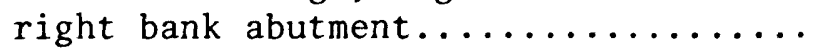

--Highwater mark on first creosote piling on left bank upstream wingwall.

U.S. Highway 45 (business route) bridge highwater mark on 14-inch creosote sign pole 40 feet east of southeast

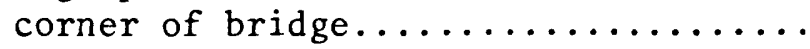

10.6

335.4

10.0

332.1

9.8

331.0

9.5

328.8

8.2

318.3

8.1

317.0

8.1

316.7

Grand Avenue bridge, highwater mark on power pole, 130 feet right of bridge, 25 feet upstream from centerline of

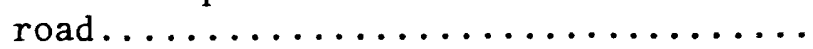

--Highwater mark on upstream right

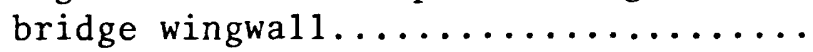

--Highwater mark on downstream wall of concrete block building, 130 feet downstream and 200 feet right of right bridge abutment..................

$\begin{array}{ll}7.1 & 312.7\end{array}$


TABLE 5.-Flood-crest stages-Continued

(Data furnished by Corps of Engineers, Mobile District)

\begin{tabular}{|c|c|c|}
\hline Stream and location & $\begin{array}{l}\text { Distance } \\
\text { upstream } \\
\text { from mouth } \\
\text { (miles) }\end{array}$ & $\begin{array}{l}\text { Elevation } \\
\text { National gec } \\
\text { vertical da } \\
\text { of } 1929 \\
\text { (feet) }\end{array}$ \\
\hline \multicolumn{3}{|l|}{ PASCAGOULA RIVER BASIN--Continued } \\
\hline \multicolumn{3}{|l|}{ Pascagoula River tributary streams--Continued } \\
\hline \\
\hline $\begin{array}{l}\text { 31st Avenue bridge, highwater mark on } \\
\text { concrete guardrail post at right bank } \\
\text { bridge abutment pile cap............. } \\
\text { - - Highwater mark on rear of church about } \\
120 \text { feet from right bank abutment, } 80 \\
\text { feet downstream from centerline of }\end{array}$ & 6.6 & 310.7 \\
\hline road $\ldots \ldots \ldots \ldots \ldots \ldots \ldots \ldots$ & 6.6 & 310.5 \\
\hline $\begin{array}{l}\text { Gulf Mobile \& Ohio Railroad, highwater } \\
\text { mark on upstream piling of fifth pile }\end{array}$ & & \\
\hline bent from right abutment........... & 6.5 & 308.3 \\
\hline $\begin{array}{l}\text { I-20 frontage road bridge, highwater } \\
\text { mark on upstream concrete pile of } \\
\text { second pile bent from left bank }\end{array}$ & & \\
\hline 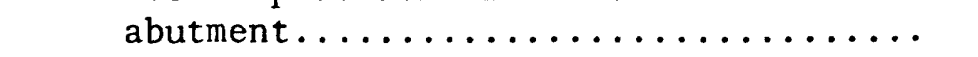 & 5.6 & 302.6 \\
\hline $\begin{array}{l}\text { 49th Avenue bridge, highwater mark on } \\
18 \text {-inch oak tree about } 50 \text { feet up- } \\
\text { stream and } 60 \text { feet left of left bank }\end{array}$ & & \\
\hline 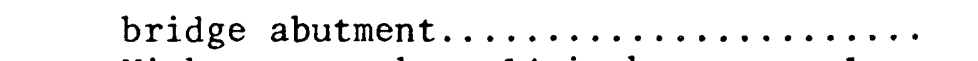 & 4.8 & 301.4 \\
\hline $\begin{array}{l}- \text {-Highwater mark on } 14 \text {-inch power pole } \\
50 \text { feet downstream and } 130 \text { feet from }\end{array}$ & & \\
\hline left bank bridge abutment.......... & 4.8 & 301.1 \\
\hline Mouth, at Okatibbee Creek mile $84.4 \ldots \ldots$ & 0.0 & \\
\hline
\end{tabular}

PEARL RIVER BASIN

Nanih Waiya Creek:

Near Louisville, Miss., upstream side of State Highway 14, 150 feet right of

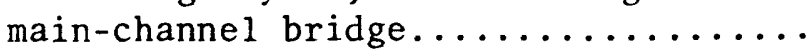

- -At downstream right abutment of main-

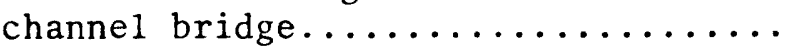

Near Fearns Springs, Miss., upstream side of State Highway 490, 1800 feet right of main-channel bridge.........

- -Downstream side of State Highway 490 , 1800 feet right of main-channel bridge.................. g/446.2 


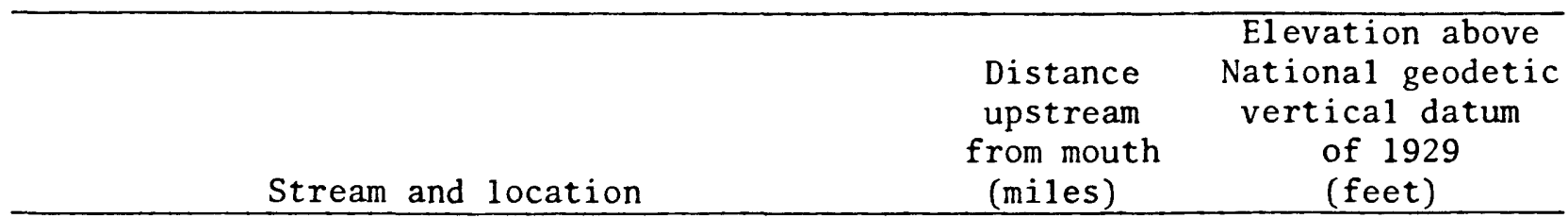

PEARL RIVER BASIN--Continued

Nanih Waiya Creek--Continued

Near Handle, Miss., upstream side of State Highway 397,1300 feet right of

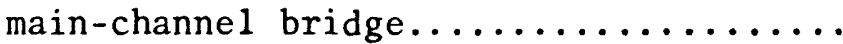
--Downstream side of State Highway 397, 1000 feet left of main-channel bridge..

$\underline{\mathrm{g}} / 442.9$

430.6

$\underline{g} / 442.9$

430.2

Pearl River:

Near Burnside, Miss., upstream side of State Highway 15, 1700 feet left of main-channel bridge..............

- -Downstream side of State Highway 15, 150 feet left of main-channel bridge....

Near Philadelphia, Miss., upstream side of State Highway 19, 4800 feet right of

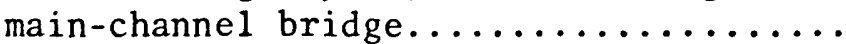

--Downstream side of State Highway 19, 300 feet right of main-channel bridge...

Edinburg, Miss., upstream side of State Highway 16, 1300 feet left of main-

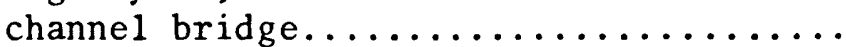

U.S. Geological Survey gaging station (02482000) right bank, 20 feet downstream from bridge on State Highway 16,

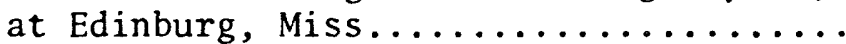

Near Edinburg, Miss., left bank, 1.5 miles downstream from State Highway 16,

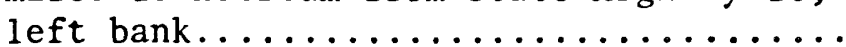

Near Sunrise, Miss., upstream side of county highway, 2100 feet right of

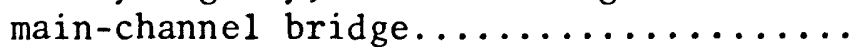

- -At Corps of Engineers profile point No. 17 , left bank, in $\mathrm{SW}_{\frac{1}{4}} \mathrm{NE} \frac{1}{4} \mathrm{sec} .1$, T. 10 N., R. 8 E..................

Near Freeny, Miss., at State Highway 488 crossing of Standing Pine Creek........ Near Carthage, Miss., upstream side of State Highway 35, 7000 feet right of

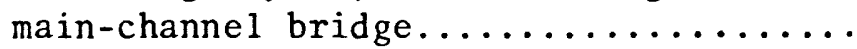

$\underline{g} / 416.2$

401.3

g/416.2

399.0

$\underline{g} / 412.9$

395.2

$\underline{g} / 412.9$

394.1

g/ 387.5

373.8

387.5

371.7

h/ 386.4

h/ 370.0

$\mathrm{g} / 378.5$

362.0

376.0

$\underline{\mathrm{h}} / 355.7$

i $/ 371.8$

h/ 352.0

g/366. 3

344.7

See footnotes at end of table. 


\begin{tabular}{|c|c|c|}
\hline Stream and location & $\begin{array}{l}\text { Distance } \\
\text { upstream } \\
\text { from mouth } \\
\text { (miles) }\end{array}$ & $\begin{array}{l}\text { Elevation al } \\
\text { National geo } \\
\text { vertical da } \\
\text { of } 1929 \\
\text { (feet) }\end{array}$ \\
\hline \multicolumn{3}{|l|}{ PEARL RIVER BASIN--Continued } \\
\hline \multicolumn{3}{|l|}{ Pearl River--Continued } \\
\hline $\begin{array}{l}\text { - Downstream side of State Highway } 35 \text {, } \\
7000 \text { feet right of main-channel bridge.. } \\
\text { U.S. Geological Survey gaging station } \\
\text { (02482550) right bank on downstream side } \\
\text { of bridge on State Highway } 35 \text {, near }\end{array}$ & $\underline{\mathrm{g}} / 366.3$ & 343.3 \\
\hline 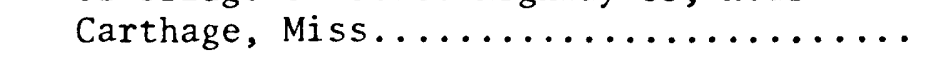 & 366.3 & 344.0 \\
\hline $\begin{array}{l}\text { Near Carthage, Miss., } 5.8 \text { river miles } \\
\text { downstream from bridge at State Highway. }\end{array}$ & & \\
\hline 35 , right bank of main channel.......... & 360.5 & $\underline{\mathrm{h}} / 335.7$ \\
\hline $\begin{array}{l}\text { Near Wiggins, Miss., } 4.3 \text { river miles up- } \\
\text { stream from bridge at State Highway } 13 \text {, }\end{array}$ & & \\
\hline Near Wiggins, Miss., upstream side of & 358.4 & $\underline{\mathrm{h}} / 335.2$ \\
\hline 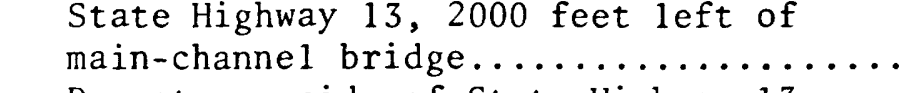 & $\underline{\mathrm{g}} / 354.1$ & 332.8 \\
\hline $\begin{array}{l}\text { - - Downstream side of State Highway } 13 \text {, } \\
\text { left bank at end of main-channel bridge. } \\
\text { Near Wiggins, Miss., } 1.8 \text { river miles } \\
\text { downstream from State Highway 13, right }\end{array}$ & $\mathrm{g} / 354.1$ & $\underline{\mathrm{h}} / 331.7$ \\
\hline $\begin{array}{l}\text { bank of main channel............... } \\
\text { Near Ofahoma, Miss., } 6.3 \text { river miles up- } \\
\text { stream from Ross Barnett Reservoir low- } \\
\text { head dam, right bank } 1400 \text { feet right on }\end{array}$ & 352.3 & $\underline{\mathrm{h} / 330.4}$ \\
\hline $\begin{array}{l}\text { right bank of Gogg Lake................. } \\
\text { Ross Barnett Reservoir low-head dam. }\end{array}$ & 348.8 & $\underline{\mathrm{h}} / 327.8$ \\
\hline right bank, 250 feet downstream......... & 342.6 & $\underline{\mathrm{h}} / 321.3$ \\
\hline $\begin{array}{l}\text { Near Ross Barnett Reservoir low-head dam } \\
1.5 \text { river miles downstream, right bank } \\
2000 \text { feet right of main channel, on }\end{array}$ & & \\
\hline 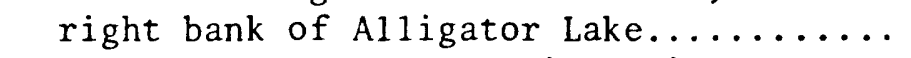 & 341.4 & $\underline{\mathrm{h} / 319.5}$ \\
\hline $\begin{array}{l}\text { Near Ratliff Ferry, } 7.8 \text { river miles up- } \\
\text { stream left bank, } 1000 \text { feet upstream } \\
\text { from Rankin-Scott County line. }\end{array}$ & & 317.3 \\
\hline Near Ratliff Ferry, 5.9 river miles up- & 338.2 & $31 / .3$ \\
\hline 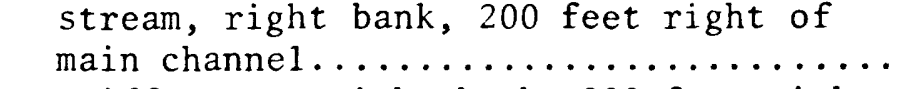 & 336.3 & $\underline{\mathrm{h} / 317.2}$ \\
\hline 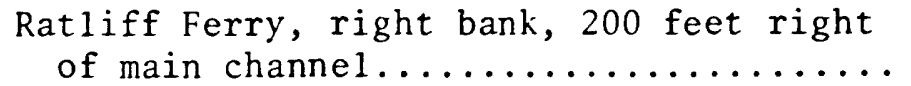 & 330.4 & 313.1 \\
\hline 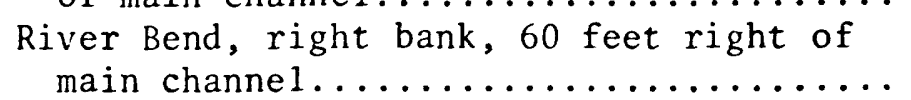 & 328.5 & 310.4 \\
\hline
\end{tabular}

See footnotes at end of table. 


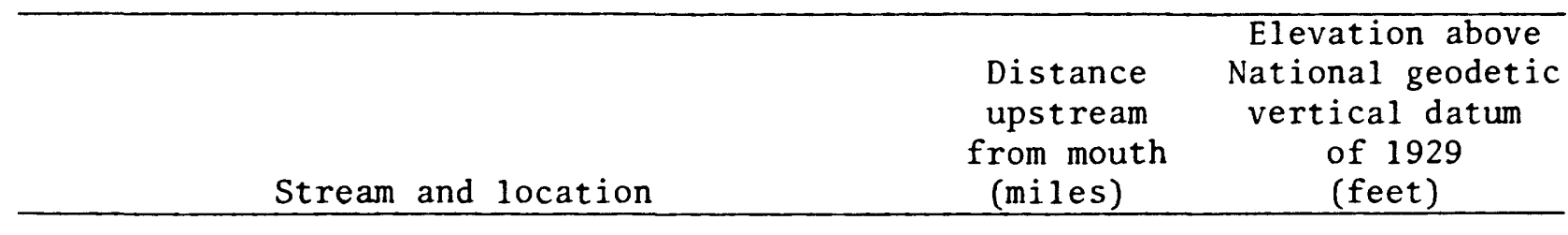

PEARL RIVER BASIN--Continued

Pearl River--Continued

Near River Bend, 2.8 river miles downstream right bank 2000 feet right, of main channel at Natchez Trace crossing

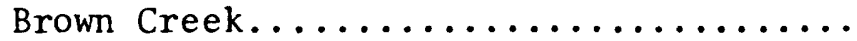
Near Canton, Miss., along Natchez Trace, 2.0 miles north of State Highway $43 \ldots .$.

Near Canton, at State Highway 43, upstream side of State Highway 43, 3500 feet left of main-channel bridge....... --Downstream side of State Highway 43, 50 feet left of main-channel bridge....

U.S. Geological Survey gaging station (02485000 discontinued) downstream side of left main pier of Meeks bridge on State Highway 43 near Canton, Miss...... Ross Barnett Reservoir dam, upstream side of dam at Control House gage...........

--Downstream side of dam, 10,500 feet

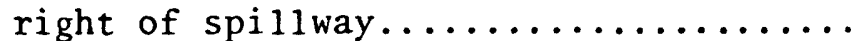

Jackson, Miss., north side of Jackson Country $\mathrm{Club}$ at maintenance buildings... Jackson, Miss., southeast side of St. Andrews Drive, 400 feet northeast from intersection with St. Haylake Drive.....

--01d Canton Road at Purple Creek........ Near Jackson, Miss., near north edge of sec. 23, T. 6 N., R. 2 E., 600 feet

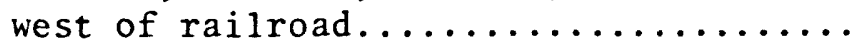

Near Jackson, Miss., on gravel road 0.5 miles west of Luckney, Miss............ Jackson, Miss., at intersection of East Northside Drive and East Cheryl........

Jackson, Miss., at Hanging Moss Creek at Interstate Highway $55 \ldots \ldots \ldots \ldots \ldots \ldots$

Near Jackson, Miss., at intersection of State Highways 25 and 475 in $N^{\frac{1}{4}} \mathrm{sec}$. 28, T. 6 N., R. 2 E............... Near Jackson, Miss., at State Highway 475,

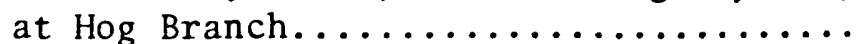
At Jackson, Miss., in SE $\frac{1}{4}$ sec. 19, T. 6 N., R. 2 E., at Lake Circle...........
325.8

305.8

321.0

301.3

$\underline{g} / 319.4$

301.3

g/319.4

299.9

319.4

300.0

301.8

299.8

301.6

288.2

299.6

287.7

298.0

287.3

296.6

286.8

296.5

286.9

295.

286.4

295.

286.1

295.

286.4

294.

285.6

294.

284.7

293.3
285.2

See footnotes at end of table. 


\begin{tabular}{|c|c|c|}
\hline Stream and location & $\begin{array}{l}\text { Distance } \\
\text { upstream } \\
\text { from mouth } \\
\text { (miles) }\end{array}$ & $\begin{array}{l}\text { Elevation ab } \\
\text { National geod } \\
\text { vertical dat } \\
\text { of } 1929 \\
\text { (feet) }\end{array}$ \\
\hline \multicolumn{3}{|l|}{ PEARL RIVER BASIN--Continued } \\
\hline \multicolumn{3}{|l|}{ Pearl River-Continued } \\
\hline $\begin{array}{l}\text { Near Jackson, Miss., in SE } \frac{1}{4} \text { sec. } 29 \text {, } \\
\text { T. } 6 \text { N., R. } 2 \text { E., upstream side of } \\
\text { State Highway } 25 \text {, at B-Line } \\
\text { Gasoline-Jiffy Stop, } 4200 \text { feet left } \\
\text { of main-channel bridge................ } \\
\text {--Downstream side of State Highway } 25\end{array}$ & 293.2 & 285.2 \\
\hline $\begin{array}{l}\text { across from B-Line Station.................. } \\
\text { Jackson, Miss., upstream side of State } \\
\text { Highway } 25,150 \text { feet northwest of } \\
\text { Lelia Drive intersection, } 3500 \text { feet }\end{array}$ & 293.2 & 284.5 \\
\hline $\begin{array}{l}\text { west of main-channel bridge............. } \\
\text {--Downstream side State Highway } 25,100\end{array}$ & 292.5 & 284.6 \\
\hline $\begin{array}{l}\text { feet south of Lelia Drive intersection.. } \\
\text { Jackson, Miss., upstream side of State }\end{array}$ & 292.5 & 283.6 \\
\hline 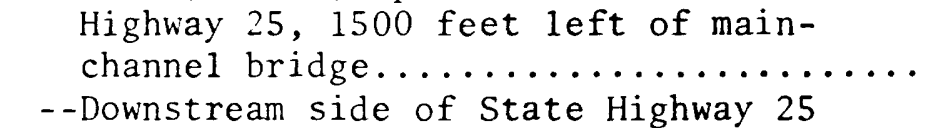 & $\underline{\mathrm{g}} / 292.4$ & 285.1 \\
\hline 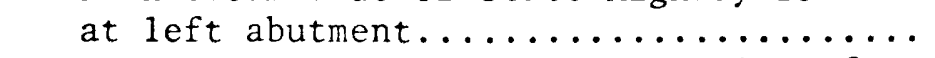 & $\underline{\mathrm{g} / 292.4}$ & 284.1 \\
\hline 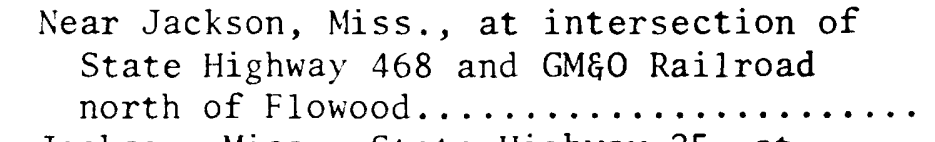 & 291.9 & 284.1 \\
\hline $\begin{array}{l}\text { Jackson, Miss., State Highway } 25 \text {, at } \\
\text { Smith-Wills Stadium................... } \\
\text { Jackson, Miss., upstream side of GME् } \\
\quad \text { Railroad, right bank, } 800 \text { feet right }\end{array}$ & 291.5 & 283.1 \\
\hline --Downstream side of GMEO Railroad, right & $\underline{\mathrm{g} / 290.6}$ & 282.3 \\
\hline $\begin{array}{l}\text { bank, } 800 \text { feet right of main channel } \ldots \\
\text { Jackson, Miss., Interstate Highway } 55 \text { in }\end{array}$ & $\underline{\mathrm{g} / 290.6}$ & 281.8 \\
\hline $\begin{array}{l}\text { NE } \frac{1}{4} \text { sec. 2, T. } 5 \text { N., R. I E., about } 1000 \\
\text { feet north of Fortification Street...... } \\
\text { Jackson, Miss., on west side of building } \\
\text { at corner of Noody and Harris Streets, } \\
800 \text { feet southwest of Fortification }\end{array}$ & 289.6 & 281.8 \\
\hline $\begin{array}{l}\text { Street inside fairgrounds levee......... } \\
\text { Jackson, Miss., upstream side of Inter- } \\
\text { state Highway } 55 \text { left bank. } 200 \text { feet }\end{array}$ & 289.2 & 279.4 \\
\hline $\begin{array}{l}\text { left of main-channel bridge................. } \\
\text {--Downstream side of Interstate Highway } \\
55 \text { at left abutment of main-channel }\end{array}$ & 288.4 & 279.9 \\
\hline 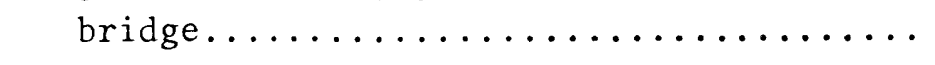 & $\mathrm{g} / 288.3$ & 279.3 \\
\hline
\end{tabular}




\begin{tabular}{|c|c|c|}
\hline Stream and location & $\begin{array}{l}\text { Distance } \\
\text { upstream } \\
\text { from mouth } \\
\text { (miles) }\end{array}$ & $\begin{array}{l}\text { Elevation ab } \\
\text { National geod } \\
\text { vertical dat } \\
\text { of } 1929 \\
\text { (feet) }\end{array}$ \\
\hline \multicolumn{3}{|l|}{ PEARL RIVER BASIN--Continued } \\
\hline Pearl River--Continued & & \\
\hline $\begin{array}{l}\text { Jackson, Miss., on Tombigbee Street, } \\
200 \text { feet east of Jefferson Street } \\
\text { across from northeast corner of } \\
\text { Mississippi Power \& Light } \\
\text { Company building inside fairgrounds }\end{array}$ & & \\
\hline $\begin{array}{l}\text { Jackson, Miss., downstream side of } \\
\text { Woodrow Wilson bridge at right }\end{array}$ & 288.2 & 279.4 \\
\hline 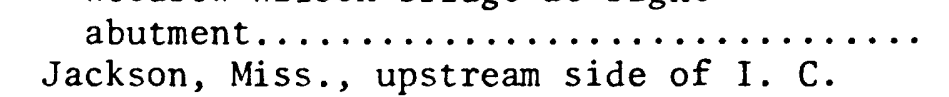 & $\underline{\mathrm{g}} / 287.6$ & 278.7 \\
\hline $\begin{array}{l}\text { Railroad, right bank.......................... } \\
\text {--Downstream side of I. C. Railroad }\end{array}$ & $\mathrm{g} / 287.2$ & 278.1 \\
\hline $\begin{array}{l}\text { at right end of bridge.................... } \\
\text { Jackson, Miss., upstream side of U.S. } \\
\text { Highway } 80 \text {, right bank, } 800 \text { feet right }\end{array}$ & $\underline{\mathrm{g}} / 287.2$ & 277.8 \\
\hline $\begin{array}{l}\text { Highway } 80 \text {, right bank, } 800 \text { feet right } \\
\text { of main-channel bridge................... } \\
\text { U.S. Geological Survey gaging station } \\
\text { (02486000) on left bank at downstream } \\
\text { side of bridge on U.S. Highway } 80 \text { at }\end{array}$ & $\underline{\mathrm{g}} / 287.0$ & 277.8 \\
\hline 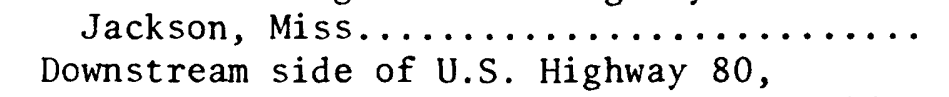 & 287.0 & 277.0 \\
\hline $\begin{array}{l}\text { right bank at end of main-channel bridge } \\
\text { Jackson, Miss., upstream side of Inter- } \\
\text { state Highway 20, right bank, } 800 \text { feet }\end{array}$ & 287.0 & 276.9 \\
\hline $\begin{array}{l}\text { right of river.............................. } \\
\text {--Downstream side of Interstate Highway } \\
20, \text { right bank, } 800 \text { feet right of main }\end{array}$ & $\underline{\mathrm{g} / 286.7}$ & 276.8 \\
\hline $\begin{array}{l}\text { Jackson, Miss., GMEO Railroad at } \\
\text { McDowel1 Road at abandoned landfill, } \\
\text { right bank, } 1000 \text { feet right of main }\end{array}$ & $\underline{g} / 286.6$ & 276.2 \\
\hline $\begin{array}{l}\text { channe } 1 \ldots \ldots \ldots \ldots \ldots \ldots \ldots \ldots \ldots \ldots \\
\text { Jackson, Miss., along GMEO Railroad } \\
\text { about } 300 \text { feet south and } 300 \text { feet west }\end{array}$ & 285.6 & 275.5 \\
\hline $\begin{array}{l}\text { Jackson, Miss., along GMEO Railroad, } 2000 \\
\text { feet north of Savannah Street inter- } \\
\text { change, right bank, } 1500 \text { feet right of } \\
\text { river at north levee of Wastewater }\end{array}$ & $\underline{3} / 28$ & 275.0 \\
\hline $\begin{array}{l}\text { Treatment plant } .1500 \text { feet south of Savannah Street inter- } \\
\text { change, } 400 \text { feet east of railroad at } \\
\text { south levee of Wastewater Treatment }\end{array}$ & 282.4 & 272.6 \\
\hline 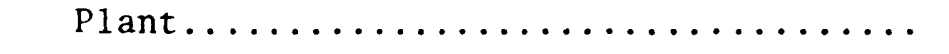 & 281.4 & 270.8 \\
\hline
\end{tabular}




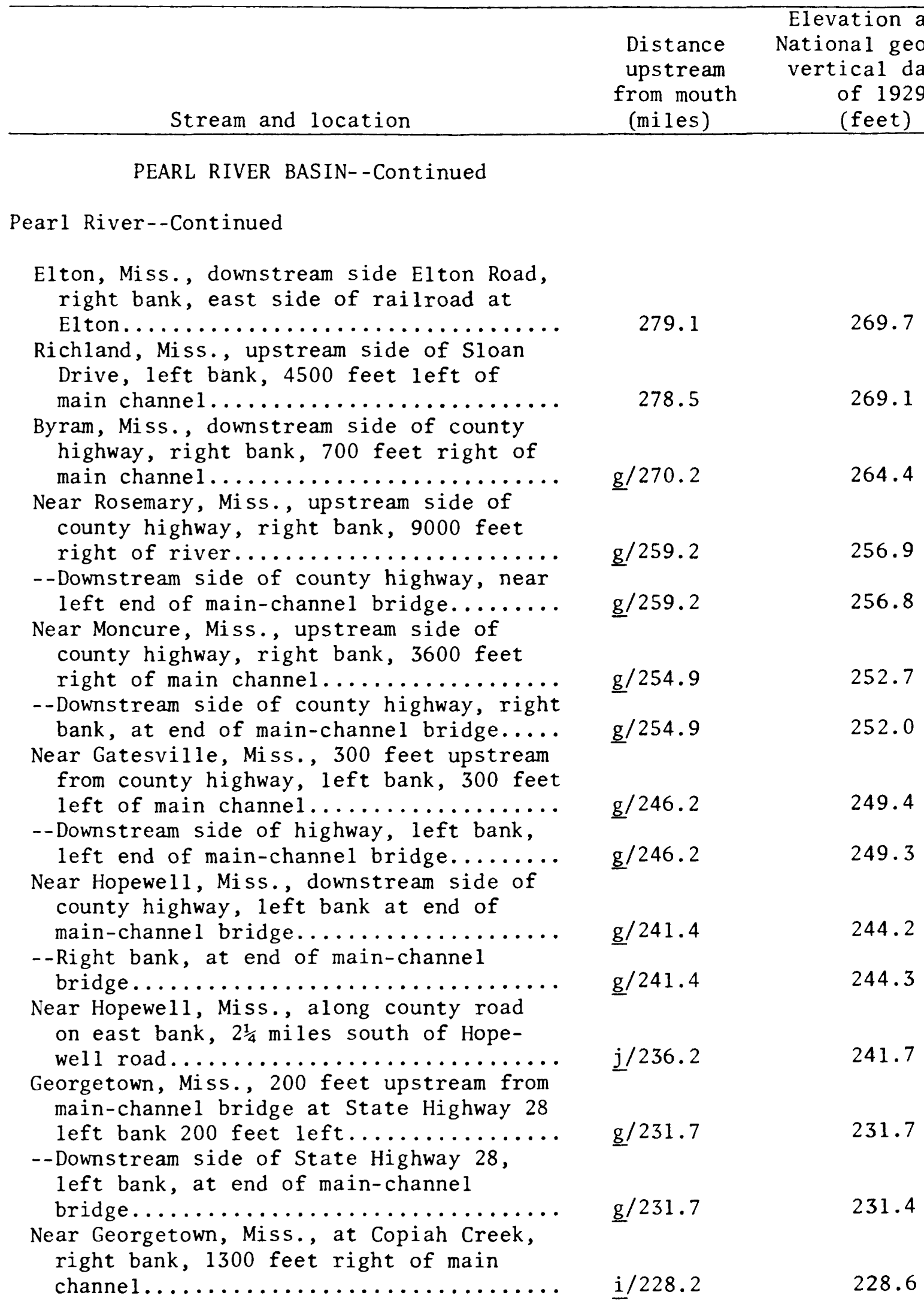

See footnotes at end of table. 


\begin{tabular}{ccc}
\hline & $\begin{array}{c}\text { Elevation above } \\
\text { upstream } \\
\text { Strom mouth } \\
\text { (miles) }\end{array}$ & $\begin{array}{c}\text { National geodetic } \\
\text { vertical datum } \\
\text { of 1929 } \\
\text { (feet) }\end{array}$ \\
\hline
\end{tabular}

PEARL RIVER BASIN--Continued

Pearl River--Continued

Near Rockport, Miss., at Keys Creek, right bank, 2100 feet right of main

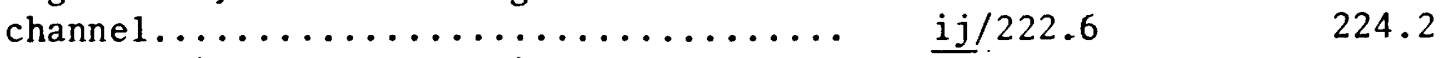

U.S. Geological Survey gaging station (02448000 discontinued) downstream side at right end of bridge on county highway near Rockport, Miss., (in abandoned gage house)...............

--Downstream left bank at end of bridge...

Near Rockport, Miss., at Dry Creek, left

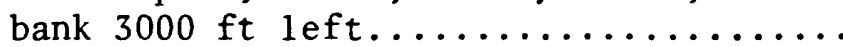

--At Pegies Creek, right bank, 2300 feet

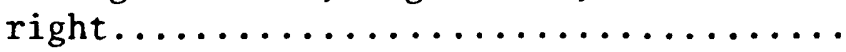

--At Saddlebags Creek, right bank, 600

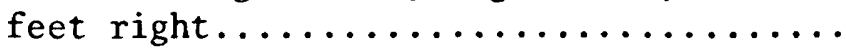

Near Ferguson, Miss., at St. Regis Paper Company pumping station, left bank.....

i $/ 204.8$

209.4

Wanilla, Miss., 7700 feet right of river, at State Highway 27 crossing of Bear

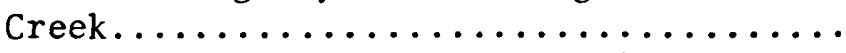

Near Rosella, Miss., upstream side of county highway, left bank, 800 feet

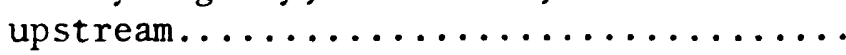

--Downstream side of county highway, under reference point near center of

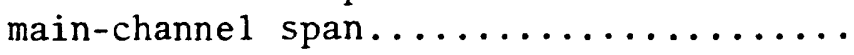

Near Monticel1o, Miss., at tributary, 1 mile north of intersection of U.S. Highway 84 and county highway, left

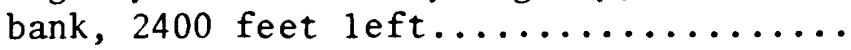

Monticello, Miss., at old bridge site, 1.1 miles upstream from U.S. Highway 84 ,

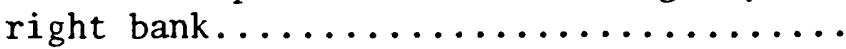

Montice1lo, Miss., upstream side U.S. Highway 84 , left bank, 700 feet 1 eft...

--400 feet downstream from U.S. Highway 84, left bank, 300 feet left..........

U.S. Geological Survey gaging station (02488500) downstream side of left pier of bridge on U.S. Highway 84 near

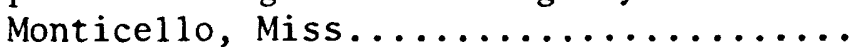




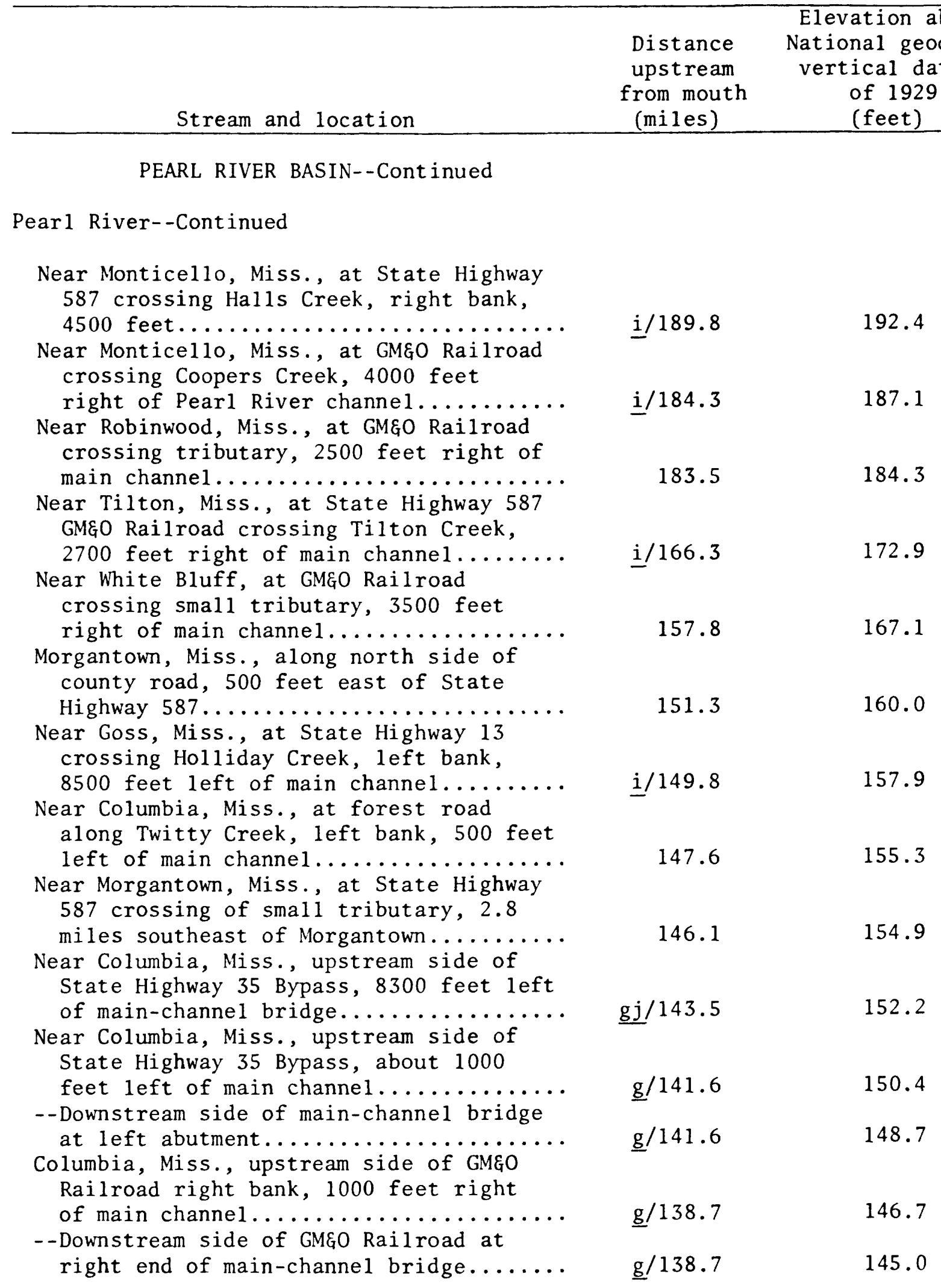

See footnotes at end of table. 


\begin{tabular}{|c|c|c|}
\hline Stream and location & $\begin{array}{l}\text { Distance } \\
\text { upstream } \\
\text { from mouth } \\
\text { (miles) }\end{array}$ & $\begin{array}{l}\text { Elevation above } \\
\text { National geodetic } \\
\text { vertical datum } \\
\text { of } 1929 \\
\text { (feet) }\end{array}$ \\
\hline
\end{tabular}

PEARL RIVER BASIN--Continued

Pearl River--Continued

Columbia, Miss., upstream side of U.S.

Highway 98, right bank about 500 feet

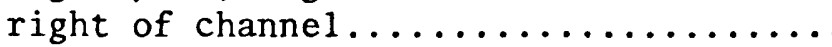

National Weather Service gaging station

$\begin{array}{ll}\mathrm{g} / 137.8 & 144.8\end{array}$

(02489000) downstream side of upstream

bridge on U.S. Highway 98 near

Columbia, Miss., wire-weight gage

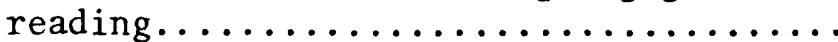

--Downstream side of U.S. Highway 98 at

right abutment of main-channel bridge...

Near Lampton, Miss., at State Highway

crossing of Upper Little Creek, 2.2

miles left of river, half a mile south

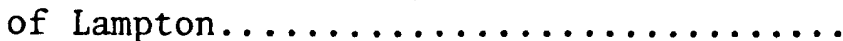

Near Hub, Miss., at State Highway 43

crossing of Lower Little Creek, 1.8

miles southwest of Hub, 1.2 miles left

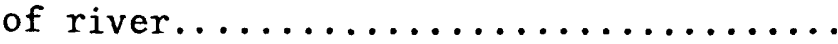

Near Sandy Hook, Miss., 1800 feet east of

railroad at upstream side of county

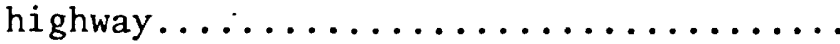

Near Marion-Pearl River County line, at

State Highway 43 crossing tributary,

left bank, 4000 feet left of main

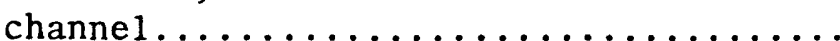

Near Bogalusa, La., gaging station near right bank at downstream side of State Highway 10, 2 miles east of Bogalusa....

Near Bogalusa, La., at Richardson's Landing on 5-inch tree, 300 feet upstream

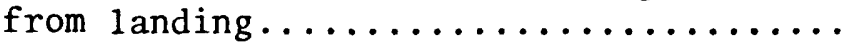

East Pearl River near Nicholson, downstream side Interstate Highway 59 near left downstream abutment.............

West Pearl River at Pearl River, La., on power pole 20 feet upstream from

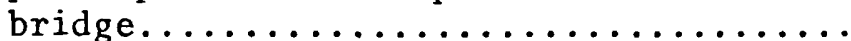

East Pearl River near Gainsville, Miss.,

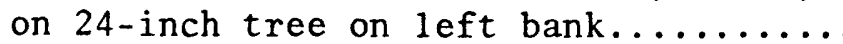

g/ 137.8

143.5

g/137.8

142.8

125.8

133.6

121.9

125.9

111.9

115.2

j/103.4

106.6

78.2

h/ 78.3

74.8

h/ 74.5

33.5

h/ 24.0

22.1

h/ 19.8

20.7

h/ 12.9

See footnotes at end of table. 


\begin{tabular}{|c|c|c|}
\hline Stream and location & $\begin{array}{l}\text { Distance } \\
\text { upstream } \\
\text { from mouth } \\
\text { (miles) }\end{array}$ & $\begin{array}{l}\text { Elevation a } \\
\text { National geo } \\
\text { vertical da } \\
\text { of } 1929 \\
\text { (feet) }\end{array}$ \\
\hline \multicolumn{3}{|l|}{ PEARL RIVER BASIN--Continued } \\
\hline \multicolumn{3}{|l|}{ Pear1 River--Continued } \\
\hline \multirow{2}{*}{\multicolumn{3}{|c|}{$\begin{array}{l}\text { West Pearl River at Davis Landing } \\
\text { transferred to power pole downstream } \\
\text { right bank end of boat ramp........... } \\
\text { East Pearl River near S1idell, La., down- } \\
\text { stream side Interstate Highway } 10 \text {, near }\end{array}$}} \\
\hline & & \\
\hline $\begin{array}{l}\text { East Pearl River near Pearlington, Miss., } \\
\text { at U.S. Highway } 90 \ldots \ldots \ldots \ldots \ldots \ldots \ldots \ldots \ldots \\
\text { West Pearl River near Pearlington, Miss., } \\
\text { at U.S. Highway } 80 \text { near downstream }\end{array}$ & 9.0 & $\underline{\mathrm{h} /} \quad 5.0$ \\
\hline 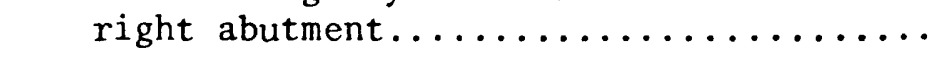 & 7.9 & $\underline{\mathrm{h} /} \quad 5.6$ \\
\hline \multicolumn{3}{|l|}{ Pearl River tributary streams: } \\
\hline \multicolumn{3}{|l|}{ Tallahaga Creek: } \\
\hline \multicolumn{3}{|l|}{$\begin{array}{l}\text { Near Louisville, Miss., upstream side } \\
\text { of State Highway 25, left bank, }\end{array}$} \\
\hline 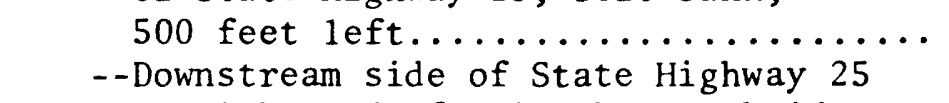 & $\underline{g} / 15.9$ & 480.5 \\
\hline $\begin{array}{l}\text { at right end of main-channel bridge... } \\
\text { Near Noxapater, Miss., upstream side of } \\
\text { State Highway } 15 \text {, right bank, } 1450\end{array}$ & g/ 15.9 & 478.2 \\
\hline 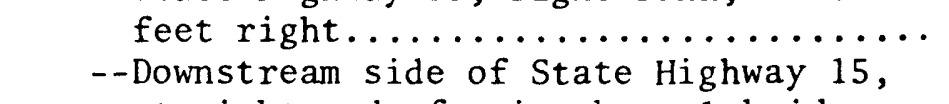 & g/ 9.7 & 445.1 \\
\hline at right end of main-channe 1 bridge... & g/ $\quad 9.7$ & 443.7 \\
\hline
\end{tabular}

Noxapater Creek:

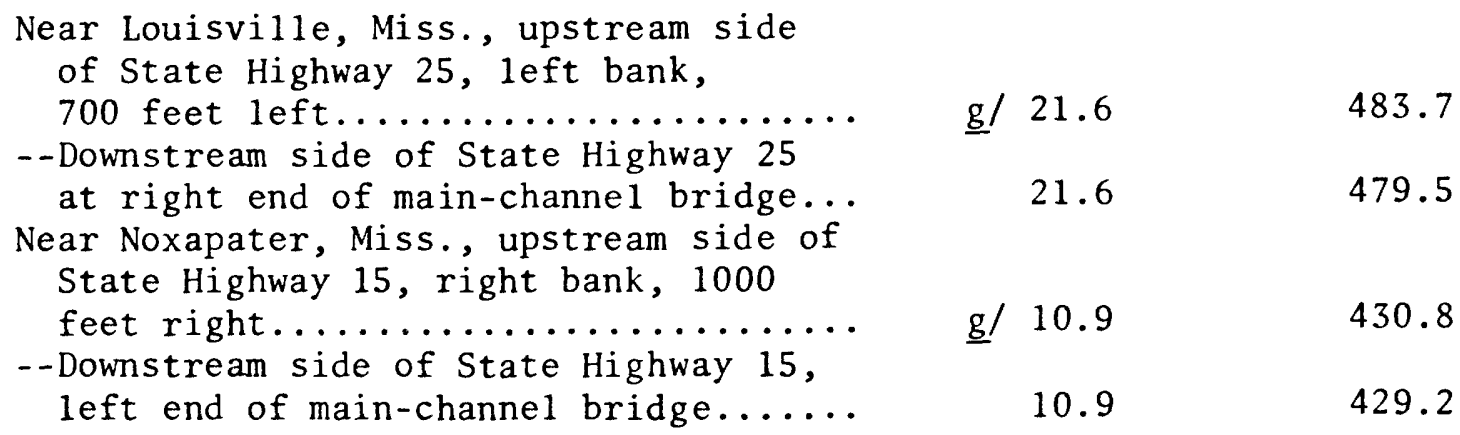

See footnotes at end of table. 
TABLE 5.-Flood-crest stages-Continued

\begin{tabular}{ccc}
\hline & Elevation above \\
& Distance & National geodetic \\
& upstream & vertical datum \\
Stream and location & from mouth & of 1929 \\
(miles) & (feet) \\
\hline
\end{tabular}

PEARL RIVER BASIN--Continued

Pearl River tributary streams--Continued

Lobutcha Creek:

Near Zama, Miss., upstream side of county highway (Massy Crossing), left bank, 2900 feet left of main channel..

--Downstream side of county highway right bank, 150 feet right of main-

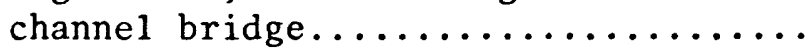

Zama, Miss., upstream side of State Highway 19, right bank, 75 feet right of main-channel bridge.............

--Downstream side of State Highway 19, 500 feet right of right end of main-

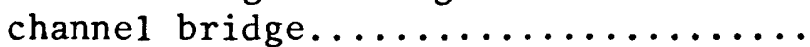
Near Renfroe, Miss., upstream side of State Highway 25, right bank, 500 feet right of main channel.............

--Downstream side of State Highway 25 at right end of main-channel bridge...

37.1

424.2

37.1

422.8

31.8

415.2

31.8

414.2

16.7

385.9

16.7

383.9

Near Carthage, Miss., upstream side of washed out county highway (Scotts Crossing), left bank, 50 feet right of main channel.................. Near Carthage, Miss., upstream side of State Highway 16, left bank, 4400 feet left of main channel.......... --Downstream side of State Highway 16 at right end of main-channel bridge...

Yockanookany River:

Ackerman, Miss., upstream side of State Highway 15, left bank, 100 feet left of tributary and 200 feet south of Illinois Central Gulf Rail-

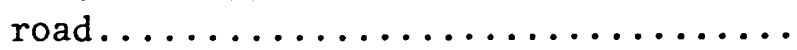
Near Fentress, Miss., upstream side of county road, left bank, 650 feet

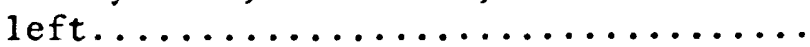

--Downstream side of county road at right end of main-channel bridge.....
515.6

$\begin{array}{ll}71.1 & 488.1\end{array}$

$\begin{array}{ll}71.1 & 485.3\end{array}$

See footnotes at end of table. 


\begin{tabular}{|c|c|c|}
\hline Stream and location & $\begin{array}{l}\text { Distance } \\
\text { upstream } \\
\text { from mouth } \\
\text { (miles) }\end{array}$ & $\begin{array}{c}\text { Elevation ab } \\
\text { National geod } \\
\text { vertical dat } \\
\text { of } 1929 \\
\text { (feet) }\end{array}$ \\
\hline \multicolumn{3}{|l|}{ PEARL RIVER BASIN--Continued } \\
\hline Pearl River tributary streams--Continued & & \\
\hline \multicolumn{3}{|l|}{ Yockanookany River--Continued } \\
\hline $\begin{array}{l}\text { Weir, Miss., upstream side of county } \\
\text { road, right bank, } 900 \text { feet right...... } \\
\text {--Downstream side of road at left end }\end{array}$ & 66.7 & 460.4 \\
\hline $\begin{array}{l}\text { of main-channel bridge............. } \\
\text { Weir, Miss., upstream side of State } \\
\text { Highway } 413 \text {, right bank, } 50 \text { feet }\end{array}$ & 66.7 & 459.8 \\
\hline --Downstream side of State Highway 413 & 66.0 & 458.3 \\
\hline $\begin{array}{l}\text { at right end of main-channel bridge... } \\
\text { McCool, Miss., upstream side of State } \\
\text { Highway 411, left bank, } 1200 \text { feet }\end{array}$ & 66.0 & 457.0 \\
\hline 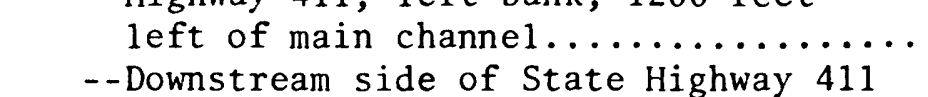 & 60.1 & 437.9 \\
\hline $\begin{array}{l}\text { at left end of main-channel bridge.... } \\
\text { Near Ethel, Miss., upstream side of } \\
\text { county highway east of Ethel, right }\end{array}$ & 60.1 & 437.0 \\
\hline $\begin{array}{l}\text { bank, } 1400 \text { feet right................. } \\
- \text {-Downstream side of county road, } 100\end{array}$ & 51.6 & 422.3 \\
\hline $\begin{array}{l}\text { feet right of main-channel bridge..... } \\
\text { Near Ethel, Miss., upstream side of }\end{array}$ & 51.6 & 421.1 \\
\hline $\begin{array}{l}\text { county highway, south of Ethel, left } \\
\text { bank, } 1000 \text { feet left......................... } \\
\text {--Downstream side of county highway, }\end{array}$ & 50.6 & 418.8 \\
\hline $\begin{array}{l}700 \text { feet left of main-channel bridge.. } \\
\text { Near Kosciusko, Miss., upstream side of } \\
\text { county highway (Munson Crossing), } 3 \\
\text { miles east of Kosciusko, right bank, }\end{array}$ & 50.6 & 416.7 \\
\hline $\begin{array}{l}2200 \text { feet right........................... } \\
\text {--Downstream side of county highway, }\end{array}$ & 45.3 & 402.4 \\
\hline $\begin{array}{l}\text { right bank, } 3600 \text { feet right........... } \\
\text { Near Kosciusko, Miss., } 400 \text { feet upstream } \\
\text { from left end of bridge at State High- } \\
\text { way } 35 \text {, and about } 300 \text { feet left of }\end{array}$ & 45.3 & 402.0 \\
\hline --Downstream side of State Highway 35 & 41.9 & 398.9 \\
\hline $\begin{array}{l}\text { at left end of bridge................ } \\
\text { U.S. Geological Survey gaging station } \\
(02484000) \text { left bank on downstream } \\
\text { side of bridge on State Highway } 35\end{array}$ & 41.9 & 397.0 \\
\hline near Kosciusko, Miss........... & 41.9 & 397.4 \\
\hline
\end{tabular}

See footnotes at end of table. 
TABLE 5.-Flood-crest stages-Continued

\begin{tabular}{ccc}
\hline & Elevation above \\
& $\begin{array}{c}\text { Distance } \\
\text { upstream } \\
\text { from mouth } \\
\text { Stream and location }\end{array}$ & $\begin{array}{c}\text { National geodetic } \\
\text { vertical datum } \\
\text { of 1929 }\end{array}$ \\
\hline
\end{tabular}

PEARL RIVER BASIN--Continued

Pearl River tributary streams--Continued

Yockanookany River--Continued

Near Kosciusko, Miss., at crest-stage gage site 5-C, right bank, 600 feet

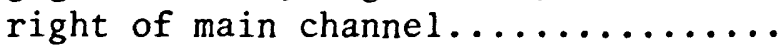

--At crest-stage gage site 4-D, right

j/ $40.9 \quad 394.0$

bank, 1000 feet right of main

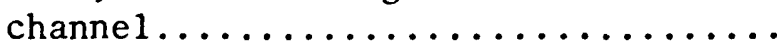

- -At crest-stage gage site 3-D, right bank, 1800 feet right of main

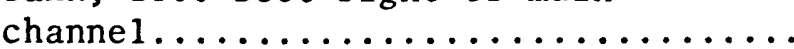

--At crest-stage gage site 2-D, right bank, 3200 feet right of main channel.

--At crest-stage gage site 1-D, right bank, 2800 feet right of main channel.

Near Thomastown, Miss., downstream side of county highway, 4 miles northeast of Thomastown, right bank, 2000 feet

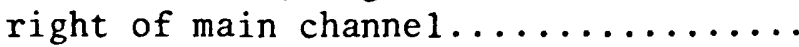
389.1

Near Thomastown, Miss., upstream side of State Highway 429, right bank, 800 feet right of main channel....... --Downstream side of State Highway 429

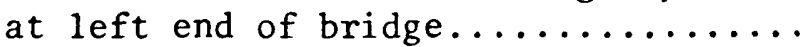

Near Ofahoma, Miss., upstream side of county highway (Red Dog Road), 8.6 river miles upstream from State Highway 16,500 feet right of main-

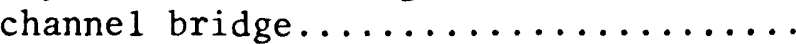

--Downstream side of main-channel bridge at left abutment............ Near Ofahoma, Miss., upstream side of State Highway 16, right bank, 5800 feet right of main channel..........

361.2

22.2

359.2

U.S. Geological Survey gaging station (02484500) near center of span on downstream side of bridge on State Highway 16 near Ofahoma, Miss........

--Downstream side of State Highway 16 near right abutment............... 


\begin{tabular}{ccc} 
& Elevation above \\
Stream and location & $\begin{array}{c}\text { Distance } \\
\text { upstream } \\
\text { from mouth } \\
\text { (miles) }\end{array}$ & $\begin{array}{c}\text { vertical datum } \\
\text { of } 1929\end{array}$ \\
\hline
\end{tabular}

\section{BIG BLACK RIVER BASIN}

\section{Big Black River:}

Near Tomnolan, Miss., downstream side of

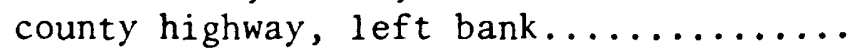

Near Stewart, Miss., upstream side of county highway, right bank, about 90 feet from main-channel bridge.......

Near Kilmichael, Miss., downstream side of State Highway 413, right bank.......

Near Vaiden, Miss., downstream State Highway 35, right bank, 1300 feet right of main-channel bridge.......... West, Miss., upstream side of State Highway 19, right bank, 1700 feet right of main channel.....................

--Downstream side of State Highway 19 at right end of main-channel bridge.....

U.S. Geological Survey gaging station (07289350) downstream side of left pier of bridge on State Highway 19

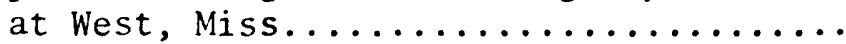

Durant, Miss., upstream side of State Highway 12, right bank, 6000 feet

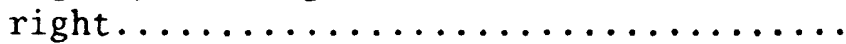

--Downstream side of State Highway 12, at $1 \mathrm{eft}$ end of main-channel bridge...... Near Goodman, Miss., upstream side of State Highway 14, right bank, 500 feet right of main channel...........

--Upstream side of State Highway 14, right bank, 6700 feet right of main-

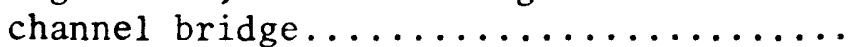

--Downstream side of State Highway 14 at left end of main-channel bridge.........

263.6

h/ 341.9

258.5

250.8

h/ 327.7

250.8

225.2

$\underline{\mathrm{h}} / 288.2$

209.0

274.6

209.0

273.6

209.0

274.0

190.8

255.1

190.8

253.0

175.2

236.7

176.0

237.0

176.0

235.1

U.S. Geological Survey gaging station (07289500 discontinued) downstream side of bridge on old (abandoned) U.S. Highway 51 at Pickens, Miss., 220 feet right of bridge.............

Pickens, Miss., upstream side of U.S. Highway 51, right bank, 3200 feet

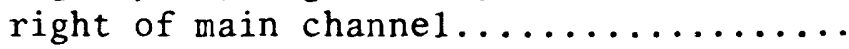

162.7

h/219.9

161.2 
TABLE 5.-Flood-crest stages-Continued

\begin{tabular}{|c|c|c|}
\hline Stream and location & $\begin{array}{l}\text { Distance } \\
\text { upstream } \\
\text { from mouth } \\
\text { (miles) }\end{array}$ & $\begin{array}{c}\text { Elevation above } \\
\text { National geodetic } \\
\text { vertical datum } \\
\text { of } 1929 \\
\text { (feet) }\end{array}$ \\
\hline
\end{tabular}

BIG BLACK RIVER BASIN--Continued

Big Black River--Continued

--Downstream side of U.S. Highway

51 at $1 \mathrm{eft}$ end of main-channel

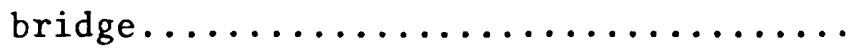

Way, Miss., upstream side of Illinois

Central Railroad, left bank, 4500

feet left of main channel.............

--Downstream side of Illinois Central

Railroad, left bank, 4500 feet left

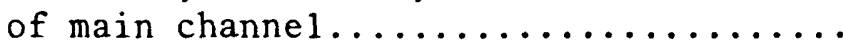

Near Way, Miss., upstream side of Inter-

state Highway 55, right bank, 3800 feet

right of main channe1..............

--Downstream side of Interstate Highway 55

at left end of main-channel bridge......

Near Canton, Miss., upstream side of State Highway 16, right bank, 3000 feet right of main-channel bridge..............

--Downstream side of State Highway 16 at right end of main-channel bridge.......

161.2

217.6

Near Bentonia, Miss., upstream side of U.S. Highway 49, right bank, 1000 feet right of main-channel bridge.........

--Downstream side of U.S. Highway 49 at right end of main-channel bridge.......

U.S. Army Corps of Engineers crest-stage gage (07289730) on downstream side of left pier of bridge on U.S. Highway 49 near Bentonia, Miss.................

Near Bentonia, Miss., at county highway at Turkeyfoot Branch, right bank,

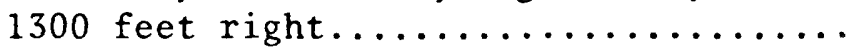

Near Nevada, Miss., at abandoned Coxs

Ferry Road, right bank, 4000 feet

right of main channel..............

Near Nevada, Miss., at abandoned Coxs

Ferry Road, left bank, 3500 feet left

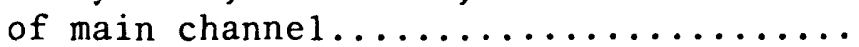

145.8

201.3

145.8

200.4

144.5

199.6

144.5

198.4

139.6

194.5

139.6

192.9

106.0

162.9

106.0

161.6

Near Nevada, Miss., at county highway near King Solomon Church, right bank,

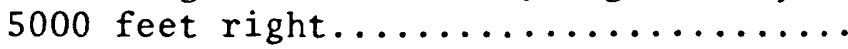




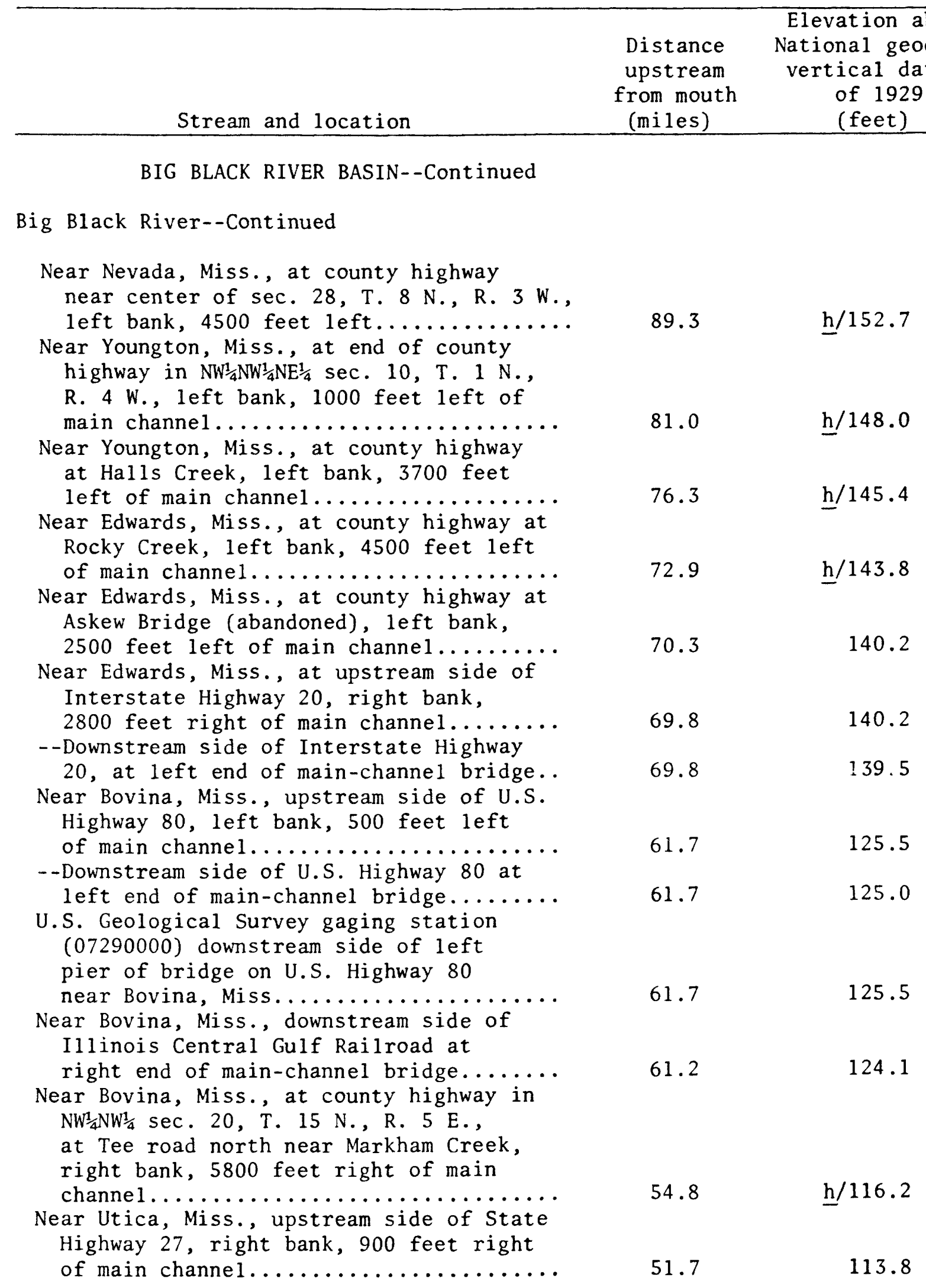

See footnotes at end of table. 


\begin{tabular}{ccc}
\hline & Elevation above \\
& Distance & National geodetic \\
Stream and location & upstream & vertical datum \\
from mouth & of 1929 \\
(miles) & (feet) \\
\hline
\end{tabular}

BIG BLACK RIVER BASIN--Continued

Big Black River--Continued

--Downstream side of State Highway 27, at right end of main-channel bridge.....

51.7

112.4

Near Utica, Miss., upstream side of

Fishers Bridge, right bank, 6000 feet

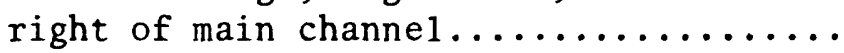

--Downstream side of Fishers Bridge, 50

feet downstream from left end of main-

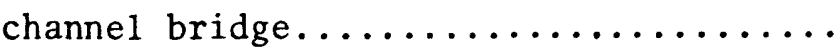

Near Rocky Springs, Miss., at end of county highway in $\mathrm{NW}^{\frac{1}{4}} \mathrm{SE}^{\frac{1}{4}} \mathrm{sec} .37$,

T. 14 N., R. 4 E., left bank, 100 feet

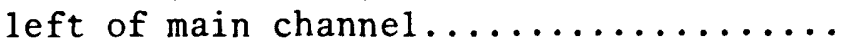

Near Port Gibson, Miss., at Hankinson

Bridge, left bank, 100 feet left of

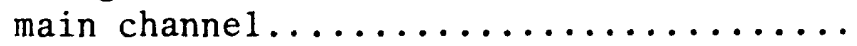

--Right bank, 1300 feet right of main

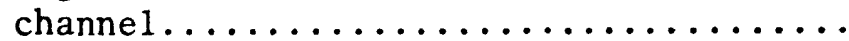

Near Port Gibson, Miss., upstream side of

left abutment of U.S. Highway 61

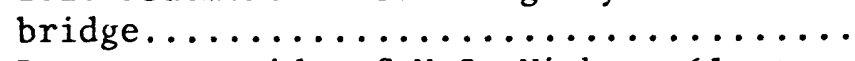

--Downstream side of U.S. Highway 61 at

right abutment of main-channel bridge...

Near Port Gibson, Miss., at Karnac Lake

Ferry, left bank, 200 feet left of

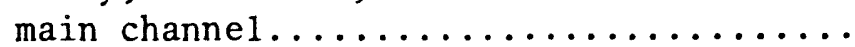

Big Black River tributary streams:

\section{Bear Creek:}

Near Gluckstadt, Miss., downstream side of U.S. Highway 51, right bank.......

Near Canton, Miss., upstream side of U.S. Highway 51, left bank, 1000 feet

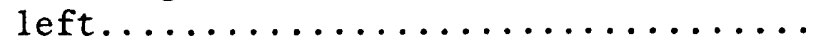

- -Downstream side of U.S. Highway 51 , at right end of main-channel bridge... Canton, Miss., upstream side of State Highway 22, left bank, 200 feet left..

--Downstream side of State Highway 22 at right end of main-channel bridge... g/ 20.0

h/ 99.4

23.0

h/ 94.3

23.0

93.8

16.0

h/ 90.6

16.0

90.3

8.3

h/ 87.4 g/ $12.0 \quad 224.0$

g/ $12.0 \quad 222.9$

g/ $10.0 \quad 217.4$

g/ $10.0 \quad 216.4$

See footnotes at end of table. 
TABLE 5.-Flood-crest stages-Continued

\begin{tabular}{ccc}
\hline & Elevation above \\
& Distance & National geodetic \\
Stream and location & upstream & vertical datum \\
from mouth & of 1929 \\
\hline
\end{tabular}

BIG BLACK RIVER BASIN--Continued

Big Black River tributary streams--Continued

Bear Creek--Continued

Near Canton, Miss., upstream side of Interstate Highway 55, left bank, 200 feet left................ g/ $9.3 \quad 215.1$

--Downstream side of Interstate Highway 55 at right end of main-channel

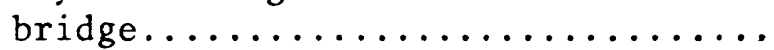

Near Canton, Miss., upstream side of county highway (old Yazoo City Road) left bank, 400 feet left.............

--Downstream side of county highway at right end of main-channel bridge.....

g/ 9.3

g/ 3.4

197.9

g/ 3.4

a Occurred Apri1 16, 1979.

b Occurred April 17, 1979.

c Occurred April 18, 1979.

d Possible meander effect.

e Occurred April 22, 1979.

$f$ Sediment range lines, U.S. Army Corps of Engineers sediment surveys.

$g$ River mile at main channel bridge.

$h$ Furnished by U.S. Army Corps of Engineers (rounded to nearest 0.1 foot).

$i$ River mile at mouth of tributary.

j River mile assigned by U.S. Geological Survey. 
TABLES

TABLE 6.-Streamflow velocities, Alabama River near Montgomery, Ala, 02420000, April 12-20, 1979

\begin{tabular}{|c|c|c|c|c|c|}
\hline Date & Time & $\begin{array}{l}\text { Point } \\
\text { velocity } \\
(\mathrm{ft} / \mathrm{s})\end{array}$ & $\begin{array}{l}\text { Average } \\
\text { velocity } \\
(\mathrm{ft} / \mathrm{s})\end{array}$ & $\begin{array}{l}\text { Stage } \\
(\mathrm{ft})\end{array}$ & $\begin{array}{c}\text { Discharge } \\
\left(\mathrm{ft}^{3} / \mathrm{s}\right)\end{array}$ \\
\hline $4-12$ & 2400 & 3.32 & 3.46 & 32.10 & 83,000 \\
\hline $4-13$ & 0600 & 4.16 & 4.15 & 34.60 & 108,000 \\
\hline $4-13$ & 1200 & 4.73 & 4.59 & 36.90 & 128,000 \\
\hline $4-13$ & 1800 & 5.00 & 4.76 & 39.40 & 142,000 \\
\hline $4-13$ & 2400 & 5.30 & 4.96 & 42.00 & 159,000 \\
\hline $4-14$ & 0600 & 5.60 & 5.15 & 45.30 & 183,000 \\
\hline $4-14$ & 1200 & 5.81 & 5.29 & 48.10 & 210,000 \\
\hline $4-14$ & 1800 & 5.92 & 5.36 & 50.00 & 229,000 \\
\hline $4-14$ & 2400 & 6.00 & 5.40 & 51.70 & 245,000 \\
\hline $4-15$ & 0600 & 6.02 & 5.42 & 52.63 & 254,000 \\
\hline $4-15$ & 1200 & 5.92 & 5.36 & 53.37 & 258,000 \\
\hline $4-15$ & 1800 & 5.85 & 5.32 & 53.87 & 260,000 \\
\hline $4-15$ & 2400 & 5.74 & 5.24 & 54.20 & 259,000 \\
\hline $4-16$ & 0600 & 5.66 & 5.19 & 54.40 & $258, \cap 00$ \\
\hline $4-16$ & 1200 & 5.50 & 5.09 & 54.50 & 254,000 \\
\hline $4-16$ & 1800 & 5.40 & 5.02 & 54.41 & 250,000 \\
\hline $4-16$ & 2400 & 5.20 & 4.87 & 54.22 & 241,000 \\
\hline $4-17$ & 0600 & 5.10 & 4.8 .3 & 53.82 & 236,000 \\
\hline $4-17$ & 1200 & 5.00 & 4.76 & 53.36 & 229,000 \\
\hline $4-17$ & 1800 & 4.84 & 4.66 & 52.92 & 221,000 \\
\hline $4-17$ & 2400 & 4.72 & 4.58 & 52.29 & 212,000 \\
\hline $4-18$ & 0600 & 4.60 & 4.50 & 51.70 & 204,000 \\
\hline $4-18$ & 1200 & 4.50 & 4.44 & 51.00 & 197,000 \\
\hline $4-18$ & 1800 & 4.48 & 4.43 & 50.00 & 189,000 \\
\hline $4-18$ & 2400 & 4.40 & 4.37 & 48.99 & 180,000 \\
\hline $4-19$ & 0600 & 4.30 & 4.31 & 48.00 & 170,000 \\
\hline $4-19$ & 1200 & 4.28 & 4.30 & 47.08 & 163,000 \\
\hline $4-19$ & $18 \cap 0$ & 4.26 & 4.28 & 46.02 & 158,000 \\
\hline $4-19$ & 2400 & 4.25 & 4.28 & 45.01 & 151,000 \\
\hline $4-20$ & 0600 & 4.20 & 4.24 & 43.80 & 143,000 \\
\hline $4-20$ & 1200 & 4.18 & 4.23 & 42.75 & 138,000 \\
\hline $4-20$ & 1800 & 4.12 & 4.19 & 41.60 & 133,000 \\
\hline $4-20$ & 2400 & 4.10 & 4.18 & $40.4 n$ & 128,000 \\
\hline
\end{tabular}

*From continuous velocity meter at stage of 15 feet, about 25 feet above stream bottom, at upstream end of center bridge pier on U.S. Highway 31 near Montgomery. 
TABLE 7.-Gage height, discharge, and accumulated runoff, flood of April 1979

[Includes data for flood of March 1979, where significant. Gage height, in feet; discharge, in cubic feet per second; accumulated runoff, in inches] Site 02400100, Terrapin Creek at Ellisville, Ala.

[Maximum discharge occurred on March 4, 1979]

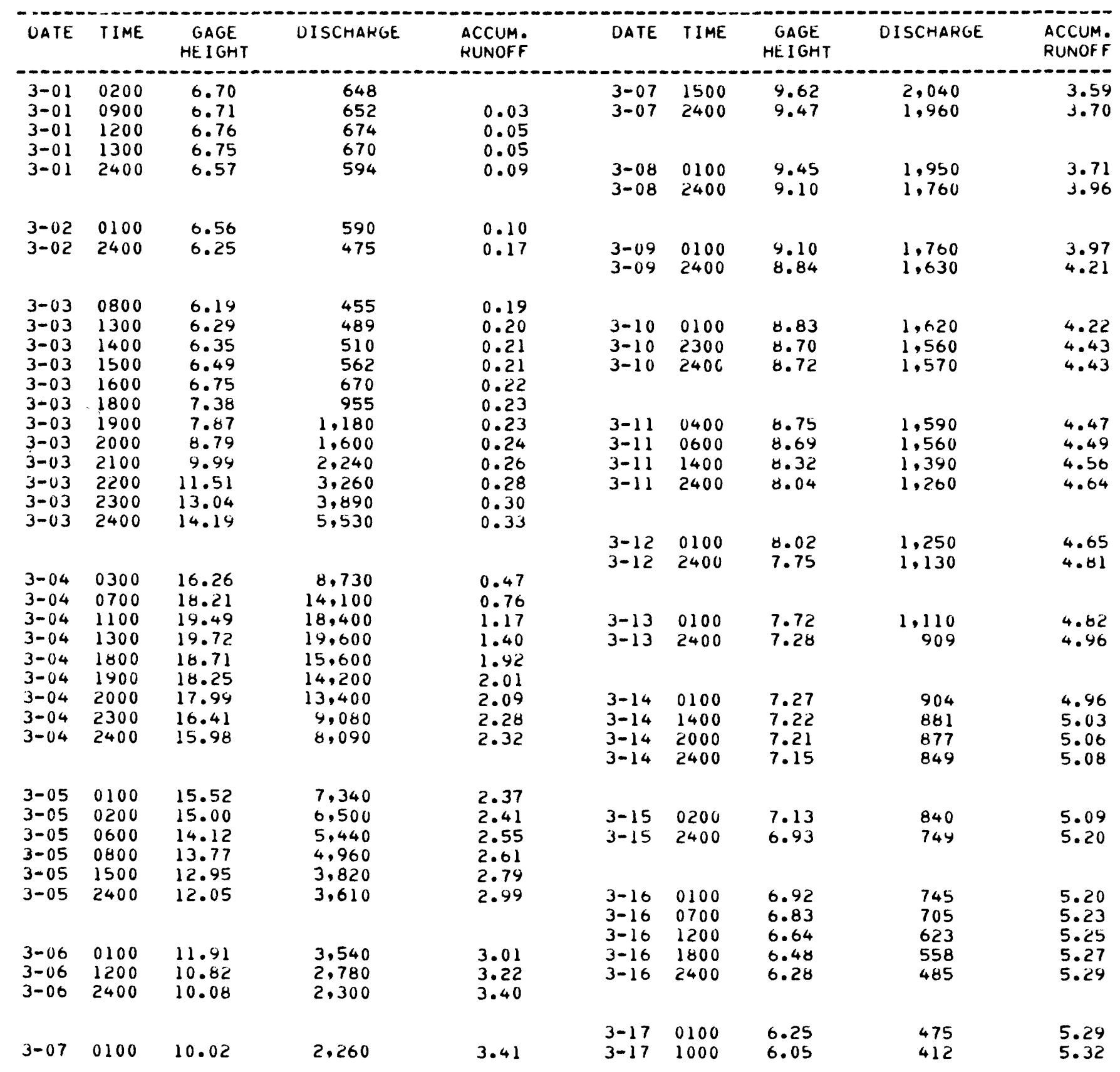


TABLE 7.-Gage height, discharge, and accumulated runoff, flood of April 1979-Continued 02400100, Terrapin Creek at Ellisville, Ala-Continued

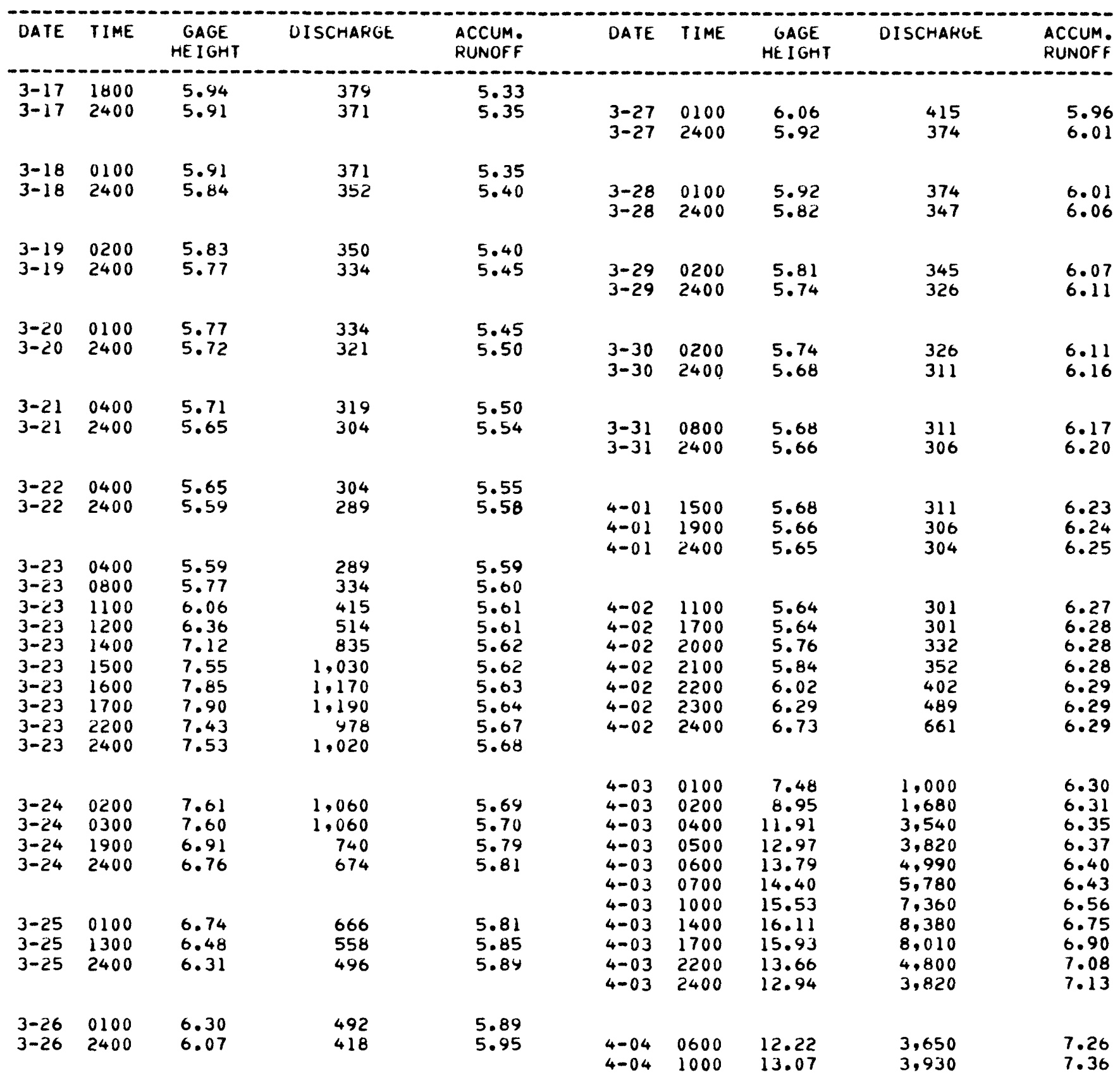


TABLE 7.-Gage height, discharge, and accumulated runoff, flood of April 1979-Continued 0240100, Terrapin Creek at Ellisville, Ala.-Continued

\begin{tabular}{|c|c|c|c|c|c|c|c|c|c|}
\hline DATE & TIME & $\begin{array}{c}\text { GAGE } \\
\text { HE I GHT }\end{array}$ & DISCHARGE & $\begin{array}{l}\text { ACCUM. } \\
\text { RUNOFF }\end{array}$ & DATE & TIME & $\begin{array}{l}\text { GAGE } \\
\text { HE IGHT }\end{array}$ & DISCHARGE & $\begin{array}{l}\text { ACCUM. } \\
\text { RUNOFF }\end{array}$ \\
\hline $\begin{array}{l}4-04 \\
4-04 \\
4-04 \\
4-04 \\
4-04 \\
4-04\end{array}$ & $\begin{array}{l}1200 \\
1400 \\
1700 \\
1800 \\
2300 \\
2400\end{array}$ & $\begin{array}{l}13.47 \\
13.57 \\
13.21 \\
12.98 \\
11.83 \\
11.64\end{array}$ & $\begin{array}{l}4,520 \\
4,670 \\
4,140 \\
3,830 \\
3,480 \\
3,350\end{array}$ & $\begin{array}{l}7.41 \\
7.46 \\
7.54 \\
7.57 \\
7.68 \\
7.70\end{array}$ & $\begin{array}{l}4-13 \\
4-13 \\
4-13 \\
4-13 \\
4-13\end{array}$ & $\begin{array}{l}0400 \\
1600 \\
2000 \\
2200 \\
2400\end{array}$ & $\begin{array}{l}17.68 \\
17.19 \\
17.26 \\
17.12 \\
16.72\end{array}$ & $\begin{array}{r}12,400 \\
11,000 \\
11,300 \\
10,800 \\
9,810\end{array}$ & $\begin{array}{l}10.29 \\
10.77 \\
11.04 \\
11.18 \\
11.30\end{array}$ \\
\hline $\begin{array}{l}4-05 \\
4-05 \\
4-05 \\
4-05\end{array}$ & $\begin{array}{l}0100 \\
0600 \\
1900 \\
2400\end{array}$ & $\begin{array}{r}11.46 \\
10.80 \\
9.93 \\
9.67\end{array}$ & $\begin{array}{l}3,220 \\
2,770 \\
2,210 \\
2,070\end{array}$ & $\begin{array}{l}7.71 \\
7.80 \\
7.99 \\
8.00\end{array}$ & $\begin{array}{l}4-14 \\
4-14 \\
4-14 \\
4-14 \\
4-14 \\
4-14\end{array}$ & $\begin{array}{l}0100 \\
0400 \\
0600 \\
1100 \\
1800 \\
2400\end{array}$ & $\begin{array}{l}16.43 \\
15.32 \\
14.68 \\
13.73 \\
12.93 \\
12.39\end{array}$ & $\begin{array}{l}9,130 \\
7,020 \\
6,120 \\
4,900 \\
3,810 \\
3,690\end{array}$ & $\begin{array}{l}11.35 \\
11.49 \\
11.56 \\
11.72 \\
11.90 \\
12.04\end{array}$ \\
\hline $\begin{array}{l}4-06 \\
4-06 \\
4-07 \\
4-07\end{array}$ & $\begin{array}{l}0100 \\
2400\end{array}$ & $\begin{array}{l}9.00 \\
8.63\end{array}$ & $\begin{array}{l}1,700 \\
1,530\end{array}$ & $\begin{array}{l}8.34 \\
8.56\end{array}$ & $\begin{array}{l}4-15 \\
4-15 \\
4-15 \\
4-15\end{array}$ & $\begin{array}{l}0100 \\
0700 \\
2100 \\
2400\end{array}$ & $\begin{array}{l}12.32 \\
11.81 \\
10.51 \\
10.30\end{array}$ & $\begin{array}{l}3,670 \\
3,470 \\
2,580 \\
2,450\end{array}$ & $\begin{array}{l}12.06 \\
12.19 \\
12.44 \\
12.48\end{array}$ \\
\hline $\begin{array}{l}4-08 \\
4-08 \\
4-08\end{array}$ & $\begin{array}{l}0100 \\
2200 \\
2400\end{array}$ & $\begin{array}{l}8.62 \\
8.41 \\
8.46\end{array}$ & $\begin{array}{l}1,530 \\
1,430 \\
1,450\end{array}$ & $\begin{array}{l}8.57 \\
8.75 \\
8.77\end{array}$ & $\begin{array}{l}4-16 \\
4-16 \\
4-16\end{array}$ & $\begin{array}{l}0100 \\
2200 \\
2400\end{array}$ & $\begin{array}{r}10.24 \\
9.52 \\
9.49\end{array}$ & $\begin{array}{l}2,410 \\
1,990 \\
1,970\end{array}$ & $\begin{array}{l}12.50 \\
12.77 \\
12.79\end{array}$ \\
\hline $\begin{array}{l}4-09 \\
4-09\end{array}$ & $\begin{array}{l}0100 \\
0200\end{array}$ & $\begin{array}{l}8.58 \\
8.92\end{array}$ & $\begin{array}{l}1,510 \\
1,660\end{array}$ & $\begin{array}{l}8.78 \\
8.74\end{array}$ & $\begin{array}{l}4-17 \\
4-17\end{array}$ & $\begin{array}{l}0100 \\
2400\end{array}$ & $\begin{array}{l}9.47 \\
9.15\end{array}$ & $\begin{array}{l}1,960 \\
1,780\end{array}$ & $\begin{array}{l}12.81 \\
13.06\end{array}$ \\
\hline $\begin{array}{l}4-09 \\
4-09 \\
4-09 \\
4-09\end{array}$ & $\begin{array}{l}0400 \\
0500 \\
1200 \\
2400\end{array}$ & $\begin{array}{l}9.68 \\
9.80 \\
8.81 \\
8.02\end{array}$ & $\begin{array}{l}2,070 \\
2,140 \\
1,610 \\
1,250\end{array}$ & $\begin{array}{l}8.81 \\
8.83 \\
8.90 \\
9.01\end{array}$ & $\begin{array}{l}4-18 \\
4-18\end{array}$ & $\begin{array}{l}0100 \\
2400\end{array}$ & $\begin{array}{l}9.14 \\
8.88\end{array}$ & $\begin{array}{l}1,780 \\
1,640\end{array}$ & $\begin{array}{l}13.07 \\
13.31\end{array}$ \\
\hline $\begin{array}{l}4-10 \\
4-10 \\
4-10\end{array}$ & $\begin{array}{l}0100 \\
1300 \\
2400\end{array}$ & $\begin{array}{l}7.97 \\
7.57 \\
7.35\end{array}$ & $\begin{array}{r}1,230 \\
1,040 \\
941\end{array}$ & $\begin{array}{l}9.01 \\
9.09 \\
9.16\end{array}$ & $\begin{array}{l}4-19 \\
4-19\end{array}$ & $\begin{array}{l}0100 \\
2400\end{array}$ & $\begin{array}{l}8.87 \\
8.63\end{array}$ & $\begin{array}{l}1,640 \\
1,530\end{array}$ & $\begin{array}{l}13.32 \\
13.54\end{array}$ \\
\hline $\begin{array}{l}4-11 \\
4-11\end{array}$ & $\begin{array}{l}0100 \\
2400\end{array}$ & $\begin{array}{l}7.33 \\
7.08\end{array}$ & $\begin{array}{l}932 \\
817\end{array}$ & $\begin{array}{l}9.16 \\
9.28\end{array}$ & $\begin{array}{l}4-20 \\
4-20 \\
4-20\end{array}$ & $\begin{array}{l}0100 \\
2300 \\
2400\end{array}$ & $\begin{array}{l}8.61 \\
8.31 \\
8.24\end{array}$ & $\begin{array}{l}1,520 \\
1,380 \\
1,350\end{array}$ & $\begin{array}{l}13.55 \\
13.74 \\
13.75\end{array}$ \\
\hline $\begin{array}{l}4-12 \\
4-12 \\
4-12\end{array}$ & $\begin{array}{l}0700 \\
0900 \\
1100\end{array}$ & $\begin{array}{l}7.03 \\
7.27 \\
7.33\end{array}$ & $\begin{array}{l}794 \\
904 \\
932\end{array}$ & $\begin{array}{l}9.32 \\
9.33 \\
9.34\end{array}$ & $\begin{array}{l}4-21 \\
4-21 \\
4-21\end{array}$ & $\begin{array}{l}0100 \\
0700 \\
2400\end{array}$ & $\begin{array}{l}8.18 \\
7.99 \\
7.81\end{array}$ & $\begin{array}{l}1.320 \\
1.240 \\
1.150\end{array}$ & $\begin{array}{l}13.70 \\
13.80 \\
13.93\end{array}$ \\
\hline $\begin{array}{l}4-12 \\
4-12 \\
4-12\end{array}$ & $\begin{array}{l}1200 \\
1300 \\
1400\end{array}$ & $\begin{array}{l}7.67 \\
8.33 \\
9.20\end{array}$ & $\begin{array}{l}1,090 \\
1,390 \\
1,810\end{array}$ & $\begin{array}{l}9.35 \\
9.35 \\
9.37\end{array}$ & $\begin{array}{l}4-22 \\
4-22\end{array}$ & $\begin{array}{l}0200 \\
2400\end{array}$ & $\begin{array}{l}7.79 \\
7.61\end{array}$ & $\begin{array}{l}1,140 \\
1,060\end{array}$ & $\begin{array}{l}13.94 \\
14.09\end{array}$ \\
\hline $\begin{array}{l}4-12 \\
4-12 \\
4-12 \\
4-12 \\
4-12\end{array}$ & $\begin{array}{l}1600 \\
1700 \\
1800 \\
2000 \\
2400\end{array}$ & $\begin{array}{l}11.44 \\
12.13 \\
12.79 \\
14.29 \\
16.22\end{array}$ & $\begin{array}{l}3,210 \\
3,630 \\
3,780 \\
5,650 \\
8,640\end{array}$ & $\begin{array}{l}9.40 \\
9.42 \\
9.44 \\
9.51 \\
9.69\end{array}$ & $\begin{array}{l}4-23 \\
4-23 \\
4-23\end{array}$ & $\begin{array}{l}0200 \\
1500 \\
2400\end{array}$ & $\begin{array}{l}7.61 \\
7.46 \\
7.27\end{array}$ & $\begin{array}{r}1,060 \\
992 \\
904\end{array}$ & $\begin{array}{l}14.10 \\
14.18 \\
14.23\end{array}$ \\
\hline $\begin{array}{l}4-13 \\
4-13\end{array}$ & $\begin{array}{l}0400 \\
0800\end{array}$ & $\begin{array}{l}17.14 \\
17.52\end{array}$ & $\begin{array}{l}10,900 \\
12,000\end{array}$ & $\begin{array}{r}9.93 \\
10.21\end{array}$ & $\begin{array}{l}4-24 \\
4-24 \\
4-24\end{array}$ & $\begin{array}{l}0100 \\
1600 \\
2400\end{array}$ & $\begin{array}{l}7.25 \\
7.06 \\
7.00\end{array}$ & $\begin{array}{l}895 \\
408 \\
780\end{array}$ & $\begin{array}{l}14.24 \\
14.31 \\
14.35\end{array}$ \\
\hline
\end{tabular}


TABLES

TABLE 7.-Gage height, discharge, and accumulated runoff, flood of April 1979-Continued 02401390, Big Canoe Creek at Ashville, Ala.

\begin{tabular}{|c|c|c|c|c|c|c|c|c|c|}
\hline DATE & TIME & $\begin{array}{c}\text { GAGE } \\
\text { HEIGHT }\end{array}$ & DISCHARGE & $\begin{array}{l}\text { ACCUM. } \\
\text { RUNOFF }\end{array}$ & DATE & TIME & $\begin{array}{c}\text { GAGE } \\
\text { HE IGHT }\end{array}$ & DISCHARGE & $\begin{array}{l}\text { ACCUM. } \\
\text { RUNOFF }\end{array}$ \\
\hline $\begin{array}{l}3-01 \\
3-01 \\
3-01 \\
3-01 \\
3-01 \\
3-01\end{array}$ & $\begin{array}{l}0200 \\
0600 \\
1300 \\
1700 \\
2200 \\
2400\end{array}$ & $\begin{array}{l}5.99 \\
6.52 \\
8.13 \\
8.30 \\
7.97 \\
7.73\end{array}$ & $\begin{array}{l}479 \\
566 \\
820 \\
845 \\
796 \\
760\end{array}$ & $\begin{array}{l}0.03 \\
0.09 \\
0.12 \\
0.16 \\
0.18\end{array}$ & $\begin{array}{l}3-10 \\
3-10 \\
3-11 \\
3-11 \\
3-11\end{array}$ & $\begin{array}{l}0100 \\
2400 \\
1100 \\
1800 \\
2400\end{array}$ & $\begin{array}{l}5.47 \\
5.30 \\
5.45 \\
5.31 \\
5.12\end{array}$ & $\begin{array}{l}403 \\
380 \\
\\
400 \\
382 \\
356\end{array}$ & $\begin{array}{l}5.05 \\
5.14 \\
5.19 \\
5.21 \\
5.24\end{array}$ \\
\hline $\begin{array}{l}3-02 \\
3-02 \\
3-02\end{array}$ & $\begin{array}{l}0100 \\
1500 \\
2400\end{array}$ & $\begin{array}{l}7.59 \\
6.31 \\
6.00\end{array}$ & $\begin{array}{l}739 \\
531 \\
480\end{array}$ & $\begin{array}{l}0.19 \\
0.28 \\
0.32\end{array}$ & $\begin{array}{l}3-12 \\
3-12\end{array}$ & $\begin{array}{l}0100 \\
2400\end{array}$ & $\begin{array}{l}5.09 \\
4.71\end{array}$ & $\begin{array}{l}352 \\
302\end{array}$ & $\begin{array}{l}5.24 \\
5.32\end{array}$ \\
\hline $\begin{array}{l}3-03 \\
3-03 \\
3-03 \\
3-03 \\
3-03 \\
3-03 \\
3-03\end{array}$ & $\begin{array}{l}0800 \\
1200 \\
1500 \\
1800 \\
1900 \\
2100 \\
2400\end{array}$ & $\begin{array}{r}6.00 \\
6.89 \\
8.40 \\
10.84 \\
11.55 \\
13.04 \\
14.89\end{array}$ & $\begin{array}{r}480 \\
631 \\
860 \\
1,310 \\
1,530 \\
2,790 \\
5,140\end{array}$ & $\begin{array}{l}0.36 \\
0.39 \\
0.41 \\
0.45 \\
0.46 \\
0.52 \\
0.65\end{array}$ & $\begin{array}{l}3-13 \\
3-13 \\
3-14 \\
3-14\end{array}$ & $\begin{array}{l}0100 \\
2400 \\
0100 \\
2400\end{array}$ & $\begin{array}{l}4.70 \\
4.49 \\
4.48 \\
4.43\end{array}$ & $\begin{array}{l}301 \\
274 \\
\\
273 \\
267\end{array}$ & $\begin{array}{l}5.32 \\
5.39 \\
5.39 \\
5.46\end{array}$ \\
\hline $\begin{array}{l}3-04 \\
3-04 \\
3-04 \\
3-04\end{array}$ & $\begin{array}{l}0700 \\
1500 \\
1800 \\
2400\end{array}$ & $\begin{array}{l}16.95 \\
17.39 \\
17.32 \\
16.80\end{array}$ & $\begin{array}{l}8,900 \\
9,940 \\
9,770 \\
8,580\end{array}$ & $\begin{array}{l}1.20 \\
2.01 \\
2.31 \\
2.89\end{array}$ & $\begin{array}{l}3-15 \\
3-15\end{array}$ & $\begin{array}{l}0100 \\
2400\end{array}$ & $\begin{array}{l}4.42 \\
4.15\end{array}$ & $\begin{array}{l}266 \\
237\end{array}$ & $\begin{array}{l}5.46 \\
5.52\end{array}$ \\
\hline & & & & & $\begin{array}{l}3-16 \\
3-16\end{array}$ & $\begin{array}{l}0100 \\
2400\end{array}$ & $\begin{array}{l}4.14 \\
3.99\end{array}$ & $\begin{array}{l}235 \\
219\end{array}$ & $\begin{array}{l}5.52 \\
5.58\end{array}$ \\
\hline $\begin{array}{l}3-05 \\
3-05 \\
3-05\end{array}$ & $\begin{array}{l}0100 \\
1300 \\
2400\end{array}$ & $\begin{array}{l}16.66 \\
14.80 \\
13.43\end{array}$ & $\begin{array}{l}8,290 \\
5,000 \\
3,210\end{array}$ & $\begin{array}{l}2.98 \\
3.77 \\
4.24\end{array}$ & $\begin{array}{l}3-17 \\
3-17\end{array}$ & $\begin{array}{l}0100 \\
2400\end{array}$ & $\begin{array}{l}3.98 \\
3.85\end{array}$ & $\begin{array}{l}218 \\
202\end{array}$ & $\begin{array}{l}5.58 \\
5.63\end{array}$ \\
\hline $\begin{array}{l}3-06 \\
3-06 \\
3-06 \\
3-06\end{array}$ & $\begin{array}{l}0100 \\
0800 \\
1600 \\
2400\end{array}$ & $\begin{array}{r}13.24 \\
11.53 \\
9.70 \\
8.37\end{array}$ & $\begin{array}{r}3.010 \\
1.510 \\
1.080 \\
856\end{array}$ & $\begin{array}{l}4.27 \\
4.43 \\
4.53 \\
4.61\end{array}$ & $\begin{array}{l}3-18 \\
3-18\end{array}$ & $\begin{array}{l}0100 \\
2400\end{array}$ & $\begin{array}{l}3.85 \\
3.75\end{array}$ & $\begin{array}{l}202 \\
190\end{array}$ & $\begin{array}{l}5.63 \\
5.68\end{array}$ \\
\hline $\begin{array}{l}3-07 \\
3-07\end{array}$ & $\begin{array}{l}0100 \\
2400\end{array}$ & $\begin{array}{l}8.25 \\
6.91\end{array}$ & $\begin{array}{l}838 \\
634\end{array}$ & $\begin{array}{l}4.62 \\
4.79\end{array}$ & $\begin{array}{l}3-19 \\
3-19\end{array}$ & $\begin{array}{l}0200 \\
2400\end{array}$ & $\begin{array}{l}3.75 \\
3.65\end{array}$ & $\begin{array}{l}190 \\
178\end{array}$ & $\begin{array}{l}5.68 \\
5.73\end{array}$ \\
\hline $\begin{array}{l}3-08 \\
3-08\end{array}$ & $\begin{array}{l}0100 \\
2400\end{array}$ & $\begin{array}{l}6.89 \\
6.10\end{array}$ & $\begin{array}{l}631 \\
496\end{array}$ & $\begin{array}{l}4.80 \\
4.93\end{array}$ & $\begin{array}{l}3-20 \\
3-20\end{array}$ & $\begin{array}{l}0100 \\
2400\end{array}$ & $\begin{array}{l}3.65 \\
3.58\end{array}$ & $\begin{array}{l}178 \\
170\end{array}$ & $\begin{array}{l}5.73 \\
5.77\end{array}$ \\
\hline $\begin{array}{l}3-09 \\
3-09\end{array}$ & $\begin{array}{l}0100 \\
2400\end{array}$ & $\begin{array}{l}6.06 \\
5.48\end{array}$ & $\begin{array}{l}490 \\
404\end{array}$ & $\begin{array}{l}4.94 \\
5.04\end{array}$ & $\begin{array}{l}3-21 \\
3-21\end{array}$ & $\begin{array}{l}0500 \\
2400\end{array}$ & $\begin{array}{l}3.58 \\
3.52\end{array}$ & $\begin{array}{l}170 \\
162\end{array}$ & $\begin{array}{l}5.78 \\
5.81\end{array}$ \\
\hline
\end{tabular}


TABLE 7.-Gage height, discharge, and accumulated runoff, flood of April 1979-Continued 02401390, Big Canoe Creek at Ashville, Ala.-Continued

\begin{tabular}{|c|c|c|c|c|c|c|c|c|c|}
\hline DATE & TIME & $\begin{array}{c}\text { GAGE } \\
\text { HEIGHT }\end{array}$ & DISCHARGE & $\begin{array}{l}\text { ACCUM. } \\
\text { RUNOFF }\end{array}$ & DATE & TIME & $\begin{array}{l}\text { GAGE } \\
\text { HEIGHT }\end{array}$ & DISCHARGE & $\begin{array}{l}\text { ACCUM. } \\
\text { RUNOFF }\end{array}$ \\
\hline $\begin{array}{l}3-22 \\
3-22\end{array}$ & $\begin{array}{l}0300 \\
2400\end{array}$ & $\begin{array}{l}3.52 \\
3.46\end{array}$ & $\begin{array}{l}162 \\
156\end{array}$ & $\begin{array}{l}5.82 \\
5.85\end{array}$ & $\begin{array}{l}4-02 \\
4-02 \\
4-02\end{array}$ & $\begin{array}{l}1900 \\
2200 \\
2400\end{array}$ & $\begin{array}{l}3.67 \\
3.82 \\
4.44\end{array}$ & $\begin{array}{l}180 \\
198 \\
268\end{array}$ & $\begin{array}{l}0.53 \\
6.53 \\
6.54\end{array}$ \\
\hline $\begin{array}{l}3-23 \\
3-23 \\
3-23 \\
3-23 \\
3-23 \\
3-23 \\
3-23 \\
3-23\end{array}$ & $\begin{array}{l}0600 \\
0800 \\
1100 \\
1300 \\
1600 \\
2000 \\
2100 \\
2400\end{array}$ & $\begin{array}{l}3.53 \\
3.90 \\
4.83 \\
6.08 \\
7.72 \\
8.34 \\
8.29 \\
7.80\end{array}$ & $\begin{array}{l}164 \\
208 \\
318 \\
493 \\
758 \\
851 \\
844 \\
770\end{array}$ & $\begin{array}{l}5.86 \\
5.86 \\
5.87 \\
5.88 \\
5.90 \\
5.94 \\
5.95 \\
5.97\end{array}$ & $\begin{array}{l}4-03 \\
4-03 \\
4-03 \\
4-03 \\
4-03 \\
4-03 \\
4-03\end{array}$ & $\begin{array}{l}0100 \\
0200 \\
0500 \\
0900 \\
1200 \\
1800 \\
2400\end{array}$ & $\begin{array}{r}5.03 \\
5.87 \\
9.00 \\
11.32 \\
12.25 \\
12.71 \\
12.31\end{array}$ & $\begin{array}{r}344 \\
461 \\
950 \\
1,430 \\
2,180 \\
2,520 \\
2,220\end{array}$ & $\begin{array}{l}6.54 \\
6.55 \\
6.57 \\
6.63 \\
6.69 \\
6.84 \\
6.99\end{array}$ \\
\hline $\begin{array}{l}3-24 \\
3-24 \\
3-24 \\
3-24\end{array}$ & $\begin{array}{l}0100 \\
1000 \\
2200 \\
2400\end{array}$ & $\begin{array}{l}7.58 \\
5.82 \\
4.95 \\
4.87\end{array}$ & $\begin{array}{l}737 \\
454 \\
334 \\
323\end{array}$ & $\begin{array}{l}5.98 \\
6.03 \\
6.08 \\
6.09\end{array}$ & $\begin{array}{l}4-04 \\
4-04 \\
4-04\end{array}$ & $\begin{array}{l}0100 \\
0700 \\
2400\end{array}$ & $\begin{array}{l}12.21 \\
11.45 \\
11.17\end{array}$ & $\begin{array}{l}2,150 \\
1,470 \\
1,390\end{array}$ & $\begin{array}{l}7.01 \\
7.12 \\
7.38\end{array}$ \\
\hline $\begin{array}{l}3-25 \\
3-25\end{array}$ & $\begin{array}{l}0100 \\
2400\end{array}$ & $\begin{array}{l}4.84 \\
4.33\end{array}$ & $\begin{array}{l}319 \\
256\end{array}$ & $\begin{array}{l}6.09 \\
6.16\end{array}$ & $\begin{array}{l}4-05 \\
4-05 \\
4-05\end{array}$ & $\begin{array}{l}0100 \\
1900 \\
2400\end{array}$ & $\begin{array}{r}11.06 \\
7.93 \\
7.39\end{array}$ & $\begin{array}{r}1.370 \\
790 \\
709\end{array}$ & $\begin{array}{l}7.40 \\
7.59 \\
7.63\end{array}$ \\
\hline $\begin{array}{l}3-26 \\
3-26\end{array}$ & $\begin{array}{l}0100 \\
2400\end{array}$ & $\begin{array}{l}4.32 \\
4.03\end{array}$ & $\begin{array}{l}255 \\
223\end{array}$ & $\begin{array}{l}6.16 \\
6.22\end{array}$ & $\begin{array}{l}4-06 \\
4-06\end{array}$ & $\begin{array}{l}0100 \\
2400\end{array}$ & $\begin{array}{l}7.30 \\
6.07\end{array}$ & $\begin{array}{l}695 \\
491\end{array}$ & $\begin{array}{l}7.64 \\
7.78\end{array}$ \\
\hline $\begin{array}{l}3-27 \\
3-27\end{array}$ & $\begin{array}{l}0100 \\
2400\end{array}$ & $\begin{array}{l}4.02 \\
3.85\end{array}$ & $\begin{array}{l}222 \\
202\end{array}$ & $\begin{array}{l}6.22 \\
6.27\end{array}$ & $\begin{array}{l}4-07 \\
4-07\end{array}$ & $\begin{array}{l}0100 \\
2400\end{array}$ & $\begin{array}{l}6.04 \\
5.37\end{array}$ & $\begin{array}{l}487 \\
390\end{array}$ & $\begin{array}{l}7.78 \\
7.88\end{array}$ \\
\hline $\begin{array}{l}3-28 \\
3-28\end{array}$ & $\begin{array}{l}0100 \\
2400\end{array}$ & $\begin{array}{l}3.85 \\
3.71\end{array}$ & $\begin{array}{l}202 \\
185\end{array}$ & $\begin{array}{l}6.27 \\
6.32\end{array}$ & $\begin{array}{l}4-08 \\
4-08 \\
4-08\end{array}$ & $\begin{array}{l}1900 \\
2300 \\
2400\end{array}$ & $\begin{array}{l}5.12 \\
5.84 \\
6.16\end{array}$ & $\begin{array}{l}356 \\
457 \\
506\end{array}$ & $\begin{array}{l}7.96 \\
7.97 \\
7.98\end{array}$ \\
\hline $\begin{array}{l}3-30 \\
3-30\end{array}$ & $\begin{array}{l}0200 \\
2400\end{array}$ & $\begin{array}{l}3.58 \\
3.48\end{array}$ & $\begin{array}{l}170 \\
158\end{array}$ & $\begin{array}{l}6.37 \\
6.41\end{array}$ & $\begin{array}{l}4-09 \\
4-09 \\
4-09 \\
4-09\end{array}$ & $\begin{array}{l}0500 \\
0900 \\
1000 \\
2400\end{array}$ & $\begin{array}{l}8.18 \\
8.64 \\
8.58 \\
6.28\end{array}$ & $\begin{array}{l}827 \\
896 \\
887 \\
526\end{array}$ & $\begin{array}{l}8.02 \\
8.05 \\
8.06 \\
8.17\end{array}$ \\
\hline $3-31$ & 2400 & 3.51 & 161 & 6.44 & $\begin{array}{l}4-10 \\
4-10 \\
4-10\end{array}$ & $\begin{array}{l}0100 \\
1400 \\
2400\end{array}$ & $\begin{array}{l}6.18 \\
5.44 \\
5.19\end{array}$ & $\begin{array}{l}510 \\
399 \\
365\end{array}$ & $\begin{array}{l}8.17 \\
8.23 \\
6.27\end{array}$ \\
\hline $4-01$ & 2400 & 3.88 & 206 & 6.49 & $\begin{array}{l}4-11 \\
4-11\end{array}$ & $\begin{array}{l}0100 \\
2400\end{array}$ & $\begin{array}{l}5.18 \\
4.82\end{array}$ & $\begin{array}{l}364 \\
317\end{array}$ & $\begin{array}{l}8.27 \\
8.36\end{array}$ \\
\hline
\end{tabular}


TABLE 7.-Gage height, discharge, and accumulated runoff, flood of April 1979-Continued 02401390, Big Canoe Creek at Ashville, Ala-Continued

\begin{tabular}{|c|c|c|c|c|c|c|c|c|c|}
\hline DATE & TIME & $\begin{array}{c}\text { GAGE } \\
\text { HEIGHT }\end{array}$ & DISCHARGE & $\begin{array}{l}\text { ACCUM. } \\
\text { RUNOFF }\end{array}$ & DATE & TIME & $\begin{array}{l}\text { GAGE } \\
\text { HEIGHT }\end{array}$ & DISCHARGE & $\begin{array}{l}\text { ACCUM. } \\
\text { RUNOFF }\end{array}$ \\
\hline & & & & & $\begin{array}{l}4-17 \\
4-17\end{array}$ & $\begin{array}{l}0100 \\
2400\end{array}$ & $\begin{array}{l}7.27 \\
6.24\end{array}$ & $\begin{array}{l}691 \\
519\end{array}$ & $\begin{array}{l}14.34 \\
14.48\end{array}$ \\
\hline \multirow[t]{2}{*}{$\begin{array}{l}4-12 \\
4-12 \\
4-12 \\
4-12 \\
4-12 \\
4-12\end{array}$} & $\begin{array}{l}0600 \\
0700 \\
1200 \\
1500 \\
1800 \\
2400\end{array}$ & $\begin{array}{r}4.73 \\
5.01 \\
8.94 \\
11.35 \\
13.28 \\
15.02\end{array}$ & $\begin{array}{r}305 \\
341 \\
941 \\
1,440 \\
3,050 \\
5,330\end{array}$ & $\begin{array}{l}8.37 \\
8.38 \\
8.41 \\
8.45 \\
8.53 \\
8.81\end{array}$ & $\begin{array}{l}4-18 \\
4-18\end{array}$ & $\begin{array}{l}0100 \\
2400\end{array}$ & $\begin{array}{l}6.20 \\
5.61\end{array}$ & $\begin{array}{l}513 \\
423\end{array}$ & $\begin{array}{l}14.48 \\
14.60\end{array}$ \\
\hline & & & & & $\begin{array}{l}4-19 \\
4-19\end{array}$ & $\begin{array}{l}0100 \\
2400\end{array}$ & $\begin{array}{l}5.59 \\
5.18\end{array}$ & $\begin{array}{l}420 \\
364\end{array}$ & $\begin{array}{l}14.60 \\
14.69\end{array}$ \\
\hline $\begin{array}{l}4-13 \\
4-13 \\
4-13\end{array}$ & $\begin{array}{l}1100 \\
1800 \\
2400\end{array}$ & $\begin{array}{l}17.81 \\
18.74 \\
18.45\end{array}$ & $\begin{array}{l}11,000 \\
13,600 \\
12,800\end{array}$ & $\begin{array}{r}9.76 \\
10.70 \\
11.54\end{array}$ & $\begin{array}{l}4-20 \\
4-20\end{array}$ & $\begin{array}{l}0100 \\
2400\end{array}$ & $\begin{array}{l}5.16 \\
4.84\end{array}$ & $\begin{array}{l}361 \\
319\end{array}$ & $\begin{array}{l}14.70 \\
14.78\end{array}$ \\
\hline $\begin{array}{l}4-14 \\
4-14 \\
4-14\end{array}$ & $\begin{array}{l}0100 \\
1600 \\
2400\end{array}$ & $\begin{array}{l}18.35 \\
15.47 \\
14.41\end{array}$ & $\begin{array}{r}12,500 \\
6,060 \\
4,440\end{array}$ & $\begin{array}{l}11.67 \\
13.06 \\
13.48\end{array}$ & $\begin{array}{l}4-21 \\
4-21\end{array}$ & $\begin{array}{l}0100 \\
2400\end{array}$ & $\begin{array}{l}4.83 \\
4.60\end{array}$ & $\begin{array}{l}318 \\
288\end{array}$ & $\begin{array}{l}14.78 \\
14.85\end{array}$ \\
\hline \multirow[t]{2}{*}{$\begin{array}{l}4-15 \\
4-15 \\
4-15 \\
4-15\end{array}$} & $\begin{array}{l}0100 \\
1500 \\
1700 \\
2400\end{array}$ & $\begin{array}{r}14.31 \\
12.04 \\
11.55 \\
9.98\end{array}$ & $\begin{array}{l}4,310 \\
2,030 \\
1.530 \\
1.130\end{array}$ & $\begin{array}{l}13.53 \\
13.99 \\
14.02 \\
14.12\end{array}$ & $\begin{array}{l}4-22 \\
4-22\end{array}$ & $\begin{array}{l}0100 \\
2400\end{array}$ & $\begin{array}{l}4.59 \\
4.44\end{array}$ & $\begin{array}{l}287 \\
268\end{array}$ & $\begin{array}{l}14.86 \\
14.92\end{array}$ \\
\hline & & & & & $\begin{array}{l}4-23 \\
4-23\end{array}$ & $\begin{array}{l}0100 \\
2400\end{array}$ & $\begin{array}{l}4.42 \\
4.32\end{array}$ & $\begin{array}{l}266 \\
255\end{array}$ & $\begin{array}{l}14.93 \\
14.99\end{array}$ \\
\hline $\begin{array}{l}4-16 \\
4-16 \\
4-16\end{array}$ & $\begin{array}{l}0100 \\
1300 \\
2400\end{array}$ & $\begin{array}{l}9.79 \\
8.13 \\
7.35\end{array}$ & $\begin{array}{r}1,090 \\
820 \\
703\end{array}$ & $\begin{array}{l}14.13 \\
14.24 \\
14.33\end{array}$ & $\begin{array}{l}4-24 \\
4-24\end{array}$ & $\begin{array}{l}0100 \\
2400\end{array}$ & $\begin{array}{l}4.31 \\
4.24\end{array}$ & $\begin{array}{l}254 \\
246\end{array}$ & $\begin{array}{l}14.99 \\
15.05\end{array}$ \\
\hline
\end{tabular}


TABLE 7.-Gage height, discharge, and accumulated runoff, flood of April 1979-Continued 02404400, Choccolocco Creek at Jackson Shoals, Ala.

\begin{tabular}{|c|c|c|c|c|c|c|c|c|c|}
\hline DATE & TIME & $\begin{array}{c}\text { GAGE } \\
\text { HEIGHT }\end{array}$ & DISCHARGE & $\begin{array}{l}\text { ACCUM. } \\
\text { RUNOFF }\end{array}$ & _DATE & - TIME & $\begin{array}{c}\text { GAGE } \\
\text { HEIGHT }\end{array}$ & ISCHARGE & $\begin{array}{r}\text { ACCUM. } \\
\text { RUNOF }\end{array}$ \\
\hline $3-2$ & 2400 & 19.49 & 955 & 0.00 & $\begin{array}{l}4-3 \\
4-3\end{array}$ & $\begin{array}{l}1300 \\
1400\end{array}$ & $\begin{array}{l}33.80 \\
33.70\end{array}$ & $\begin{array}{l}19700 \\
19500\end{array}$ & $\begin{array}{l}0.78 \\
0.84\end{array}$ \\
\hline $\begin{array}{l}3-3 \\
3-3\end{array}$ & $\begin{array}{l}1500 \\
1700\end{array}$ & $\begin{array}{l}19.45 \\
19.63\end{array}$ & $\begin{array}{r}933 \\
1040\end{array}$ & $\begin{array}{l}0.04 \\
0.05\end{array}$ & $4-3$ & 2400 & 30.53 & 13400 & 1.27 \\
\hline $3-3$ & 2000 & 20.56 & 1640 & 0.07 & $4-4$ & 0100 & 30.10 & 12800 & 1.31 \\
\hline $3-3$ & 2100 & 21.21 & 2130 & 0.07 & $4-4$ & 0500 & 29.24 & 11500 & 1.46 \\
\hline $3-3$ & 2200 & 22.87 & 3550 & 0.09 & $4-4$ & 1600 & 29.71 & 12200 & 1.89 \\
\hline $3-3$ & 2300 & 24.76 & 5570 & 0.10 & $4-4$ & 2400 & 27.76 & 9460 & 2.13 \\
\hline $3-3$ & 2400 & 26.81 & 8150 & 0.13 & & & & & \\
\hline $3-4$ & 0500 & & & & $4-5$ & 0100 & 27.49 & 9090 & 2.16 \\
\hline $3-4$ & 1400 & 37.76 & 30100 & 0.45 & $4-5$ & 1300 & 25.49 & 6450 & 2.41 \\
\hline $3-4$ & 2400 & 35.55 & 23800 & $\begin{array}{l}1.02 \\
2.08\end{array}$ & $4-5$ & 2400 & 24.43 & 5180 & 2.59 \\
\hline & & & & & $4-6$ & 0100 & 24.32 & 5060 & 2.61 \\
\hline $\begin{array}{l}3-5 \\
3-5\end{array}$ & 0100 & 35.19 & 22900 & 2.16 & $4-6$ & 2400 & 22.97 & 3640 & 2.88 \\
\hline $\begin{array}{l}3-5 \\
3-5\end{array}$ & 1000 & 32.56 & 17300 & 2.65 & & & & & \\
\hline $3-5$ & 2400 & 28.24 & 10100 & 3.11 & $\begin{array}{l}4-7 \\
4-7\end{array}$ & $\begin{array}{l}0100 \\
2400\end{array}$ & $\begin{array}{l}22.94 \\
22.20\end{array}$ & $\begin{array}{l}3620 \\
2950\end{array}$ & $\begin{array}{l}2.89 \\
3.10\end{array}$ \\
\hline $3-6$ & 0100 & 27.98 & 9770 & 3.14 & & & & & \\
\hline $3-6$ & 1000 & 26.78 & 8110 & 3.37 & $4-10$ & 2400 & 21.45 & 2320 & 0.00 \\
\hline $3-6$ & 2400 & 25.81 & 6850 & 3.68 & & & & & \\
\hline $3-7$ & 0100 & 25.76 & 6790 & 3.70 & $\begin{array}{l}4-11 \\
4-11\end{array}$ & $\begin{array}{l}0200 \\
2400\end{array}$ & $\begin{array}{l}21.44 \\
21.11\end{array}$ & 2050 & $\begin{array}{l}0.01 \\
0.16\end{array}$ \\
\hline $3-7$ & 2400 & 24.24 & 4970 & 4.07 & & & & & \\
\hline & & & & & $4-12$ & 0800 & 21.16 & 2090 & 0.21 \\
\hline $\begin{array}{l}3-8 \\
3-8\end{array}$ & 0100 & 24.18 & 4910 & 4.08 & $4-12$ & 1200 & 22.09 & 2860 & 0.25 \\
\hline $3-8$ & 2000 & 22.84 & 3530 & 4.30 & $4-12$ & 1300 & 22.60 & 3310 & 0.26 \\
\hline 3- 8 & 2100 & 22.00 & 2780 & 4.31 & $4-12$ & 1500 & 24.70 & 5500 & 0.30 \\
\hline $3-8$ & 2200 & 22.75 & 3450 & 4.32 & $4-12$ & 2200 & 33.32 & 18700 & 0.71 \\
\hline 3- 8 & 2400 & 22.57 & 3280 & 4.34 & $4-12$ & 2400 & 34.15 & 20400 & 0.84 \\
\hline 3- 9 & 0100 & 22.52 & 3248 & 4.35 & $4-13$ & 0900 & 35.52 & 23800 & 1.53 \\
\hline 3- 9 & 2400 & 21.79 & 2600 & 4.54 & $4-13$ & 1900 & 37.76 & 30100 & 2.49 \\
\hline $4-1$ & 2400 & 18.99 & 685 & 0.00 & $4-13$ & 2400 & 36.92 & 27600 & 2.94 \\
\hline & & & & & $4-14$ & 0100 & 36.79 & 27200 & 3.02 \\
\hline $\begin{array}{l}4-2 \\
4-2\end{array}$ & 1900 & 18.98 & 680 & 0.04 & $4-14$ & 2100 & 30.59 & 13500 & 3.89 \\
\hline $\begin{array}{l}4-2 \\
4-2\end{array}$ & 2000 & 19.09 & 735 & 0.04 & & & & & \\
\hline 4- 2 & 2100 & 19.36 & 883 & 0.05 & $4-15$ & 0100 & 29.54 & 12000 & 4.04 \\
\hline 4- 2 & 2200 & 19.96 & 1240 & 0.05 & $4-15$ & 0600 & 28.76 & 10900 & 4.22 \\
\hline $4-2$ & 2300 & 21.42 & 2300 & 0.06 & $4-15$ & 2400 & 26.68 & 7980 & 4.68 \\
\hline $4-2$ & 2400 & 23.60 & 4270 & 0.07 & & & & & \\
\hline $4-3$ & 0400 & 30.20 & 12900 & 0.24 & $\begin{array}{l}4-16 \\
4-16\end{array}$ & $\begin{array}{l}0100 \\
2400\end{array}$ & $\begin{array}{l}26.58 \\
24.57\end{array}$ & $\begin{array}{l}7850 \\
5340\end{array}$ & $\begin{array}{l}4.70 \\
5.09\end{array}$ \\
\hline $4-3$ & 0800 & 32.82 & 17800 & 0.46 & & & & & \\
\hline
\end{tabular}


TABLE 7.-Gage height, discharge, and accumulated runoff, flood of April 1979-Continued 02407000, Coosa River at Childersburg, Ala.

\begin{tabular}{|c|c|c|c|c|c|c|c|c|c|}
\hline DATE & - TIME & $\begin{array}{r}\text { GAGE } \\
\text { HEIGH! }\end{array}$ & DISCHARGE & $\begin{array}{l}\text { ACCUM. } \\
\text { RUNOFF }\end{array}$ & -DATE & -IIME & $\begin{array}{c}\text { GAGE } \\
\text { HE IGHT }\end{array}$ & DISCHARGE & $\begin{array}{l}\text { ACCUM. } \\
\text { RUNOFF }\end{array}$ \\
\hline $3-2$ & 2400 & 16.18 & 33500 & 0.00 & $4-11$ & 2400 & 15.76 & 31800 & 0.00 \\
\hline $\begin{array}{l}3-3 \\
3-\quad 3 \\
3-3 \\
3-3\end{array}$ & $\begin{array}{l}0600 \\
1200 \\
1800 \\
2400\end{array}$ & $\begin{array}{l}16.17 \\
16.21 \\
16.25 \\
19.52\end{array}$ & $\begin{array}{l}34106 \\
34800 \\
36100 \\
65300\end{array}$ & $\begin{array}{l}0.04 \\
0.08 \\
0.12 \\
0.19\end{array}$ & $\begin{array}{l}4-12 \\
4-12 \\
4-12 \\
4-12\end{array}$ & $\begin{array}{l}0600 \\
1200 \\
1800 \\
2400\end{array}$ & $\begin{array}{l}14.87 \\
15.96 \\
19.54 \\
21.61\end{array}$ & $\begin{array}{l}19000 \\
43700 \\
77400 \\
84400\end{array}$ & $\begin{array}{l}0.02 \\
0.07 \\
0.16 \\
0.25\end{array}$ \\
\hline $\begin{array}{l}3-4 \\
3-4 \\
3-4 \\
3-4\end{array}$ & $\begin{array}{l}0600 \\
1200 \\
1800 \\
2400\end{array}$ & $\begin{array}{l}21.53 \\
22.79 \\
24.32 \\
25.10\end{array}$ & $\begin{array}{r}78100 \\
91300 \\
110000 \\
117000\end{array}$ & $\begin{array}{l}0.28 \\
0.38 \\
0.50 \\
0.63\end{array}$ & $\begin{array}{l}4-13 \\
4-13 \\
4-13 \\
4-13\end{array}$ & $\begin{array}{l}0600 \\
1200 \\
1800 \\
2400\end{array}$ & $\begin{array}{l}22.89 \\
24.87 \\
26.58 \\
27.87\end{array}$ & $\begin{array}{r}93300 \\
115000 \\
132000 \\
146000\end{array}$ & $\begin{array}{l}0.35 \\
0.48 \\
0.63 \\
0.79\end{array}$ \\
\hline $\begin{array}{l}3-5 \\
3-5 \\
3-5 \\
3-5 \\
3-5\end{array}$ & $\begin{array}{l}0300 \\
0600 \\
1200 \\
1800 \\
2400\end{array}$ & $\begin{array}{l}25.16 \\
25.12 \\
24.82 \\
24.53 \\
24.22\end{array}$ & $\begin{array}{l}119000 \\
116000 \\
111000 \\
111000 \\
107000\end{array}$ & $\begin{array}{l}0.69 \\
0.76 \\
0.88 \\
1.00 \\
1.12\end{array}$ & $\begin{array}{l}4-14 \\
4-14 \\
4-14 \\
4-14 \\
4-14\end{array}$ & $\begin{array}{l}0600 \\
1200 \\
1500 \\
1800 \\
2400\end{array}$ & $\begin{array}{l}28.68 \\
28.92 \\
28.99 \\
28.96 \\
28.82\end{array}$ & $\begin{array}{l}150000 \\
148000 \\
149000 \\
148000 \\
148000\end{array}$ & $\begin{array}{l}0.95 \\
1.12 \\
1.20 \\
1.28 \\
1.45\end{array}$ \\
\hline $\begin{array}{l}3-6 \\
3-6 \\
3-6 \\
3-6\end{array}$ & $\begin{array}{l}0600 \\
1200 \\
1800 \\
2400\end{array}$ & $\begin{array}{l}23.89 \\
23.47 \\
23.15 \\
22.93\end{array}$ & $\begin{array}{r}107000 \\
101000 \\
98400 \\
95600\end{array}$ & $\begin{array}{l}1.24 \\
1.35 \\
1.46 \\
1.57\end{array}$ & $\begin{array}{l}4-15 \\
4-15 \\
4-15 \\
4-15\end{array}$ & $\begin{array}{l}0600 \\
1200 \\
1800 \\
2400\end{array}$ & $\begin{array}{l}28.49 \\
28.11 \\
27.69 \\
27.28\end{array}$ & $\begin{array}{l}145000 \\
141000 \\
138000 \\
134000\end{array}$ & $\begin{array}{l}1.61 \\
1.76 \\
1.92 \\
2.06\end{array}$ \\
\hline $\begin{array}{l}3-7 \\
3-7 \\
3-7 \\
3-7\end{array}$ & $\begin{array}{l}0600 \\
1200 \\
1800 \\
2400\end{array}$ & $\begin{array}{l}22.73 \\
22.60 \\
22.52 \\
22.44\end{array}$ & $\begin{array}{l}95000 \\
91900 \\
91200 \\
91600\end{array}$ & $\begin{array}{l}1.67 \\
1.78 \\
1.88 \\
1.98\end{array}$ & $\begin{array}{l}4-16 \\
4-16 \\
4-16 \\
4-16\end{array}$ & $\begin{array}{l}0600 \\
1200 \\
1800 \\
2400\end{array}$ & $\begin{array}{l}26.87 \\
26.31 \\
25.65 \\
24.93\end{array}$ & $\begin{array}{l}133000 \\
127000 \\
118000 \\
110000\end{array}$ & $\begin{array}{l}2.21 \\
2.35 \\
2.48 \\
2.61\end{array}$ \\
\hline $\begin{array}{l}3-8 \\
3-8 \\
3-8 \\
3-8\end{array}$ & $\begin{array}{l}0600 \\
1200 \\
1800 \\
2400\end{array}$ & $\begin{array}{l}22.33 \\
22.12 \\
22.09 \\
22.03\end{array}$ & $\begin{array}{l}91800 \\
87100 \\
88800 \\
88400\end{array}$ & $\begin{array}{l}2.08 \\
2.18 \\
2.27 \\
2.37\end{array}$ & $\begin{array}{l}4-17 \\
4-17 \\
4-17 \\
4-17\end{array}$ & $\begin{array}{l}0600 \\
1200 \\
1800 \\
2400\end{array}$ & $\begin{array}{l}24.02 \\
23.30 \\
22.47 \\
21.91\end{array}$ & $\begin{array}{r}101000 \\
97400 \\
87700 \\
84500\end{array}$ & $\begin{array}{l}2.72 \\
2.83 \\
2.92 \\
3.02\end{array}$ \\
\hline $\begin{array}{l}3-9 \\
3-9 \\
3-9 \\
3-9\end{array}$ & $\begin{array}{l}0600 \\
1200 \\
1800 \\
2400\end{array}$ & $\begin{array}{l}21.95 \\
21.68 \\
21.21 \\
20.91\end{array}$ & $\begin{array}{l}88700 \\
84100 \\
76900 \\
76700\end{array}$ & $\begin{array}{l}2.47 \\
2.56 \\
2.65 \\
2.73\end{array}$ & $\begin{array}{l}4-18 \\
4-18 \\
4-18 \\
4-18\end{array}$ & $\begin{array}{l}0600 \\
1200 \\
1800 \\
2400\end{array}$ & $\begin{array}{l}21.56 \\
21.15 \\
20.70 \\
20.46\end{array}$ & $\begin{array}{l}82100 \\
77100 \\
74400 \\
74500\end{array}$ & $\begin{array}{l}3.11 \\
3.19 \\
3.28 \\
3.36\end{array}$ \\
\hline $\begin{array}{l}3-10 \\
3-10 \\
3-10 \\
3-10\end{array}$ & $\begin{array}{l}0600 \\
1200 \\
1800 \\
2400\end{array}$ & $\begin{array}{l}20.74 \\
20.37 \\
19.99 \\
19.93\end{array}$ & $\begin{array}{l}75688 \\
70500 \\
68800 \\
70100\end{array}$ & $\begin{array}{l}2.82 \\
2.90 \\
2.97 \\
3.05\end{array}$ & $\begin{array}{l}4-19 \\
4-19 \\
4-19 \\
4-19\end{array}$ & $\begin{array}{l}0600 \\
1200 \\
1800 \\
2400\end{array}$ & $\begin{array}{l}20.29 \\
19.99 \\
19.70 \\
19.48\end{array}$ & $\begin{array}{l}74200 \\
70500 \\
64700 \\
65900\end{array}$ & $\begin{array}{l}3.44 \\
3.52 \\
3.59 \\
3.66\end{array}$ \\
\hline $\begin{array}{l}3-11 \\
3-11 \\
3-11 \\
3-11\end{array}$ & $\begin{array}{l}0600 \\
1200 \\
1800 \\
2400\end{array}$ & $\begin{array}{l}19.96 \\
19.89 \\
19.86 \\
19.79\end{array}$ & $\begin{array}{l}71100 \\
69100 \\
68100 \\
67700\end{array}$ & $\begin{array}{l}3.13 \\
3.21 \\
3.28 \\
3.36\end{array}$ & $\begin{array}{l}4-20 \\
4-20 \\
4-20 \\
4-20\end{array}$ & $\begin{array}{l}0600 \\
1200 \\
1800 \\
2400\end{array}$ & $\begin{array}{l}19.33 \\
19.28 \\
19.27 \\
19.25\end{array}$ & $\begin{array}{l}65000 \\
64700 \\
64600 \\
64500\end{array}$ & $\begin{array}{l}3.73 \\
3.81 \\
3.88 \\
3.95\end{array}$ \\
\hline $\begin{array}{l}3-12 \\
3-12 \\
3-12 \\
3-12\end{array}$ & $\begin{array}{l}0600 \\
1200 \\
1800 \\
2400\end{array}$ & $\begin{array}{l}19.71 \\
19.59 \\
19.43 \\
19.34\end{array}$ & $\begin{array}{l}68800 \\
64700 \\
62400 \\
63500\end{array}$ & $\begin{array}{l}3.43 \\
3.51 \\
3.57 \\
3.64\end{array}$ & $\begin{array}{l}4-21 \\
4-21 \\
4-21 \\
4-21\end{array}$ & $\begin{array}{l}0600 \\
1200 \\
1800 \\
2400\end{array}$ & $\begin{array}{l}19.22 \\
19.18 \\
18.97 \\
18.81\end{array}$ & $\begin{array}{l}64400 \\
63400 \\
60000 \\
58900\end{array}$ & $\begin{array}{l}4.02 \\
4.09 \\
4.16 \\
4.22\end{array}$ \\
\hline $\begin{array}{l}3-13 \\
3-13 \\
3-13 \\
3-13\end{array}$ & $\begin{array}{l}0600 \\
1200 \\
1800 \\
2400\end{array}$ & $\begin{array}{l}19.30 \\
19.32 \\
19.19 \\
19.06\end{array}$ & $\begin{array}{l}63300 \\
62600 \\
60300 \\
59600\end{array}$ & $\begin{array}{l}3.71 \\
3.78 \\
3.85 \\
3.92\end{array}$ & $\begin{array}{l}4-22 \\
4-22 \\
4-22 \\
4-22\end{array}$ & $\begin{array}{l}0600 \\
1200 \\
1800 \\
2400\end{array}$ & $\begin{array}{l}18.78 \\
18.74 \\
18.71 \\
18.71\end{array}$ & $\begin{array}{l}59700 \\
59600 \\
60200 \\
60200\end{array}$ & $\begin{array}{l}4.29 \\
4.36 \\
4.42 \\
4.49\end{array}$ \\
\hline $\begin{array}{l}3-14 \\
3-14 \\
3-14 \\
3-14\end{array}$ & $\begin{array}{l}0600 \\
1200 \\
1800 \\
2400\end{array}$ & $\begin{array}{l}19.03 \\
18.97 \\
18.79 \\
18.69\end{array}$ & $\begin{array}{l}60200 \\
59900 \\
50000 \\
58600\end{array}$ & $\begin{array}{l}3.98 \\
4.05 \\
4.12 \\
4.18\end{array}$ & $\begin{array}{l}4-23 \\
4-23 \\
4-23 \\
4-23\end{array}$ & $\begin{array}{l}0600 \\
1200 \\
1800 \\
2400\end{array}$ & $\begin{array}{l}18.68 \\
18.19 \\
17.76 \\
17.33\end{array}$ & $\begin{array}{l}59300 \\
49100 \\
50200 \\
40700\end{array}$ & $\begin{array}{l}4.55 \\
4.61 \\
4.66 \\
4.71\end{array}$ \\
\hline
\end{tabular}


TABLE 7.-Gage height, discharge, and accumulated runoff, flood of April 1979-Continued 02411000, Coosa River at Jordan Dam

\begin{tabular}{|c|c|c|c|c|c|c|c|c|c|}
\hline DATE & - TIME & $\begin{array}{l}\text { GAGE } \\
\text { HEIGHT }\end{array}$ & DISSCHARGE & $\begin{array}{l}\text { ACCUM. } \\
\text { RUNOFF }\end{array}$ & DATE & TIME & $\begin{array}{l}\text { GAGE } \\
\text { IEIGHT }\end{array}$ & DISCHARGE & $\begin{array}{l}\text { ACCUM. } \\
\text { RUNOFF }\end{array}$ \\
\hline 3- 2 & 2400 & c & 40700 & 0.00 & $\begin{array}{l}4-12 \\
4-12\end{array}$ & $\begin{array}{l}0600 \\
1200\end{array}$ & $\begin{array}{l}\text { c } \\
\text { c }\end{array}$ & $\begin{array}{l}32600 \\
39900\end{array}$ & $\begin{array}{l}0.03 \\
0.07\end{array}$ \\
\hline $3-3$ & 0400 & c & 40700 & 0.02 & $4-12$ & 1800 & c & 80000 & 0.14 \\
\hline $3-3$ & 0600 & c & 34000 & 0.04 & $4-12$ & $24 Q 0$ & c & 191000 & 0.31 \\
\hline $3-3$ & 1200 & c & 34000 & 0.07 & & & & & \\
\hline $3-3$ & 1400 & c & 49000 & 0.08 & $4-13$ & 0400 & c & 195000 & 0.43 \\
\hline $3-3$ & 1600 & c & 56200 & 0.10 & $4-13$ & 0900 & c & 199000 & 0.58 \\
\hline $3-3$ & 1800 & c & 65100 & 0.12 & $4-13$ & 1200 & c & 215000 & 0.68 \\
\hline \multirow[t]{2}{*}{$3-3$} & 2400 & c & 146000 & 0.25 & $4-13$ & 1500 & c & 267000 & 0.80 \\
\hline & & & & & $4-13$ & 1800 & c & 305000 & 0.94 \\
\hline $3-4$ & 0600 & c & 251000 & 0.48 & $4-13$ & 2000 & c & 316000 & 1.04 \\
\hline $3-4$ & 0800 & c & 253000 & 0.56 & $4-13$ & 2100 & c & 301000 & 1.08 \\
\hline $3-4$ & 1000 & c & 253000 & 0.63 & $4-13$ & 2200 & c & 308000 & 1.13 \\
\hline $3-4$ & 1200 & c & 251000 & 0.71 & $4-13$ & 2300 & c & 311000 & 1.18 \\
\hline $3-4$ & 1800 & c & 227000 & 0.92 & $4-13$ & 2400 & c & 308000 & 1.22 \\
\hline \multirow[t]{2}{*}{$3-4$} & 2400 & c & 190000 & 1.09 & & & & & \\
\hline & & & & & $4-14$ & 0400 & c & 298000 & 1.41 \\
\hline 3- 5 & 0600 & c & 163000 & 1.24 & $4-14$ & 0800 & c & 264000 & 1.57 \\
\hline $3-5$ & 1200 & c & 158000 & 1.38 & $4-14$ & 1200 & c & 239000 & 1.71 \\
\hline $3-5$ & 1800 & c & 136000 & 1.51 & $4-14$ & 1800 & c & 211000 & 1.90 \\
\hline $3-5$ & 2200 & c & 101000 & 1.57 & $4-14$ & 2400 & c & 186000 & 2.07 \\
\hline \multirow[t]{2}{*}{$3-5$} & 2400 & c & 110000 & 1.60 & & & & & \\
\hline & & & & & $4-15$ & 0600 & c & 174000 & 2.23 \\
\hline $3-6$ & 0100 & c & 114000 & 1.62 & $4-15$ & 0900 & c & 147000 & 2.30 \\
\hline $3-6$ & 0200 & c & 120000 & 1.64 & $4-15$ & 1200 & c & 155000 & 2.37 \\
\hline $3-6$ & 0600 & c & 114000 & 1.71 & $4-15$ & 1800 & c & 149000 & 2.50 \\
\hline $3-6$ & 1200 & c & 114000 & 1.81 & $4-15$ & 2400 & c & 148000 & 2.64 \\
\hline $3-6$ & 1800 & c & 101000 & 1.90 & & & & & \\
\hline $3-6$ & 2100 & c & 92500 & 1.94 & $4-16$ & 0600 & c & 147000 & 2.77 \\
\hline \multirow[t]{2}{*}{$3-6$} & 2400 & c & 99700 & 1.99 & $4-16$ & 1200 & c & 137000 & 2.90 \\
\hline & & & & & $4-16$ & 1800 & c & 136000 & 3.02 \\
\hline $3-7$ & 0200 & c & 99700 & 2.02 & $4-16$ & 2400 & c & 119000 & 3.13 \\
\hline $3-7$ & 0300 & c & 105000 & 2.04 & & & & & \\
\hline $3-7$ & 1200 & c & 96200 & 2.17 & $4-17$ & 0600 & c & 115000 & 3.24 \\
\hline $3-7$ & 1800 & c & 93000 & 2.25 & $4-17$ & 1200 & c & 115000 & 3.34 \\
\hline \multirow[t]{2}{*}{$3-7$} & 2400 & c & 95000 & 2.34 & $4-17$ & 1800 & c & 105000 & 3.44 \\
\hline & & & & & $4-17$ & 2400 & c & 96700 & 3.52 \\
\hline \multirow[t]{5}{*}{$4-11$} & 2400 & c & 35600 & 0.00 & & & & & \\
\hline & & & & & $4-18$ & 0600 & c & 99900 & 3.62 \\
\hline & & & & & $4-18$ & 1200 & c & 79100 & 3.69 \\
\hline & & & & & $4-18$ & 1800 & c & 79900 & 3.76 \\
\hline & & & & & $4-18$ & 2400 & c & 82000 & 3.84 \\
\hline
\end{tabular}

c No gage height record. 
TABLES

TABLE 7.-Gage height, discharge, and accumulated runoff, flood of April 1979-Continued 02412000, Tallapoosa River near Heflin, Ala.

\begin{tabular}{|c|c|c|c|c|c|c|c|c|c|}
\hline DATE & TIME & $\begin{array}{l}\text { GAGE } \\
\text { HEIGHT }\end{array}$ & DISCHARGE & $\begin{array}{l}\text { ACCUM. } \\
\text { RUNOFF }\end{array}$ & DATE & TIME & $\begin{array}{c}\text { GAGE } \\
\text { HEIGHT }\end{array}$ & DISCHARGE & $\begin{array}{l}\text { ACCUM. } \\
\text { RUNOFF }\end{array}$ \\
\hline $\begin{array}{l}3-01 \\
3-01\end{array}$ & $\begin{array}{l}0100 \\
2400\end{array}$ & $\begin{array}{l}5.77 \\
5.48\end{array}$ & $\begin{array}{l}905 \\
818\end{array}$ & 0.07 & $\begin{array}{l}3-06 \\
3-06\end{array}$ & $\begin{array}{l}1200 \\
2400\end{array}$ & $\begin{array}{l}26.24 \\
23.87\end{array}$ & $\begin{array}{l}18,600 \\
13,400\end{array}$ & $\begin{array}{l}4.13 \\
4.80\end{array}$ \\
\hline $\begin{array}{l}3-02 \\
3-02\end{array}$ & $\begin{array}{l}0100 \\
2400\end{array}$ & $\begin{array}{l}5.47 \\
5.22\end{array}$ & $\begin{array}{l}815 \\
740\end{array}$ & $\begin{array}{l}0.07 \\
0.13\end{array}$ & $\begin{array}{l}3-07 \\
3-07 \\
3-07\end{array}$ & $\begin{array}{l}0100 \\
1300 \\
1600\end{array}$ & $\begin{array}{l}23.61 \\
16.51 \\
11.38\end{array}$ & $\begin{array}{r}12,900 \\
6,010 \\
3,280\end{array}$ & $\begin{array}{l}4.85 \\
5.24 \\
5.29\end{array}$ \\
\hline $\begin{array}{l}3-03 \\
3-03 \\
3-03 \\
3-03 \\
3-03 \\
3-03\end{array}$ & $\begin{array}{l}1700 \\
1800 \\
1900 \\
2000 \\
2100 \\
2400\end{array}$ & $\begin{array}{r}5.31 \\
5.49 \\
5.98 \\
6.97 \\
8.37 \\
14.47\end{array}$ & $\begin{array}{r}767 \\
821 \\
968 \\
1,310 \\
1,930 \\
4,780\end{array}$ & $\begin{array}{l}0.18 \\
0.18 \\
0.19 \\
0.19 \\
0.19 \\
0.23\end{array}$ & $\begin{array}{l}3-07 \\
3-07 \\
3-07 \\
3-08 \\
3-08\end{array}$ & $\begin{array}{l}1800 \\
2100 \\
2400 \\
\\
0100 \\
2400\end{array}$ & $\begin{array}{r}8.90 \\
7.72 \\
7.44 \\
\\
7.38 \\
6.65\end{array}$ & $\begin{array}{l}2,160 \\
1,630 \\
1,500 \\
\\
1,480 \\
1,190\end{array}$ & $\begin{array}{l}5.31 \\
5.33 \\
5.35 \\
\\
5.35 \\
5.46\end{array}$ \\
\hline $\begin{array}{l}3-04 \\
3-04 \\
3-04 \\
3-04\end{array}$ & $\begin{array}{l}0400 \\
0900 \\
1200 \\
2400\end{array}$ & $\begin{array}{l}20.68 \\
26.26 \\
26.62 \\
26.59\end{array}$ & $\begin{array}{r}9,060 \\
18,700 \\
19,600 \\
19,500\end{array}$ & $\begin{array}{l}0.33 \\
0.57 \\
0.77 \\
1.59\end{array}$ & $\begin{array}{l}3-09 \\
3-09\end{array}$ & $\begin{array}{l}0100 \\
2400\end{array}$ & $\begin{array}{l}6.63 \\
6.21\end{array}$ & $\begin{array}{l}1,180 \\
1,040\end{array}$ & $\begin{array}{l}5.46 \\
5.55\end{array}$ \\
\hline $\begin{array}{l}3-05 \\
3-05\end{array}$ & $\begin{array}{l}2000 \\
2400\end{array}$ & $\begin{array}{l}27.13 \\
27.13\end{array}$ & $\begin{array}{l}20,800 \\
20,800\end{array}$ & $\begin{array}{l}2.99 \\
3.28\end{array}$ & $\begin{array}{l}3-10 \\
3-10\end{array}$ & $\begin{array}{l}0100 \\
2400\end{array}$ & $\begin{array}{l}6.19 \\
6.04\end{array}$ & $\begin{array}{r}1,030 \\
987\end{array}$ & $\begin{array}{l}5.56 \\
5.64\end{array}$ \\
\hline $3-06$ & 0500 & 27.14 & 20,900 & 3.65 & $\begin{array}{l}3-11 \\
3-11\end{array}$ & $\begin{array}{l}1900 \\
2400\end{array}$ & $\begin{array}{l}6.23 \\
6.17\end{array}$ & $\begin{array}{l}1,050 \\
1,030\end{array}$ & $\begin{array}{l}5.70 \\
5.72\end{array}$ \\
\hline
\end{tabular}


TABLE 7.-Gage height, discharge, and accumulated runoff, flood of April 1979-Continued 02412000, Tallapoosa River near Heflin, Ala.-Continued

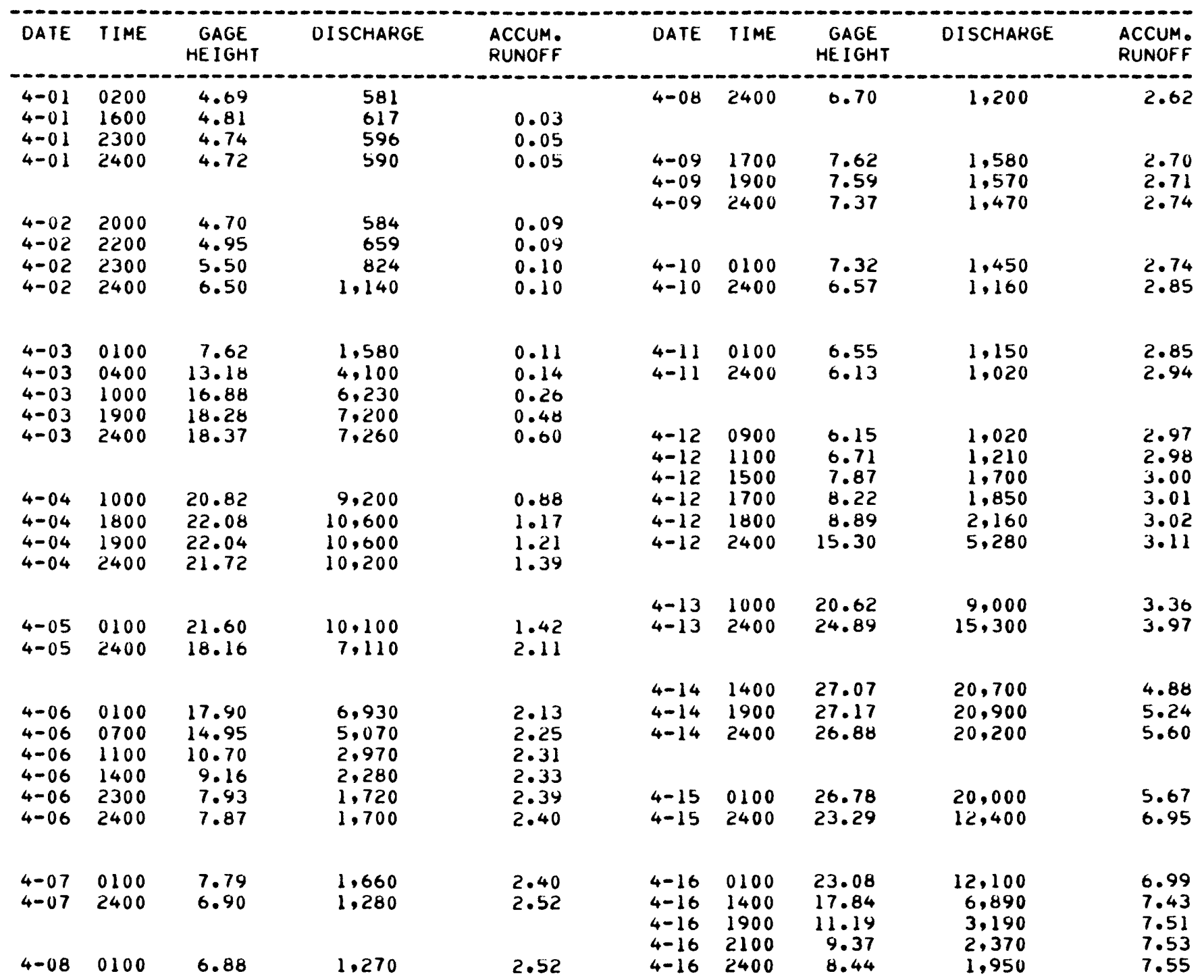


TABLES

TABLE 7.-Gage height, discharge, and accumulated runoff, flood of April 1979-Continued 02413300, Little Tallapoosa River near Newell, Ala.

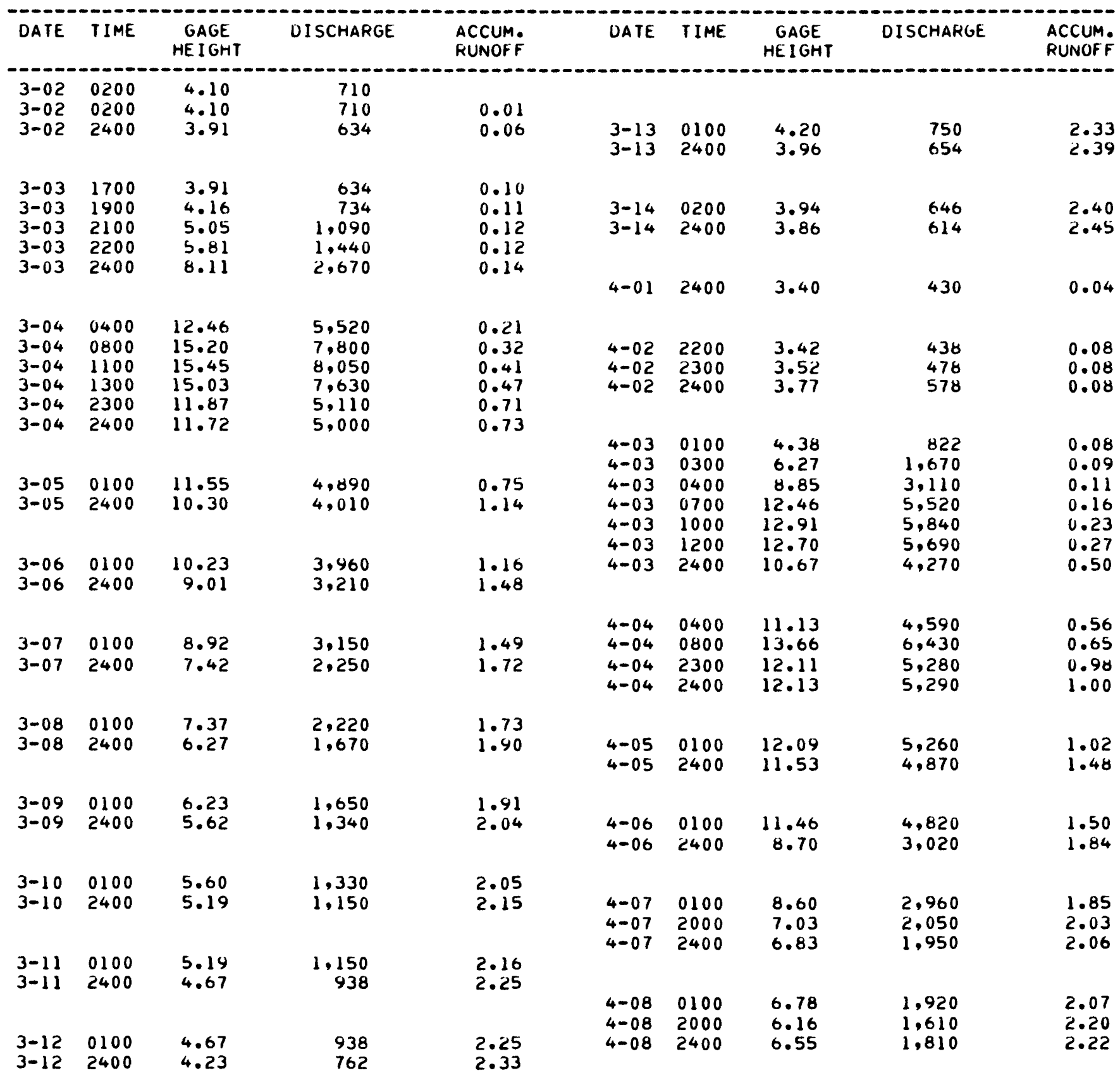


TABLE 7.-Gage height, discharge, and accumulated runoff, flood of April 1979-Continued 02413300, Little Tallapoosa River near Newell, Ala-Continued

\begin{tabular}{|c|c|c|c|c|c|c|c|c|c|}
\hline DATE & TIME & $\begin{array}{c}\text { GAGE } \\
\text { HE IGHT }\end{array}$ & OISCHARGE & $\begin{array}{l}\text { ACCUM. } \\
\text { RUNOFF }\end{array}$ & DATE & TIME & $\begin{array}{l}\text { GAGE } \\
\text { HEIGHT }\end{array}$ & DISCHARGE & $\begin{array}{l}\text { ACCUM. } \\
\text { RUNOFF }\end{array}$ \\
\hline $\begin{array}{l}4-09 \\
4-09\end{array}$ & $\begin{array}{l}0100 \\
2400\end{array}$ & $\begin{array}{l}6.64 \\
5.91\end{array}$ & $\begin{array}{l}1,850 \\
1,490\end{array}$ & $\begin{array}{l}2.23 \\
2.38\end{array}$ & $\begin{array}{l}4-13 \\
4-13\end{array}$ & $\begin{array}{l}1800 \\
2400\end{array}$ & $\begin{array}{l}16.66 \\
16.10\end{array}$ & $\begin{array}{l}9,930 \\
8,940\end{array}$ & $\begin{array}{l}3.40 \\
3.61\end{array}$ \\
\hline $\begin{array}{l}4-10 \\
4-10\end{array}$ & $\begin{array}{l}0100 \\
2400\end{array}$ & $\begin{array}{l}5.88 \\
5.31\end{array}$ & $\begin{array}{l}1,470 \\
1,190\end{array}$ & $\begin{array}{l}2.38 \\
2.50\end{array}$ & $\begin{array}{l}4-14 \\
4-14\end{array}$ & $\begin{array}{l}0700 \\
2400\end{array}$ & $\begin{array}{l}16.20 \\
15.81\end{array}$ & $\begin{array}{l}9,100 \\
8.490\end{array}$ & $\begin{array}{l}3.85 \\
4.44\end{array}$ \\
\hline $\begin{array}{l}4-11 \\
4-11\end{array}$ & $\begin{array}{l}0100 \\
2400\end{array}$ & $\begin{array}{l}5.29 \\
4.82\end{array}$ & $\begin{array}{r}1,190 \\
998\end{array}$ & $\begin{array}{l}2.51 \\
2.00\end{array}$ & $\begin{array}{l}4-15 \\
4-15\end{array}$ & $\begin{array}{l}0100 \\
2400\end{array}$ & $\begin{array}{l}15.79 \\
12.78\end{array}$ & $\begin{array}{l}8,470 \\
5,750\end{array}$ & $\begin{array}{l}4.47 \\
5.11\end{array}$ \\
\hline $\begin{array}{l}4-12 \\
4-12 \\
4-12 \\
4-12\end{array}$ & $\begin{array}{l}1000 \\
1700 \\
1800 \\
1900\end{array}$ & $\begin{array}{l}4.76 \\
5.29 \\
5.79 \\
7.03\end{array}$ & $\begin{array}{r}974 \\
1.190 \\
1.430 \\
2.050\end{array}$ & $\begin{array}{l}2.64 \\
2.67 \\
2.67 \\
2.68\end{array}$ & $\begin{array}{l}4-16 \\
4-16 \\
4-16\end{array}$ & $\begin{array}{l}0100 \\
1800 \\
2400\end{array}$ & $\begin{array}{r}12.61 \\
9.43 \\
8.75\end{array}$ & $\begin{array}{l}5,630 \\
3,460 \\
3,050\end{array}$ & $\begin{array}{l}5.13 \\
5.42 \\
5.49\end{array}$ \\
\hline $\begin{array}{l}4-12 \\
4-12 \\
4-12 \\
4-12\end{array}$ & $\begin{array}{l}2000 \\
2200 \\
2300 \\
2400\end{array}$ & $\begin{array}{r}8.77 \\
10.13 \\
10.00 \\
11.96\end{array}$ & $\begin{array}{l}3,060 \\
3,890 \\
3,800 \\
5,170\end{array}$ & $\begin{array}{l}2.69 \\
2.72 \\
2.74 \\
2.76\end{array}$ & $\begin{array}{l}4-17 \\
4-17\end{array}$ & $\begin{array}{l}0100 \\
2400\end{array}$ & $\begin{array}{l}8.67 \\
7.63\end{array}$ & $\begin{array}{r}3,000 \\
2,380\end{array}$ & $\begin{array}{l}5.50 \\
5.73\end{array}$ \\
\hline & & & & & $\begin{array}{l}4-18 \\
4-18\end{array}$ & $\begin{array}{l}0100 \\
2400\end{array}$ & $\begin{array}{l}7.60 \\
6.77\end{array}$ & $\begin{array}{l}2,360 \\
1,920\end{array}$ & $\begin{array}{l}5.74 \\
5.93\end{array}$ \\
\hline $\begin{array}{l}4-13 \\
4-13 \\
4-13 \\
4-13\end{array}$ & $\begin{array}{l}0500 \\
1000 \\
1400 \\
1500\end{array}$ & $\begin{array}{l}14.24 \\
16.67 \\
17.80 \\
17.80\end{array}$ & $\begin{array}{r}6,920 \\
9,950 \\
12,700 \\
12,700\end{array}$ & $\begin{array}{l}2.88 \\
3.04 \\
3.23 \\
3.28\end{array}$ & $\begin{array}{l}4-19 \\
4-19\end{array}$ & $\begin{array}{l}0100 \\
2400\end{array}$ & $\begin{array}{l}6.70 \\
6.05\end{array}$ & $\begin{array}{l}1,880 \\
1,560\end{array}$ & $\begin{array}{l}5.94 \\
6.09\end{array}$ \\
\hline
\end{tabular}


TABLES

TABLE 7.-Gage height, discharge, and accumulated runoff, flood of April 1979-Continued 02414500, Tallapoosa River at Wadley, Ala.

\begin{tabular}{|c|c|c|c|c|c|c|c|c|c|}
\hline DATE & TIME & $\begin{array}{c}\text { GAGE } \\
\text { HEIGHT }\end{array}$ & DISCHARGE & $\begin{array}{l}\text { ACCUM . } \\
\text { RUNOFF }\end{array}$ & DATE. & TIME & $\begin{array}{c}\text { GAGE } \\
\text { HE IGHT }\end{array}$ & DISCHARGE & $\begin{array}{l}\text { ACCUM. } \\
\text { RUNOFF }\end{array}$ \\
\hline $3-2$ & 2400 & 5.51 & 3500 & 0.00 & $\begin{array}{l}4-8 \\
4-8\end{array}$ & $\begin{array}{l}2000 \\
2400\end{array}$ & $\begin{array}{l}7.37 \\
7.67\end{array}$ & $\begin{array}{l}6630 \\
7240\end{array}$ & $\begin{array}{l}2.44 \\
2.47\end{array}$ \\
\hline $3-3$ & 1745 & 5.53 & 3530 & 0.06 & & & & & \\
\hline $3-3$ & 2100 & 6.06 & 4350 & 0.07 & $4-9$ & 0100 & 7.75 & 7420 & 2.47 \\
\hline $3-3$ & 2400 & 6.98 & 5890 & 0.09 & $\begin{array}{l}4-9 \\
4-9\end{array}$ & $\begin{array}{l}0900 \\
2400\end{array}$ & $\begin{array}{l}8.57 \\
7.67\end{array}$ & $\begin{array}{l}9280 \\
7240\end{array}$ & $\begin{array}{l}2.54 \\
2.64\end{array}$ \\
\hline $3-4$ & 0130 & 7.99 & 7950 & 0.10 & & & & & \\
\hline $3-4$ & 0630 & 13.01 & 19500 & 0.19 & $4-10$ & 2400 & 7.10 & 6110 & 2.78 \\
\hline $3-4$ & 0915 & 15.67 & 25700 & 0.26 & & & & & \\
\hline $3-4$ & 1000 & 16.90 & 28700 & 0.28 & $4-11$ & 2400 & 6.61 & 5250 & 2.90 \\
\hline $3-4$ & 1430 & 26.67 & 67600 & 0.55 & & & & & \\
\hline $3-4$ & 1815 & 28.23 & 76200 & 0.83 & $4-12$ & 1200 & 6.47 & 5010 & 2.95 \\
\hline $3-4$ & 1845 & 28.12 & 75600 & 0.85 & $4-12$ & 1700 & 6.88 & 5710 & 2.98 \\
\hline $3-4$ & 2400 & 26.17 & 64900 & 1.18 & $4-12$ & 2100 & 8.89 & 10000 & 3.02 \\
\hline & & & & & $4-12$ & 2400 & 11.81 & 16700 & 3.07 \\
\hline $3-5$ & 0015 & 26.03 & 64100 & 1.19 & & & & & \\
\hline $3-5$ & 0400 & 23.55 & 51500 & 1.38 & $4-13$ & 0100 & 13.41 & 20400 & 3.08 \\
\hline $3-5$ & 1000 & 21.84 & 43300 & 1.62 & $4-13$ & 0600 & 17.55 & 30400 & 3.23 \\
\hline $3-5$ & 2400 & 18.84 & 33800 & 2.06 & $4-13$ & 1200 & 22.04 & 40100 & 3.45 \\
\hline & & & & & $4-13$ & 1800 & 27.75 & 73600 & 3.86 \\
\hline $3-6$ & 0015 & 18.76 & 33600 & 2.07 & $4-13$ & 2300 & 30.29 & 87500 & 4.27 \\
\hline $3-6$ & 1015 & 17.52 & 30300 & 2.35 & $4-13$ & 2400 & 30.35 & 87800 & 4.35 \\
\hline $3-6$ & 2130 & 16.39 & 27400 & 2.63 & & & & & \\
\hline & & & & & $4-14$ & 0200 & 30.57 & 89100 & 4.52 \\
\hline $3-7$ & 0915 & 15.71 & 25800 & 2.92 & $4-14$ & 0400 & 29.94 & 85600 & 4.68 \\
\hline $3-7$ & 0930 & 15.67 & 25700 & 2.92 & $4-14$ & 1200 & 26.77 & 68200 & 5.19 \\
\hline $3-7$ & 2400 & 13.94 & 21600 & 3.22 & $4-14$ & 2400 & 22.02 & 44000 & 5.68 \\
\hline $3-8$ & 0030 & 13.84 & 21400 & 3.23 & $4-15$ & 0300 & 21.37 & 41500 & 5.80 \\
\hline $3-8$ & 1045 & 11.62 & 16300 & 3.38 & $4-15$ & 0900 & 20.50 & 38800 & 6.02 \\
\hline $3-8$ & 1745 & 8.55 & 9240 & 3.44 & $4-15$ & 2400 & 19.05 & 34400 & 6.50 \\
\hline $3-9$ & 0900 & 7.33 & 6550 & 3.54 & $4-16$ & 1200 & 17.44 & 30100 & 6.83 \\
\hline $3-9$ & 0915 & 7.32 & 6530 & 3.54 & $4-16$ & 1800 & 16.31 & 27200 & 6.99 \\
\hline 3- 9 & 2400 & 6.92 & 5790 & 3.62 & $4-16$ & 2400 & 15.08 & 24300 & 7.12 \\
\hline $4-2$ & 2400 & 4.89 & 2610 & 0.00 & $4-17$ & 0600 & 14.02 & 21800 & 7.24 \\
\hline & 0200 & 4.96 & 2700 & 0.01 & $4-17$ & 1200 & 12.63 & 18600 & 7.35 \\
\hline $4-3$ & 0600 & 6.16 & 4510 & 0.02 & $4-17$ & 1800 & 10.13 & 12900 & 7.42 \\
\hline $4-3$ & 1000 & 12.82 & 19100 & 0.09 & $4-17$ & 2400 & 8.85 & 9920 & 7.48 \\
\hline $4-3$ & 1200 & 14.67 & 23300 & 0.14 & $4-18$ & 2400 & 8.01 & 7990 & 7.66 \\
\hline $4-3$ & 1400 & 16.28 & 27200 & 0.19 & $4-10$ & 2400 & 0.01 & 00000 & 1.00 \\
\hline $4-3$ & 2000 & 18.39 & 32600 & 0.37 & & & & & \\
\hline $4-3$ & 2400 & 17.62 & 30600 & 0.48 & $4-19$ & 0100 & 7.96 & 7880 & 7.66 \\
\hline $4-4$ & 0345 & 16.30 & 27200 & 0.57 & & 2400 & 7.45 & 6790 & 7.81 \\
\hline $4-4$ & 1200 & 17.77 & 31000 & 0.82 & $4-20$ & 240.0 & 7.08 & 6070 & 7.94 \\
\hline $4-4$ & 1945 & 18.94 & 34100 & 1.06 & & & & & \\
\hline $4-4$ & 2400 & 18.22 & 32200 & 1.19 & $4-21$ & 0100 & 7.04 & 6000 & 7.95 \\
\hline $4-5$ & $120 \rho$ & 15.35 & 24900 & 1.47 & $4-21$ & 2400 & 6.73 & 5450 & 8.07 \\
\hline $4-5$ & 2400 & 14.35 & 22600 & 1.73 & $4-22$ & 2400 & 6.53 & 5110 & 8.18 \\
\hline $\begin{array}{l}4-6 \\
4-6\end{array}$ & $\begin{array}{l}0900 \\
2400\end{array}$ & $\begin{array}{l}13.85 \\
11.81\end{array}$ & $\begin{array}{l}21400 \\
16700\end{array}$ & $\begin{array}{l}1.91 \\
2.14\end{array}$ & & & & & \\
\hline $4-7$ & 2400 & 7.96 & 7880 & 2.32 & & & & & \\
\hline
\end{tabular}


TABLE 7.--Gage height, discharge, and accumulated runoff, flood of April 1979-Continued 02420000, Alabama River near Montgomery, Ala.

\begin{tabular}{|c|c|c|c|c|c|c|c|c|c|}
\hline DATE & TIME & $\begin{array}{c}\text { GAGE } \\
\text { HEIGHT }\end{array}$ & DISCHARGE & $\begin{array}{l}\text { ACCUM. } \\
\text { RUNOFF }\end{array}$ & DATE & TIME & $\begin{array}{c}\text { GAGE } \\
\text { HEIGHT }\end{array}$ & DISCHARGE & $\begin{array}{l}\text { ACCUM. } \\
\text { RUNOFF }\end{array}$ \\
\hline $2-28$ & 2400 & 32.88 & 77700 & $\ldots \ldots$ & $3-9$ & 2400 & 40.98 & 142000 & $\ldots$ \\
\hline $3-1$ & 0600 & 32.20 & 74600 & $\ldots$ & $3-10$ & 0600 & 40.38 & 138000 & .. \\
\hline $3-1$ & 1200 & 32.02 & 72000 & $\ldots \ldots$ & $3-10$ & 1200 & 39.87 & 135000 & $\ldots$ \\
\hline $3-1$ & 1800 & 31.75 & 68700 & $\ldots \ldots$ & $3-10$ & 1800 & 39.40 & 132000 & $\ldots$ \\
\hline $3-1$ & 2400 & 31.41 & 66400 & $\ldots \ldots$ & $3-10$ & 2400 & 39.05 & 129000 & $\ldots$ \\
\hline $3-2$ & 0600 & 31.25 & 62400 & $\ldots \ldots$ & $3-11$ & 0600 & 38.65 & 126000 & $\ldots$ \\
\hline $3-2$ & 1200 & 31.10 & 60300 & $\ldots \ldots$ & $3-11$ & 1200 & 38.10 & 124000 & $\ldots$ \\
\hline $3-2$ & 1800 & 30.91 & 58100 & $\ldots \ldots$ & $3-11$ & 1800 & 37.60 & 120000 & $\ldots$ \\
\hline $3-2$ & 2400 & 30.74 & 59800 & $\ldots \ldots$ & $3-11$ & 2400 & 37.20 & 118000 & $\ldots \ldots$ \\
\hline $3-3$ & 0600 & 30.58 & 58600 & . & $3-12$ & 0600 & 36.91 & 117000 & \\
\hline $3-3$ & 1200 & 30.40 & 60500 & $\ldots \ldots$ & $3-12$ & 1200 & 36.78 & 116000 & $\ldots$ \\
\hline $3-3$ & 1800 & 30.30 & 60600 & $\ldots \ldots$ & $3-12$ & 1800 & 36.60 & 115000 & $\ldots$ \\
\hline $3-3$ & 2400 & 31.90 & 71000 & $\ldots \ldots$ & $3-12$ & 2400 & 36.30 & 112000 & ..... \\
\hline $3-4$ & 0600 & 34.90 & 100000 & $\ldots \ldots$ & $3-13$ & 0600 & 35.92 & 110000 & \\
\hline $3-4$ & 1200 & 38.20 & 127000 & $\ldots \ldots$ & $3-13$ & 1200 & 35.69 & 109000 & $\ldots$ \\
\hline $3-4$ & 1800 & 41.00 & 143000 & $\ldots \ldots$ & $3-13$ & 1800 & 35.48 & 108000 & ...... \\
\hline $3-4$ & 2400 & 43.70 & 160000 & $\ldots \ldots$ & $3-13$ & 2400 & 35.20 & 105000 & $\ldots \ldots$ \\
\hline $3-5$ & 0600 & 45.68 & 172000 & $\ldots \ldots$ & $3-14$ & 0600 & $\ldots$ & 102000 & \\
\hline $3-5$ & 1200 & 46.80 & 179000 & $\ldots \ldots$ & $3-14$ & 1200 & $\ldots \ldots$ & 101000 & $\ldots$ \\
\hline $3-5$ & 1800 & 47.90 & 185000 & $\ldots \ldots$ & $3-14$ & 1800 & .... & 100000 & $\ldots$ \\
\hline $3-5$ & 2400 & 48.30 & 183000 & $\ldots \ldots$ & $3-14$ & 2400 & $\ldots$ & 97300 & $\ldots \ldots$ \\
\hline $3-6$ & 0600 & 48.40 & 181000 & $\ldots \ldots$ & $3-15$ & 0600 & $\ldots$ & 94100 & $\ldots$ \\
\hline $3-6$ & 1200 & 48.40 & 179000 & $\ldots \ldots$ & $3-15$ & 1200 & $\ldots$ & 94100 & $\ldots$ \\
\hline $3-6$ & 1800 & 48.10 & 176000 & $\ldots \ldots$ & $3-15$ & 1800 & $\ldots \ldots$ & 89900 & $\ldots$ \\
\hline $3-6$ & 2400 & 47.83 & 174000 & $\ldots \ldots$ & $3-15$ & 2400 & $\ldots \ldots$ & 86200 & $\ldots$ \\
\hline 3- 7 & 0600 & 47.28 & 171000 & $\ldots \ldots$ & $3-16$ & 0600 & $\ldots$ & 84300 & \\
\hline $3-7$ & 1200 & 46.60 & 169000 & $\ldots \ldots$ & $3-16$ & 1200 & $\ldots \ldots$ & 79700 & $\ldots$ \\
\hline $3-7$ & 1800 & 45.97 & 167000 & $\ldots \ldots$ & $3-16$ & 1800 & $\ldots$. & 75600 & $\ldots$ \\
\hline $3-7$ & 2400 & 45.28 & 163000 & $\ldots \ldots$ & $3-16$ & 2400 & $\ldots \ldots$ & 73000 & $\cdots$ \\
\hline $3-8$ & 0600 & 44.60 & 160000 & $\ldots$ & $3-17$ & 0600 & $\ldots$ & 67300 & \\
\hline $3-8$ & 1200 & 44.05 & 156000 & $\ldots \ldots$ & $3-17$ & 1200 & $\ldots \ldots$ & 65600 & $\ldots$ \\
\hline $3-8$ & 1800 & 43.56 & 154000 & $\ldots \ldots$ & $3-17$ & 1800 & $\ldots$. & 63000 & ...... \\
\hline $3-8$ & 2400 & 43.00 & 151000 & $\ldots \ldots$ & $3-17$ & 2400 & $\ldots$ & 61600 & $\ldots \ldots$ \\
\hline 3- 9 & 0600 & 42.50 & 148000 & $\ldots$ & $3-18$ & 0600 & & 58000 & \\
\hline $3-9$ & 1200 & 41.90 & 146000 & $\ldots \ldots$ & $3-18$ & 1200 & .... & 53700 & $\ldots$ \\
\hline 3- 9 & 1800 & 41.50 & 144000 & $\ldots \ldots$ & $3-18$ & 1800 & .... & 53400 & \\
\hline
\end{tabular}


TABLES

TABLE 7.-Gage height, discharge, and accumulated runoff, flood of April 1979-Continued 02420000, Alabama River near Montgomery, Ala-Continued

\begin{tabular}{|c|c|c|c|c|c|c|c|c|c|}
\hline DATE & TIME & $\begin{array}{c}\text { GAGE } \\
\text { HEIGHT }\end{array}$ & DISCHARGE & $\begin{array}{l}\text { ACCUM. } \\
\text { RUNOFF }\end{array}$ & DATE & TIME & $\begin{array}{c}\text { GAGE } \\
\text { HEIGHT }\end{array}$ & DISCHARGE & $\begin{array}{l}\text { ACCUM. } \\
\text { RUNOFF }\end{array}$ \\
\hline $3-18$ & 2400 & $\ldots$ & 52100 & $\ldots \ldots$ & $\begin{array}{l}3-27 \\
3-27\end{array}$ & $\begin{array}{l}1800 \\
2400\end{array}$ & $\begin{array}{c}\ldots \ldots \\
\ldots \ldots\end{array}$ & $\begin{array}{l}29000 \\
28500\end{array}$ & $\begin{array}{l}\ldots \ldots \\
\ldots \ldots\end{array}$ \\
\hline $3-19$ & 0600 & $\ldots \ldots$ & 51600 & $\ldots \ldots$ & & & & & \\
\hline $3-19$ & 1200 & $\ldots$ & 50300 & $\ldots \ldots$ & $3-28$ & 0600 & $\ldots \ldots$ & 34500 & $\ldots \ldots$ \\
\hline $3-19$ & 1800 & $\ldots$ & 49400 & $\ldots \ldots$ & $3-28$ & 1200 & $\ldots$. & 29500 & $\ldots \ldots$ \\
\hline $3-19$ & 2400 & $\ldots \ldots$ & 47200 & $\ldots \ldots$ & $\begin{array}{l}3-28 \\
3-28\end{array}$ & $\begin{array}{l}1800 \\
2400\end{array}$ & $\ldots$ & $\begin{array}{l}27700 \\
36000\end{array}$ & $\ldots \ldots$ \\
\hline $3-20$ & 0600 & $\ldots$ & 45400 & $\ldots \ldots$ & & & & & \\
\hline $3-20$ & 1200 & $\ldots \ldots$ & 45200 & $\ldots \ldots$ & $3-29$ & 0600 & $\ldots$. & 32800 & $\ldots \ldots$ \\
\hline $3-20$ & 1800 & $\ldots \ldots$ & 45500 & $\ldots \ldots$ & $3-29$ & 1200 & $\ldots \ldots$ & 31400 & $\ldots \ldots$ \\
\hline $3-20$ & 2400 & $\ldots$ & 47400 & $\ldots \ldots$ & $\begin{array}{l}3-29 \\
3-29\end{array}$ & $\begin{array}{l}1800 \\
2400\end{array}$ & $\ldots$ & $\begin{array}{l}28400 \\
26800\end{array}$ & $\ldots \ldots$ \\
\hline $3-21$ & 0600 & $\ldots$ & 40800 & $\ldots \ldots$ & & & & & \\
\hline $3-21$ & 1200 & $\ldots$ & 39200 & $\ldots \ldots$ & $3-30$ & 0600 & $\ldots \ldots$ & 33200 & $\ldots \ldots$ \\
\hline $3-21$ & 1800 & $\ldots$. & 41400 & $\ldots \ldots$ & $3-30$ & 1200 & $\ldots \ldots$ & 28800 & $\ldots \ldots$ \\
\hline $3-21$ & 2400 & $\ldots$ & 40200 & ..... & $\begin{array}{l}3-30 \\
3-30\end{array}$ & $\begin{array}{l}1800 \\
2400\end{array}$ & $\ldots$ & $\begin{array}{l}29700 \\
28600\end{array}$ & $\begin{array}{l}\cdots \cdots \\
\ldots \ldots\end{array}$ \\
\hline $3-22$ & 0600 & $\ldots$ & 40200 & $\ldots \ldots$ & & & & & \\
\hline $3-22$ & 1200 & $\ldots$ & 42400 & $\ldots \ldots$ & $3-31$ & 0600 & $\ldots$. & 21800 & $\ldots \ldots$ \\
\hline $3-22$ & 1800 & $\ldots \ldots$ & 45400 & $\ldots \ldots$ & $3-31$ & 1200 & $\ldots \ldots$ & 27500 & $\ldots \ldots$ \\
\hline $3-22$ & 2400 & $\ldots$ & 45800 & $\ldots \ldots$ & $\begin{array}{l}3-31 \\
3-31\end{array}$ & $\begin{array}{l}1800 \\
2400\end{array}$ & $\ldots$ & $\begin{array}{l}22400 \\
18500\end{array}$ & $\ldots \ldots$ \\
\hline $3-23$ & 0600 & $\ldots$ & 46900 & $\ldots \ldots$ & & & & & \\
\hline $3-23$ & 1200 & $\ldots \ldots$ & 49400 & $\ldots \ldots$ & $4-1$ & 0600 & $\ldots$. & 16800 & $\ldots$ \\
\hline $3-23$ & 1800 & $\ldots$ & 51900 & $\ldots \ldots$ & $4-1$ & 1200 & $\ldots$. & 18500 & $\ldots \ldots$ \\
\hline $3-23$ & 2400 & $\ldots$ & 52600 & $\ldots \ldots$ & $\begin{array}{l}4-1 \\
4-1\end{array}$ & $\begin{array}{l}1800 \\
2400\end{array}$ & $\ldots$ & $\begin{array}{l}16800 \\
17300\end{array}$ & $\ldots \ldots$ \\
\hline $3-24$ & 0600 & $\ldots$ & 52200 & $\ldots \ldots$ & & & & & \\
\hline $3-24$ & 1200 & $\ldots \ldots$ & 51000 & $\ldots \ldots$ & 4- 2 & 0600 & $\ldots \ldots$ & 16600 & $\ldots$ \\
\hline $3-24$ & 1800 & $\ldots$ & 50000 & $\ldots \ldots$ & $4-2$ & 1200 & $\ldots$. & 16600 & $\ldots \ldots$ \\
\hline $3-24$ & 2400 & $\ldots$ & 46200 & $\ldots \ldots$ & $\begin{array}{l}4-2 \\
4-2\end{array}$ & $\begin{array}{l}1800 \\
2400\end{array}$ & $\ldots$ & $\begin{array}{l}17200 \\
23300\end{array}$ & $\ldots \ldots$ \\
\hline $3-25$ & 0600 & $\ldots$ & 43700 & $\ldots \ldots$ & & & & & \\
\hline $3-25$ & 1200 & $\ldots$ & 40400 & $\ldots \ldots$ & $4-3$ & 0600 & $\ldots \ldots$ & 43600 & $\ldots$ \\
\hline $3-25$ & 1800 & $\ldots$ & 41400 & $\ldots \ldots$ & $4-3$ & 1200 & $\ldots \ldots$ & 80800 & $\ldots$ \\
\hline $3-25$ & 2400 & $\ldots$ & 39100 & $\ldots \ldots$ & $\begin{array}{l}4-3 \\
4-3\end{array}$ & $\begin{array}{l}1800 \\
2400\end{array}$ & $\ldots \ldots$ & $\begin{array}{l}105000 \\
121000\end{array}$ & $\ldots \ldots$ \\
\hline $3-26$ & 0600 & $\ldots \ldots$ & 38300 & $\ldots \ldots$ & & & & & \\
\hline $3-26$ & 1200 & $\ldots$. & 32000 & $\ldots \ldots$ & $4-4$ & 0600 & 38.10 & 118000 & $\ldots$ \\
\hline $3-26$ & 1800 & $\ldots \ldots$ & 29100 & $\ldots \ldots$ & $4-4$ & 1200 & 39.70 & 118000 & $\ldots .$. \\
\hline $3-26$ & 2400 & .... & 35400 & $\ldots \ldots$ & $\begin{array}{l}4-4 \\
4-4\end{array}$ & $\begin{array}{l}1800 \\
2400\end{array}$ & $\begin{array}{l}40.95 \\
42.05\end{array}$ & $\begin{array}{l}119000 \\
121000\end{array}$ & $\begin{array}{l}\ldots \ldots \\
\ldots \ldots\end{array}$ \\
\hline $3-27$ & 0600 & $\ldots$ & 33200 & $\ldots \ldots$ & & & & & \\
\hline $3-27$ & 1200 & $\ldots \ldots$ & 32100 & $\ldots \ldots$ & 4- 5 & 0600 & 42.55 & 125000 & $\ldots$ \\
\hline
\end{tabular}


TABLE 7.-Gage height, discharge, and accumulated runoff, flood of April 1979-Continued 02420000, Alabama River near Montgomery, Ala-Continued

\begin{tabular}{|c|c|c|c|c|c|c|c|c|c|}
\hline DATE & TIME & $\begin{array}{c}\text { GAGE } \\
\text { HEIGHT }\end{array}$ & DISCHARGE & $\begin{array}{l}\text { ACCUM. } \\
\text { RUNOFF }\end{array}$ & DATE & TIME & $\begin{array}{c}\text { GAGE } \\
\text { HEIGHT }\end{array}$ & DISCHARGE & $\begin{array}{l}\text { ACCUM. } \\
\text { RUNOFF }\end{array}$ \\
\hline $4-5$ & 1200 & 43.02 & 128000 & $\ldots \ldots$ & $4-14$ & 0600 & 45.30 & 183000 & $\ldots \ldots$ \\
\hline $4-5$ & 1800 & 43.32 & 126000 & $\ldots \ldots$ & $4-14$ & 1200 & 48.10 & 210000 & $\ldots \ldots$ \\
\hline $4-5$ & 2400 & 43.35 & 126000 & $\ldots \ldots$ & $\begin{array}{l}4-14 \\
4-14\end{array}$ & $\begin{array}{l}1800 \\
2400\end{array}$ & $\begin{array}{l}50.00 \\
51.70\end{array}$ & $\begin{array}{l}229000 \\
245000\end{array}$ & $\begin{array}{l}\ldots \ldots \\
\ldots \ldots\end{array}$ \\
\hline $4-6$ & 0600 & 43.30 & 126000 & $\ldots \ldots$ & & & & & $\cdots \cdots$ \\
\hline $4-6$ & 1200 & 43.25 & 131000 & $\ldots \ldots$ & $4-15$ & 0600 & 52.63 & 254000 & $\ldots \ldots$ \\
\hline $4-6$ & 1800 & 42.82 & 142000 & $\ldots \ldots$ & $4-15$ & 1200 & 53.37 & 258000 & $\ldots \ldots$ \\
\hline $4-6$ & 2400 & 42.46 & 144000 & $\ldots \ldots$ & $\begin{array}{l}4-15 \\
4-15\end{array}$ & $\begin{array}{l}1800 \\
2400\end{array}$ & $\begin{array}{l}53.87 \\
54.20\end{array}$ & $\begin{array}{l}260000 \\
259000\end{array}$ & $\begin{array}{l}\ldots \ldots \\
\ldots \ldots\end{array}$ \\
\hline $4-7$ & 0600 & 42.42 & 142000 & $\ldots \ldots$ & & & & & \\
\hline $4-7$ & 1200 & 41.45 & 140000 & $\ldots \ldots$ & $4-16$ & 0600 & 54.40 & 258000 & $\ldots .$. \\
\hline $4-7$ & 1800 & 41.05 & 140000 & $\ldots \ldots$ & $4-16$ & 1200 & 54.50 & 254000 & $\ldots \ldots$ \\
\hline $4-7$ & 2400 & 40.38 & 140000 & $\ldots \ldots$ & $\begin{array}{l}4-16 \\
4-16\end{array}$ & $\begin{array}{l}1800 \\
2400\end{array}$ & $\begin{array}{l}54.41 \\
54.22\end{array}$ & $\begin{array}{l}250000 \\
241000\end{array}$ & $\ldots \ldots$ \\
\hline $4-8$ & 0600 & 39.75 & 135000 & $\ldots \ldots$ & & & & & \\
\hline $4-8$ & 1200 & 39.15 & 130000 & $\ldots \ldots$ & $4-17$ & 0600 & 53.82 & 236000 & $\ldots \ldots$ \\
\hline $4-8$ & 1800 & 38.52 & 126000 & $\ldots \ldots$ & $4-17$ & 1200 & 53.36 & 229000 & $\ldots \ldots$ \\
\hline $4-8$ & 2400 & 37.87 & 123000 & $\ldots \ldots$ & $\begin{array}{l}4-17 \\
4-17\end{array}$ & $\begin{array}{l}1800 \\
2400\end{array}$ & $\begin{array}{l}52.92 \\
52.29\end{array}$ & $\begin{array}{l}221000 \\
212000\end{array}$ & $\begin{array}{l}\cdots \cdots \\
\cdots \cdots\end{array}$ \\
\hline $4-9$ & 0600 & 37.20 & 120000 & $\ldots \ldots$ & & & & & \\
\hline $4-9$ & 1200 & 36.69 & 116000 & $\ldots \ldots$ & $4-18$ & 0600 & 51.70 & 204000 & ...... \\
\hline $4-9$ & 1800 & 36.00 & 112000 & $\ldots \ldots$ & $4-18$ & 1200 & 51.00 & 197000 & $\ldots \ldots$ \\
\hline $4-9$ & 2400 & 35.52 & 108000 & $\ldots \ldots$ & $\begin{array}{l}4-18 \\
4-18\end{array}$ & $\begin{array}{l}1800 \\
2400\end{array}$ & $\begin{array}{l}50.00 \\
48.99\end{array}$ & $\begin{array}{l}189000 \\
180000\end{array}$ & $\ldots \ldots$ \\
\hline $4-10$ & 0600 & 34.90 & 104000 & $\ldots \ldots$ & & & & & \\
\hline $4-10$ & 1200 & 34.40 & 99400 & $\ldots \ldots$ & $4-19$ & 0600 & 48.00 & 170000 & $\ldots$ \\
\hline $4-10$ & 1800 & 33.89 & 96100 & $\ldots \ldots$ & $4-19$ & 1200 & 47.08 & 163000 & $\ldots$ \\
\hline $4-10$ & 2400 & 33.30 & 92300 & $\ldots \ldots$ & $\begin{array}{l}4-19 \\
4-19\end{array}$ & $\begin{array}{l}1800 \\
2400\end{array}$ & $\begin{array}{l}46.02 \\
45.10\end{array}$ & $\begin{array}{l}158000 \\
151000\end{array}$ & $\cdots$ \\
\hline $4-11$ & 0600 & 32.82 & 86700 & $\ldots \ldots$ & & & & & \\
\hline $4-11$ & 1200 & 32.48 & 83300 & $\ldots \ldots$ & $4-20$ & 0600 & 43.80 & 143000 & $\ldots$ \\
\hline $4-11$ & 1800 & 32.10 & 80600 & $\ldots \ldots$ & $4-20$ & 1200 & 42.75 & 138000 & $\ldots \ldots$ \\
\hline $4-11$ & 2400 & 32.72 & 77700 & $\ldots \ldots$ & $\begin{array}{l}4-20 \\
4-20\end{array}$ & $\begin{array}{l}1800 \\
2400\end{array}$ & $\begin{array}{l}41.60 \\
40.40\end{array}$ & $\begin{array}{l}133000 \\
128000\end{array}$ & $\ldots \ldots$ \\
\hline $4-12$ & 0600 & 32.38 & 75000 & $\cdots \cdots$ & & & & & \\
\hline $4-12$ & 1200 & 31.00 & 68800 & $\ldots \ldots$ & $4-21$ & 0600 & 39.50 & 125000 & $\ldots$ \\
\hline $4-12$ & 1800 & 30.92 & 69400 & $\ldots \ldots$ & $4-21$ & 1200 & 38.26 & 124000 & ...... \\
\hline $4-12$ & 2400 & 32.10 & 83000 & $\ldots$ & $\begin{array}{l}4-21 \\
4-21\end{array}$ & $\begin{array}{l}1800 \\
2400\end{array}$ & $\begin{array}{l}37.30 \\
36.20\end{array}$ & $\begin{array}{l}116000 \\
112000\end{array}$ & $\ldots \ldots$ \\
\hline $4-13$ & 0600 & 34.60 & 108000 & $\ldots \ldots$ & & & & & \\
\hline $4-13$ & 1200 & 36.90 & 128000 & $\ldots \ldots$ & & & & & \\
\hline $4-13$ & 1800 & 39.40 & 142000 & $\ldots \ldots$ & & & & & \\
\hline $4-13$ & 2400 & 42.00 & 159000 & ..... & & & & & \\
\hline
\end{tabular}


TABLE 7.-Gage height, discharge, and accumulated runoff, flood of April 1979-Continued 02422500, Mulberry Creek at Jones, Ala.

\begin{tabular}{|c|c|c|c|c|c|c|c|c|c|}
\hline DATE. & TIME & $\begin{array}{c}\text { GAGE } \\
\text { HEIGHT }\end{array}$ & ISCHARGE & $\begin{array}{l}\text { ACCUM. } \\
\text { RUNOFF }\end{array}$ & DATE & TIME & $\begin{array}{c}\text { GAGE } \\
\text { HEIGHT }\end{array}$ & ISCHARGE & $\begin{array}{l}\text { ACCUM. } \\
\text { RUNOFF }\end{array}$ \\
\hline $3-2$ & 2400 & 3.35 & 352 & 0.00 & $4-11$ & 2400 & 3.66 & 920 & 0.00 \\
\hline $3-3$ & 1100 & 3.41 & 374 & 0.03 & $4-12$ & 1100 & 3.63 & 902 & 0.07 \\
\hline $3-3$ & 1400 & 3.59 & 446 & 0.04 & $4-12$ & 1600 & 3.76 & 980 & 0.11 \\
\hline $3-3$ & 1600 & 3.97 & 625 & 0.05 & $4-12$ & 1800 & 3.96 & 1100 & 0.13 \\
\hline $3-3$ & 1800 & 5.48 & 1510 & 0.07 & $4-12$ & 1900 & 4.29 & 1200 & 0.14 \\
\hline $3-3$ & 2000 & 7.26 & 2640 & 0.11 & $4-12$ & 2100 & 10.53 & 5070 & 0.21 \\
\hline $3-3$ & 2100 & 8.53 & 3640 & 0.14 & $4-12$ & 2300 & 13.66 & 7260 & 0.32 \\
\hline $3-3$ & 2200 & 10.63 & 5140 & 0.18 & $4-12$ & 2400 & 14.70 & 8060 & 0.38 \\
\hline $3-3$ & 2400 & 15.45 & 8660 & 0.31 & & & & & \\
\hline & & & & & $4-13$ & 0400 & 16.83 & 9850 & 0.67 \\
\hline $3-4$ & 0400 & 19.90 & 13100 & 0.70 & $4-13$ & 1200 & 17.49 & 10500 & 1.30 \\
\hline $3-4$ & 1100 & 20.92 & 14200 & 1.44 & $4-13$ & 1700 & 20.23 & 13500 & 1.80 \\
\hline $3-4$ & 1800 & 22.37 & 16000 & 2.27 & $4-13$ & 2400 & 23.99 & 18500 & 2.77 \\
\hline $3-4$ & 2000 & 22.06 & 15600 & 2.50 & & & & & \\
\hline $3-4$ & 2400 & 18.94 & 12000 & 2.86 & $4-14$ & 0500 & 25.26 & 20900 & 3.55 \\
\hline & & & & & $4-14$ & 1500 & 12.03 & 6128 & 4.00 \\
\hline $3-5$ & 0100 & 17.74 & 10700 & 2.94 & $4-14$ & 1900 & 8.84 & 4000 & 4.12 \\
\hline $3-5$ & 0500 & 11.73 & 5910 & 3.12 & $4-14$ & 2400 & 7.07 & 2920 & 4.23 \\
\hline $3-5$ & 0900 & 8.36 & 3640 & 3.23 & & & & & \\
\hline $3-5$ & 1200 & 6.90 & 2980 & 3.29 & $4-15$ & 0100 & 6.86 & 2770 & 4.25 \\
\hline $3-5$ & 1700 & 5.86 & 2000 & 3.37 & $4-15$ & 0800 & 5.99 & 2160 & 4.36 \\
\hline $3-5$ & 2400 & 5.27 & 1890 & 3.47 & $4-15$ & 2400 & 5.07 & 1610 & 4.55 \\
\hline $3-6$ & 0100 & 5.19 & 1788 & 3.48 & $4-16$ & 0100 & 5.02 & 1580 & 4.57 \\
\hline $3-6$ & 2100 & 4.34 & 1330 & 3.68 & $4-16$ & 2400 & 4.36 & 1180 & 4.77 \\
\hline $3-6$ & 2400 & 4.27 & 1290 & 3.71 & & & & & \\
\hline & & & & & $4-17$ & 0100 & 4.34 & 1170 & 4.78 \\
\hline $3-7$ & 0100 & 4.26 & 1280 & 3.72 & $4-17$ & 2400 & 3.99 & 962 & 4.94 \\
\hline $3-7$ & 2000 & 3.97 & 1110 & 3.87 & & & & & \\
\hline $3-7$ & 2400 & 3.94 & 1090 & 3.98 & $\begin{array}{l}4-18 \\
4-18\end{array}$ & $\begin{array}{l}0100 \\
2400\end{array}$ & $\begin{array}{l}3.97 \\
3.72\end{array}$ & $\begin{array}{l}950 \\
800\end{array}$ & $\begin{array}{l}4.95 \\
5.09\end{array}$ \\
\hline $3-8$ & 0100 & 3.92 & 1080 & 3.91 & & & & & \\
\hline $3-8$ & 2400 & 3.65 & 914 & 4.07 & $\begin{array}{l}4-19 \\
4-19\end{array}$ & $\begin{array}{l}0200 \\
2400\end{array}$ & $\begin{array}{l}3.71 \\
3.53\end{array}$ & $\begin{array}{l}794 \\
695\end{array}$ & $\begin{array}{l}5.10 \\
5.21\end{array}$ \\
\hline $3-9$ & 0100 & 3.64 & 908 & & & & & & \\
\hline $3-9$ & 2400 & 3.43 & 782 & 4.21 & & & & & \\
\hline
\end{tabular}


TABLE 7.-Gage height, discharge, and accumulated runoff, flood of April 1979-Continued 02423425, Cahaba River near Cahaba Heights, Ala.

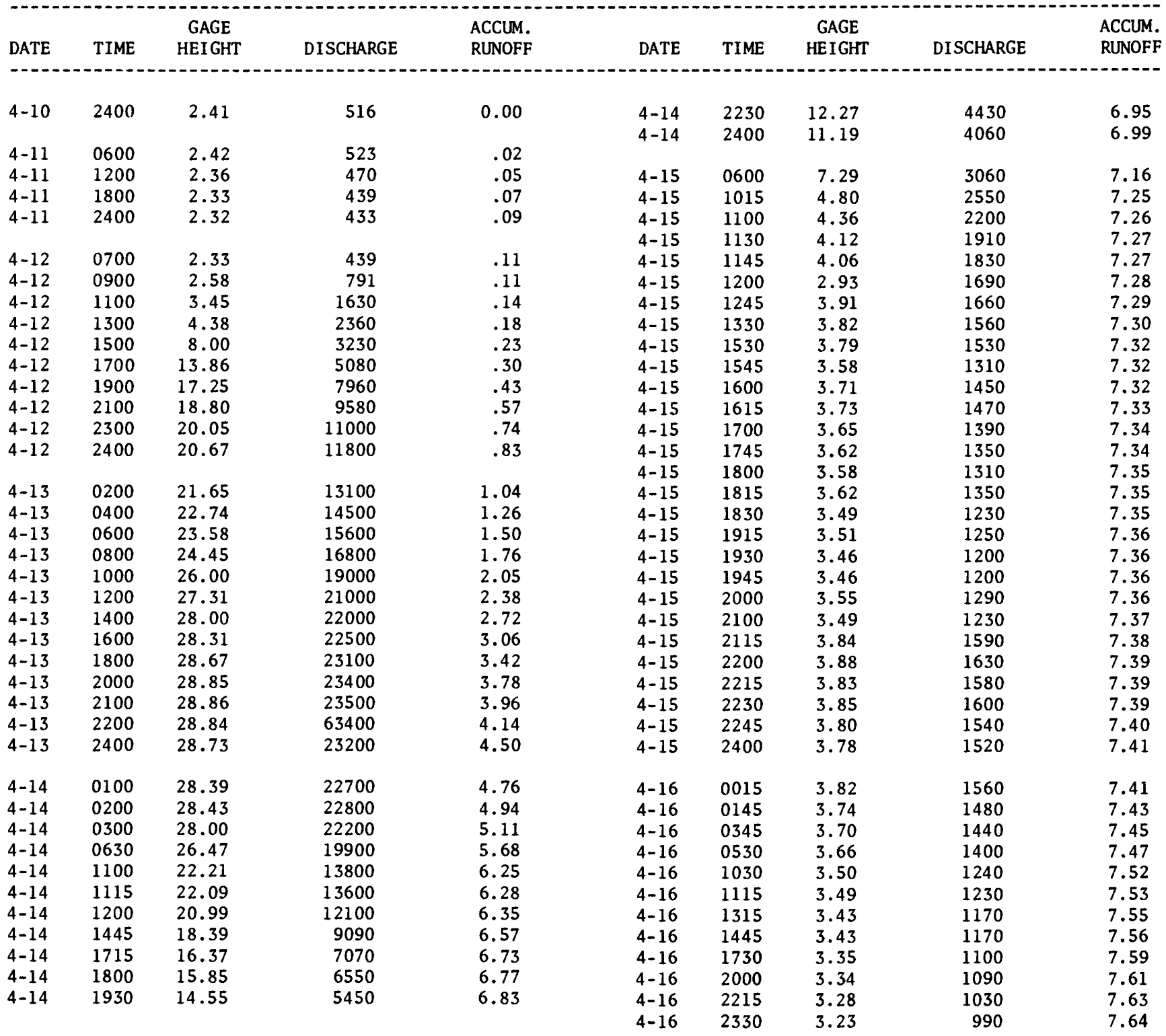


TABLES

TABLE 7.-Gage height, discharge, and accumulated runoff, flood of April 1979-Continued 02423425, Cahaba River near Cahaba Heights, Ala.-Continued

\begin{tabular}{|c|c|c|c|c|c|c|c|c|c|}
\hline & & GAGE & & ACCUM. & & & GAGE & & ACCUM. \\
\hline DATE & TIME & HEIGHT & DISCHARGE & RUNOFF & DATE & TIME & HEIGHT & DISCHARGE & RUNOFF \\
\hline \multirow[t]{2}{*}{$4-16$} & 2400 & 3.27 & 1030 & 7.64 & $4-19$ & 0745 & 2.48 & 437 & 7.93 \\
\hline & & & & & $4-19$ & 0815 & 2.48 & 437 & 7.93 \\
\hline $4-17$ & 0045 & 3.25 & 1010 & 7.65 & $4-19$ & 0845 & 2.43 & 405 & 7.93 \\
\hline $4-17$ & 0100 & 3.26 & 1020 & 7.65 & $4-19$ & 1215 & 2.41 & 392 & 7.94 \\
\hline $4-17$ & 0430 & 3.20 & 963 & 7.67 & $4-19$ & 1530 & 2.38 & 374 & 7.95 \\
\hline $4-17$ & 1015 & 3.08 & 865 & 7.72 & $4-19$ & 1630 & 2.40 & 386 & 7.96 \\
\hline $4-17$ & 1200 & 3.08 & 865 & 7.73 & $4-19$ & 2130 & 2.37 & 368 & 7.97 \\
\hline $4-17$ & 1245 & 3.04 & 833 & 7.73 & $4-19$ & 2200 & 2.38 & 374 & 7.97 \\
\hline $4-17$ & 1300 & 3.07 & 857 & 7.73 & $4-19$ & 2215 & 2.36 & 362 & 7.97 \\
\hline $4-17$ & 1400 & 3.03 & 824 & 7.74 & $4-19$ & 2400 & 2.36 & 362 & 7.98 \\
\hline \multirow[t]{2}{*}{$4-17$} & 2400 & 2.92 & 738 & 7.80 & & & & & \\
\hline & & & & & $4-20$ & 0430 & 2.37 & 368 & 7.99 \\
\hline $4-18$ & 0015 & 2.92 & 738 & 7.80 & $4-20$ & 0530 & 2.41 & 392 & 7.99 \\
\hline $4-18$ & 0100 & 2.89 & 715 & 7.81 & $4-20$ & 0745 & 2.42 & 399 & 8.00 \\
\hline $4-18$ & 0115 & 2.85 & 685 & 7.81 & $4-20$ & 0800 & 2.39 & 380 & 8.00 \\
\hline $4-18$ & 0130 & 2.86 & 692 & 7.81 & $4-20$ & 0900 & 2.38 & 374 & 8.00 \\
\hline $4-18$ & 0230 & 2.81 & 655 & 7.81 & $4-20$ & 0915 & 2.34 & 351 & 8.00 \\
\hline $4-18$ & 0415 & 2.78 & 634 & 7.82 & $4-20$ & 1045 & 2.34 & 351 & 8.01 \\
\hline $4-18$ & 0445 & 2.79 & 641 & 7.82 & $4-20$ & 1115 & 2.32 & 339 & 8.01 \\
\hline $4-18$ & 0845 & 2.75 & 613 & 7.84 & $4-20$ & 1145 & 2.32 & 339 & 8.01 \\
\hline $4-18$ & 0900 & 2.72 & 592 & 7.85 & $4-20$ & 1215 & 2.30 & 327 & 8.01 \\
\hline $4-18$ & 1045 & 2.71 & 585 & 7.85 & $4-20$ & 1500 & 2.30 & 327 & 8.02 \\
\hline $4-18$ & 1115 & 2.69 & 571 & 7.86 & $4-20$ & 1715 & 2.29 & 322 & 8.02 \\
\hline $4-18$ & 1130 & 2.71 & 585 & 7.86 & $4-20$ & 2015 & 2.27 & 311 & 8.03 \\
\hline $4-18$ & 1430 & 2.67 & 558 & 7.87 & $4-20$ & 2400 & 2.27 & 311 & 8.04 \\
\hline $4-18$ & 1545 & 2.66 & 552 & 7.87 & & & & & \\
\hline $4-18$ & 1600 & 2.60 & 512 & 7.88 & $4-21$ & 0245 & 2.27 & 311 & 8.05 \\
\hline $4-18$ & 1800 & 2.51 & 456 & 7.88 & $4-21$ & 0345 & 2.29 & 322 & 8.05 \\
\hline $4-18$ & 1930 & 2.48 & 437 & 7.89 & $4-21$ & 0900 & 2.29 & 322 & 8.06 \\
\hline $4-18$ & 2000 & 2.49 & 444 & 7.89 & $4-21$ & 0915 & 2.28 & 316 & 8.06 \\
\hline $4-18$ & 2045 & 2.47 & 431 & 7.89 & $4-21$ & 1045 & 2.26 & 305 & 8.07 \\
\hline \multirow[t]{2}{*}{$4-18$} & 2400 & 2.46 & 424 & 7.90 & $4-21$ & 1115 & 2.24 & 295 & 8.07 \\
\hline & & & & & $4-21$ & 1415 & 2.23 & 289 & 8.08 \\
\hline $4-19$ & 0115 & 2.45 & 418 & 7.91 & $4-21$ & 1445 & 2.21 & 278 & 8.08 \\
\hline $4-19$ & 0145 & 2.47 & 431 & 7.91 & $4-21$ & 2345 & 2.21 & 278 & 8.10 \\
\hline $4-19$ & 0300 & 2.48 & 437 & 7.91 & $4-21$ & 2400 & 2.22 & 284 & 8.10 \\
\hline $4-19$ & 0315 & 2.46 & 424 & 7.91 & & & & & \\
\hline $4-19$ & 0400 & 2.48 & 437 & 7.92 & $4-22$ & 0500 & 2.24 & 295 & 8.11 \\
\hline $4-19$ & 0500 & 2.47 & 431 & 7.92 & $4-22$ & 0745 & 2.26 & 305 & 8.11 \\
\hline $4-19$ & 0515 & 2.49 & 444 & 7.92 & $4-22$ & 0845 & 2.26 & 305 & 8.12 \\
\hline $4-19$ & 0530 & 2.48 & 437 & 7.92 & $4-22$ & 0900 & 2.23 & 289 & 8.12 \\
\hline $4-19$ & 0600 & 2.50 & 450 & 7.92 & $4-22$ & 0930 & 2.23 & 289 & 8.12 \\
\hline $4-19$ & 0700 & 2.50 & 450 & 7.93 & $4-22$ & 1030 & 2.19 & 268 & 8.12 \\
\hline
\end{tabular}


TABLE 7.-Gage height, discharge, and accumulated runoff, flood of April 1979-Continued 02423425, Cahaba River near Cahaba Heights, Ala-Continued

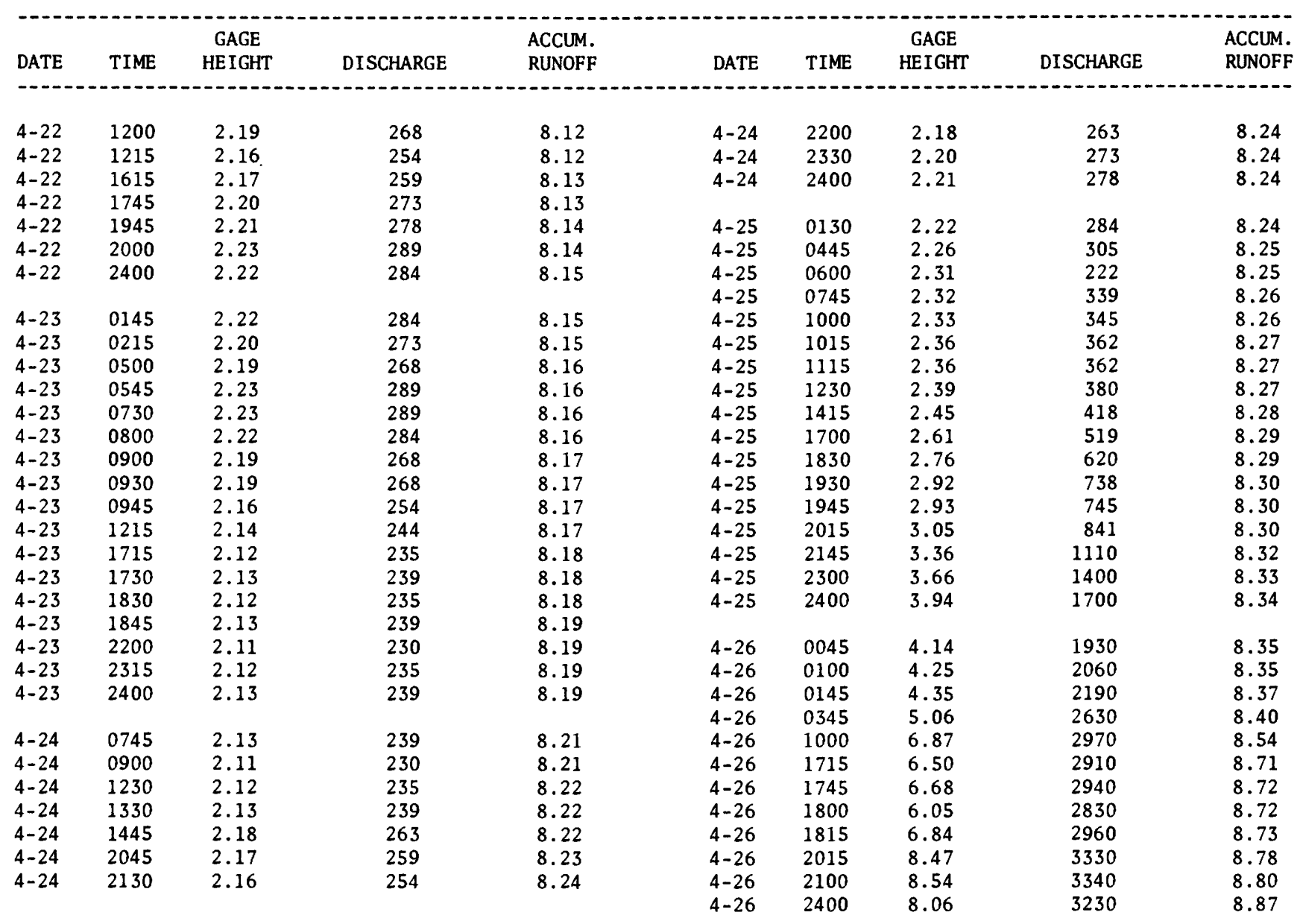


TABLES

TABLE 7.-Gage height, discharge, and accumulated runoff, flood of April 1979-Continued 02424000, Cahaba River at Centreville, Ala.

\begin{tabular}{|c|c|c|c|c|c|c|c|c|c|}
\hline DATE & TIME & $\begin{array}{l}\text { GAGE } \\
\text { HE IGHT }\end{array}$ & DISCHARGE & $\begin{array}{l}\text { ACCUM. } \\
\text { RUNOFF }\end{array}$ & DATE & TIME & $\begin{array}{c}\text { GAGE } \\
\text { HEIGHT }\end{array}$ & DISCHARGE & $\begin{array}{l}\text { ACCUM. } \\
\text { RUNOFF }\end{array}$ \\
\hline $\begin{array}{l}4-11 \\
4-11 \\
4-11\end{array}$ & $\begin{array}{l}0600 \\
1200 \\
1800\end{array}$ & $\begin{array}{l}7.17 \\
6.98 \\
6.81\end{array}$ & $\begin{array}{l}2600 \\
2490 \\
2400\end{array}$ & $\begin{array}{l}.02 \\
.05 \\
.07\end{array}$ & $\begin{array}{l}4-16 \\
4-16 \\
4-16 \\
4-16\end{array}$ & $\begin{array}{l}0600 \\
1200 \\
1800 \\
2400\end{array}$ & $\begin{array}{l}24.47 \\
21.65 \\
18.13 \\
14.99\end{array}$ & $\begin{array}{r}17900 \\
11000 \\
8040 \\
6430\end{array}$ & $\begin{array}{l}6.15 \\
6.25 \\
6.32 \\
6.38\end{array}$ \\
\hline $4-11$ & 2400 & 6.66 & 2310 & .09 & $\begin{array}{l}4-17 \\
4-17\end{array}$ & $\begin{array}{l}0600 \\
1200\end{array}$ & $\begin{array}{l}12.94 \\
11.85\end{array}$ & $\begin{array}{l}5860 \\
5460\end{array}$ & $\begin{array}{l}6.43 \\
6.48\end{array}$ \\
\hline $\begin{array}{l}4-12 \\
4-12 \\
4-12\end{array}$ & $\begin{array}{l}0600 \\
0800 \\
1200\end{array}$ & $\begin{array}{l}6.55 \\
6.51 \\
7.62\end{array}$ & $\begin{array}{l}2250 \\
2230 \\
3420\end{array}$ & $\begin{array}{l}.11 \\
.12 \\
.14\end{array}$ & $\begin{array}{l}4-17 \\
4-17\end{array}$ & $\begin{array}{l}1800 \\
2400\end{array}$ & $\begin{array}{l}11.18 \\
10.66\end{array}$ & $\begin{array}{l}5060 \\
4760\end{array}$ & $\begin{array}{l}6.53 \\
6.57\end{array}$ \\
\hline $\begin{array}{l}4-12 \\
4-12 \\
4-12 \\
4-12\end{array}$ & $\begin{array}{l}1400 \\
1600 \\
1800 \\
2000\end{array}$ & $\begin{array}{r}9.90 \\
13.58 \\
17.58 \\
22.26\end{array}$ & $\begin{array}{r}6350 \\
11100 \\
17000 \\
24500\end{array}$ & $\begin{array}{l}.16 \\
.19 \\
.24 \\
.31\end{array}$ & $\begin{array}{l}4-18 \\
4-18 \\
4-18 \\
4-18\end{array}$ & $\begin{array}{l}0600 \\
1200 \\
1800 \\
2400\end{array}$ & $\begin{array}{r}10.26 \\
9.93 \\
9.62 \\
9.31\end{array}$ & $\begin{array}{l}4490 \\
4400 \\
4180 \\
3970\end{array}$ & $\begin{array}{l}6.61 \\
6.65 \\
6.69\end{array}$ \\
\hline $4-12$ & 2200 & 25.78 & 29800 & .40 & & & & & \\
\hline $4-12$ & 2400 & 28.01 & 33800 & .51 & $\begin{array}{l}4-19 \\
4-19\end{array}$ & $\begin{array}{l}1200 \\
2400\end{array}$ & $\begin{array}{l}8.69 \\
8.17\end{array}$ & $\begin{array}{l}3550 \\
3210\end{array}$ & $\begin{array}{l}6.79 \\
6.85\end{array}$ \\
\hline $\begin{array}{l}4-13 \\
4-13 \\
4-13 \\
4-13\end{array}$ & $\begin{array}{l}0400 \\
0800 \\
1200 \\
1600\end{array}$ & $\begin{array}{l}30.38 \\
31.41 \\
32.96 \\
34.11\end{array}$ & $\begin{array}{l}42700 \\
48300 \\
57900 \\
68100\end{array}$ & $\begin{array}{l}.76 \\
1.05 \\
1.40 \\
1.81\end{array}$ & $\begin{array}{l}4-20 \\
4-20\end{array}$ & $\begin{array}{l}1200 \\
2400\end{array}$ & $\begin{array}{l}7.86 \\
7.63\end{array}$ & $\begin{array}{l}3020 \\
2880\end{array}$ & $\begin{array}{l}6.90 \\
6.96\end{array}$ \\
\hline $\begin{array}{l}4-13 \\
4-13 \\
4-13\end{array}$ & $\begin{array}{l}2000 \\
2300 \\
2400\end{array}$ & $\begin{array}{l}34.82 \\
35.03 \\
35.03\end{array}$ & $\begin{array}{l}75800 \\
78400 \\
78400\end{array}$ & $\begin{array}{l}2.27 \\
2.62 \\
2.74\end{array}$ & $\begin{array}{l}4-21 \\
4-21\end{array}$ & $\begin{array}{l}1200 \\
2400\end{array}$ & $\begin{array}{l}7.34 \\
7.11\end{array}$ & $\begin{array}{l}2700 \\
2570\end{array}$ & $\begin{array}{l}7.00 \\
7.05\end{array}$ \\
\hline $\begin{array}{l}4-14 \\
4-14 \\
4-14 \\
4-14 \\
4-14\end{array}$ & $\begin{array}{l}0200 \\
0600 \\
1200 \\
1800 \\
2400\end{array}$ & $\begin{array}{l}34.84 \\
34.46 \\
33.31 \\
31.77 \\
30.70\end{array}$ & $\begin{array}{l}76100 \\
71600 \\
60700 \\
50400 \\
44300\end{array}$ & $\begin{array}{l}2.97 \\
3.40 \\
3.95 \\
4.41 \\
4.81\end{array}$ & $\begin{array}{l}4-22 \\
4-22\end{array}$ & $\begin{array}{l}1200 \\
2400\end{array}$ & $\begin{array}{l}6.98 \\
6.88\end{array}$ & $\begin{array}{l}2490 \\
2430\end{array}$ & $\begin{array}{l}7.10 \\
7.14\end{array}$ \\
\hline $\begin{array}{l}4-15 \\
4-15 \\
4-15 \\
4-15\end{array}$ & $\begin{array}{l}0600 \\
1200 \\
1800 \\
2400\end{array}$ & $\begin{array}{l}29.84 \\
28.90 \\
27.77 \\
26.36\end{array}$ & $\begin{array}{l}40000 \\
35400 \\
30900 \\
24700\end{array}$ & $\begin{array}{l}5.17 \\
5.49 \\
5.77 \\
5.99\end{array}$ & & & & & \\
\hline
\end{tabular}


TABLE 7.-Gage height, discharge, and accumulated runoff, flood of April 1979-Continued 02425000, Cahaba River near Marion Jct., Ala.

\begin{tabular}{|c|c|c|c|c|c|c|c|c|c|}
\hline DATE & TIME & $\begin{array}{c}\text { GAGE } \\
\text { HEIGHT }\end{array}$ & DISCHARGE & $\begin{array}{l}\text { ACCUM. } \\
\text { RUNOFF }\end{array}$ & DATE & TIME & $\begin{array}{c}\text { GAGE } \\
\text { HEIGHT }\end{array}$ & DISCHARGE & $\begin{array}{l}\text { ACCUM. } \\
\text { RUNOFF }\end{array}$ \\
\hline $4-10$ & 2400 & 15.59 & 8770 & 0.00 & $\begin{array}{l}4-16 \\
4-16\end{array}$ & $\begin{array}{l}0100 \\
2400\end{array}$ & $\begin{array}{l}40.77 \\
39.05\end{array}$ & $\begin{array}{l}69500 \\
48900\end{array}$ & $\begin{array}{l}2.97 \\
3.96\end{array}$ \\
\hline $4-11$ & 0100 & 15.47 & 8690 & 0.01 & & & & & \\
\hline $4-11$ & 2400 & 12.31 & 6470 & 0.14 & $\begin{array}{l}4-17 \\
4-17\end{array}$ & $\begin{array}{l}0100 \\
2400\end{array}$ & $\begin{array}{l}38.96 \\
37.24\end{array}$ & $\begin{array}{l}47800 \\
35200\end{array}$ & $\begin{array}{l}4.00 \\
4.71\end{array}$ \\
\hline $4-12$ & 1300 & 11.66 & 6070 & 0.21 & & & & & \\
\hline $4-12$ & 1800 & 11.88 & 6220 & 0.23 & $4-18$ & 0100 & 37.15 & 34900 & 4.74 \\
\hline $\begin{array}{l}4-12 \\
4-12\end{array}$ & $\begin{array}{l}2100 \\
2400\end{array}$ & $\begin{array}{l}15.94 \\
19.36\end{array}$ & $\begin{array}{r}9360 \\
12000\end{array}$ & $\begin{array}{l}0.26 \\
0.29\end{array}$ & $4-18$ & 2400 & 33.81 & 27600 & 5.38 \\
\hline $4-13$ & 2400 & 27.44 & 19500 & 0.70 & $\begin{array}{l}4-19 \\
4-19\end{array}$ & $\begin{array}{l}0100 \\
2400\end{array}$ & $\begin{array}{l}33.60 \\
28.16\end{array}$ & $\begin{array}{l}27300 \\
20300\end{array}$ & $\begin{array}{l}5.32 \\
5.73\end{array}$ \\
\hline $\begin{array}{l}4-14 \\
4-14 \\
4-14\end{array}$ & $\begin{array}{l}1800 \\
2200 \\
2400\end{array}$ & $\begin{array}{l}35.91 \\
38.01 \\
38.97\end{array}$ & $\begin{array}{l}31000 \\
39200 \\
47900\end{array}$ & $\begin{array}{l}1.19 \\
1.33 \\
1.41\end{array}$ & $\begin{array}{l}4-20 \\
4-20\end{array}$ & $\begin{array}{l}0100 \\
2400\end{array}$ & $\begin{array}{l}27.89 \\
22.48\end{array}$ & $\begin{array}{l}20000 \\
14500\end{array}$ & $\begin{array}{l}5.75 \\
6.04\end{array}$ \\
\hline $\begin{array}{l}4-15 \\
4-15 \\
4-15 \\
4-15\end{array}$ & $\begin{array}{l}0600 \\
1400 \\
1800 \\
2400\end{array}$ & $\begin{array}{l}40.64 \\
41.13 \\
41.08 \\
40.82\end{array}$ & $\begin{array}{l}68000 \\
73900 \\
73300 \\
70100\end{array}$ & $\begin{array}{l}1.77 \\
2.29 \\
2.54 \\
2.91\end{array}$ & $\begin{array}{l}4-21 \\
4-21\end{array}$ & $\begin{array}{l}0100 \\
2400\end{array}$ & $\begin{array}{l}22.30 \\
18.60\end{array}$ & $\begin{array}{l}14300 \\
11400\end{array}$ & $\begin{array}{l}6.05 \\
6.28\end{array}$ \\
\hline
\end{tabular}


TABLES

TABLE 7.-Gage height, discharge, and accumulated runoff, flood of April 1979-Continued 02429500, Alabama River at Claiborne, Ala.

\begin{tabular}{|c|c|c|c|c|c|c|c|c|c|}
\hline DATE & TIME & $\begin{array}{c}\text { GAGE } \\
\text { HEIGHT }\end{array}$ & DISCHARGE & $\begin{array}{l}\text { ACCUM. } \\
\text { RUNOFF }\end{array}$ & DATE & TIME & $\begin{array}{l}\text { GAGE } \\
\text { HEIGHT }\end{array}$ & DISCHARGE & $\begin{array}{l}\text { ACCUM. } \\
\text { RUNOFF }\end{array}$ \\
\hline \multirow[t]{2}{*}{$2-28$} & 2400 & 41.60 & 118000 & 0.00 & $3-14$ & 0600 & 48.30 & 169000 & 3.49 \\
\hline & & & & & $3-14$ & 1200 & 48.00 & 165000 & 3.56 \\
\hline $3-1$ & 0600 & 41.70 & 118000 & .05 & $3-14$ & 1800 & 47.70 & 162000 & 3.63 \\
\hline $3-1$ & 1200 & 41.73 & 117000 & .10 & $3-14$ & 2400 & 47.40 & 156000 & 3.70 \\
\hline $3-1$ & 1800 & 41.70 & 116000 & .15 & & & & & \\
\hline 3- 1 & 2400 & 41.62 & 114000 & .20 & $\begin{array}{l}3-15 \\
3-15\end{array}$ & $\begin{array}{l}0600 \\
1200\end{array}$ & $\begin{array}{l}47.00 \\
46.65\end{array}$ & $\begin{array}{l}152000 \\
150000\end{array}$ & $\begin{array}{l}3.76 \\
3.82\end{array}$ \\
\hline $3-2$ & 0600 & 41.50 & 111000 & .24 & $3-15$ & 1800 & 46.20 & 144000 & 3.88 \\
\hline 3- 2 & 1200 & 41.25 & 109000 & .29 & $3-15$ & 2400 & 45.85 & 141000 & 3.94 \\
\hline $3-2$ & 1800 & 40.90 & 103000 & .33 & & & & & \\
\hline \multirow{2}{*}{$3-2$} & 2400 & 40.45 & 101000 & .38 & $3-16$ & 0600 & 45.40 & 137000 & 4.00 \\
\hline & & & & & $3-16$ & 1200 & 44.98 & 131000 & 4.06 \\
\hline $3-3$ & 0600 & 40.00 & 96300 & .42 & $3-16$ & 1800 & 44.50 & 127000 & 4.11 \\
\hline $3-3$ & 1200 & 39.33 & 93300 & .46 & $3-16$ & 2400 & 44.00 & 123000 & 4.16 \\
\hline $3-3$ & 1800 & 39.20 & 102000 & .50 & & & & & \\
\hline \multirow[t]{2}{*}{$3-3$} & 2400 & 39.50 & 115000 & .55 & $3-17$ & 0600 & 43.46 & 119000 & 4.21 \\
\hline & & & & & $3-17$ & 1200 & 42.95 & 114000 & 4.26 \\
\hline $3-4$ & 0600 & 40.16 & 119000 & .60 & $3-17$ & 1800 & 42.40 & 110000 & 4.31 \\
\hline $3-4$ & 1200 & 40.80 & 125000 & .65 & $3-17$ & 2400 & 41.85 & 106000 & 4.35 \\
\hline $3-4$ & 1800 & 41.52 & 128000 & .70 & & & & & \\
\hline \multirow[t]{2}{*}{$3-4$} & 2400 & 42.20 & 132000 & .76 & $3-18$ & 0600 & 41.20 & 103000 & 4.40 \\
\hline & & & & & $3-18$ & 1200 & 40.55 & 98200 & 4.44 \\
\hline $3-5$ & 0600 & 42.70 & 134000 & .82 & $3-18$ & 1800 & 39.80 & 93300 & 4.48 \\
\hline $3-5$ & 1200 & 43.20 & 138000 & .88 & $3-18$ & 2400 & 39.00 & 91800 & 4.52 \\
\hline $3-5$ & 1800 & 43.70 & 142000 & .94 & & & & & \\
\hline \multirow[t]{2}{*}{$3-5$} & 2400 & 44.20 & 145000 & 1.00 & $3-19$ & 0600 & 38.30 & 87700 & 4.55 \\
\hline & & & & & 3-19 & 1200 & 37.45 & 82000 & 4.59 \\
\hline 3- 6 & 0600 & 44.62 & 148000 & 1.06 & $3-19$ & 1800 & 36.62 & 77500 & 4.62 \\
\hline 3- 6 & 1200 & 45.02 & 150000 & 1.12 & $3-19$ & 2400 & 35.74 & 75500 & 4.65 \\
\hline 3- 6 & 1800 & 45.40 & 154000 & 1.19 & & & & & \\
\hline \multirow[t]{2}{*}{ 3- 6} & 2400 & 45.75 & 158000 & 1.25 & $3-20$ & 0600 & 34.90 & 71900 & 4.68 \\
\hline & & & & & $3-20$ & 1200 & 34.15 & 68900 & 4.71 \\
\hline $3-7$ & 0600 & 46.10 & 162000 & 1.32 & $3-20$ & 1800 & 33.50 & 67100 & 4.74 \\
\hline $3-7$ & 1200 & 46.40 & 165000 & 1.39 & $3-20$ & 2400 & 32.92 & 64800 & 4.77 \\
\hline 3- 7 & 1800 & 46.70 & 168000 & 1.46 & & & & & \\
\hline \multirow[t]{2}{*}{ 3- 7} & 2400 & 46.95 & 171000 & 1.54 & $3-21$ & 0600 & 32.20 & 61300 & 4.79 \\
\hline & & & & & $3-21$ & 1200 & 31.35 & 56900 & 4.82 \\
\hline $3-8$ & 0600 & 47.18 & 173000 & 1.61 & $3-21$ & 1800 & 30.20 & 51700 & 4.84 \\
\hline $3-8$ & 1200 & 47.45 & 175000 & 1.68 & $3-21$ & 2400 & 29.18 & 50800 & 4.86 \\
\hline $3-8$ & 1800 & 47.65 & 175000 & 1.76 & & & & & \\
\hline $3-8$ & 2400 & 47.89 & 176000 & 1.83 & $3-22$ & 0600 & 28.65 & 51400 & 4.88 \\
\hline 3- 9 & 0600 & 48.10 & 180000 & 1.91 & $3-22$ & 1200 & 28.28 & 51800 & 4.90 \\
\hline $3-9$ & 1200 & 48.30 & 183000 & 1.98 & $3-22$ & 1800 & 27.95 & 50800 & 4.93 \\
\hline 3- 9 & 1800 & 48.50 & 185000 & 2.06 & $3-22$ & 2400 & 27.82 & 53000 & 4.95 \\
\hline 3- 9 & 2400 & 48.70 & 188000 & 2.14 & $3-23$ & 0600 & 28.05 & 56500 & 4.97 \\
\hline & 0600 & 48.85 & 190000 & & $\begin{array}{l}3-23 \\
3-23\end{array}$ & 1200 & $\begin{array}{l}28.05 \\
28.65\end{array}$ & $\begin{array}{l}56500 \\
62600\end{array}$ & $\begin{array}{l}4.97 \\
5.00\end{array}$ \\
\hline $3-10$ & 1200 & 49.00 & 192000 & $\begin{array}{l}2.22 \\
2.30\end{array}$ & $3-23$ & 1800 & 29.25 & 65000 & 5.03 \\
\hline $3-10$ & 1800 & 49.25 & 197000 & $\begin{array}{l}2.30 \\
2.39\end{array}$ & $3-23$ & 2400 & 29.90 & 66500 & 5.05 \\
\hline \multirow{2}{*}{$3-10$} & 2400 & 49.36 & 196000 & 2.47 & & & & & \\
\hline & & & & & $3-24$ & 0600 & 30.54 & 67900 & 5.08 \\
\hline $3-11$ & 0600 & 49.48 & 196000 & 2.55 & $3-24$ & 1200 & 30.85 & 69800 & 5.11 \\
\hline $3-11$ & 1200 & 49.57 & 197000 & 2.64 & $3-24$ & 1800 & 31.25 & 71000 & 5.14 \\
\hline $3-11$ & 1800 & 49.58 & 195000 & 2.72 & $3-24$ & 2400 & 31.72 & 71700 & 5.17 \\
\hline $3-11$ & 2400 & 49.60 & 193000 & 2.80 & $3-25$ & 0600 & 31.95 & 71300 & 5.20 \\
\hline $3-12$ & 0600 & 49.58 & 191000 & 2.88 & $3-25$ & 1200 & 32.10 & 70600 & 5.23 \\
\hline $3-12$ & 1200 & 49.55 & 191000 & 2.96 & $3-25$ & 1800 & 32.20 & 69700 & 5.26 \\
\hline $3-12$ & 1800 & 49.50 & 188000 & 3.04 & $3-25$ & 2400 & 31.95 & 65800 & 5.29 \\
\hline \multirow[t]{2}{*}{$3-12$} & 2400 & 49.37 & 185000 & 3.12 & & & & & \\
\hline & & & & & $3-26$ & 0600 & 31.95 & 62400 & $\begin{array}{l}5.32 \\
5.34\end{array}$ \\
\hline $3-13$ & $\begin{array}{l}0600 \\
1200\end{array}$ & $\begin{array}{l}49.20 \\
49.00\end{array}$ & $\begin{array}{l}183000 \\
180000\end{array}$ & 3.20 & $\begin{array}{l}3-20 \\
3-26\end{array}$ & $\begin{array}{l}1200 \\
1800\end{array}$ & $\begin{array}{l}31.52 \\
31.00\end{array}$ & $\begin{array}{l}61500 \\
56900\end{array}$ & $\begin{array}{l}5.34 \\
5.37\end{array}$ \\
\hline $3-13$ & $\begin{array}{l}1200 \\
1800\end{array}$ & $\begin{array}{l}49.00 \\
48.80\end{array}$ & $\begin{array}{l}180000 \\
176000\end{array}$ & 3.27 & $3-26$ & 2400 & 30.02 & 53600 & 5.39 \\
\hline $3-13$ & 2400 & 48.55 & $\begin{array}{l}176000 \\
172000\end{array}$ & $\begin{array}{l}3.35 \\
3.42\end{array}$ & & & & & \\
\hline
\end{tabular}


TABLE 7.-Gage height, discharge, and accumulated runoff, flood of April 1979-Continued 02429500, Alabama River at Claiborne, Ala.-Continued

\begin{tabular}{|c|c|c|c|c|c|c|c|c|c|}
\hline DATE & TIME & $\begin{array}{c}\text { GAGE } \\
\text { HE IGHT }\end{array}$ & DISCHARGE & $\begin{array}{l}\text { ACCUM, } \\
\text { RUNOFF }\end{array}$ & DATE & TIME & $\begin{array}{c}\text { GAGE } \\
\text { HEIGHT }\end{array}$ & DISCHARGE & $\begin{array}{l}\text { ACCUM } \\
\text { RUNOFI }\end{array}$ \\
\hline $\begin{array}{l}3-27 \\
3-27 \\
3-27 \\
3-27\end{array}$ & $\begin{array}{l}0600 \\
1200 \\
1800 \\
2400\end{array}$ & $\begin{array}{l}29.18 \\
28.25 \\
27.63 \\
26.88\end{array}$ & $\begin{array}{l}50300 \\
47400 \\
45600 \\
43400\end{array}$ & $\begin{array}{l}5.41 \\
5.43 \\
5.45 \\
5.47\end{array}$ & $\begin{array}{l}4-9 \\
4-9 \\
4-9 \\
4-9\end{array}$ & $\begin{array}{l}0600 \\
1200 \\
1800 \\
2400\end{array}$ & $\begin{array}{l}47.45 \\
47.62 \\
47.75 \\
47.85\end{array}$ & $\begin{array}{l}171000 \\
173000 \\
175000 \\
176000\end{array}$ & $\begin{array}{l}7.30 \\
7.37 \\
7.45 \\
7.52\end{array}$ \\
\hline $\begin{array}{l}3-28 \\
3-28 \\
3-28 \\
3-28\end{array}$ & $\begin{array}{l}0600 \\
1200 \\
1800 \\
2400\end{array}$ & $\begin{array}{l}26.60 \\
26.38 \\
25.75 \\
24.80\end{array}$ & $\begin{array}{l}46600 \\
45400 \\
40800 \\
36900\end{array}$ & $\begin{array}{l}5.49 \\
5.51 \\
5.52 \\
5.54\end{array}$ & $\begin{array}{l}4-10 \\
4-10 \\
4-10 \\
4-10\end{array}$ & $\begin{array}{l}0600 \\
1200 \\
1800 \\
2400\end{array}$ & $\begin{array}{l}47.90 \\
47.95 \\
47.95 \\
47.90\end{array}$ & $\begin{array}{l}176000 \\
173000 \\
172000 \\
171000\end{array}$ & $\begin{array}{l}7.60 \\
7.67 \\
7.74 \\
7.81\end{array}$ \\
\hline $\begin{array}{l}3-29 \\
3-29 \\
3-29 \\
3-29\end{array}$ & $\begin{array}{l}0600 \\
1200 \\
1800 \\
2400\end{array}$ & $\begin{array}{l}24.25 \\
23.60 \\
23.37 \\
23.45\end{array}$ & $\begin{array}{l}35500 \\
37800 \\
38400 \\
47300\end{array}$ & $\begin{array}{l}5.55 \\
5.57 \\
5.59 \\
5.61\end{array}$ & $\begin{array}{l}4-11 \\
4-11 \\
4-11 \\
4-11\end{array}$ & $\begin{array}{l}0600 \\
1200 \\
1800 \\
2400\end{array}$ & $\begin{array}{l}47.85 \\
47.72 \\
47.55 \\
47.40\end{array}$ & $\begin{array}{l}171000 \\
168000 \\
166000 \\
162000\end{array}$ & $\begin{array}{l}7.89 \\
7.96 \\
8.03 \\
8.10\end{array}$ \\
\hline $\begin{array}{l}3-30 \\
3-30 \\
3-30 \\
3-30\end{array}$ & $\begin{array}{l}0600 \\
1200 \\
1800 \\
2400\end{array}$ & $\begin{array}{l}23.75 \\
23.88 \\
24.25 \\
24.35\end{array}$ & $\begin{array}{l}41100 \\
41900 \\
43400 \\
42500\end{array}$ & $\begin{array}{l}5.62 \\
5.64 \\
5.66 \\
5.68\end{array}$ & $\begin{array}{l}4-12 \\
4-12 \\
4-12 \\
4-12\end{array}$ & $\begin{array}{l}0600 \\
1200 \\
1800 \\
2400\end{array}$ & $\begin{array}{l}47.15 \\
46.80 \\
46.50 \\
46.15\end{array}$ & $\begin{array}{l}158000 \\
153000 \\
150000 \\
147000\end{array}$ & $\begin{array}{l}8.16 \\
8.23 \\
8.29 \\
8.35\end{array}$ \\
\hline $\begin{array}{l}3-31 \\
3-31 \\
3-31 \\
3-31\end{array}$ & $\begin{array}{l}0600 \\
1200 \\
1800 \\
2400\end{array}$ & $\begin{array}{l}24.40 \\
24.43 \\
24.43 \\
24.22\end{array}$ & $\begin{array}{l}42600 \\
42300 \\
42300 \\
38300\end{array}$ & $\begin{array}{l}5.70 \\
5.71 \\
5.73 \\
5.75\end{array}$ & $\begin{array}{l}4-13 \\
4-13 \\
4-13 \\
4-13\end{array}$ & $\begin{array}{l}0600 \\
1200 \\
1800 \\
2400\end{array}$ & $\begin{array}{l}45.75 \\
45.30 \\
44.90 \\
44.45\end{array}$ & $\begin{array}{l}141000 \\
137000 \\
132000 \\
128000\end{array}$ & $\begin{array}{l}8.41 \\
8.47 \\
8.53 \\
8.58\end{array}$ \\
\hline $\begin{array}{l}4-1 \\
4-1 \\
4-1 \\
4-1\end{array}$ & $\begin{array}{l}0600 \\
1200 \\
1800 \\
2400\end{array}$ & $\begin{array}{l}23.75 \\
22.95 \\
22.05 \\
21.25\end{array}$ & $\begin{array}{l}34600 \\
32600 \\
31900 \\
29900\end{array}$ & $\begin{array}{l}5.76 \\
5.78 \\
5.79 \\
5.80\end{array}$ & $\begin{array}{l}4-14 \\
4-14 \\
4-14 \\
4-14\end{array}$ & $\begin{array}{l}0600 \\
1200 \\
1800 \\
2400\end{array}$ & $\begin{array}{l}44.05 \\
43.70 \\
43.50 \\
43.35\end{array}$ & $\begin{array}{l}126000 \\
125000 \\
125000 \\
126000\end{array}$ & $\begin{array}{l}8.63 \\
8.69 \\
8.74 \\
8.79\end{array}$ \\
\hline $\begin{array}{l}4-2 \\
4-2 \\
4-2 \\
4-2\end{array}$ & $\begin{array}{l}0600 \\
1200 \\
1800 \\
2400\end{array}$ & $\begin{array}{l}20.72 \\
20.42 \\
20.15 \\
20.90\end{array}$ & $\begin{array}{l}29300 \\
29800 \\
29700 \\
42700\end{array}$ & $\begin{array}{l}5.81 \\
5.83 \\
5.84 \\
5.86\end{array}$ & $\begin{array}{l}4-15 \\
4-15 \\
4-15 \\
4-15\end{array}$ & $\begin{array}{l}0600 \\
1200 \\
1800 \\
2400\end{array}$ & $\begin{array}{l}43.30 \\
43.32 \\
43.40 \\
43.55\end{array}$ & $\begin{array}{l}128000 \\
130000 \\
132000 \\
133000\end{array}$ & $\begin{array}{l}8.85 \\
8.90 \\
8.96 \\
9.01\end{array}$ \\
\hline $\begin{array}{l}4-3 \\
4-3 \\
4-3 \\
4-3\end{array}$ & $\begin{array}{l}0600 \\
1200 \\
1800 \\
2400\end{array}$ & $\begin{array}{l}\mathrm{c} \\
\mathrm{c} \\
\mathrm{c} \\
\mathrm{c}\end{array}$ & $\begin{array}{l}60000 \\
73000 \\
84000 \\
96000\end{array}$ & $\begin{array}{l}5.88 \\
5.91 \\
5.95 \\
5.99\end{array}$ & $\begin{array}{l}4-16 \\
4-16 \\
4-16 \\
4-16\end{array}$ & $\begin{array}{l}0600 \\
1200 \\
1800 \\
2400\end{array}$ & $\begin{array}{l}43.75 \\
44.00 \\
44.25 \\
44.50\end{array}$ & $\begin{array}{l}136000 \\
139000 \\
142000 \\
144000\end{array}$ & $\begin{array}{l}9.07 \\
9.13 \\
9.19 \\
9.25\end{array}$ \\
\hline $\begin{array}{l}4-4 \\
4-4 \\
4-4 \\
4-4\end{array}$ & $\begin{array}{l}0600 \\
1200 \\
1800 \\
2400\end{array}$ & $\begin{array}{l}\mathrm{c} \\
\mathrm{c} \\
\mathrm{c} \\
\mathrm{c}\end{array}$ & $\begin{array}{l}103000 \\
110000 \\
118000 \\
125000\end{array}$ & $\begin{array}{l}6.03 \\
6.08 \\
6.13 \\
6.18\end{array}$ & $\begin{array}{l}4-17 \\
4-17 \\
4-17 \\
4-17\end{array}$ & $\begin{array}{l}0600 \\
1200 \\
1800 \\
2400\end{array}$ & $\begin{array}{l}44.83 \\
45.20 \\
45.55 \\
45.94\end{array}$ & $\begin{array}{l}149000 \\
152000 \\
157000 \\
162000\end{array}$ & $\begin{array}{l}9.31 \\
9.38 \\
9.44 \\
9.51\end{array}$ \\
\hline $\begin{array}{l}4-5 \\
4-5 \\
4-5 \\
4-5\end{array}$ & $\begin{array}{l}0600 \\
1200 \\
1800 \\
2400\end{array}$ & $\begin{array}{l}42.00 \\
42.60 \\
43.20 \\
43.75\end{array}$ & $\begin{array}{l}131000 \\
136000 \\
140000 \\
145000\end{array}$ & $\begin{array}{l}6.24 \\
6.30 \\
6.35 \\
6.42\end{array}$ & $\begin{array}{l}4-18 \\
4-18 \\
4-18 \\
4-18\end{array}$ & $\begin{array}{l}0600 \\
1200 \\
1800 \\
2400\end{array}$ & $\begin{array}{l}46.30 \\
46.75 \\
47.25 \\
47.65\end{array}$ & $\begin{array}{l}167000 \\
172000 \\
177000 \\
182000\end{array}$ & $\begin{array}{l}9.58 \\
9.66 \\
9.73 \\
9.81\end{array}$ \\
\hline $\begin{array}{l}4-6 \\
4-6 \\
4-6 \\
4-6\end{array}$ & $\begin{array}{l}0600 \\
1200 \\
1800 \\
2400\end{array}$ & $\begin{array}{l}44.25 \\
44.75 \\
45.10 \\
45.40\end{array}$ & $\begin{array}{l}147000 \\
151000 \\
153000 \\
154000\end{array}$ & $\begin{array}{l}6.48 \\
6.54 \\
6.61 \\
6.67\end{array}$ & $\begin{array}{l}4-19 \\
4-19 \\
4-19 \\
4-19\end{array}$ & $\begin{array}{l}0600 \\
1200 \\
1800 \\
2400\end{array}$ & $\begin{array}{l}48.05 \\
48.45 \\
48.80 \\
49.10\end{array}$ & $\begin{array}{l}185000 \\
190000 \\
195000 \\
198000\end{array}$ & $\begin{array}{r}9.89 \\
9.97 \\
10.05 \\
10.13\end{array}$ \\
\hline $\begin{array}{l}4-7 \\
4-7 \\
4-7 \\
4-7\end{array}$ & $\begin{array}{l}0600 \\
1200 \\
1800 \\
2400\end{array}$ & $\begin{array}{l}45.70 \\
46.00 \\
46.25 \\
46.45\end{array}$ & $\begin{array}{l}158000 \\
161000 \\
162000 \\
164000\end{array}$ & $\begin{array}{l}6.74 \\
6.81 \\
6.87 \\
6.94\end{array}$ & $\begin{array}{l}4-20 \\
4-20 \\
4-20 \\
4-20\end{array}$ & $\begin{array}{l}0600 \\
1200 \\
1800 \\
2400\end{array}$ & $\begin{array}{l}49.45 \\
49.75 \\
50.00 \\
50.25\end{array}$ & $\begin{array}{l}201000 \\
205000 \\
208000 \\
209000\end{array}$ & $\begin{array}{l}10.22 \\
10.30 \\
10.39 \\
10.48\end{array}$ \\
\hline $\begin{array}{l}4-8 \\
4-8 \\
4-8 \\
4-8\end{array}$ & $\begin{array}{l}0600 \\
1200 \\
1800 \\
2400\end{array}$ & $\begin{array}{l}46.67 \\
46.85 \\
47.10 \\
47.30\end{array}$ & $\begin{array}{l}166000 \\
166000 \\
169000 \\
170000\end{array}$ & $\begin{array}{l}7.01 \\
7.08 \\
7.16 \\
7.23\end{array}$ & $4-21$ & 0600 & 50.50 & 212000 & 10.57 \\
\hline
\end{tabular}


TABLE 7.-Gage height, discharge, and accumulated runoff, flood of April 1979-Continued 02429500, Alabama River at Claiborne, Ala-Continued

\begin{tabular}{|c|c|c|c|c|c|c|c|c|c|}
\hline DATE & TIME & $\begin{array}{c}\text { GAGE } \\
\text { HEIGHT }\end{array}$ & DISCHARGE & $\begin{array}{l}\text { ACCUM. } \\
\text { RUNOFF }\end{array}$ & DATE & TIME & $\begin{array}{c}\text { GAGE } \\
\text { HEIGHT }\end{array}$ & DISCHARGE & $\begin{array}{l}\text { ACCUM. } \\
\text { RUNOFF }\end{array}$ \\
\hline $\begin{array}{l}4-21 \\
4-21 \\
4-21\end{array}$ & $\begin{array}{l}1200 \\
1800 \\
2400\end{array}$ & $\begin{array}{l}50.68 \\
50.85 \\
51.00\end{array}$ & $\begin{array}{l}212000 \\
214000 \\
216000\end{array}$ & $\begin{array}{l}10.66 \\
10.75 \\
10.84\end{array}$ & $\begin{array}{l}4-26 \\
4-26 \\
4-26 \\
4-26\end{array}$ & $\begin{array}{l}0600 \\
1200 \\
1800 \\
2400\end{array}$ & $\begin{array}{l}47.80 \\
47.24 \\
46.56 \\
45.95\end{array}$ & $\begin{array}{l}155000 \\
149000 \\
143000 \\
137000\end{array}$ & $\begin{array}{l}12.25 \\
12.32 \\
12.38 \\
12.43\end{array}$ \\
\hline $\begin{array}{l}4-22 \\
4-22 \\
4-22 \\
4-22\end{array}$ & $\begin{array}{l}0600 \\
1200 \\
1800 \\
2400\end{array}$ & $\begin{array}{l}51.13 \\
51.24 \\
51.28 \\
51.29\end{array}$ & $\begin{array}{l}216000 \\
217000 \\
216000 \\
214000\end{array}$ & $\begin{array}{l}10.93 \\
11.02 \\
11.11 \\
11.20\end{array}$ & $\begin{array}{l}4-27 \\
4-27 \\
4-27 \\
4-27\end{array}$ & $\begin{array}{l}0600 \\
1200 \\
1800 \\
2400\end{array}$ & $\begin{array}{l}45.35 \\
44.80 \\
44.30 \\
43.88\end{array}$ & $\begin{array}{l}132000 \\
127000 \\
126000 \\
125000\end{array}$ & $\begin{array}{l}12.49 \\
12.54 \\
12.60 \\
12.65\end{array}$ \\
\hline $\begin{array}{l}4-23 \\
4-23 \\
4-23 \\
4-23\end{array}$ & $\begin{array}{l}0600 \\
1200 \\
1800 \\
2400\end{array}$ & $\begin{array}{l}51.29 \\
51.25 \\
51.18 \\
51.07\end{array}$ & $\begin{array}{l}214000 \\
213000 \\
210000 \\
207000\end{array}$ & $\begin{array}{l}11.30 \\
11.39 \\
11.47 \\
11.56\end{array}$ & $\begin{array}{l}4-28 \\
4-28 \\
4-28 \\
4-28\end{array}$ & $\begin{array}{l}0600 \\
1200 \\
1800 \\
2400\end{array}$ & $\begin{array}{l}43.55 \\
43.30 \\
43.15 \\
43.05\end{array}$ & $\begin{array}{l}124000 \\
123000 \\
123000 \\
124000\end{array}$ & $\begin{array}{l}12.70 \\
12.75 \\
12.81 \\
12.86\end{array}$ \\
\hline $\begin{array}{l}4-24 \\
4-24 \\
4-24 \\
4-24\end{array}$ & $\begin{array}{l}0600 \\
1200 \\
1800 \\
2400\end{array}$ & $\begin{array}{l}50.92 \\
50.71 \\
50.45 \\
50.15\end{array}$ & $\begin{array}{l}205000 \\
200000 \\
195000 \\
190000\end{array}$ & $\begin{array}{l}11.65 \\
11.73 \\
11.82 \\
11.90\end{array}$ & $\begin{array}{l}4-29 \\
4-29 \\
4-29 \\
4-29\end{array}$ & $\begin{array}{l}0600 \\
1200 \\
1800 \\
2400\end{array}$ & $\begin{array}{l}42.95 \\
42.93 \\
42.93 \\
42.97\end{array}$ & $\begin{array}{l}124000 \\
126000 \\
126000 \\
127000\end{array}$ & $\begin{array}{l}12.91 \\
12.96 \\
13.02 \\
13.07\end{array}$ \\
\hline $\begin{array}{l}4-25 \\
4-25 \\
4-25 \\
4-25\end{array}$ & $\begin{array}{l}0600 \\
1200 \\
1800 \\
2400\end{array}$ & $\begin{array}{l}49.70 \\
49.35 \\
48.85 \\
48.35\end{array}$ & $\begin{array}{l}183000 \\
175000 \\
169000 \\
162000\end{array}$ & $\begin{array}{l}11.97 \\
12.05 \\
12.12 \\
12.19\end{array}$ & $\begin{array}{l}4-30 \\
4-30 \\
4-30 \\
4-30\end{array}$ & $\begin{array}{l}0600 \\
1200 \\
1800 \\
2400\end{array}$ & $\begin{array}{l}43.00 \\
43.05 \\
43.10 \\
43.15\end{array}$ & $\begin{array}{l}127000 \\
128000 \\
128000 \\
128000\end{array}$ & $\begin{array}{l}13.12 \\
13.18 \\
13.23 \\
13.29\end{array}$ \\
\hline
\end{tabular}


TABLE 7.-Gage height, discharge, and accumulated runoff, flood of April 1979-Continued 02439400, Buttahatchie River near Aberdeen, Miss.

\begin{tabular}{|c|c|c|c|c|c|c|c|c|c|}
\hline DATE & TIME & $\begin{array}{l}\text { GAGE } \\
\text { HEIGHT }\end{array}$ & DISCHARGE & $\begin{array}{l}\text { ACCUMULATED } \\
\text { RUNDFF }\end{array}$ & DATE & TIME & $\begin{array}{l}\text { GAGE } \\
\text { HEIGHT }\end{array}$ & DISCHARGE & $\begin{array}{l}\text { ACCUMUL_ATED } \\
\text { RUNOFF }\end{array}$ \\
\hline $04-12$ & 0100 & 11.30 & 2,630 & 0.00 & $04-15$ & 2400 & 15.62 & 9,500 & 2.16 \\
\hline $04-12$ & 0600 & 12.91 & 3,920 & $0.0: 3$ & & & & & \\
\hline $\begin{array}{l}04-12 \\
04-12\end{array}$ & $\begin{array}{l}1200 \\
1800\end{array}$ & $\begin{array}{l}14.08 \\
14.09\end{array}$ & $\begin{array}{l}5,790 \\
5,810\end{array}$ & $\begin{array}{l}0.013 \\
0.15\end{array}$ & $\begin{array}{l}04-16 \\
04-16\end{array}$ & $\begin{array}{l}1200 \\
2400\end{array}$ & $\begin{array}{l}14.99 \\
14.47\end{array}$ & $\begin{array}{l}7,840 \\
6,610\end{array}$ & $\begin{array}{l}2.36 \\
2.53\end{array}$ \\
\hline $04-12$ & 2400 & 14.97 & 7,780 & 0.23 & & & & & \\
\hline & & & & & $04-17$ & 2400 & 13.34 & 4,520 & 2.80 \\
\hline $04-13$ & 1200 & 16.01 & 10,600 & 0.45 & & & & & \\
\hline $04-13$ & 2400 & 17.13 & 14,900 & 0.75 & $04-18$ & 2400 & 12.25 & 3,320 & 2.98 \\
\hline $04-14$ & 0600 & 17.51 & 16,700 & 0.74 & $04-19$ & 2400 & 11.35 & 2,660 & 3.12 \\
\hline $04-14$ & 1200 & 17.70 & 17,700 & 1.14 & & & & & \\
\hline $04-14$ & 1400 & 17.74 & 17,900 & 1.21 & $04-20$ & 2400 & 10.52 & 2,210 & 3.24 \\
\hline $04-14$ & 1800 & 17.71 & 17,700 & 1.35 & & & & & \\
\hline $04-14$ & 2400 & 17.48 & 16,600 & 1.55 & $04-21$ & 2400 & 9.82 & 1,870 & 3.34 \\
\hline 04-15 & 1200 & 16.53 & 12,500 & 1.90 & $04-22$ & 2400 & 9.39 & 1,680 & 3.42 \\
\hline
\end{tabular}


TABLES

TABLE 7.-Gage height, discharge, and accumulated runoff, flood of April 1979-Continued 02441000, Tibbee Creek near Tibbee, Miss.

\begin{tabular}{|c|c|c|c|c|c|c|c|c|c|}
\hline DIATE & TIME & $\begin{array}{l}\text { GAGE } \\
\text { HEIGHT }\end{array}$ & DISCHARGE & $\begin{array}{c}\text { ACCUMUUI_ATED } \\
\text { RUINIJFF }\end{array}$ & DATE & TIME & $\begin{array}{l}\text { GAGE } \\
\text { HEIGHT }\end{array}$ & DI SCHAFGE & $\begin{array}{l}\text { ACCUMMUL_ATEO } \\
\text { RUNOFF }\end{array}$ \\
\hline $04-11$ & 2400 & 12.17 & 2,960 & 0.00 & & & & & \\
\hline $\begin{array}{l}04-12 \\
04-12\end{array}$ & $\begin{array}{l}0130 \\
0300\end{array}$ & $\begin{array}{l}12.01 \\
13.47\end{array}$ & $\begin{array}{l}2,900 \\
3,430\end{array}$ & $\begin{array}{l}0.00 \\
0.01\end{array}$ & $\begin{array}{l}04-15 \\
04-15\end{array}$ & $\begin{array}{l}1200 \\
2400\end{array}$ & $\begin{array}{l}23.85 \\
23.10\end{array}$ & $\begin{array}{r}11,500 \\
9,270\end{array}$ & $\begin{array}{l}2.43 \\
2.67\end{array}$ \\
\hline $04-12$ & 0600 & 17.93 & 5,090 & 0.03 & $04-16$ & 1200 & 22.27 & 7,800 & 2.33 \\
\hline $\begin{array}{l}04-12 \\
04-12\end{array}$ & $\begin{array}{l}0900 \\
1200\end{array}$ & $\begin{array}{l}20.16 \\
21.47\end{array}$ & $\begin{array}{l}6,140 \\
6,960\end{array}$ & $\begin{array}{l}0.06 \\
0.09\end{array}$ & $04-16$ & 2400 & 20.93 & 6,560 & 2.77 \\
\hline $04-12$ & 1600 & 22.62 & 8,690 & 0.14 & $04-17$ & 1200 & 18.99 & 5,570 & 3.09 \\
\hline $\begin{array}{l}04-12 \\
04-12\end{array}$ & $\begin{array}{l}2000 \\
2400\end{array}$ & $\begin{array}{l}23.74 \\
24.66\end{array}$ & $\begin{array}{l}11,100 \\
14,500\end{array}$ & $\begin{array}{l}0.20 \\
0.23\end{array}$ & $04-17$ & 2400 & 16.57 & 4,550 & 3.13 \\
\hline $\begin{array}{l}04-13 \\
04-13\end{array}$ & $\begin{array}{l}0300 \\
0600\end{array}$ & $\begin{array}{l}25.58 \\
26.51\end{array}$ & $\begin{array}{l}19,000 \\
24,600\end{array}$ & $\begin{array}{l}0.36 \\
0.46\end{array}$ & $\begin{array}{l}04-18 \\
04-18\end{array}$ & $\begin{array}{l}1200 \\
2400\end{array}$ & $\begin{array}{l}13.62 \\
10.70\end{array}$ & $\begin{array}{l}3,560 \\
2,420\end{array}$ & $\begin{array}{l}3.26 \\
3.31\end{array}$ \\
\hline $\begin{array}{l}04-13 \\
04-13 \\
04-13\end{array}$ & $\begin{array}{l}1200 \\
1000 \\
2400\end{array}$ & $\begin{array}{l}27.29 \\
27.51 \\
27.37\end{array}$ & $\begin{array}{l}30,300 \\
32,100 \\
30,900\end{array}$ & $\begin{array}{l}0.72 \\
1.02 \\
1.32\end{array}$ & $\begin{array}{l}04-19 \\
04-19\end{array}$ & $\begin{array}{l}1200 \\
2400\end{array}$ & $\begin{array}{l}8.50 \\
6.45\end{array}$ & $\begin{array}{r}1,570 \\
964\end{array}$ & $\begin{array}{l}3.35 \\
3.313\end{array}$ \\
\hline $04-14$ & 0600 & 2607 & 27.900 & 150 & $04-20$ & 2400 & 4.99 & 563 & 3.4 .1 \\
\hline $\begin{array}{l}04-14 \\
04-14\end{array}$ & $\begin{array}{l}1200 \\
2400\end{array}$ & $\begin{array}{l}26.30 \\
25.00\end{array}$ & $\begin{array}{l}23,800 \\
16,000\end{array}$ & $\begin{array}{l}1.3 . \\
1.34 \\
2.22\end{array}$ & $04-21$ & 2400 & 4.61 & 477 & 3.42 \\
\hline & & & & & $04-22$ & 2400 & 4.36 & 422 & 3.44 \\
\hline
\end{tabular}


TABLE 7.-Gage height, discharge, and accumulated runoff, flood of April 1979-Continued 02441500, Tombigbee River at Columbus, Miss.

\begin{tabular}{|c|c|c|c|c|c|c|c|c|c|}
\hline DATE & TIME & $\begin{array}{l}\text { GAGE } \\
\text { HEIGHT }\end{array}$ & DISCHARGE & $\begin{array}{c}\text { ACCOUMULATED } \\
\text { FUNOFF }\end{array}$ & DATE & TIME & $\begin{array}{l}\text { GAGEE } \\
\text { HEIGHT }\end{array}$ & DISCHARGE & $\begin{array}{l}\text { ACCIUMULATED } \\
\text { RUINOFF }\end{array}$ \\
\hline $\begin{array}{l}04-11 \\
04-11 \\
04-11\end{array}$ & $\begin{array}{l}0100 \\
1200 \\
2400\end{array}$ & $\begin{array}{l}15.04 \\
14.47 \\
13.67\end{array}$ & $\begin{array}{l}15,400 \\
14,500 \\
13,400\end{array}$ & $\begin{array}{l}0.00 \\
0.05 \\
0.11\end{array}$ & $\begin{array}{l}04-16 \\
04-16\end{array}$ & $\begin{array}{l}1200 \\
2400\end{array}$ & $\begin{array}{l}33.43 \\
32.68\end{array}$ & $\begin{array}{l}64,100 \\
59,200\end{array}$ & $\begin{array}{l}2.33 \\
2.58\end{array}$ \\
\hline $\begin{array}{l}04-12 \\
04-12 \\
04-12\end{array}$ & $\begin{array}{l}0300 \\
0600 \\
0900\end{array}$ & $\begin{array}{l}13.82 \\
16.38 \\
18.93\end{array}$ & $\begin{array}{l}13,600 \\
17,400 \\
21,600\end{array}$ & $\begin{array}{l}0.12 \\
0.14 \\
0.16\end{array}$ & $\begin{array}{l}04-17 \\
04-17\end{array}$ & $\begin{array}{l}1200 \\
2400\end{array}$ & $\begin{array}{l}31.88 \\
30.82\end{array}$ & $\begin{array}{l}57,200 \\
52,800\end{array}$ & $\begin{array}{l}2.32 \\
3.05\end{array}$ \\
\hline $\begin{array}{l}04-12 \\
04-12 \\
04-12\end{array}$ & $\begin{array}{l}1200 \\
1800 \\
2400\end{array}$ & $\begin{array}{l}21.13 \\
24.85 \\
27.63\end{array}$ & $\begin{array}{l}25,500 \\
33,100 \\
40,800\end{array}$ & $\begin{array}{l}0.13 \\
0.24 \\
0.32\end{array}$ & $\begin{array}{l}04-18 \\
04-18\end{array}$ & $\begin{array}{l}1200 \\
2400\end{array}$ & $\begin{array}{l}29.51 \\
27.98\end{array}$ & $\begin{array}{l}47,800 \\
42,800\end{array}$ & $\begin{array}{l}3.26 \\
3.45\end{array}$ \\
\hline $\begin{array}{l}04-13 \\
04-13\end{array}$ & $\begin{array}{l}0600 \\
1200\end{array}$ & $\begin{array}{l}30.53 \\
32.50\end{array}$ & $\begin{array}{l}49,800 \\
58,100\end{array}$ & $\begin{array}{l}0.42 \\
0.53\end{array}$ & $\begin{array}{l}04-19 \\
04-19\end{array}$ & $\begin{array}{l}1200 \\
2400\end{array}$ & $\begin{array}{l}26.08 \\
23.83\end{array}$ & $\begin{array}{l}37,800 \\
32,600\end{array}$ & $\begin{array}{l}3.61 \\
3.76\end{array}$ \\
\hline $\begin{array}{l}04-13 \\
04-13\end{array}$ & $\begin{array}{l}1800 \\
2400\end{array}$ & $\begin{array}{l}33.85 \\
34.62\end{array}$ & $\begin{array}{l}67,000 \\
72,900\end{array}$ & $\begin{array}{l}0.36 \\
0.30\end{array}$ & $\begin{array}{l}04-20 \\
04-20\end{array}$ & $\begin{array}{l}1200 \\
2400\end{array}$ & $\begin{array}{l}21.30 \\
18.72\end{array}$ & $\begin{array}{l}27,300 \\
22,500\end{array}$ & $\begin{array}{l}3.843 \\
3.99\end{array}$ \\
\hline $\begin{array}{l}04-14 \\
04-14 \\
04-14\end{array}$ & $\begin{array}{l}0600 \\
1400 \\
1800\end{array}$ & $\begin{array}{l}35.16 \\
35.33 \\
35.21\end{array}$ & $\begin{array}{l}78,100 \\
80,400 \\
78,800\end{array}$ & $\begin{array}{l}0.96 \\
1.13 \\
1.29\end{array}$ & $\begin{array}{l}04-21 \\
04-21\end{array}$ & $\begin{array}{l}1200 \\
2400\end{array}$ & $\begin{array}{l}16.25 \\
13.89\end{array}$ & $\begin{array}{l}18,200 \\
14,500\end{array}$ & $\begin{array}{l}4.07 \\
4.14\end{array}$ \\
\hline $04-14$ & 2400 & 35.10 & 77,300 & 1.45 & $\begin{array}{l}04-22 \\
04-22\end{array}$ & $\begin{array}{l}1200 \\
2400\end{array}$ & $\begin{array}{r}11.26 \\
9.22\end{array}$ & $\begin{array}{r}10,800 \\
8,090\end{array}$ & $\begin{array}{l}4.19 \\
4.23\end{array}$ \\
\hline $\begin{array}{l}04-15 \\
04-15\end{array}$ & $\begin{array}{l}1200 \\
2400\end{array}$ & $\begin{array}{l}34.64 \\
34.07\end{array}$ & $\begin{array}{l}73,000 \\
68,500\end{array}$ & $\begin{array}{l}1.76 \\
2.05\end{array}$ & $04-23$ & 2400 & 7.79 & 6,250 & 4.29 \\
\hline
\end{tabular}


TABLES

TABLE 7.-Gage height, discharge, and accumulated runoff, flood of April 1979-Continued 02443500, Luxapallila Creek near Columbus, Miss.

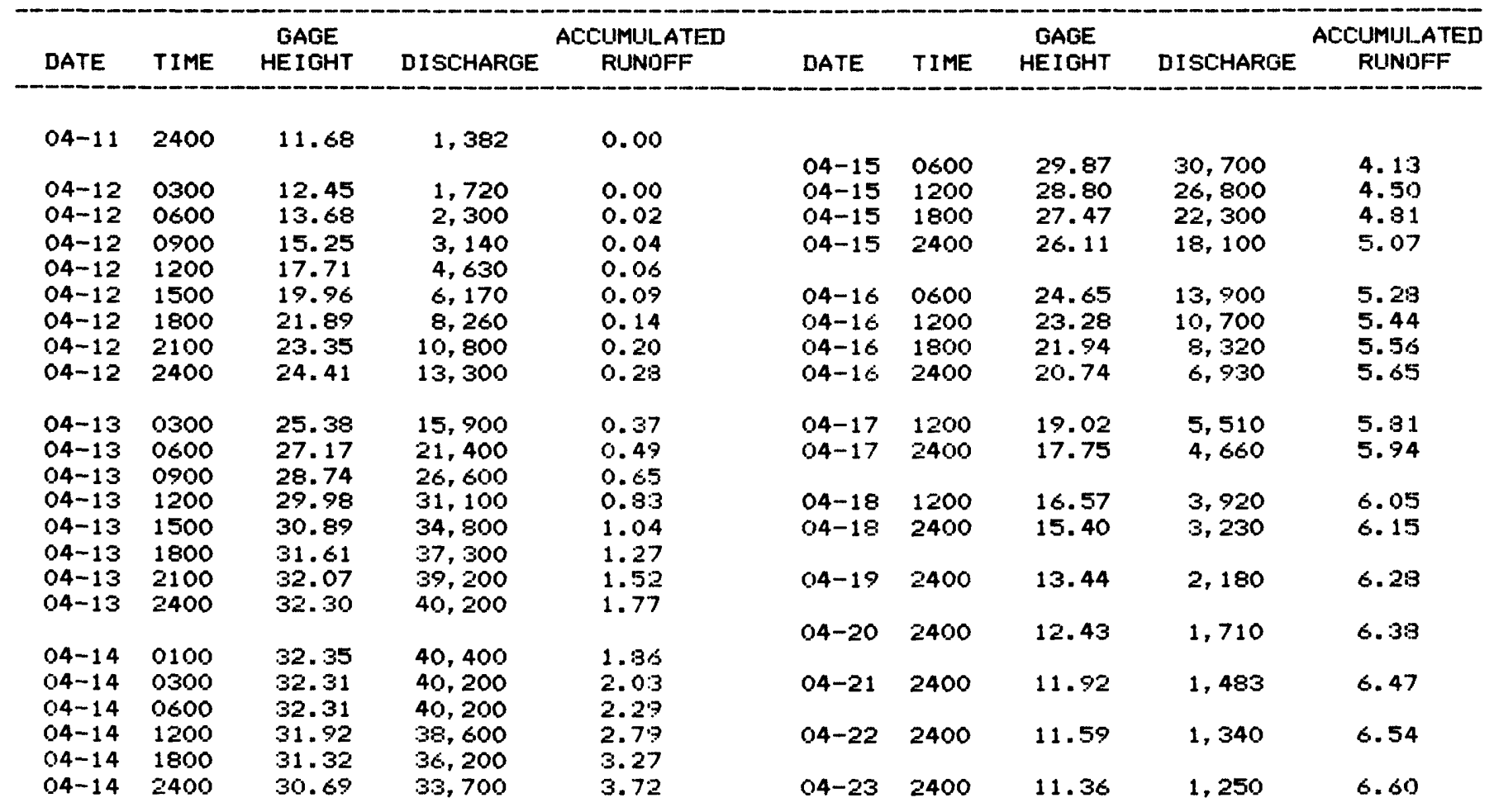


TABLE 7.-Gage height, discharge, and accumulated runoff, flood of April 1979-Continued 02446500, Sipsey River near Elrod, Ala.

\begin{tabular}{|c|c|c|c|c|c|c|c|c|c|}
\hline DATE & TIME & $\begin{array}{l}\text { GAGE } \\
\text { HEIGHT }\end{array}$ & DISCHARGE & $\begin{array}{l}\text { ACCUM. } \\
\text { RUNOFF }\end{array}$ & DATE & TIME & $\begin{array}{c}\text { GAGE } \\
\text { HE I GHT }\end{array}$ & DISCHARGE & $\begin{array}{l}\text { ACCUM. } \\
\text { RUNOFF }\end{array}$ \\
\hline $\begin{array}{l}3-01 \\
3-01 \\
3-01\end{array}$ & $\begin{array}{l}0200 \\
1700 \\
2400\end{array}$ & $\begin{array}{l}13.88 \\
14.06 \\
14.20\end{array}$ & $\begin{array}{l}3,210 \\
3,680 \\
4,100\end{array}$ & $\begin{array}{l}0.17 \\
0.25\end{array}$ & $\begin{array}{l}3-16 \\
3-16\end{array}$ & $\begin{array}{l}0200 \\
2400\end{array}$ & $\begin{array}{l}12.35 \\
12.17\end{array}$ & $\begin{array}{l}1,280 \\
1,190\end{array}$ & $\begin{array}{l}4.64 \\
4.72\end{array}$ \\
\hline $\begin{array}{l}3-02 \\
3-02 \\
3-02\end{array}$ & $\begin{array}{l}0600 \\
2200 \\
2400\end{array}$ & $\begin{array}{l}14.23 \\
14.09 \\
14.06\end{array}$ & $\begin{array}{l}4,190 \\
3,770 \\
3,680\end{array}$ & $\begin{array}{l}0.33 \\
0.52 \\
0.54\end{array}$ & $\begin{array}{l}3-17 \\
3-17\end{array}$ & $\begin{array}{l}0100 \\
2400\end{array}$ & $\begin{array}{l}12.16 \\
11.93\end{array}$ & $\begin{array}{l}1,180 \\
1,090\end{array}$ & $\begin{array}{l}4.72 \\
4.80\end{array}$ \\
\hline & & & & & $\begin{array}{l}3-18 \\
3-18\end{array}$ & $\begin{array}{l}0100 \\
2400\end{array}$ & $\begin{array}{l}11.92 \\
11.66\end{array}$ & $\begin{array}{l}1,080 \\
1,010\end{array}$ & $\begin{array}{l}4.80 \\
4.87\end{array}$ \\
\hline $\begin{array}{l}3-03 \\
3-03 \\
3-03\end{array}$ & $\begin{array}{l}1200 \\
1800 \\
2400\end{array}$ & $\begin{array}{l}14.10 \\
14.41 \\
14.99\end{array}$ & $\begin{array}{l}3,800 \\
4,730 \\
7,250\end{array}$ & $\begin{array}{l}0.67 \\
0.75 \\
0.86\end{array}$ & $\begin{array}{l}3-19 \\
3-19\end{array}$ & $\begin{array}{l}0100 \\
2400\end{array}$ & $\begin{array}{l}11.64 \\
11 \cdot 35\end{array}$ & $\begin{array}{r}1.000 \\
943\end{array}$ & $\begin{array}{l}4.88 \\
4.94\end{array}$ \\
\hline $\begin{array}{l}3-04 \\
3-04 \\
3-04\end{array}$ & $\begin{array}{l}0800 \\
1000 \\
2400\end{array}$ & $\begin{array}{l}15.46 \\
15.48 \\
15.21\end{array}$ & $\begin{array}{l}9,600 \\
9,700 \\
8,350\end{array}$ & $\begin{array}{l}1.07 \\
1.13 \\
1.51\end{array}$ & $\begin{array}{l}3-20 \\
3-20\end{array}$ & $\begin{array}{l}0100 \\
2400\end{array}$ & $\begin{array}{l}11.33 \\
11.06\end{array}$ & $\begin{array}{l}939 \\
891\end{array}$ & $\begin{array}{l}4.95 \\
5.01\end{array}$ \\
\hline $\begin{array}{l}3-05 \\
3-05 \\
3-05\end{array}$ & $\begin{array}{l}0100 \\
2200 \\
2400\end{array}$ & $\begin{array}{l}15.19 \\
14.93 \\
14.92\end{array}$ & $\begin{array}{l}8,250 \\
6,950 \\
6,900\end{array}$ & $\begin{array}{l}1.53 \\
2.00 \\
2.04\end{array}$ & $\begin{array}{l}3-21 \\
3-21\end{array}$ & $\begin{array}{l}0100 \\
2400\end{array}$ & $\begin{array}{l}11.06 \\
10.92\end{array}$ & $\begin{array}{l}891 \\
866\end{array}$ & $\begin{array}{l}5.01 \\
5.07\end{array}$ \\
\hline $\begin{array}{l}3-06 \\
3-06 \\
3-06\end{array}$ & $\begin{array}{l}0100 \\
2000 \\
2400\end{array}$ & $\begin{array}{l}14.92 \\
14.71 \\
14.81\end{array}$ & $\begin{array}{l}6,900 \\
5,850 \\
6,350\end{array}$ & $\begin{array}{l}2.06 \\
2.42 \\
2.49\end{array}$ & $\begin{array}{l}3-22 \\
3-22\end{array}$ & $\begin{array}{l}0100 \\
2400\end{array}$ & $\begin{array}{l}10.90 \\
10.65\end{array}$ & $\begin{array}{l}862 \\
817\end{array}$ & $\begin{array}{l}5.07 \\
5.13\end{array}$ \\
\hline & & & & & $3-23$ & 2400 & $11 \cdot 12$ & 902 & 5.19 \\
\hline $\begin{array}{l}3-07 \\
3-07\end{array}$ & $\begin{array}{l}1000 \\
2400\end{array}$ & $\begin{array}{l}15.06 \\
14.86\end{array}$ & $\begin{array}{l}7,600 \\
6,600\end{array}$ & $\begin{array}{l}2.70 \\
3.01\end{array}$ & $\begin{array}{l}3-24 \\
3-24\end{array}$ & $\begin{array}{l}1200 \\
2400\end{array}$ & $\begin{array}{l}11.19 \\
11.14\end{array}$ & $\begin{array}{l}914 \\
905\end{array}$ & $\begin{array}{l}5.23 \\
5.26\end{array}$ \\
\hline $\begin{array}{l}3-08 \\
3-08\end{array}$ & $\begin{array}{l}0100 \\
2400\end{array}$ & $\begin{array}{l}14.85 \\
14.40\end{array}$ & $\begin{array}{l}6,550 \\
4,700\end{array}$ & $\begin{array}{l}3.03 \\
3.40\end{array}$ & $\begin{array}{l}3-25 \\
3-25\end{array}$ & $\begin{array}{l}0200 \\
2400\end{array}$ & $\begin{array}{l}11.13 \\
11.05\end{array}$ & $\begin{array}{l}903 \\
889\end{array}$ & $\begin{array}{l}5.26 \\
5.32\end{array}$ \\
\hline $\begin{array}{l}3-09 \\
3-09\end{array}$ & $\begin{array}{l}0100 \\
2400\end{array}$ & $\begin{array}{l}14.38 \\
14.04\end{array}$ & $\begin{array}{l}4,640 \\
3,620\end{array}$ & $\begin{array}{l}3.41 \\
3.70\end{array}$ & $3-26$ & 2400 & 11.21 & 918 & 5.39 \\
\hline \multirow[t]{2}{*}{$\begin{array}{l}3-10 \\
3-10\end{array}$} & $\begin{array}{l}0100 \\
2400\end{array}$ & $\begin{array}{l}14.03 \\
13.77\end{array}$ & $\begin{array}{l}3,590 \\
2,990\end{array}$ & $\begin{array}{l}3.71 \\
3.93\end{array}$ & $3-27$ & 2400 & 11.86 & 1.060 & 5.46 \\
\hline & & & & & $3-28$ & 2400 & 12.56 & 1,410 & 5.55 \\
\hline $\begin{array}{l}3-11 \\
3-11\end{array}$ & $\begin{array}{l}0100 \\
2400\end{array}$ & $\begin{array}{l}13.76 \\
13.51\end{array}$ & $\begin{array}{l}2,970 \\
2,510\end{array}$ & $\begin{array}{l}3.94 \\
4.13\end{array}$ & $\begin{array}{l}3-29 \\
3-29\end{array}$ & $\begin{array}{l}2100 \\
2400\end{array}$ & $\begin{array}{l}12.76 \\
12.75\end{array}$ & $\begin{array}{l}1,570 \\
1,570\end{array}$ & $\begin{array}{l}5.64 \\
5.66\end{array}$ \\
\hline $\begin{array}{l}3-12 \\
3-12\end{array}$ & $\begin{array}{l}0100 \\
2400\end{array}$ & $\begin{array}{l}13.49 \\
13.20\end{array}$ & $\begin{array}{l}2,470 \\
2,030\end{array}$ & $\begin{array}{l}4.13 \\
4.29\end{array}$ & $\begin{array}{l}3-30 \\
3-30\end{array}$ & $\begin{array}{l}0100 \\
2400\end{array}$ & $\begin{array}{l}12.74 \\
12.42\end{array}$ & $\begin{array}{l}1,560 \\
1,320\end{array}$ & $\begin{array}{l}5.66 \\
5.76\end{array}$ \\
\hline $\begin{array}{l}3-13 \\
3-13\end{array}$ & $\begin{array}{l}0100 \\
2400\end{array}$ & $\begin{array}{l}13.19 \\
12.89\end{array}$ & $\begin{array}{l}2,020 \\
1.690\end{array}$ & $\begin{array}{l}4.29 \\
4.42\end{array}$ & $\begin{array}{l}3-31 \\
3-31\end{array}$ & $\begin{array}{l}0100 \\
2400\end{array}$ & $\begin{array}{l}12.40 \\
12.09\end{array}$ & $\begin{array}{l}1,310 \\
1.150\end{array}$ & $\begin{array}{l}5.76 \\
5.85\end{array}$ \\
\hline $\begin{array}{l}3-14 \\
3-14\end{array}$ & $\begin{array}{l}0100 \\
2400\end{array}$ & $\begin{array}{l}12.87 \\
12.59\end{array}$ & $\begin{array}{l}1,670 \\
1,430\end{array}$ & $\begin{array}{l}4.43 \\
4.53\end{array}$ & $\begin{array}{l}4-01 \\
4-01\end{array}$ & $\begin{array}{l}0100 \\
2400\end{array}$ & $\begin{array}{l}12.09 \\
12.02\end{array}$ & $\begin{array}{l}1,150 \\
1,120\end{array}$ & $\begin{array}{l}5.85 \\
5.93\end{array}$ \\
\hline $\begin{array}{l}3-15 \\
3-15\end{array}$ & $\begin{array}{l}0100 \\
2400\end{array}$ & $\begin{array}{l}12.58 \\
12.36\end{array}$ & $\begin{array}{l}1,430 \\
1,290\end{array}$ & $\begin{array}{l}4.54 \\
4.63\end{array}$ & $\begin{array}{l}4-02 \\
4-02\end{array}$ & $\begin{array}{l}2300 \\
2400\end{array}$ & $\begin{array}{l}12.14 \\
12.18\end{array}$ & $\begin{array}{l}1,170 \\
1,190\end{array}$ & $\begin{array}{l}6.00 \\
6.01\end{array}$ \\
\hline
\end{tabular}


TABLE 7.-Gage height, discharge, and accumulated runoff, flood of April 1979-Continued 02446500, Sipsey River near Elrod, Ala.-Continued

\begin{tabular}{|c|c|c|c|c|c|c|c|c|c|}
\hline DATE & TIME & $\begin{array}{l}\text { GAGE } \\
\text { HEIGHT }\end{array}$ & DISCHARGE & $\begin{array}{l}\text { ACCUM. } \\
\text { RUNOFF }\end{array}$ & DATE & TIME & $\begin{array}{l}\text { GAGE } \\
\text { HEIGHT }\end{array}$ & DISCHARGE & $\begin{array}{l}\text { ACCUM. } \\
\text { RUNOFF }\end{array}$ \\
\hline $4-03$ & 2400 & 12.85 & 1.660 & 6.11 & $\begin{array}{l}4-15 \\
4-15 \\
4-15\end{array}$ & $\begin{array}{l}1200 \\
2300 \\
2400\end{array}$ & $\begin{array}{l}16.86 \\
16.59 \\
16.56\end{array}$ & $\begin{array}{l}17,200 \\
15,500 \\
15,400\end{array}$ & $\begin{array}{l}11.10 \\
11.63 \\
11.68\end{array}$ \\
\hline $4-04$ & 2400 & 13.06 & 1,870 & 6.24 & & & & & \\
\hline $\begin{array}{l}4-05 \\
4-05\end{array}$ & $\begin{array}{l}0100 \\
2400\end{array}$ & $\begin{array}{l}13.06 \\
12.83\end{array}$ & $\begin{array}{l}1,870 \\
1,640\end{array}$ & $\begin{array}{l}6.25 \\
6.36\end{array}$ & $\begin{array}{l}4-16 \\
4-16\end{array}$ & $\begin{array}{l}0100 \\
2400\end{array}$ & $\begin{array}{l}16.52 \\
15.36\end{array}$ & $\begin{array}{r}15,100 \\
9,100\end{array}$ & $\begin{array}{l}11.72 \\
12.54\end{array}$ \\
\hline $4-06$ & 2400 & 12.97 & 1.770 & 6.48 & $\begin{array}{l}4-17 \\
4-17 \\
4-17\end{array}$ & $\begin{array}{l}0100 \\
2100 \\
2400\end{array}$ & $\begin{array}{l}15.31 \\
14.65 \\
14.59\end{array}$ & $\begin{array}{l}8,850 \\
5,600 \\
5,360\end{array}$ & $\begin{array}{l}12.57 \\
12.98 \\
13.03\end{array}$ \\
\hline $\begin{array}{l}4-07 \\
4-08\end{array}$ & 2400 & 13.50 & $\begin{array}{l}2.040 \\
2.490\end{array}$ & 6.78 & $\begin{array}{l}4-18 \\
4-18\end{array}$ & $\begin{array}{l}0100 \\
2400\end{array}$ & $\begin{array}{l}14.57 \\
14.17\end{array}$ & $\begin{array}{l}5,280 \\
4,010\end{array}$ & $\begin{array}{l}13.05 \\
13.36\end{array}$ \\
\hline $\begin{array}{l}4-09 \\
4-09 \\
4-09\end{array}$ & $\begin{array}{l}0800 \\
2000 \\
2400\end{array}$ & $\begin{array}{l}13.54 \\
13.42 \\
13.36\end{array}$ & $\begin{array}{l}2,560 \\
2,360 \\
2.270\end{array}$ & $\begin{array}{l}6.85 \\
6.93\end{array}$ & $\begin{array}{l}4-19 \\
4-19\end{array}$ & $\begin{array}{l}0100 \\
2400\end{array}$ & $\begin{array}{l}14.16 \\
13.85\end{array}$ & $\begin{array}{l}3,980 \\
3,150\end{array}$ & $\begin{array}{l}13.37 \\
13.62\end{array}$ \\
\hline & & 13.36 & 2,270 & 6.96 & $\begin{array}{l}4-20 \\
4-20\end{array}$ & $\begin{array}{l}0100 \\
2400\end{array}$ & $\begin{array}{l}13.85 \\
13.55\end{array}$ & $\begin{array}{l}3.150 \\
2.580\end{array}$ & $\begin{array}{l}13.63 \\
13.82\end{array}$ \\
\hline $\begin{array}{l}4-10 \\
4-10\end{array}$ & $\begin{array}{l}0100 \\
2400\end{array}$ & $\begin{array}{l}13.35 \\
13.02\end{array}$ & $\begin{array}{l}2.250 \\
1,820\end{array}$ & $\begin{array}{l}6.97 \\
7.11\end{array}$ & $\begin{array}{l}4-21 \\
4-21\end{array}$ & $\begin{array}{l}0100 \\
2400\end{array}$ & $\begin{array}{l}13.54 \\
13.24\end{array}$ & $\begin{array}{l}2,560 \\
2,090\end{array}$ & $\begin{array}{l}13.83 \\
13.99\end{array}$ \\
\hline $\begin{array}{l}4-11 \\
4-11\end{array}$ & $\begin{array}{l}0100 \\
240.0\end{array}$ & $\begin{array}{l}13.00 \\
12.62\end{array}$ & $\begin{array}{l}1,800 \\
1,460\end{array}$ & $\begin{array}{l}7.11 \\
7.22\end{array}$ & $4-22$ & 0100 & 13.23 & 2,070 & 13.99 \\
\hline $\begin{array}{l}4-12 \\
4-12 \\
4-12 \\
4-12 \\
4-12\end{array}$ & $\begin{array}{l}0400 \\
1000 \\
1400 \\
1700 \\
2400\end{array}$ & $\begin{array}{l}12.55 \\
13.30 \\
13.99 \\
14.95 \\
17.07\end{array}$ & $\begin{array}{r}1,410 \\
2,170 \\
3,480 \\
7,050 \\
18,400\end{array}$ & $\begin{array}{l}7.24 \\
7.27 \\
7.31 \\
7.36 \\
7.64\end{array}$ & $\begin{array}{l}4-22 \\
4-23 \\
4-23\end{array}$ & $\begin{array}{l}0100 \\
2400\end{array}$ & $\begin{array}{l}12.91 \\
12.89 \\
12.56\end{array}$ & $\begin{array}{l}1,710 \\
1.690 \\
1.410\end{array}$ & $\begin{array}{l}14.13 \\
14.23\end{array}$ \\
\hline $\begin{array}{l}4-13 \\
4-13 \\
4-13\end{array}$ & $\begin{array}{l}0800 \\
0900 \\
2400\end{array}$ & $\begin{array}{l}17.85 \\
17.81 \\
17.32\end{array}$ & $\begin{array}{l}23,100 \\
22,900 \\
19,900\end{array}$ & $\begin{array}{l}8.16 \\
8.23 \\
9.18\end{array}$ & $\begin{array}{l}4-24 \\
4-24\end{array}$ & $\begin{array}{l}0100 \\
2400\end{array}$ & $\begin{array}{l}12.54 \\
12.34\end{array}$ & $\begin{array}{l}1,400 \\
1.270\end{array}$ & $\begin{array}{l}14.24 \\
14.33\end{array}$ \\
\hline $\begin{array}{l}4-14 \\
4-14\end{array}$ & $\begin{array}{l}0100 \\
2400\end{array}$ & $\begin{array}{l}17.28 \\
16.86\end{array}$ & $\begin{array}{l}19,700 \\
17,200\end{array}$ & $\begin{array}{r}9.24 \\
10.48\end{array}$ & $\begin{array}{l}4-25 \\
4-25\end{array}$ & $\begin{array}{l}0100 \\
2400\end{array}$ & $\begin{array}{l}12.33 \\
12.15\end{array}$ & $\begin{array}{l}1,270 \\
1.180\end{array}$ & $\begin{array}{l}14.33 \\
14.42\end{array}$ \\
\hline
\end{tabular}


TABLE 7.-Gage height, discharge, and accumulated runoff, flood of April 1979-Continued 02448000, Noxubee River at Macon, Miss.

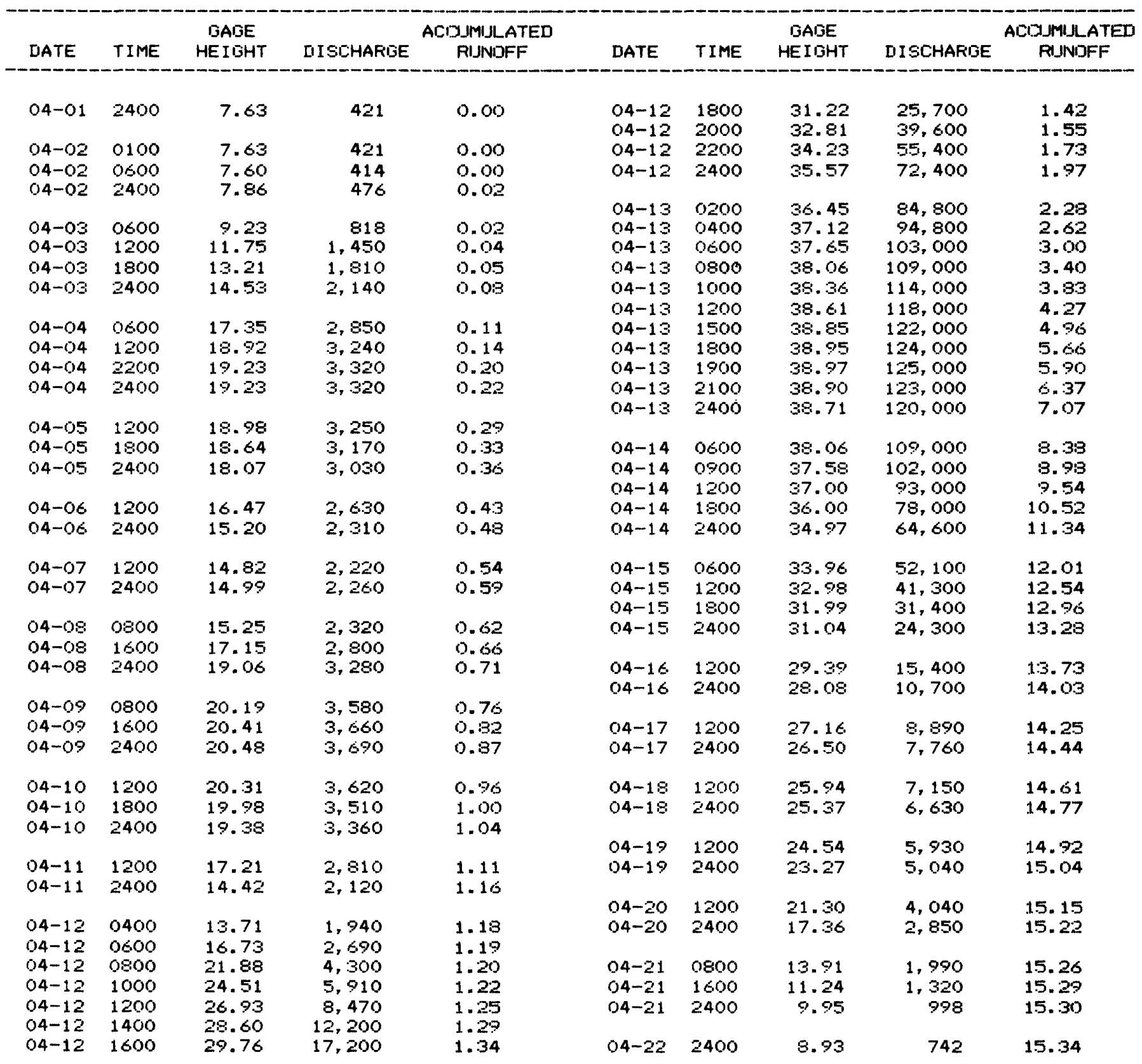


TABLES

TABLE 7.-Gage height, discharge, and accumulated runoff, flood of April 1979-Continued 02448500, Noxubee River near Geiger, Ala.

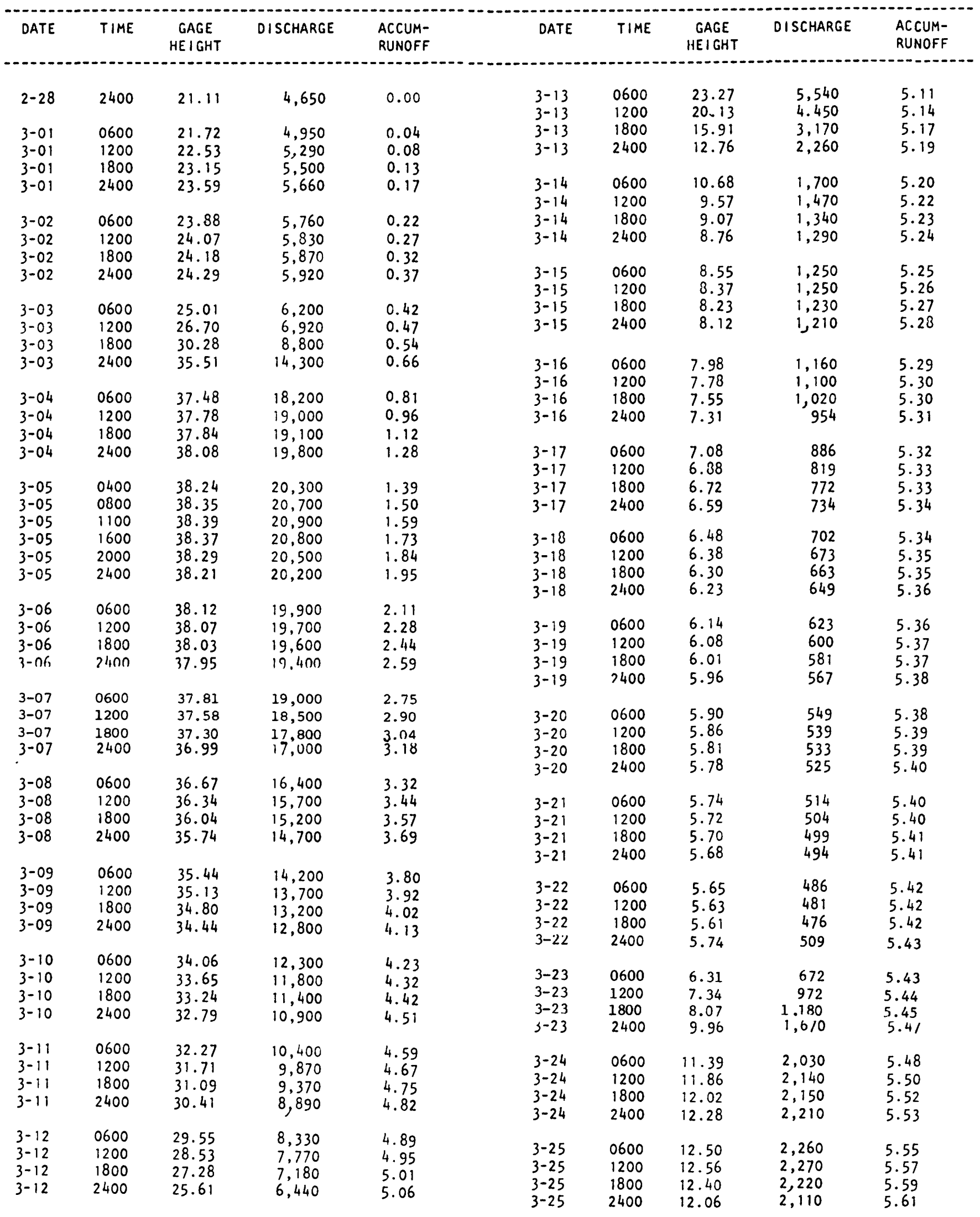


TABLE 7.-Gage height, discharge, and accumulated runoff, flood of April 1979-Continued 02448500, Noxubee River near Geiger, Ala.-Continued

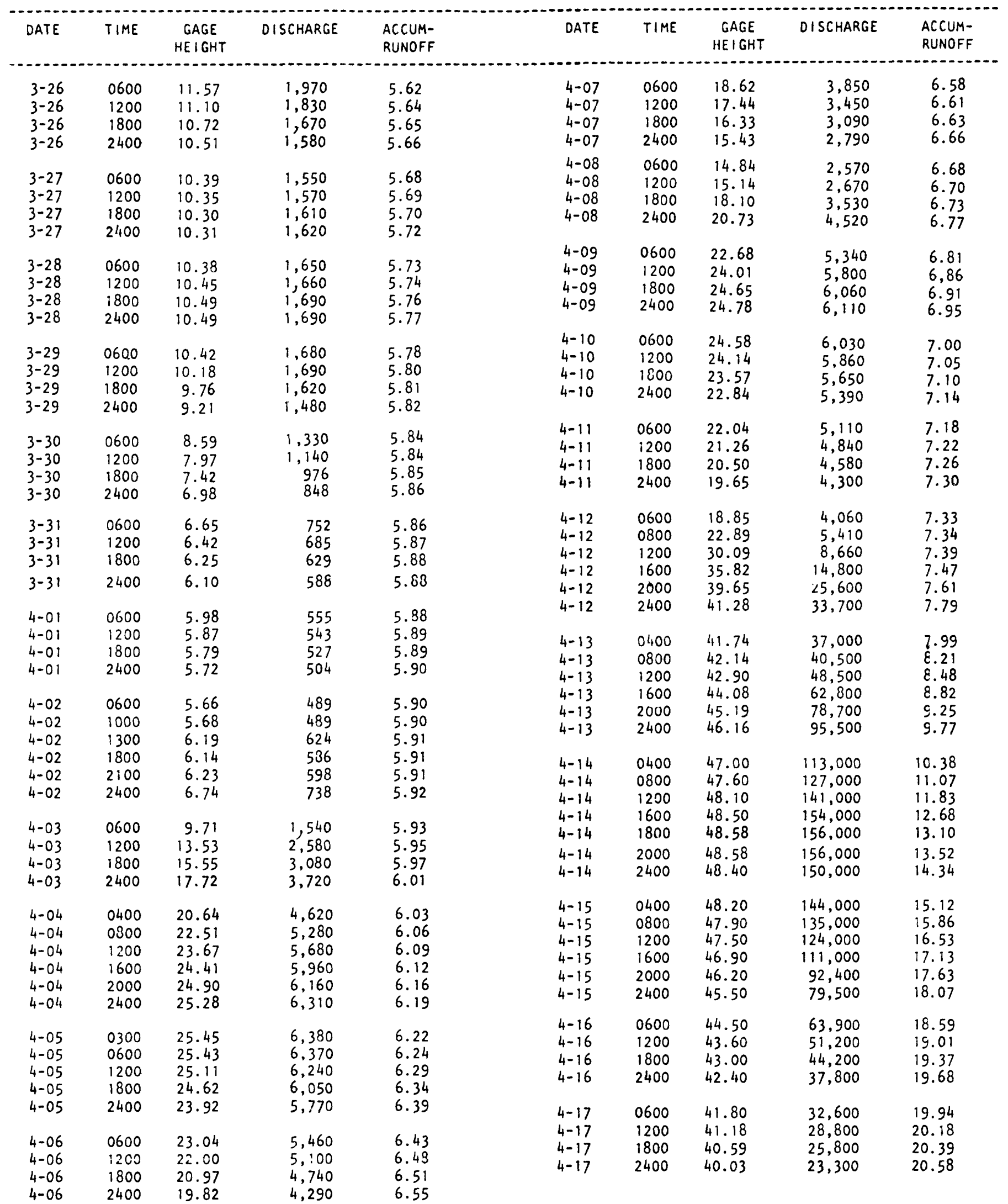


TABLES

TABLE 7.-Gage height, discharge, and accumulated runoff, flood of April 1979-Continued 02448500, Noxubee River near Geiger, Ala.-Continued

\begin{tabular}{|c|c|c|c|c|c|c|c|c|c|}
\hline DATE & TIME & $\begin{array}{l}\text { GAGE } \\
\text { HEIGHT }\end{array}$ & DISCHARGE & $\begin{array}{l}\text { ACCUM- } \\
\text { RUNOFF }\end{array}$ & DATE & TIME & $\begin{array}{c}\text { GAGE } \\
\text { HEIGHT }\end{array}$ & DISCHARGE & $\begin{array}{l}\text { ACCUM- } \\
\text { RUNOFF }\end{array}$ \\
\hline $\begin{array}{l}4-18 \\
4-18 \\
4-18 \\
4-18\end{array}$ & $\begin{array}{l}0600 \\
1200 \\
1800 \\
2400\end{array}$ & $\begin{array}{l}39.48 \\
38.98 \\
38.49 \\
38.02\end{array}$ & $\begin{array}{l}21,200 \\
19,500 \\
18,000 \\
16,600\end{array}$ & $\begin{array}{l}20.75 \\
20.91 \\
21.06 \\
21.19\end{array}$ & $\begin{array}{l}4-25 \\
4-25 \\
4-25 \\
4-25\end{array}$ & $\begin{array}{l}0600 \\
1200 \\
1800 \\
2400\end{array}$ & $\begin{array}{l}7.97 \\
8.15 \\
8.35 \\
8.43\end{array}$ & $\begin{array}{l}1,180 \\
1,290 \\
1,360 \\
1,420\end{array}$ & $\begin{array}{l}22.66 \\
22.67 \\
22.68 \\
22.69\end{array}$ \\
\hline $\begin{array}{l}4-19 \\
4-19 \\
4-19 \\
4-19\end{array}$ & $\begin{array}{l}0600 \\
1200 \\
1800 \\
2400\end{array}$ & $\begin{array}{l}37.54 \\
37.07 \\
36.59 \\
36.14\end{array}$ & $\begin{array}{l}15,600 \\
14,500 \\
13,600 \\
12,900\end{array}$ & $\begin{array}{l}21.32 \\
21.44 \\
21.55 \\
21.65\end{array}$ & $\begin{array}{l}4-26 \\
4-26 \\
4-26 \\
4-26\end{array}$ & $\begin{array}{l}0600 \\
1200 \\
1800 \\
2400\end{array}$ & $\begin{array}{l}8.58 \\
8.64 \\
8.66 \\
9.62\end{array}$ & $\begin{array}{l}1,490 \\
1,510 \\
1,510 \\
1,750\end{array}$ & $\begin{array}{l}22.70 \\
22.72 \\
22.73 \\
22.74\end{array}$ \\
\hline $\begin{array}{l}4-20 \\
4-20 \\
4-20 \\
4-20\end{array}$ & $\begin{array}{l}0600 \\
1200 \\
1800 \\
2400\end{array}$ & $\begin{array}{l}35.68 \\
35.21 \\
34.72 \\
34.20\end{array}$ & $\begin{array}{l}12,300 \\
11,600 \\
11,000 \\
10,500\end{array}$ & $\begin{array}{l}21.75 \\
21.85 \\
21.94 \\
22.02\end{array}$ & $\begin{array}{l}4-27 \\
4-27 \\
4-27 \\
4-27\end{array}$ & $\begin{array}{l}0600 \\
1200 \\
1800 \\
2400\end{array}$ & $\begin{array}{l}9.68 \\
9.43 \\
9.25 \\
9.00\end{array}$ & $\begin{array}{l}1,770 \\
1,700 \\
1,660 \\
1,600\end{array}$ & $\begin{array}{l}22.76 \\
22.77 \\
22.79 \\
22.80\end{array}$ \\
\hline $\begin{array}{l}4-21 \\
4-21 \\
4-21 \\
4-21\end{array}$ & $\begin{array}{l}0600 \\
1200 \\
1800 \\
2400\end{array}$ & $\begin{array}{l}33.66 \\
33.07 \\
32.41 \\
31.59\end{array}$ & $\begin{array}{l}9,930 \\
9,390 \\
8,810 \\
8,190\end{array}$ & $\begin{array}{l}22.60 \\
22.18 \\
22.25 \\
22.32\end{array}$ & $\begin{array}{l}4-28 \\
4-28 \\
4-28 \\
4-28\end{array}$ & $\begin{array}{l}0600 \\
1200 \\
1800 \\
2400\end{array}$ & $\begin{array}{l}8.74 \\
8.42 \\
8.13 \\
7.87\end{array}$ & $\begin{array}{l}1,530 \\
1,450 \\
1,380 \\
1,320\end{array}$ & $\begin{array}{l}22.81 \\
22.82 \\
22.83 \\
22.85\end{array}$ \\
\hline $\begin{array}{l}4-22 \\
4-22 \\
4-22 \\
4-22\end{array}$ & $\begin{array}{l}0600 \\
1200 \\
1800 \\
2400\end{array}$ & $\begin{array}{l}30.50 \\
29.06 \\
27.30 \\
25.21\end{array}$ & $\begin{array}{l}7,360 \\
6,430 \\
5,680 \\
4,960\end{array}$ & $\begin{array}{l}22.38 \\
22.44 \\
22.48 \\
22.52\end{array}$ & $\begin{array}{l}4-29 \\
4-29 \\
4-29 \\
4-29\end{array}$ & $\begin{array}{l}0600 \\
1200 \\
1300 \\
2400\end{array}$ & $\begin{array}{l}7.64 \\
7.44 \\
7.23 \\
7.05\end{array}$ & $\begin{array}{l}1,260 \\
1,210 \\
1,140 \\
1,100\end{array}$ & $\begin{array}{l}22.86 \\
22.87 \\
22.87 \\
22.88\end{array}$ \\
\hline $\begin{array}{l}4-23 \\
4-23 \\
4-23\end{array}$ & $\begin{array}{l}0600 \\
1200 \\
1800\end{array}$ & $\begin{array}{l}22.75 \\
20.01 \\
16.95\end{array}$ & $\begin{array}{l}3,930 \\
3,210 \\
2,400\end{array}$ & $\begin{array}{l}22.55 \\
22.58 \\
22.60\end{array}$ & $\begin{array}{l}4-30 \\
4-30\end{array}$ & $\begin{array}{l}0600 \\
1200\end{array}$ & $\begin{array}{l}6.88 \\
6.72\end{array}$ & $\begin{array}{l}1,050 \\
1,000\end{array}$ & $\begin{array}{l}22.89 \\
22.90\end{array}$ \\
\hline 4-23 & 2400 & 13.92 & 1,710 & 22.61 & $\begin{array}{l}4-30 \\
4-30\end{array}$ & $\begin{array}{l}1800 \\
2400\end{array}$ & $\begin{array}{l}6.57 \\
6.44\end{array}$ & $\begin{array}{l}955 \\
916\end{array}$ & $\begin{array}{l}22.91 \\
22.92\end{array}$ \\
\hline $\begin{array}{l}4-24 \\
4-24 \\
4-24 \\
4-24\end{array}$ & $\begin{array}{l}0600 \\
1200 \\
1800 \\
2400\end{array}$ & $\begin{array}{r}11.46 \\
9.65 \\
8.46 \\
7.98\end{array}$ & $\begin{array}{l}1,300 \\
1,200 \\
1,130 \\
1,110\end{array}$ & $\begin{array}{l}22.62 \\
22.63 \\
22.64 \\
22.65\end{array}$ & & & & & \\
\hline
\end{tabular}


TABLE 7.-Gage height, discharge, and accumulated runoff, flood of April 1979-Continued 02449000, Tombigbee River at Gainesville, Ala.

\begin{tabular}{|c|c|c|c|c|c|c|c|c|c|}
\hline DATE & TIME & $\begin{array}{c}\text { GAGE } \\
\text { HEIGHT }\end{array}$ & DISCHARGE & $\begin{array}{l}\text { ACCUM. } \\
\text { RUNOFF }\end{array}$ & DATE & TIME & $\begin{array}{c}\text { GAGE } \\
\text { HEIGHT }\end{array}$ & DISCHARGE & $\begin{array}{l}\text { ACCUM. } \\
\text { RUNOFF }\end{array}$ \\
\hline $2-28$ & 2400 & 31.58 & 46200 & 0.00 & $3-17$ & 1200 & 16.38 & 12700 & 3.88 \\
\hline $3-1$ & 1200 & 30.68 & 46900 & .10 & $3-18$ & 1200 & 15.58 & 10700 & 3.93 \\
\hline $3-2$ & 1200 & 30.49 & 46900 & .30 & $3-19$ & 1200 & 14.50 & 8490 & 3.97 \\
\hline $\begin{array}{l}3-3 \\
3-3\end{array}$ & $\begin{array}{l}1200 \\
2400\end{array}$ & $\begin{array}{l}31.67 \\
37.18\end{array}$ & $\begin{array}{l}49000 \\
63800\end{array}$ & $\begin{array}{l}.51 \\
.65\end{array}$ & $3-20$ & 1200 & 14.45 & 8240 & 4.01 \\
\hline $\begin{array}{l}3-4 \\
3-4 \\
3-4 \\
3-4\end{array}$ & $\begin{array}{l}0600 \\
1200 \\
1800 \\
2400\end{array}$ & $\begin{array}{l}39.06 \\
41.57 \\
42.34 \\
42.80\end{array}$ & $\begin{array}{l}72900 \\
87400 \\
91300 \\
93800\end{array}$ & $\begin{array}{r}.73 \\
.82 \\
.92 \\
1.02\end{array}$ & $\begin{array}{l}3-22 \\
3-23\end{array}$ & 1200 & $\begin{array}{l}13.93 \\
14.28 \\
15.67\end{array}$ & 7270 & $\begin{array}{l}4.07 \\
4.12\end{array}$ \\
\hline $\begin{array}{l}3-5 \\
3-5 \\
3-5 \\
3-5 \\
3-5\end{array}$ & $\begin{array}{l}0600 \\
1200 \\
1600 \\
1800 \\
2400\end{array}$ & $\begin{array}{l}43.12 \\
43.26 \\
43.15 \\
43.35 \\
43.40\end{array}$ & $\begin{array}{l}94700 \\
95600 \\
96100 \\
95700 \\
96000\end{array}$ & $\begin{array}{l}1.12 \\
1.22 \\
1.29 \\
1.32 \\
1.42\end{array}$ & $\begin{array}{l}3-24 \\
3-25 \\
3-26\end{array}$ & $\begin{array}{l}1200 \\
1200 \\
1200\end{array}$ & $\begin{array}{l}19.29 \\
21.95 \\
22.44\end{array}$ & $\begin{array}{l}20200 \\
25700 \\
27000\end{array}$ & $\begin{array}{l}4.21 \\
4.32 \\
4.44\end{array}$ \\
\hline $\begin{array}{l}3-6 \\
3-6 \\
3-6 \\
3-6\end{array}$ & $\begin{array}{l}0600 \\
1200 \\
1800 \\
2400\end{array}$ & $\begin{array}{l}43.44 \\
43.32 \\
43.29 \\
43.21\end{array}$ & $\begin{array}{l}96200 \\
95400 \\
95100 \\
94600\end{array}$ & $\begin{array}{l}1.52 \\
1.62 \\
1.72 \\
1.82\end{array}$ & $\begin{array}{l}3-27 \\
3-28\end{array}$ & $\begin{array}{l}1200 \\
1200\end{array}$ & $\begin{array}{l}22.74 \\
21.74\end{array}$ & $\begin{array}{l}27400 \\
25100\end{array}$ & $\begin{array}{l}4.56 \\
4.67\end{array}$ \\
\hline $3-7$ & 1200 & 42.87 & 91600 & 2.02 & $3-29$ & 1200 & 19.37 & 18800 & 4.75 \\
\hline $3-8$ & 1200 & 41.78 & 86700 & 2.39 & $\begin{array}{l}3-30 \\
3-31\end{array}$ & $\begin{array}{l}1200 \\
1200\end{array}$ & $\begin{array}{l}16.75 \\
16.34\end{array}$ & $\begin{array}{l}13800 \\
13000\end{array}$ & $\begin{array}{l}4.81 \\
4.87\end{array}$ \\
\hline 3- 9 & 1200 & 40.86 & 81600 & 2.74 & $4-1$ & & 15.07 & 10500 & 4.91 \\
\hline $\begin{array}{l}3-10 \\
3-11\end{array}$ & $\begin{array}{l}1200 \\
1200\end{array}$ & $\begin{array}{l}38.96 \\
35.69\end{array}$ & $\begin{array}{l}71900 \\
60000\end{array}$ & $\begin{array}{l}3.03 \\
3.29\end{array}$ & $4-2$ & 1200 & 15.57 & 12400 & 4.96 \\
\hline $3-12$ & 1200 & 31.79 & 46100 & 3.49 & $\begin{array}{l}4-3 \\
4-4\end{array}$ & $\begin{array}{l}1200 \\
1200\end{array}$ & 21.48 & 23500 & 5.06 \\
\hline $3-13$ & 1200 & 26.80 & 31600 & 3.63 & $4-5$ & 1200 & 29.01 & 41400 & 5.40 \\
\hline $3-14$ & 1200 & 21.34 & 20700 & 3.72 & $4-6$ & 1200 & 28.84 & 40900 & 5.57 \\
\hline $\begin{array}{l}3-15 \\
3-16\end{array}$ & 1200 & 17.79 & 14000 & 3.78 & $4-7$ & 1200 & 28.15 & 38900 & 5.74 \\
\hline
\end{tabular}


TABLES

TABLE 7.-Gage height, discharge, and accumulated runoff, flood of April 1979-Continued 02449000, Tombigbee River at Gainesville, Ala.-Continued

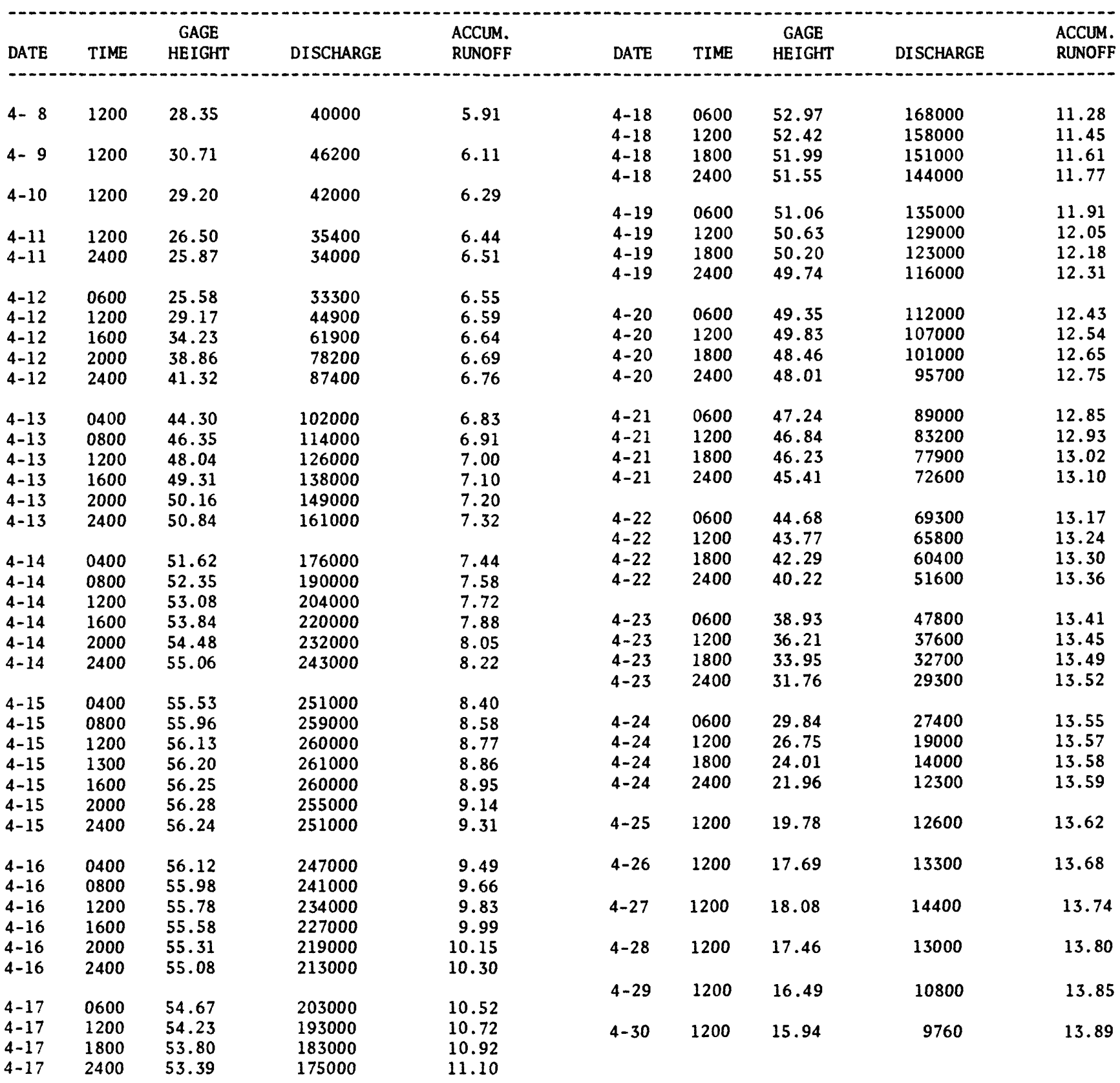


TABLE 7.-Gage height, discharge, and accumulated runoff, flood of April 1979-Continued 02449245, Brush Creek near Eutaw, Ala.

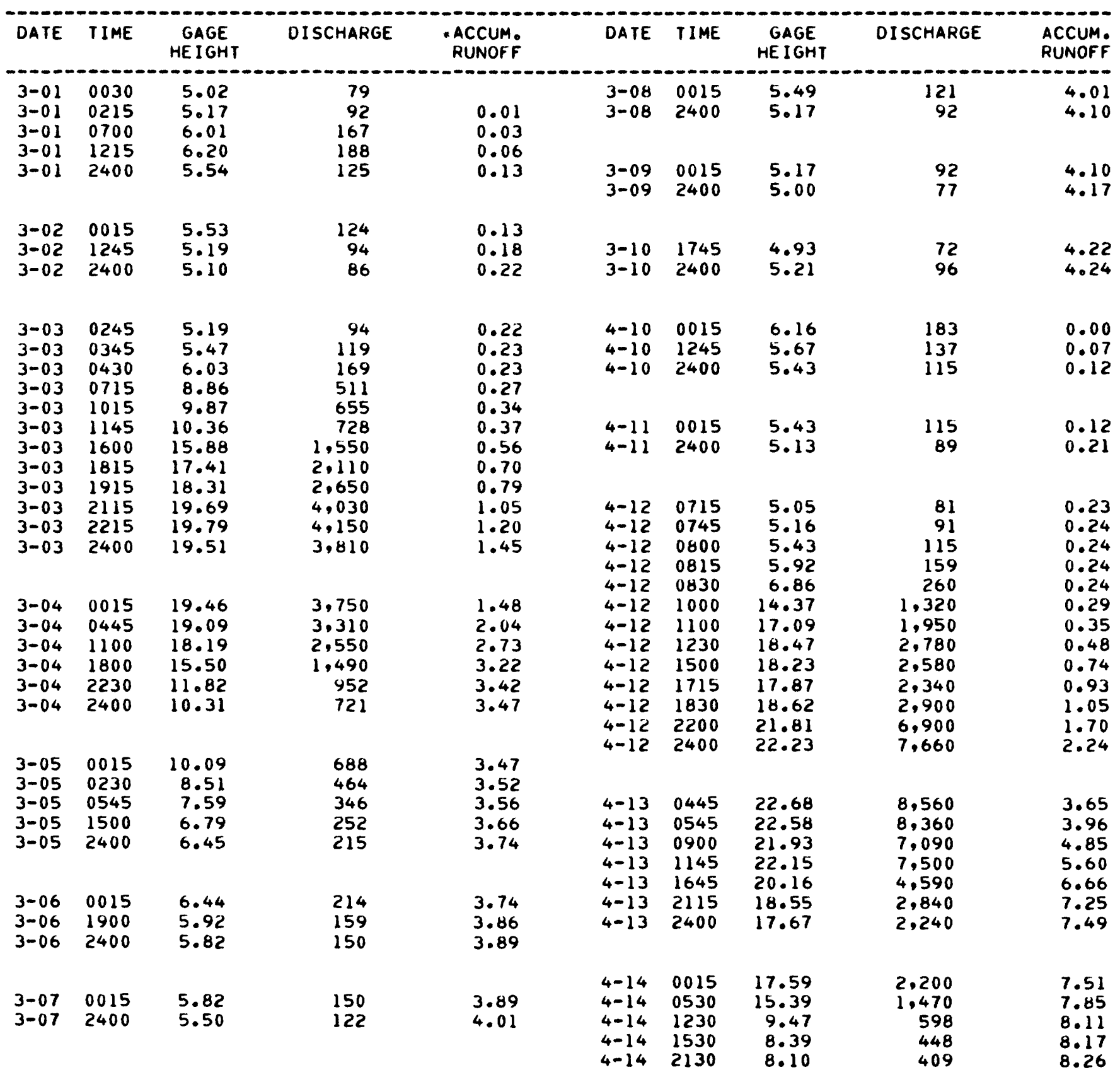


TABLE 7.-Gage height, discharge, and accumulated runoff, flood of April 1979-Continued 02449245, Brush Creek near Eutaw, Ala.-Continued

\begin{tabular}{|c|c|c|c|c|c|c|c|c|c|}
\hline DATE & TIME & $\begin{array}{l}\text { GAGE } \\
\text { HEIGHT }\end{array}$ & UISCHARGE & $\begin{array}{l}\text { ACCUM. } \\
\text { RUNOFF }\end{array}$ & DATE & TIME & $\begin{array}{c}\text { GAGE } \\
\text { HE IGHT }\end{array}$ & DISCHARGE & $\begin{array}{l}\text { ACCUM, } \\
\text { RUNOFF }\end{array}$ \\
\hline $4-14$ & 2400 & 8.22 & 425 & 8.29 & $\begin{array}{l}4-18 \\
4-18\end{array}$ & $\begin{array}{l}0015 \\
2400\end{array}$ & $\begin{array}{l}7.93 \\
6.25\end{array}$ & $\begin{array}{l}387 \\
193\end{array}$ & $\begin{array}{l}9.74 \\
9.99\end{array}$ \\
\hline $\begin{array}{l}4-15 \\
4-15\end{array}$ & $\begin{array}{l}2200 \\
2400\end{array}$ & $\begin{array}{l}9.70 \\
9.74\end{array}$ & $\begin{array}{l}631 \\
637\end{array}$ & $\begin{array}{l}8.73 \\
8.77\end{array}$ & & & & & \\
\hline $\begin{array}{l}4-16 \\
4-16\end{array}$ & $\begin{array}{l}0745 \\
2400\end{array}$ & $\begin{array}{l}9.77 \\
9.30\end{array}$ & $\begin{array}{l}641 \\
573\end{array}$ & $\begin{array}{l}8.95 \\
9.32\end{array}$ & $\begin{array}{l}4-19 \\
4-19 \\
4-19\end{array}$ & $\begin{array}{l}0015 \\
1715 \\
2400\end{array}$ & $\begin{array}{l}6.25 \\
5.17 \\
4.85\end{array}$ & $\begin{array}{r}193 \\
92 \\
67\end{array}$ & $\begin{array}{r}9.99 \\
10.08 \\
10.10\end{array}$ \\
\hline $\begin{array}{l}4-17 \\
4-17\end{array}$ & $\begin{array}{l}0015 \\
2400\end{array}$ & $\begin{array}{l}9.29 \\
7.95\end{array}$ & $\begin{array}{l}572 \\
389\end{array}$ & $\begin{array}{l}9.32 \\
9.74\end{array}$ & $\begin{array}{l}4-20 \\
4-20 \\
4-20\end{array}$ & $\begin{array}{l}0015 \\
0830 \\
2400\end{array}$ & $\begin{array}{l}4.84 \\
4.68 \\
4.59\end{array}$ & $\begin{array}{l}66 \\
55 \\
49\end{array}$ & $\begin{array}{l}10.10 \\
10.11 \\
10.14\end{array}$ \\
\hline
\end{tabular}


TABLE 7.-Gage height, discharge, and accumulated runoff, flood of April 1979-Continued 02461500, Valley Creek near Bessemer, Ala.

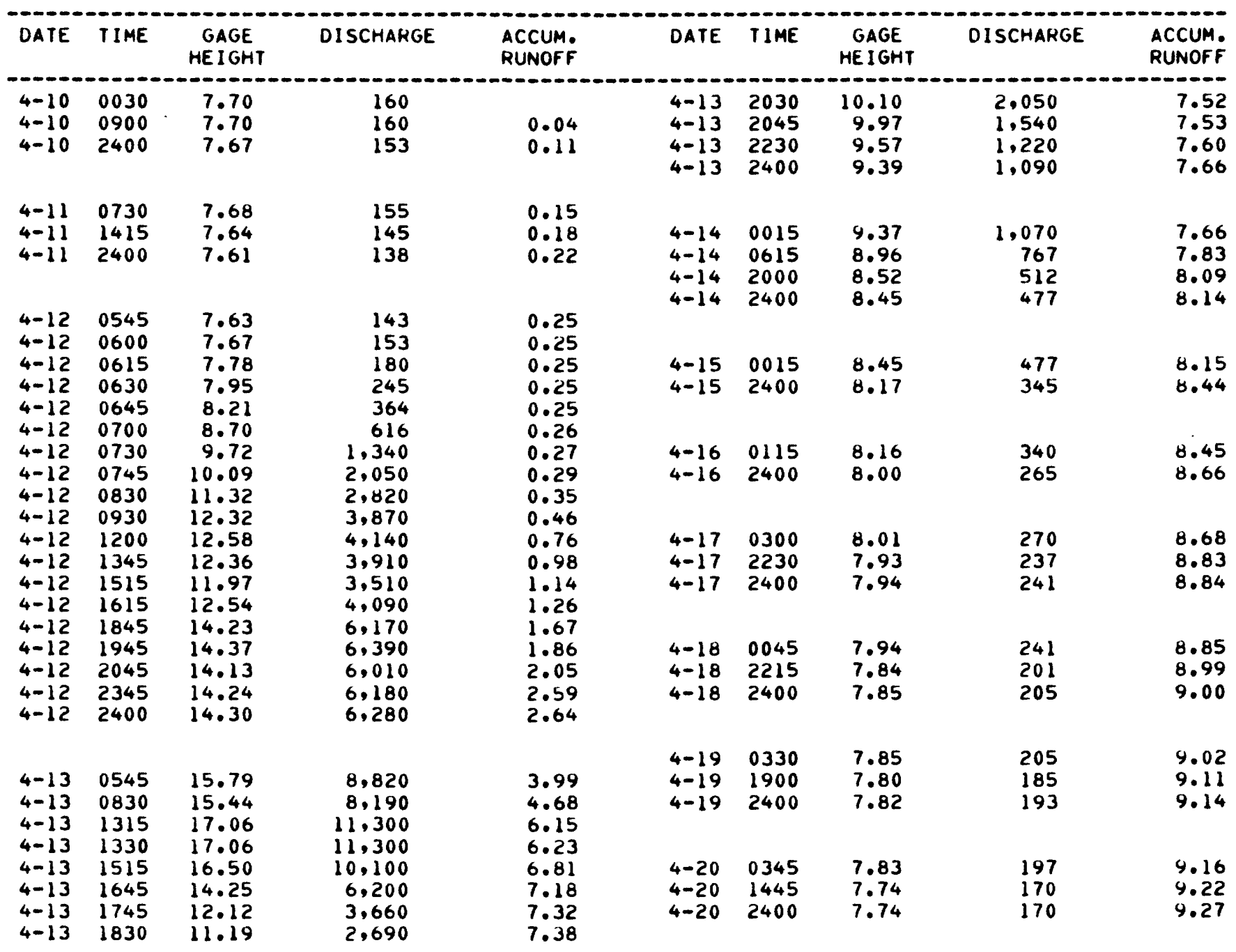


TABLES

TABLE 7.-Gage height, discharge, and accumulated runoff, flood of April 1979-Continued 02462000, Valley Creek near Oak Grove, Ala.

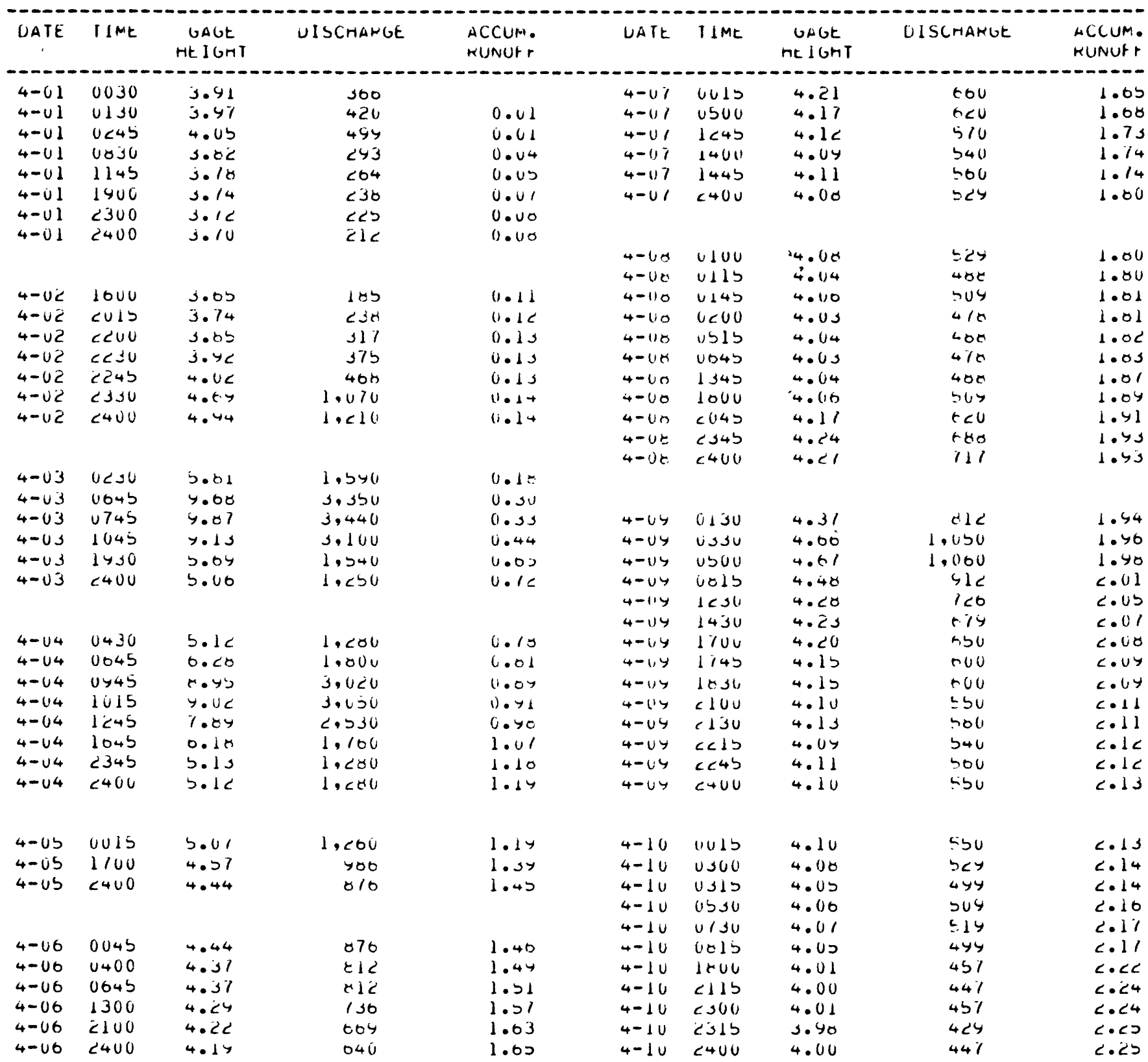


TABLE 7.-Gage height, discharge, and accumulated runoff, flood of April 1979-Continued 02462000, Valley Creek near Oak Grove, Ala.-Continued

\begin{tabular}{|c|c|c|c|c|c|c|c|c|c|}
\hline UAIE & TIME & $\begin{array}{l}\text { uAut } \\
\text { He l GRI }\end{array}$ & UlSCMAKut. & $\begin{array}{l}\text { mCCUM. } \\
\text { rurut }\end{array}$ & UAIt & I I ME & $\begin{array}{c}\text { GAGE } \\
\text { ME IGHI }\end{array}$ & UISCMAKUt & $\begin{array}{l}\text { ACLUM. } \\
\text { KunUt }\end{array}$ \\
\hline--- & - & $=-$ & . & - & 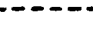 & 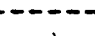 & $---m$ & -- & $-\infty-\infty$ \\
\hline $4-11$ & 0430 & 4.00 & 447 & $2 \cdot c 1$ & $4-10$ & 0115 & 4.21 & 711 & $10 \cdot<j$ \\
\hline $4-11$ & $1<36$ & 3.47 & 420 & $2 \cdot 31$ & $4-18$ & $c<1)$ & 4.11 & 500 & $10 \cdot 37$ \\
\hline $4-11$ & 1030 & 3.74 & נצנ & $2 \cdot 3 c$ & $4-10$ & $c<4 b$ & $4.1<$ & 510 & $10 \cdot 31$ \\
\hline $4-11$ & 1945 & 3.53 & $4 U C$ & $2 \cdot 34$ & $4-18$ & $\angle 400$ & 4.11 & 500 & 10.38 \\
\hline \multirow{2}{*}{$4-11$} & $<400$ & 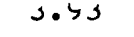 & 304 & C.so & & & & & \\
\hline & & & & & $4-1 y$ & $1<45$ & 4.13 & 900 & 10.40 \\
\hline $4-12$ & $v<45$ & 5.41 & 300 & $\dot{c} \cdot g$ & $4-14$ & 11345 & $4 \cdot 07$ & $=40$ & 10.40 \\
\hline $4-12$ & 0045 & $3 \cdot>1$ & 300 & $2 \cdot \sin$ & $4-1 y$ & טכ 1 כט & 4.11 & sou & $10 \cdot 41$ \\
\hline $4-12$ & U/15 & 4.04 & $4 \pi 8$ & $2 \cdot 3 c$ & $4-14$ & טנד טu & 4.00 & $2<y$ & $10 \cdot 4 c$ \\
\hline $4-12$ & vovo & $4 \cdot C E$ & $1<0$ & C.sy & $4-1 y$ & 0030 & 4.10 & 500 & 10.43 \\
\hline $4-16$ & 0415 & $4 \cdot\langle y$ & 130 & $2 \cdot 411$ & $4-1 y$ & 1045 & 4.05 & 4yy & 10.41 \\
\hline $4-12$ & 1000 & 4.40 & 694 & $c \cdot 41$ & $4-1 y$ & 1030 & 4.01 & -14 & 10.40 \\
\hline $4-1 C$ & 1130 & 5.00 & $1.5<0$ & 2.43 & $4-1 y$ & $<\cup 4 b$ & ל. & 4Yל & 10.50 \\
\hline $4-12$ & $1<15$ & 1.44 & $c \cdot s c b$ & 2.44 & $4-1 y$ & $<400$ & 4.03 & +10 & $10.5<$ \\
\hline $4-12$ & 1400 & 14.10 & טלניניט & $2 \cdot 2 c$ & & & & & \\
\hline $4-12$ & 1000 & $\langle U \cdot c y$ & $\alpha \cdot c b 0$ & 2.02 & & & & & \\
\hline \multirow[t]{3}{*}{$4-1 c$} & $\angle 400$ & Cs.t & 10.000 & 3.41 & $4-c u$ & U1ju & 4.114 & 400 & $10 \cdot b<$ \\
\hline & & & & & $4-20$ & 0150 & 4.06 & 441 & כל. 10 \\
\hline & & & & & $4-c u$ & 1000 & $4.0 \mathrm{C}$ & 400 & Iu.bl \\
\hline $4-13$ & 0430 & cb.ue & $1<\cdot i v 0$ & כיצ.3 & $4-<0$ & 1500 & 4.00 & 447 & צל 10 \\
\hline $4-13$ & 0745 & $2 t .01$ & $14,5 \cup 0$ & $4 \cdot 43$ & $4-<v$ & b & 4.00 & 447 & 10.01 \\
\hline $4-13$ & $1<15$ & $C>.14$ & $c^{4,000}$ & 5.40 & $4-<v$ & 1030 & 4.03 & 470 & 10.01 \\
\hline $4-13$ & 1015 & Cy.cu & 20,500 & 0.47 & $4-60$ & $\angle U O U$ & 4.01 & 457 & 10.01 \\
\hline $4-13$ & 1730 & $<y .80$ & $\angle 0,000$ & 1.01 & $4-<U$ & $\angle 400$ & 3.40 & 411 & 10.03 \\
\hline $4-13$ & 2345 & $<0.10$ & 10,400 & $6 \cdot<0$ & & & & & \\
\hline \multirow[t]{3}{*}{$4-13$} & 2400 & 20.50 & 10.000 & o.se & & & & & \\
\hline & & & & & $4-<1$ & 1.030 & 4.00 & 447 & 10.01 \\
\hline & & & & & $4-<1$ & $1 \cup 0 U$ & $3 . y y$ & 450 & 10.60 \\
\hline $4-14$ & 0015 & 20.50 & 1 I ל ל & $M .30$ & $4-21$ & 2400 & 3.48 & $4<y$ & 10.74 \\
\hline $4-14$ & 1315 & $c c .10$ & $4,<7 u$ & r.. 14 & & & & & \\
\hline $4-14$ & 0130 & 10.01 & 3.010 & $y \cdot 1,3$ & & & & & \\
\hline $4-14$ & $\checkmark 745$ & 0.04 & 2.070 & 9.11 & $4-C c$ & 0700 & 3.90 & $4<y$ & 10.71 \\
\hline $4-14$ & 1730 & 0.10 & $2 \cdot 030$ & 4.30 & $4-C C$ & 1100 & 3.44 & 343 & 10.14 \\
\hline \multirow[t]{3}{*}{$4-14$} & $<400$ & ל. & $1,0<0$ & 4.43 & $+-<c$ & 1015 & $s . y s$ & 304 & 10.01 \\
\hline & & & & & $4-c .<$ & 1030 & 3.40 & 411 & $10.0<$ \\
\hline & & & & & $4-2 c$ & $\angle 400$ & 4.110 & 447 & b. \\
\hline $4-15$ & 0015 & ל.tל & 1,010 & 4.43 & & & & & \\
\hline $4-15$ & 1345 & 4.45 & $1 \cdot \csc$ & 4.03 & & & & & \\
\hline \multirow[t]{3}{*}{$4-1 b$} & 2400 & 4.75 & 1,110 & 4.10 & $4-<3$ & U 15 & 4.10 & טכל & $10.0 \%$ \\
\hline & & & & & $4-<3$ & 4715 & 4.15 & t.uU & 10.04 \\
\hline & & & & & $4-<3$ & uruu & 4.14 & 590 & $10 \cdot 40$ \\
\hline $4-10$ & 0045 & $4.7 t$ & 1,120 & Y. 71 & $4-<3$ & 1400 & 3.90 & $4<4$ & נצ. 10 \\
\hline \multirow[t]{2}{*}{$4-10$} & $\angle 400$ & 4.44 & 010 & 10.01 & $4-<3$ & $\cos 0$ & 3. & 384 & $10 \cdot 4$ \\
\hline & & & & & $4-<3$ & $\angle 400$ & 3.91 & 300 & $10 \cdot 41$ \\
\hline $4-17$ & ל11 & 4.45 & 085 & $10.0 \mathrm{~s}$ & & & & & \\
\hline \multirow[t]{3}{*}{$4-17$} & 2400 & $4 .<0$ & 707 & $10 \cdot c c$ & $4-<4$ & $1 / 1 \mathrm{~b}$ & 3.41 & 306 & 11.04 \\
\hline & & & & & $4-<4$ & culb & 3.94 & 3y & 11.6 \\
\hline & & & & & $4-<4$ & $\angle 400$ & 4.01 & 4 & 11.01 \\
\hline
\end{tabular}


TABLES

TABLE 7.-Gage height, discharge, and accumulated runoff, flood of April 1979-Continued 02462000, Valley Creek near Oak Grove, Ala-Continued

\begin{tabular}{|c|c|c|c|c|c|c|c|c|c|}
\hline DATE & TIME & $\begin{array}{l}\text { GAGE } \\
\text { HEIGHT }\end{array}$ & UISCHAKGE & $\begin{array}{l}\text { ACCUM. } \\
\text { RUNOFF }\end{array}$ & DATE & TIME & $\begin{array}{c}\text { GAGE } \\
H E ̈ \perp G H T\end{array}$ & UISCHAKGE & $\begin{array}{l}\text { ACCUM. } \\
\text { RUNURF }\end{array}$ \\
\hline $\begin{array}{l}4-25 \\
4-25 \\
4-25 \\
4-25 \\
4-25 \\
4-25 \\
4-25 \\
4-25\end{array}$ & $\begin{array}{l}0300 \\
0500 \\
1100 \\
1315 \\
1730 \\
2015 \\
2345 \\
2400\end{array}$ & $\begin{array}{l}4.61 \\
4.08 \\
3.57 \\
3.49 \\
4.15 \\
4.28 \\
4.49 \\
4.48\end{array}$ & $\begin{array}{l}457 \\
529 \\
420 \\
430 \\
600 \\
726 \\
921 \\
912\end{array}$ & $\begin{array}{l}11.08 \\
11.09 \\
11.12 \\
11.13 \\
11.15 \\
11.17 \\
11.21 \\
11.21\end{array}$ & $\begin{array}{l}4-27 \\
4-27 \\
4-27 \\
4-27 \\
4-27 \\
4-27 \\
4-27 \\
4-27\end{array}$ & $\begin{array}{l}0015 \\
0115 \\
0215 \\
0245 \\
0715 \\
1130 \\
2 \$ 30 \\
2400\end{array}$ & $\begin{array}{l}5.44 \\
7.25 \\
7.62 \\
7.52 \\
5.04 \\
4.55 \\
4.26 \\
4.25\end{array}$ & $\begin{array}{r}1,420 \\
2,240 \\
2,400 \\
2,360 \\
1,270 \\
470 \\
707 \\
646\end{array}$ & $\begin{array}{l}11.48 \\
11.50 \\
11.53 \\
11.04 \\
11.02 \\
11.07 \\
11.78 \\
11.78\end{array}$ \\
\hline $\begin{array}{l}4-26 \\
4-26 \\
4-26 \\
4-26 \\
4-26 \\
4-26 \\
4-26 \\
4-26\end{array}$ & $\begin{array}{l}0400 \\
0615 \\
1400 \\
1400 \\
2130 \\
2300 \\
2315 \\
2400\end{array}$ & $\begin{array}{l}4.93 \\
5.54 \\
4.51 \\
4.35 \\
4.39 \\
4.39 \\
4.44 \\
5.44\end{array}$ & $\begin{array}{r}1,200 \\
1,470 \\
438 \\
793 \\
831 \\
831 \\
876 \\
1,420\end{array}$ & $\begin{array}{l}11.25 \\
11.29 \\
11.38 \\
11.43 \\
11.45 \\
11.46 \\
11.47 \\
11.48\end{array}$ & $\begin{array}{l}4-2 y \\
4-29 \\
4-2 y \\
4-2 y \\
4-2 y\end{array}$ & $\begin{array}{l}0045 \\
0730 \\
0015 \\
1215 \\
2400\end{array}$ & $\begin{array}{l}4.08 \\
4.05 \\
4.02 \\
3.94 \\
3.98\end{array}$ & $\begin{array}{l}529 \\
499 \\
468 \\
438 \\
429\end{array}$ & $\begin{array}{l}11.79 \\
11.83 \\
11.84 \\
11.87 \\
11.94\end{array}$ \\
\hline
\end{tabular}


TABLE 7.-Gage height, discharge, and accumulated runoff, flood of April 1979-Continued 02464000, North River near Samantha, Ala.

\begin{tabular}{|c|c|c|c|c|c|c|c|c|c|}
\hline DATE & TIME & $\begin{array}{l}\text { GAGE } \\
\text { HEIGHT }\end{array}$ & DISCHARGE & $\begin{array}{l}\text { ACCUM. } \\
\text { RUNOFF }\end{array}$ & DATE & TIME & $\begin{array}{l}\text { GAGE } \\
\text { HE IGHT }\end{array}$ & DISCHARGE & $\begin{array}{l}\text { ACCUM. } \\
\text { RUNOFF }\end{array}$ \\
\hline $\begin{array}{l}4-10 \\
4-10 \\
4-10 \\
4-11 \\
4-11\end{array}$ & $\begin{array}{l}0200 \\
0100 \\
2400 \\
0200 \\
2400\end{array}$ & $\begin{array}{l}3.00 \\
3.00 \\
2.79 \\
\\
2.79 \\
2.69\end{array}$ & $\begin{array}{l}500 \\
500 \\
385\end{array}$ & $\begin{array}{l}0.08 \\
0.14\end{array}$ & $\begin{array}{l}4-15 \\
4-15 \\
4-15 \\
4-15 \\
4-15 \\
4-15\end{array}$ & $\begin{array}{l}0100 \\
0400 \\
0700 \\
0900 \\
1100 \\
2400\end{array}$ & $\begin{array}{r}20.13 \\
15.34 \\
8.90 \\
6.85 \\
6.22 \\
5.06\end{array}$ & $\begin{array}{r}10,200 \\
7,060 \\
3,680 \\
2,550 \\
2,200 \\
1,580\end{array}$ & $\begin{array}{l}7.49 \\
7.67 \\
7.77 \\
7.81 \\
7.84 \\
8.01\end{array}$ \\
\hline $\begin{array}{l}4-12 \\
4-12 \\
4-12 \\
4-12 \\
4-12 \\
4-12 \\
4-12 \\
4-12 \\
4-12 \\
4-12\end{array}$ & $\begin{array}{l}0500 \\
0600 \\
0800 \\
0900 \\
1000 \\
1100 \\
1200 \\
1600 \\
2300 \\
2400\end{array}$ & $\begin{array}{r}2.66 \\
3.17 \\
4.95 \\
7.75 \\
9.60 \\
10.57 \\
13.42 \\
25.21 \\
29.25 \\
29.44\end{array}$ & $\begin{array}{r}321 \\
594 \\
1,530 \\
3,040 \\
4,060 \\
4,570 \\
5,990 \\
13,700 \\
17,900 \\
18,100\end{array}$ & $\begin{array}{l}0.15 \\
0.15 \\
0.17 \\
0.19 \\
0.22 \\
0.25 \\
0.29 \\
0.61 \\
1.43 \\
1.56\end{array}$ & $\begin{array}{l}4-16 \\
4-16 \\
4-17 \\
4-17\end{array}$ & $\begin{array}{l}0100 \\
2400 \\
0100 \\
2400\end{array}$ & $\begin{array}{l}5.04 \\
4.13 \\
4.08 \\
3.61 \\
\\
3.60 \\
3.28\end{array}$ & $\begin{array}{r}1,570 \\
1,120 \\
1,090 \\
836 \\
\\
830 \\
654\end{array}$ & $\begin{array}{l}8.02 \\
8.23 \\
\\
8.24 \\
8.39 \\
\\
8.40 \\
8.52\end{array}$ \\
\hline $\begin{array}{l}4-13 \\
4-13 \\
4-13\end{array}$ & $\begin{array}{l}1000 \\
2200 \\
2400\end{array}$ & $\begin{array}{l}30.55 \\
30.16 \\
30.00\end{array}$ & $\begin{array}{l}19,400 \\
18,900 \\
18,700\end{array}$ & $\begin{array}{l}2.88 \\
4.50 \\
4.76\end{array}$ & $\begin{array}{l}4-19 \\
4-19\end{array}$ & $\begin{array}{l}0100 \\
2400\end{array}$ & $\begin{array}{l}3.27 \\
3.03\end{array}$ & $\begin{array}{l}649 \\
517\end{array}$ & $\begin{array}{l}8.5 \\
8.6\end{array}$ \\
\hline & & & & & $\begin{array}{l}4-20 \\
4-20\end{array}$ & $\begin{array}{l}0100 \\
2400\end{array}$ & $\begin{array}{l}3.02 \\
2.86\end{array}$ & $\begin{array}{l}511 \\
423\end{array}$ & $\begin{array}{l}8.6 \\
8.6\end{array}$ \\
\hline $\begin{array}{l}4-14 \\
4-14 \\
4-14\end{array}$ & $\begin{array}{l}0100 \\
1500 \\
2400\end{array}$ & $\begin{array}{l}29.91 \\
26.72 \\
21.17\end{array}$ & $\begin{array}{l}18,600 \\
15,100 \\
10,900\end{array}$ & $\begin{array}{l}4.89 \\
6.60 \\
7.42\end{array}$ & $\begin{array}{l}4-21 \\
4-21\end{array}$ & $\begin{array}{l}0100 \\
2400\end{array}$ & $\begin{array}{l}2.85 \\
2.75\end{array}$ & $\begin{array}{l}418 \\
365\end{array}$ & $\begin{array}{l}8.70 \\
8.76\end{array}$ \\
\hline
\end{tabular}


TABLES

TABLE 7.-Gage height, discharge, and accumulated runoff, flood of April 1979-Continued 02465000, Black Warrior River at Northport, Ala.

\begin{tabular}{|c|c|c|c|c|c|c|c|c|c|}
\hline DATE & TIME & $\begin{array}{l}\text { GAGE } \\
\text { HEIGHT }\end{array}$ & DISCHARGE & $\begin{array}{l}\text { ACCUM- } \\
\text { RUNOFF }\end{array}$ & DATE & TIME & $\begin{array}{c}\text { GAGE } \\
\text { HEI GHT }\end{array}$ & DISCHARGE & $\begin{array}{l}\text { ACCUM- } \\
\text { RUNDFF }\end{array}$ \\
\hline $4-11$ & 2400 & 42.67 & 12300 & & $\begin{array}{l}4-14 \\
4-14\end{array}$ & $\begin{array}{l}2200 \\
2400\end{array}$ & $\begin{array}{l}60.49 \\
60.15\end{array}$ & $\begin{array}{l}150000 \\
144000\end{array}$ & $\begin{array}{l}3.80 \\
3.89\end{array}$ \\
\hline $\begin{array}{l}4-12 \\
4-12 \\
4-12 \\
4-12 \\
4-12\end{array}$ & $\begin{array}{l}0600 \\
1200 \\
1600 \\
2000 \\
2400\end{array}$ & $\begin{array}{l}42.48 \\
44.78 \\
51.90 \\
58.48 \\
61.30\end{array}$ & $\begin{array}{r}11100 \\
28500 \\
102000 \\
173000 \\
199000\end{array}$ & $\begin{array}{l}0.02 \\
0.08 \\
0.21 \\
0.43 \\
0.68\end{array}$ & $\begin{array}{l}4-15 \\
4-15 \\
4-15 \\
4-15\end{array}$ & $\begin{array}{l}0600 \\
1200 \\
1800 \\
2400\end{array}$ & $\begin{array}{l}59.40 \\
58.18 \\
57.28 \\
55.67\end{array}$ & $\begin{array}{r}136000 \\
123000 \\
117000 \\
99500\end{array}$ & $\begin{array}{l}4.16 \\
4.39 \\
4.62 \\
4.81\end{array}$ \\
\hline $\begin{array}{l}4-13 \\
4-13 \\
4-13 \\
4-13 \\
4-13\end{array}$ & $\begin{array}{l}0400 \\
0800 \\
0900 \\
1000 \\
1100\end{array}$ & $\begin{array}{l}62.87 \\
64.16 \\
64.90 \\
65.99 \\
66.80\end{array}$ & $\begin{array}{l}209000 \\
214000 \\
230000 \\
269000 \\
272000\end{array}$ & $\begin{array}{l}0.95 \\
1.23 \\
1.30 \\
1.39 \\
1.48\end{array}$ & $\begin{array}{l}4-16 \\
4-16 \\
4-16 \\
4-16\end{array}$ & $\begin{array}{l}0600 \\
1200 \\
1800 \\
2400\end{array}$ & $\begin{array}{l}54.44 \\
52.52 \\
51.11 \\
48.17\end{array}$ & $\begin{array}{l}90000 \\
74800 \\
66600 \\
46300\end{array}$ & $\begin{array}{l}4.98 \\
5.13 \\
5.26 \\
5.35\end{array}$ \\
\hline $\begin{array}{l}4-13 \\
4-13 \\
4-13 \\
4-13\end{array}$ & $\begin{array}{l}1200 \\
1600 \\
2000 \\
2400\end{array}$ & $\begin{array}{l}66.85 \\
66.17 \\
65.59 \\
64.97\end{array}$ & $\begin{array}{l}265000 \\
245000 \\
239000 \\
231000\end{array}$ & $\begin{array}{l}1.56 \\
1.88 \\
2.18 \\
2.48\end{array}$ & $\begin{array}{l}4-17 \\
4-17 \\
4-17 \\
4-17 \\
4-17\end{array}$ & $\begin{array}{l}0200 \\
0400 \\
0600 \\
1200 \\
1400\end{array}$ & $\begin{array}{l}47.12 \\
46.02 \\
45.34 \\
45.14 \\
45.20\end{array}$ & $\begin{array}{l}39400 \\
31000 \\
30400 \\
31400 \\
31900\end{array}$ & $\begin{array}{l}5.37 \\
5.39 \\
5.41 \\
5.47 \\
5.49\end{array}$ \\
\hline $\begin{array}{l}4-14 \\
4-14 \\
4-14 \\
4-14 \\
4-14 \\
4-14\end{array}$ & $\begin{array}{l}0600 \\
1200 \\
1400 \\
1600 \\
1800 \\
2000\end{array}$ & $\begin{array}{l}63.87 \\
62.49 \\
61.86 \\
61.46 \\
61.07 \\
60.80\end{array}$ & $\begin{array}{l}209000 \\
197000 \\
188000 \\
175000 \\
169000 \\
162000\end{array}$ & $\begin{array}{l}2.88 \\
3.26 \\
3.38 \\
3.49 \\
3.60 \\
3.71\end{array}$ & $\begin{array}{l}4-17 \\
4-17 \\
4-17 \\
4-17 \\
4-17\end{array}$ & $\begin{array}{l}1600 \\
1800 \\
2000 \\
2200 \\
2400\end{array}$ & $\begin{array}{l}45.53 \\
47.06 \\
44.94 \\
44.33 \\
44.21\end{array}$ & $\begin{array}{l}34500 \\
48500 \\
29800 \\
24900 \\
24000\end{array}$ & $\begin{array}{l}5.51 \\
5.54 \\
5.56 \\
5.58 \\
5.59\end{array}$ \\
\hline
\end{tabular}


TABLE 7.-Gage height, discharge, and accumulated runoff, flood of April 1979-Continued 02465286, Cribbs Mill Creek at $2 \mathrm{~d}$ Avenue in Tuscaloosa, Ala.

\begin{tabular}{|c|c|c|c|c|c|c|c|c|c|}
\hline DATE & TIME & $\begin{array}{c}\text { GAGE } \\
\text { HEIGHT }\end{array}$ & DISCHARGE & $\begin{array}{l}\text { ACCUM. } \\
\text { RUNOFF }\end{array}$ & DATE & TIME & $\begin{array}{c}\text { GAGE } \\
\text { HEIGHT }\end{array}$ & DISCHARGE & $\begin{array}{l}\text { ACCUM. } \\
\text { RUNOFF }\end{array}$ \\
\hline $4-11$ & 0030 & 0.52 & 4.0 & 0.00 & $4-12$ & 2015 & 4.86 & 412 & 2.75 \\
\hline $4-11$ & 1400 & .52 & 4.0 & .03 & $4-12$ & 2045 & 4.01 & 301 & 2.85 \\
\hline \multirow[t]{2}{*}{$4-11$} & 2400 & .51 & 3.7 & .05 & $4-12$ & 2115 & 3.70 & 264 & 2.92 \\
\hline & & & & & $4-12$ & 2145 & 3.63 & 256 & 3.00 \\
\hline $4-12$ & 0600 & .59 & 5.7 & .07 & $4-12$ & 2200 & 3.67 & 260 & 3.03 \\
\hline $4-12$ & 0615 & 1.17 & 31 & .07 & $4-12$ & 2230 & 3.47 & 237 & 3.10 \\
\hline $4-12$ & 0630 & 1.94 & 85 & .08 & $4-12$ & 2300 & 3.00 & 185 & 3.16 \\
\hline $4-12$ & 0645 & 2.02 & 92 & .10 & $4-12$ & 2315 & 2.80 & 165 & 3.18 \\
\hline $4-12$ & 0700 & 2.83 & 168 & .12 & $4-12$ & 2330 & 2.68 & 153 & 3.20 \\
\hline $4-12$ & 0715 & 3.19 & 206 & .15 & $4-12$ & 2345 & 2.77 & 162 & 3.23 \\
\hline $4-12$ & 0745 & 3.40 & 229 & .21 & $4-12$ & 2400 & 2.74 & 159 & 3.25 \\
\hline $4-12$ & 0800 & 3.77 & 272 & .25 & & & & & \\
\hline $4-12$ & 0830 & 4.12 & 316 & .34 & $4-13$ & 0015 & 2.99 & 184 & 3.27 \\
\hline $4-12$ & 0845 & 4.01 & 301 & .38 & $4-13$ & 0030 & 4.07 & 309 & 3.32 \\
\hline $4-12$ & 0915 & 3.48 & 238 & .45 & $4-13$ & 0045 & 5.22 & 463 & 3.38 \\
\hline $4-12$ & 0945 & 2.63 & 148 & .50 & $4-13$ & 0115 & 5.92 & 576 & 3.54 \\
\hline $4-12$ & 1000 & 2.47 & 132 & .52 & $4-13$ & 0200 & 5.67 & 534 & 3.77 \\
\hline $4-12$ & 1015 & 2.93 & 178 & .54 & $4-13$ & 0215 & 4.95 & 424 & 3.83 \\
\hline $4-12$ & 1030 & 3.43 & 232 & .57 & $4-13$ & 0230 & 3.81 & 277 & 3.87 \\
\hline $4-12$ & 1100 & 5.02 & 433 & .68 & $4-13$ & 0245 & 3.26 & 214 & 3.90 \\
\hline $4-12$ & 1115 & 5.25 & 468 & .75 & $4-13$ & 0300 & 2.97 & 182 & 3.92 \\
\hline $4-12$ & 1200 & 4.81 & 405 & .93 & $4-13$ & 0330 & 2.88 & 173 & 3.97 \\
\hline $4-12$ & 1215 & 4.18 & 323 & .97 & $4-13$ & 0345 & 2.92 & 177 & 4.00 \\
\hline $4-12$ & 1230 & 3.39 & 228 & 1.01 & $4-13$ & 0430 & 2.73 & 158 & 4.07 \\
\hline $4-12$ & 1245 & 2.90 & 175 & 1.03 & $4-13$ & 0515 & 2.32 & 119 & 4.12 \\
\hline $4-12$ & 1300 & 2.58 & 143 & 1.05 & $4-13$ & 0530 & 2.24 & 112 & 4.14 \\
\hline $4-12$ & 1330 & 2.16 & 104 & 1.08 & $4-13$ & 0600 & 2.16 & 104 & 4.17 \\
\hline $4-12$ & 1345 & 2.01 & 91 & 1.10 & $4-13$ & 0615 & 2.21 & 109 & 4.18 \\
\hline $4-12$ & 1430 & 1.71 & 67 & 1.13 & $4-13$ & 0630 & 2.20 & 108 & 4.20 \\
\hline $4-12$ & 1445 & 1.78 & 72 & 1.14 & $4-13$ & 0645 & 2.26 & 113 & 4.22 \\
\hline $4-12$ & 1500 & 1.91 & 83 & 1.15 & $4-13$ & 0700 & 3.31 & 219 & 4.25 \\
\hline $4-12$ & 1515 & 1.78 & 72 & 1.16 & $4-13$ & 0730 & 6.24 & 633 & 4.40 \\
\hline $4-12$ & 1530 & 1.94 & 85 & 1.17 & $4-13$ & 0745 & 7.50 & 870 & 4.52 \\
\hline $4-12$ & 1545 & 2.02 & 92 & 1.18 & $4-13$ & 0800 & 8.29 & 1040 & 4.66 \\
\hline $4-12$ & 1600 & 2.94 & 179 & 1.21 & $4-13$ & 0815 & 8.83 & 1160 & 4.83 \\
\hline $4-12$ & 1615 & 3.82 & 278 & 1.25 & $4-13$ & 0845 & 9.27 & 1270 & 5.17 \\
\hline $4-12$ & 1630 & 4.95 & 424 & 1.31 & $4-13$ & 0900 & 9.21 & 1250 & 5.35 \\
\hline $4-12$ & 1645 & 6.05 & 599 & 1.39 & $4-13$ & 0915 & 8.77 & 1150 & 5.51 \\
\hline $4-12$ & 1715 & 7.24 & 818 & 1.61 & $4-13$ & 0930 & 7.62 & 894 & 5.64 \\
\hline $4-12$ & 1730 & 7.68 & 906 & 1.74 & $4-13$ & 1000 & 5.47 & 501 & 5.81 \\
\hline $4-12$ & 1800 & 7.86 & 942 & 2.00 & $4-13$ & 1015 & 4.19 & 325 & 5.85 \\
\hline $4-12$ & 1830 & 7.17 & 804 & 2.24 & $4-13$ & 1030 & 3.50 & 240 & 5.89 \\
\hline $4-12$ & 1900 & 6.15 & 617 & 2.42 & $4-13$ & 1045 & 3.12 & 198 & 5.92 \\
\hline $4-12$ & 1945 & 5.10 & 445 & 2.63 & $4-13$ & 1100 & 2.81 & 166 & 5.94 \\
\hline
\end{tabular}


TABLES

TABLE 7.-Gage height, discharge, and accumulated runoff, flood of April 1979-Continued 02465286, Cribbs Mill Creek at 2d Avenue in Tuscaloosa, Ala-Continued

\begin{tabular}{|c|c|c|c|c|c|c|c|c|c|}
\hline DATE & TIME & $\begin{array}{c}\text { GAGE } \\
\text { HEIGHT }\end{array}$ & DISCHARGE & $\begin{array}{l}\text { ACCUM. } \\
\text { RUNOFF }\end{array}$ & DATE & TIME & $\begin{array}{c}\text { GAGE } \\
\text { HEIGHT }\end{array}$ & DISCHARGE & $\begin{array}{l}\text { ACCUM. } \\
\text { RUNOFF }\end{array}$ \\
\hline $4-13$ & 1145 & 2.29 & 116 & 5.99 & $4-14$ & 1300 & 0.93 & 18 & 6.47 \\
\hline $4-13$ & 1200 & 2.22 & 110 & 6.01 & $4-14$ & 2400 & .85 & 15 & 6.57 \\
\hline $4-13$ & 1215 & 2.09 & 98 & 6.02 & & & & & \\
\hline $4-13$ & 1300 & 1.84 & 77 & 6.06 & $4-15$ & 1200 & .80 & 13 & 6.66 \\
\hline $4-13$ & 1400 & 1.64 & 62 & 6.10 & 4-15 & 2400 & .77 & 11 & 6.74 \\
\hline $4-13$ & 1500 & 1.50 & 52 & 6.13 & & & & & \\
\hline $4-13$ & 1615 & 1.41 & 46 & 6.16 & $4-16$ & 1200 & .74 & 10 & 6.82 \\
\hline $4-13$ & 1630 & 1.37 & 43 & 6.17 & $4-16$ & 2400 & .73 & 10 & 6.89 \\
\hline $4-13$ & 1645 & 1.37 & 43 & 6.17 & & & & & \\
\hline $4-13$ & 1845 & 1.28 & 38 & 6.22 & $4-17$ & 1300 & .70 & 9.0 & 6.96 \\
\hline $4-13$ & 2400 & 1.09 & 26 & 6.31 & $4-17$ & 2400 & .70 & 9.0 & 7.01 \\
\hline
\end{tabular}


TABLE 7.-Gage height, discharge, and accumulated runoff, flood of April 1979-Continued 02465493, Elliotts Creek at Moundville, Ala.

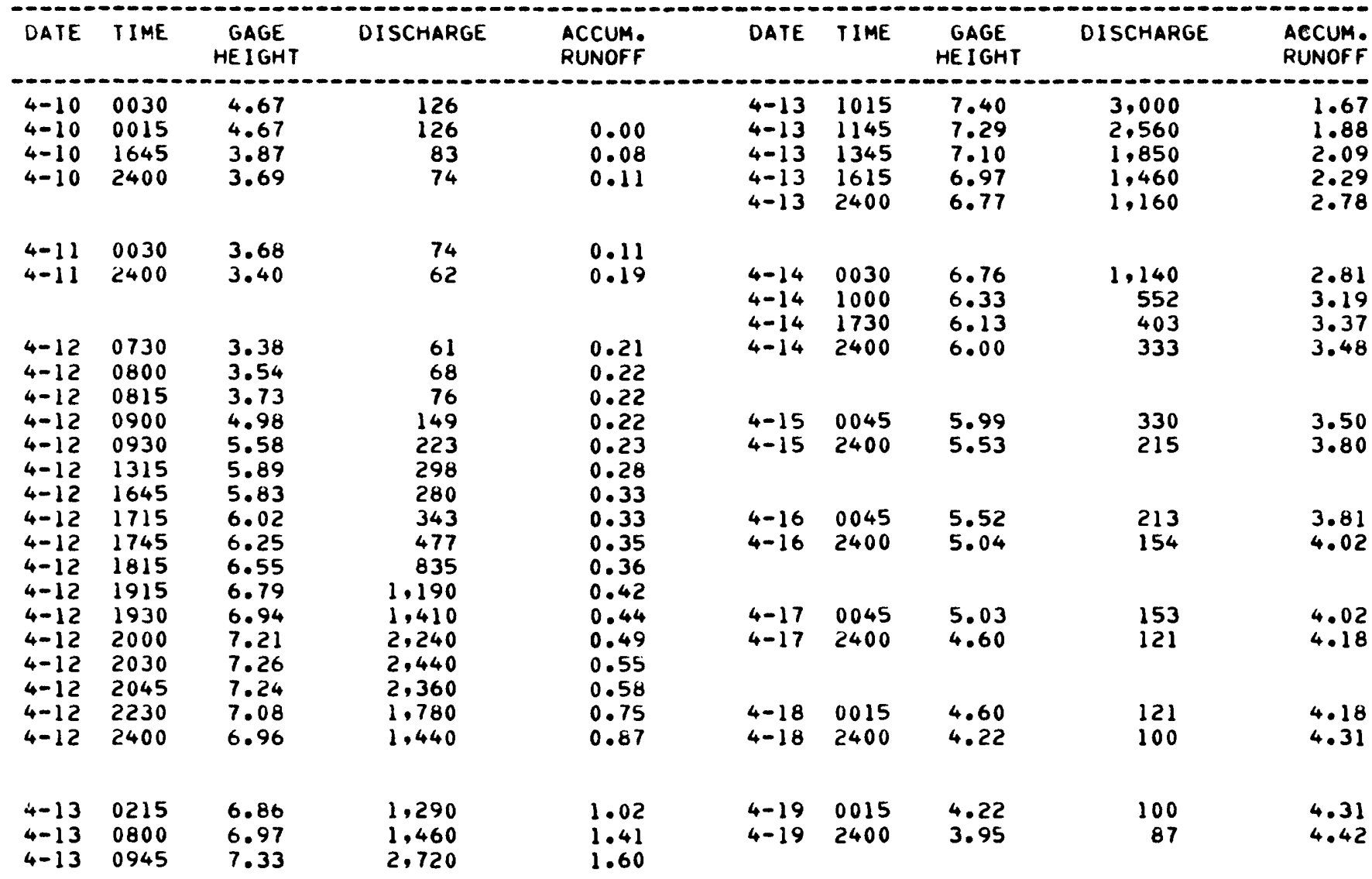


TABLES

TABLE 7.-Gage height, discharge, and accumulated runoff, flood of April 1979-Continued 02467000, Tombigbee River at Demopolis lock and dam, Ala.

\begin{tabular}{|c|c|c|c|c|c|c|c|c|c|}
\hline DATE & TIME & $\begin{array}{c}\text { GAGE } \\
\text { HEIGHT }\end{array}$ & DISCHARGE & $\begin{array}{l}\text { ACCUM. } \\
\text { RUNOFF }\end{array}$ & DATE & TIME & $\begin{array}{c}\text { GAGE } \\
\text { HEIGHT }\end{array}$ & DISCHARGE & $\begin{array}{l}\text { ACCUM. } \\
\text { RUNOFF }\end{array}$ \\
\hline $2-28$ & 2400 & 23.18 & 85600 & 0.00 & $\begin{array}{l}3-9 \\
3-9\end{array}$ & $\begin{array}{l}0600 \\
1200\end{array}$ & $\begin{array}{l}27.93 \\
27.94\end{array}$ & $\begin{array}{l}170000 \\
170000\end{array}$ & $\begin{array}{l}2.41 \\
2.51\end{array}$ \\
\hline $3-1$ & 0600 & 23.10 & 84000 & .05 & 3- 9 & 1800 & 27.97 & 171000 & 2.61 \\
\hline $3-1$ & 1200 & 23.01 & 82200 & .10 & $3-9$ & 2400 & 27.94 & 170000 & 2.72 \\
\hline $3-1$ & 1800 & 22.91 & 80300 & .15 & & & & & \\
\hline $3-1$ & 2400 & 22.82 & 78600 & .20 & $\begin{array}{l}3-10 \\
3-10\end{array}$ & $\begin{array}{l}0600 \\
1200\end{array}$ & $\begin{array}{l}27.89 \\
27.80\end{array}$ & $\begin{array}{l}169000 \\
164000\end{array}$ & $\begin{array}{l}2.82 \\
2.92\end{array}$ \\
\hline $3-2$ & 0600 & 22.70 & 76300 & .24 & $3-10$ & 1800 & 27.71 & 165000 & 3.02 \\
\hline $3-2$ & 1200 & 22.59 & 74200 & .29 & $3-10$ & 2400 & 27.55 & 160000 & 3.11 \\
\hline $3-2$ & 1800 & 22.50 & 72500 & .33 & & & & & \\
\hline \multirow[t]{2}{*}{$3-2$} & 2400 & 22.40 & 70600 & .37 & $3-11$ & 0600 & 27.36 & 156000 & 3.21 \\
\hline & & & & & $3-11$ & 1200 & 27.13 & 154000 & 3.30 \\
\hline & 0600 & 22.34 & 69500 & .42 & $3-11$ & 1800 & 26.87 & 151000 & 3.39 \\
\hline $3-3$ & 1200 & 22.39 & 70400 & .46 & $3-11$ & 2400 & 26.52 & 145000 & 3.48 \\
\hline $3-3$ & 1800 & 22.76 & 77400 & .50 & & & & & \\
\hline \multirow[t]{2}{*}{$3-3$} & 2400 & 23.56 & 93200 & .56 & $3-12$ & 0600 & 26.13 & 140000 & 3.56 \\
\hline & & & & & $3-12$ & 1200 & 25.64 & 133000 & 3.64 \\
\hline $3-4$ & 0600 & 24.09 & 104000 & .62 & $3-12$ & 1800 & 25.19 & 125000 & 3.72 \\
\hline $3-4$ & 1200 & 24.43 & 111000 & .69 & $3-12$ & 2400 & 24.63 & 114000 & 3.79 \\
\hline $3-4$ & 1800 & 24.73 & 117000 & .76 & & & & & \\
\hline \multirow[t]{2}{*}{$3-4$} & 2400 & 24.92 & 120000 & .83 & $3-13$ & 0600 & 24.15 & 105000 & 3.85 \\
\hline & & & & & $3-13$ & 1200 & 23.71 & 96200 & 3.91 \\
\hline $3-5$ & 0600 & 25.13 & 125000 & .91 & $3-13$ & 1800 & 23.24 & 86800 & 3.96 \\
\hline $3-5$ & 1200 & 25.44 & 130000 & .99 & $3-13$ & 2400 & 22.80 & 78200 & 4.01 \\
\hline $3-5$ & 1800 & 25.70 & 133000 & 1.07 & & & & & \\
\hline \multirow[t]{2}{*}{$3-5$} & 2400 & 26.08 & 141000 & 1.15 & $3-14$ & 0600 & 22.37 & 70000 & 4.05 \\
\hline & & & & & $3-14$ & 1200 & 21.98 & 62600 & 4.09 \\
\hline $3-6$ & 0600 & 26.34 & 145000 & 1.24 & $3-14$ & 1800 & 21.61 & 56000 & 4.12 \\
\hline $3-6$ & 1200 & 26.54 & 147000 & 1.33 & $3-14$ & 2400 & 21.35 & 51300 & 4.15 \\
\hline $3-6$ & 1800 & 26.74 & 151000 & 1.42 & & & & & \\
\hline \multirow[t]{2}{*}{ 3- 6} & 2400 & 26.92 & 152000 & 1.51 & $3-15$ & 0600 & 21.10 & 46800 & 4.18 \\
\hline & & & & & $3-15$ & 1200 & 20.93 & 43700 & 4.21 \\
\hline $3-7$ & 0600 & 27.11 & 155000 & 1.61 & $3-15$ & 1800 & 20.66 & 39100 & 4.23 \\
\hline $3-7$ & 1200 & 27.25 & 158000 & 1.70 & $3-15$ & 2400 & 20.46 & 35900 & 4.25 \\
\hline $3-7$ & 1800 & 27.39 & 160000 & 1.80 & & & & & \\
\hline \multirow[t]{2}{*}{$3-7$} & 2400 & 27.55 & 165000 & 1.90 & $3-16$ & 0600 & 20.30 & 33300 & 4.27 \\
\hline & & & & & $3-16$ & 1200 & 20.21 & 32000 & 4.29 \\
\hline $3-8$ & 0600 & 27.63 & 166000 & 2.00 & $3-16$ & 1800 & 20.17 & 31400 & 4.31 \\
\hline $3-8$ & 1200 & 27.71 & 168000 & 2.10 & $3-16$ & 2400 & 20.15 & 31000 & 4.33 \\
\hline $3-8$ & 1800 & 27.81 & 169000 & 2.20 & & & & & \\
\hline $3-8$ & 2400 & 27.88 & 170000 & 2.30 & $3-17$ & 0600 & 20.15 & 31000 & 4.35 \\
\hline
\end{tabular}


TABLE 7.-Gage height, discharge, and accumulated runoff, flood of April 1979-Continued 02467000, Tombigbee River at Demopolis lock and dam, Ala-Continued

\begin{tabular}{|c|c|c|c|c|c|c|c|c|c|}
\hline DATE & TIME & $\begin{array}{c}\text { GAGE } \\
\text { HEIGHT }\end{array}$ & DISCHARGE & $\begin{array}{l}\text { ACCUM, } \\
\text { RUNOFF }\end{array}$ & DATE & TIME & $\begin{array}{c}\text { GAGE } \\
\text { HEIGHT }\end{array}$ & DISCHARGE & $\begin{array}{l}\text { ACCUM } \\
\text { RUNOF }\end{array}$ \\
\hline $3-17$ & 1200 & 20.12 & 30600 & 4.37 & $3-26$ & 0600 & 20.85 & 42400 & 4.98 \\
\hline $3-17$ & 1800 & 20.09 & 30200 & 4.39 & $3-26$ & 1200 & 20.83 & 42000 & 5.00 \\
\hline $3-17$ & 2400 & 19.99 & 28600 & 4.40 & $\begin{array}{l}3-26 \\
3-26\end{array}$ & $\begin{array}{l}1800 \\
2400\end{array}$ & $\begin{array}{l}20.83 \\
20.89\end{array}$ & $\begin{array}{l}42000 \\
43000\end{array}$ & $\begin{array}{l}5.03 \\
5.05\end{array}$ \\
\hline $3-18$ & 0600 & 20.01 & 29000 & 4.42 & & & & & \\
\hline $3-18$ & 1200 & 19.94 & 27900 & 4.44 & $3-27$ & 0600 & 20.85 & 42400 & 5.08 \\
\hline $3-18$ & 1800 & 19.91 & 27400 & 4.45 & $3-27$ & 1200 & 20.86 & 42500 & 5.10 \\
\hline $3-18$ & 2400 & 19.78 & 25500 & 4.47 & $3-27$ & 1800 & 20.84 & 42200 & 5.13 \\
\hline $3-19$ & 0600 & 19.72 & 24600 & 4.48 & $3-27$ & 2400 & 20.80 & 41500 & 5.15 \\
\hline $3-19$ & 1200 & 19.64 & 23400 & 4.50 & $3-28$ & 0600 & 20.77 & 41000 & 5.18 \\
\hline $3-19$ & 1800 & 19.67 & 23800 & 4.51 & $3-28$ & 1200 & 20.75 & 40600 & 5.20 \\
\hline $3-19$ & 2400 & 19.68 & 24000 & 4.53 & $\begin{array}{l}3-28 \\
3-28\end{array}$ & 1800 & 20.72 & 40100 & $\begin{array}{l}5.23 \\
5.25\end{array}$ \\
\hline $3-20$ & 0600 & 19.73 & 24800 & 4.54 & $2-20$ & 2400 & 20.73 & 40300 & 3.25 \\
\hline $3-20$ & 1200 & 19.72 & 24600 & 4.56 & $3-29$ & 0600 & 20.70 & 39800 & 5.28 \\
\hline $3-20$ & 1800 & 19.73 & 24800 & 4.57 & $3-29$ & 1200 & 20.65 & 39000 & 5.30 \\
\hline $3-20$ & 2400 & 19.61 & 23000 & 4.59 & $3-29$ & 1800 & 20.51 & 36700 & 5.32 \\
\hline & & & & & $3-29$ & 2400 & 20.28 & 33000 & 5.34 \\
\hline $3-21$ & 0600 & 19.52 & 21700 & 4.60 & & & & & \\
\hline $3-21$ & 1200 & 19.50 & 21400 & 4.61 & $3-30$ & 0600 & 20.11 & 30400 & 5.36 \\
\hline $3-21$ & 1800 & 19.51 & 21500 & 4.62 & $3-30$ & 1200 & 19.98 & 28500 & 5.38 \\
\hline $3-21$ & 2400 & 19.56 & 22200 & 4.64 & $\begin{array}{l}3-30 \\
3-30\end{array}$ & $\begin{array}{l}1800 \\
2400\end{array}$ & $\begin{array}{l}20.03 \\
20.00\end{array}$ & $\begin{array}{l}29200 \\
28800\end{array}$ & $\begin{array}{l}5.40 \\
5.41\end{array}$ \\
\hline $3-22$ & 0600 & 19.60 & 22800 & 4.65 & & & & & \\
\hline $3-22$ & 1200 & 19.61 & 23000 & 4.67 & $3-31$ & 0600 & 19.95 & 28000 & 5.43 \\
\hline $3-22$ & 1800 & 19.56 & 22200 & 4.68 & $3-31$ & 1200 & 19.90 & 27300 & 5.45 \\
\hline $3-22$ & 2400 & 19.59 & 22700 & 4.69 & $3-31$ & 1800 & 19.82 & 26100 & 5.46 \\
\hline $3-23$ & 0600 & 19.64 & 23400 & 4.71 & $3-31$ & 2400 & 19.66 & 23700 & 5.48 \\
\hline $3-23$ & 1200 & 19.77 & 25400 & 4.72 & $4-1$ & 0600 & 19.54 & 22000 & 5.49 \\
\hline $3-23$ & 1800 & 19.94 & 27900 & 4.74 & $4-1$ & 1200 & 19.39 & 19900 & 5.50 \\
\hline $3-23$ & 2400 & 20.17 & 31400 & 4.76 & $4-1$ & 1800 & 19.13 & 16200 & 5.51 \\
\hline & & & & & $4-1$ & 2400 & 19.00 & 14500 & 5.52 \\
\hline $3-24$ & 0600 & 20.33 & 33000 & 4.78 & & & & & \\
\hline $3-24$ & 1200 & 20.43 & 35400 & 4.80 & $4-2$ & 0600 & 19.25 & 17900 & 5.53 \\
\hline $3-24$ & 1800 & 20.60 & 38100 & 4.82 & $4-2$ & 1200 & 19.44 & 20600 & 5.54 \\
\hline $3-24$ & 2400 & 20.79 & 41300 & 4.85 & $4-2$ & 1800 & 19.62 & 23100 & 5.56 \\
\hline & & & & & $4-2$ & 2400 & 19.91 & 27400 & 5.57 \\
\hline $3-25$ & 0600 & 20.89 & 43000 & 4.87 & & & & & \\
\hline $3-25$ & 1200 & 20.90 & 43200 & 4.90 & $4-3$ & 0600 & 20.70 & 39800 & 5.60 \\
\hline 3-25 & 1800 & 20.88 & 42900 & 4.93 & $4-3$ & 1200 & 21.10 & 46800 & 5.63 \\
\hline $3-25$ & 2400 & 20.78 & 41200 & 4.95 & $4-3$ & 1800 & 21.35 & 51300 & 5.66 \\
\hline & & & & & $4-3$ & 2400 & 21.73 & 58100 & 5.69 \\
\hline
\end{tabular}


TABLE 7.-Gage height, discharge, and accumulated runoff, flood of April 1979-Continued 02467000, Tombigbee River at Demopolis lock and dam, Ala-Continued

\begin{tabular}{|c|c|c|c|c|c|c|c|c|c|}
\hline DATE & TIME & $\begin{array}{c}\text { GAGE } \\
\text { HEIGHT }\end{array}$ & DISCHARGE & $\begin{array}{l}\text { ACCUM. } \\
\text { RUNOFF }\end{array}$ & DATE & TIME & $\begin{array}{c}\text { GAGE } \\
\text { HEIGHT }\end{array}$ & DISCHARGE & $\begin{array}{l}\text { ACCUM. } \\
\text { RUNOFF }\end{array}$ \\
\hline $\begin{array}{l}4-4 \\
4-4 \\
4-4 \\
4-4\end{array}$ & $\begin{array}{l}0600 \\
1200 \\
1800 \\
2400\end{array}$ & $\begin{array}{l}22.12 \\
22.41 \\
22.68 \\
22.85\end{array}$ & $\begin{array}{l}65300 \\
70800 \\
75900 \\
79200\end{array}$ & $\begin{array}{l}5.73 \\
5.77 \\
5.82 \\
5.87\end{array}$ & $\begin{array}{l}4-13 \\
4-13 \\
4-13 \\
4-13\end{array}$ & $\begin{array}{l}0600 \\
1200 \\
1800 \\
2400\end{array}$ & $\begin{array}{l}24.23 \\
24.85 \\
25.34 \\
25.78\end{array}$ & $\begin{array}{l}107000 \\
119000 \\
129000 \\
138000\end{array}$ & $\begin{array}{l}7.38 \\
7.45 \\
7.52 \\
7.61\end{array}$ \\
\hline $\begin{array}{l}4-5 \\
4-5 \\
4-5 \\
4-5\end{array}$ & $\begin{array}{l}0600 \\
1200 \\
1800 \\
2400\end{array}$ & $\begin{array}{l}22.96 \\
23.04 \\
23.08 \\
23.11\end{array}$ & $\begin{array}{l}81200 \\
82800 \\
83600 \\
84200\end{array}$ & $\begin{array}{l}5.92 \\
5.97 \\
6.02 \\
6.07\end{array}$ & $\begin{array}{l}4-14 \\
4-14 \\
4-14 \\
4-14\end{array}$ & $\begin{array}{l}0600 \\
1200 \\
1800 \\
2400\end{array}$ & $\begin{array}{l}26.29 \\
26.96 \\
27.54 \\
28.17\end{array}$ & $\begin{array}{l}146000 \\
158000 \\
168000 \\
175000\end{array}$ & $\begin{array}{l}7.70 \\
7.79 \\
7.89 \\
8.00\end{array}$ \\
\hline $\begin{array}{l}4-6 \\
4-6 \\
4-6 \\
4-6\end{array}$ & $\begin{array}{l}0600 \\
1200 \\
1800 \\
2400\end{array}$ & $\begin{array}{l}23.11 \\
23.10 \\
23.11 \\
23.11\end{array}$ & $\begin{array}{l}84200 \\
84000 \\
84200 \\
84200\end{array}$ & $\begin{array}{l}6.12 \\
6.17 \\
6.22 \\
6.27\end{array}$ & $\begin{array}{l}4-15 \\
4-15 \\
4-15 \\
4-15\end{array}$ & $\begin{array}{l}0600 \\
1200 \\
1800 \\
2400\end{array}$ & $\begin{array}{l}28.85 \\
29.64 \\
30.49 \\
31.42\end{array}$ & $\begin{array}{l}183000 \\
192000 \\
202000 \\
212000\end{array}$ & $\begin{array}{l}8.11 \\
8.23 \\
8.35 \\
8.48\end{array}$ \\
\hline $\begin{array}{l}4-7 \\
4-7 \\
4-7 \\
4-7\end{array}$ & $\begin{array}{l}0600 \\
1200 \\
1800 \\
2400\end{array}$ & $\begin{array}{l}23.07 \\
23.04 \\
22.89 \\
22.76\end{array}$ & $\begin{array}{l}83400 \\
82800 \\
79900 \\
77400\end{array}$ & $\begin{array}{l}6.32 \\
6.37 \\
6.42 \\
6.47\end{array}$ & $\begin{array}{l}4-16 \\
4-16 \\
4-16 \\
4-16\end{array}$ & $\begin{array}{l}0600 \\
1200 \\
1800 \\
2400\end{array}$ & $\begin{array}{l}32.37 \\
33.31 \\
34.18 \\
34.93\end{array}$ & $\begin{array}{l}242000 \\
261000 \\
271000 \\
286000\end{array}$ & $\begin{array}{l}8.62 \\
8.78 \\
8.94 \\
9.12\end{array}$ \\
\hline $\begin{array}{l}4-8 \\
4-8 \\
4-8 \\
4-8\end{array}$ & $\begin{array}{l}0600 \\
1200 \\
1800 \\
2400\end{array}$ & $\begin{array}{l}22.65 \\
22.62 \\
22.58 \\
22.65\end{array}$ & $\begin{array}{l}75400 \\
74800 \\
74000 \\
75400\end{array}$ & $\begin{array}{l}6.51 \\
6.56 \\
6.60 \\
6.65\end{array}$ & $\begin{array}{l}4-17 \\
4-17 \\
4-17 \\
4-17\end{array}$ & $\begin{array}{l}0600 \\
1200 \\
1800 \\
2400\end{array}$ & $\begin{array}{l}35.53 \\
36.03 \\
36.45 \\
36.72\end{array}$ & $\begin{array}{l}298000 \\
306000 \\
320000 \\
335000\end{array}$ & $\begin{array}{l}9.30 \\
9.48 \\
9.67 \\
9.88\end{array}$ \\
\hline $\begin{array}{l}4-9 \\
4-9 \\
4-9 \\
4-9\end{array}$ & $\begin{array}{l}0600 \\
1200 \\
1800 \\
2400\end{array}$ & $\begin{array}{l}22.72 \\
22.70 \\
22.65 \\
22.61\end{array}$ & $\begin{array}{l}76700 \\
76300 \\
75400 \\
74600\end{array}$ & $\begin{array}{l}6.69 \\
6.74 \\
6.79 \\
6.83\end{array}$ & $\begin{array}{l}4-18 \\
4-18 \\
4-18 \\
4-18\end{array}$ & $\begin{array}{l}0600 \\
1200 \\
1800 \\
2400\end{array}$ & $\begin{array}{l}36.90 \\
37.00 \\
37.03 \\
36.95\end{array}$ & $\begin{array}{l}341000 \\
343000 \\
340000 \\
325000\end{array}$ & $\begin{array}{l}10.08 \\
10.29 \\
10.49 \\
10.69\end{array}$ \\
\hline $\begin{array}{l}4-10 \\
4-10 \\
4-10 \\
4-10\end{array}$ & $\begin{array}{l}0600 \\
1200 \\
1800 \\
2400\end{array}$ & $\begin{array}{l}22.54 \\
22.40 \\
22.32 \\
22.20\end{array}$ & $\begin{array}{l}73300 \\
70600 \\
69100 \\
66800\end{array}$ & $\begin{array}{l}6.87 \\
6.92 \\
6.96 \\
7.00\end{array}$ & $\begin{array}{l}4-19 \\
4-19 \\
4-19 \\
4-19\end{array}$ & $\begin{array}{l}0600 \\
1200 \\
1800 \\
2400\end{array}$ & $\begin{array}{l}36.83 \\
36.66 \\
36.42 \\
36.15\end{array}$ & $\begin{array}{l}311000 \\
298000 \\
286000 \\
285000\end{array}$ & $\begin{array}{l}10.88 \\
11.06 \\
11.23 \\
11.40\end{array}$ \\
\hline $\begin{array}{l}4-11 \\
4-11 \\
4-11 \\
4-11\end{array}$ & $\begin{array}{l}0600 \\
1200 \\
1800 \\
2400\end{array}$ & $\begin{array}{l}22.07 \\
21.92 \\
21.82 \\
21.75\end{array}$ & $\begin{array}{l}64300 \\
61600 \\
59800 \\
58500\end{array}$ & $\begin{array}{l}7.04 \\
7.08 \\
7.11 \\
7.15\end{array}$ & $\begin{array}{l}4-20 \\
4-20 \\
4-20 \\
4-20\end{array}$ & $\begin{array}{l}0600 \\
1200 \\
1800 \\
2400\end{array}$ & $\begin{array}{l}35.85 \\
35.57 \\
35.20 \\
34.82\end{array}$ & $\begin{array}{l}282000 \\
279000 \\
275000 \\
267000\end{array}$ & $\begin{array}{l}11.57 \\
11.74 \\
11.91 \\
12.07\end{array}$ \\
\hline $\begin{array}{l}4-12 \\
4-12 \\
4-12 \\
4-12\end{array}$ & $\begin{array}{l}0600 \\
1200 \\
1800 \\
2400\end{array}$ & $\begin{array}{l}21.68 \\
21.72 \\
22.18 \\
23.37\end{array}$ & $\begin{array}{l}57200 \\
58000 \\
66400 \\
89400\end{array}$ & $\begin{array}{l}7.18 \\
7.22 \\
7.26 \\
7.31\end{array}$ & $\begin{array}{l}4-21 \\
4-21 \\
4-21 \\
4-21\end{array}$ & $\begin{array}{l}0600 \\
1200 \\
1800 \\
2400\end{array}$ & $\begin{array}{l}34.42 \\
34.01 \\
33.59 \\
33.14\end{array}$ & $\begin{array}{l}257000 \\
247000 \\
242000 \\
234000\end{array}$ & $\begin{array}{l}12.22 \\
12.37 \\
12.52 \\
12.66\end{array}$ \\
\hline
\end{tabular}


TABLE 7.-Gage height, discharge, and accumulated runoff, flood of April 1979-Continued 02467000, Tombigbee River at Demopolis lock and dam, Ala.-Continued

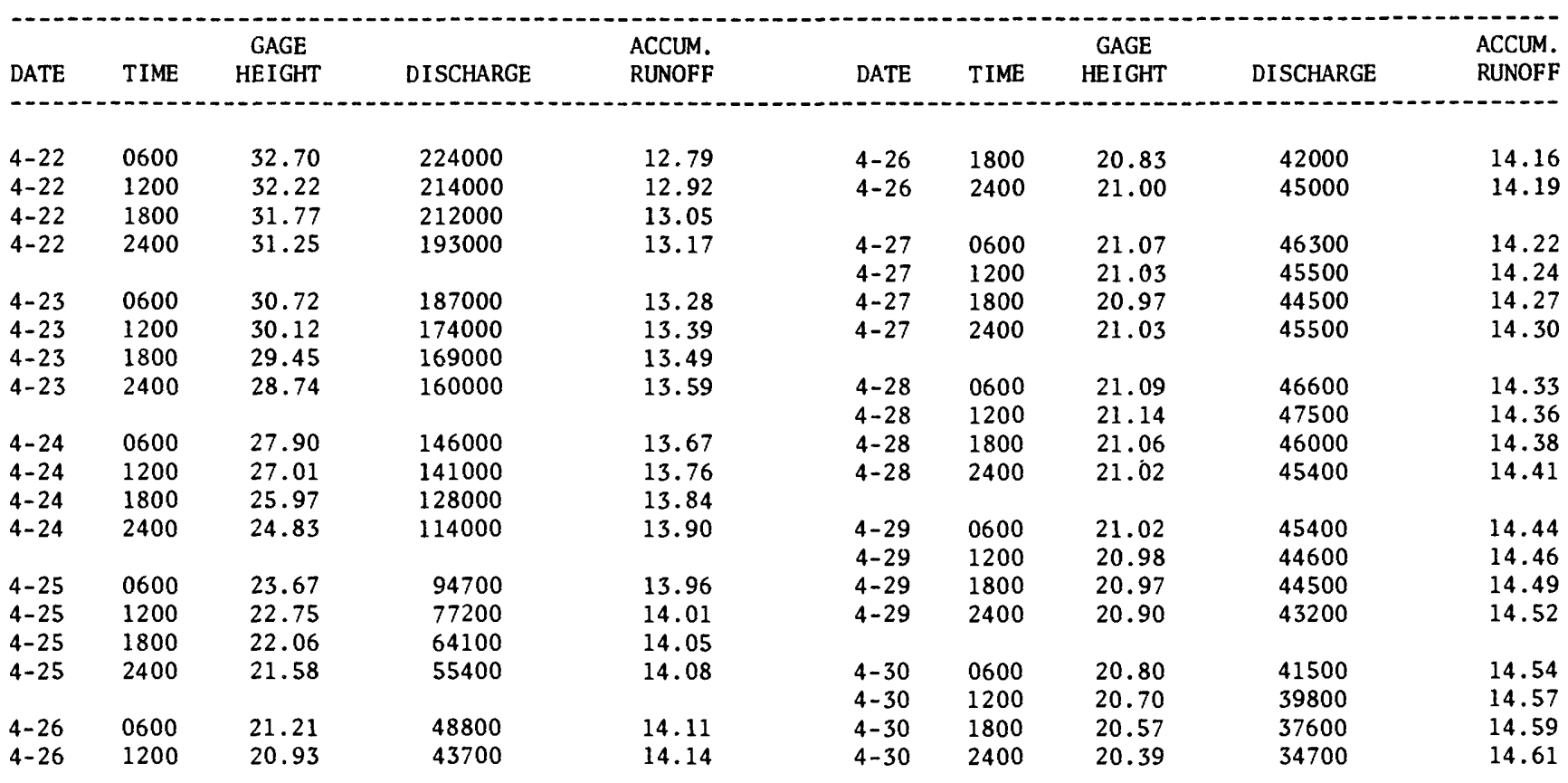


TABLES

TABLE 7.-Gage height, discharge, and accumulated runoff, flood of April 1979-Continued 02467500, Sucarnoochee River at Livingston, Ala.

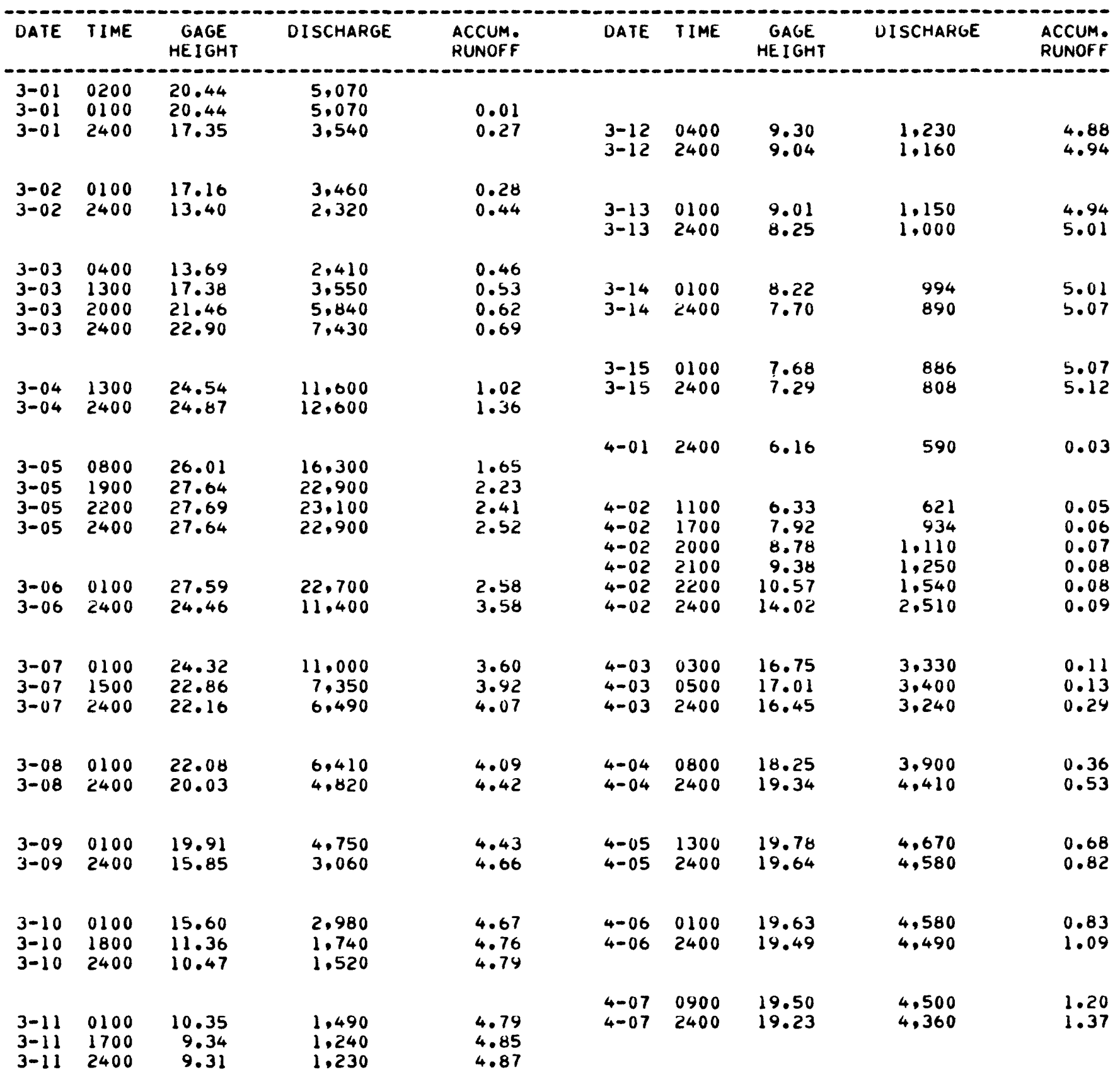


TABLE 7.-Gage height, discharge, and accumulated runoff, flood of April 1979-Continued 02467500, Sucarnoochee River at Livingston, Ala-Continued

\begin{tabular}{|c|c|c|c|c|c|c|c|c|c|}
\hline UATE & TIME & $\begin{array}{c}\text { GAGE } \\
\text { HE IGHT }\end{array}$ & DISCHARGE & $\begin{array}{l}\text { ACCUM. } \\
\text { RUNOFF }\end{array}$ & UATE & TIME & $\begin{array}{l}\text { GAGE } \\
\text { HEIGHT }\end{array}$ & DISCHARGE & $\begin{array}{l}\text { ACCUM. } \\
\text { RUNOFF }\end{array}$ \\
\hline $\begin{array}{l}4-08 \\
4-08 \\
4-08 \\
4-08\end{array}$ & $\begin{array}{l}1200 \\
1800 \\
2000 \\
2400\end{array}$ & $\begin{array}{l}18.92 \\
19.72 \\
19.65 \\
19.36\end{array}$ & $\begin{array}{l}4,200 \\
4,630 \\
4,590 \\
4,420\end{array}$ & $\begin{array}{l}1.50 \\
1.57 \\
1.59 \\
1.63\end{array}$ & $\begin{array}{l}4-15 \\
4-15 \\
4-15\end{array}$ & $\begin{array}{l}0100 \\
1700 \\
2400\end{array}$ & $\begin{array}{l}31.84 \\
27.72 \\
25.95\end{array}$ & $\begin{array}{l}49,100 \\
23,200 \\
16,100\end{array}$ & $\begin{array}{l}7.57 \\
8.98 \\
9.32\end{array}$ \\
\hline $\begin{array}{l}4-09 \\
4-09\end{array}$ & $\begin{array}{l}0100 \\
2400\end{array}$ & $\begin{array}{l}19.30 \\
18.60\end{array}$ & $\begin{array}{l}4,390 \\
4,040\end{array}$ & $\begin{array}{l}1.65 \\
1.89\end{array}$ & & & & & \\
\hline $\begin{array}{l}4-10 \\
4-10\end{array}$ & $\begin{array}{l}0100 \\
2400\end{array}$ & $\begin{array}{l}18.59 \\
18.06\end{array}$ & $\begin{array}{l}4,040 \\
3,820\end{array}$ & $\begin{array}{l}1.90 \\
2.13\end{array}$ & $\begin{array}{l}4-16 \\
4-16 \\
4-16\end{array}$ & $\begin{array}{l}0100 \\
1100 \\
2400\end{array}$ & $\begin{array}{l}25.73 \\
23.90 \\
22.62\end{array}$ & $\begin{array}{r}15,200 \\
9,760 \\
6,950\end{array}$ & $\begin{array}{l}9.36 \\
9.66 \\
9.93\end{array}$ \\
\hline $\begin{array}{l}4-11 \\
4-11\end{array}$ & $\begin{array}{l}0100 \\
2400\end{array}$ & $\begin{array}{l}18.04 \\
17.90\end{array}$ & $\begin{array}{l}3,820 \\
3,760\end{array}$ & $\begin{array}{l}2.14 \\
2.36\end{array}$ & $\begin{array}{l}4-17 \\
4-17\end{array}$ & $\begin{array}{l}0100 \\
2400\end{array}$ & $\begin{array}{l}22.54 \\
20.81\end{array}$ & $\begin{array}{l}6,870 \\
5,330\end{array}$ & $\begin{array}{r}9.95 \\
10.30\end{array}$ \\
\hline $\begin{array}{l}4-12 \\
4-12 \\
4-12\end{array}$ & $\begin{array}{l}0900 \\
2000 \\
2400\end{array}$ & $\begin{array}{l}18.25 \\
20.36 \\
22.71\end{array}$ & $\begin{array}{l}3,900 \\
5,020 \\
7,050\end{array}$ & $\begin{array}{l}2.45 \\
2.58 \\
2.64\end{array}$ & $\begin{array}{l}4-18 \\
4-18\end{array}$ & $\begin{array}{l}0100 \\
2400\end{array}$ & $\begin{array}{l}20.73 \\
17.92\end{array}$ & $\begin{array}{l}5,270 \\
3,770\end{array}$ & $\begin{array}{l}10.31 \\
10.58\end{array}$ \\
\hline & & & & & $\begin{array}{l}4-19 \\
4-19\end{array}$ & $\begin{array}{l}0100 \\
2400\end{array}$ & $\begin{array}{l}17.75 \\
12.51\end{array}$ & $\begin{array}{l}3,700 \\
2,050\end{array}$ & $\begin{array}{l}10.59 \\
10.76\end{array}$ \\
\hline $\begin{array}{l}4-13 \\
4-13 \\
4-13 \\
4-13\end{array}$ & $\begin{array}{l}0600 \\
1000 \\
2200 \\
2400\end{array}$ & $\begin{array}{l}25.24 \\
26.41 \\
30.03 \\
30.68\end{array}$ & $\begin{array}{l}13,700 \\
17,900 \\
35,600 \\
40,200\end{array}$ & $\begin{array}{l}2.81 \\
2.98 \\
3.80 \\
4.00\end{array}$ & $\begin{array}{l}4-20 \\
4-20 \\
4-20\end{array}$ & $\begin{array}{l}0100 \\
1700 \\
2400\end{array}$ & $\begin{array}{r}12.30 \\
9.89 \\
9.35\end{array}$ & $\begin{array}{l}1,990 \\
1,370 \\
1,240\end{array}$ & $\begin{array}{l}10.76 \\
10.83 \\
10.85\end{array}$ \\
\hline $\begin{array}{l}4-14 \\
4-14 \\
4-14\end{array}$ & $\begin{array}{l}1000 \\
1300 \\
2000\end{array}$ & $\begin{array}{l}33.31 \\
33.47 \\
32.79\end{array}$ & $\begin{array}{l}60,900 \\
62,200 \\
56,700\end{array}$ & $\begin{array}{l}5.36 \\
5.63 \\
6.90\end{array}$ & $\begin{array}{l}4-21 \\
4-21\end{array}$ & $\begin{array}{l}0100 \\
2400\end{array}$ & $\begin{array}{l}9.29 \\
8.53\end{array}$ & $\begin{array}{l}1,220 \\
1,060\end{array}$ & $\begin{array}{l}10.85 \\
10.92\end{array}$ \\
\hline
\end{tabular}


TABLE 7.-Gage height, discharge, and accumulated runoff, flood of April 1979-Continued 02468500, Chickasaw Bogue near Linden, Ala.

[Maximum discharge occurred on March 4, 1979]

\begin{tabular}{|c|c|c|c|c|c|c|c|c|c|}
\hline DATE & TIME & $\begin{array}{c}\text { GAGE } \\
\text { HE IGHT }\end{array}$ & DISCHARGE & $\begin{array}{l}\text { ACCUM. } \\
\text { RUNOFF }\end{array}$ & DATE & TIME & $\begin{array}{c}\text { GAGE } \\
\text { HEIGHT }\end{array}$ & DISCHARGE & $\begin{array}{l}\text { ACCUM } \\
\text { RUNOFI }\end{array}$ \\
\hline $3-2$ & 2400 & 5.33 & 74 & 0.00 & $4-11$ & 2400 & 5.97 & 162 & 0.00 \\
\hline $3-3$ & 0900 & 5.23 & 65 & 0.00 & $4-12$ & 1400 & 5.68 & 116 & 0.01 \\
\hline $3-3$ & 1400 & 5.27 & 68 & 0.01 & $4-12$ & 1500 & 4.67 & 30 & 0.01 \\
\hline $3-3$ & 1500 & 6.09 & 185 & 0.01 & $4-12$ & 1600 & 5.66 & 113 & 0.01 \\
\hline $3-3$ & 1600 & 6.72 & 343 & 0.01 & $4-12$ & 1900 & 5.72 & 122 & 0.01 \\
\hline $3-3$ & 1700 & 7.86 & 729 & 0.01 & $4-12$ & 2000 & 6.34 & 243 & 0.01 \\
\hline $3-3$ & 1800 & 9.47 & 1370 & 0.02 & $4-12$ & 2100 & 7.59 & 625 & 0.02 \\
\hline $3-3$ & 2000 & 13.66 & 3400 & 0.06 & $4-12$ & 2200 & 9.61 & 1430 & 0.03 \\
\hline $3-3$ & 2400 & 20.01 & 8210 & 0.26 & $\begin{array}{l}4-12 \\
4-12\end{array}$ & $\begin{array}{l}2300 \\
2400\end{array}$ & $\begin{array}{l}12.98 \\
16.22\end{array}$ & $\begin{array}{l}2990 \\
5050\end{array}$ & $\begin{array}{l}0.04 \\
0.07\end{array}$ \\
\hline $3-4$ & 0300 & 23.31 & 13100 & 0.50 & & & & & \\
\hline $3-4$ & 0500 & 26.08 & 19000 & 0.72 & $4-13$ & 0300 & 20.65 & 9050 & 0.24 \\
\hline $3-4$ & 0800 & 29.13 & 33300 & 1.32 & $4-13$ & 0900 & 25.13 & 16800 & 0.84 \\
\hline $3-4$ & 1200 & 30.18 & 38700 & 2.25 & $4-13$ & 1300 & 25.80 & 18200 & 1.28 \\
\hline $3-4$ & 2000 & 28.11 & 28200 & 3.61 & $4-13$ & 1600 & 25.63 & 17800 & 1.60 \\
\hline $3-4$ & 2400 & 26.56 & 20600 & 4.10 & $4-13$ & 2400 & 24.14 & 14800 & 2.31 \\
\hline $3-5$ & 0100 & 26.17 & 19300 & 4.22 & $4-14$ & 0100 & 23.89 & 14300 & 2.40 \\
\hline $3-5$ & 1300 & 19.38 & 7580 & 4.77 & $4-14$ & 1800 & 14.78 & 4070 & 2.81 \\
\hline $3-5$ & 2100 & 13.84 & 3500 & 4.93 & $4-14$ & 2300 & 11.14 & 2080 & 2.88 \\
\hline $3-5$ & 2400 & 11.79 & 2400 & 4.98 & $4-14$ & 2400 & 10.69 & 1880 & 2.89 \\
\hline
\end{tabular}


TABLE 7.-Gage height, discharge, and accumulated runoff, flood of April 1979-Continued 02475500, Chunky Creek near Chunky, Miss.

[Maximum discharge occurred on March 4, 1979]

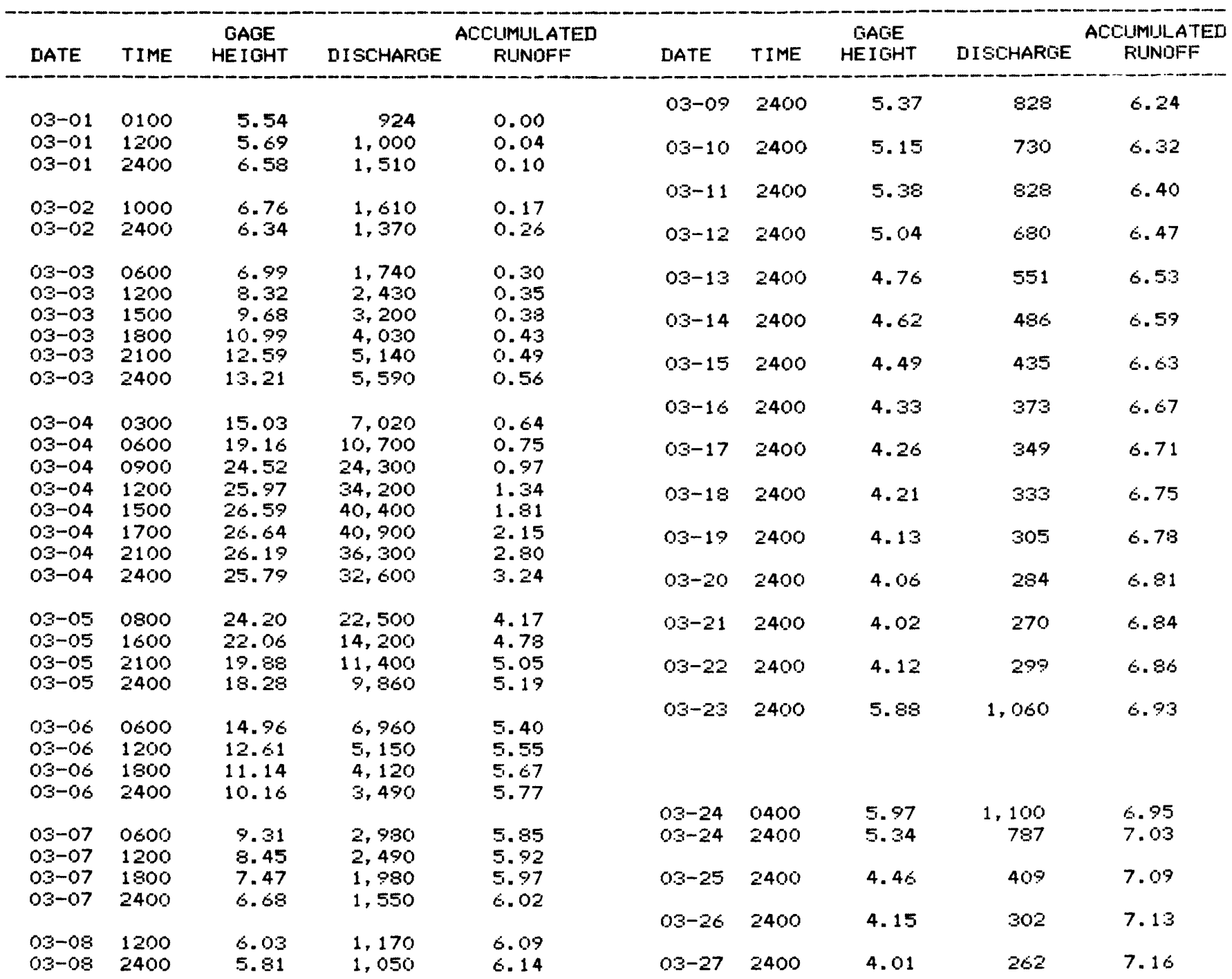


TABLE 7.-Gage height, discharge, and accumulated runoff, flood of April 1979-Continued 02475500, Chunky Creek near Chunky, Miss.-Continued

\begin{tabular}{|c|c|c|c|c|c|c|c|c|c|}
\hline DATE & TIME & $\begin{array}{l}\text { GAGE } \\
\text { HEIGHT }\end{array}$ & DISCHARGE & $\begin{array}{l}\text { ACCUMULATED } \\
\text { RUNIOFF }\end{array}$ & DATE & TIME & $\begin{array}{l}\text { GAGE } \\
\text { HEIGHT }\end{array}$ & DISEHAFIBE & $\begin{array}{l}\text { ACCUMULATED } \\
\text { RUNIFF }\end{array}$ \\
\hline $03-28$ & 2400 & $\begin{array}{l}3.93 \\
3.86\end{array}$ & 240 & $\begin{array}{l}7.18 \\
7.20\end{array}$ & $\begin{array}{l}04-12 \\
04-12 \\
04-12\end{array}$ & $\begin{array}{l}0600 \\
1400 \\
2400\end{array}$ & $\begin{array}{l}5.81 \\
5.63 \\
9.88\end{array}$ & $\begin{array}{r}979 \\
890 \\
3,320\end{array}$ & $\begin{array}{l}9.58 \\
9.61 \\
9.70\end{array}$ \\
\hline $03-30$ & 2400 & 3.83 & 213 & 7.23 & $\begin{array}{l}04-13 \\
04-13\end{array}$ & $\begin{array}{l}0100 \\
0300\end{array}$ & $\begin{array}{l}10.23 \\
12.07\end{array}$ & $\begin{array}{l}3,530 \\
4,760\end{array}$ & $\begin{array}{l}9.72 \\
9.75\end{array}$ \\
\hline $03-31$ & 2400 & 3.82 & 210 & 7.25 & $\begin{array}{l}04-13 \\
04-13\end{array}$ & $\begin{array}{l}0600 \\
0900\end{array}$ & $\begin{array}{l}13.26 \\
15.20\end{array}$ & $\begin{array}{l}5,630 \\
7,160\end{array}$ & $\begin{array}{l}9.82 \\
9.90\end{array}$ \\
\hline $04-01$ & 2400 & 3.85 & 215 & 7.27 & $\begin{array}{l}04-13 \\
04-13\end{array}$ & $\begin{array}{l}1200 \\
1500\end{array}$ & $\begin{array}{l}20.07 \\
24.12\end{array}$ & $\begin{array}{l}11,600 \\
22,100\end{array}$ & $\begin{array}{l}10.02 \\
10.23\end{array}$ \\
\hline $\begin{array}{l}04-02 \\
04-02\end{array}$ & $\begin{array}{l}0800 \\
1600\end{array}$ & $\begin{array}{l}3.89 \\
4.85\end{array}$ & $\begin{array}{l}255 \\
555\end{array}$ & $\begin{array}{l}7.213 \\
7.29\end{array}$ & $\begin{array}{l}04-13 \\
04-13\end{array}$ & $\begin{array}{l}1800 \\
2100\end{array}$ & $\begin{array}{l}25.38 \\
25.99\end{array}$ & $\begin{array}{l}27,500 \\
34,400\end{array}$ & $\begin{array}{l}10.56 \\
10.96\end{array}$ \\
\hline $04-02$ & 2400 & 5.17 & 700 & 7.31 & $04-13$ & 2400 & 26.14 & 35,800 & 11.40 \\
\hline $\begin{array}{l}04-03 \\
04-03\end{array}$ & $\begin{array}{l}1200 \\
2400\end{array}$ & $\begin{array}{l}7.43 \\
8.09\end{array}$ & $\begin{array}{l}1,910 \\
2,270\end{array}$ & $\begin{array}{l}7.38 \\
7.43\end{array}$ & $\begin{array}{l}04-14 \\
04-14 \\
04-14\end{array}$ & $\begin{array}{l}0100 \\
0300 \\
0600\end{array}$ & $\begin{array}{l}26.17 \\
25.96 \\
25.58\end{array}$ & $\begin{array}{l}36,100 \\
34,100 \\
30,800\end{array}$ & $\begin{array}{l}11.56 \\
11.85 \\
12.26\end{array}$ \\
\hline $\begin{array}{l}04-04 \\
04-04\end{array}$ & $\begin{array}{l}1200 \\
2400\end{array}$ & $\begin{array}{r}9.34 \\
10.06\end{array}$ & $\begin{array}{l}2,980 \\
3,430\end{array}$ & $\begin{array}{l}7.62 \\
7.78\end{array}$ & $\begin{array}{l}04-14 \\
04-14 \\
04-14\end{array}$ & $\begin{array}{l}0900 \\
1200 \\
1500\end{array}$ & $\begin{array}{l}25.10 \\
24.60 \\
24.16\end{array}$ & $\begin{array}{l}27,700 \\
24,800 \\
22,300\end{array}$ & $\begin{array}{l}12.63 \\
12.96 \\
13.26\end{array}$ \\
\hline $\begin{array}{l}04-05 \\
04-05 \\
04-05\end{array}$ & $\begin{array}{l}1200 \\
2000 \\
2400\end{array}$ & $\begin{array}{l}11.80 \\
12.32 \\
12.19\end{array}$ & $\begin{array}{l}4,570 \\
4,940 \\
4,850\end{array}$ & $\begin{array}{l}7.93 \\
8.14 \\
8.22\end{array}$ & $\begin{array}{l}04-14 \\
04-14 \\
04-14\end{array}$ & $\begin{array}{l}1800 \\
2100 \\
2400\end{array}$ & $\begin{array}{l}23.51 \\
22.78 \\
21.69\end{array}$ & $\begin{array}{l}19,000 \\
16,100 \\
13,500\end{array}$ & $\begin{array}{l}13.52 \\
13.74 \\
13.93\end{array}$ \\
\hline $\begin{array}{l}04-06 \\
04-06 \\
04-06 \\
04-06\end{array}$ & $\begin{array}{l}0600 \\
1200 \\
1800 \\
2400\end{array}$ & $\begin{array}{r}11.72 \\
10.84 \\
10.07 \\
9.31\end{array}$ & $\begin{array}{l}4,520 \\
3,930 \\
3,430 \\
2,960\end{array}$ & $\begin{array}{l}8.34 \\
8.45 \\
8.54 \\
8.62\end{array}$ & $\begin{array}{l}04-15 \\
04-15 \\
04-15 \\
04-15\end{array}$ & $\begin{array}{l}0600 \\
1200 \\
1800 \\
2400\end{array}$ & $\begin{array}{l}19.00 \\
15.74 \\
13.23 \\
11.56\end{array}$ & $\begin{array}{r}10,500 \\
7,610 \\
5,610 \\
4,410\end{array}$ & $\begin{array}{l}14.23 \\
14.46 \\
14.63 \\
14.76\end{array}$ \\
\hline $\begin{array}{l}04-07 \\
04-07\end{array}$ & $\begin{array}{l}1200 \\
2400\end{array}$ & $\begin{array}{l}7.17 \\
5.62\end{array}$ & $\begin{array}{r}1,770 \\
895\end{array}$ & $\begin{array}{l}8.74 \\
8.81\end{array}$ & $\begin{array}{l}04-16 \\
04-16 \\
04-16\end{array}$ & $\begin{array}{l}0600 \\
1200 \\
2400\end{array}$ & $\begin{array}{r}10.49 \\
9.61 \\
7.62\end{array}$ & $\begin{array}{l}3,700 \\
3,140 \\
2,000\end{array}$ & $\begin{array}{l}14.36 \\
14.94 \\
15.07\end{array}$ \\
\hline $\begin{array}{l}04-08 \\
04-08 \\
04-08\end{array}$ & $\begin{array}{l}0700 \\
1200 \\
2400\end{array}$ & $\begin{array}{l}5.37 \\
5.54 \\
6.96\end{array}$ & $\begin{array}{r}774 \\
852 \\
1,640\end{array}$ & $\begin{array}{l}8.83 \\
8.85 \\
8.91\end{array}$ & $\begin{array}{l}04-17 \\
04-17\end{array}$ & $\begin{array}{l}1200 \\
2400\end{array}$ & $\begin{array}{l}6.20 \\
5.64\end{array}$ & $\begin{array}{r}1,180 \\
885\end{array}$ & $\begin{array}{l}15.15 \\
15.21\end{array}$ \\
\hline $\begin{array}{l}04-09 \\
04-09 \\
04-09\end{array}$ & $\begin{array}{l}1200 \\
1800 \\
2400\end{array}$ & $\begin{array}{l}7.82 \\
8.08 \\
8.34\end{array}$ & $\begin{array}{l}2,110 \\
2,250 \\
2,390\end{array}$ & $\begin{array}{l}9.01 \\
9.06 \\
9.12\end{array}$ & $\begin{array}{l}04-19 \\
04-19\end{array}$ & $\begin{array}{l}1200 \\
2400\end{array}$ & $\begin{array}{l}5.33 \\
5.13\end{array}$ & $\begin{array}{l}739 \\
655\end{array}$ & $\begin{array}{l}15.25 \\
15.23\end{array}$ \\
\hline $\begin{array}{l}04-10 \\
04-10 \\
04-10\end{array}$ & $\begin{array}{l}1000 \\
1700 \\
2400\end{array}$ & $\begin{array}{l}8.51 \\
8.41 \\
8.19\end{array}$ & $\begin{array}{l}2,490 \\
2,430 \\
2,310\end{array}$ & $\begin{array}{l}9.22 \\
9.30 \\
9.37\end{array}$ & $\begin{array}{l}04-19 \\
04-19\end{array}$ & $\begin{array}{l}1200 \\
2400\end{array}$ & $\begin{array}{l}4.96 \\
4.84\end{array}$ & $\begin{array}{l}573 \\
520\end{array}$ & $\begin{array}{l}15.31 \\
15.34\end{array}$ \\
\hline \multirow[t]{2}{*}{$\begin{array}{l}04-11 \\
04-11 \\
04-11\end{array}$} & $\begin{array}{l}0600 \\
1200 \\
2400\end{array}$ & $\begin{array}{l}7.88 \\
7.54 \\
6.27\end{array}$ & $\begin{array}{l}2,140 \\
1,960 \\
1,230\end{array}$ & $\begin{array}{l}9.42 \\
9.47 \\
9.56\end{array}$ & $\begin{array}{l}04-21 \\
04-21\end{array}$ & $\begin{array}{l}1200 \\
2400\end{array}$ & $\begin{array}{l}4.60 \\
4.54\end{array}$ & $\begin{array}{l}420 \\
398\end{array}$ & $\begin{array}{l}15.41 \\
15.43\end{array}$ \\
\hline & & & & & $\begin{array}{l}04-22 \\
04-22\end{array}$ & $\begin{array}{l}1200 \\
2400\end{array}$ & $\begin{array}{l}4.48 \\
4.45\end{array}$ & $\begin{array}{l}376 \\
366\end{array}$ & $\begin{array}{l}15.45 \\
15.47\end{array}$ \\
\hline
\end{tabular}


TABLE 7.-Gage height, discharge, and accumulated runoff, flood of April 1979-Continued 02476500, Sowashee Creek at Meridian, Miss.

\begin{tabular}{|c|c|c|c|c|c|c|c|c|c|}
\hline DATE & TIME & $\begin{array}{l}\text { GAGE } \\
\text { HEIGHT }\end{array}$ & DISCHARGE & $\begin{array}{c}\text { ACCUMULATED } \\
\text { RUNDFF }\end{array}$ & DATE & TIME & $\begin{array}{l}\text { GAGE } \\
\text { HEIGHT }\end{array}$ & DISCHARGE & $\begin{array}{l}\text { ACCUMIUL_ATED } \\
\text { RUNIDFF }\end{array}$ \\
\hline $04-11$ & 0100 & 3.49 & 177 & 0.00 & $04-14$ & 1200 & 5.68 & 414 & 3.71 \\
\hline $\begin{array}{l}04-11 \\
04-11\end{array}$ & $\begin{array}{l}0600 \\
1200\end{array}$ & $\begin{array}{l}3.45 \\
3.41\end{array}$ & $\begin{array}{l}170 \\
164\end{array}$ & $\begin{array}{l}0.02 \\
0.05\end{array}$ & $04-14$ & 2400 & 4.36 & 273 & 4.03 \\
\hline $\begin{array}{l}04-11 \\
04-11\end{array}$ & $\begin{array}{l}1800 \\
2400\end{array}$ & $\begin{array}{l}3.36 \\
3.31\end{array}$ & $\begin{array}{l}150 \\
148\end{array}$ & $\begin{array}{l}0.08 \\
0.11\end{array}$ & $\begin{array}{l}04-15 \\
04-15\end{array}$ & $\begin{array}{l}1200 \\
2400\end{array}$ & $\begin{array}{l}3.90 \\
3.61\end{array}$ & $\begin{array}{l}224 \\
190\end{array}$ & $\begin{array}{l}4.12 \\
4.17\end{array}$ \\
\hline $\begin{array}{l}04-12 \\
04-12\end{array}$ & $\begin{array}{l}0600 \\
1200\end{array}$ & $\begin{array}{l}3.29 \\
6.30\end{array}$ & $\begin{array}{l}145 \\
490\end{array}$ & $\begin{array}{l}0.13 \\
0.19\end{array}$ & $04-16$ & 2400 & 3.40 & 158 & 4.32 \\
\hline $\begin{array}{l}04-12 \\
04-12\end{array}$ & $\begin{array}{l}1800 \\
2400\end{array}$ & $\begin{array}{r}7.84 \\
16.11\end{array}$ & $\begin{array}{r}693 \\
2,400\end{array}$ & $\begin{array}{l}0.29 \\
0.57\end{array}$ & $04-17$ & 2400 & 3.26 & 135 & 4.42 \\
\hline & & & & & $04-18$ & 2400 & 3.17 & 119 & 4.51 \\
\hline $04-13$ & 0730 & 22.87 & 7,270 & 1.65 & & & & & \\
\hline $\begin{array}{l}04-13 \\
04-13\end{array}$ & $\begin{array}{l}0900 \\
1200\end{array}$ & $\begin{array}{l}22.50 \\
21.12\end{array}$ & $\begin{array}{l}6,900 \\
5,630\end{array}$ & $\begin{array}{l}1.97 \\
2.53\end{array}$ & $04-19$ & 2400 & 3.11 & 108 & 4.59 \\
\hline $04-13$ & 1500 & 19.15 & 4,110 & 2.97 & $04-20$ & 2400 & 3.10 & 106 & 4.67 \\
\hline $04-13$ & 1800 & 16.61 & 2,620 & 3.27 & & & & & \\
\hline $04-13$ & 2100 & 13.97 & 1,750 & 3.47 & $04-21$ & 2400 & 3.06 & 98.0 & 4.74 \\
\hline $04-$ & 2400 & 11.72 & 30 & - & $04-22$ & 2400 & 3.14 & 109 & 4.82 \\
\hline
\end{tabular}


TABLE 7.-Gage height, discharge, and accumulated runoff, flood of April 1979-Continued 02476600, Okatibbee Creek at Arundel, Miss.

\begin{tabular}{|c|c|c|c|c|c|c|c|c|c|}
\hline DATE & TIME & $\begin{array}{l}\text { GADE } \\
\text { HEISIHT }\end{array}$ & IISCHARTE & $\begin{array}{c}\text { ACCUMUULATED } \\
\text { FIUNDIFF }\end{array}$ & DIATE & TIME & $\begin{array}{l}\text { GAGE } \\
\text { HEIOHT }\end{array}$ & DISCHAFIEE & $\begin{array}{c}\text { ACCUMUILATED } \\
\text { FUUNOFF }\end{array}$ \\
\hline $\begin{array}{l}03-01 \\
03-01\end{array}$ & $\begin{array}{l}0100 \\
2400\end{array}$ & $\begin{array}{l}11.07 \\
11.17\end{array}$ & $\begin{array}{l}1,740 \\
1,770\end{array}$ & $\begin{array}{l}0.00 \\
0.18\end{array}$ & $\begin{array}{l}03-14 \\
03-15\end{array}$ & 2400 & 10.99 & $\begin{array}{l}1,780 \\
1,720\end{array}$ & $\begin{array}{l}4.73 \\
5.12\end{array}$ \\
\hline $\begin{array}{l}03-02 \\
03-02\end{array}$ & $\begin{array}{l}1200 \\
2400\end{array}$ & $\begin{array}{l}10.70 \\
10.36\end{array}$ & $\begin{array}{l}1,630 \\
1,530\end{array}$ & $\begin{array}{l}0.27 \\
0.36\end{array}$ & $03-16$ & 2400 & 10.84 & 1,670 & 5.30 \\
\hline $\begin{array}{l}03-03 \\
03-03 \\
03-03 \\
03-03 \\
03-03\end{array}$ & $\begin{array}{l}0600 \\
1200 \\
1800 \\
2100 \\
2400\end{array}$ & $\begin{array}{l}10.69 \\
11.86 \\
14.19 \\
13.93 \\
19.84\end{array}$ & $\begin{array}{r}1,630 \\
2,000 \\
2,820 \\
8,380 \\
11,600\end{array}$ & $\begin{array}{l}0.40 \\
0.45 \\
0.51 \\
0.59 \\
0.7 .3\end{array}$ & $\begin{array}{l}03-17 \\
03-18 \\
03-19\end{array}$ & $\begin{array}{l}2400 \\
2400 \\
2400\end{array}$ & $\begin{array}{l}10.63 \\
10.53\end{array}$ & $\begin{array}{l}1,640 \\
1,610 \\
1,580\end{array}$ & $\begin{array}{l}5.4: 3 \\
5.66 \\
5.83\end{array}$ \\
\hline $\begin{array}{l}03-04 \\
03-04 \\
03-04 \\
03-04 \\
03-04 \\
03-04\end{array}$ & $\begin{array}{l}0300 \\
0600 \\
1200 \\
1330 \\
1800 \\
2400\end{array}$ & $\begin{array}{l}20.02 \\
20.15 \\
20.69 \\
20.71 \\
20.35 \\
19.37\end{array}$ & $\begin{array}{r}12,400 \\
13,000 \\
15,600 \\
15,700 \\
13,900 \\
9,850\end{array}$ & $\begin{array}{l}0.89 \\
1.06 \\
1.45 \\
1.56 \\
1.86 \\
2.13\end{array}$ & $\begin{array}{c}03-20 \\
03-21 \\
03-22 \\
03-23\end{array}$ & $\begin{array}{l}2400 \\
2400 \\
2400 \\
2400\end{array}$ & $\begin{array}{c}10.47 \\
10.39 \\
7.48 \\
9.47\end{array}$ & $\begin{array}{l}1,560 \\
1,540 \\
1,280 \\
1,280\end{array}$ & $\begin{array}{l}6.00 \\
6.17 \\
6.33 \\
6.47\end{array}$ \\
\hline $\begin{array}{l}03-05 \\
03-05 \\
03-05 \\
03-05\end{array}$ & $\begin{array}{l}0600 \\
1200 \\
1800 \\
2400\end{array}$ & $\begin{array}{l}18.29 \\
17.29 \\
16.48 \\
15.82\end{array}$ & $\begin{array}{l}6,580 \\
4,510 \\
3,850 \\
3,500\end{array}$ & $\begin{array}{l}2.41 \\
2.56 \\
2.67 \\
2.77\end{array}$ & $\begin{array}{l}03-24 \\
03-25\end{array}$ & $\begin{array}{l}2400 \\
2400\end{array}$ & $\begin{array}{r}9.91 \\
10.29\end{array}$ & $\begin{array}{l}1,400 \\
1,510\end{array}$ & $\begin{array}{l}6.61 \\
6.77\end{array}$ \\
\hline $\begin{array}{l}03-06 \\
03-06\end{array}$ & $\begin{array}{l}1200 \\
2400\end{array}$ & $\begin{array}{l}14.77 \\
14.07\end{array}$ & $\begin{array}{l}3,050 \\
2,790\end{array}$ & $\begin{array}{l}2.95 \\
3.11\end{array}$ & $\begin{array}{l}03-26 \\
03-27\end{array}$ & $\begin{array}{l}2400 \\
2400\end{array}$ & $\begin{array}{l}10.40 \\
10.37\end{array}$ & $\begin{array}{l}1,540 \\
1,530\end{array}$ & $\begin{array}{l}6.74 \\
7.10\end{array}$ \\
\hline $\begin{array}{l}03-07 \\
03-07\end{array}$ & $\begin{array}{l}1200 \\
2400\end{array}$ & $\begin{array}{l}13.51 \\
13.11\end{array}$ & $\begin{array}{l}2,570 \\
2,420\end{array}$ & $\begin{array}{l}3.25 \\
3.39\end{array}$ & $03-28$ & 2400 & 10.30 & 1,510 & 7.27 \\
\hline $\begin{array}{l}03-08 \\
03-08\end{array}$ & $\begin{array}{l}1200 \\
2400\end{array}$ & $\begin{array}{l}12.85 \\
12.59\end{array}$ & $\begin{array}{l}2,330 \\
2,240\end{array}$ & $\begin{array}{l}3.52 \\
3.64\end{array}$ & $\begin{array}{c}03-29 \\
03-30\end{array}$ & $\begin{array}{l}2400 \\
2400\end{array}$ & $\begin{array}{l}10.22 \\
10.15\end{array}$ & $\begin{array}{l}1,490 \\
1,470\end{array}$ & $\begin{array}{l}7.43 \\
7.59\end{array}$ \\
\hline $03-09$ & 2400 & 12.12 & 2,080 & 3.813 & $03-31$ & 2400 & 7.92 & 887 & 7.72 \\
\hline $03-10$ & 2400 & 12.00 & 2,040 & 4.10 & $04-01$ & 2400 & 5.50 & 585 & 7.80 \\
\hline $03-11$ & 2400 & 11.83 & 1,980 & 4.32 & $04-02$ & 2400 & 9.43 & 1,270 & 7.90 \\
\hline $03-12$ & 2400 & 11.56 & 1,900 & $4.5: 3$ & $\begin{array}{l}04-03 \\
04-03\end{array}$ & $\begin{array}{l}1200 \\
2400\end{array}$ & $\begin{array}{l}12.69 \\
14.10\end{array}$ & $\begin{array}{l}2,280 \\
2,790\end{array}$ & $\begin{array}{l}8.00 \\
8.14\end{array}$ \\
\hline
\end{tabular}


TABLE 7.-Gage height, discharge, and accumulated runoff, flood of April 1979-Continued 02476600, Okatibbee Creek at Arundel, Miss.-Continued

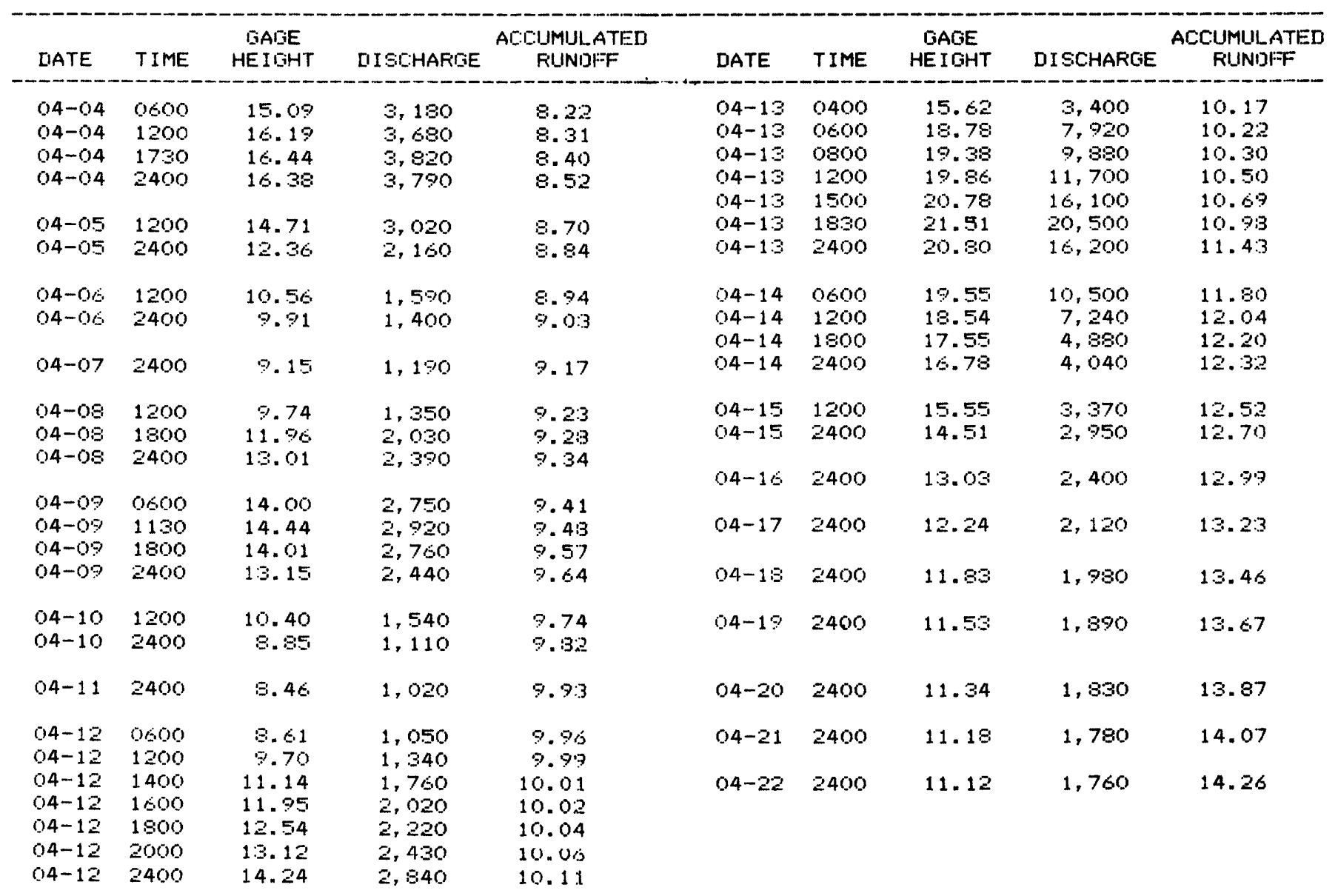


TABLES

TABLE 7.-Gage height, discharge, and accumulated runoff, flood of April 1979-Continued 02477000 , Chickasawhay River at Enterprise, Miss.

\begin{tabular}{|c|c|c|c|c|c|c|c|c|c|}
\hline DATE & TIME & $\begin{array}{l}\text { GAGE } \\
\text { HEIGHT }\end{array}$ & DISCHARGE & $\begin{array}{c}\text { ACCUMUJ_ATED } \\
\text { RUNOFF }\end{array}$ & LIATE & TIME & $\begin{array}{c}\text { GAGEE } \\
\text { HEIGHT }\end{array}$ & [ISCHAFIGE & $\begin{array}{c}\text { ACCOUMUILATED } \\
\text { FUINOJFF }\end{array}$ \\
\hline $\begin{array}{l}03-01 \\
03-01 \\
03-01\end{array}$ & $\begin{array}{l}0100 \\
1200 \\
2400\end{array}$ & $\begin{array}{l}17.31 \\
16.74 \\
16.55\end{array}$ & $\begin{array}{l}4,400 \\
4,160 \\
4,080\end{array}$ & $\begin{array}{l}0.00 \\
0.07 \\
0.16\end{array}$ & $\begin{array}{l}03-08 \\
03-08 \\
03-09\end{array}$ & $\begin{array}{l}1200 \\
2400 \\
2400\end{array}$ & $\begin{array}{l}19.67 \\
18.46 \\
16.72\end{array}$ & $\begin{array}{l}5,450 \\
4,900 \\
4,150\end{array}$ & $\begin{array}{l}5.03 \\
5.19 \\
5.37\end{array}$ \\
\hline $\begin{array}{l}03-02 \\
03-02\end{array}$ & $\begin{array}{l}1200 \\
2400\end{array}$ & $\begin{array}{l}16.12 \\
15.84\end{array}$ & $\begin{array}{l}3,900 \\
3,780\end{array}$ & $\begin{array}{l}0.24 \\
0.32\end{array}$ & $03-10$ & 2400 & $15.2 \%$ & 3,560 & 5.53 \\
\hline $\begin{array}{l}03-03 \\
03-03 \\
03-03\end{array}$ & $\begin{array}{l}0600 \\
0900 \\
1200\end{array}$ & $\begin{array}{l}18.55 \\
20.89 \\
25.68\end{array}$ & $\begin{array}{r}4,940 \\
6,130 \\
10,000\end{array}$ & $\begin{array}{l}0.31 \\
0.37 \\
0.43\end{array}$ & $09-11$ & 2400 & 14.78 & $\begin{array}{l}3,350 \\
3,090\end{array}$ & 5.67 \\
\hline $\begin{array}{l}03-03 \\
03-03 \\
03-03\end{array}$ & $\begin{array}{l}1500 \\
1800 \\
2400\end{array}$ & $\begin{array}{l}27.44 \\
29.37 \\
30.53\end{array}$ & $\begin{array}{l}11,900 \\
14,200 \\
15,800\end{array}$ & $\begin{array}{l}0.47 \\
0.55 \\
0.71\end{array}$ & $\begin{array}{c}03-13 \\
03-14\end{array}$ & 2400 & 13.44 & $\begin{array}{l}2,840 \\
2,700\end{array}$ & 5.92 \\
\hline $\begin{array}{l}03-04 \\
03-04 \\
03-04 \\
03-04 \\
03-04 \\
03-04 \\
03-04 \\
03-04\end{array}$ & $\begin{array}{l}0300 \\
0600 \\
0900 \\
1200 \\
1500 \\
1800 \\
2100 \\
2400\end{array}$ & $\begin{array}{l}30.97 \\
31.12 \\
32.62 \\
33.80 \\
35.23 \\
37.03 \\
38.82 \\
40.39\end{array}$ & $\begin{array}{l}16,400 \\
16,600 \\
19,000 \\
20,900 \\
23,800 \\
28,600 \\
34,600 \\
40,900\end{array}$ & $\begin{array}{l}0.79 \\
0.87 \\
0.96 \\
1.07 \\
1.13 \\
1.31 \\
1.47 \\
1.67\end{array}$ & $\begin{array}{l}03-15 \\
03-16 \\
03-17 \\
03-13 \\
03-19\end{array}$ & $\begin{array}{l}2400 \\
2400 \\
2400 \\
2400 \\
2400\end{array}$ & $\begin{array}{l}12.74 \\
12.41 \\
12.22 \\
12.06 \\
11.89\end{array}$ & $\begin{array}{l}2,580 \\
2,460 \\
2,390 \\
2,340 \\
2,280\end{array}$ & $\begin{array}{l}6.15 \\
6.25 \\
6.35 \\
6.44 \\
6.54\end{array}$ \\
\hline $\begin{array}{l}03-0.5 \\
03-05 \\
03-0.5 \\
03-0.5 \\
03-0.5 \\
03-0.5 \\
03-0.5 \\
03-05\end{array}$ & $\begin{array}{l}0300 \\
0700 \\
0900 \\
1200 \\
1500 \\
1500 \\
2100 \\
2400\end{array}$ & $\begin{array}{l}41.37 \\
41.88 \\
41.85 \\
41.61 \\
41.35 \\
40.90 \\
40.25 \\
39.50\end{array}$ & $\begin{array}{l}46,300 \\
49,700 \\
49,500 \\
47,900 \\
46,200 \\
43,500 \\
40,200 \\
37,100\end{array}$ & $\begin{array}{l}1.87 \\
2.21 \\
2.313 \\
2.63 \\
2.87 \\
3.10 \\
3.31 \\
3.51\end{array}$ & $\begin{array}{c}03-20 \\
03-21 \\
03-22 \\
03-23\end{array}$ & $\begin{array}{l}2400 \\
2400 \\
2400 \\
2400\end{array}$ & $\begin{array}{l}11.76 \\
11.64 \\
11.64 \\
13.02\end{array}$ & $\begin{array}{l}2,230 \\
2,190 \\
2,190 \\
2,690\end{array}$ & $\begin{array}{l}6.63 \\
6.72 \\
6.31 \\
6.91\end{array}$ \\
\hline $\begin{array}{l}03-06 \\
03-06 \\
03-06 \\
03-06\end{array}$ & $\begin{array}{l}0 \leqslant 00 \\
1200 \\
1800 \\
2400\end{array}$ & $\begin{array}{l}37.76 \\
35.24 \\
32.69 \\
30.39\end{array}$ & $\begin{array}{l}31,000 \\
23,800 \\
19,100 \\
15,600\end{array}$ & $\begin{array}{l}3.96 \\
4.1: 3 \\
4.35 \\
4.53\end{array}$ & $\begin{array}{l}03-24 \\
03-25 \\
03-26\end{array}$ & $\begin{array}{l}2400 \\
2400 \\
2400\end{array}$ & $\begin{array}{l}12.20 \\
11.80\end{array}$ & $\begin{array}{l}2,740 \\
2,380 \\
2,240\end{array}$ & $\begin{array}{l}7.02 \\
7.12 \\
7.22\end{array}$ \\
\hline $\begin{array}{l}03-07 \\
03-07 \\
03-07 \\
03-07\end{array}$ & $\begin{array}{l}0600 \\
1200 \\
1800 \\
2400\end{array}$ & $\begin{array}{l}28.10 \\
26.00 \\
23.43 \\
21.75\end{array}$ & $\begin{array}{r}12,600 \\
10,300 \\
8,190 \\
6,790\end{array}$ & $\begin{array}{l}4.67 \\
4.77 \\
4.83 \\
4.96\end{array}$ & $\begin{array}{l}03-27 \\
03-28\end{array}$ & 2400 & 11.59 & 2,170 & 7.31 \\
\hline
\end{tabular}


TABLE 7.-Gage height, discharge, and accumulated runoff, flood of April 1979-Continued 02477000, Chickasawhay River at Enterprise, Miss.-Continued

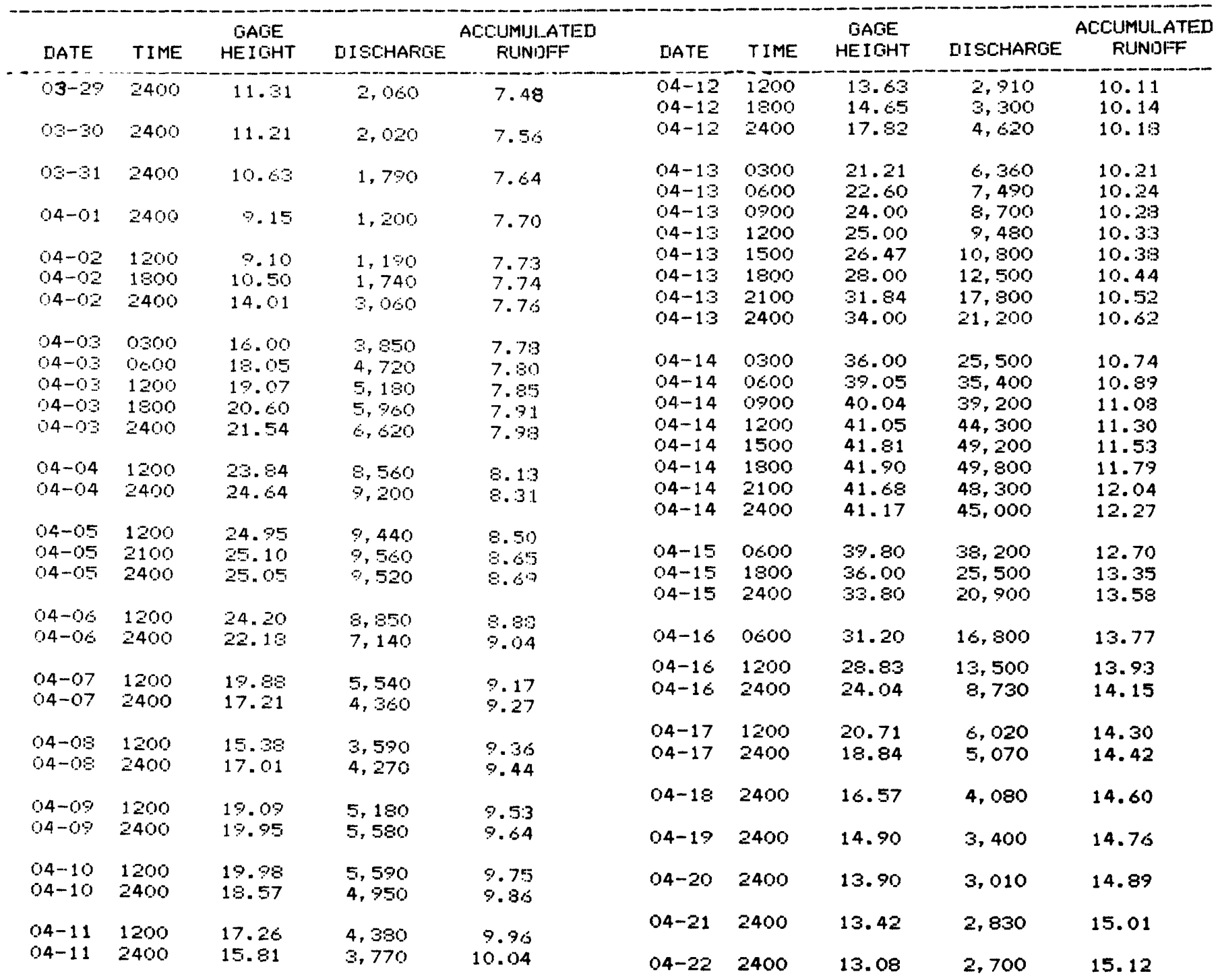


TABLE 7.-Gage height, discharge, and accumulated runoff, flood of April 1979-Continued 02478500, Chickasawhay River at Leakesville, Miss.

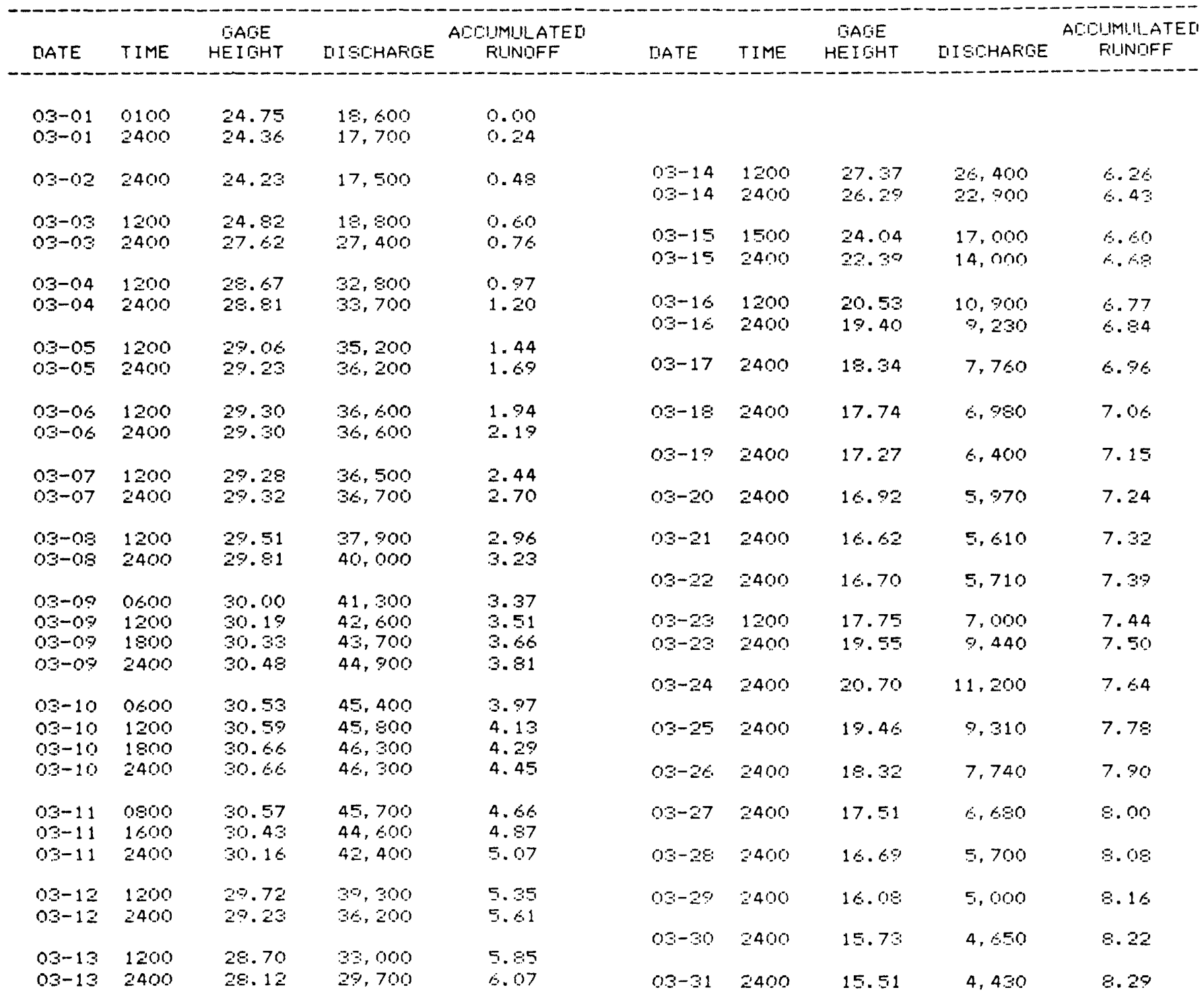


TABLE 7.-Gage height, discharge, and accumulated runoff, flood of April 1979-Continued 02478500, Chickasawhay River at Leakesville, Miss.-Continued

\begin{tabular}{|c|c|c|c|c|c|c|c|c|c|}
\hline IATE & TIME & $\begin{array}{l}\text { GAGE } \\
\text { HEIGHT }\end{array}$ & DISCHARGE & $\begin{array}{c}\text { ACCUMUULATEO } \\
\text { RIINOFF }\end{array}$ & IIATE & TIME & $\begin{array}{l}\text { GAGE } \\
\text { HEIGHT }\end{array}$ & DISCHAFGE & $\begin{array}{l}\text { ACCIMUULATED } \\
\text { RUINIIFF }\end{array}$ \\
\hline $04-01$ & 2400 & 15.45 & 4,380 & 8.35 & $04-14$ & 2400 & 22.22 & 13,700 & 12.62 \\
\hline $04-02$ & 0100 & 15.45 & 4,380 & 8.35 & $04-15$ & 2400 & 21.39 & 12,300 & 12.80 \\
\hline $04-02$ & 2400 & 15.58 & 4,500 & 8.41 & $04-16$ & 2400 & 21.70 & 12,800 & $12 . \% 7$ \\
\hline $04-03$ & 2400 & 23.12 & 15,100 & 8.54 & $94-17$ & 2400 & 22.79 & 14,700 & 13.16 \\
\hline $\begin{array}{l}04-04 \\
04-04\end{array}$ & $\begin{array}{l}1200 \\
2400\end{array}$ & $\begin{array}{l}26.95 \\
28.99\end{array}$ & $\begin{array}{l}24,800 \\
31,100\end{array}$ & $\begin{array}{l}\text { E.69: } \\
\text { E. } 98\end{array}$ & $04-19$ & 2400 & 24.24 & 17,500 & 13.39 \\
\hline $\begin{array}{l}04-05 \\
04-05\end{array}$ & $\begin{array}{l}1200 \\
2400\end{array}$ & $\begin{array}{l}29.20 \\
29.75\end{array}$ & $\begin{array}{l}36,000 \\
39,600\end{array}$ & $\begin{array}{l}9.11 \\
9.37\end{array}$ & $04-19$ & 2400 & 25.70 & 21,100 & 13.55 \\
\hline $04-05$ & 0500 & $2 \%, 85$ & 40,200 & $\because 49$ & $04-20$ & 2400 & 26.85 & $=4.600$ & 13.97 \\
\hline $04-06$ & 2400 & $2 \% \cdot 5$ & 39,200 & $\because 91$ & $04-21$ & $\approx 400$ & 27.02 & 25,100 & 14.31 \\
\hline $\begin{array}{l}04-07 \\
04-077\end{array}$ & $\begin{array}{l}1200 \\
2400\end{array}$ & $\begin{array}{l}20.16 \\
20.76\end{array}$ & $\begin{array}{l}95,800 \\
30,400\end{array}$ & $\begin{array}{l}10.17 \\
10.41\end{array}$ & $0 n-2=$ & $\therefore 450$ & 26.21 & 22,600 & 14.54 \\
\hline $\begin{array}{l}04-06 \\
04-09\end{array}$ & $\begin{array}{l}1200 \\
2400\end{array}$ & $\begin{array}{l}29.39 \\
29.02\end{array}$ & $\begin{array}{l}31,100 \\
29,200\end{array}$ & $\begin{array}{l}10.85 \\
10.84\end{array}$ & $\begin{array}{l}04-23 \\
04-23\end{array}$ & $\begin{array}{l}1200 \\
2400\end{array}$ & $\begin{array}{l}25.16 \\
25.03\end{array}$ & $\begin{array}{l}19,600 \\
15,100\end{array}$ & $\begin{array}{l}14.79 \\
14.91\end{array}$ \\
\hline $\begin{array}{l}04-09 \\
04-09\end{array}$ & $\begin{array}{l}1200 \\
2400\end{array}$ & $\begin{array}{l}27.64 \\
27.25\end{array}$ & $\begin{array}{l}27,500 \\
25,900\end{array}$ & $\begin{array}{l}11.04 \\
11.2=\end{array}$ & $\begin{array}{l}04-24 \\
04-25\end{array}$ & $\begin{array}{l}2400 \\
2400\end{array}$ & $\begin{array}{l}1 \% .15 \\
15.66\end{array}$ & $\begin{array}{l}8,590 \\
5,190\end{array}$ & $\begin{array}{l}15.07 \\
15.1 \%\end{array}$ \\
\hline $\begin{array}{l}04-10 \\
04-10\end{array}$ & $\begin{array}{l}1200 \\
2400\end{array}$ & $\begin{array}{l}2 \leq 02 \\
2 \leq 01\end{array}$ & $\begin{array}{l}24,800 \\
23,900\end{array}$ & $\begin{array}{l}11.40 \\
11.56\end{array}$ & 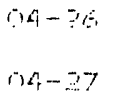 & $\begin{array}{l}\therefore 400 \\
\therefore 400\end{array}$ & $\begin{array}{l}19.72 \\
19.59\end{array}$ & $\begin{array}{l}8,-800 \\
8,000\end{array}$ & $\begin{array}{l}15.30 \\
15.42\end{array}$ \\
\hline $0.4-11$ & 2400 & 25.99 & $\because 2,000$ & 11.80 & $04-9$ & $\therefore 400$ & 18.18 & 7,550 & 15.52 \\
\hline $04-12$ & 2400 & 24.55 & $1 \%, 100$ & 12.16 & $04-29$ & $\therefore 400$ & 17.50 & 6.770 & 15.62 \\
\hline $04-12$ & 2400 & 23.65 & 16,300 & 12.41 & $04-30$ & 2400 & 1628 & 5,170 & 15.71 \\
\hline
\end{tabular}


TABLES

TABLE 7.-Gage height, discharge, and accumulated runoff, flood of April 1979-Continued 02482000, Pearl River at Edinburg, Miss.

\begin{tabular}{|c|c|c|c|c|c|c|c|c|c|}
\hline DATE & TIME & $\begin{array}{c}\text { GAGE } \\
\text { HEIGHT }\end{array}$ & IIISCHARGE & $\begin{array}{c}\text { ACCUMIILATED } \\
\text { RUNOFF }\end{array}$ & DATE & TIME & $\begin{array}{l}\text { GAGE } \\
\text { HEIGHT }\end{array}$ & II SCHARGE & $\begin{array}{c}\text { ACCUMULATED } \\
\text { RUNOFF: }\end{array}$ \\
\hline $03-03$ & 0100 & 20.26 & 5,450 & 0.00 & $09-16$ & 1200 & 11.99 & 1,700 & 4.91 \\
\hline $03-03$ & 0800 & 20.76 & 5,890 & 0.06 & $03-16$ & 2400 & 11.67 & 1,610 & 4.94 \\
\hline $03-03$ & 1600 & 21.97 & 7,170 & 0.15 & & & & & \\
\hline $03-03$ & 2400 & 23.06 & 8,770 & 0.26 & $0:-17$ & 1200 & 11.39 & 1,530 & 4.97 \\
\hline $03-04$ & 0800 & 23.71 & 10,100 & 0.39 & $9-17$ & 2400 & 11.14 & 1,470 & 5.00 \\
\hline $\begin{array}{l}03-04 \\
03-04\end{array}$ & $\begin{array}{l}1600 \\
2400\end{array}$ & $\begin{array}{l}24.32 \\
25.45\end{array}$ & $\begin{array}{l}11,700 \\
17,200\end{array}$ & $\begin{array}{l}0.54 \\
0.74\end{array}$ & $\begin{array}{l}0 s-18 \\
05-10\end{array}$ & $\begin{array}{l}1200 \\
2400\end{array}$ & $\begin{array}{l}10.88 \\
10.61\end{array}$ & $\begin{array}{l}1,400 \\
1,330\end{array}$ & $\begin{array}{l}5.03 \\
5.06\end{array}$ \\
\hline $\begin{array}{l}03-0.5 \\
03-0.5 \\
03-05\end{array}$ & $\begin{array}{l}0800 \\
1600 \\
2100\end{array}$ & $\begin{array}{l}26.05 \\
26.33 \\
26.45\end{array}$ & $\begin{array}{l}22,100 \\
25,000 \\
26,300\end{array}$ & $\begin{array}{l}1.02 \\
1.34 \\
1.56\end{array}$ & $\begin{array}{l}09-19 \\
03-19\end{array}$ & $\begin{array}{l}1200 \\
2400\end{array}$ & $\begin{array}{l}10.30 \\
10.00\end{array}$ & $\begin{array}{l}1,260 \\
1,180\end{array}$ & $\begin{array}{l}5.09 \\
5.11\end{array}$ \\
\hline $03-05$ & 2400 & 26.40 & 25,800 & 1.70 & $\begin{array}{l}03-20 \\
03-20\end{array}$ & $\begin{array}{l}1200 \\
2400\end{array}$ & $\begin{array}{l}9.67 \\
9.37\end{array}$ & $\begin{array}{l}1,110 \\
1,050\end{array}$ & $\begin{array}{l}5.14 \\
5.16\end{array}$ \\
\hline $0.3-06$ & 0800 & 26.37 & 25,400 & 2.05 & & & & & \\
\hline $03-06$ & 1600 & 26.14 & 23,000 & 2.313 & $03-21$ & 1200 & 9.06 & 984 & $5.1: 3$ \\
\hline $03-06$ & 2400 & 25.81 & 20,000 & 2.63 & $05-21$ & 2400 & 5.76 & 924 & 5.20 \\
\hline $03-07$ & 0800 & 25.43 & 17,100 & 2.94 & $03-22$ & 1200 & 8.50 & 873 & 5.22 \\
\hline $\begin{array}{l}03-07 \\
03-07\end{array}$ & $\begin{array}{l}1600 \\
2400\end{array}$ & $\begin{array}{l}24.91 \\
24.35\end{array}$ & $\begin{array}{l}14,000 \\
11,800\end{array}$ & $\begin{array}{l}3.15 \\
3.3: 3\end{array}$ & $03-22$ & 2400 & $8.5 \%$ & 879 & 5.24 \\
\hline & & & & & $03-23$ & 1200 & 9.85 & 1,150 & 5.20 \\
\hline $\begin{array}{l}03-08 \\
03-08\end{array}$ & $\begin{array}{l}1200 \\
2400\end{array}$ & $\begin{array}{l}23.51 \\
22.60\end{array}$ & $\begin{array}{l}9,620 \\
8,040\end{array}$ & $\begin{array}{l}3.55 \\
3.74\end{array}$ & $03-23$ & 2400 & 9.78 & 1,130 & 5.23 \\
\hline $\begin{array}{l}03-09 \\
03-09\end{array}$ & $\begin{array}{l}1200 \\
2400\end{array}$ & $\begin{array}{l}21.66 \\
20.85\end{array}$ & $\begin{array}{l}6,800 \\
5,970\end{array}$ & $\begin{array}{l}3.89 \\
4.02\end{array}$ & $\begin{array}{l}03-24 \\
03-24\end{array}$ & $\begin{array}{l}1200 \\
2400\end{array}$ & $\begin{array}{l}10.19 \\
10.59\end{array}$ & $\begin{array}{l}1,230 \\
1,330\end{array}$ & $\begin{array}{l}5.31 \\
5.33\end{array}$ \\
\hline $\begin{array}{l}03-10 \\
03-10\end{array}$ & $\begin{array}{l}1200 \\
2400\end{array}$ & $\begin{array}{l}20.06 \\
19.41\end{array}$ & $\begin{array}{l}5,290 \\
4,840\end{array}$ & $\begin{array}{l}4.14 \\
4.24\end{array}$ & $\begin{array}{l}03-25 \\
03-25\end{array}$ & $\begin{array}{l}1200 \\
2400\end{array}$ & $\begin{array}{l}10.51 \\
10.18\end{array}$ & $\begin{array}{l}1,310 \\
1,230\end{array}$ & $\begin{array}{l}5.36 \\
5.39\end{array}$ \\
\hline $\begin{array}{l}00-11 \\
00-11\end{array}$ & $\begin{array}{l}1200 \\
2400\end{array}$ & $\begin{array}{l}18.66 \\
17.91\end{array}$ & $\begin{array}{l}4,380 \\
3,980\end{array}$ & $\begin{array}{l}4.34 \\
4.42\end{array}$ & $\begin{array}{l}03-26 \\
03-26\end{array}$ & $\begin{array}{l}1200 \\
2400\end{array}$ & $\begin{array}{l}9.81 \\
9.60\end{array}$ & $\begin{array}{l}1,140 \\
1,100\end{array}$ & $\begin{array}{l}5.41 \\
5.43\end{array}$ \\
\hline $02-12$ & 1200 & 17.12 & $3,5 \% 0$ & 4.50 & $03-27$ & 2400 & 9.39 & 1,050 & 5.48 \\
\hline $03-12$ & 2400 & 16.30 & 3,230 & 4.57 & $03-28$ & 2400 & 9.37 & 1,050 & 5.52 \\
\hline $\begin{array}{l}03-13 \\
03-13\end{array}$ & $\begin{array}{l}1200 \\
2400\end{array}$ & $\begin{array}{l}15.46 \\
14.68\end{array}$ & $\begin{array}{l}2,800 \\
2,580\end{array}$ & $\begin{array}{l}4.64 \\
4.67\end{array}$ & $03-29$ & 2400 & 9.40 & 1,050 & 5.57 \\
\hline $0:-14$ & 1200 & 13.99 & 2,330 & 4.74 & $03-30$ & 2400 & 9.38 & 1,050 & 5.61 \\
\hline $09-14$ & 2400 & 13.37 & 2,130 & 4.79 & $03-31$ & 2400 & 9.20 & 1,010 & 5.65 \\
\hline $\begin{array}{l}03-15 \\
03-15\end{array}$ & $\begin{array}{l}0200 \\
2400\end{array}$ & $\begin{array}{l}12.83 \\
12.38\end{array}$ & $\begin{array}{l}1,950 \\
1,910\end{array}$ & $\begin{array}{l}4.80 \\
4.87\end{array}$ & $04-01$ & 2400 & 8.86 & 944 & 5.69 \\
\hline
\end{tabular}


TABLE 7.-Gage height, discharge, and accumulated runoff, flood of April 1979-Continued 02482000, Pearl River at Edinburg, Miss.-Continued

\begin{tabular}{|c|c|c|c|c|c|c|c|c|c|}
\hline DATE & TIME & $\begin{array}{l}\text { GAGE } \\
\text { HEIGHT }\end{array}$ & DISCHARGE & $\begin{array}{l}\text { ACCUMUI_ATED } \\
\text { RUNIOFF }\end{array}$ & DATE & TIME & $\begin{array}{c}\text { GAGE } \\
\text { HEIGHT }\end{array}$ & DISCHARGE & $\begin{array}{l}\text { ACCUMULATED } \\
\text { RUNOFF }\end{array}$ \\
\hline $\begin{array}{l}04-02 \\
04-02\end{array}$ & $\begin{array}{l}1200 \\
2400\end{array}$ & $\begin{array}{l}8.79 \\
9.21\end{array}$ & $\begin{array}{r}930 \\
1,010\end{array}$ & $\begin{array}{l}5.71 \\
5.73\end{array}$ & $\begin{array}{l}04-15 \\
04-15 \\
04-15\end{array}$ & $\begin{array}{l}0800 \\
1600 \\
2400\end{array}$ & $\begin{array}{l}29.41 \\
28.87 \\
28.39\end{array}$ & $\begin{array}{l}68,100 \\
60,200 \\
53,200\end{array}$ & $\begin{array}{l}13.01 \\
13.90 \\
14.613\end{array}$ \\
\hline 04-03 & 1200 & 11.84 & 1,660 & 5.76 & & & & & \\
\hline $04-03$ & 2400 & 12.45 & 1,830 & 5.84 & $\begin{array}{l}04-16 \\
04-16\end{array}$ & $\begin{array}{l}1200 \\
2400\end{array}$ & $\begin{array}{l}27.52 \\
26.63\end{array}$ & $\begin{array}{l}40,800 \\
28,500\end{array}$ & $\begin{array}{l}15.65 \\
16.37\end{array}$ \\
\hline $04-04$ & 2400 & 16.01 & 3,100 & 5.91 & $04-17$ & 1200 & 25.75 & 19,500 & 16.87 \\
\hline $\begin{array}{l}04-05 \\
04-05\end{array}$ & $\begin{array}{l}1200 \\
2400\end{array}$ & 16.77 & $\begin{array}{l}3,430 \\
3,750\end{array}$ & 5.97 & $04-17$ & 2400 & 24.76 & 13,300 & 17.21 \\
\hline 04-06 & 1200 & 17.66 & 3,860 & 6.13 & $\begin{array}{l}04-18 \\
04-18\end{array}$ & $\begin{array}{l}1200 \\
2400\end{array}$ & $\begin{array}{l}23.68 \\
22.59\end{array}$ & $\begin{array}{r}10,000 \\
8,020\end{array}$ & $\begin{array}{l}17.45 \\
17.64\end{array}$ \\
\hline $04-07$ & 2400 & 17.44 & 3,750 & 6.21 & $\begin{array}{l}04-19 \\
04-19\end{array}$ & $\begin{array}{l}1200 \\
2400\end{array}$ & $\begin{array}{l}21.53 \\
20.63\end{array}$ & $\begin{array}{l}6,640 \\
5,760\end{array}$ & $\begin{array}{l}17.79 \\
17.92\end{array}$ \\
\hline $04-07$ & 2400 & 17.34 & 3,700 & 6.36 & $\begin{array}{l}04-20 \\
04-20\end{array}$ & $\begin{array}{l}1200 \\
2400\end{array}$ & $\begin{array}{l}19.76 \\
18.91\end{array}$ & $\begin{array}{l}5,070 \\
4,520\end{array}$ & $\begin{array}{l}18.03 \\
18.13\end{array}$ \\
\hline $04-08$ & 2400 & 19.31 & 4,770 & 6.53 & $\begin{array}{l}04-21 \\
04-21\end{array}$ & $\begin{array}{l}1200 \\
2400\end{array}$ & $\begin{array}{l}18.05 \\
17.10\end{array}$ & $\begin{array}{l}4,060 \\
3,580\end{array}$ & $\begin{array}{l}18.22 \\
18.30\end{array}$ \\
\hline $\begin{array}{l}04-09 \\
04-09\end{array}$ & $\begin{array}{l}1200 \\
2400\end{array}$ & $\begin{array}{l}19.51 \\
19.70\end{array}$ & $\begin{array}{l}4,900 \\
5,030\end{array}$ & $\begin{array}{l}6.63 \\
6.7: 3\end{array}$ & $04-22$ & 1200 & 16.06 & 3,130 & 18.37 \\
\hline $\begin{array}{l}04-10 \\
04-10\end{array}$ & $\begin{array}{l}1200 \\
1900\end{array}$ & $\begin{array}{l}17.94 \\
20.00\end{array}$ & $\begin{array}{l}5,200 \\
5,240\end{array}$ & $\begin{array}{l}6.84 \\
6.90\end{array}$ & $04-22$ & 2400 & 15.11 & 2,740 & 18.43 \\
\hline $04-10$ & 2400 & 19.97 & 5,220 & $\begin{array}{l}6.70 \\
6.95\end{array}$ & $\begin{array}{l}04-23 \\
04-23\end{array}$ & $\begin{array}{l}1200 \\
2400\end{array}$ & $\begin{array}{l}14.33 \\
13.68\end{array}$ & $\begin{array}{l}2,450 \\
2,230\end{array}$ & $\begin{array}{l}18.48 \\
18.53\end{array}$ \\
\hline $\begin{array}{l}04-11 \\
04-11\end{array}$ & $\begin{array}{l}1200 \\
2400\end{array}$ & $\begin{array}{l}19.74 \\
19.40\end{array}$ & $\begin{array}{l}5,060 \\
4,830\end{array}$ & $\begin{array}{l}7.05 \\
7.16\end{array}$ & $\begin{array}{l}04-24 \\
04-24\end{array}$ & $\begin{array}{l}1200 \\
2400\end{array}$ & $\begin{array}{l}13.16 \\
12.77\end{array}$ & $\begin{array}{l}2,050 \\
1,930\end{array}$ & $\begin{array}{l}18.57 \\
18.61\end{array}$ \\
\hline $\begin{array}{l}04-12 \\
04-12 \\
04-12\end{array}$ & $\begin{array}{l}0800 \\
1600 \\
2400\end{array}$ & $\begin{array}{l}20.52 \\
24.41 \\
25.85\end{array}$ & $\begin{array}{r}5,660 \\
12,000 \\
20,300\end{array}$ & $\begin{array}{l}7.23 \\
7.35 \\
7.57\end{array}$ & $\begin{array}{l}04-25 \\
04-25\end{array}$ & $\begin{array}{l}1200 \\
2400\end{array}$ & $\begin{array}{l}12.41 \\
12.11\end{array}$ & $\begin{array}{l}1,820 \\
1,720\end{array}$ & $\begin{array}{l}18.65 \\
18.69\end{array}$ \\
\hline $\begin{array}{l}04-13 \\
04-13 \\
04-13\end{array}$ & $\begin{array}{l}0800 \\
1600 \\
2400\end{array}$ & $\begin{array}{l}26.45 \\
27.37 \\
28.77\end{array}$ & $\begin{array}{l}26,100 \\
38,600 \\
58,700\end{array}$ & $\begin{array}{l}7.89 \\
8.34 \\
9.01\end{array}$ & $\begin{array}{l}04-26 \\
04-26\end{array}$ & $\begin{array}{l}1200 \\
2400\end{array}$ & $\begin{array}{l}11.82 \\
11.55\end{array}$ & $\begin{array}{l}1,620 \\
1,540\end{array}$ & $\begin{array}{l}18.72 \\
18.76\end{array}$ \\
\hline $04-14$ & 0400 & 29.41 & 68,100 & 9.45 & $\begin{array}{l}04-27 \\
04-27\end{array}$ & $\begin{array}{l}1200 \\
2400\end{array}$ & $\begin{array}{l}11.25 \\
10.96\end{array}$ & $\begin{array}{l}1,450 \\
1,360\end{array}$ & $\begin{array}{l}18.79 \\
18.82\end{array}$ \\
\hline $\begin{array}{l}04-14 \\
04-14 \\
04-14 \\
04-14 \\
04-14\end{array}$ & $\begin{array}{l}0800 \\
1200 \\
1500 \\
1900 \\
2400\end{array}$ & $\begin{array}{l}29.62 \\
29.96 \\
30.06 \\
30.00 \\
29.83\end{array}$ & $\begin{array}{l}71,300 \\
76,300 \\
77,900 \\
77,000 \\
74,400\end{array}$ & $\begin{array}{r}9.9: 3 \\
10.44 \\
10.84 \\
11.37 \\
12.03\end{array}$ & $\begin{array}{l}04-28 \\
04-28\end{array}$ & $\begin{array}{l}1200 \\
2400\end{array}$ & $\begin{array}{l}10.64 \\
10.35\end{array}$ & $\begin{array}{l}1,270 \\
1,190\end{array}$ & $\begin{array}{l}18.84 \\
18.87\end{array}$ \\
\hline
\end{tabular}


TABLE 7.-Gage height, discharge, and accumulated runoff, flood of April 1979-Continued 02482550, Pearl River at Carthage, Miss.

\begin{tabular}{|c|c|c|c|c|c|c|c|c|c|}
\hline DATE & TIME & $\begin{array}{l}\text { GAGE } \\
\text { HEIGHT }\end{array}$ & DISCHARGE & $\begin{array}{c}\text { ACCUMULATED } \\
\text { RUNOFF }\end{array}$ & DATE & TIME & $\begin{array}{l}\text { GAGE } \\
\text { HEIGHT }\end{array}$ & DISCHARGE & $\begin{array}{l}\text { ACCUMUL } \\
\text { RUND }\end{array}$ \\
\hline $\begin{array}{l}03-03 \\
03-03\end{array}$ & $\begin{array}{l}0100 \\
1200\end{array}$ & $\begin{array}{l}19.40 \\
20.83\end{array}$ & $\begin{array}{r}7,730 \\
11,100\end{array}$ & $\begin{array}{l}0.00 \\
0.13\end{array}$ & $\begin{array}{l}03-14 \\
03-14\end{array}$ & $\begin{array}{l}1200 \\
2400\end{array}$ & $\begin{array}{l}14.65 \\
14.07\end{array}$ & $\begin{array}{l}4,150 \\
3,750\end{array}$ & $\begin{array}{l}4.64 \\
4.67\end{array}$ \\
\hline $03-03$ & 2400 & 22.45 & 15,600 & 0.31 & $\begin{array}{l}03-15 \\
03-15\end{array}$ & $\begin{array}{l}1200 \\
2400\end{array}$ & $\begin{array}{l}13.48 \\
12.92\end{array}$ & $\begin{array}{l}3,370 \\
3,030\end{array}$ & $\begin{array}{l}4.74 \\
4.79\end{array}$ \\
\hline $\begin{array}{l}03-04 \\
03-04 \\
03-04\end{array}$ & $\begin{array}{l}0300 \\
1200 \\
2400\end{array}$ & $\begin{array}{l}22.49 \\
22.45 \\
22.68\end{array}$ & $\begin{array}{l}15,800 \\
15,600 \\
16,800\end{array}$ & $\begin{array}{l}0.37 \\
0.53 \\
0.75\end{array}$ & $\begin{array}{l}03-16 \\
03-16\end{array}$ & $\begin{array}{l}1200 \\
2400\end{array}$ & $\begin{array}{l}12.43 \\
11.99\end{array}$ & $\begin{array}{l}2,750 \\
2,510\end{array}$ & $\begin{array}{l}4.82 \\
4.66\end{array}$ \\
\hline $\begin{array}{l}03-05 \\
03-05\end{array}$ & $\begin{array}{l}1200 \\
2400\end{array}$ & $\begin{array}{l}23.04 \\
23.78\end{array}$ & $\begin{array}{l}18,900 \\
25,100\end{array}$ & $\begin{array}{l}1.00 \\
1.30\end{array}$ & $\begin{array}{r}03-17 \\
03-17\end{array}$ & $\begin{array}{l}1200 \\
2400\end{array}$ & $\begin{array}{l}11.64 \\
11.38\end{array}$ & $\begin{array}{l}2,330 \\
2,100\end{array}$ & $\begin{array}{l}4.89 \\
4.93\end{array}$ \\
\hline $\begin{array}{l}03-06 \\
03-06 \\
03-06\end{array}$ & $\begin{array}{l}0800 \\
1500 \\
2400\end{array}$ & $\begin{array}{l}24.21 \\
24.35 \\
24.29\end{array}$ & $\begin{array}{l}29,500 \\
31,000 \\
30,400\end{array}$ & $\begin{array}{l}1.55 \\
1.80 \\
2.11\end{array}$ & $\begin{array}{l}03-18 \\
03-18\end{array}$ & $\begin{array}{l}1200 \\
2400\end{array}$ & $\begin{array}{l}11.00 \\
10.08\end{array}$ & $\begin{array}{l}2,060 \\
1,900\end{array}$ & $\begin{array}{l}4.96 \\
4.98\end{array}$ \\
\hline $\begin{array}{l}03-07 \\
03-07\end{array}$ & $\begin{array}{l}1200 \\
2400\end{array}$ & $\begin{array}{l}24.00 \\
23.60\end{array}$ & $\begin{array}{l}27,300 \\
23,400\end{array}$ & $\begin{array}{l}2.51 \\
2.86\end{array}$ & $\begin{array}{l}05-19 \\
03-19\end{array}$ & $\begin{array}{l}1200 \\
2400\end{array}$ & $\begin{array}{l}10.63 \\
10.41\end{array}$ & $\begin{array}{l}1,850 \\
1,750\end{array}$ & $\begin{array}{l}5.01 \\
5.03\end{array}$ \\
\hline $\begin{array}{l}03-08 \\
03-08\end{array}$ & $\begin{array}{l}1200 \\
2400\end{array}$ & $\begin{array}{l}23.13 \\
22.57\end{array}$ & $\begin{array}{l}19,500 \\
16,200\end{array}$ & $\begin{array}{l}3.16 \\
3.41\end{array}$ & $\begin{array}{l}03-20 \\
03-20\end{array}$ & $\begin{array}{l}1200 \\
2400\end{array}$ & $\begin{array}{r}10.17 \\
9.94\end{array}$ & $\begin{array}{l}1,670 \\
1,550\end{array}$ & $\begin{array}{l}5.06 \\
5.09\end{array}$ \\
\hline $\begin{array}{l}03-09 \\
03-09\end{array}$ & $\begin{array}{l}1200 \\
2400\end{array}$ & $\begin{array}{l}21.84 \\
20.95\end{array}$ & $\begin{array}{l}13,500 \\
11,400\end{array}$ & $\begin{array}{l}3.61 \\
3.78\end{array}$ & $\begin{array}{l}03-21 \\
03-21\end{array}$ & $\begin{array}{l}1200 \\
2400\end{array}$ & $\begin{array}{l}9.70 \\
9.48\end{array}$ & $\begin{array}{l}1,510 \\
1,440\end{array}$ & $\begin{array}{l}5.10 \\
5.12\end{array}$ \\
\hline $\begin{array}{l}03-10 \\
03-10\end{array}$ & $\begin{array}{l}1200 \\
2400\end{array}$ & $\begin{array}{l}20.04 \\
19.32\end{array}$ & $\begin{array}{l}9,670 \\
8,540\end{array}$ & $\begin{array}{l}3.93 \\
4.05\end{array}$ & $\begin{array}{l}03-22 \\
03-22\end{array}$ & $\begin{array}{l}1200 \\
2400\end{array}$ & $\begin{array}{l}9.26 \\
9.45\end{array}$ & $\begin{array}{l}1,360 \\
1,430\end{array}$ & $\begin{array}{l}5.14 \\
5.16\end{array}$ \\
\hline $\begin{array}{l}03-11 \\
03-11\end{array}$ & $\begin{array}{l}1200 \\
2400\end{array}$ & $\begin{array}{l}18.61 \\
17.88\end{array}$ & $\begin{array}{l}7,630 \\
6,860\end{array}$ & $\begin{array}{l}4.17 \\
4.27\end{array}$ & $\begin{array}{l}03-23 \\
03-23\end{array}$ & $\begin{array}{l}1200 \\
2400\end{array}$ & $\begin{array}{l}10.10 \\
10.70\end{array}$ & $\begin{array}{l}1,640 \\
1,080\end{array}$ & $\begin{array}{l}5.13 \\
5.21\end{array}$ \\
\hline $\begin{array}{l}03-12 \\
03-12\end{array}$ & $\begin{array}{l}1200 \\
2400\end{array}$ & $\begin{array}{l}17.18 \\
16.50\end{array}$ & $\begin{array}{l}6,170 \\
5,570\end{array}$ & $\begin{array}{l}4.36 \\
4.44\end{array}$ & $\begin{array}{l}03-24 \\
03-24\end{array}$ & $\begin{array}{l}1200 \\
2400\end{array}$ & $\begin{array}{l}11.09 \\
11.26\end{array}$ & $\begin{array}{l}2,060 \\
2,140\end{array}$ & $\begin{array}{l}5.23 \\
5.26\end{array}$ \\
\hline $\begin{array}{l}03-13 \\
03-13\end{array}$ & $\begin{array}{l}1200 \\
2400\end{array}$ & $\begin{array}{l}15.87 \\
15.26\end{array}$ & $\begin{array}{l}5,060 \\
4,590\end{array}$ & $\begin{array}{l}4.51 \\
4.58\end{array}$ & $\begin{array}{l}03-25 \\
03-25 \\
03-25\end{array}$ & $\begin{array}{l}0800 \\
1600 \\
2400\end{array}$ & $\begin{array}{l}11.30 \\
11.33 \\
11.31\end{array}$ & $\begin{array}{l}2,160 \\
2,180 \\
2,160\end{array}$ & $\begin{array}{l}5.23 \\
5.30 \\
5.32\end{array}$ \\
\hline
\end{tabular}


TABLE 7.-Gage height, discharge, and accumulated runoff, flood of April 1979-Continued 02482550, Pearl River at Carthage, Miss.-Continued

\begin{tabular}{|c|c|c|c|c|c|c|c|c|c|}
\hline DATE & TIME & $\begin{array}{c}\text { GAGE } \\
\text { HEIGHT }\end{array}$ & DISCHARGE & $\begin{array}{l}\text { ACCUMUI_ATED } \\
\text { RUNDFF }\end{array}$ & DATE & TIME & $\begin{array}{l}\text { GAGE } \\
\text { HEIGHT }\end{array}$ & DISCHARGE & $\begin{array}{l}\text { ACCUMULA } \\
\text { RUNIOF }\end{array}$ \\
\hline $\begin{array}{l}03-26 \\
03-26\end{array}$ & $\begin{array}{l}1200 \\
2400\end{array}$ & $\begin{array}{l}11.10 \\
10.84\end{array}$ & $\begin{array}{l}2,000 \\
1,940\end{array}$ & $\begin{array}{l}5.35 \\
5.98\end{array}$ & $\begin{array}{l}04-06 \\
04-06\end{array}$ & $\begin{array}{l}1200 \\
2400\end{array}$ & $\begin{array}{l}15.28 \\
15.55\end{array}$ & $\begin{array}{l}4,610 \\
4,810\end{array}$ & $\begin{array}{l}5.90 \\
6.06\end{array}$ \\
\hline $\begin{array}{l}03-27 \\
03-27\end{array}$ & $\begin{array}{l}1200 \\
2400 \\
1200\end{array}$ & $\begin{array}{l}10.55 \\
10.36 \\
10.10\end{array}$ & $\begin{array}{l}1,820 \\
1,740 \\
1,640\end{array}$ & $\begin{array}{l}5.40 \\
5.43 \\
5.45\end{array}$ & $\begin{array}{r}04-07 \\
04-07 \\
04-07\end{array}$ & $\begin{array}{l}0500 \\
1600 \\
2400\end{array}$ & $\begin{array}{l}15.68 \\
15.71 \\
15.71\end{array}$ & $\begin{array}{l}4,900 \\
4,940 \\
4,940\end{array}$ & $\begin{array}{l}6.10 \\
6.15 \\
6.1 \%\end{array}$ \\
\hline $\begin{array}{l}03-28 \\
03-29 \\
03-29\end{array}$ & $\begin{array}{l}2400 \\
1200 \\
2400\end{array}$ & $\begin{array}{l}9.84 \\
9.60 \\
7.49\end{array}$ & $\begin{array}{l}1,500 \\
1,480 \\
1,440\end{array}$ & $\begin{array}{l}5.47 \\
5.50 \\
5.52\end{array}$ & $\begin{array}{r}04-08 \\
04-08 \\
04-08\end{array}$ & $\begin{array}{l}0000 \\
1600 \\
2400\end{array}$ & $\begin{array}{l}15.70 \\
15.51 \\
17.22\end{array}$ & $\begin{array}{l}4,50 \\
5,580 \\
6,210\end{array}$ & $\begin{array}{l}6.24 \\
6.28 \\
6.34\end{array}$ \\
\hline $\begin{array}{l}03-30 \\
03-30\end{array}$ & $\begin{array}{l}1200 \\
2400\end{array}$ & $\begin{array}{l}9.39 \\
9.36\end{array}$ & $\begin{array}{l}1,410 \\
1,400\end{array}$ & $\begin{array}{l}5.5 .4 \\
5.55\end{array}$ & $\begin{array}{r}04-09 \\
04-09\end{array}$ & $\begin{array}{l}1200 \\
2400\end{array}$ & $\begin{array}{l}17.86 \\
16.25\end{array}$ & $\begin{array}{l}6,840 \\
7,240\end{array}$ & $\begin{array}{l}6.43 \\
6.53\end{array}$ \\
\hline $\begin{array}{l}0 z-31 \\
0 ;-31\end{array}$ & $\begin{array}{l}1200 \\
2400\end{array}$ & $\begin{array}{l}9.2 \% \\
9.24\end{array}$ & $\begin{array}{l}1,300 \\
1,360\end{array}$ & $\begin{array}{l}5.57 \\
5.59\end{array}$ & $\begin{array}{l}04-10 \\
04-10\end{array}$ & $\begin{array}{l}1200 \\
2400\end{array}$ & $\begin{array}{l}18.40 \\
18.38\end{array}$ & $\begin{array}{l}7,460 \\
7,370\end{array}$ & $\begin{array}{l}6.63 \\
6.73\end{array}$ \\
\hline $\begin{array}{l}04-01 \\
04-01\end{array}$ & $\begin{array}{l}1200 \\
2400\end{array}$ & $\begin{array}{l}7.16 \\
9.07\end{array}$ & $\begin{array}{l}1,300 \\
1,310\end{array}$ & $\begin{array}{l}5.61 \\
5.63\end{array}$ & $\begin{array}{l}04-11 \\
04-11\end{array}$ & $\begin{array}{l}1200 \\
2400\end{array}$ & $\begin{array}{l}19.28 \\
18.25\end{array}$ & $\begin{array}{l}7,220 \\
7,240\end{array}$ & $\begin{array}{l}6.83 \\
6.93\end{array}$ \\
\hline $\begin{array}{l}04-02 \\
04-02\end{array}$ & $\begin{array}{l}1200 \\
2400\end{array}$ & $\begin{array}{l}9.04 \\
9.36\end{array}$ & $\begin{array}{l}1,300 \\
1,400\end{array}$ & $\begin{array}{l}5.65 \\
5.67\end{array}$ & $\begin{array}{l}04-12 \\
04-12 \\
04-12\end{array}$ & $\begin{array}{l}0300 \\
0600 \\
0800 \\
0000\end{array}$ & $\begin{array}{l}18.60 \\
1 \% .08 \\
1 \% .51\end{array}$ & $\begin{array}{l}7,620 \\
6,210 \\
6,820 \\
0\end{array}$ & $\begin{array}{l}6.90 \\
6.93 \\
7.00 \\
7.01\end{array}$ \\
\hline $\begin{array}{r}04-03 \\
04-03\end{array}$ & $\begin{array}{l}0800 \\
1600\end{array}$ & $\begin{array}{l}10.69 \\
11.35\end{array}$ & $\begin{array}{l}1,970 \\
2,100\end{array}$ & $\begin{array}{l}5.60 \\
5.70\end{array}$ & $\begin{array}{l}04-12 \\
04-12 \\
04-12\end{array}$ & $\begin{array}{l}000 \\
1000 \\
1100\end{array}$ & $\begin{array}{l}19.86 \\
20.26 \\
20.85\end{array}$ & $\begin{array}{r}9,400 \\
10,100 \\
10,800\end{array}$ & $\begin{array}{l}7.01 \\
7.02 \\
7.04\end{array}$ \\
\hline $04-03$ & 2400 & 11.9 & $\Xi 480$ & 5.72 & $\begin{array}{l}04-12 \\
04-12\end{array}$ & $\begin{array}{l}1200 \\
1300\end{array}$ & $\begin{array}{l}21.06 \\
21.46\end{array}$ & $\begin{array}{l}11,600 \\
12,500\end{array}$ & $\begin{array}{l}7.05 \\
7.06\end{array}$ \\
\hline $\begin{array}{l}04-04 \\
04-04\end{array}$ & $\begin{array}{l}1200 \\
2400\end{array}$ & $\begin{array}{l}13.09 \\
14.19\end{array}$ & $\begin{array}{l}3,600 \\
3,300\end{array}$ & $\begin{array}{l}5.7 \% \\
5.82\end{array}$ & $\begin{array}{l}04-12 \\
04-12\end{array}$ & $\begin{array}{l}1400 \\
1500\end{array}$ & $\begin{array}{l}21.95 \\
22.21\end{array}$ & $\begin{array}{l}13,600 \\
14,600\end{array}$ & $\begin{array}{l}7.08 \\
7.09\end{array}$ \\
\hline $\begin{array}{l}04-05 \\
04-05\end{array}$ & $\begin{array}{l}1200 \\
2400\end{array}$ & $\begin{array}{l}14.54 \\
14.95\end{array}$ & $\begin{array}{l}4,070 \\
4,360\end{array}$ & $\begin{array}{l}5.67 \\
5.93\end{array}$ & $\begin{array}{l}04-12 \\
04-12 \\
04-12\end{array}$ & $\begin{array}{l}1600 \\
1700 \\
1800\end{array}$ & $\begin{array}{l}22.57 \\
22.85 \\
23.09\end{array}$ & $\begin{array}{l}16,200 \\
17,800 \\
19,200\end{array}$ & $\begin{array}{l}7.11 \\
7.13 \\
7.15\end{array}$ \\
\hline
\end{tabular}


TABLES

TABLE 7.-Gage height, discharge, and accumulated runoff, flood of April 1979-Continued 02482550, Pearl River at Carthage, Miss.-Continued

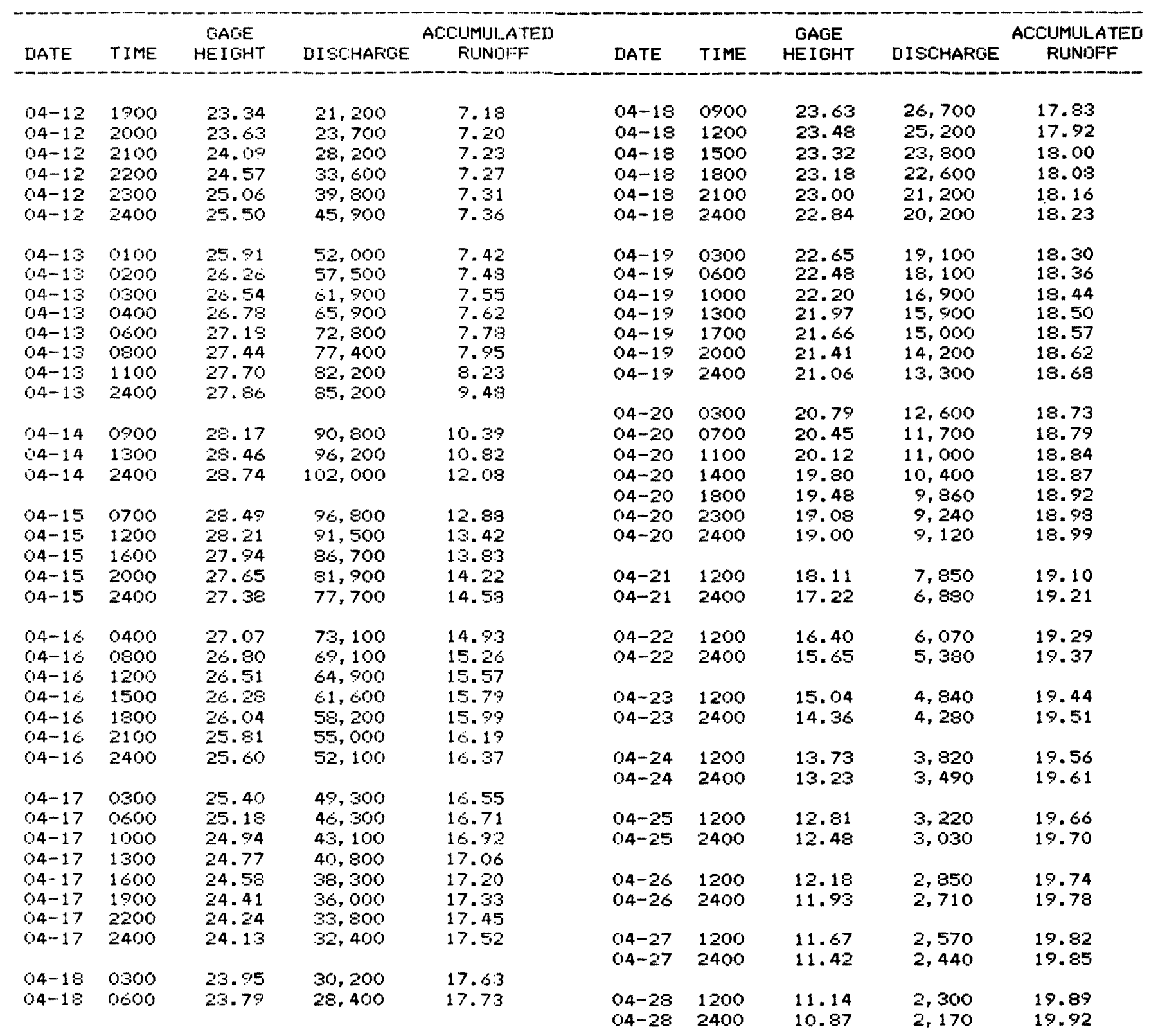


TABLE 7.-Gage height, discharge, and accumulated runoff, flood of April 1979-Continued 02483000, Tuscolameta Creek at Walnut Grove, Miss.

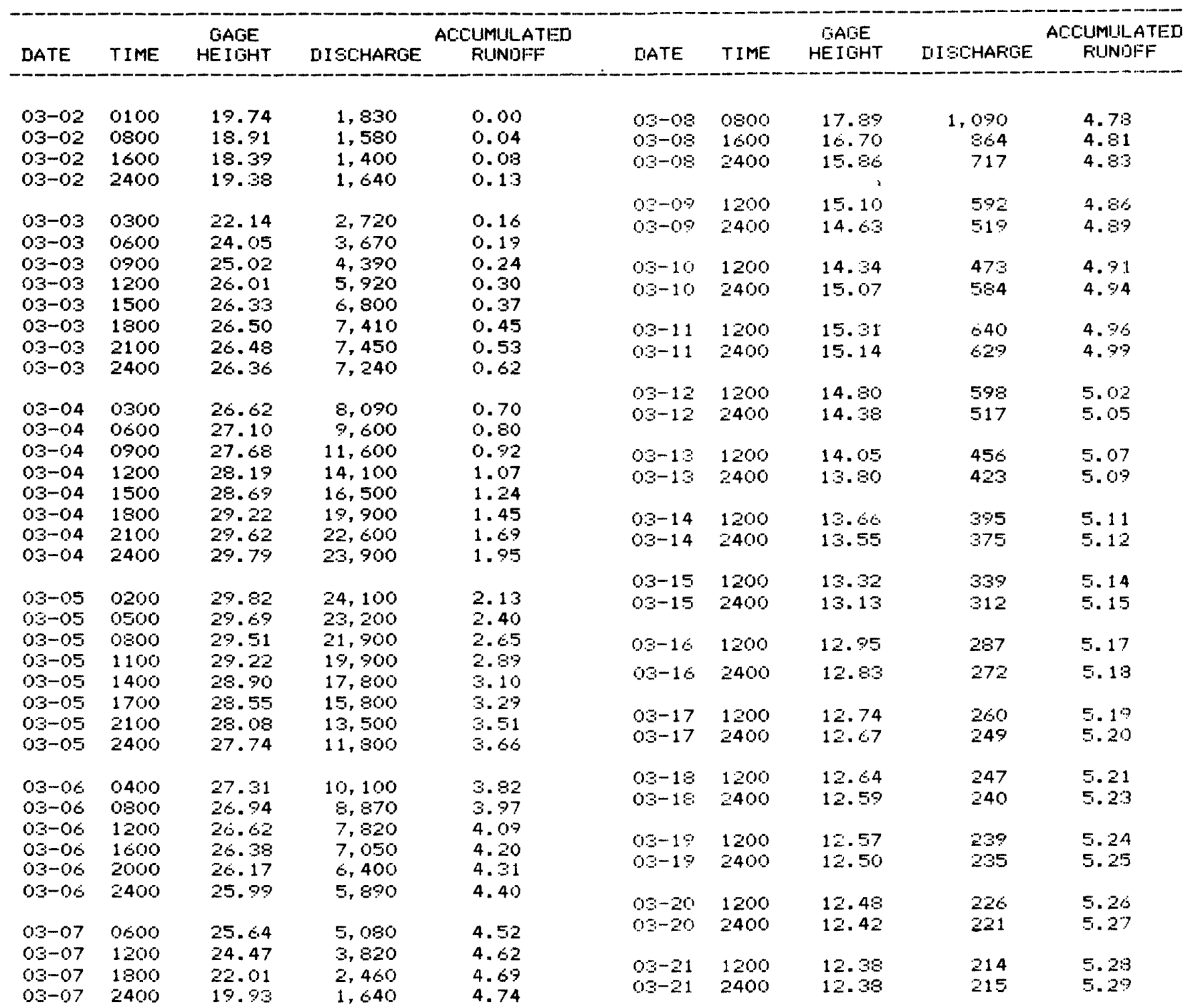


TABLES

TABLE 7.-Gage height, discharge, and accumulated runoff, flood of April 1979-Continued

02483000, Tuscolameta Creek at Walnut Grove, Miss.-Continued

\begin{tabular}{|c|c|c|c|c|c|c|c|c|c|}
\hline IIATE & TIME & $\begin{array}{c}\text { GAGE } \\
\text { HEIGHT }\end{array}$ & DISCHARGE & $\begin{array}{c}\text { ACCUMUILATED } \\
\text { RUINDFF= }\end{array}$ & DATE & TIME & $\begin{array}{l}\text { GAGE } \\
\text { HEIGHT }\end{array}$ & DISCHARGE & $\begin{array}{c}\text { ACCUMULATED } \\
\text { RUNIJFF }\end{array}$ \\
\hline $\begin{array}{l}03-22 \\
03-22\end{array}$ & $\begin{array}{l}1200 \\
2400\end{array}$ & $\begin{array}{l}12.39 \\
14.36\end{array}$ & $\begin{array}{l}219 \\
446\end{array}$ & $\begin{array}{l}5.30 \\
5.31\end{array}$ & $\begin{array}{l}04-05 \\
04-05\end{array}$ & $\begin{array}{l}1200 \\
2400\end{array}$ & $\begin{array}{l}26.23 \\
26.08\end{array}$ & $\begin{array}{l}6,670 \\
6,220\end{array}$ & $\begin{array}{l}6.84 \\
7.13\end{array}$ \\
\hline $\begin{array}{l}03-23 \\
03-20 \\
0-23 \\
0-20\end{array}$ & $\begin{array}{l}0800 \\
1400 \\
1900\end{array}$ & $\begin{array}{l}17.52 \\
18.05 \\
17.90 \\
17.76\end{array}$ & $\begin{array}{l}1,070 \\
1,200 \\
1,200\end{array}$ & $\begin{array}{l}5.34 \\
5.36 \\
5.30 \\
5.41\end{array}$ & $\begin{array}{l}04-06 \\
04-06\end{array}$ & $\begin{array}{l}1200 \\
2400\end{array}$ & $\begin{array}{l}25.84 \\
25.10\end{array}$ & $\begin{array}{l}5,500 \\
4,370\end{array}$ & $\begin{array}{l}7.40 \\
7.62\end{array}$ \\
\hline $03-23$ & 2400 & 17.76 & 1,340 & 5.41 & $04-07$ & 0800 & 22.41 & 2,700 & 7.73 \\
\hline $\begin{array}{l}03-24 \\
03-24\end{array}$ & $\begin{array}{l}1200 \\
2400\end{array}$ & $\begin{array}{l}17.55 \\
16.63\end{array}$ & $\begin{array}{l}1,300 \\
1,120\end{array}$ & $\begin{array}{l}5.47 \\
5.53\end{array}$ & $\begin{array}{l}04-07 \\
04-07\end{array}$ & $\begin{array}{l}1600 \\
2400\end{array}$ & $\begin{array}{l}19.20 \\
16.69\end{array}$ & $\begin{array}{r}1,440 \\
863\end{array}$ & $\begin{array}{l}7.79 \\
7.83\end{array}$ \\
\hline $\begin{array}{l}03-25 \\
03-25\end{array}$ & $\begin{array}{l}1200 \\
2400\end{array}$ & $\begin{array}{l}15.17 \\
14.17\end{array}$ & $\begin{array}{l}791 \\
509\end{array}$ & $\begin{array}{l}5.57 \\
5.60\end{array}$ & $\begin{array}{l}04-08 \\
04-08 \\
04-08\end{array}$ & $\begin{array}{l}0800 \\
1600 \\
2400\end{array}$ & $\begin{array}{l}15.40 \\
20.06 \\
21.98\end{array}$ & $\begin{array}{r}667 \\
1,840 \\
2,650\end{array}$ & $\begin{array}{l}7.85 \\
7.89 \\
7.95\end{array}$ \\
\hline $\begin{array}{l}03-26 \\
03-26\end{array}$ & $\begin{array}{l}1200 \\
2400\end{array}$ & $\begin{array}{l}13.49 \\
13.02\end{array}$ & $\begin{array}{l}389 \\
316\end{array}$ & $\begin{array}{l}5.62 \\
5.63\end{array}$ & $\begin{array}{l}04-09 \\
04-09\end{array}$ & $\begin{array}{l}1200 \\
2400\end{array}$ & $\begin{array}{l}22.95 \\
22.82\end{array}$ & $\begin{array}{l}3,160 \\
3,160\end{array}$ & $\begin{array}{l}8.07 \\
8.23\end{array}$ \\
\hline $\begin{array}{l}03-27 \\
03-27\end{array}$ & $\begin{array}{l}1200 \\
2400\end{array}$ & $\begin{array}{l}12.74 \\
12.56\end{array}$ & $\begin{array}{l}281 \\
251\end{array}$ & $\begin{array}{l}5.65 \\
5.66\end{array}$ & $\begin{array}{l}04-10 \\
04-10\end{array}$ & $\begin{array}{l}1200 \\
2400\end{array}$ & $\begin{array}{l}22.81 \\
22.06\end{array}$ & $\begin{array}{l}3,190 \\
2,820\end{array}$ & $\begin{array}{l}8.37 \\
8.51\end{array}$ \\
\hline $\begin{array}{l}03-28 \\
03-28\end{array}$ & $\begin{array}{l}1200 \\
2400\end{array}$ & $\begin{array}{l}12.44 \\
12.33\end{array}$ & $\begin{array}{l}232 \\
219\end{array}$ & $\begin{array}{l}5.67 \\
5.63\end{array}$ & $\begin{array}{l}04-11 \\
04-11\end{array}$ & $\begin{array}{l}1200 \\
2400\end{array}$ & $\begin{array}{l}20.29 \\
17.89\end{array}$ & $\begin{array}{l}1,980 \\
1,210\end{array}$ & $\begin{array}{l}8.62 \\
8.67\end{array}$ \\
\hline $03-29$ & 1200 & 12.25 & 221 & 5.67 & & & & & \\
\hline $03-29$ & 2400 & 12.16 & 205 & 5.70 & $\begin{array}{l}04-12 \\
04-12\end{array}$ & $\begin{array}{l}0300 \\
0600\end{array}$ & $\begin{array}{l}19.49 \\
19.26\end{array}$ & $\begin{array}{l}1,300 \\
1,460\end{array}$ & $\begin{array}{l}8.70 \\
8.72\end{array}$ \\
\hline $\begin{array}{l}03-30 \\
03-30\end{array}$ & $\begin{array}{l}1200 \\
2400\end{array}$ & $\begin{array}{l}12.13 \\
12.08\end{array}$ & $\begin{array}{l}193 \\
18 \%\end{array}$ & $\begin{array}{l}5.71 \\
5.72\end{array}$ & $\begin{array}{l}04-12 \\
04-12 \\
04-12\end{array}$ & $\begin{array}{l}0800 \\
0900 \\
1000\end{array}$ & $\begin{array}{l}21.66 \\
23.30 \\
24.43\end{array}$ & $\begin{array}{l}2,590 \\
3,350 \\
3,910\end{array}$ & $\begin{array}{l}5.73 \\
8.75 \\
8.76\end{array}$ \\
\hline $03-31$ & 1200 & 1200 & 187 & $5.7: 3$ & $04-12$ & 1100 & 25.17 & 4,460 & 8.77 \\
\hline $03-31$ & 2400 & 12.12 & 191 & 5.74 & $\begin{array}{l}04-12 \\
04-12\end{array}$ & $\begin{array}{l}1200 \\
1300\end{array}$ & $\begin{array}{l}25.56 \\
25.79\end{array}$ & $\begin{array}{l}4,910 \\
5,320\end{array}$ & $\begin{array}{l}8.79 \\
8.81\end{array}$ \\
\hline $\begin{array}{l}04-01 \\
04-01\end{array}$ & $\begin{array}{l}1200 \\
2400\end{array}$ & $\begin{array}{l}12.14 \\
12.21\end{array}$ & $\begin{array}{l}197 \\
202\end{array}$ & $\begin{array}{l}5.74 \\
5.75\end{array}$ & $\begin{array}{l}04-12 \\
04-12 \\
04-12\end{array}$ & $\begin{array}{l}1400 \\
1500 \\
1600\end{array}$ & $\begin{array}{l}25.86 \\
25.92 \\
25.62\end{array}$ & $\begin{array}{l}5,480 \\
5,430 \\
5,110\end{array}$ & $\begin{array}{l}8.8: 3 \\
8.85 \\
8.87\end{array}$ \\
\hline $\begin{array}{l}04-02 \\
04-02 \\
04-02\end{array}$ & $\begin{array}{l}0500 \\
1600 \\
2400\end{array}$ & $\begin{array}{l}12.46 \\
14.51 \\
17.89\end{array}$ & $\begin{array}{r}228 \\
568 \\
1,240\end{array}$ & $\begin{array}{l}5.76 \\
5.77 \\
5.30\end{array}$ & $\begin{array}{l}04-12 \\
04-12 \\
04-12\end{array}$ & $\begin{array}{l}1700 \\
1800 \\
1900\end{array}$ & $\begin{array}{l}25.35 \\
26.10 \\
26.41\end{array}$ & $\begin{array}{l}4,840 \\
6,240 \\
7,140\end{array}$ & $\begin{array}{l}8.39 \\
8.91 \\
8.94\end{array}$ \\
\hline & & & & 5.81 & $\begin{array}{l}04-12 \\
04-12\end{array}$ & $\begin{array}{l}2000 \\
2100\end{array}$ & $\begin{array}{l}26.65 \\
26.87\end{array}$ & $\begin{array}{l}7,870 \\
9,560\end{array}$ & $\begin{array}{l}8.97 \\
9.00\end{array}$ \\
\hline $\begin{array}{l}04-03 \\
04-03\end{array}$ & $\begin{array}{l}0800 \\
1600\end{array}$ & $\begin{array}{l}2.60 \\
23.05\end{array}$ & $\begin{array}{l}2,970 \\
3,230\end{array}$ & $\begin{array}{l}5.86 \\
5.96\end{array}$ & $04-12$ & 2200 & 27.00 & 8,980 & 9.03 \\
\hline $04-03$ & 2400 & 25.00 & 4,430 & 6.07 & $\begin{array}{l}04-12 \\
04-12\end{array}$ & $\begin{array}{l}2300 \\
2400\end{array}$ & $\begin{array}{l}27.02 \\
26.99\end{array}$ & $\begin{array}{l}9,070 \\
9,000\end{array}$ & $\begin{array}{l}9.00 \\
9.10\end{array}$ \\
\hline $\begin{array}{l}04-04 \\
04-04\end{array}$ & $\begin{array}{l}1200 \\
2400\end{array}$ & $\begin{array}{l}25.50 \\
26.02\end{array}$ & $\begin{array}{l}5,200 \\
6,170\end{array}$ & $\begin{array}{l}6.27 \\
6.55\end{array}$ & & & & & \\
\hline
\end{tabular}


TABLE 7.-Gage height, discharge, and accumulated runoff, flood of April 1979-Continued 02483000, Tuscolameta Creek at Walnut Grove, Miss.-Continued

\begin{tabular}{|c|c|c|c|c|c|c|c|c|c|}
\hline DATE & TIME & $\begin{array}{l}\text { GAGE } \\
\text { HEIGHT }\end{array}$ & DISCHARGE & $\begin{array}{c}\text { ACCUMUI_ATED } \\
\text { RUNDFF }\end{array}$ & DATE & TIME & $\begin{array}{l}\text { GAGE } \\
\text { HEIGHT }\end{array}$ & DISCHARGE & $\begin{array}{l}\text { ACCUMUI } \\
\text { RUNI }\end{array}$ \\
\hline $\begin{array}{l}04-13 \\
04-13 \\
04-13 \\
04-13 \\
04-13 \\
04-13 \\
04-13 \\
04-13\end{array}$ & $\begin{array}{l}0100 \\
0200 \\
0300 \\
0400 \\
0600 \\
0800 \\
1100 \\
2400\end{array}$ & $\begin{array}{l}26.98 \\
26.94 \\
26.94 \\
26.93 \\
26.96 \\
27.12 \\
27.62 \\
29.71\end{array}$ & $\begin{array}{r}9,010 \\
8,950 \\
9,010 \\
9,020 \\
9,170 \\
9,700 \\
11,400 \\
23,300\end{array}$ & $\begin{array}{r}9.13 \\
9.17 \\
9.20 \\
9.23 \\
9.30 \\
9.37 \\
9.47 \\
10.34\end{array}$ & $\begin{array}{l}04-19 \\
04-19 \\
04-19 \\
04-19 \\
04-19 \\
04-19 \\
04-19\end{array}$ & $\begin{array}{l}0300 \\
0600 \\
1000 \\
1300 \\
1700 \\
2000 \\
2400\end{array}$ & $\begin{array}{r}13.97 \\
13.87 \\
13.78 \\
13.46 \\
13.34 \\
13.28 \\
13.22\end{array}$ & $\begin{array}{l}405 \\
392 \\
380 \\
345 \\
332 \\
324 \\
317\end{array}$ & $\begin{array}{l}13.79 \\
13.78 \\
13.79 \\
13.79 \\
13.80 \\
13.80 \\
13.81\end{array}$ \\
\hline $\begin{array}{l}04-14 \\
04-14 \\
04-14\end{array}$ & $\begin{array}{l}0300 \\
1300 \\
2400\end{array}$ & $\begin{array}{l}29.76 \\
29.40 \\
29.39\end{array}$ & $\begin{array}{l}23,600 \\
21,000 \\
14,900\end{array}$ & $\begin{array}{l}10.61 \\
11.45 \\
12.19\end{array}$ & $\begin{array}{l}04-20 \\
04-20 \\
04-20 \\
04-20 \\
04-20\end{array}$ & $\begin{array}{l}0300 \\
0700 \\
1100 \\
1400 \\
1800\end{array}$ & $\begin{array}{l}13.20 \\
13.16 \\
13.10 \\
13.07 \\
13.02\end{array}$ & $\begin{array}{l}313 \\
307 \\
299 \\
294 \\
289\end{array}$ & $\begin{array}{l}13.81 \\
13.82 \\
13.82 \\
13.82 \\
13.8: 3\end{array}$ \\
\hline $\begin{array}{l}04-15 \\
04-15 \\
04-15 \\
04-15 \\
04-15\end{array}$ & $\begin{array}{l}0700 \\
1200 \\
1600 \\
2000 \\
2400\end{array}$ & $\begin{array}{l}27.67 \\
27.24 \\
26.92 \\
26.67 \\
26.45\end{array}$ & $\begin{array}{r}11,500 \\
9,810 \\
8,760 \\
7,920 \\
7,230\end{array}$ & $\begin{array}{l}12.54 \\
12.74 \\
12.813 \\
13.01 \\
13.12\end{array}$ & $\begin{array}{l}04-20 \\
04-20 \\
04-21 \\
04-21\end{array}$ & $\begin{array}{l}2300 \\
2400 \\
1200 \\
2400\end{array}$ & $\begin{array}{l}12.97 \\
12.97 \\
12.87 \\
12.73\end{array}$ & $\begin{array}{l}282 \\
282\end{array}$ & $\begin{array}{l}13.83 \\
13.83 \\
13.85 \\
13.86\end{array}$ \\
\hline $\begin{array}{l}04-16 \\
04-16 \\
04-16 \\
04-16 \\
04-16\end{array}$ & $\begin{array}{l}0400 \\
0800 \\
1200 \\
1500 \\
1800\end{array}$ & $\begin{array}{l}26.26 \\
26.11 \\
25.90 \\
25.64 \\
25.11\end{array}$ & $\begin{array}{l}6,630 \\
6,160 \\
5,580 \\
5,020 \\
4,280\end{array}$ & $\begin{array}{l}13.2: 3 \\
13 .: 32 \\
13.41 \\
13.47 \\
13.5: 3\end{array}$ & $\begin{array}{l}04-22 \\
04-22 \\
04-23 \\
04-23\end{array}$ & $\begin{array}{l}1200 \\
2400 \\
1200 \\
2400\end{array}$ & $\begin{array}{l}12.65 \\
12.65 \\
12.76 \\
12.97\end{array}$ & $\begin{array}{l}245 \\
246 \\
262 \\
291\end{array}$ & $\begin{array}{l}13.87 \\
13.88 \\
13.89 \\
13.91\end{array}$ \\
\hline $\begin{array}{l}04-16 \\
04-16\end{array}$ & $\begin{array}{l}2100 \\
2400\end{array}$ & $\begin{array}{l}24.14 \\
22.81\end{array}$ & $\begin{array}{l}3,490 \\
2,750\end{array}$ & $\begin{array}{l}13.57 \\
13.61\end{array}$ & $\begin{array}{l}04-24 \\
04-24\end{array}$ & $\begin{array}{l}1200 \\
2400\end{array}$ & $\begin{array}{l}13.28 \\
13.48\end{array}$ & $\begin{array}{l}347 \\
397\end{array}$ & $\begin{array}{l}13.92 \\
13.94\end{array}$ \\
\hline $\begin{array}{l}04-17 \\
04-17 \\
04-17 \\
04-17\end{array}$ & $\begin{array}{l}0300 \\
0600 \\
1000 \\
1300\end{array}$ & $\begin{array}{l}21.52 \\
20.27 \\
19.01 \\
18.13\end{array}$ & $\begin{array}{l}2,160 \\
1,700 \\
1,310 \\
1,100\end{array}$ & $\begin{array}{l}13.63 \\
13.65 \\
13.69 \\
13.67\end{array}$ & $\begin{array}{l}04-25 \\
04-25\end{array}$ & $\begin{array}{l}120 C \\
2400\end{array}$ & $\begin{array}{l}13.51 \\
13.46\end{array}$ & $\begin{array}{l}420 \\
415\end{array}$ & $\begin{array}{l}13.9 .5 \\
13.97\end{array}$ \\
\hline $\begin{array}{l}04-17 \\
04-17\end{array}$ & $\begin{array}{l}1600 \\
1900\end{array}$ & $\begin{array}{l}17.37 \\
16.75\end{array}$ & $\begin{array}{r}1,100 \\
950 \\
832\end{array}$ & $\begin{array}{l}13.07 \\
13.70 \\
13.71\end{array}$ & $\begin{array}{l}04-26 \\
04-26\end{array}$ & $\begin{array}{l}1200 \\
2400\end{array}$ & $\begin{array}{l}13.31 \\
13.29\end{array}$ & $\begin{array}{l}400 \\
371\end{array}$ & $\begin{array}{l}13.99 \\
14.01\end{array}$ \\
\hline $\begin{array}{l}04-17 \\
04-17\end{array}$ & $\begin{array}{l}2200 \\
2400\end{array}$ & $\begin{array}{l}16.28 \\
16.01\end{array}$ & $\begin{array}{l}750 \\
703\end{array}$ & $\begin{array}{l}13.72 \\
13.73\end{array}$ & $\begin{array}{l}04-27 \\
04-27\end{array}$ & $\begin{array}{l}1200 \\
2400\end{array}$ & $\begin{array}{l}13.28 \\
13.06\end{array}$ & $\begin{array}{l}415 \\
428\end{array}$ & $\begin{array}{l}14.0: 3 \\
14.05\end{array}$ \\
\hline $\begin{array}{l}04-18 \\
04-18 \\
04-18 \\
04-18 \\
04-18\end{array}$ & $\begin{array}{l}0300 \\
0600 \\
0900 \\
1200 \\
1500\end{array}$ & $\begin{array}{l}15.61 \\
15.17 \\
14.85 \\
14.63 \\
14.47\end{array}$ & $\begin{array}{l}638 \\
573 \\
528 \\
498 \\
474\end{array}$ & $\begin{array}{l}13.7: 3 \\
13.74 \\
13.75 \\
13.75 \\
13.76\end{array}$ & $\begin{array}{l}04-28 \\
04-28\end{array}$ & $\begin{array}{l}1200 \\
2400\end{array}$ & $\begin{array}{l}12.79 \\
12.57\end{array}$ & $\begin{array}{l}342 \\
280\end{array}$ & $\begin{array}{l}14.065 \\
14.013\end{array}$ \\
\hline $\begin{array}{l}04-18 \\
04-18 \\
04-18\end{array}$ & $\begin{array}{l}1800 \\
2100 \\
2400\end{array}$ & $\begin{array}{l}14.33 \\
14.20 \\
14.08\end{array}$ & $\begin{array}{l}454 \\
438 \\
421\end{array}$ & $\begin{array}{l}13.76 \\
13.77 \\
13.77\end{array}$ & & & & & \\
\hline
\end{tabular}


TABLES

TABLE 7.-Gage height, discharge, and accumulated runoff, flood of April 1979-Continued 02484000, Yockanookany River near Kosciusko, Miss.

\begin{tabular}{|c|c|c|c|c|c|c|c|c|c|}
\hline DATE & TIME & $\begin{array}{l}\text { GAGE } \\
\text { HEIGHT }\end{array}$ & DISCHARGE & $\begin{array}{l}\text { ACCUMULATED } \\
\text { RUNDFF }\end{array}$ & DATE & TIME & $\begin{array}{l}\text { GAGE } \\
\text { HEIGHT }\end{array}$ & DISCHARGE & $\begin{array}{l}\text { ACCUMULATED } \\
\text { RUNOFF }\end{array}$ \\
\hline $\begin{array}{l}03-03 \\
03-03 \\
03-03 \\
03-03\end{array}$ & $\begin{array}{l}0100 \\
0800 \\
1600 \\
2400\end{array}$ & $\begin{array}{r}9.87 \\
11.56 \\
13.42 \\
14.31\end{array}$ & $\begin{array}{r}898 \\
1,490 \\
3,510 \\
5,070\end{array}$ & $\begin{array}{l}0.00 \\
0.04 \\
0.13 \\
0.30\end{array}$ & $\begin{array}{l}03-16 \\
03-16\end{array}$ & $\begin{array}{l}1200 \\
2400\end{array}$ & $\begin{array}{l}6.03 \\
5.97\end{array}$ & $\begin{array}{l}251 \\
244\end{array}$ & $\begin{array}{l}3.16 \\
3.18\end{array}$ \\
\hline $03-03$ & 2400 & 14.31 & 5,070 & 0.30 & $\begin{array}{l}03-17 \\
03-17\end{array}$ & $\begin{array}{l}1200 \\
2400\end{array}$ & $\begin{array}{l}5.85 \\
5.77\end{array}$ & $\begin{array}{l}230 \\
220\end{array}$ & $\begin{array}{l}3.19 \\
3.20\end{array}$ \\
\hline $\begin{array}{l}03-04 \\
03-04\end{array}$ & $\begin{array}{l}1600 \\
2400\end{array}$ & $\begin{array}{l}14.42 \\
14.43\end{array}$ & $\begin{array}{l}5,280 \\
5,300\end{array}$ & $\begin{array}{l}0.73 \\
0.94\end{array}$ & $\begin{array}{l}03-18 \\
03-18\end{array}$ & $\begin{array}{l}1200 \\
2400\end{array}$ & $\begin{array}{l}5.71 \\
5.64\end{array}$ & $\begin{array}{l}214 \\
206\end{array}$ & $\begin{array}{l}3.22 \\
3.23\end{array}$ \\
\hline $\begin{array}{l}03-0.5 \\
03-05 \\
03-05\end{array}$ & $\begin{array}{l}0800 \\
1600 \\
2400\end{array}$ & $\begin{array}{l}14.91 \\
15.20 \\
15.05\end{array}$ & $\begin{array}{l}6,300 \\
6,970 \\
6,610\end{array}$ & $\begin{array}{l}1.17 \\
1.43 \\
1.70\end{array}$ & $\begin{array}{l}03-19 \\
03-19\end{array}$ & $\begin{array}{l}1200 \\
2400\end{array}$ & $\begin{array}{l}5.60 \\
5.55\end{array}$ & $\begin{array}{l}202 \\
196\end{array}$ & $\begin{array}{l}3.24 \\
3.25\end{array}$ \\
\hline $\begin{array}{l}03-06 \\
03-06\end{array}$ & $\begin{array}{l}0800 \\
1600\end{array}$ & $\begin{array}{l}14.68 \\
14.21\end{array}$ & $\begin{array}{l}5,800 \\
4,870\end{array}$ & $\begin{array}{l}1.94 \\
2.15\end{array}$ & $\begin{array}{l}03-20 \\
03-20\end{array}$ & $\begin{array}{l}1200 \\
2400\end{array}$ & $\begin{array}{l}5.51 \\
5.46\end{array}$ & $\begin{array}{l}192 \\
187\end{array}$ & $\begin{array}{l}3.27 \\
3.28\end{array}$ \\
\hline $03-06$ & 2400 & 13.71 & 3,970 & 2.33 & $03-21$ & 1200 & 5.46 & 187 & 3.29 \\
\hline $\begin{array}{l}03-07 \\
03-07\end{array}$ & $\begin{array}{l}0800 \\
1600\end{array}$ & $\begin{array}{l}13.16 \\
12.38\end{array}$ & $\begin{array}{l}3,130 \\
2,150\end{array}$ & $\begin{array}{l}2.47 \\
2.57\end{array}$ & $03-21$ & 2400 & 5.43 & 183 & 3.30 \\
\hline $03-07$ & 2400 & 11.35 & 1,380 & 2.64 & $\begin{array}{l}03-22 \\
03-22\end{array}$ & $\begin{array}{l}1200 \\
2400\end{array}$ & $\begin{array}{l}5.46 \\
7.71\end{array}$ & $\begin{array}{l}187 \\
486\end{array}$ & $\begin{array}{l}3.31 \\
3.33\end{array}$ \\
\hline $\begin{array}{l}03-08 \\
03-08\end{array}$ & $\begin{array}{l}1200 \\
2400\end{array}$ & $\begin{array}{l}9.29 \\
8.58\end{array}$ & $\begin{array}{l}768 \\
635\end{array}$ & $\begin{array}{l}2.71 \\
2.75\end{array}$ & $\begin{array}{l}03-23 \\
03-23\end{array}$ & $\begin{array}{l}0800 \\
1600\end{array}$ & $\begin{array}{r}9.82 \\
11.62\end{array}$ & $\begin{array}{r}886 \\
1,520\end{array}$ & $\begin{array}{l}3.36 \\
3.40\end{array}$ \\
\hline $\begin{array}{l}03-09 \\
03-09\end{array}$ & $\begin{array}{l}1200 \\
2400\end{array}$ & $\begin{array}{l}8.09 \\
7.69\end{array}$ & $\begin{array}{l}549 \\
483\end{array}$ & $\begin{array}{l}2.73 \\
2.81\end{array}$ & $03-23$ & 2400 & 12.76 & 2,610 & 3.49 \\
\hline $\begin{array}{l}03-10 \\
03-10\end{array}$ & $\begin{array}{l}1200 \\
2400\end{array}$ & $\begin{array}{l}7.39 \\
7.79\end{array}$ & $\begin{array}{l}436 \\
499\end{array}$ & $\begin{array}{l}2.84 \\
2.87\end{array}$ & $\begin{array}{l}03-24 \\
03-24 \\
03-24\end{array}$ & $\begin{array}{l}0600 \\
1200 \\
2400\end{array}$ & $\begin{array}{l}12.92 \\
12.80 \\
12.52\end{array}$ & $\begin{array}{l}2,810 \\
2,660 \\
2,310\end{array}$ & $\begin{array}{l}3.57 \\
3.65 \\
3.79\end{array}$ \\
\hline $\begin{array}{l}03-11 \\
03-11\end{array}$ & $\begin{array}{l}1200 \\
2400\end{array}$ & $\begin{array}{l}8.57 \\
8.95\end{array}$ & $\begin{array}{l}633 \\
703\end{array}$ & $\begin{array}{l}2.90 \\
2.94\end{array}$ & $\begin{array}{l}0.3-25 \\
03-25\end{array}$ & $\begin{array}{l}1200 \\
2400\end{array}$ & $\begin{array}{l}12.42 \\
12.17\end{array}$ & $\begin{array}{l}2,190 \\
1,930\end{array}$ & $\begin{array}{l}3.93 \\
4.05\end{array}$ \\
\hline $\begin{array}{l}03-12 \\
03-12\end{array}$ & $\begin{array}{l}1200 \\
2400\end{array}$ & $\begin{array}{l}8.52 \\
7.96\end{array}$ & $\begin{array}{l}624 \\
527\end{array}$ & $\begin{array}{l}2.98 \\
3.02\end{array}$ & $\begin{array}{l}03-26 \\
03-26 \\
03-26\end{array}$ & $\begin{array}{l}0800 \\
1600 \\
2400\end{array}$ & $\begin{array}{r}11.47 \\
9.93 \\
8.24\end{array}$ & $\begin{array}{r}1,440 \\
912 \\
575\end{array}$ & $\begin{array}{l}4.12 \\
4.16 \\
4.19\end{array}$ \\
\hline $\begin{array}{l}03-13 \\
03-13\end{array}$ & $\begin{array}{l}1200 \\
2400\end{array}$ & $\begin{array}{l}7.51 \\
7.13\end{array}$ & $\begin{array}{l}455 \\
397\end{array}$ & $\begin{array}{l}3.04 \\
3.07\end{array}$ & $\begin{array}{l}03-27 \\
03-27\end{array}$ & $\begin{array}{l}1200 \\
2400\end{array}$ & $\begin{array}{l}7.09 \\
6.61\end{array}$ & $\begin{array}{l}392 \\
324\end{array}$ & $\begin{array}{l}4.22 \\
4.24\end{array}$ \\
\hline $\begin{array}{l}03-14 \\
03-14\end{array}$ & $\begin{array}{l}1200 \\
2400\end{array}$ & $\begin{array}{l}6.86 \\
6.63\end{array}$ & $\begin{array}{l}359 \\
327\end{array}$ & $\begin{array}{l}3.09 \\
3.11\end{array}$ & $\begin{array}{l}03-38 \\
03-28\end{array}$ & $\begin{array}{l}1200 \\
2400\end{array}$ & $\begin{array}{l}6.32 \\
6.09\end{array}$ & $\begin{array}{l}285 \\
258\end{array}$ & $\begin{array}{l}4.26 \\
4.28\end{array}$ \\
\hline $\begin{array}{l}03-15 \\
03-15\end{array}$ & $\begin{array}{l}1200 \\
2400\end{array}$ & $\begin{array}{l}6.40 \\
6.17\end{array}$ & $\begin{array}{l}297 \\
268\end{array}$ & $\begin{array}{l}3.13 \\
3.15\end{array}$ & $\begin{array}{l}03-29 \\
03-29\end{array}$ & $\begin{array}{l}1200 \\
2400\end{array}$ & $\begin{array}{l}5.92 \\
5.79\end{array}$ & $\begin{array}{l}239 \\
223\end{array}$ & $\begin{array}{l}4.29 \\
4.30\end{array}$ \\
\hline
\end{tabular}


TABLE 7.-Gage height, discharge, and accumulated runoff, flood of April 1979-Continued 02484000, Yockanookany River near Kosciusko, Miss.-Continued

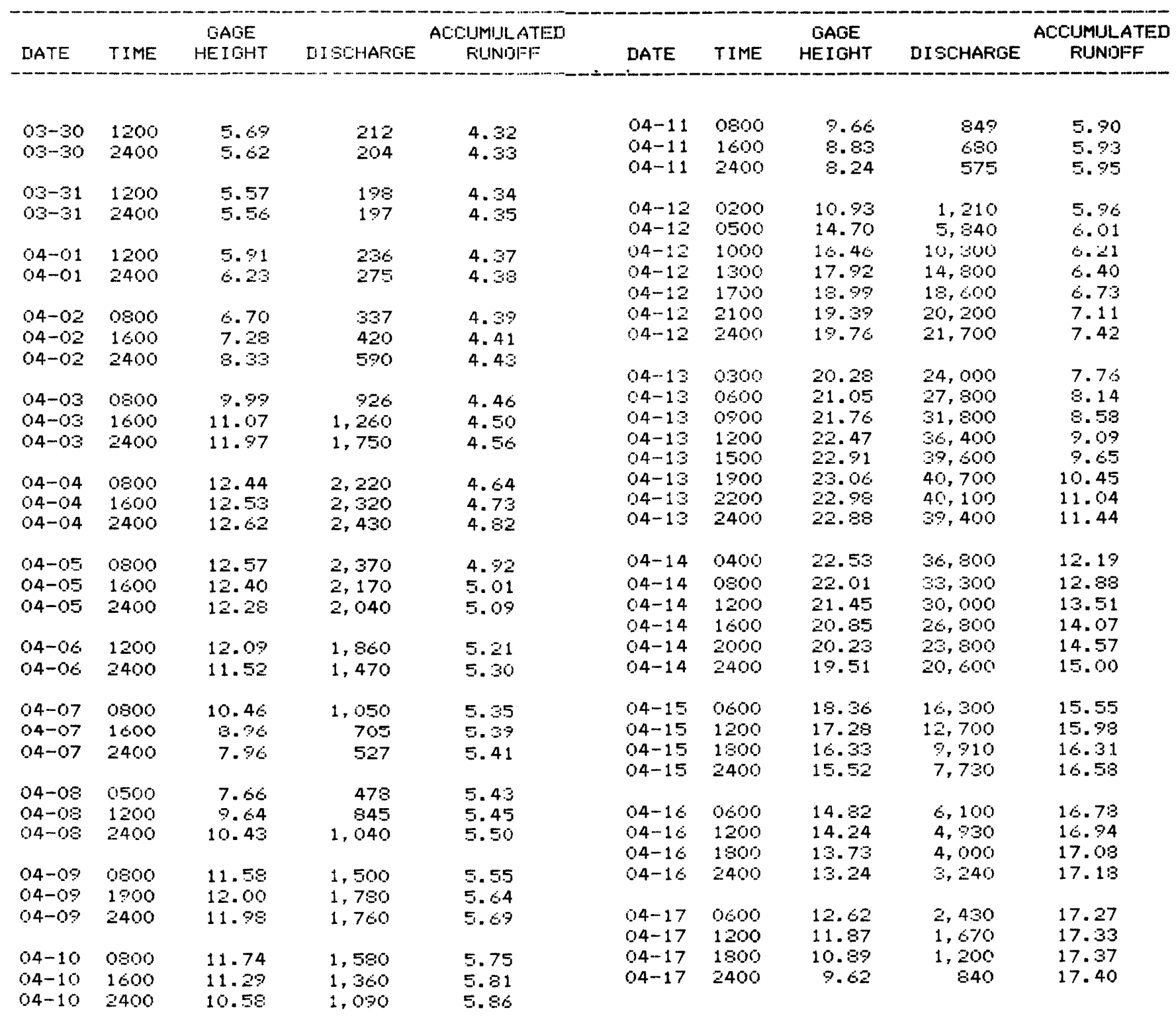


TABLES

TABLE 7.-Gage height, discharge, and accumulated runoff, flood of April 1979-Continued 02484000, Yockanookany River near Kosciusko, Miss.-Contimued

\begin{tabular}{|c|c|c|c|c|c|c|c|c|c|}
\hline DATE & TIME & $\begin{array}{l}\text { GAGE } \\
\text { HEIGHT }\end{array}$ & DISCHAFGE & $\begin{array}{c}\text { ACCUMULATED } \\
\text { FUNOFF }\end{array}$ & DATE & TIME & $\begin{array}{l}\text { GAGE } \\
\text { HEIGHT }\end{array}$ & DISCHARGE & $\begin{array}{c}\text { ACCUMULATED } \\
\text { RUNDFF }\end{array}$ \\
\hline $\begin{array}{l}04-18 \\
04-16 \\
04-18\end{array}$ & $\begin{array}{l}0800 \\
1600 \\
2400\end{array}$ & $\begin{array}{l}8.29 \\
7.64 \\
7.22\end{array}$ & $\begin{array}{l}583 \\
475 \\
411\end{array}$ & $\begin{array}{l}17.43 \\
17.45 \\
17.47\end{array}$ & $\begin{array}{l}04-24 \\
04-24 \\
04-24\end{array}$ & $\begin{array}{l}0600 \\
1200 \\
2400\end{array}$ & $\begin{array}{l}7.06 \\
7.01 \\
6.90\end{array}$ & $\begin{array}{l}387 \\
380 \\
364\end{array}$ & $\begin{array}{l}17.65 \\
17.66 \\
17.68\end{array}$ \\
\hline $\begin{array}{l}04-19 \\
04-19\end{array}$ & $\begin{array}{l}1200 \\
2400\end{array}$ & $\begin{array}{l}6.73 \\
6.49\end{array}$ & $\begin{array}{l}341 \\
308\end{array}$ & $\begin{array}{l}17.49 \\
17.51\end{array}$ & $\begin{array}{l}04-25 \\
04-25 \\
04-25\end{array}$ & $\begin{array}{l}1200 \\
2000 \\
2400\end{array}$ & $\begin{array}{l}6.95 \\
7.14 \\
7.10\end{array}$ & $\begin{array}{l}371 \\
399 \\
393\end{array}$ & $\begin{array}{l}17.70 \\
17.72 \\
17.73\end{array}$ \\
\hline $\begin{array}{l}04-20 \\
04-20\end{array}$ & $\begin{array}{l}1200 \\
2400\end{array}$ & $\begin{array}{l}6.29 \\
6.13\end{array}$ & $\begin{array}{l}283 \\
263\end{array}$ & $\begin{array}{l}17.53 \\
17.54\end{array}$ & $\begin{array}{l}04-26 \\
04-26\end{array}$ & $\begin{array}{l}1200 \\
2400\end{array}$ & $\begin{array}{l}7.02 \\
6.77\end{array}$ & $\begin{array}{l}381 \\
346\end{array}$ & $\begin{array}{l}17.75 \\
17.77\end{array}$ \\
\hline $\begin{array}{l}04-21 \\
04-21\end{array}$ & $\begin{array}{l}1200 \\
2400\end{array}$ & $\begin{array}{l}5.97 \\
5.88\end{array}$ & $\begin{array}{l}244 \\
233\end{array}$ & $\begin{array}{l}17.56 \\
17.57\end{array}$ & $\begin{array}{l}04-27 \\
04-27\end{array}$ & $\begin{array}{l}1200 \\
2400\end{array}$ & $\begin{array}{l}6.39 \\
6.03\end{array}$ & $\begin{array}{l}296 \\
251\end{array}$ & $\begin{array}{l}17.79 \\
17.81\end{array}$ \\
\hline $\begin{array}{l}04-22 \\
04-22\end{array}$ & $\begin{array}{l}1200 \\
2400\end{array}$ & $\begin{array}{l}5.79 \\
5.98\end{array}$ & $\begin{array}{l}223 \\
245\end{array}$ & $\begin{array}{l}17.59 \\
17.60\end{array}$ & $\begin{array}{l}04-28 \\
04-28\end{array}$ & $\begin{array}{l}1200 \\
2400\end{array}$ & $\begin{array}{l}5.72 \\
5.45\end{array}$ & $\begin{array}{l}215 \\
186\end{array}$ & $\begin{array}{l}17.82 \\
17.83\end{array}$ \\
\hline $\begin{array}{l}04-23 \\
04-23\end{array}$ & $\begin{array}{l}1200 \\
2400\end{array}$ & $\begin{array}{l}6.63 \\
7.03\end{array}$ & $\begin{array}{l}327 \\
383\end{array}$ & $\begin{array}{l}17.62 \\
17.64\end{array}$ & & & & & \\
\hline
\end{tabular}


TABLE 7.-Gage height, discharge, and accumulated runoff, flood of April 1979-Continued 02484500, Yockanookany River near Ofahoma, Miss.

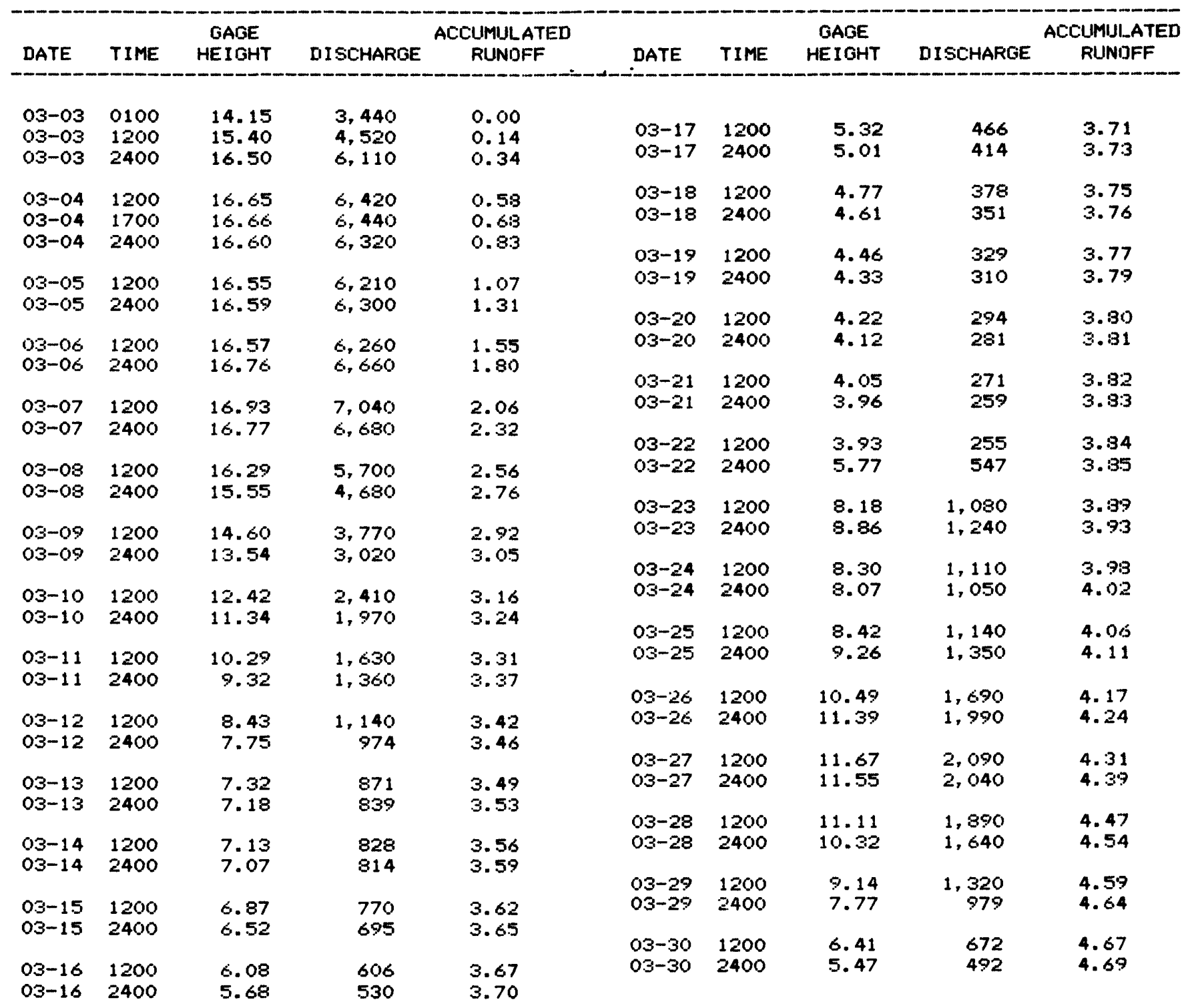


TABLE 7.-Gage height, discharge, and accumulated runoff, flood of April 1979-Continued 02484500, Yockanookany River near Ofahoma, Miss.-Continued

\begin{tabular}{|c|c|c|c|c|c|c|c|c|c|}
\hline DATE & TIME & $\begin{array}{l}\text { GAGE } \\
\text { HEIGHT }\end{array}$ & DI SCHARGE & $\begin{array}{l}\text { ACCUMUILATED } \\
\text { RUNDFF }\end{array}$ & DATE & TIME & $\begin{array}{l}\text { GAGE } \\
\text { HEIGHT }\end{array}$ & DISCHARGE & $\begin{array}{c}\text { ACCUMULLATED } \\
\text { RUNOFF }\end{array}$ \\
\hline $03-31$ & 1200 & 4.93 & 401 & 4.71 & $04-12$ & 0200 & 11.58 & 2,060 & 6.23 \\
\hline $03-31$ & 2400 & 4.63 & 354 & 4.72 & $\begin{array}{l}04-12 \\
04-12\end{array}$ & $\begin{array}{l}0300 \\
0400\end{array}$ & $\begin{array}{l}11.71 \\
12.01\end{array}$ & $\begin{array}{l}2,110 \\
2,240\end{array}$ & $\begin{array}{l}6.24 \\
6.24\end{array}$ \\
\hline $\begin{array}{l}04-01 \\
04-01\end{array}$ & 2400 & $\begin{array}{l}4.43 \\
4.32\end{array}$ & $\begin{array}{l}327 \\
308\end{array}$ & $\begin{array}{l}4.74 \\
4.75\end{array}$ & $04-12$ & 0500 & 12.37 & 2,390 & 6.25 \\
\hline & & & 508 & 4.75 & $\begin{array}{l}04-12 \\
04-12\end{array}$ & $\begin{array}{l}0600 \\
0700\end{array}$ & $\begin{array}{l}12.74 \\
13.19\end{array}$ & $\begin{array}{l}2,580 \\
2,820\end{array}$ & $\begin{array}{l}6.26 \\
6.27\end{array}$ \\
\hline $04-02$ & 1200 & 4.37 & 316 & 4.76 & $04-12$ & 0800 & 13.78 & 3,170 & 6.21 \\
\hline $04-02$ & 2400 & 4.70 & 365 & 4.77 & $04-12$ & 0900 & 14.58 & 3,760 & 6.29 \\
\hline $\begin{array}{l}04-03 \\
04-03\end{array}$ & $\begin{array}{l}1200 \\
2400\end{array}$ & $\begin{array}{l}6.76 \\
7.64\end{array}$ & $\begin{array}{l}746 \\
947\end{array}$ & $\begin{array}{l}4.80 \\
4.83\end{array}$ & $\begin{array}{l}04-12 \\
04-12 \\
04-12\end{array}$ & $\begin{array}{l}1000 \\
1100 \\
1200\end{array}$ & $\begin{array}{l}15.52 \\
16.25 \\
16.78\end{array}$ & $\begin{array}{l}4,650 \\
5,620 \\
6,700\end{array}$ & $\begin{array}{l}6.30 \\
6.32 \\
6.34\end{array}$ \\
\hline $\begin{array}{l}04-04 \\
04-04\end{array}$ & $\begin{array}{l}1200 \\
2400\end{array}$ & $\begin{array}{l}8.94 \\
9.44\end{array}$ & $\begin{array}{l}1,260 \\
1,390\end{array}$ & $\begin{array}{l}4.87 \\
4.92\end{array}$ & $\begin{array}{l}04-12 \\
04-12 \\
04-12\end{array}$ & $\begin{array}{l}1300 \\
1400 \\
1500\end{array}$ & $\begin{array}{l}17.19 \\
17.57 \\
18.04\end{array}$ & $\begin{array}{r}7,710 \\
8,800 \\
10,300\end{array}$ & $\begin{array}{l}6.34 \\
6.39 \\
6.42\end{array}$ \\
\hline $\begin{array}{l}04-05 \\
04-05\end{array}$ & $\begin{array}{l}1200 \\
2400\end{array}$ & $\begin{array}{r}9.62 \\
10.25\end{array}$ & $\begin{array}{l}1,440 \\
1,620\end{array}$ & $\begin{array}{l}4.9: 3 \\
5.0: 3\end{array}$ & $\begin{array}{l}04-12 \\
04-12 \\
04-12\end{array}$ & $\begin{array}{l}1600 \\
1700 \\
1800\end{array}$ & $\begin{array}{l}18.63 \\
19.04 \\
19.36\end{array}$ & $\begin{array}{l}12,500 \\
14,300 \\
15,800\end{array}$ & $\begin{array}{l}6.45 \\
6.50 \\
6.55\end{array}$ \\
\hline $\begin{array}{l}04-06 \\
04-06\end{array}$ & $\begin{array}{l}1200 \\
2400\end{array}$ & $\begin{array}{l}11.31 \\
11.95\end{array}$ & $\begin{array}{l}1,960 \\
2,210\end{array}$ & $\begin{array}{l}5.10 \\
5.18\end{array}$ & $\begin{array}{l}04-12 \\
04-12 \\
04-12\end{array}$ & $\begin{array}{l}1900 \\
2000 \\
2100\end{array}$ & $\begin{array}{l}19.68 \\
19.91 \\
20.12\end{array}$ & $\begin{array}{l}17,400 \\
18,600 \\
19,800\end{array}$ & $\begin{array}{l}6.60 \\
6.66 \\
6.72\end{array}$ \\
\hline $\begin{array}{l}04-07 \\
04-07\end{array}$ & $\begin{array}{l}1200 \\
2400\end{array}$ & $\begin{array}{l}12.13 \\
12.04\end{array}$ & $\begin{array}{l}2,290 \\
2,250\end{array}$ & $\begin{array}{l}5.27 \\
5.36\end{array}$ & $\begin{array}{l}04-12 \\
04-12 \\
04-12\end{array}$ & $\begin{array}{l}2200 \\
2300 \\
2400\end{array}$ & $\begin{array}{l}20.31 \\
20.48 \\
20.56\end{array}$ & $\begin{array}{l}21,000 \\
22,000 \\
22,600\end{array}$ & $\begin{array}{l}6.78 \\
6.85 \\
6.92\end{array}$ \\
\hline $\begin{array}{l}04-08 \\
04-08\end{array}$ & $\begin{array}{l}1200 \\
2400\end{array}$ & $\begin{array}{l}12.04 \\
14.08\end{array}$ & $\begin{array}{l}2,250 \\
3,390\end{array}$ & $\begin{array}{l}5.44 \\
5.55\end{array}$ & $\begin{array}{l}04-13 \\
04-13\end{array}$ & $\begin{array}{l}0100 \\
0200\end{array}$ & $\begin{array}{l}20.70 \\
20.84\end{array}$ & $\begin{array}{l}23,500 \\
24,400\end{array}$ & $\begin{array}{l}7.00 \\
7.07\end{array}$ \\
\hline $\begin{array}{l}04-09 \\
04-09\end{array}$ & $\begin{array}{l}1100 \\
2400\end{array}$ & $\begin{array}{l}15.27 \\
14.21\end{array}$ & $\begin{array}{l}4,380 \\
3,480\end{array}$ & $\begin{array}{l}5.69 \\
5.85\end{array}$ & $\begin{array}{l}04-13 \\
04-13 \\
04-13\end{array}$ & $\begin{array}{l}0300 \\
0400 \\
0600\end{array}$ & $\begin{array}{l}20.91 \\
21.04 \\
21.13\end{array}$ & $\begin{array}{l}24,900 \\
25,800 \\
26,500\end{array}$ & $\begin{array}{l}7.15 \\
7.2: 3 \\
7.40\end{array}$ \\
\hline $\begin{array}{l}04-10 \\
04-10 \\
04-10 \\
04-10 \\
04-10\end{array}$ & $\begin{array}{l}0100 \\
0300 \\
0600 \\
0900 \\
1200\end{array}$ & $\begin{array}{l}14.09 \\
13.84 \\
13.48 \\
13.14 \\
12.83\end{array}$ & $\begin{array}{l}3,390 \\
3,220 \\
2,990 \\
2,790 \\
2,620\end{array}$ & $\begin{array}{l}5.86 \\
5.813 \\
5.91 \\
5.94 \\
5.97\end{array}$ & $\begin{array}{l}04-13 \\
04-13 \\
04-13 \\
04-13 \\
04-13\end{array}$ & $\begin{array}{l}0800 \\
1100 \\
1400 \\
1900 \\
2400\end{array}$ & $\begin{array}{l}21.23 \\
21.30 \\
21.41 \\
21.59 \\
21.86\end{array}$ & $\begin{array}{l}27,200 \\
27,800 \\
28,600 \\
30,100 \\
32,300\end{array}$ & $\begin{array}{l}7.57 \\
7.34 \\
8.11 \\
8.513 \\
9.013\end{array}$ \\
\hline $\begin{array}{l}04-10 \\
04-10 \\
04-10\end{array}$ & $\begin{array}{l}1600 \\
2100 \\
2400\end{array}$ & $\begin{array}{l}12.49 \\
12.15 \\
11.97\end{array}$ & $\begin{array}{l}2,450 \\
2,300 \\
2,220\end{array}$ & $\begin{array}{l}6.00 \\
6.04 \\
6.06\end{array}$ & $\begin{array}{l}04-14 \\
04-14 \\
04-14\end{array}$ & $\begin{array}{l}0400 \\
0700 \\
0700\end{array}$ & $\begin{array}{l}22.08 \\
22.30 \\
22.48\end{array}$ & $\begin{array}{l}34,300 \\
36,300 \\
38,000\end{array}$ & $\begin{array}{r}9.51 \\
9.84 \\
10.01\end{array}$ \\
\hline $\begin{array}{l}04-11 \\
04-11 \\
04-11\end{array}$ & $\begin{array}{l}0600 \\
1200 \\
2400\end{array}$ & $\begin{array}{l}11.68 \\
11.42 \\
11.31\end{array}$ & $\begin{array}{l}2,100 \\
2,000 \\
1,960\end{array}$ & $\begin{array}{l}6.10 \\
6.14 \\
6.22\end{array}$ & $\begin{array}{l}04-14 \\
04-14 \\
04-14 \\
04-14 \\
04-14 \\
04-14\end{array}$ & $\begin{array}{l}1000 \\
1300 \\
1700 \\
2100 \\
2300 \\
2400\end{array}$ & $\begin{array}{l}22.53 \\
22.74 \\
23.02 \\
23.22 \\
23.26 \\
23.25\end{array}$ & $\begin{array}{l}38,500 \\
40,700 \\
43,700 \\
45,900 \\
46,400 \\
46,200\end{array}$ & $\begin{array}{l}10.20 \\
10.57 \\
11.13 \\
11.70 \\
12.00 \\
12.14\end{array}$ \\
\hline
\end{tabular}


TABLE 7.-Gage height, discharge, and accumulated runoff, flood of April 1979-Continued 02484500, Yockanookany River near Ofahoma, Miss.-Continued

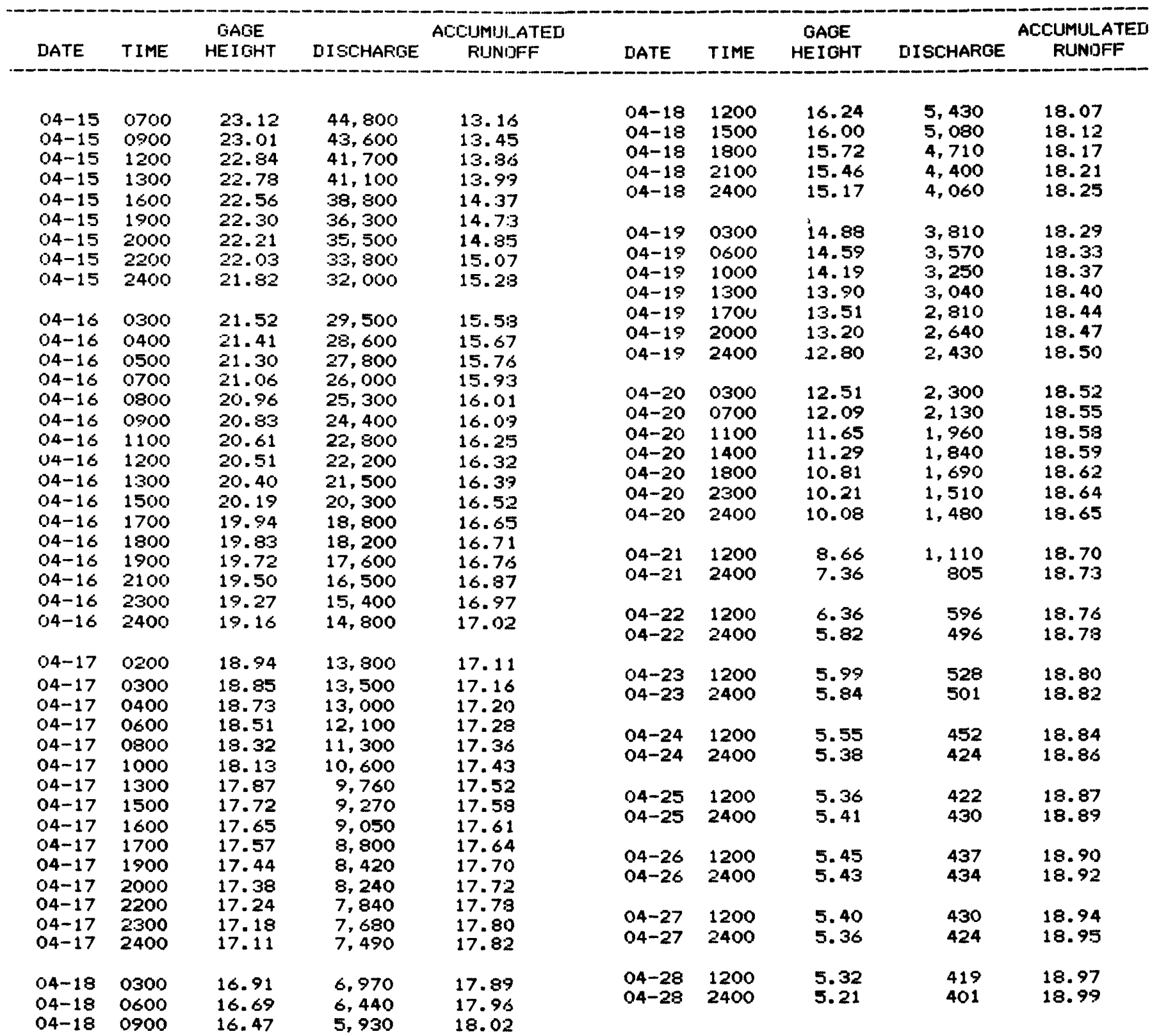


TABLE 7.-Gage height, discharge, and accumulated runoff, flood of April 1979-Continued 02486000, Pearl River at Jackson, Miss.

\begin{tabular}{|c|c|c|c|c|c|c|c|c|c|}
\hline DATE & TIME & $\begin{array}{l}\text { GAGE } \\
\text { HEIGHT }\end{array}$ & DISCHARGE & $\begin{array}{c}\text { ACCUMUILATED } \\
\text { RUNIFF }\end{array}$ & DATE & TIME & $\begin{array}{l}\text { GAGEE } \\
\text { HEIGHT }\end{array}$ & DISCHARGE & $\begin{array}{l}\text { ACCUMMULATED } \\
\text { RUNDFF }\end{array}$ \\
\hline $\begin{array}{l}03-02 \\
03-02 \\
03-02\end{array}$ & $\begin{array}{l}0100 \\
1030 \\
2400\end{array}$ & $\begin{array}{l}31.67 \\
30.89 \\
31.30\end{array}$ & $\begin{array}{l}27,000 \\
24,600 \\
25,800\end{array}$ & $\begin{array}{l}0.00 \\
0.12 \\
0.29\end{array}$ & $\begin{array}{l}03-19 \\
03-19\end{array}$ & $\begin{array}{l}1200 \\
2400\end{array}$ & $\begin{array}{l}11.81 \\
10.75\end{array}$ & $\begin{array}{l}3,500 \\
3,010\end{array}$ & $\begin{array}{l}5.17 \\
5.21\end{array}$ \\
\hline $\begin{array}{l}03-03 \\
03-03\end{array}$ & $\begin{array}{l}1200 \\
2400\end{array}$ & $\begin{array}{l}32.27 \\
33.12\end{array}$ & $\begin{array}{l}29,000 \\
32,100\end{array}$ & $\begin{array}{l}0.45 \\
0.64\end{array}$ & $\begin{array}{l}03-20 \\
03-20\end{array}$ & $\begin{array}{l}1600 \\
2400\end{array}$ & $\begin{array}{r}10.12 \\
9.22\end{array}$ & $\begin{array}{l}2,720 \\
2,320\end{array}$ & $\begin{array}{l}5.23 \\
5.24\end{array}$ \\
\hline $03-04$ & 2400 & 33.57 & 33,800 & 1.03 & $03-21$ & 2400 & 8.71 & $2,0 \% 0$ & 5.27 \\
\hline $03-05$ & 2400 & 33.99 & 35,500 & 1.45 & $\begin{array}{l}03-22 \\
03-22\end{array}$ & $\begin{array}{l}1200 \\
2400\end{array}$ & $\begin{array}{r}8.83 \\
10.21\end{array}$ & $\begin{array}{l}2,150 \\
2,760\end{array}$ & $\begin{array}{l}5.23 \\
5.30\end{array}$ \\
\hline $\begin{array}{l}03-06 \\
03-06\end{array}$ & $\begin{array}{l}1600 \\
2400\end{array}$ & $\begin{array}{l}34.51 \\
34.33\end{array}$ & $\begin{array}{l}38,500 \\
37,400\end{array}$ & $\begin{array}{l}1.74 \\
1.87\end{array}$ & $\begin{array}{l}03-23 \\
03-23\end{array}$ & $\begin{array}{l}1200 \\
2400\end{array}$ & $\begin{array}{l}10.29 \\
12.88\end{array}$ & $\begin{array}{l}2,800 \\
4,010\end{array}$ & $\begin{array}{l}5.31 \\
5.33\end{array}$ \\
\hline $\begin{array}{l}03-07 \\
03-07\end{array}$ & $\begin{array}{l}0300 \\
2400\end{array}$ & $\begin{array}{l}34.22 \\
34.41\end{array}$ & $\begin{array}{l}36,800 \\
37,900\end{array}$ & $\begin{array}{l}1.95 \\
2.34\end{array}$ & $\begin{array}{l}03-24 \\
03-24\end{array}$ & $\begin{array}{l}1200 \\
2400\end{array}$ & $\begin{array}{l}13.69 \\
15.65\end{array}$ & $\begin{array}{l}4,400 \\
5,340\end{array}$ & $\begin{array}{l}5.36 \\
5.39\end{array}$ \\
\hline $\begin{array}{l}03-08 \\
03-08\end{array}$ & $\begin{array}{l}1600 \\
2400\end{array}$ & $\begin{array}{l}34.63 \\
34.58\end{array}$ & $\begin{array}{l}39,200 \\
38,700\end{array}$ & $\begin{array}{l}2.65 \\
2.81\end{array}$ & $03-25$ & 2400 & 16.83 & 5,920 & 5.46 \\
\hline $03-09$ & 2400 & 34.66 & 39,400 & 3.23 & $\begin{array}{l}03-26 \\
03-26\end{array}$ & $\begin{array}{l}0900 \\
2400\end{array}$ & $\begin{array}{l}17.00 \\
15.10\end{array}$ & $\begin{array}{l}6,000 \\
5,080\end{array}$ & $\begin{array}{l}5.4: 3 \\
5.52\end{array}$ \\
\hline $03-10$ & 2400 & 34.40 & 37,800 & 3.74 & $03-27$ & 2400 & 13.81 & 4,460 & $5.5: 3$ \\
\hline $03-11$ & 2400 & 32.11 & 28,400 & 4.14 & & & & & \\
\hline $\begin{array}{l}03-12 \\
03-12 \\
03-13\end{array}$ & $\begin{array}{l}1200 \\
2400 \\
2400\end{array}$ & $\begin{array}{l}30.31 \\
28.87 \\
26.97\end{array}$ & $\begin{array}{l}22,900 \\
18,900 \\
15,300\end{array}$ & $\begin{array}{l}4.27 \\
4.42 \\
4.62\end{array}$ & $\begin{array}{l}03-28 \\
03-29 \\
03-29\end{array}$ & $\begin{array}{l}2400 \\
1200 \\
2400\end{array}$ & $\begin{array}{l}13.61 \\
13.46 \\
12.66\end{array}$ & $\begin{array}{l}4,360 \\
4,290 \\
3,900\end{array}$ & $\begin{array}{l}5.63 \\
5.66 \\
5.69\end{array}$ \\
\hline $\begin{array}{l}03-14 \\
03-14\end{array}$ & $\begin{array}{l}1200 \\
2400\end{array}$ & $\begin{array}{l}26.17 \\
25.37\end{array}$ & $\begin{array}{l}14,100 \\
13,100\end{array}$ & $\begin{array}{l}4.71 \\
4.79\end{array}$ & $\begin{array}{l}03-30 \\
03-31\end{array}$ & $\begin{array}{l}2400 \\
2400\end{array}$ & $\begin{array}{c}11.06 \\
9.72\end{array}$ & $\begin{array}{l}3,150 \\
2,540\end{array}$ & $\begin{array}{l}5.73 \\
5.76\end{array}$ \\
\hline $\begin{array}{l}03-15 \\
03-15\end{array}$ & $\begin{array}{l}1200 \\
2400\end{array}$ & $\begin{array}{l}24.37 \\
22.86\end{array}$ & $\begin{array}{l}13,100 \\
10,300\end{array}$ & $\begin{array}{l}4.87 \\
4.94\end{array}$ & $\begin{array}{l}04-01 \\
04-01\end{array}$ & $\begin{array}{l}1200 \\
2400\end{array}$ & $\begin{array}{l}8.79 \\
7.19\end{array}$ & $\begin{array}{l}2,130 \\
1,450\end{array}$ & $\begin{array}{l}5.78 \\
5.79\end{array}$ \\
\hline $03-16$ & 2400 & 19.21 & 7,310 & 5.05 & $\begin{array}{l}04-02 \\
04-02\end{array}$ & $\begin{array}{l}1200 \\
2400\end{array}$ & $\begin{array}{l}7.73 \\
9.01\end{array}$ & $\begin{array}{l}1,680 \\
2,220\end{array}$ & $\begin{array}{l}5.80 \\
5.81\end{array}$ \\
\hline $\begin{array}{l}03-17 \\
03-17 \\
03-17\end{array}$ & $\begin{array}{l}0800 \\
1600 \\
2400\end{array}$ & $\begin{array}{l}18.11 \\
16.73 \\
14.85\end{array}$ & $\begin{array}{l}6,570 \\
5,870 \\
4,960\end{array}$ & $\begin{array}{l}5.07 \\
5.10 \\
5.12\end{array}$ & $\begin{array}{l}04-03 \\
04-03\end{array}$ & $\begin{array}{l}1200 \\
2400\end{array}$ & $\begin{array}{l}13.14 \\
15.91\end{array}$ & $\begin{array}{l}4,130 \\
5,470\end{array}$ & $\begin{array}{l}5.83 \\
5.36\end{array}$ \\
\hline $\begin{array}{l}03-18 \\
03-18\end{array}$ & $\begin{array}{l}1200 \\
2400\end{array}$ & $\begin{array}{l}12.72 \\
12.13\end{array}$ & $\begin{array}{l}3,930 \\
3,650\end{array}$ & $\begin{array}{l}5.15 \\
5.17\end{array}$ & $\begin{array}{l}04-04 \\
04-04\end{array}$ & $\begin{array}{l}1200 \\
2400\end{array}$ & $\begin{array}{l}18.86 \\
21.17\end{array}$ & $\begin{array}{l}7,070 \\
8,740\end{array}$ & $\begin{array}{l}5.89 \\
5.94\end{array}$ \\
\hline
\end{tabular}


TABLE 7.-Gage height, discharge, and accumulated runoff, flood of April 1979-Continued 02486000, Pearl River at Jackson, Miss.-Continued

\begin{tabular}{|c|c|c|c|c|c|c|c|c|c|}
\hline DATE & TIME & $\begin{array}{l}\text { GSACIE } \\
\text { HEIGHT }\end{array}$ & DISCHARGE & $\begin{array}{c}\text { ACCUMIUL_ATED } \\
\text { RUINOFF }\end{array}$ & DATE & TIME & $\begin{array}{l}\text { GAGE } \\
\text { HEIGHT }\end{array}$ & DISCHARGE & $\begin{array}{l}\text { ACCIUMUILATED } \\
\text { RLINIJFF }\end{array}$ \\
\hline $04-05$ & 1200 & 23.62 & 11,000 & 6.00 & $04-17$ & 0300 & 43.07 & 125,000 & 11.79 \\
\hline $04-0.5$ & 2400 & 25.53 & 13,300 & 6.07 & $\begin{array}{l}04-17 \\
04-17\end{array}$ & $\begin{array}{l}0600 \\
0900\end{array}$ & $\begin{array}{l}43.16 \\
43.23\end{array}$ & $\begin{array}{l}126,000 \\
127,000\end{array}$ & $\begin{array}{l}11.93 \\
12.17\end{array}$ \\
\hline $04-06$ & 1200 & 26.67 & 14,800 & 6.16 & $04-17$ & 1200 & 43.28 & 128,000 & 12.36 \\
\hline $04-06$ & 2400 & 26.79 & 15,000 & 6.25 & $\begin{array}{l}04-17 \\
04-17\end{array}$ & $\begin{array}{l}1500 \\
1800\end{array}$ & $\begin{array}{l}43.28 \\
43.22\end{array}$ & $\begin{array}{l}128,000 \\
127,000\end{array}$ & $\begin{array}{l}12.55 \\
12.74\end{array}$ \\
\hline $04-07$ & 2400 & 26.15 & 14,100 & 6.42 & $04-17$ & 2400 & 42.98 & 125,000 & 13.12 \\
\hline $04-08$ & 1400 & 25.78 & 13,600 & 6.52 & $04-18$ & 0800 & 42.60 & 118,000 & $13.4: 3$ \\
\hline $04-08$ & 2400 & --- & 14,000 & 6.59 & $\begin{array}{l}04-13 \\
04-18\end{array}$ & $\begin{array}{l}1200 \\
1800\end{array}$ & $\begin{array}{l}42.16 \\
41.70\end{array}$ & $\begin{array}{l}112,000 \\
106,000\end{array}$ & $\begin{array}{l}13.82 \\
14.15\end{array}$ \\
\hline $04-09$ & 2400 & $-\cdots$ & 15,500 & 6.76 & $04-18$ & 2400 & 41.31 & 102,000 & 14.46 \\
\hline $\begin{array}{l}04-10 \\
04-10\end{array}$ & $\begin{array}{l}1700 \\
2400\end{array}$ & $\begin{array}{l}27.64 \\
27.78\end{array}$ & $\begin{array}{l}16,300 \\
16,500\end{array}$ & $\begin{array}{l}6.90 \\
6.90\end{array}$ & $\begin{array}{l}04-19 \\
04-19\end{array}$ & $\begin{array}{l}0600 \\
1200\end{array}$ & $\begin{array}{l}40.94 \\
40.54\end{array}$ & $\begin{array}{l}97,300 \\
92,800\end{array}$ & $\begin{array}{l}14.76 \\
15.05\end{array}$ \\
\hline $\begin{array}{l}04-11 \\
04-11\end{array}$ & $\begin{array}{l}2300 \\
2400\end{array}$ & $\begin{array}{l}27.61 \\
28.44\end{array}$ & $\begin{array}{l}16,200 \\
17,900\end{array}$ & $\begin{array}{l}7.14 \\
7.15\end{array}$ & $\begin{array}{l}04-19 \\
04-19\end{array}$ & $\begin{array}{l}1800 \\
2400\end{array}$ & $\begin{array}{l}39.89 \\
39.11\end{array}$ & $\begin{array}{l}35,700 \\
77,100\end{array}$ & $\begin{array}{l}15.31 \\
15.56\end{array}$ \\
\hline $04-12$ & 0600 & 30.05 & 22,100 & 7.21 & $\begin{array}{l}04-20 \\
04-20\end{array}$ & $\begin{array}{l}0800 \\
1600\end{array}$ & $\begin{array}{l}38.15 \\
37.28\end{array}$ & $\begin{array}{l}67,400 \\
59,200\end{array}$ & $\begin{array}{l}15.35 \\
16.10\end{array}$ \\
\hline $\begin{array}{l}04-12 \\
04-12\end{array}$ & $\begin{array}{l}1200 \\
1800\end{array}$ & $\begin{array}{l}31.10 \\
32.98\end{array}$ & $\begin{array}{l}25,200 \\
31,500\end{array}$ & $\begin{array}{l}7.23 \\
7.37\end{array}$ & $04-20$ & 2400 & 36.16 & 49,700 & 16.32 \\
\hline $04-12$ & 2400 & 33.93 & 35,200 & 7.47 & $\begin{array}{l}04-21 \\
04-21\end{array}$ & $\begin{array}{l}0800 \\
1600\end{array}$ & $\begin{array}{l}35.27 \\
34.57\end{array}$ & $\begin{array}{l}43,400 \\
38,900\end{array}$ & $\begin{array}{l}16.50 \\
16.67\end{array}$ \\
\hline $\begin{array}{l}04-13 \\
04-13\end{array}$ & $\begin{array}{l}0600 \\
1200\end{array}$ & $\begin{array}{l}35.05 \\
35.59\end{array}$ & $\begin{array}{l}41,900 \\
45,600\end{array}$ & $\begin{array}{l}7.513 \\
7.72\end{array}$ & $04-21$ & 2400 & 33.55 & 33,700 & 16.81 \\
\hline $\begin{array}{l}04-13 \\
04-13\end{array}$ & $\begin{array}{l}1800 \\
2400\end{array}$ & $\begin{array}{l}35.97 \\
36.56\end{array}$ & $\begin{array}{l}48,300 \\
53,000\end{array}$ & $\begin{array}{l}7.86 \\
8.01\end{array}$ & $\begin{array}{l}04-22 \\
04-22 \\
04-22\end{array}$ & $\begin{array}{l}1200 \\
2100 \\
2400\end{array}$ & $\begin{array}{l}32.19 \\
31.79 \\
31.96\end{array}$ & $\begin{array}{l}28,700 \\
27,400 \\
27,900\end{array}$ & $\begin{array}{l}17.00 \\
17.13 \\
17.17\end{array}$ \\
\hline $\begin{array}{l}04-14 \\
04-14\end{array}$ & 0600 & 37.64 & 62,500 & 6.13 & & & & & \\
\hline $04-14$ & 1200 & 38.90 & 74,900 & 8.39 & $04-23$ & 1200 & 30.58 & 23,600 & 17.32 \\
\hline $\begin{array}{l}04-14 \\
04-14\end{array}$ & $\begin{array}{l}1800 \\
2400\end{array}$ & $\begin{array}{l}39.67 \\
40.42\end{array}$ & $\begin{array}{l}83,200 \\
91,500\end{array}$ & $\begin{array}{l}8.63 \\
8.89\end{array}$ & $04-23$ & 2400 & 29.32 & 20,100 & 17.45 \\
\hline $\begin{array}{l}04-15 \\
04-15\end{array}$ & $\begin{array}{l}0600 \\
1200\end{array}$ & $\begin{array}{l}41.20 \\
41.83\end{array}$ & $\begin{array}{l}100,000 \\
108,000\end{array}$ & $\begin{array}{l}9.17 \\
9.49\end{array}$ & $\begin{array}{l}04-24 \\
04-24\end{array}$ & $\begin{array}{l}1200 \\
2400\end{array}$ & $\begin{array}{l}25.51 \\
27.51\end{array}$ & $\begin{array}{l}18,000 \\
16,100\end{array}$ & $\begin{array}{l}17.57 \\
17.67\end{array}$ \\
\hline $\begin{array}{l}04-15 \\
04-15\end{array}$ & $\begin{array}{l}1800 \\
2400\end{array}$ & $\begin{array}{l}42.25 \\
42.49\end{array}$ & $\begin{array}{l}113,000 \\
116,000\end{array}$ & $\begin{array}{r}9.82 \\
10.16\end{array}$ & $\begin{array}{l}04-25 \\
04-25\end{array}$ & $\begin{array}{l}1200 \\
2400\end{array}$ & $\begin{array}{l}26.50 \\
25.07\end{array}$ & $\begin{array}{l}14,600 \\
12,700\end{array}$ & $\begin{array}{l}17.76 \\
17.85\end{array}$ \\
\hline $\begin{array}{l}04-16 \\
04-16 \\
04-16\end{array}$ & $\begin{array}{l}0600 \\
1200 \\
1800\end{array}$ & $\begin{array}{l}42.62 \\
42.78 \\
42.85\end{array}$ & $\begin{array}{l}118,000 \\
121,000 \\
122,000\end{array}$ & $\begin{array}{l}10.51 \\
10.87 \\
11.24\end{array}$ & $\begin{array}{l}04-26 \\
04-26\end{array}$ & $\begin{array}{l}1200 \\
2400\end{array}$ & $\begin{array}{l}23.56 \\
21.63\end{array}$ & $\begin{array}{r}11,000 \\
9,100\end{array}$ & $\begin{array}{l}17.92 \\
17.9: 3\end{array}$ \\
\hline $04-16$ & 2400 & 42.95 & 123,000 & 11.60 & $\begin{array}{l}04-27 \\
04-27\end{array}$ & $\begin{array}{l}1200 \\
2400\end{array}$ & $\begin{array}{l}19.76 \\
17.32\end{array}$ & $\begin{array}{l}7,680 \\
6,160\end{array}$ & $\begin{array}{l}18.03 \\
18.07\end{array}$ \\
\hline & & & & & $\begin{array}{l}04-28 \\
04-28\end{array}$ & $\begin{array}{l}1200 \\
2400\end{array}$ & $\begin{array}{l}15.08 \\
13.31\end{array}$ & $\begin{array}{l}5,070 \\
4,210\end{array}$ & $\begin{array}{l}18.10 \\
18.1: 3\end{array}$ \\
\hline
\end{tabular}


TABLE 7.-Gage height, discharge, and accumulated runoff, flood of April 1979-Continued 02488500, Pearl River at Monticello, Miss.

\begin{tabular}{|c|c|c|c|c|c|c|c|c|c|}
\hline DATE & TIME & $\begin{array}{l}\text { GAGE } \\
\text { HEIGHT }\end{array}$ & DISCHARGE & $\begin{array}{l}\text { ACCUMULATED } \\
\text { RIJNDFF }\end{array}$ & DATE & TIME & $\begin{array}{l}\text { GAGE } \\
\text { HEIGHT }\end{array}$ & DISCHARGE & $\begin{array}{l}\text { ACCUMILAATED } \\
\text { RUNOFF }\end{array}$ \\
\hline $04-01$ & 2400 & 11.19 & 6,330 & 0.00 & & & & & \\
\hline $\begin{array}{l}04-02 \\
04-02\end{array}$ & $\begin{array}{l}1200 \\
2400\end{array}$ & $\begin{array}{l}10.97 \\
12.50\end{array}$ & $\begin{array}{l}6,070 \\
7,950\end{array}$ & $\begin{array}{l}0.02 \\
0.04\end{array}$ & $\begin{array}{l}04-16 \\
04-16\end{array}$ & $\begin{array}{l}1200 \\
2400\end{array}$ & $\begin{array}{l}25.51 \\
26.66\end{array}$ & $\begin{array}{l}43,800 \\
52,200\end{array}$ & $\begin{array}{l}2.15 \\
2.33\end{array}$ \\
\hline $\begin{array}{l}04-03 \\
04-03\end{array}$ & $\begin{array}{l}1200 \\
2400\end{array}$ & $\begin{array}{l}15.77 \\
18.91\end{array}$ & $\begin{array}{l}12,400 \\
16,900\end{array}$ & $\begin{array}{l}0.013 \\
0.14\end{array}$ & $\begin{array}{l}04-17 \\
04-17\end{array}$ & $\begin{array}{l}1200 \\
2400\end{array}$ & $\begin{array}{l}28.23 \\
29.79\end{array}$ & $\begin{array}{l}64,700 \\
78,400\end{array}$ & $\begin{array}{l}2.54 \\
2.81\end{array}$ \\
\hline $\begin{array}{l}04-04 \\
04-04\end{array}$ & $\begin{array}{l}1200 \\
2400\end{array}$ & $\begin{array}{l}21.14 \\
21.77\end{array}$ & $\begin{array}{l}21,300 \\
22,800\end{array}$ & $\begin{array}{l}0.21 \\
0.29\end{array}$ & $\begin{array}{l}04-18 \\
04-18\end{array}$ & $\begin{array}{l}1200 \\
2400\end{array}$ & $\begin{array}{l}31.22 \\
32.41\end{array}$ & $\begin{array}{r}91,900 \\
104,000\end{array}$ & $\begin{array}{l}3.12 \\
3.49\end{array}$ \\
\hline $04-0.5$ & 2400 & 21.84 & 23,000 & 0.46 & $\begin{array}{l}04-19 \\
04-19\end{array}$ & $\begin{array}{l}1200 \\
2400\end{array}$ & $\begin{array}{l}33.40 \\
33.95\end{array}$ & $\begin{array}{l}114,000 \\
120,000\end{array}$ & $\begin{array}{l}3.38 \\
4.31\end{array}$ \\
\hline $04-06$ & 2400 & 21.00 & 21,000 & 0.62 & $\begin{array}{l}04-20 \\
04-20\end{array}$ & $\begin{array}{l}1200 \\
2400\end{array}$ & $\begin{array}{l}34.08 \\
33.87\end{array}$ & $\begin{array}{l}122,000 \\
120,000\end{array}$ & $\begin{array}{l}4.76 \\
5.21\end{array}$ \\
\hline $04-07$ & 2400 & 20.23 & 19,300 & 0.77 & $04-21$ & 2400 & 32.77 & 108,000 & 6.05 \\
\hline $04-08$ & 2400 & 19.94 & 18,700 & 0.91 & $04-22$ & 2400 & 31.56 & 95,300 & 6.80 \\
\hline $\begin{array}{l}04-09 \\
04-10\end{array}$ & 2400 & $\begin{array}{l}19.54 \\
19.23\end{array}$ & 17,900 & $\begin{array}{l}1.04 \\
1.17\end{array}$ & $04-23$ & 2400 & 30.37 & 83,700 & 7.46 \\
\hline $\begin{array}{l}04-10 \\
04-11\end{array}$ & $\begin{array}{l}2400 \\
2400\end{array}$ & $\begin{array}{l}19.23 \\
19.27\end{array}$ & $\begin{array}{l}17,400 \\
17,400\end{array}$ & $\begin{array}{l}1.17 \\
1.30\end{array}$ & $04-24$ & 2400 & 29.06 & 71,800 & 8.03 \\
\hline $04-12$ & 2400 & 19.77 & 18,300 & 1.43 & $\begin{array}{l}04-25 \\
04-26\end{array}$ & $\begin{array}{l}2400 \\
2400\end{array}$ & $\begin{array}{l}27.87 \\
26.85\end{array}$ & $\begin{array}{l}61,700 \\
53,600\end{array}$ & $\begin{array}{l}8.53 \\
8.95\end{array}$ \\
\hline $\begin{array}{l}04-13 \\
04-13\end{array}$ & $\begin{array}{l}1200 \\
2400\end{array}$ & $\begin{array}{l}20.42 \\
21.27\end{array}$ & $\begin{array}{l}19,700 \\
21,600\end{array}$ & $\begin{array}{l}1.50 \\
1.58\end{array}$ & $04-27$ & 2400 & 20.85 & $\begin{array}{l}33,600 \\
45,800\end{array}$ & 9.79 \\
\hline $\begin{array}{l}04-14 \\
04-14\end{array}$ & $\begin{array}{l}1200 \\
2400\end{array}$ & $\begin{array}{l}22.12 \\
22.93\end{array}$ & $\begin{array}{l}24,200 \\
27,500\end{array}$ & $\begin{array}{l}1.66 \\
1.76\end{array}$ & $04-28$ & 2400 & 24.32 & 35,800 & 9.62 \\
\hline $\begin{array}{l}04-15 \\
04-15\end{array}$ & $\begin{array}{l}1200 \\
2400\end{array}$ & $\begin{array}{l}23.78 \\
24.56\end{array}$ & $\begin{array}{l}32,400 \\
37,300\end{array}$ & $\begin{array}{l}1.87 \\
2.00\end{array}$ & $04-30$ & 2400 & $\begin{array}{l}21.49 \\
16.18\end{array}$ & $\begin{array}{l}22,000 \\
11,400\end{array}$ & 9.96 \\
\hline
\end{tabular}


TABLE 7.-Gage height, discharge, and accumulated runoff, flood of April 1979-Continued 02489500, Pearl River near Bogalusa, La.

\begin{tabular}{|c|c|c|c|c|c|c|c|c|c|}
\hline DATE & TIME & $\begin{array}{l}\text { GAGE } \\
\text { HEIGHT }\end{array}$ & DISCHARGE & $\begin{array}{l}\text { ACCUMIJI_ATED } \\
\text { RUNOFF }\end{array}$ & DATE & TIME & $\begin{array}{l}\text { GAGE } \\
\text { HEIGHT }\end{array}$ & DISCHARGE & $\begin{array}{l}\text { ACCUMIJLATED } \\
\text { RUNIOFF }\end{array}$ \\
\hline $04-02$ & 2400 & 14.36 & 8,280 & 0.00 & $04-19$ & 2400 & 19.67 & 40,200 & 2.94 \\
\hline $\begin{array}{l}04-03 \\
04-03 \\
04-03\end{array}$ & $\begin{array}{l}0600 \\
1200 \\
1800\end{array}$ & $\begin{array}{l}14.74 \\
15.83 \\
17.25\end{array}$ & $\begin{array}{r}8,930 \\
10,100 \\
15,000\end{array}$ & $\begin{array}{l}0.01 \\
0.02 \\
0.04\end{array}$ & $\begin{array}{l}04-20 \\
04-20\end{array}$ & $\begin{array}{l}1600 \\
2400\end{array}$ & $\begin{array}{l}19.90 \\
20.10\end{array}$ & $\begin{array}{l}45,000 \\
49,400\end{array}$ & $\begin{array}{l}3.10 \\
3.19\end{array}$ \\
\hline $\begin{array}{l}04-03 \\
04-0.3\end{array}$ & $\begin{array}{l}2100 \\
2400\end{array}$ & $\begin{array}{l}17.87 \\
18.55\end{array}$ & $\begin{array}{l}17,800 \\
22,900\end{array}$ & $\begin{array}{l}0.05 \\
0.06\end{array}$ & $\begin{array}{l}04-21 \\
04-21\end{array}$ & $\begin{array}{l}1200 \\
2400\end{array}$ & $\begin{array}{l}20.53 \\
21.23\end{array}$ & $\begin{array}{l}59,400 \\
76,400\end{array}$ & $\begin{array}{l}3.34 \\
3.53\end{array}$ \\
\hline $\begin{array}{l}04-04 \\
04-04 \\
04-04\end{array}$ & $\begin{array}{l}0600 \\
1400 \\
2000\end{array}$ & $\begin{array}{l}19.18 \\
19.67 \\
19.82\end{array}$ & $\begin{array}{l}31,200 \\
40,200 \\
43,300\end{array}$ & $\begin{array}{l}0.10 \\
0.17 \\
0.23\end{array}$ & $\begin{array}{l}04-22 \\
04-22\end{array}$ & $\begin{array}{l}1200 \\
2400\end{array}$ & $\begin{array}{l}21.97 \\
22.61\end{array}$ & $\begin{array}{r}95,000 \\
112,000\end{array}$ & $\begin{array}{l}3.77 \\
4.06\end{array}$ \\
\hline $04-04$ & 2400 & 19.85 & 44,000 & 0.27 & $04-23$ & 0600 & 22.80 & 117,000 & 4.22 \\
\hline $04-05$ & 2400 & 19.70 & 40,800 & 0.51 & $\begin{array}{l}04-23 \\
04-23\end{array}$ & $\begin{array}{l}1600 \\
2400\end{array}$ & $\begin{array}{l}23.15 \\
23.20\end{array}$ & $\begin{array}{l}127,000 \\
128,000\end{array}$ & $\begin{array}{l}4.51 \\
4.75\end{array}$ \\
\hline $\begin{array}{l}04-06 \\
04-07\end{array}$ & $\begin{array}{l}2400 \\
2400\end{array}$ & $\begin{array}{l}19.56 \\
19.44\end{array}$ & $\begin{array}{l}38,000 \\
35,800\end{array}$ & $\begin{array}{l}0.73 \\
0.93\end{array}$ & $\begin{array}{l}04-24 \\
04-24 \\
04-24\end{array}$ & $\begin{array}{l}0300 \\
1600 \\
2400\end{array}$ & $\begin{array}{l}23.23 \\
23.20 \\
23.04\end{array}$ & $\begin{array}{l}129,000 \\
128,000 \\
124,000\end{array}$ & $\begin{array}{l}4.84 \\
5.23 \\
5.46\end{array}$ \\
\hline $04-08$ & 2400 & 19.35 & 34,100 & $1.1: 3$ & $\begin{array}{l}04-25 \\
04-25\end{array}$ & $\begin{array}{l}1200 \\
2400\end{array}$ & $\begin{array}{l}22.75 \\
22.40\end{array}$ & $\begin{array}{l}116,000 \\
106,000\end{array}$ & $\begin{array}{l}5.80 \\
6.11\end{array}$ \\
\hline $04-09$ & 2400 & 19.25 & 32,400 & 1.32 & $04-26$ & 2400 & 21.60 & 85,700 & 6.65 \\
\hline $\begin{array}{l}04-10 \\
04-11\end{array}$ & $\begin{array}{l}2400 \\
2400\end{array}$ & $\begin{array}{l}19.10 \\
18.92\end{array}$ & $\begin{array}{l}29,900 \\
27,300\end{array}$ & $\begin{array}{l}1.47 \\
1.65\end{array}$ & $04-27$ & 2400 & 20.95 & 69,500 & 7.09 \\
\hline $04-12$ & 2400 & 18.80 & 25,700 & 1.80 & $04-28$ & 2400 & 20.57 & 60,400 & 7.45 \\
\hline $04-13$ & 2400 & 18.66 & 24,100 & 1.94 & $\begin{array}{l}04-29 \\
04-30\end{array}$ & $\begin{array}{l}2400 \\
2400\end{array}$ & $\begin{array}{l}20.32 \\
20.15\end{array}$ & $\begin{array}{l}54,500 \\
50,600\end{array}$ & $\begin{array}{l}7.77 \\
8.06\end{array}$ \\
\hline $04-14$ & 2400 & 18.65 & 24,000 & 2.013 & $05-01$ & 2400 & 19.97 & 46,500 & 8.34 \\
\hline $04-15$ & 2400 & 18.80 & 25,700 & 2.21 & $05-02$ & 2400 & 19.65 & 39,800 & 8.58 \\
\hline $04-16$ & 2400 & 19.01 & 28,600 & 2.37 & $05-03$ & 2400 & 18.52 & 22,600 & 8.75 \\
\hline $04-17$ & 2400 & 19.23 & 32,000 & 2.54 & $05-04$ & 2400 & 16.74 & 13,400 & 8.86 \\
\hline $04-18$ & 2400 & 19.46 & 36,100 & 2.73 & $05-05$ & 2400 & 16.29 & 12,200 & 8.93 \\
\hline
\end{tabular}


TABLE 7.-Gage height, discharge, and accumulated runoff, flood of April 1979-Continued 07289350, Big Black River at West, Miss.

\begin{tabular}{|c|c|c|c|c|c|c|c|c|c|}
\hline DATE & TIME & $\begin{array}{l}\text { GAGE } \\
\text { HEIGHT }\end{array}$ & IISCHARGE & $\begin{array}{l}\text { ACCIMYIJLATED } \\
\text { FIJNOFF }\end{array}$ & DATE & TIME & $\begin{array}{l}\text { GAGE } \\
\text { HEIGHT }\end{array}$ & DISCHARGE & $\begin{array}{l}\text { ACCIJMIJLATED } \\
\text { FIJNDFF }\end{array}$ \\
\hline $\begin{array}{l}04-01 \\
04-01\end{array}$ & $\begin{array}{l}0100 \\
2400\end{array}$ & $\begin{array}{l}7.58 \\
8.25\end{array}$ & $\begin{array}{l}798 \\
928\end{array}$ & $\begin{array}{l}0.00 \\
0.03\end{array}$ & $\begin{array}{l}04-12 \\
04-12 \\
04-12\end{array}$ & $\begin{array}{l}1500 \\
1800 \\
2400\end{array}$ & $\begin{array}{l}20.20 \\
21.68 \\
23.12\end{array}$ & $\begin{array}{l}13,800 \\
22,600 \\
35,200\end{array}$ & $\begin{array}{l}1.37 \\
1.46 \\
1.73\end{array}$ \\
\hline $\begin{array}{l}04-02 \\
04-02 \\
04-02\end{array}$ & $\begin{array}{l}1200 \\
1800 \\
2400\end{array}$ & $\begin{array}{l}10.57 \\
12.43 \\
13.45\end{array}$ & $\begin{array}{l}1,440 \\
1,920 \\
2,220\end{array}$ & $\begin{array}{l}0.05 \\
0.06 \\
0.08\end{array}$ & $\begin{array}{l}04-13 \\
04-13 \\
04-13\end{array}$ & $\begin{array}{l}0600 \\
1200 \\
1600\end{array}$ & $\begin{array}{l}23.89 \\
24.21 \\
24.27\end{array}$ & $\begin{array}{l}43,500 \\
47,200 \\
48,000\end{array}$ & $\begin{array}{l}2.10 \\
2.5: 3 \\
2.3: 3\end{array}$ \\
\hline $\begin{array}{l}04-03 \\
04-03 \\
04-03 \\
04-03\end{array}$ & $\begin{array}{l}0600 \\
1200 \\
1800 \\
2400\end{array}$ & $\begin{array}{l}14.28 \\
14.77 \\
15.06 \\
15.35\end{array}$ & $\begin{array}{l}2,470 \\
2,630 \\
2,730 \\
2,850\end{array}$ & $\begin{array}{l}0.11 \\
0.13 \\
0.16 \\
0.13\end{array}$ & $\begin{array}{l}04-13 \\
04-13 \\
04-13\end{array}$ & $\begin{array}{l}1800 \\
2030 \\
2400\end{array}$ & $\begin{array}{l}24.27 \\
24.20 \\
24.14\end{array}$ & $\begin{array}{l}48,000 \\
45,400 \\
46,400\end{array}$ & $\begin{array}{l}2.93 \\
3.17 \\
3.42\end{array}$ \\
\hline $\begin{array}{l}04-04 \\
04-04\end{array}$ & $\begin{array}{l}1200 \\
2400\end{array}$ & $\begin{array}{l}15.91 \\
16.41\end{array}$ & $\begin{array}{l}3,070 \\
3,330\end{array}$ & $\begin{array}{l}0.24 \\
0.30\end{array}$ & $\begin{array}{l}04-14 \\
04-14 \\
04-14 \\
04-14\end{array}$ & $\begin{array}{l}0600 \\
1200 \\
1800 \\
2400\end{array}$ & $\begin{array}{l}23.79 \\
23.42 \\
23.08 \\
22.71\end{array}$ & $\begin{array}{l}42,400 \\
38,300 \\
34,800 \\
31,100\end{array}$ & $\begin{array}{l}3.34 \\
4.22 \\
4.56 \\
4.37\end{array}$ \\
\hline $\begin{array}{l}04-05 \\
04-05\end{array}$ & $\begin{array}{l}1200 \\
2400\end{array}$ & $\begin{array}{l}16.97 \\
17.36\end{array}$ & $\begin{array}{l}3,730 \\
4,230\end{array}$ & $\begin{array}{l}0.36 \\
0.44\end{array}$ & $\begin{array}{l}04-15 \\
04-15\end{array}$ & $\begin{array}{l}1200 \\
2400\end{array}$ & $\begin{array}{l}21.93 \\
20.99\end{array}$ & $\begin{array}{l}24,400 \\
18,000\end{array}$ & $\begin{array}{l}5.40 \\
5.30\end{array}$ \\
\hline $\begin{array}{l}04-06 \\
04-06 \\
04-06\end{array}$ & $\begin{array}{l}1200 \\
1400 \\
2400\end{array}$ & $\begin{array}{l}17.47 \\
17.49 \\
17.48\end{array}$ & $\begin{array}{l}4,420 \\
4,450 \\
4,440\end{array}$ & $\begin{array}{l}0.52 \\
0.54 \\
0.61\end{array}$ & $04-16$ & 2400 & 19.53 & 10,700 & 6.34 \\
\hline $04-07$ & 2400 & 17.09 & 3,850 & 0.76 & $04-17$ & 2400 & 18.38 & 6,670 & 6.67 \\
\hline $04-08$ & 2400 & 16.70 & 3,520 & 0.90 & $04-18$ & 2400 & 17.20 & 3,970 & 6.87 \\
\hline $04-09$ & 2400 & 16.23 & 3,230 & 1.03 & $04-19$ & 2400 & 15.83 & 3,040 & 7.00 \\
\hline $04-10$ & 2400 & 15.11 & 2,750 & 1.14 & $\begin{array}{l}04-20 \\
04-20\end{array}$ & $\begin{array}{l}1200 \\
2400\end{array}$ & $\begin{array}{l}14.26 \\
11.39\end{array}$ & $\begin{array}{l}2,460 \\
1,620\end{array}$ & $\begin{array}{l}7.05 \\
7.09\end{array}$ \\
\hline $04-11$ & 2400 & 14.50 & 2,540 & 1.24 & $\begin{array}{l}04-21 \\
04-21\end{array}$ & $\begin{array}{l}1200 \\
2400\end{array}$ & $\begin{array}{l}8.76 \\
7.72\end{array}$ & $\begin{array}{l}990 \\
781\end{array}$ & $\begin{array}{l}7.12 \\
7.13\end{array}$ \\
\hline $\begin{array}{l}04-12 \\
04-12 \\
04-12\end{array}$ & $\begin{array}{l}0300 \\
0600 \\
1200\end{array}$ & $\begin{array}{l}15.91 \\
17.35 \\
18.44\end{array}$ & $\begin{array}{l}3,070 \\
4,210 \\
6,850\end{array}$ & $\begin{array}{l}1.25 \\
1.27 \\
1.32\end{array}$ & $\begin{array}{l}04-22 \\
04-22\end{array}$ & $\begin{array}{l}1200 \\
2400\end{array}$ & $\begin{array}{l}7.27 \\
7.22\end{array}$ & $\begin{array}{l}696 \\
687\end{array}$ & $\begin{array}{l}7.15 \\
7.16\end{array}$ \\
\hline
\end{tabular}


TABLE 7.-Gage height, discharge, and accumulated runoff, flood of April 1979-Continued 07290000, Big Black River near Bovina, Miss.

\begin{tabular}{|c|c|c|c|c|c|c|c|c|c|}
\hline DATE & TIME & $\begin{array}{l}\text { GAGE } \\
\text { HEIGHT }\end{array}$ & DISCHARGE & $\begin{array}{c}\text { ACCUMIU_ATED } \\
\text { RUMOFF }\end{array}$ & DATE & TIME & $\begin{array}{l}\text { GAGE } \\
\text { HEIGHT }\end{array}$ & DISCHARGE & $\begin{array}{l}\text { ACCUMULATED } \\
\text { RUNDFF }\end{array}$ \\
\hline $04-02$ & 2400 & 15.13 & 3,580 & 0.00 & & & & & \\
\hline $\begin{array}{l}04-03 \\
04-03\end{array}$ & $\begin{array}{l}1200 \\
2400\end{array}$ & $\begin{array}{l}15.77 \\
18.30\end{array}$ & $\begin{array}{l}3,890 \\
5,150\end{array}$ & $\begin{array}{l}0.02 \\
0.0 .5\end{array}$ & $\begin{array}{l}04-16 \\
04-16 \\
04-16 \\
04-16\end{array}$ & $\begin{array}{l}0600 \\
1200 \\
1900 \\
2400\end{array}$ & $\begin{array}{l}40.42 \\
40.50 \\
40.56 \\
40.51\end{array}$ & $\begin{array}{l}80,200 \\
82,000 \\
83,300 \\
82,200\end{array}$ & $\begin{array}{l}3.00 \\
3.27 \\
3.59 \\
3.81\end{array}$ \\
\hline $04-04$ & 1200 & 22.10 & 7,310 & 0.09 & & & & & \\
\hline $04-04$ & 2400 & 23.06 & 7,890 & 0.14 & $\begin{array}{l}04-17 \\
04-17\end{array}$ & $\begin{array}{l}0600 \\
1100\end{array}$ & $\begin{array}{l}40.52 \\
40.44\end{array}$ & $\begin{array}{l}82,400 \\
80,700\end{array}$ & $\begin{array}{l}4.09 \\
4.31\end{array}$ \\
\hline $\begin{array}{l}04-05 \\
04-0.5\end{array}$ & $\begin{array}{l}0600 \\
2400\end{array}$ & $\begin{array}{l}23.12 \\
22.87\end{array}$ & $\begin{array}{l}7,920 \\
7,770\end{array}$ & $\begin{array}{l}0.17 \\
0.25\end{array}$ & $\begin{array}{l}04-17 \\
04-17\end{array}$ & $\begin{array}{l}1800 \\
2400\end{array}$ & $\begin{array}{l}40.32 \\
40.23\end{array}$ & $\begin{array}{l}78,000 \\
76,100\end{array}$ & $\begin{array}{l}4.62 \\
4.87\end{array}$ \\
\hline $04-06$ & 2400 & 22.46 & 7,530 & 0.35 & $\begin{array}{l}04-18 \\
04-18\end{array}$ & $\begin{array}{l}1200 \\
2400\end{array}$ & $\begin{array}{l}39.97 \\
39.67\end{array}$ & $\begin{array}{l}70,400 \\
64,100\end{array}$ & $\begin{array}{l}5.36 \\
5.80\end{array}$ \\
\hline $04-07$ & 2400 & 21.93 & 7,210 & 0.44 & & & & & \\
\hline $04-08$ & 2400 & 22.10 & 7,310 & 0.54 & $\begin{array}{l}04-19 \\
04-19\end{array}$ & $\begin{array}{l}1200 \\
2400\end{array}$ & $\begin{array}{l}39.33 \\
38.97\end{array}$ & $\begin{array}{l}56,900 \\
49,700\end{array}$ & $\begin{array}{l}6.20 \\
6.55\end{array}$ \\
\hline $\begin{array}{l}04-09 \\
04-09\end{array}$ & $\begin{array}{l}1200 \\
2400\end{array}$ & $\begin{array}{l}23.47 \\
24.06\end{array}$ & $\begin{array}{l}8,130 \\
8,490\end{array}$ & $\begin{array}{l}0.59 \\
0.65\end{array}$ & $04-20$ & 2400 & 38.32 & 36,900 & 7.13 \\
\hline $04-10$ & 2400 & 24.66 & 8,850 & $0.7 \mathrm{~s}$ & $04-21$ & 2400 & 37.48 & 28,400 & 7.56 \\
\hline $\begin{array}{l}04-11 \\
04-11\end{array}$ & $\begin{array}{l}1200 \\
2400\end{array}$ & $\begin{array}{l}24.95 \\
26.72\end{array}$ & $\begin{array}{r}9,020 \\
10,100\end{array}$ & $\begin{array}{l}0.32 \\
0.33\end{array}$ & $\begin{array}{l}04-22 \\
04-23\end{array}$ & $\begin{array}{l}2400 \\
2400\end{array}$ & $\begin{array}{l}36.33 \\
35.03\end{array}$ & $\begin{array}{l}24,000 \\
20,500\end{array}$ & $\begin{array}{l}7.91 \\
8.20\end{array}$ \\
\hline $\begin{array}{l}04-12 \\
04-12 \\
04-12\end{array}$ & $\begin{array}{l}0600 \\
1200 \\
1800\end{array}$ & $\begin{array}{l}28.59 \\
29.88 \\
32.84\end{array}$ & $\begin{array}{l}11,300 \\
12,400 \\
16,200\end{array}$ & $\begin{array}{l}0.92 \\
0.76 \\
1.01\end{array}$ & $\begin{array}{l}04-24 \\
04-24\end{array}$ & $\begin{array}{l}1200 \\
2400\end{array}$ & $\begin{array}{l}34.23 \\
33.37\end{array}$ & $\begin{array}{l}18,800 \\
17,100\end{array}$ & $\begin{array}{l}8.3: 3 \\
8.45\end{array}$ \\
\hline $04-12$ & 2400 & 34.10 & 18,500 & 1.05 & $\begin{array}{l}04-25 \\
04-25\end{array}$ & $\begin{array}{l}1200 \\
2400\end{array}$ & $\begin{array}{l}32.59 \\
31.89\end{array}$ & $\begin{array}{l}15,800 \\
14,700\end{array}$ & $\begin{array}{l}8.56 \\
8.615\end{array}$ \\
\hline $\begin{array}{l}04-13 \\
04-13 \\
04-13 \\
04-13\end{array}$ & $\begin{array}{l}0600 \\
1200 \\
1800 \\
2400\end{array}$ & $\begin{array}{l}34.64 \\
35.28 \\
35.80 \\
36.65\end{array}$ & $\begin{array}{l}19,600 \\
21,100 \\
22,500 \\
25,100\end{array}$ & $\begin{array}{l}1.13 \\
1.19 \\
1.27 \\
1.34\end{array}$ & $\begin{array}{l}04-26 \\
04-26\end{array}$ & $\begin{array}{l}1200 \\
2400\end{array}$ & $\begin{array}{l}31.23 \\
30.60\end{array}$ & $\begin{array}{l}13,800 \\
13,100\end{array}$ & $\begin{array}{l}8.75 \\
8.84\end{array}$ \\
\hline $\begin{array}{l}04-14 \\
04-14\end{array}$ & $\begin{array}{l}0600 \\
1200\end{array}$ & $\begin{array}{l}37.69 \\
38.42\end{array}$ & $\begin{array}{l}29,400 \\
38,700\end{array}$ & $\begin{array}{l}1.43 \\
1.55\end{array}$ & $\begin{array}{l}04-27 \\
04-27\end{array}$ & $\begin{array}{l}1200 \\
2400\end{array}$ & $\begin{array}{l}29.95 \\
29.26\end{array}$ & $\begin{array}{l}12,500 \\
11,800\end{array}$ & $\begin{array}{l}8.93 \\
9.01\end{array}$ \\
\hline $\begin{array}{l}04-14 \\
04-14\end{array}$ & $\begin{array}{l}1800 \\
2400\end{array}$ & $\begin{array}{l}38.85 \\
39.25\end{array}$ & $\begin{array}{l}47,300 \\
55,300\end{array}$ & $\begin{array}{l}1.69 \\
1.36\end{array}$ & $\begin{array}{l}04-28 \\
04-28\end{array}$ & $\begin{array}{l}1200 \\
2400\end{array}$ & $\begin{array}{l}28.56 \\
27.74\end{array}$ & $\begin{array}{l}11,300 \\
10,700\end{array}$ & $\begin{array}{l}9.09 \\
9.16\end{array}$ \\
\hline $\begin{array}{l}04-15 \\
04-15 \\
04-15 \\
04-15\end{array}$ & $\begin{array}{l}0600 \\
1200 \\
1800 \\
2400\end{array}$ & $\begin{array}{l}39.53 \\
39.79 \\
40.04\end{array}$ & $\begin{array}{l}61,100 \\
66,600 \\
71,900\end{array}$ & $\begin{array}{l}2.05 \\
2.26 \\
2.49\end{array}$ & $\begin{array}{l}04-29 \\
04-29\end{array}$ & $\begin{array}{l}1200 \\
2400\end{array}$ & $\begin{array}{l}26.32 \\
23.13\end{array}$ & $\begin{array}{l}9,760 \\
7,770\end{array}$ & $\begin{array}{l}9.23 \\
9.213\end{array}$ \\
\hline $04-15$ & 2400 & 40.25 & 76,500 & 2.74 & $\begin{array}{l}04-30 \\
04-30\end{array}$ & $\begin{array}{l}1200 \\
2400\end{array}$ & $\begin{array}{l}18.99 \\
15.79\end{array}$ & $\begin{array}{l}5,240 \\
3,580\end{array}$ & $\begin{array}{l}9.33 \\
9.36\end{array}$ \\
\hline
\end{tabular}


TABLE 8.-Ground-water levels in selected observation wells showing effects of flood of April 1979 in Alabama and Mississippi

\begin{tabular}{|c|c|c|c|c|c|}
\hline $\begin{array}{c}\text { Sice } \\
\text { Number }\end{array}$ & Location and Well Number & $\begin{array}{l}\text { Cased Depth } \\
\text { (feet) }\end{array}$ & $\begin{array}{l}\text { Well Depth } \\
\text { (feet) }\end{array}$ & $\begin{array}{l}\text { Formation and } \\
\text { Lithology }\end{array}$ & $\begin{array}{l}\text { Factors influencing } \\
\text { ground-water condition }\end{array}$ \\
\hline $1 \mathrm{~W}$ & B1bb County, AL 325622087075501 & 80 & 404 & $\mid \begin{array}{c}\text { Cambrian and ordoviclan } \\
\text { (dolomite) }\end{array}$ & $\begin{array}{l}\text { Inflleration and } \\
\text { loading from } \\
\text { Cahaba River }\end{array}$ \\
\hline $2 \mathrm{~W}$ & Elmore County, AL 323757086013901 & 63 & 402 & $\begin{array}{l}\text { Augen gniess } \\
\text { (metamorph1c rocks) }\end{array}$ & Local precipltation \\
\hline $3 \mathrm{w}$ & Greene County, AL 325005087532001 & 395 & 407 & Eutaw Formation (sand) & Local precipitation \\
\hline 5W & Hale County, AL 324205087352801 & 258 & 278 & Eutaw Formation (sand) & Local precipitation \\
\hline $6 \mathrm{~W}$ & $\begin{array}{l}\text { Jefferson County, AL } \\
\qquad 332605086523001\end{array}$ & 68 & 140 & $\begin{array}{l}\text { Bangor Limes tone } \\
\text { (limes tone) }\end{array}$ & Local precipitation \\
\hline $7 \mathrm{~W}$ & $\begin{array}{l}\text { Marengo County, AL } \\
\qquad 323055087504101\end{array}$ & 860 & 900 & Eutaw Formation (sand) & $\begin{array}{l}\text { Loading from } \\
\text { Tomblgbee River }\end{array}$ \\
\hline $8 \mathrm{~W}$ & $\begin{array}{l}\text { P1ckens County, AL } \\
330116088113101\end{array}$ & 71 & 91 & Eutaw Formation (sand) & $\begin{array}{l}\text { Loading fron } \\
\text { Tombigbee River }\end{array}$ \\
\hline $9 \mathrm{~W}$ & $\begin{array}{l}\text { Plckens County, AL } \\
330117088180301\end{array}$ & 117 & 137 & Eutaw Formation (sand) & $\begin{array}{l}\text { Loading from } \\
\text { Tombigbee River }\end{array}$ \\
\hline $11 \mathrm{~W}$ & Sumter County, AL 325212088121601 & 370 & 390 & Eutaw Formacton (sand) & $\begin{array}{l}\text { Loading from } \\
\text { Tombigbee River, } \\
1.35 \text { miles west of } \\
\text { River }\end{array}$ \\
\hline $12 \mathrm{~W}$ & Sumter Councy, AL 325215088111301 & 350 & 370 & Eutaw Fonnation (sand) & $\begin{array}{l}\text { Loading from } \\
\text { Tombigbee River, } \\
0.35 \text { mile west of } \\
\text { River }\end{array}$ \\
\hline $15 \mathrm{~W}$ & $\begin{array}{l}\text { Noxubee County, Miss. (near } \\
\text { P1ckensville, AL) } 331426088192202\end{array}$ & 20 & 30 & $\begin{array}{l}\text { Alluvial Deposits } \\
\text { (sand and grave } 1 \text { ) }\end{array}$ & $\begin{array}{l}\text { Local precipitacion } \\
\text { and recharge from } \\
\text { Tombigbee Rlver }\end{array}$ \\
\hline
\end{tabular}


TABLE 8.-Ground-water levels, flood of April 1979-Continued Site 1W (325622087075501), Well at Centreville, Ala. (Centreville Gin \& Cotton Co.)

[Water level, in feet, below land-surface datum at indicated time. 1979]

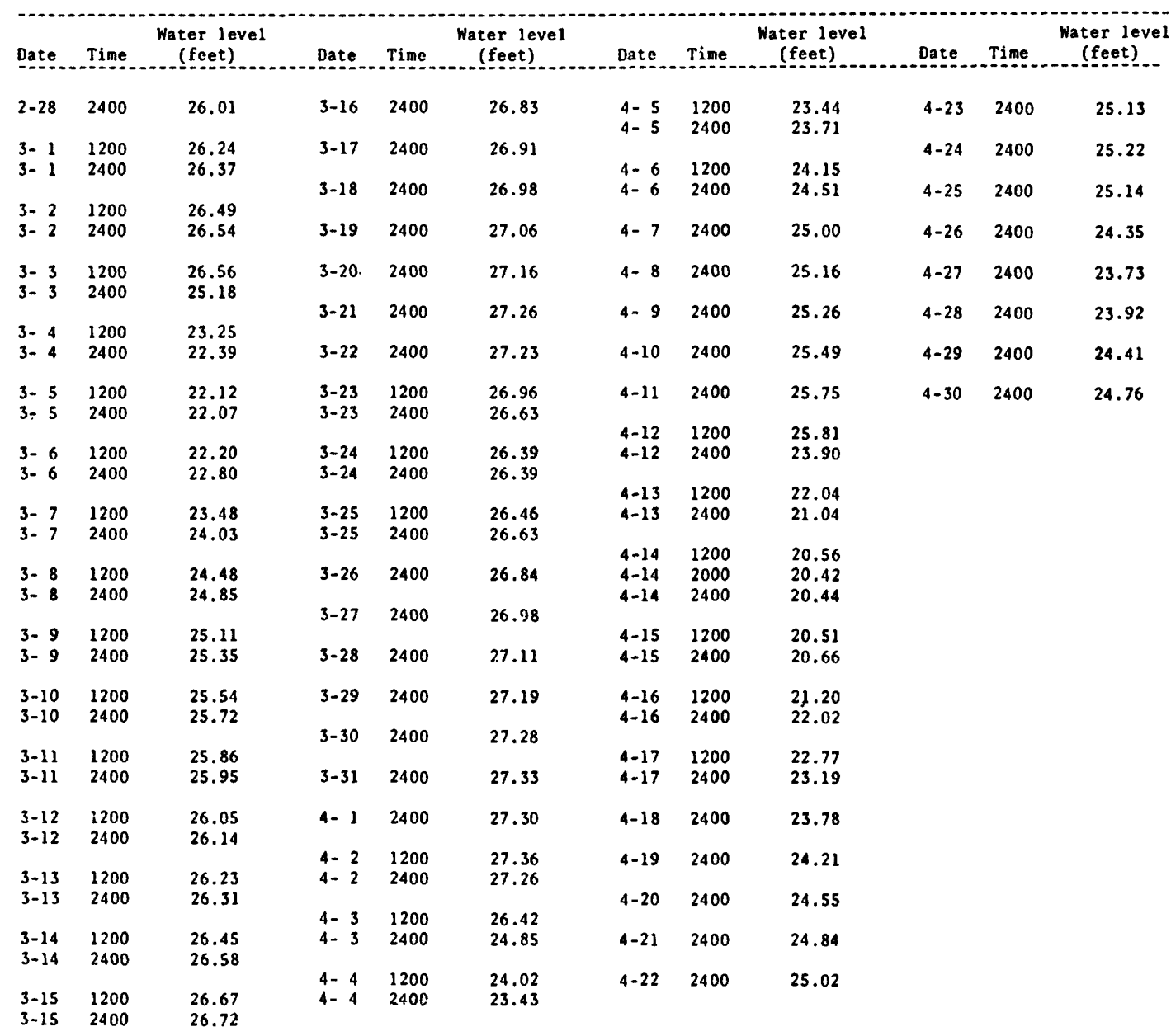


TABLE 8.-Ground-water levels, flood of April 1979-Continued Site 2W (323757086013901), Well at Elmore County High School, Eclectic, Ala.

[Water levels, in feet, below land surface datum (altitude 557.5 feet) at indicated time]

\begin{tabular}{|c|c|c|c|c|c|c|c|c|c|c|c|}
\hline Date & Time & $\begin{array}{l}\text { Water level } \\
\text { (feet) }\end{array}$ & Date & Time & $\begin{array}{l}\text { Water level } \\
\text { (feet) }\end{array}$ & Date & Time & $\begin{array}{c}\text { Water level } \\
\text { (feet) }\end{array}$ & Date & Time & $\begin{array}{l}\text { Water level } \\
\text { (feet) }\end{array}$ \\
\hline $2-28$ & 2400 & 8.20 & $3-15$ & 2400 & 7.96 & $3-30$ & 2400 & 8.01 & $4-14$ & 2400 & 7.37 \\
\hline $3-1$ & 2400 & 8.18 & $3-16$ & 2400 & 7.96 & $3-31$ & 2400 & 8.00 & $4-15$ & 2400 & 7.34 \\
\hline $3-2$ & 2400 & 8.15 & $3-17$ & 2400 & 7.94 & 4- 1 & 2400 & 7.99 & $4-16$ & 2400 & 7.35 \\
\hline $3-3$ & 2400 & 8.02 & $3-18$ & 2400 & 7.92 & 4- 2 & 2400 & 8.02 & $4-17$ & 2400 & 7.37 \\
\hline $3-4$ & 2400 & 7.97 & $3-19$ & 2400 & 7.94 & $4-3$ & 2400 & 7.81 & $4-18$ & 2400 & 7.39 \\
\hline $3-5$ & 2400 & 7.95 & $3-20$ & 2400 & 7.95 & 4- 4 & 2400 & 7.70 & $4-19$ & 2400 & 7.42 \\
\hline 3- 6 & 2400 & 7.92 & $3-21$ & 2400 & 7.98 & $4-5$ & 2400 & 7.73 & $4-20$ & 2400 & 7.47 \\
\hline 3- 7 & 2400 & 7.91 & $3-22$ & 2400 & 8.00 & $4-6$ & 2400 & 7.72 & $4-21$ & 2400 & 7.51 \\
\hline 3- 8 & 2400 & 7.97 & $3-23$ & 2400 & 7.94 & $4-7$ & 2400 & 7.70 & $4-22$ & 2400 & 7.55 \\
\hline 3- 9 & 2400 & 7.98 & $3-24$ & 2400 & 7.97 & $4-8$ & 2400 & 7.61 & $4-23$ & 2400 & 7.57 \\
\hline $3-10$ & 2400 & 7.97 & $3-25$ & 2400 & 8.02 & 4- 9 & 2400 & 7.64 & $4-24$ & 2400 & 7.51 \\
\hline $3-11$ & 2400 & 7.97 & $3-26$ & 2400 & 8.04 & $4-10$ & 2400 & 7.64 & $4-25$ & 2400 & 7.28 \\
\hline $3-12$ & 2400 & 7.96 & $3-27$ & 2400 & 8.04 & $4-11$ & 2400 & 7.64 & $4-26$ & 2400 & 7.23 \\
\hline $3-13$ & 2400 & 7.94 & $3-28$ & 2400 & 8.04 & $4-12$ & 2400 & 7.59 & $4-27$ & 2400 & 7.21 \\
\hline \multirow[t]{3}{*}{$3-14$} & 2400 & 7.97 & $3-29$ & 2400 & 8.02 & $4-13$ & 2400 & 7.41 & $4-28$ & 2400 & 7.21 \\
\hline & & & & & & & & & $4-29$ & 2400 & 7.21 \\
\hline & & & & & & & & & $4-30$ & 2400 & 7.23 \\
\hline
\end{tabular}


TABLE 8.-Ground-water levels, flood of April 1979-Continued

Site 3W (325005087532001), Well at Eutaw, Ala. (Greene County warehouse) [Water level, in feet, below land-surface datum (altitude 110.1 feet NGVD) at indicated time]

\begin{tabular}{|c|c|c|c|c|c|c|c|c|c|c|c|}
\hline Date & Time & $\begin{array}{c}\text { Water level } \\
\text { (feet) }\end{array}$ & Date & Time & $\begin{array}{c}\text { Water level } \\
\text { (feet) }\end{array}$ & Date & Time & $\begin{array}{c}\text { Water level } \\
\text { (feet) }\end{array}$ & Date & Time & $\begin{array}{c}\text { Water level } \\
\text { (feet) }\end{array}$ \\
\hline $2-28$ & 2400 & 44.12 & $3-15$ & 2400 & 43.53 & $3-31$ & 2400 & 43.71 & $4-15$ & 2400 & 43.63 \\
\hline $3-1$ & 2400 & 44.08 & $3-16$ & 2400 & 43.58 & $4-1$ & 2400 & 43.63 & $4-16$ & 2400 & 43.58 \\
\hline $3-2$ & 1200 & 44.07 & $3-17$ & 2400 & 43.60 & $4-2$ & 2400 & 43.63 & $4-17$ & 2400 & 43.58 \\
\hline $3-2$ & 2400 & 44.08 & $3-18$ & 2400 & 43.62 & $4-3$ & 2400 & 44.05 & $4-18$ & 1200 & 43.54 \\
\hline $3-3$ & 1200 & 43.91 & $3-19$ & 2400 & 43.66 & $4-4$ & 2400 & 44.18 & $4-18$ & 2400 & 43.57 \\
\hline $3-4$ & 2400 & 43.81 & $3-20$ & 2400 & 43.72 & $4-5$ & 2400 & 44.19 & $4-19$ & 2400 & 43.57 \\
\hline $3-5$ & 2400 & 43.83 & $3-21$ & 2400 & 43.73 & $4-6$ & 2400 & 44.14 & $4-20$ & 2400 & 43.54 \\
\hline $3-6$ & 1200 & 43.84 & $3-22$ & 2400 & 43.66 & $4-7$ & 2400 & 44.00 & $4-21$ & 2400 & 43.50 \\
\hline $3-7$ & 2400 & 43.80 & $3-23$ & 2400 & 43.58 & $4-8$ & 2400 & 43.77 & $4-22$ & 2400 & 43.42 \\
\hline $3-8$ & 2400 & 43.73 & $3-24$ & 2400 & 43.57 & $4-9$ & 2400 & 43.69 & $4-23$ & 2400 & 43.38 \\
\hline $3-9$ & 2400 & 43.72 & $3-25$ & 2400 & 43.57 & $4-10$ & 2400 & 43.69 & $4-24$ & 2400 & 43.39 \\
\hline $3-10$ & 2400 & 43.69 & $3-26$ & 2400 & 43.63 & $4-11$ & $\cdot 2400$ & 43.74 & $4-25$ & 2400 & 43.38 \\
\hline $3-11$ & 0500 & 43.68 & $3-27$ & 2400 & 43.65 & $4-12$ & 0700 & 43.76 & $4-26$ & 2400 & 43.37 \\
\hline $3-11$ & 2400 & 43.63 & $3-28$ & 2400 & 43.67 & $4-12$ & 2400 & 43.73 & $4-27$ & 2400 & 43.40 \\
\hline $3-12$ & 2400 & 43.58 & $3-29$ & 2400 & 43.70 & $4-13$ & 1400 & 43.59 & $4-28$ & 2400 & 43.40 \\
\hline $3-13$ & 2400 & 43.54 & $3-30$ & 2400 & 43.74 & $4-13$ & 2400 & 43.61 & $4-29$ & 0500 & 43.44 \\
\hline $3-14$ & 2400 & 43.51 & & & & $4-14$ & 2400 & 43.67 & $4-29$ & 2400 & 43.42 \\
\hline & & & & & & & & & $4-30$ & 2400 & 43.42 \\
\hline
\end{tabular}


TABLE 8.-Ground-water levels, flood of April 1979-Continued Site 5W (324205087352801), Well at City of Greensboro, Ala. [Water level, in feet, below land-surface datum (altitude 257 feet) at indicated time]

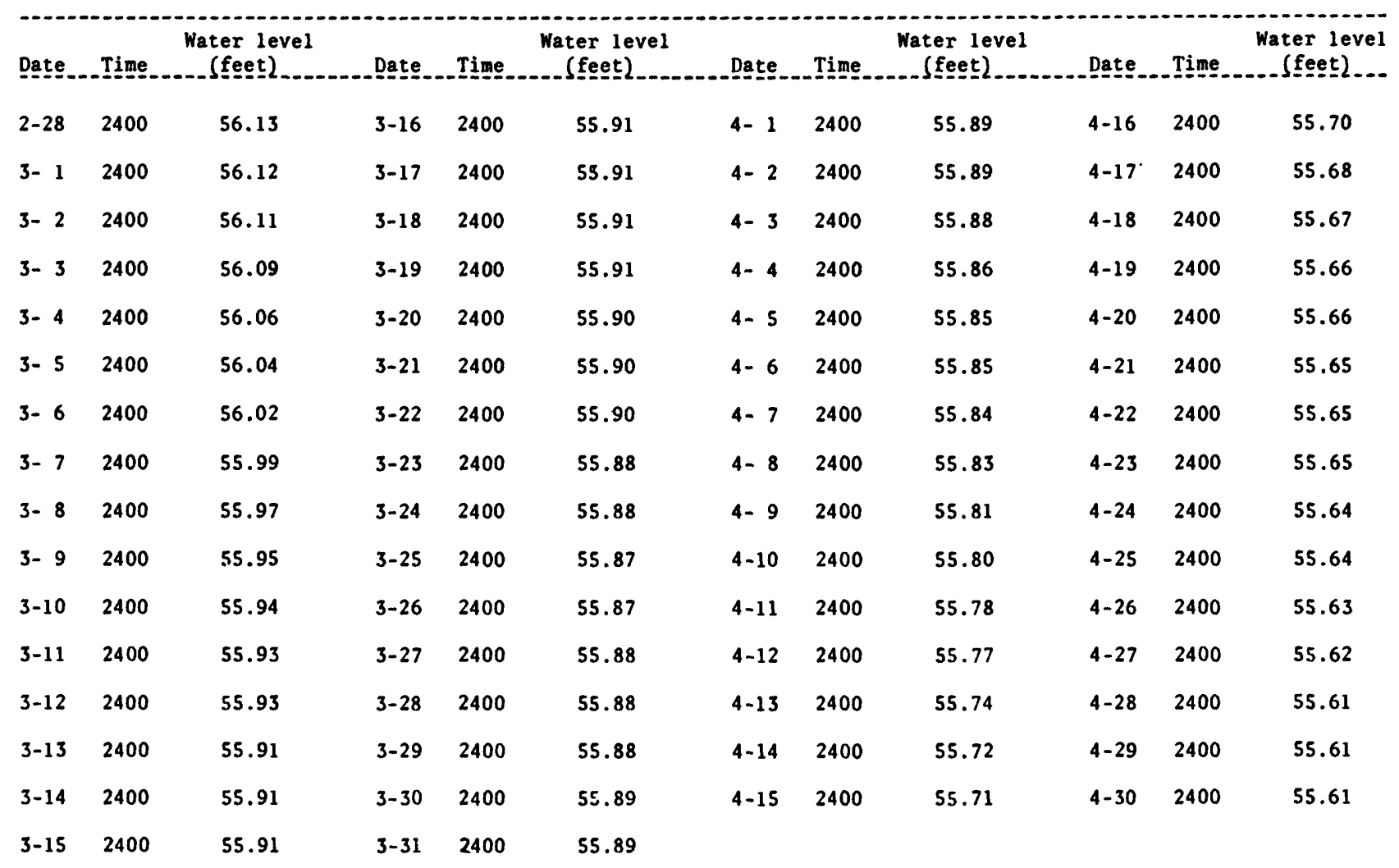


TABLE 8.-Ground-water levels, flood of April 1979-Continued

Site 6W (332605086523001), Well near Oxmoor, Ala. (at County Road 42)

[Water level, in feet, below land-surface datum at indicated time]

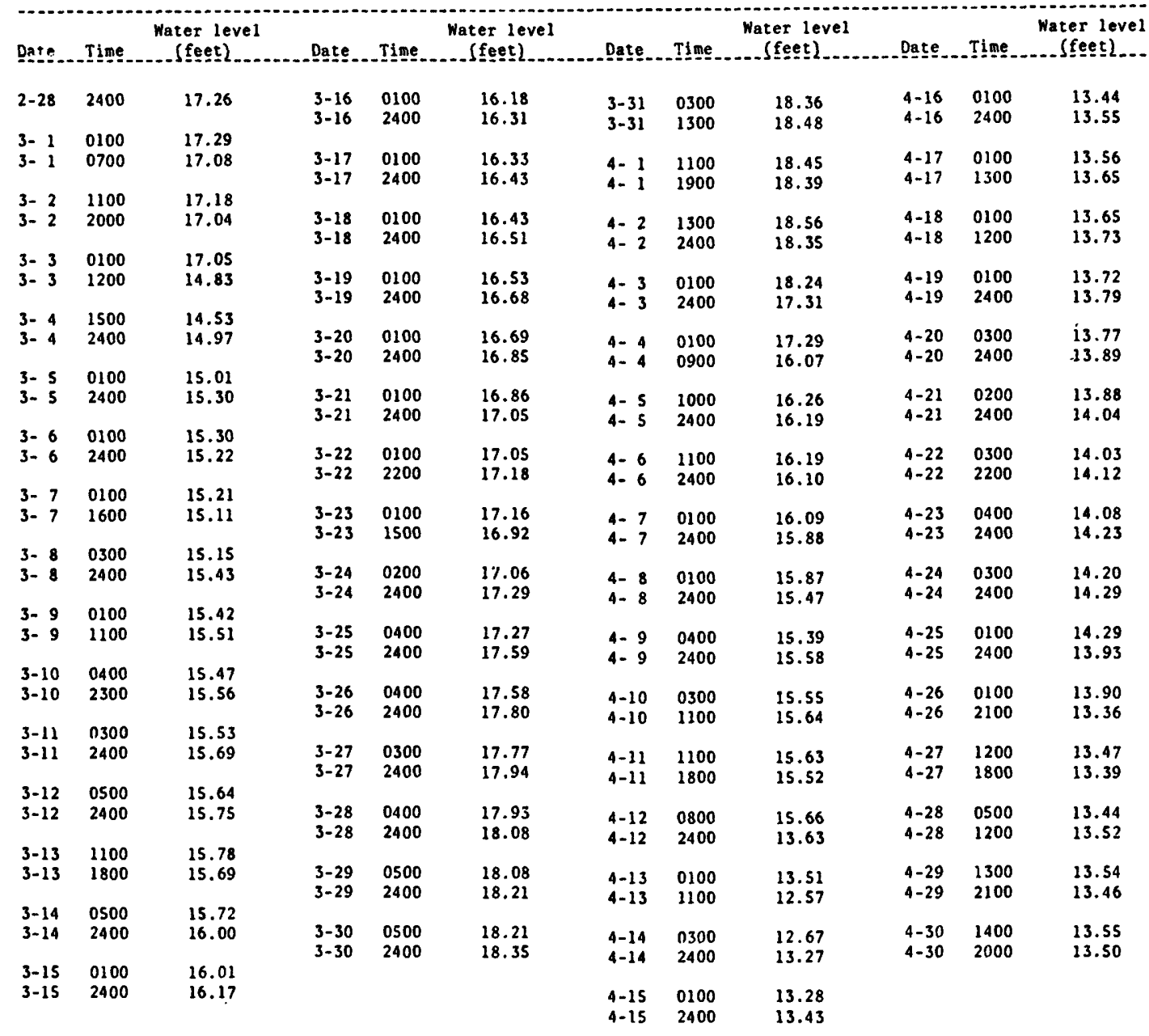


TABLE 8.-Ground-water levels, flood of April 1979-Continued Site 7W (323055087504101), Well at J. C. Webb Compress Co., Demopolis, Ala.

[Water level, in feet, below land-surface datum (altitude 110 feet) at indicated time]

\begin{tabular}{|c|c|c|c|c|c|c|c|c|c|c|c|}
\hline Dete & Tine & $\begin{array}{c}\text { Water level } \\
\text { (feet) }\end{array}$ & Date & Time & $\begin{array}{l}\text { Water level } \\
\text { (feet) }\end{array}$ & Date & Time & $\begin{array}{l}\text { Water level } \\
\text { (feet) }\end{array}$ & Date & Time & $\begin{array}{l}\text { Water level } \\
\text { (feet) }\end{array}$ \\
\hline $2-28$ & 2400 & 14.67 & $\begin{array}{l}3-16 \\
3-16\end{array}$ & $\begin{array}{l}1200 \\
2400\end{array}$ & $\begin{array}{l}15.41 \\
15.36\end{array}$ & $3-31$ & 2400 & 14.88 & $4-17$ & 2400 & 8.34 \\
\hline $\begin{array}{l}\text { 3- } 1 \\
\text { 3- } 1\end{array}$ & $\begin{array}{l}1200 \\
2400\end{array}$ & $\begin{array}{l}14.81 \\
15.02\end{array}$ & $3-17$ & 2400 & 15.38 & $4-1$ & 2400 & 14.63 & $\begin{array}{l}4-18 \\
4-18\end{array}$ & $\begin{array}{l}1200 \\
2400\end{array}$ & $\begin{array}{l}7.95 \\
7.68\end{array}$ \\
\hline 3- 2 & 2400 & 15.26 & $3-18$ & 2400 & 15.13 & 4- 2 & 2400 & 14.41 & $4-19$ & 2400 & 7.58 \\
\hline $3-3$ & 2400 & 15.33 & $3-19$ & 2400 & 14.89 & $4-3$ & 2400 & 14.04 & $\begin{array}{l}4-20 \\
4-20\end{array}$ & $\begin{array}{l}0300 \\
2400\end{array}$ & $\begin{array}{l}7.57 \\
7.76\end{array}$ \\
\hline $3-4$ & 2400 & 15.05 & $3-20$ & 2400 & 14.75 & $4-4$ & 2400 & 13.92 & $\begin{array}{l}4-21 \\
4-21\end{array}$ & $\begin{array}{l}1200 \\
2400\end{array}$ & $\begin{array}{l}7.95 \\
8.16\end{array}$ \\
\hline 3- 5 & 2400 & 14.46 & $3-21$ & 2400 & 14.65 & $4-5$ & 2400 & 14.31 & $4-22$ & 2400 & 8.70 \\
\hline $3-6$ & 2400 & 14.19 & $3-22$ & 2400 & 14.65 & $4-6$ & 2400 & 14.12 & $4-23$ & 2400 & 9.96 \\
\hline 3- 7 & 2400 & 13.70 & $3-23$ & 2400 & 14.41 & $4-7$ & 2400 & 14.03 & $4-24$ & 2400 & 11.42 \\
\hline $3-8$ & 2400 & 13.40 & $\begin{array}{l}3-24 \\
3-24\end{array}$ & $\begin{array}{l}1600 \\
2400\end{array}$ & $\begin{array}{l}14.41 \\
14.54\end{array}$ & 4- 8 & 2400 & 14.00 & $4-25$ & 2400 & 12.23 \\
\hline 3- 9 & 2400 & 13.36 & $\begin{array}{l}3-25 \\
3-25\end{array}$ & $\begin{array}{l}1200 \\
2400\end{array}$ & $\begin{array}{l}14.58 \\
14.49\end{array}$ & 4- 9 & 2400 & 14.12 & $4-26$ & 2400 & 12.61 \\
\hline $3-10$ & 2400 & 13.61 & $3-26$ & 2400 & 14.28 & $4-10$ & 2400 & 14.18 & $4-27$ & 2400 & 12.73 \\
\hline $3-11$ & 2400 & 13.93 & $3-27$ & 2400 & 14.27 & $4-11$ & 2400 & 14.38 & $4-28$ & 2400 & 13.07 \\
\hline $3-12$ & 2400 & 14.31 & $3-28$ & 2400 & 14.61 & $4-12$ & 2400 & 13.89 & $4-29$ & 2400 & 13.10 \\
\hline $3-13$ & 2400 & 14.79 & $3-29$ & 2400 & 15.01 & $4-13$ & 2400 & 13.43 & $4-30$ & 2400 & 13.53 \\
\hline $3-14$ & 2400 & 15.37 & $3-30$ & 2400 & 15.07 & $4-14$ & 2400 & 12.67 & & & \\
\hline \multirow[t]{2}{*}{$3-15$} & 2400 & 15.46 & & & & $4-15$ & 2400 & 11.78 & & & \\
\hline & & & & & & $4-16$ & 2400 & 10.14 & & & \\
\hline
\end{tabular}


TABLE 8.-Ground-water levels, flood of April 1979-Continued

Site 8W (330116088113101) (USCOE), Well on Tombigbee River approximately halfway between Aliceville and Gainesville lock and dam at river mile 259.2 above mouth of Tombigbee River

[Water level, in feet, below land-surface datum (135.6 feet NGVD) at indicated time]

\begin{tabular}{|c|c|c|c|c|c|c|c|c|c|c|c|}
\hline Date & Time & $\begin{array}{c}\text { Water level } \\
\text { (feet) }\end{array}$ & Date & Time & $\begin{array}{c}\text { Water level } \\
\text { (feet) }\end{array}$ & Date & Time & $\begin{array}{c}\text { Water level } \\
\text { (feet) }\end{array}$ & Date & Time & $\begin{array}{c}\text { Water level } \\
\text { (feet) }\end{array}$ \\
\hline $2-28$ & 2400 & 17.36 & $3-15$ & 2400 & 17.42 & $3-30$ & 2400 & 17.34 & $4-15$ & 2400 & 14.02 \\
\hline $3-1$ & 2400 & 17.34 & $3-16$ & 2400 & 17.42 & $3-31$ & 2400 & 17.32 & $4-16$ & 2400 & 13.96 \\
\hline $3-2$ & 2400 & 17.36 & $3-17$ & 2400 & 17.42 & $4-1$ & 2400 & 17.32 & $4-17$ & 2400 & 14.00 \\
\hline $3-3$ & 2400 & 17.04 & $3-18$ & 2400 & 17.44 & $4-2$ & 2400 & 17.23 & $4-18$ & 2400 & 14.13 \\
\hline $3-4$ & 2400 & 16.78 & $3-19$ & 2400 & 17.44 & $4-3$ & 2400 & 17.14 & $4-19$ & 2400 & 14.34 \\
\hline $3-5$ & 2400 & 16.49 & $3-20$ & 2400 & 17.43 & $4-4$ & 2400 & 17.06 & $4-20$ & 2400 & 14.62 \\
\hline $3-6$ & 2400 & 16.40 & $3-21$ & 2400 & 17.42 & $4-5$ & 2400 & 16.99 & $4-21$ & 2400 & 15.03 \\
\hline $3-7$ & 0300 & 16.39 & $3-22$ & 2400 & 17.36 & $4-6$ & 2400 & 16.95 & $4-22$ & 2400 & 15.57 \\
\hline $3-7$ & 1800 & 16.39 & $3-23$ & 1300 & 17.31 & $4-7$ & 2400 & 16.90 & $4-23$ & 2400 & 15.93 \\
\hline $3-7$ & 2400 & 16.41 & $3-23$ & 2400 & 17.33 & $4-8$ & 2400 & 16.77 & $4-24$ & 2400 & 16.18 \\
\hline $3-8$ & 2400 & 16.47 & $3-24$ & 2400 & 17.30 & $4-9$ & 2400 & 16.80 & $4-25$ & 2400 & 16.29 \\
\hline $3-9$ & 2400 & 16.59 & $3-25$ & 2400 & 17.29 & $4-10$ & 2400 & 16.84 & $4-26$ & 2400 & 16.34 \\
\hline $3-10$ & 2400 & 16.73 & $3-26$ & 2400 & 17.27 & $4-11$ & 2400 & 16.89 & $4-27$ & 2400 & 16.40 \\
\hline $3-11$ & 2400 & 16.91 & $3-27$ & 2400 & 17.28 & $4-12$ & 2400 & 16.16 & $4-28$ & 2400 & 16.46 \\
\hline $3-12$ & 2400 & 17.09 & $3-28$ & 2400 & 17.31 & $4-13$ & 2400 & 14.80 & $4-29$ & 2400 & 16.49 \\
\hline $3-13$ & 2400 & 17.24 & $3-29$ & 2400 & 17.36 & $4-14$ & 2400 & 14.21 & $4-30$ & 2400 & 16.53 \\
\hline $3-14$ & 2400 & 17.37 & & & & & & & & & \\
\hline
\end{tabular}


TABLE 8.-Ground-water levels, flood of April 1979-Continued

Site 9W (330117088180301), Well 0.7 mile west of Tombigbee River, 3.5 miles southwest of Pickensville at river mile 286.0 above mouth of Tombigbee River

[Water level, in feet, below land-surface datum (139.80 feet NGVD) at indicated time]

\begin{tabular}{llcllcccccccc}
\hline Date & Time & $\begin{array}{c}\text { Water level } \\
\text { (feet) }\end{array}$ & Date & Time & $\begin{array}{c}\text { Water level } \\
\text { (feet) }\end{array}$ & Date & Time & $\begin{array}{c}\text { Water 1evel } \\
\text { (feet) }\end{array}$ & $\begin{array}{c}\text { Date } \\
\text { Dime }\end{array}$ & $\begin{array}{c}\text { Water level } \\
\text { (feet) }\end{array}$ \\
\hline $3-21$ & 2400 & 4.08 & $4-2$ & 2400 & 4.06 & $4-12$ & 1800 & 2.92 & $4-19$ & 2400 & 3.25 \\
$3-22$ & 2400 & 4.01 & $4-3$ & 2400 & 3.96 & $4-12$ & 2400 & 3.04 & $4-20$ & 2400 & 3.59 \\
$3-23$ & 2400 & 4.02 & $4-4$ & 2400 & 3.99 & $4-13$ & 0600 & 2.77 & $4-21$ & 2400 & 3.68 \\
$3-24$ & 2400 & 4.04 & $4-5$ & 2400 & 4.03 & $4-13$ & 1200 & 2.21 & $4-22$ & 2400 & 3.68 \\
$3-25$ & 2400 & 4.05 & $4-6$ & 2400 & 4.04 & $4-13$ & 2400 & 1.44 & $4-23$ & 2400 & 3.68 \\
$3-26$ & 2400 & 4.09 & $4-7$ & 2400 & 4.04 & $4-14$ & 1200 & 1.13 & $4-25$ & 2400 & 3.66 \\
$3-27$ & 2400 & 4.10 & $4-8$ & 2400 & 3.90 & $4-14$ & 2400 & 1.06 & $4-26$ & 2400 & 3.64 \\
$3-28$ & 2400 & 4.11 & $4-9$ & 2400 & 3.94 & $4-15$ & 1200 & 1.05 & $4-27$ & 2400 & 3.67 \\
$3-29$ & 2400 & 4.12 & $4-10$ & 2400 & 3.96 & $4-15$ & 2400 & 1.15 & $4-28$ & 2400 & 3.69 \\
$3-30$ & 2400 & 4.11 & $4-11$ & 2400 & 3.97 & $4-16$ & 2400 & 1.55 & $4-29$ & 2400 & 3.72 \\
$3-31$ & 2400 & 4.12 & $4-12$ & 0600 & 3.81 & $4-17$ & 2400 & 2.09 & $4-30$ & 2400 & 3.75 \\
$4-1$ & 2400 & 4.11 & $4-12$ & 1200 & 3.51 & $4-18$ & 2400 & 2.67 & & &
\end{tabular}


TABLE 8.-Ground-water levels, flood of April 1979-Continued

Site 11W (325212088121601), Well 1.35 miles west of Tombigbee River, 4.2 miles northwest of Gainesville at river mile 240.9 above mouth of Tombigbee River

[Water level, in feet, below land-surface datum (140.6 feet) at indicated time]

\begin{tabular}{|c|c|c|c|c|c|c|c|c|c|c|c|}
\hline Date & Time & $\begin{array}{l}\text { Water level } \\
\text { (feet) }\end{array}$ & Date & Time & $\begin{array}{l}\text { Water level } \\
\text { (feet) }\end{array}$ & Date & Time & $\begin{array}{l}\text { Water level } \\
\text { (feet) }\end{array}$ & Date & Time & $\begin{array}{c}\text { Water level } \\
\text { (feet) }\end{array}$ \\
\hline $2-28$ & 2400 & 14.55 & $3-14$ & 2400 & 14.27 & $3-30$ & 2400 & 14.45 & $4-15$ & 2400 & 13.62 \\
\hline $3-1$ & 2400 & 14.54 & $3-15$ & 2400 & 14.30 & $3-31$ & 2400 & 14.39 & $4-16$ & 2400 & 13.55 \\
\hline $3-2$ & 2400 & 14.51 & $3-16$ & 2400 & 14.31 & $4-1$ & 2400 & 14.40 & $4-17$ & 2400 & 13.56 \\
\hline $3-3$ & 1200 & 14.40 & $3-17$ & 2400 & 14.30 & $4-2$ & 2400 & 14.32 & $4-18$ & 2400 & 13.57 \\
\hline $3-3$ & 2000 & 14.25 & $3-18$ & 2400 & 14.30 & $4-3$ & 2400 & 14.36 & $4-19$ & 2400 & 13.58 \\
\hline $3-3$ & 2400 & 14.35 & $3-19$ & 2400 & 14.30 & 4-4 & 2400 & 14.40 & $4-20$ & 2400 & 13.59 \\
\hline $3-4$ & 2400 & 14.44 & $3-20$ & 2400 & 14.30 & 4-5 & 2400 & 14.45 & $4-21$ & 2400 & 13.60 \\
\hline $3-5$ & 2400 & 14.43 & $3-21$ & 2400 & 14.32 & $4-6$ & 2400 & 14.45 & $4-22$ & 2400 & 13.60 \\
\hline $3-6$ & 2400 & 14.37 & $3-22$ & 2400 & 14.24 & $4-7$ & 2400 & 14.42 & $4-23$ & 2400 & 13.64 \\
\hline $3-7$ & 2400 & 14.32 & $3-23$ & 0300 & 14.19 & $4-8$ & 2400 & 14.31 & $4-24$ & 2400 & 13.63 \\
\hline $3-8$ & 2400 & 14.31 & $3-23$ & 2400 & 14.26 & $4-9$ & 2400 & 14.36 & $4-25$ & 2400 & 13.62 \\
\hline $3-9$ & 2400 & 14.29 & $3-24$ & 2400 & 14.30 & $4-10$ & -2400 & 14.36 & $4-26$ & 2400 & 13.68 \\
\hline $3-10$ & 2400 & 14.27 & $3-25$ & 2400 & 14.35 & $4-11$ & 2400 & 14.36 & $4-27$ & 2400 & 13.75 \\
\hline $3-11$ & 2400 & 14.27 & $3-26$ & 2400 & 14.38 & $4-12$ & 1800 & 14.01 & $4-28$ & 2400 & 13.83 \\
\hline $3-12$ & 2400 & 14.24 & $3-27$ & 2400 & 14.40 & $4-12$ & 2400 & 14.17 & $4-29$ & 2400 & 13.88 \\
\hline $3-13$ & 1900 & 14.20 & $3-28$ & 2400 & 14.42 & $4-13$ & 2400 & 14.13 & $4-30$ & 2400 & 13.94 \\
\hline $3-13$ & 2400 & 14.22 & $3-29$ & 2400 & 14.42 & $4-14$ & 2400 & 13.89 & & & \\
\hline
\end{tabular}


TABLE 8.-Ground-water levels, flood of April 1979-Continued

Site 12W (325215088111301), Well 0.35 mile west of Tombigbee River, 3.7 miles northwest of Gainesville at river mile 240.9 above mouth of Tombigbee River

[Water level, in feet, below land-surface datum (162.82 feet, NGVD) at indicated time]

\begin{tabular}{|c|c|c|c|c|c|c|c|c|c|c|c|}
\hline Date & Time & $\begin{array}{c}\text { Water level } \\
\text { (feet) }\end{array}$ & Date & Time & $\begin{array}{l}\text { Water level } \\
\text { (feet) }\end{array}$ & Date & Time & $\begin{array}{l}\text { Water level } \\
\text { (feet) }\end{array}$ & Date & Time & $\begin{array}{c}\text { Water level } \\
\text { (feet) }\end{array}$ \\
\hline $2-28$ & 2400 & 37.42 & $3-18$ & 2400 & 37.41 & $4-4$ & 2400 & 37.32 & $4-16$ & 2400 & 35.66 \\
\hline $3-1$ & 2400 & 37.43 & $3-19$ & 2400 & 37.40 & $4-5$ & 2400 & 37.35 & $4-17$ & 1200 & 35.62 \\
\hline $3-2$ & 2400 & 37.42 & $3-20$ & 2400 & 37.40 & $4-6$ & 2400 & 37.38 & $4-17$ & 2400 & 35.59 \\
\hline $3-3$ & 2400 & 37.25 & $3-21$ & 2400 & 37.39 & $4-7$ & 2400 & 37.36 & $4-18$ & 2400 & 35.62 \\
\hline $3-4$ & 2400 & 37.33 & $3-22$ & 2400 & 37.31 & $4-8$ & 2400 & 37.24 & $4-19$ & 2400 & 35.73 \\
\hline $3-5$ & 2400 & 37.32 & $3-23$ & 2400 & 37.31 & $4-9$ & 2400 & 37.29 & $4-20$ & 2400 & 35.89 \\
\hline $3-6$ & 2400 & 37.27 & $3-24$ & 2400 & 37.35 & $4-10$ & 2400 & 37.30 & $4-21$ & 2400 & 36.11 \\
\hline $3-7$ & 2400 & 37.23 & $3-25$ & 2400 & 37.41 & $4-11$ & 2400 & 37.32 & $4-22$ & 2400 & 36.30 \\
\hline $3-8$ & 2400 & 37.30 & $3-26$ & 2400 & 37.44 & $4-12$ & 0700 & 37.32 & $4-23$ & 2400 & 36.47 \\
\hline $3-9$ & 2400 & 37.33 & $3-27$ & 2400 & 37.46 & $4-12$ & 1200 & 37.21 & $4-24$ & 2400 & 36.57 \\
\hline $3-10$ & 2400 & 37.34 & $3-28$ & 2400 & 37.48 & $4-12$ & 2400 & 37.14 & $4-25$ & 3400 & 36.62 \\
\hline $3-11$ & 2400 & 37.34 & $3-29$ & 2400 & 37.47 & $4-13$ & 1200 & 36.98 & $4-26$ & 2400 & 36.72 \\
\hline $3-12$ & 2400 & 37.36 & $3-30$ & 2400 & 37.46 & $4-13$ & 2400 & 36.82 & $4-27$ & 2400 & 36.80 \\
\hline $3-13$ & 2400 & 37.36 & $3-31$ & 2400 & 37.38 & $4-14$ & 1200 & 36.62 & $4-28$ & 2400 & 36.87 \\
\hline $3-14$ & 2400 & 37.43 & $4-1$ & 2400 & 37.36 & $4-14$ & 2400 & 36.34 & $4-29$ & 2400 & 36.93 \\
\hline $3-15$ & 2400 & 37.43 & $4-2$ & 2400 & 37.28 & $4-15$ & 1200 & 36.11 & $4-30$ & 2400 & 36.97 \\
\hline $3-16$ & 2400 & 37.42 & $4-3$ & 1900 & 37.31 & $4-15$ & 2400 & 35.90 & & & \\
\hline $3-17$ & 2400 & 37.41 & $4-3$ & 2400 & 37.25 & & & & & & \\
\hline
\end{tabular}


TABLE 8.-Ground-water levels, flood of April 1979-Continued

Site 15W (331426088192202), Well 3.4 miles west of Pickensville, Ala. (Noxubee County, Miss.) at river mile 292.0 above mouth of Tombigbee River

[Water level, in feet, below land-surface datum (146.64 feet) at indicated time]

\begin{tabular}{|c|c|c|c|c|c|c|c|c|c|c|c|}
\hline Date & Time & $\begin{array}{l}\text { Water level } \\
\text { (feet) }\end{array}$ & Date & Time & $\begin{array}{l}\text { Water level } \\
\text { (feet) }\end{array}$ & Date & Time & $\begin{array}{l}\text { Water level } \\
\text { (feet) }\end{array}$ & Date & Time & $\begin{array}{l}\text { Water level } \\
\text { (feet) }\end{array}$ \\
\hline $2-28$ & 2400 & 4.30 & $3-18$ & 2400 & 4.32 & $4-4$ & 2400 & 4.63 & $4-14$ & 1200 & 1.29 \\
\hline $3-1$ & 2400 & 4.20 & $3-19$ & 2400 & 4.39 & 4-5 & 2400 & 4.56 & $4-14$ & 2400 & 1.44 \\
\hline $3-2$ & 2400 & 4.17 & $3-20$ & 2400 & 4.46 & $4-6$ & 2400 & 4.56 & $4-15$ & 1200 & 1.57 \\
\hline $3-3$ & 2400 & 3.17 & $3-21$ & 2400 & 4.52 & $4-7$ & 2400 & 4.58 & $4-15$ & 2400 & 1.70 \\
\hline $3-4$ & 1700 & 3.05 & $3-22$ & 2100 & 4.57 & $4-8$ & 2400 & 4.51 & $4-16$ & 2400 & 1.93 \\
\hline $3-4$ & 2400 & 3.08 & $3-22$ & 2400 & 4.52 & $4-9$ & 2400 & 4.50 & $4-17$ & 2400 & 2.19 \\
\hline $3-5$ & 2400 & 3.19 & $3-23$ & 0200 & 4.50 & $4-10$ & 2400 & 4.49 & $4-18$ & 2400 & 2.44 \\
\hline $3-6$ & 2400 & 3.30 & $3-23$ & 2400 & 4.56 & $4-11$ & 2400 & 4.54 & $4-19$ & 2400 & 2.66 \\
\hline $3-7$ & 2400 & 3.43 & $3-24$ & 2400 & 4.61 & $4-12$ & 0500 & 4.57 & $4-20$ & 2400 & 2.84 \\
\hline $3-8$ & 2400 & 3.56 & $3-25$ & 2400 & 4.67 & $4-12$ & 1200 & 4.00 & $4-21$ & 2400 & 3.01 \\
\hline $3-9$ & 2400 & 3.66 & $3-26$ & 2400 & 4.72 & $4-12$ & 1800 & 1.68 & $4-22$ & 2400 & 3.13 \\
\hline $3-10$ & 2400 & 3.72 & $3-27$ & 2400 & 4.77 & $4-12$ & 2400 & 1.03 & $4-23$ & 2400 & 3.26 \\
\hline $3-11$ & 2400 & 3.79 & $3-28$ & 2400 & 4.82 & $4-13$ & 0600 & 0.89 & $4-24$ & 2400 & 3.35 \\
\hline $3-12$ & 2400 & 3.86 & $3-29$ & 2400 & 4.86 & $4-13$ & 0800 & 0.52 & $4-25$ & 2400 & 3.45 \\
\hline $3-13$ & 2400 & 3.93 & $3-30$ & 2400 & 4.92 & $4-13$ & 1000 & 0.71 & $4-26$ & 2400 & 3.58 \\
\hline $3-14$ & 2400 & 4.04 & $3-31$ & 2400 & 4.95 & $4-13$ & 1200 & 0.82 & $4-27$ & 2400 & 3.70 \\
\hline $3-15$ & 2400 & 4.11 & $4-1$ & 2400 & 4.98 & $4-13$ & 1800 & 1.04 & $4-28$ & 2400 & 3.81 \\
\hline $3-16$ & 2400 & 4.18 & $4-2$ & 2400 & 5.01 & $4-13$ & 2400 & 1.15 & $4-29$ & 2400 & 3.92 \\
\hline $3-17$ & 2400 & 4.20 & $1-3$ & 2400 & 4.85 & & & & $4-30$ & 2400 & 4.03 \\
\hline
\end{tabular}


TABLES

TABLE 9.-Specific conductance and temperature of samples at selected sites along the Intracoastal Waterway at the mouth of Mobile Bay, April 28-29, 1979

\begin{tabular}{|c|c|c|c|c|c|c|c|}
\hline $\begin{array}{r}\text { Station } \\
\text { number }\end{array}$ & Location & $\begin{array}{l}\text { Date } \\
1979\end{array}$ & $\begin{array}{c}\text { Time } \\
\text { (hours) }\end{array}$ & $\begin{array}{l}\text { Weather } \\
\text { conditions }\end{array}$ & $\begin{array}{l}\text { nepth } \\
\text { (feet) }\end{array}$ & $\begin{array}{c}\text { Specific } \\
\text { conductance } \\
\text { (micromhos per } \\
\text { centimeter) } \\
\end{array}$ & $\begin{array}{c}\text { Water } \\
\text { temperature } \\
\text { (degrees } \\
\text { Celsius) } \\
\end{array}$ \\
\hline 1 & $\begin{array}{l}\text { Marker } 18 \text { in Bon Secour } \\
\text { River near Mimi's } \\
\text { Restaurant }\end{array}$ & April 28 & 1510 & $\begin{array}{l}\text { Calm, warm, } \\
\text { clear }\end{array}$ & $\begin{array}{c}\text { Surface } \\
5 \\
6\end{array}$ & $\begin{array}{l}2,400 \\
2,600 \\
2,600\end{array}$ & $\begin{array}{l}23.5 \\
21.5 \\
21.5\end{array}$ \\
\hline 2 & $\begin{array}{l}\text { Post with staff gage in } \\
\text { Bon Secour River out } \\
\text { from lower end of boat } \\
\text { dock on right bank }\end{array}$ & April 28 & 1500 & $\begin{array}{l}\text { Calm, warm, } \\
\text { clear }\end{array}$ & $\begin{array}{c}\text { Surface } \\
5\end{array}$ & $\begin{array}{l}2,7 \cap 0 \\
2,900\end{array}$ & $\begin{array}{l}22 \cdot 5 \\
21 \cdot 5\end{array}$ \\
\hline 3 & $\begin{array}{l}\text { Buoy } 136 \text { on Intracoastal } \\
\text { Waterway }\end{array}$ & April 28 & $142 n$ & $\begin{array}{l}\text { Calm, clear, } \\
\text { warm }\end{array}$ & $\begin{array}{c}\text { Surface } \\
5 \\
10 \\
12\end{array}$ & $\begin{array}{l}3,200 \\
3,200 \\
3,200 \\
3,200\end{array}$ & $\begin{array}{l}22.5 \\
22.0 \\
22.0 \\
21.5\end{array}$ \\
\hline 4 & $\begin{array}{l}\text { State Hwy. } 59 \text { crossing } \\
\text { Intracoastal Waterway }\end{array}$ & April 28 & 1350 & $\begin{array}{l}\text { Calm, warm, } \\
\text { clear }\end{array}$ & $\begin{array}{c}\text { Surface } \\
5 \\
10 \\
12\end{array}$ & $\begin{array}{l}3,000 \\
3,000 \\
3,000 \\
3, n 00\end{array}$ & $\begin{array}{l}22.0 \\
22.0 \\
22.0 \\
22.0\end{array}$ \\
\hline 5 & $\begin{array}{l}\text { Buoy } 147 \text { on Intra- } \\
\text { coastal Waterway }\end{array}$ & April 28 & $132 n$ & $\begin{array}{l}\text { Calm, warm, } \\
\text { clear }\end{array}$ & $\begin{array}{c}\text { Surf ace } \\
5 \\
10 \\
14\end{array}$ & $\begin{array}{l}3,200 \\
3,200 \\
3,200 \\
3,200\end{array}$ & $\begin{array}{l}22.5 \\
21.5 \\
21.5 \\
21.5\end{array}$ \\
\hline 6 & $\begin{array}{l}\text { Ruoy } 159 \text { on Intracoastal } \\
\text { Waterway - Mobile Bay }\end{array}$ & April 28 & 1300 & $\begin{array}{l}\text { Calm, warm } \\
\text { clear }\end{array}$ & $\begin{array}{c}\text { Surface } \\
5 \\
10\end{array}$ & $\begin{array}{l}2,900 \\
2,900 \\
3,000\end{array}$ & $\begin{array}{l}21 \cdot 5 \\
21 \cdot 5 \\
21.0\end{array}$ \\
\hline 7 & $\begin{array}{l}\text { Buoy } 167 \text { on Intracoastal } \\
\text { Waterway--Mobile Bay }\end{array}$ & April 28 & 1255 & $\begin{array}{l}\text { Calm, warm, } \\
\text { clear }\end{array}$ & $\left|\begin{array}{c}\text { Surface } \\
5 \\
6\end{array}\right|$ & $\begin{array}{l}2,800 \\
2,800 \\
2,850\end{array}$ & $\begin{array}{l}21.5 \\
21.5 \\
21.5\end{array}$ \\
\hline \multirow[t]{2}{*}{8} & $\begin{array}{l}\text { Buoy } 175 \text { on Intracoastal } \\
\text { Waterway--Mobile Bay }\end{array}$ & April 28 & 0915 & $\begin{array}{l}\text { Calm, warm, } \\
\text { clear }\end{array}$ & \begin{tabular}{|c|} 
Surface \\
5 \\
10 \\
14
\end{tabular} & $\begin{array}{l}1,700 \\
1,700 \\
1,750 \\
1,800\end{array}$ & $\begin{array}{l}21.5 \\
20.5 \\
20.5 \\
20.5\end{array}$ \\
\hline & & April 28 & 1245 & $\begin{array}{l}\text { Calm, warm } \\
\text { clear }\end{array}$ & $\begin{array}{c}\text { Surface } \\
5 \\
8\end{array}$ & $\begin{array}{l}1,700 \\
1,750 \\
1,750\end{array}$ & $\begin{array}{l}21.0 \\
21.0 \\
21.0\end{array}$ \\
\hline 9 & $\begin{array}{l}\text { Buoy } 183 \text { on Intracoastal } \\
\text { Waterway--Mobile Bay }\end{array}$ & April 28 & $095 n$ & $\begin{array}{l}\text { Calm, warm, } \\
\text { clear }\end{array}$ & $\begin{array}{c}\text { Surface } \\
5 \\
10 \\
12\end{array}$ & $\begin{array}{l}1,350 \\
1,350 \\
1,400 \\
1,400\end{array}$ & $\begin{array}{l}21.0 \\
20.5 \\
20.5 \\
20.5\end{array}$ \\
\hline 9 & & April 28 & 1235 & $\begin{array}{l}\text { Calm, warm, } \\
\text { clear }\end{array}$ & $\begin{array}{c}\text { Surface } \\
5 \\
8\end{array}$ & $\begin{array}{l}1,500 \\
1,500 \\
1,500\end{array}$ & $\begin{array}{l}21.0 \\
21.0 \\
21.0\end{array}$ \\
\hline 9 & & April 29 & 1120 & $\begin{array}{l}\text { Windy, cool, } \\
\text { cloudy }\end{array}$ & $\begin{array}{c}\text { Surface } \\
5 \\
10 \\
13\end{array}$ & $\begin{array}{l}2,400 \\
2,450 \\
2,400 \\
2,400\end{array}$ & $\begin{array}{l}20.5 \\
21.0 \\
21.0 \\
21.0\end{array}$ \\
\hline
\end{tabular}


TABLE 9.-Specific conductance and temperature of samples at selected sites along the Intracoastal Waterway at the mouth of Mobile Bay, April 28-29, 1979-Continued

\begin{tabular}{|c|c|c|c|c|c|c|c|}
\hline $\begin{array}{l}\text { Station } \\
\text { number }\end{array}$ & Location & $\begin{array}{l}\text { Date } \\
1979\end{array}$ & $\begin{array}{l}\text { Time } \\
\text { (hours) }\end{array}$ & $\begin{array}{l}\text { Weather } \\
\text { conditions }\end{array}$ & $\begin{array}{r}\text { Depth } \\
\text { (feet) }\end{array}$ & $\begin{array}{c}\text { Specific } \\
\text { conductance } \\
\text { (micromhos per } \\
\text { centimeter) }\end{array}$ & $\begin{array}{c}\text { Water } \\
\text { temperature } \\
\text { (degrees } \\
\text { Celsius) } \\
\end{array}$ \\
\hline 10 & $\begin{array}{l}\text { Buoy } 191 \text { on Intracoastal } \\
\text { Waterway--Mobile Bay }\end{array}$ & April 28 & 1225 & $\begin{array}{l}\text { Calm, warm, } \\
\text { clear }\end{array}$ & \begin{tabular}{|c} 
Surface \\
5 \\
8
\end{tabular} & $\begin{array}{l}1,300 \\
1,300 \\
1,300\end{array}$ & $\begin{array}{l}21.0 \\
21.0 \\
21.0\end{array}$ \\
\hline 11 & $\begin{array}{l}\text { Buoy } 195 \text { on Intracoastal } \\
\text { Waterway--Mobile Bay }\end{array}$ & April 28 & 1010 & $\begin{array}{l}\text { Calm, warm, } \\
\text { clear }\end{array}$ & $\begin{array}{c}\text { Surface } \\
5 \\
10 \\
12\end{array}$ & $\begin{array}{l}1,550 \\
1,500 \\
1,600 \\
1,600\end{array}$ & $\begin{array}{l}20.5 \\
20.5 \\
20.0 \\
20.0\end{array}$ \\
\hline 11 & & April 29 & 1030 & $\begin{array}{l}\text { Windy, cool, } \\
\text { cloudy }\end{array}$ & $\begin{array}{c}\text { Surface } \\
5 \\
10 \\
14\end{array}$ & $\begin{array}{l}1,400 \\
1,400 \\
1,400 \\
1,400\end{array}$ & $\begin{array}{l}20.5 \\
20.5 \\
20.0 \\
20.0\end{array}$ \\
\hline 12 & $\begin{array}{l}\text { Buoy } 205 \text { on Intracoastal } \\
\text { Waterway--Mobile Bay }\end{array}$ & April 28 & 1020 & $\begin{array}{l}\text { Calm, warm, } \\
\text { clear }\end{array}$ & $\begin{array}{c}\text { Surface } \\
5 \\
10 \\
11\end{array}$ & $\begin{array}{l}200 \\
200 \\
200 \\
200\end{array}$ & $\begin{array}{l}20.5 \\
20.5 \\
20.0 \\
20.0\end{array}$ \\
\hline 12 & & April 29 & 0945 & $\begin{array}{l}\text { Windy, cool, } \\
\text { cloudy }\end{array}$ & $\begin{array}{c}\text { Surface } \\
5 \\
10 \\
14\end{array}$ & $\begin{array}{l}1,800 \\
1,800 \\
1,800 \\
1,800\end{array}$ & $\begin{array}{l}20.5 \\
20.5 \\
20.5 \\
20.5\end{array}$ \\
\hline 13 & $\begin{array}{l}\text { Buoy } 215 \text { on Intracoastal } \\
\text { Waterway--Mobile Bay }\end{array}$ & April 28 & 1030 & $\begin{array}{l}\text { Calm, warm, } \\
\text { clear }\end{array}$ & $\begin{array}{c}\text { Surface } \\
5 \\
10 \\
12\end{array}$ & $\begin{array}{l}500 \\
500 \\
600 \\
600\end{array}$ & $\begin{array}{l}20.5 \\
20.5 \\
20.0 \\
20.0\end{array}$ \\
\hline 14 & $\begin{array}{l}\text { Buoy } 223 \text { on Intracoastal } \\
\text { Waterway--Mobile Bay }\end{array}$ & April 28 & 1045 & $\begin{array}{l}\text { Calm, warm, } \\
\text { clear }\end{array}$ & \begin{tabular}{|c} 
Surface \\
5 \\
10 \\
13
\end{tabular} & $\begin{array}{l}400 \\
400 \\
500 \\
600\end{array}$ & $\begin{array}{l}21.0 \\
20.5 \\
2 n .5 \\
20.0\end{array}$ \\
\hline 15 & $\begin{array}{l}\text { Buoy } 231 \text { on Intracoastal } \\
\text { Waterway--Mobile Bay }\end{array}$ & April 28 & 1055 & $\begin{array}{l}\text { Little windy, } \\
\text { warm, clear }\end{array}$ & $\begin{array}{c}\text { Surface } \\
5 \\
10 \\
14\end{array}$ & $\begin{array}{l}250 \\
250 \\
300 \\
300\end{array}$ & $\begin{array}{l}20.5 \\
20.5 \\
20.0 \\
20.0\end{array}$ \\
\hline 16 & $\begin{array}{l}\text { Between tower (oil } \\
\text { derrick) at Dauphin } \\
\text { Island and tower at Fort } \\
\text { Morgan on Intracoastal } \\
\text { Waterway--Mobile Bay }\end{array}$ & April 28 & 1120 & $\begin{array}{l}\text { Little windy, } \\
\text { warm, clear }\end{array}$ & $\begin{array}{c}\text { Surface } \\
5 \\
10 \\
15 \\
20 \\
25 \\
30 \\
35\end{array}$ & $\begin{array}{r}300 \\
300 \\
300 \\
2,000 \\
40,000 \\
41,000 \\
43,000 \\
45,000\end{array}$ & $\begin{array}{l}20.0 \\
20.0 \\
20.0 \\
19.5 \\
20.5 \\
20.5 \\
20.5 \\
20.5\end{array}$ \\
\hline 17 & $\begin{array}{l}2.2 \text { Miles north of } \\
\text { Buoy } 205 \text { on Intracoastal } \\
\text { Waterway--Mobile Bay }\end{array}$ & April 29 & 0955 & $\begin{array}{l}\text { Windy, cool, } \\
\text { cloudy }\end{array}$ & $\begin{array}{c}\text { Surface } \\
5 \\
10 \\
11\end{array}$ & $\begin{array}{l}150 \\
150 \\
150 \\
150\end{array}$ & $\begin{array}{l}19.5 \\
19.5 \\
19.5 \\
19.5\end{array}$ \\
\hline 18 & $\begin{array}{l}2.2 \text { Miles south of } \\
\text { Buoy } 205 \text { on Intracoastal } \\
\text { Waterway--Mobile Bay }\end{array}$ & April 29 & 1015 & $\begin{array}{l}\text { Windy, cool, } \\
\text { cloudy }\end{array}$ & $\begin{array}{c}\text { Surface } \\
5 \\
10\end{array}$ & $\begin{array}{l}2,200 \\
2,200 \\
2,250\end{array}$ & $\begin{array}{l}20.0 \\
20.0 \\
20.0\end{array}$ \\
\hline
\end{tabular}


TABLE 9.-Specific conductance and temperature of samples at selected sites along the Intracoastal Waterway at the mouth of Mobile Bay, April 28-29, 1979-Continued

\begin{tabular}{|c|c|c|c|c|c|c|c|}
\hline $\begin{array}{l}\text { Station } \\
\text { number }\end{array}$ & Location & $\begin{array}{l}\text { Date } \\
1979\end{array}$ & $\begin{array}{l}\text { Time } \\
\text { (hours) }\end{array}$ & $\begin{array}{l}\text { Weather } \\
\text { conditions }\end{array}$ & $\begin{array}{l}\text { Depth } \\
\text { (feet) }\end{array}$ & $\begin{array}{c}\text { Specific } \\
\text { conductance } \\
\text { (micromhos per } \\
\text { centimeter) }\end{array}$ & $\begin{array}{c}\text { Water } \\
\text { temperature } \\
\text { (degrees } \\
\text { Celsius) } \\
\end{array}$ \\
\hline 19 & $\begin{array}{l}2.2 \text { Miles north of Ruoy } \\
195 \text { on Intracoastal } \\
\text { Waterway--Mobile Bay }\end{array}$ & April 29 & 1040 & $\begin{array}{l}\text { Windy, cool, } \\
\text { cloudy }\end{array}$ & $\begin{array}{c}\text { Surface } \\
5 \\
10\end{array}$ & $\begin{array}{l}200 \\
250 \\
250\end{array}$ & $\begin{array}{l}20.0 \\
20.0 \\
20.0\end{array}$ \\
\hline 20 & $\begin{array}{l}2.4 \text { Miles south of Buoy } \\
195 \text { on Intracoastal } \\
\text { Waterway--Mobile Bay }\end{array}$ & April 29 & 1055 & $\begin{array}{l}\text { Windy, cool, } \\
\text { cloudy }\end{array}$ & $\begin{array}{c}\text { Surface } \\
5 \\
9\end{array}$ & $\begin{array}{l}1,300 \\
1,300 \\
1,300\end{array}$ & $\begin{array}{l}20.0 \\
20.0 \\
20.0\end{array}$ \\
\hline 21 & $\begin{array}{l}2.2 \text { Miles north of } \\
\text { Buoy } 183 \text { on Intracoastal } \\
\text { Waterway--Mobile Bay }\end{array}$ & April 29 & 1130 & $\begin{array}{l}\text { Windy, cool, } \\
\text { cloudy }\end{array}$ & $\begin{array}{c}\text { Surface } \\
5 \\
10\end{array}$ & $\begin{array}{l}500 \\
550 \\
550\end{array}$ & $\begin{array}{l}21.0 \\
20.5 \\
20.5\end{array}$ \\
\hline 22 & $\begin{array}{l}2.1 \text { Miles south of } \\
\text { Buoy } 183 \text { on Intracoastal } \\
\text { Waterway--Mobile Bay }\end{array}$ & April 29 & 1145 & $\begin{array}{l}\text { Windy, cool, } \\
\text { cloudy }\end{array}$ & $\begin{array}{c}\text { Surface } \\
5 \\
8\end{array}$ & $\begin{array}{l}2,150 \\
2,200 \\
2,150\end{array}$ & $\begin{array}{l}20.5 \\
20.5 \\
20.0\end{array}$ \\
\hline
\end{tabular}


TABLE 10.-Aerial photographs obtained at or near the crest of the flood, April 1979

\begin{tabular}{lcccc}
\hline $\begin{array}{l}\text { Flight line } \\
\text { number }\end{array}$ & Date & Flight & l/Type \\
in figiure 47 & Stream and location & April & height & or \\
film
\end{tabular}

MOBILE RIVER BASIN

Black Warrior River Basin

1-2 Black Warrior River, Oliver Lock and Dam at Tuscaloosa to Interstate Highway 59 at Fosters

14

3,000

$\mathrm{B} / \mathrm{W}$

TOMBIGBEE RIVER BASIN

3-4 Tombigbee River, Cochrane to Epes

16

6,000

$\mathrm{B} / \mathrm{W}$

5-6 Noxubee River, Ala.-Miss. State line to mouth at Tombigbee River

16

6,000

$\mathrm{B} / \mathrm{W}$

7-19 Demoporis, confluence of Black Warrior and and Tambigbee Rivers

18

$6,000 \quad B / W$

ALABAMA RIVER BASIN

$20-21$

Alabama River, confluence of the Coosa and Tallapoosa Rivers to Jones Bluff Lock and Dam

16

8,500

$\mathrm{B} / \mathrm{W}$

$22-31$

Alabama River, Jones Bluff Lock and Dam to mouth of Cahaba River

$18 \quad 6,000 \quad \mathrm{~B} / \mathrm{W}$

PEARL RIVER BASIN

32 Pearl River-Burnside (SR 15), Philadelphia (SR 19), Edinburg (SR 16), Carthage (SR 35), Wiggins (SR 13)

14

6,000

$\mathrm{B} / \mathrm{W}$

$33-35$

Pearl River-Ross Barnett Reservoir to Byram

16

5,000

$\mathrm{B} / \mathrm{W}$ IR

$36-38$

Pearl River-Ross Barnett Reservoir to Byram

17

3,000

$\mathrm{B} / \mathrm{W}$ IR

$39-40$

Pearl River-Ross Barnett Reservoir to Byram

17

12,000

B/W IR

41 Pear1 River-Byram, Rosemary, Moncure, Gatesville, Hopewell (all county highways); Georgetown (SR 28); Rockport (county highway); Wanilla (Illinois Central Railroad and county highway); Monticello (U.S. 84)

$6,000 \quad \mathrm{~B} / \mathrm{W}$ IR

I/ $B / W$ denotes black and white film.

$B / W$ IR denotes black and white infrared film. 